\title{
Proposal for an Experiment to Measure Mixing, CP Violation and Rare Decays in Charm and Beauty Particle Decays at the Fermilab Collider - $\mathrm{BTeV}^{\dagger}$
}

May 2000

A. Kulyavtsev, M. Procario,,$^{\ddagger}$ J. Russ, and J. You

Carnegie Mellon University, Pittsburgh, PA 15213, USA

J. Cumalat

University of Colorado, High Energy Physics, Campus Box 390,

Boulder, CO 80309, USA

J. A. Appel, C. N. Brown, J. Butler, H. Cheung, D. Christian, G. Chiodini,

S. Cihangir, I. Gaines, P. Garbincius, L. Garren, E. E. Gottschalk,

G. Jackson, P. Kasper, P. H. Kasper, R. Kutschke, S. W. Kwan, P. Lebrun,

P. McBride, L. Stutte, and J. Yarba

Fermilab, PO Box 500, Batavia, IL 60510, USA

P. Avery, and M. Lohner

University of Florida, Gainesville, FL 32611, USA

R. A. Burnstein, D. M. Kaplan, L. M. Lederman, H. A. Rubin, and C. White

Illinois Institute of Technology, Chicago, IL 60616, USA

D. Kim, M. Selen, and J. Wiss

University of Illinois at Urbana-Champaign, High Energy Physics,

441 Loomis Lab. of Physics, 1110 W. Green St., Urbana, IL 61801-3080, USA

R. W. Gardner and D. R. Rust

Physics Department, Indiana University

Bloomington, Indiana, 47405, USA

E. Casimiro, D. Menasce, L. Moroni, D. Pedrini, and S. Sala

INFN and University of Milano, Italy

G. Boca, G. Liguori, and P. Torre

Dipartimento di Fisica Nucleare e Teorica, Universita' di Pavia and INFN,

Sez. di Pavia, Italy

A. A. Derevschikov, Y. M. Goncharenko, V. Yu. Khodyrev, A. P. Meschanin,

L. V. Nogach, K. E. Shestermanov, L. F. Soloviev, and A. N. Vasiliev

Institute of High Energy Physics (IHEP), Protvino, Moscow Region, Russia

C. Newsom

Department of Physics \& Astronomy,

The University of Iowa, Iowa City, IA 52242-1479

\footnotetext{
${ }^{\dagger}$ Spokespersons: Joel Butler and Sheldon Stone

${ }^{\ddagger}$ Currently at Department of Energy
} 
Y. Kubota, R. Poling, A. Smith and B. Speakman

University of Minnesota, High Energy Physics, Tate Laboratory of Physics,

116 Church St. S.E., Minneapolis, MN 55455, USA

T. Y. Chen

Nanjing University, Dept. of Physics, Nanjing 210008, China

V. Papavassiliou

New Mexico State University

K. Honscheid, and H. Kagan

Ohio State University,

HEP Group, Dept. of Experimental or Theoretical Physics,

Smith Lab, 174 W. 18th Ave., Columbus, OH 43210, USA

W. Selove

University of Pennsylvania, Philadelphia, PA 19104, USA

A. Lopez

University of Puerto Rico, Mayaguez, Puerto Rico

T. Coan

Southern Methodist University, Dallas, TX 75275, USA

M. Alam

State University of New York at Albany,

Dept. of Physics, Albany, NY 12222, USA

X. Q. Yu

University of Science and Technology of China, Dept. of Modern Physics,

Joint Institute for High Energy Physics, Hefei, Anhui 230027, China

M. He

Shandong University, High Energy Physics Group,

Jinan, Shandong 250100, China

S. Shapiro (emeritus)

Stanford Linear Accelerator Center, PO Box 4349, Stanford, CA 94309, USA

M. Artuso, G. Majumder, R. Mountain, T. Skwarnicki, S. Stone,

J. C. Wang, and A. Wolf

Syracuse University, 201 Physics Bldg., Syracuse, NY 13244-1130, USA

K. Cho, T. Handler and R. Mitchell

University of Tennessee, Knoxville, TN 37996-1200, USA

A. Napier

Tufts University, High Energy Physics, Science \& Technology Center,

4 Colby St., Medford, MA 02155, USA

D. D. Koetke

Valparaiso University, Neils Science Center, Valparaiso, IN 46383, USA

W. Johns, P. Sheldon, K. Stenson, and M. Webster

Vanderbilt University, Department of Physics and Astronomy,

Nashville, TN 37235, USA 
M. Sheaff

University of Wisconsin, Phenomenology Inst., Dept. of Physics, 1150 University Ave., Madison, WI 53706, USA

J. Slaughter

Yale University, High Energy Physics, 219 Prospect St., 554 JWG, Box 6666, New Haven, CT 06511, USA

S. Menary

York University, Dept. of Physics

4700 Keele St., Toronto, ON M3J 1P3, Canada 


\section{Contents}

0 Organization of the BTeV Proposal $\quad 1$

I Physics Case $\quad 2$

1 The Physics Case for BTeV 3

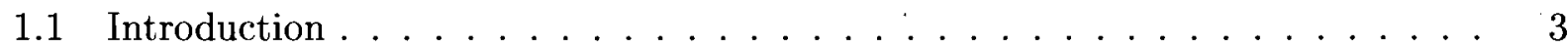

1.2 The CKM Matrix . . . . . . . . . . . . . . . . . . . . . . . . 4

1.2 .1 Unitarity Triangles . . . . . . . . . . . . . . . . . . 4

$1.2 .2 \quad$ Neutral $B$ Mixing . . . . . . . . . . . . . . . . 6

1.2.3 Current Status of the CKM Matrix . . . . . . . . . . . . . 7

1.3 CP Violation in Charged $B$ Decays . . . . . . . . . . . . . . . . . 8

$1.4 \mathrm{CP}$ Violation Formalism in Neutral $B$ decays . . . . . . . . . . . . . . 9

1.5 Techniques for Determining $\beta \ldots \ldots \ldots \ldots$

1.5.1 Removal of Two of the $\beta$ Ambiguities . . . . . . . . . . . . 14

1.6 Comment on Penguin Amplitude . . . . . . . . . . . . . . . 17

1.7 Techniques for Determining $\alpha \ldots \ldots \ldots \ldots \ldots$

1.8 Techniques for Determining $\gamma \ldots \ldots \ldots \ldots$

1.8.1 Measurement of $\gamma$ Using Time-Dependent CP violation in $B_{s}$ Decays . 20

1.8.2 Measurement of $\gamma$ Using Charged $B$ Decay Rates . . . . . . . . . . . 21

1.8.3 Measurement of $\gamma$ Using $B \rightarrow K \pi$ and $B \rightarrow \pi \pi$ Decay Rates and

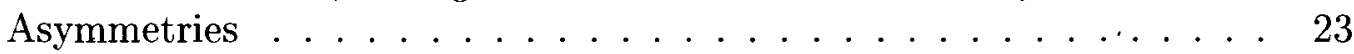

1.8.4 Measurement of $\gamma$ Using CP Asymmetries in $\boldsymbol{B}^{\circ} \rightarrow \boldsymbol{\pi}^{+} \boldsymbol{\pi}^{-}$and $\boldsymbol{B}_{\boldsymbol{s}} \boldsymbol{\rightarrow}$ $\boldsymbol{K}^{+} \boldsymbol{K}^{-} \ldots \ldots \ldots \ldots \ldots 24$

1.8.5 Opportunities with $B_{s}$ Mesons if $\Delta \Gamma$ is $\sim 10 \% \ldots \ldots 28$

1.9 Summary of CKM Tests . . . . . . . . . . . . . . . . . . . . . . . 29

1.10 Rare Decays as Probes beyond the Standard Model . . . . . . . . . . . . . . . 33

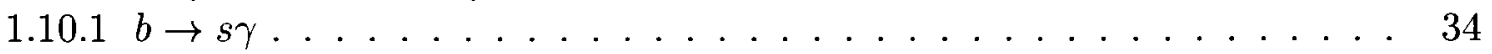

1.10 .2 The Exclusive Decays $K^{*} \gamma$ and $\rho \gamma \ldots \ldots \ldots$

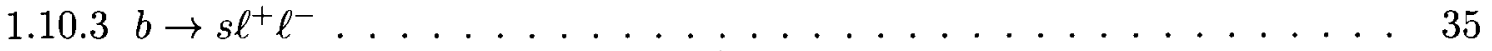

1.11 The Search for Mixing and CP Violation in Charm Decays . . . . . . . . . . 37 
II Detector Description

2 Key Design Considerations for the BTeV Detector 45

2.1 Rationale for a Forward Detector . . . . . . . . . . . . . . . 46

2.1.1 The $b \bar{b}$ Production Cross-Section . . . . . . . . . . . . . . 46

2.1.2 Characteristics of Hadronic $\boldsymbol{b}$ Production . . . . . . . . . . . 48

3 Co Experimental Area and Analysis Magnet 53

3.1 The C0 Experimental Area . . . . . . . . . . . . . . . . . 53

3.2 The $\mathrm{BTeV} / \mathrm{Co}$ Spectrometer Magnet . . . . . . . . . . . . . 54

3.3 The Muon Toroid Magnets . . . . . . . . . . . . . . . . . . . . . . 59

3.4 The Spectrometer Beampipes . . . . . . . . . . . . . . 60

4 Pixel Vertex Detector $\quad 61$

4.1 Introduction . . . . . . . . . . . . . . . . . . 61

4.2 Detector Specifications . . . . . . . . . . . . . . . . . 61

4.3 Sensor, Readout-Chips, and Their Bonding . . . . . . . . . . . . 62

4.4 Multichip Assemblies and High Density Interconnections . . . . . . . . . . . 64

4.5 Cooling Support, Shielding, and Housing . . . . . . . . . . . . . . 65

4.6 Total Material . . . . . . . . . . . . . . . . . . . . 67

4.7 Test Beam Results . . . . . . . . . . . . . . . . . . . 68

4.8 Summary . . . . . . . . . . . . . . . . . . 70

5 Forward Tracking System $\quad \cdot \quad \mathbf{7 2}$

5.1 Introduction . . . . . . . . . . . . . . . . 72

5.2 General Description . . . . . . . . . . . . . . . . . 72

5.3 Forward Straw Tracker . . . . . . . . . . . . . . . . . . . . . 74

5.3.1 Detector Description and Layout . . . . . . . . . . . . . . 74

5.3.2 Front End Electronics and Drift Time Measurement . . . . . . . . 75

5.3 .3 Technical issues . . . . . . . . . . . . . . . . . 75

5.4 Forward Silicon Tracker . . . . . . . . . . . . . . . . 76

5.4 .1 Detector Description and Layout . . . . . . . . . . . . 76

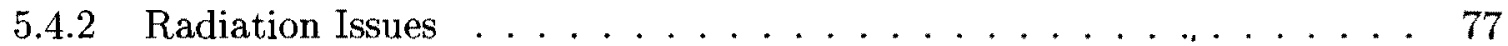

5.4 .3 Readout Electronics . . . . . . . . . . . . . . . . . 77

5.5 Forward Tracker Performance . . . . . . . . . . . . . . . . . 79

6 Charged Particle Identification System $\quad 81$

6.1 Requirements . . . . . . . . . . . . . . . . . . . . 81

6.2 RICH radiators . . . . . . . . . . . . . . . . . . . 82

6.3 RICH dimensions . . . . . . . . . . . . . . . . . . . . . 83

6.4 Photo-detectors . . . . . . . . . . . . . . . . . . . . 85

6.5 Expected resolution for the aerogel radiator . . . . . . . . . . . . 88

6.6 Expected physics performance . . . . . . . . . . . . . . . . . . . . . 89 
$\begin{array}{lll}7 & \text { Electromagnetic Calorimetry } & 93\end{array}$

7.1 Introduction . . . . . . . . . . . . . . . . 93

7.2 Choice of An Electromagnetic Calorimeter Based on $\mathrm{PbWO}_{4}$ Crystals . . . . 93

7.3 Description of the BTeV Calorimeter . . . . . . . . . . . . . . . 94

7.4 Effects of Radiation . . . . . . . . . . . . . . . 996

7.5 Expected Resolution . . . . . . . . . . . . . . . . . . . . 997 97

7.6 Examples: Photon Efficiencies in $\boldsymbol{B}^{\circ} \rightarrow \boldsymbol{K}^{*} \gamma$ and $B^{\rightarrow} \rho \pi \ldots \ldots 98$

7.7 Crystal Production . . . . . . . . . . . . . . . . . . 100

8 Muon Detector 103

8.1 General Design Considerations . . . . . . . . . . . . . . . . . 103

8.2 Baseline Muon System . . . . . . . . . . . . . . . . . . . . . . . . . . . 104

8.2 .1 Baseline Geometry . . . . . . . . . . . . . . . . . . . . . . 104

8.2 .2 Baseline Detector . . . . . . . . . . . . . . . . . . 105

8.2 .3 Front-end Electronics . . . . . . . . . . . . . . . . . . . . 106

8.3 Trigger Studies . . . . . . . . . . . . . . . . . . . 108

8.3.1 GEANT simulation description . . . . . . . . . . . . . 109

8.3.2 Properties of additional "noise" hits . . . . . . . . . . . . . 109

8.3 .3 A muon "tracking" trigger . . . . . . . . . . . . . . . . . . . 110

8.3 .4 Simplified Tracking Trigger . . . . . . . . . . . . . . . 113

8.3 .5 Trigger Summary . . . . . . . . . . . . . . . . . . . 113

9 The BTeV Trigger . 116

9.1 Goals and Requirements for the BTeV Trigger System . . . . . . . . . 116

9.1.1 Rejection Requirement . . . . . . . . . . . . . . . . . . 117

9.1 .2 Efficiency Requirement . . . . . . . . . . . . . . . . . . 117

9.1.3 Rate and Deadtime Requirement . . . . . . . . . . . . . . . . 117

$9.2 \mathrm{BTeV}$ Trigger Strategy . . . . . . . . . . . . . . . . . . . . . 117

9.3 Trigger System Implementation . . . . . . . . . . . . . . . . . . . 120

9.3 .1 Trigger System Overview . . . . . . . . . . . . . . . . . . . . . 120

9.3 .2 Level 1 Vertex Trigger . . . . . . . . . . . . . . . . . . . . . 121

9.4 Levels $2 / 3 \ldots \ldots \ldots \ldots$. . . . . . . . . . . . . . . . . . . 132

10 Data Acquisition System 135

10.1 Data Acquisition . . . . . . . . . . . . . . . . . 135

10.1.1 Detector Multiplexing . . . . . . . . . . . . . . . . . 136

10.1.2 Detector Data Links . . . . . . . . . . . . . . . . 136

10.1 .3 L1 and Switch Input Buffers _. . . . . . . . . . . . . 137

10.1.4 Ring Buffer Interconnects . . . . . . . . . . . . . . . 137

10.1.5 Staged Readout . . . . . . . . . . . . . . . . . . . . . . . . 137

10.1.6 Data Switch . . . . . . . . . . . . . . . . . . . . 138

10.1 .7 Control Network . . . . . . . . . . . . . . . . 138

10.1 .8 Switch Output Buffers . . . . . . . . . . . . . . . . . 138 
10.1 .9 Data Logging . . . . . . . . . . . . . . . . . . . . . . 139

10.1.10 Detector Control Links . . . . . . . . . . . . . . . . . . 139

10.1.11 Diagnostics . . . . . . . . . . . . . . . . . . . . . . 139

10.1.12 General Operation . . . . . . . . . . . . . . . . . . . . 139

10.1.13 Component Placement . . . . . . . . . . . . . . . . . 140

10.2 Event Supervisor and Monitor . . . . . . . . . . . . . . . 140

10.3 Level $2 / 3$ Trigger . . . . . . . . . . . . . . . . . . . . . . 141

10.4 Data Logging and Online Event Analysis . . . . . . . . . . . . . 143

10.5 Front End Electronics . . . . . . . . . . . . . . . . . . . 143

10.5.1 Infrastructure and Other Support Systems . . . . . . . . . . . 143

10.5.2 Software for Module and Subsystem Development and the Online Data Acquisition and Readout System . . . . . . . . . . 148

11 Plan for Deployment in C0 $\quad 149$

11.1 Detector Installation . . . . . . . . . . . . . . . . . . . . 149

11.2 Staging . . . . . . . . . . . . . . . . . 150

11.3 Commissioning Plan and Goals . . . . . . . . . . . . . . 150

III Physics Simulations $\quad 152$

12 Description of Simulation and Analysis Tools 153

12.1 BTeVGeant . . . . . . . . . . . . . . . . . . . . . . 154

12.1.1 Design Considerations for BTeVGeant . . . . . . . . . . 155

12.1.2 The BTeV Detector Geometry in BTeVGeant . . . . . . . . . . 156

12.1.3 The Magnetic Field Model . . . . . . . . . . . . . . 158

12.1.4 Tracking Accuracy . . . . . . . . . . . . . . . . 158

12.1 .5 Calorimetry Cuts . . . . . . . . . . . . . . 158

12.1 .6 Hits and Digitizations . . . . . . . . . . . . . . . . . 169

12.1 .7 The $1 /$ O layer . . . . . . . . . . . . . . . . . . . . 169

12.1.8 Track Pattern Recognition and The Kalman Filter . . . . . . . . . 170

12.1 .9 Optimization for Production . . . . . . . . . . . . . . 171

12.1.10 BTeVGeant Production Úsing a PC Farm . . . . . . . . . . . . . 172

12.2 MCFast Simulation Package . . . . . . . . . . . . . . . . . 173

12.2.1 Charged Particle Tracking in MCFast . . . . . . . . . . . 174

12.2 .2 Vertexing Package . . . . . . . . . . . . . . . . . 175

12.2.3 Simulation of Secondary Hadronic Interactions $\ldots \ldots \ldots . . . .177$

12.2.4 The MCFast showering subpackage . . . . . . . . . . . . 177

12.2.5 BTeV Geometry Description for MCFast . . . . . . . . . . . . . 181

12.3 Physics Event Generators . . . . . . . . . . . . . . . . . . . . . . 183

12.4 ECAL Cluster Finder Package . . . . . . . . . . . . . . . . . . 184

12.4.1 Event Information from the ECAL Detector . . . . . . . . . . . 184

12.4.2 Definition of the Cluster Finder Output Data . . . . . . . . . . . 185 
12.4.3 General Algorithm . . . . . . . . . . . . . . . . . . 186

12.4.4 Important Corrections . . . . . . . . . . . . . . . . . . . 188

12.4.5 Implementation and Performance . . . . . . . . . . . . . . . . . 189

12.4.6 Electron Identification using the ECAL Cluster Finder . . . . . . . . . 190

13 Comparison of MCFast and BTeVGeant for the Calorimeter and Tracker 195

13.1 MCFast vs. BTeVGeant showering . . . . . . . . . . . . 195

13.2 MCFast vs. GEANT tracking . . . . . . . . . . . . . . . . . 200

13.2 .1 Introduction . . . . . . . . . . . . . . . 200

13.2 .2 Occupancies. . . . . . . . . . . . . . . . . . . . 201

13.2.3 Efficiency and Resolution for $B^{0} \rightarrow \pi^{+} \pi^{-} \ldots \ldots \ldots$. . . . . 204

14 Trigger Simulations for Levels 1 and 2 and a brief discussion of Level 3207

14.1 Introduction . . . . . . . . . . . . . . . . . . . . 207

14.2 Level 1 Trigger . . . . . . . . . . . . . . . . . . . . 208

14.2.1 Level 1 Trigger Studies . . . . . . . . . . . . . . . . . . . 211

14.2 .2 Pixel Noise and Inefficiency Studies . . . . . . . . . . . . . . 213

14.2 .3 Multiple-Interaction Studies . . . . . . . . . . . . . . 217

14.3 Status of the Level 2 Trigger . . . . . . . . . . . . . 218

14.3.1 Brief description of the Level 2 Algorithm . . . . . . . . . . 218

14.3.2 Timing . . . . . . . . . . . . . . . . . . . . . 219

14.3.3 Signal efficiency and background rejection . . . . . . . . . . 220

14.4 Level 3 Trigger Strategy . . . . . . . . . . . . . . . . . . 222

15 Flavor Tagging $\quad \mathbf{2 2 5}$

15.1 Introduction . . . . . . . . . . . . . . . . . . . 225

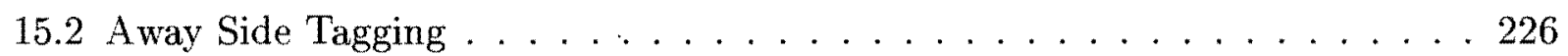

15.2.1 Lepton Tagging . . . . . . . . . . . . . . . . . . . . . . . 226

15.2 .2 Kaon Tagging. . . . . . . . . . . . . . . . . . 226

15.2.3 Vertex Charge Tagging . . . . . . . . . . . . . . . . 227

15.2.4 Combining Away Side Tagging Methods . . . . . . . . . . . . 227

15.3 Same Side Tagging . . . . . . . . . . . . . . . . . . . . . . . . . . 228

15.4 Summary of Tagging . . . . . . . . . . . . . . . . . . 228

16 Specific Physics Final States $\quad 230$

16.1 A study of $B^{o} \rightarrow \pi^{+} \pi^{-}$(and $\left.B_{s} \rightarrow K^{+} K^{-}\right) \ldots \ldots \ldots 231$

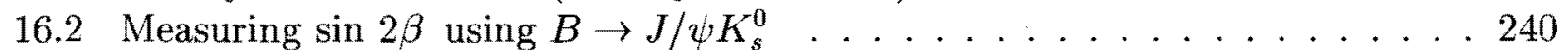

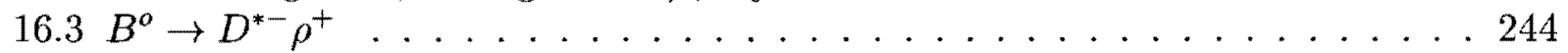

16.3.1 Introduction . . . . . . . . . . . . . . . 244

16.3 .2 Analysis . . . . . . . . . . . . . . . . . . . . . . 244

16.3.3 Background from Charm . . . . . . . . . . . . . . . 251

16.3.4 Effect of Multiple Interactions . . . . . . . . . . . . . . 251

16.3.5 The CLEO result . . . . . . . . . . . . . . . . . . 254 
16.3.6 Comparison and Summary $\ldots \ldots \ldots \ldots \ldots . \ldots \ldots$

16.3.7 Implications for Measurement of $\alpha \ldots \ldots \ldots \ldots \ldots \ldots$

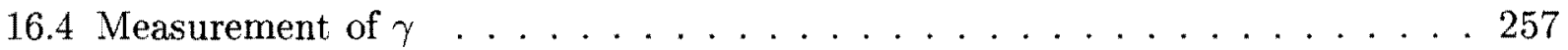

16.4.1 CP Violation in $B_{s} \rightarrow D_{s} K^{-} \ldots \ldots \ldots \ldots \ldots \ldots \ldots \ldots \ldots \ldots$

16.4.2 CP Violation in $B^{ \pm} \rightarrow D^{o} K^{ \pm} \ldots \ldots \ldots \ldots \ldots \ldots \ldots$

16.4.3 CP Violation in $B \rightarrow K \pi \ldots \ldots \ldots \ldots . \ldots \ldots 272$

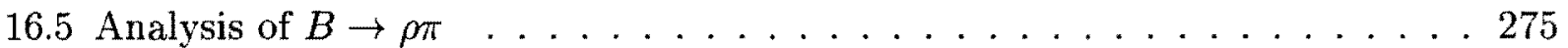

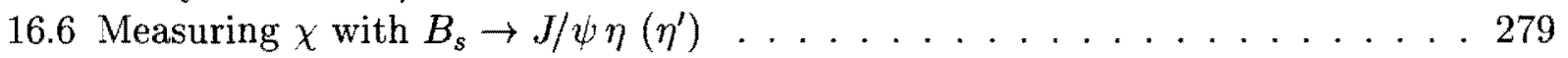

16.6.1 Introduction . . . . . . . . . . . . . 279

16.6.2 Signal Selection . . . . . . . . . . . . . . . . . . 279

16.6.3 Background Estimation . . . . . . . . . . . . . . 282

16.6.4 Sensitivity to $\sin (2 \chi) \ldots \ldots \ldots \ldots \ldots \ldots 284$

16.7 Measurement of $B_{s}$ Mixing Using $B_{s} \rightarrow D_{s}^{-} \pi^{+}$and $B_{s} \rightarrow J / \psi \bar{K}^{* 0} \ldots \ldots 285$

16.7.1 Yields, Resolutions and Signal-to-Background Ratios . . . . . . . . 285

16.7.2 Computation of the $x_{s}$ Reach . . . . . . . . . . . 289

16.8 Flavor-changing Rare $B$ Meson Decays . . . . . . . . . . . . . . 293

16.8.1 The Physics Significance of Rare $B$ Meson Decays . . . . . . . . 293

16.8.2 Exclusive Channel $B^{0} \rightarrow K^{* 0} \mu^{+} \mu^{-} \ldots \ldots \ldots \ldots . \ldots . \ldots 293$

16.8.3 Exclusive Channel $B^{+} \rightarrow K^{+} \mu^{+} \mu^{-} \ldots \ldots \ldots \ldots$. . . . . . 299

16.8.4 Inclusive $\left(B_{d}, B_{u}\right) \rightarrow X_{s} \mu^{+} \mu^{-} \ldots \ldots \ldots \ldots . \ldots \ldots 1$

16.9 Consequences of Degraded Detector Performance . . . . . . . . . . . . 303

16.9.1 Introduction . . . . . . . . . . . . . . . . . . . 303

16.9.2 Trigger Performance . . . . . . . . . . . . . . . . . . . 303

16.9.3 Decay Time Resolution . . . . . . . . . . . . . . . . . . 303

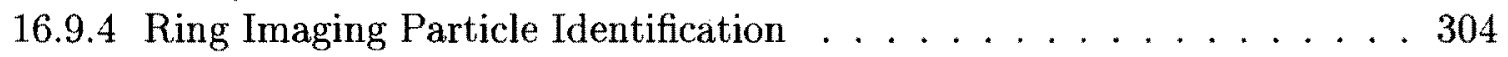

16.9.5 Electromagnetic Calorimeter . . . . . . . . . . . . . . 305

$\begin{array}{ll}\text { IV Summary of Physics reach } & 308\end{array}$

17 Summary of Physics Reach and Comparisons With Other Experiments 309

17.1 Sensitivities to CP Violating Angles . . . . . . . . . . . . . . . . 309

17.2 Sensitivity to $B_{s}$ Mixing . . . . . . . . . . . . . . . 310

17.3 Reach in Rare Decays . . . . . . . . . . . . . . . . . . . . . . . 310

17.4 Comparison with CDF, D0, CMS, and ATLAS . . . . . . . . 311

17.5 Comparison with $e^{+} e^{-}$B Factories . . . . . . . . . . . 311

17.6 Comparison with LHC-b . . . . . . . . . . . . . . . . 312

17.6 .1 General Comparisons . . . . . . . . . . . . . . . 312

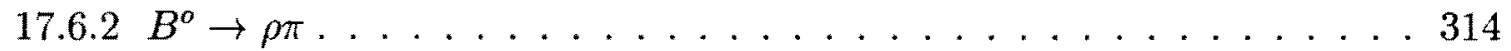

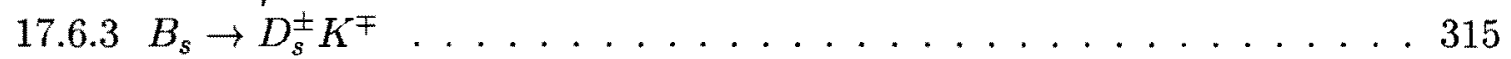

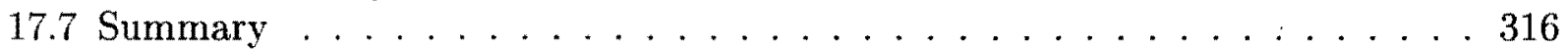


$\begin{array}{llr}\text { V Cost Summary } & 319\end{array}$

18 Cost Summary $\quad 320$

18.1 Methodology . . . . . . . . . . . . . . . . . . . . . . 320

18.1.1 Documentation . . . . . . . . . . . . . . . . 320

18.1 .2 Inclusivity . . . . . . . . . . . . . . . . . 320

18.1.3 Labor Costs . . . . . . . . . . . . . . . . . . . . . . 321

18.1.4 Handling of Inflation and Deflation . . . . . . . . . . . . 321

18.1.5 Contingency . . . . . . . . . . . . . . . . . 322

18.2 The Detector Cost Estimate . . . . . . . . . . . . . . . . . . . . . . 322

18.2.1 Vertex and Toroidal Magnets and Beam Pipe . . . . . . . . . . . 322

18.2 .2 Pixel Detector . . . . . . . . . . . . . . . . . . . 323

18.2 .3 RICH Detector . . . . . . . . . . . . . . . . . . . . . . 323

18.2.4 Electromagnetic Calorimeter Detector . . . . . . . . . . . . . . 323

18.2.5 Muon Detector . . . . . . . . . . . . . . . . . . . . . . . 324

18.2.6 Forward Tracker Straw Detector . . . . . . . . . . . . . . . . 324

18.2.7 Forward Tracker Silicon Microstrip Detector . . . . . . . . . . . . 324

18.2 .8 Unassigned . . . . . . . . . . . . . . . . . . . . . 325

18.2.9 Level 1 and Global Triggers . . . . . . . . . . . . . . . 325

18.2.10 Data Acquisition Electronics and Links . . . . . . . . . . . 325

18.2.11 Level 2/3 Processor Array . . . . . . . . . . . . . . . . . . . 325

18.2.12 Analysis and Simulation Facilities . . . . . . . . . . . 326

18.2.13 Controls/Monitoring and Timing . . . . . . . . . . 326

18.2.14 Control Room Electronics and Equipment Infrastructure . . . . . . . . 326

18.2.15 Control, Monitoring and Event Readout Software . . . . . . . . . 326

18.2.16 Algorithm and Other Software and Integration Facilities . . . . . . 327

18.2.17 Test Stands and Test Equipment . . . . . . . . . . . . . . 327

18.2.18 Unassigned . . . . . . . . . . . . . . . . . 327

18.2.19 System Installation, Integration Testing and Commissioning . . . . . 327

$18.2 .20 \mathrm{BTeV}$ Project Management . . . . . . . . . . . . . . 328

18.3 The Offline Computing Cost Estimate . . . . . . . . . . . . . . . 328

18.3.1 Analysis and Simulation Facilities . . . . . . . . . . . . . . . 328

18.3.2 Algorithm and Other Software and Integration . . . . . . . . 328

18.4 Cost Summaries . . . . . . . . . . . . . . . . . . . . . . . . . . . 329

18.4.1 Detector Cost Summary . . . . . . . . . . . . . . . . . . . 329

18.4.2 Computing Cost Summary . . . . . . . . . . . . . . . 329

A Detector Design Details and R\&D Plans 331

A.1 Pixel Detector . . . . . . . . . . . . . . . . . . . . . . . . 332

A.1.1 Sensor Design and Simulation . . . . . . . . . . . . . 333

A.1.2 Test Beam Results . . . . . . . . . . . . . . . . . . 346

A.1.3 Electromagnetic Pickup Study . . . . . . . . . . . . . 360 
A.1.4 FPIX2 Radiation Damage Tests ... . . . . . . . . . . 363

A.2 RICH Design and R\&D . . . . . . . . . . . . . . . . . . 367

A.2.1 Hybrid Photodiode R\&D . . . . . . . . . . . . . . . 367

A.2.2 HPD High Voltage System . . . . . . . . . . . . . . . 368

A.2.3 Photon Detector Mechanical Design . . . . . . . . . . . . . 368

A.2.4 HPD Readout Electronics . . . . . . . . . . . . . . . . . 369

A.2.5 Silica Aerogel Radiator . . . . . . . . . . . . . . . . . . 372

A.2.6 Alternative Photon Detector System: MAPMTs . . . . . . . . . 378

A.2.7 RICH Simulation and Reconstruction Software . . . . . . . . . . 380

A.3 Electromagnetic Calorimetry . . . . . . . . . . . . . . . . . . 383

A.3.1 Introduction . . . . . . . . . . . . . . 383

A.3.2 Radiation Tolerance and Radiation Levels . . . . . . . . . . . . . 383

A.3.3 Design Parameters . . . . . . . . . . . . . . . . . . . . . . . 390

A.3.4 Mechanical Systems . . . . . . . . . . . . . . . . . 395

A.3.5 Crystal Acquisition . . . . . . . . . . . . . . . . . . 396

A.3.6 BTeV Electromagnetic Calorimeter R\&D Program . . . . . . . . . . 399

A.4 Muon Detector Design, R\&D and Test Beam Results . . . . . . . . . . . . 404

A.4.1 Detector Design and Fabrication . . . . . . . . . . . . . . . 404

A.4.2 Front End Electronics . . . . . . . . . . . . . . . . . . . 407

A.4.3 Future prototyping . . . . . . . . . . . . . . . . . . 412

A.5 Level 1 Trigger Timing Studies . . . . . . . . . . . . . . . . . . . . 414

A.5.1 Introduction . . . . . . . . . . . . . . . . 414

A.5.2 Timing Studies . . . . . . . . . . . . . . . . 415

B Roadmap: Answers to PAC Questions $4 \mathbf{4 1 8}$

B.1 Physics Studies . . . . . . . . . . . . . . . . . . . . . . . . . . . . . 419

B.1.1 $B \rightarrow \rho \pi$ (BTeVGeant study) . . . . . . . . . . . . . . 419

B.1.2 $B_{s} \rightarrow D_{s} K$ (BTeVGeant study) . . . . . . . . . . 420

B.1.3 $B \rightarrow D^{*} \rho$ (BTeVGeant study) . . . . . . . . . . . . 420

B.1.4 $B \rightarrow J / \psi K_{s}$ (MCFast study) . . . . . . . . . . . . 421

B.1.5 $B^{-} \rightarrow \overline{D^{0}} K^{-}$(MCFast study) . . . . . . . . . . . 421

B.1.6 $x_{s}$ from $B_{s} \rightarrow D_{s} \pi$ (BTeVGeant study) $\ldots \ldots \ldots \ldots \ldots \ldots$

B.2 Trigger/Detector Studies . . . . . . . . . . . . . . . . . . 422

B.2.1 Vertex Trigger . . . . . . . . . . . . . . . . . . . 422

B.2.2 Pixel R\&D . . . . . . . . . . . . . . . . . . . . . 423

B.2.3 EMCal . . . . . . . . . . . . . . . . . . . . 425

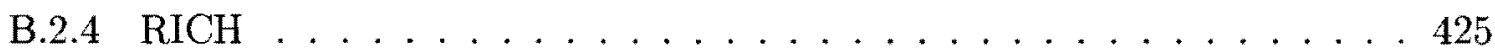

B.2.5 Muon Trigger . . . . . . . . . . . . . . . . . . . . 425

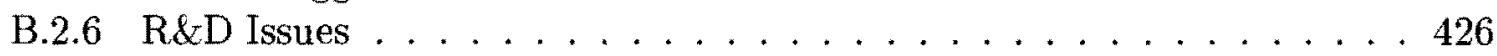

B.2.7 Cost/Performance Trade-offs . . . . . . . . . . . . . . . 426

B.2.8 Degradation of Performance and Physics Reach . . . . . . . . . . . 427

B.3 Comparisons to Other Experiments . . . . . . . . . . . . . . . 427 
B.4 Staging and Commissioning Scenarios . . . . . . . . . . . 427 


\section{Chapter 0}

\section{Organization of the BTeV Proposal}

This proposal consists of five parts and two appendices. The first part provides a detailed physics justification for the $\mathrm{BTeV}$ experiment. The second part presents the considerations that drive the detector design, followed by a description of the detector itself. The third part summarizes our simulation results which demonstrate that the design does enable us to achieve our physics goals. The fourth part compares BTeV's physics reach to that of other experiments which will be active in B physics in the same time period. The fifth part gives a very brief, high level summary of the cost estimate for $\mathrm{BTeV}$.

Appendix $\mathrm{A}$ has additional technical details about many of the detector subsystems and $R \& D$ plans; it is intended to be read primarily by experts in each area. Appendix B contains a roadmap which describes the location in the proposal of the answers to questions posed to the BTeV collaboration by the Fermilab Program Advisory Committee in June of 1999.

Two additional documents accompany this proposal. The first is an executive summary that delineates all the basic elements of the proposal and the cost estimate. The second, a really large collection of material, is the $\mathrm{BTeV}$ cost estimate in WBS format. 


\section{Part I}

\section{Physics Case}




\section{Chapter 1}

\section{The Physics Case for BTeV}

\section{$1.1 \quad$ Introduction}

Experimental particle physics seeks answers to many questions about nature. Some central issues include:

- How are fermion masses generated?

- Why is there a family structure?

- Why are there three families rather than one?

The Standard Model [1] describes these phenomena quite well. Thus far all predictions are consistent with experiment. Symmetries and symmetry violations are crucially important physics phenomena. Weak decays are known to violate parity, $\mathrm{P}$, and the product of chargeconjugation and parity, CP [2]. That the three family structure allows CP violation to occur naturally via quark mixing is an important clue that we are on the right track. However, the Standard Model is more of a description than an explanation.

The magnitude of $\mathrm{CP}$ violation is intimately tied to the question of "baryogenesis," or how did the Universe get rid of the anti-baryons. A possible solution was first proposed by Sakharov [3]. It requires three ingredients: CP violation, lack of thermal equilibrium at some time and baryon non-conservation. The Standard Model provides the third component via quantum corrections to anomaly diagrams. Inflation can provide the lack of thermal equilibrium. Although the Standard Model incorporates CP violation, it is believed that the amount is far too small. Of course we may find that the Standard Model explanation is incorrect.

We describe here a program of measurements that need to be performed in order to test whether the Standard Model indeed describes quark mixing and CP violation. There are many important experimental measurements to be made. We will describe the reasons why these measurements are crucial. We will also point out the important tests that probe for physics beyond the Standard Model. 
There are many other interesting and important physics topics concerning issues of heavy quark production, the phenomenology of weak decays, etc., that we do not discuss here. It should be kept in mind that other areas of interesting physics can be addressed by BTeV.

\subsection{The CKM Matrix}

The physical point-like states of nature that have both strong and electroweak interactions, the quarks, are mixtures of base states described by the Cabibbo-Kobayashi-Maskawa (CKM) matrix [4],

$$
\left(\begin{array}{c}
d^{\prime} \\
s^{\prime} \\
b^{\prime}
\end{array}\right)=\left(\begin{array}{lll}
V_{u d} & V_{u s} & V_{u b} \\
V_{c d} & V_{c s} & V_{c b} \\
V_{t d} & V_{t s} & V_{t b}
\end{array}\right)\left(\begin{array}{c}
d \\
s \\
b
\end{array}\right)
$$

The unprimed states are the mass eigenstates, while the primed states denote the weak eigenstates. The $V_{i j}$ 's are complex numbers that can be represented by four independent real quantities. These numbers are fundamental constants of nature that need to be determined from experiment, like any other fundamental constant such as $\alpha$ or $G$. In the Wolfenstein approximation the matrix is written as [5]

$$
V_{C K M}=\left(\begin{array}{ccc}
1-\lambda^{2} / 2 & \lambda & A \lambda^{3}\left(\rho-i \eta\left(1-\lambda^{2} / 2\right)\right) \\
-\lambda & 1-\lambda^{2} / 2-i \eta A^{2} \lambda^{4} & A \lambda^{2}\left(1+i \eta \lambda^{2}\right) \\
A \lambda^{3}(1-\rho-i \eta) & -A \lambda^{2} & 1
\end{array}\right)
$$

This expression is accurate to order $\lambda^{3}$ in the real part and $\lambda^{5}$ in the imaginary part. It is necessary to express the matrix to this order to have a complete formulation of the physics we wish to pursue. The constants $\lambda$ and $A$ have been measured using semileptonic $s$ and $b$ decays $[6] ; \lambda \approx 0.22$ and $A \approx 0.8$. The phase $\eta$ allows for $C P$ violation. There are experimental constraints on $\rho$ and $\eta$ that will be discussed below.

\subsubsection{Unitarity Triangles}

The unitarity of the CKM matrix ${ }^{1}$ allows us to construct six relationships. These equations may be thought of as triangles in the complex plane. They are shown in Fig. 1.1

In the bd triangle, the one usually considered, the angles are all thought to be relatively large. It is described by:

$$
V_{u b} V_{u d}^{*}+V_{c b} V_{c d}^{*}+V_{t b} V_{t d}^{*}=0
$$

To a good approximation

$$
\left|V_{u d}^{*}\right| \approx\left|V_{t b}\right| \approx 1
$$

which implies

$$
\frac{V_{u b}}{V_{c b}}+\frac{V_{t d}^{*}}{V_{c b}}+V_{c d}^{*}=0
$$

\footnotetext{
${ }^{1}$ Unitarity implies that any pair of rows or columns are orthogonal.
} 

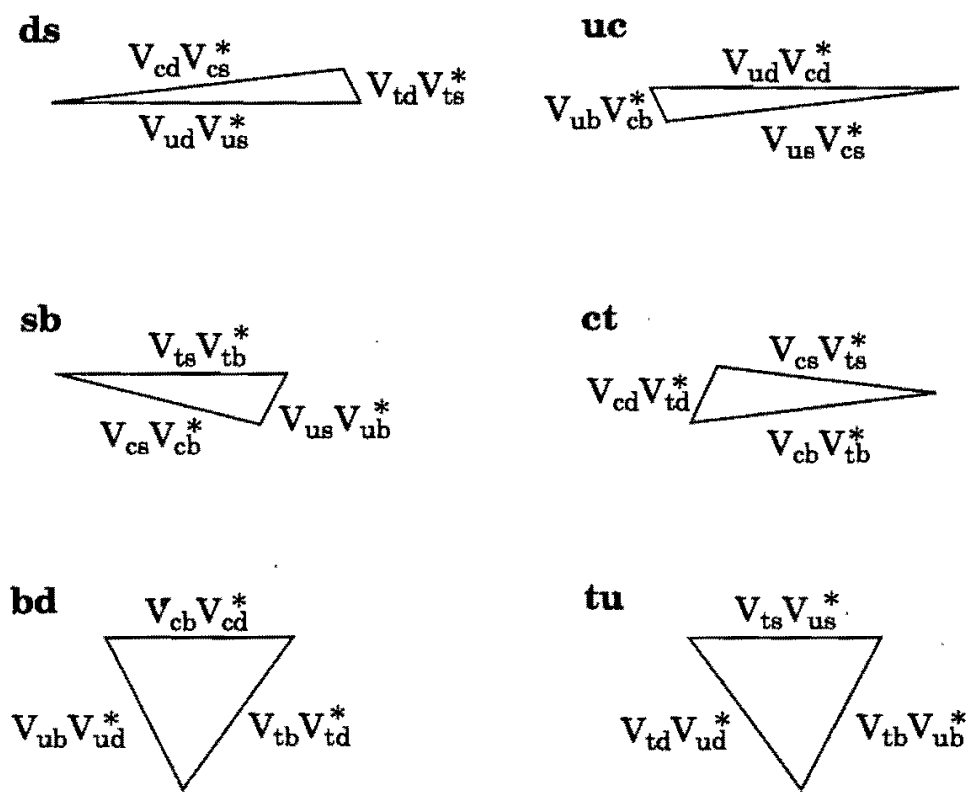

Figure 1.1: The six CKM triangles. The bold labels, i.e ds refer to the rows or columns used in the unitarity relationship.

Since $V_{c d}^{*}=\lambda$, we can define a triangle with sides

$$
\begin{aligned}
& \left|\frac{V_{t d}}{A \lambda^{3}}\right|=\sqrt{(\rho-1)^{2}+\eta^{2}}=\frac{1}{\lambda}\left|\frac{V_{t d}}{V_{t s}}\right| \\
& \left|\frac{V_{u b}}{A \lambda^{3}}\right|=\sqrt{\rho^{2}+\eta^{2}}=\frac{1}{\lambda}\left|\frac{V_{u b}}{V_{c b}}\right| .
\end{aligned}
$$

This CKM triangle is depicted in Fig. 1.2.

We know two sides already: the base is defined as unity and the left side is determined within a relatively large error by the measurements of $\left|V_{u b} / V_{c b}\right|[7]$. The right side can be determined using mixing measurements in the neutral $B$ system. However, there is a very large error due to the uncertainty in $f_{B}$, the $B$-meson decay constant. Later we will discuss other measurements that can determine this side. The figure also shows the angles $\alpha, \beta$, and $\gamma$. These angles can be determined by measuring CP violation in the $B$ system.

Aleksan, Kayser and London [8] have shown that the CKM matrix can be expressed in terms of four independent phases. These are taken as:

$$
\begin{array}{ll}
\beta=\arg \left(-\frac{V_{t b} V_{t d}^{*}}{V_{c b} V_{c d}^{*}}\right), & \gamma=\arg \left(-\frac{V_{u b}^{*} V_{u d}}{V_{c b}^{*} V_{c d}}\right), \\
\chi=\arg \left(-\frac{V_{c s}^{*} V_{c b}}{V_{t s}^{*} V_{t b}}\right), & \chi^{\prime}=\arg \left(-\frac{V_{u d}^{*} V_{u s}}{V_{c d}^{*} V_{c s}}\right),
\end{array}
$$




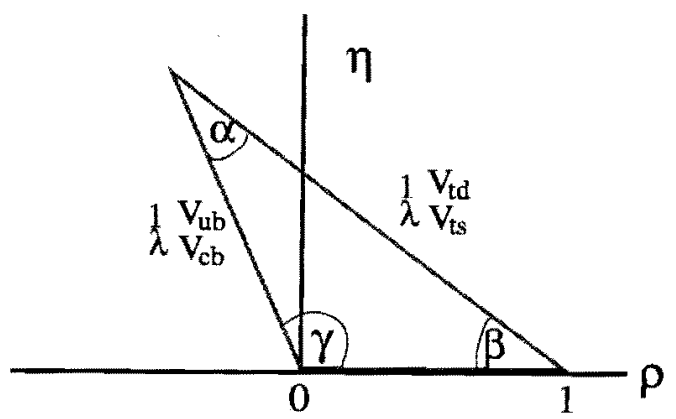

Figure 1.2: The CKM triangle shown in the $\rho-\eta$ plane. The left side is determined by $\left|V_{u b} / V_{c b}\right|$ and the right side can be determined using mixing in the neutral $B$ system. The angles can be found by making measurements of $\mathrm{CP}$ violation in $B$ decays.

where we have changed the confusing notation of Aleksan et al from $\epsilon, \epsilon^{\prime}$ to $\chi$ and $\chi^{\prime}$. We will address the usefulness of this parameterization in section 1.9.

\subsubsection{Neutral $B$ Mixing}

Neutral $B$ mesons can transform to their anti-particles before they decay. The diagrams for $B_{d}$ mixing are shown in Fig. 1.3. (The diagrams for $B_{s}$ mixing are similar with $s$ quarks replacing $d$ quarks.) Although $u, c$ and $t$ quark exchanges are all shown, the $t$ quark plays a dominant role, mainly due to its mass, since the amplitude of this process grows with the mass of the exchanged fermion.

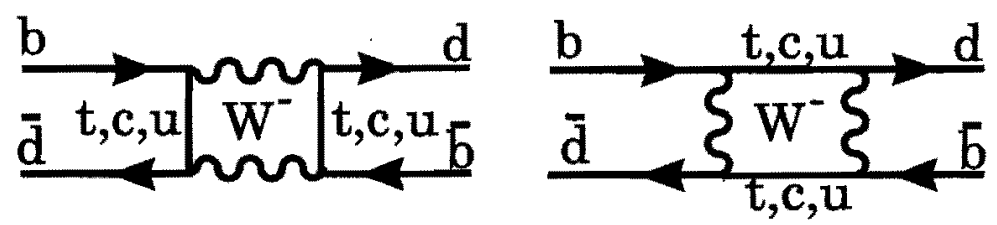

Figure 1.3: The two diagrams for $B_{d}$ mixing.

The probability of $B^{\circ}$ mixing is given by [9]

$$
\begin{gathered}
r=\frac{N\left(\bar{B}^{\circ}\right)}{N\left(B^{o}\right)}=\frac{x^{2}}{2+x^{2}}, \text { where } \\
x \equiv \frac{\Delta m}{\Gamma}=\frac{G_{F}^{2}}{6 \pi^{2}} B_{B} f_{B}^{2} m_{B} \tau_{B}\left|V_{t b}^{*} V_{t d}\right|^{2} m_{t}^{2} F\left(\frac{m_{t}^{2}}{M_{W}^{2}}\right) \eta_{Q C D}
\end{gathered}
$$

where $B_{B} f_{B}^{2}$ is related to the probability of the $d$ and $\bar{b}$ quarks forming a hadron and must be estimated theoretically; $F$ is a known function which increases approximately as $m_{t}^{2}$, and $\eta_{Q C D}$ is a $Q C D$ correction, with a value $\approx 0.8[10]$. By far the largest uncertainty arises 
from the unknown decay constant, $f_{B}$. This number gives the coupling between the $B$ and the $W^{-}$. It could in principle be determined by finding the decay rate of $B^{+} \rightarrow \mu^{+} \nu$ or $B^{+} \rightarrow \tau^{+} \nu$, both of which are very difficult to measure. Since

$$
\left|V_{t b}^{*} V_{t d}\right|^{2} \propto|(1-\rho-i \eta)|^{2}=(\rho-1)^{2}+\eta^{2}
$$

measuring mixing gives a circle centered at $(1,0)$ in the $\rho-\eta$ plane. The best recent mixing measurements have come from a variety of sources [11], yielding a value (for $B_{d}$ ) of $\Delta m=$ $(0.464 \pm 0.018) \times 10^{12} \hbar s^{-1}$.

The right-hand side of the triangle can be determined by measuring $B_{s}$ mixing using the ratio

$$
\frac{\Delta m_{s}}{\Delta m_{d}}=\left(\frac{B_{s}}{B}\right)\left(\frac{f_{B_{s}}}{f_{B}}\right)^{2}\left(\frac{m_{B_{s}}}{m_{B}}\right)\left|\frac{V_{t s}}{V_{t d}}\right|^{2},
$$

where

$$
\left|\frac{V_{t d}}{V_{t s}}\right|^{2}=\lambda^{2}\left[(\rho-1)^{2}+\eta^{2}\right] .
$$

The large uncertainty in using the $B_{d}$ mixing measurement to constrain $\rho$ and $\eta$ is largely removed since many sources of theoretical uncertainty cancel in the ratio of the first two factors in equation (1.13), which is believed to be known to $\pm 20 \%$ [12].

\subsubsection{Current Status of the CKM Matrix}

Measurements of $\left|V_{u b} / V_{c b}\right|$ probe $\rho^{2}+\eta^{2}$ and thus form a circular constraint in the $\rho-\eta$ plane centered at $(0,0)$. Similarly, mixing measurements form a circular constraint centered on $(1,0)$.

The fact that the CKM matrix is complex allows CP violation. There is a constraint on $\rho$ and $\eta$ given by the $K_{L}^{o}$ CP violation measurement $(\epsilon)$, given by [13]

$$
\eta\left[(1-\rho) A^{2}(1.4 \pm 0.2)+0.35\right] A^{2} \frac{B_{K}}{0.75}=(0.30 \pm 0.06)
$$

where the errors arise from uncertainties on $m_{t}$ and $m_{c}$.

The constraints on $\rho$ versus $\eta$ from the $V_{u b} / V_{c b}$ measurement, $\epsilon$ and $B$ mixing are shown in Fig. 1.4. The bands represent $1 \sigma$ errors, for the measurements, and a $95 \%$ confidence level upper limit on $B_{s}$ mixing. The width of the $B_{d}$ mixing band is caused mainly by the uncertainty on $f_{B}$, taken here as $240^{\prime}>f_{B}>160 \mathrm{MeV}$. Other parameters include $\left|V_{c b}\right|=0.0381 \pm 0.0021,\left|V_{u b} / V_{c b}\right|=0.085 \pm 0.019[14]$, limit on $\Delta m_{s}>12.4 \mathrm{ps}^{-1}$, and the ratio $f_{B_{s}} \sqrt{B_{B_{s}}} / f_{B_{d}} \sqrt{B_{B_{d}}} \leq 1.25[15]$.

The width of the $\epsilon$ band is caused by errors in $A, m_{t}, m_{c}$ and $B_{K}$. Here $B_{K}$ is taken as $0.80 \pm 0.15$ according to Buras [16].

Recent measurements of $\epsilon^{\prime} / \epsilon$ determine $\eta$ directly [2]. However, the theoretical errors are so large that all that can be said is that the measurement is consistent with the allowed region. We caution the reader that this plot is only a guide, since the measured quantities 


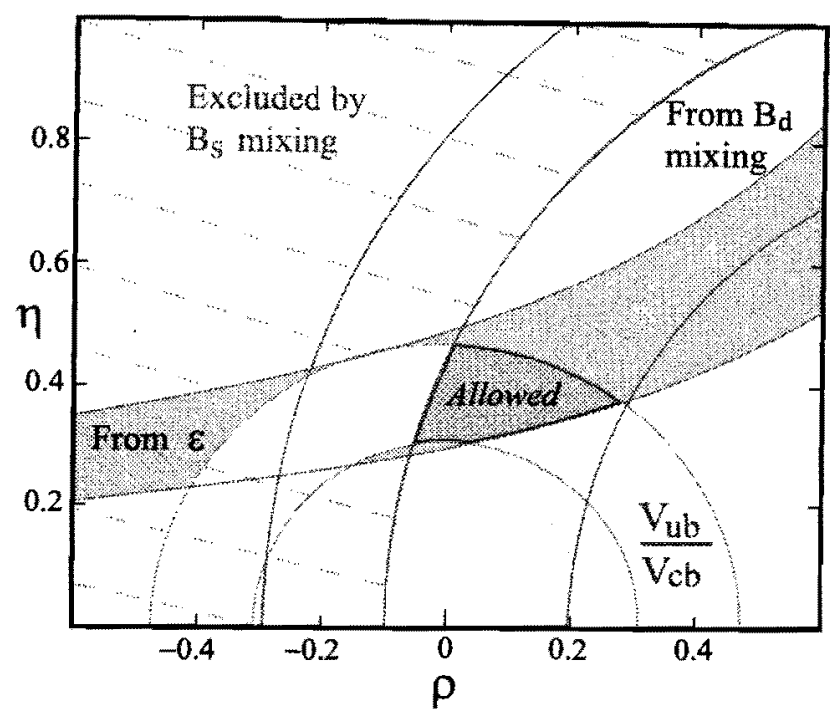

Figure 1.4: The regions in $\rho-\eta$ space (shaded) consistent with measurements of CP violation in $K_{L}^{o}$ decay $(\epsilon), V_{u b} / V_{c b}$ in semileptonic $B$ decay, $B_{d}^{o}$ mixing, and the excluded region from limits on $B_{s}^{o}$ mixing. The allowed region is defined by the overlap of the 3 permitted areas, and is where the apex of the CKM triangle sits.

all have large or even dominant errors due to theoretical models, and the bands are only $\pm 1 \sigma$ wide. This analysis is in good agreement with that of Rosner [15]. and in substantial agreement with the analysis of Plaszczynski and Schune [17], but not in agreement with Caravaglios et al [18], who extract what we view as unreasonably small errors from the data.

\subsection{CP Violation in Charged $B$ Decays}

The theoretical basis of the study of CP violation in $B$ decays was given in a series of papers by Carter and Sanda, and Bigi and Sanda [19]. We start with charged $B$ decays. Consider the final states $f^{ \pm}$which can be reached by two distinct weak processes with amplitudes $\mathcal{A}$ and $\mathcal{B}$, respectively.

$$
\mathcal{A}=a_{s} e^{i \theta_{s}} a_{w} e^{i \theta_{w}}, \quad \mathcal{B}=b_{s} e^{i \delta_{s}} b_{w} e^{i \delta_{w s}}
$$

The strong phases are denoted by the subscript $s$ and weak phases are denoted by the subscript $w$. Under the CP operation the strong phases are invariant but the weak phases change sign, so

$$
\overline{\mathcal{A}}=a_{s} e^{i \theta_{s}} a_{w} e^{-i \theta_{w}}, \quad \overline{\mathcal{B}}=b_{s} e^{i \delta_{s}} b_{w} e^{-i \delta_{w}}
$$

The rate difference is

$$
\begin{aligned}
\Gamma-\bar{\Gamma} & =|\mathcal{A}+\mathcal{B}|^{2}-|\overline{\mathcal{A}}+\overline{\mathcal{B}}|^{2} \\
& =2 a_{s} a_{w} b_{s} b_{w} \sin \left(\delta_{s}-\theta_{s}\right) \sin \left(\delta_{w}-\theta_{w}\right)
\end{aligned}
$$


A weak phase difference is guaranteed in the appropriate decay mode (different CKM phases), but the strong phase difference is not; it is very difficult to predict the magnitude of strong phase differences.

As an example consider the possibility of observing CP violation by measuring a rate difference between $B^{-} \rightarrow K^{-} \pi^{\circ}$ and $B^{+} \rightarrow K^{+} \pi^{\circ}$. The $K^{-} \pi^{\circ}$ final state can be reached either by tree or penguin diagrams as shown in Fig. 1.5. The tree diagram has an imaginary
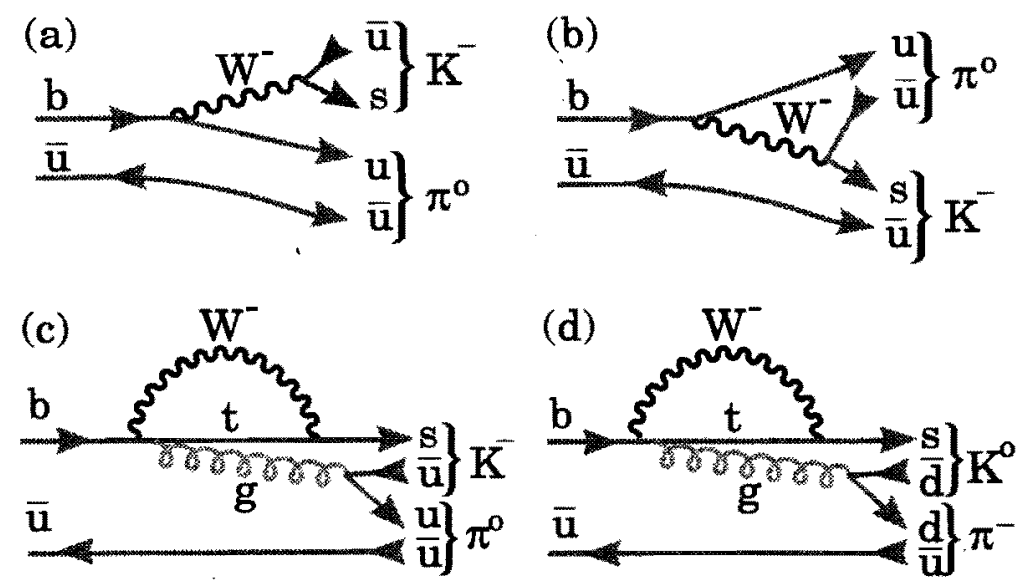

Figure 1.5: Diagrams for $B^{-} \rightarrow K^{-} \pi^{\circ}$, (a) and (b) are tree level diagrams where (b) is color suppressed; (c) is a penguin diagram. (d) shows $B^{-} \rightarrow K^{o} \pi^{-}$, which cannot be produced via a tree diagram.

part coming from the $V_{u b}$ coupling, while the penguin term does not, thus insuring a weak phase difference. This type of CP violation is called "direct." Note also that the process $B^{-} \rightarrow K^{\circ} \pi^{-}$can only be produced by the penguin diagram in Fig. $1.5(\mathrm{~d})$. Therefore, in this simple example, we do not expect a rate difference between $B^{-} \rightarrow K^{\circ} \pi^{-}$and $B^{+} \rightarrow K^{\circ} \pi^{+}$. (There have been suggestions that rescattering effects may contribute here and produce a rate asymmetry, see section 1.8 .)

\subsection{CP Violation Formalism in Neutral $B$ decays}

For neutral mesons we can construct the CP eigenstates

$$
\begin{aligned}
& \left|B_{1}^{o}\right\rangle=\frac{1}{\sqrt{2}}\left(\left|B^{o}\right\rangle+\left|\bar{B}^{o}\right\rangle\right) \\
& \left|B_{2}^{o}\right\rangle=\frac{1}{\sqrt{2}}\left(\left|B^{o}\right\rangle-\left|\bar{B}^{o}\right\rangle\right)
\end{aligned}
$$

where

$$
\begin{aligned}
& C P\left|B_{1}^{o}\right\rangle=\left|B_{1}^{o}\right\rangle, \\
& C P\left|B_{2}^{o}\right\rangle=-\left|B_{2}^{o}\right\rangle .
\end{aligned}
$$


Since $B^{o}$ and $\bar{B}^{o}$ can mix, the mass eigenstates are superpositions of $a\left|B^{o}\right\rangle+b\left|\bar{B}^{o}\right\rangle$ which obey the Schrödinger equation

$$
i \frac{d}{d t}\left(\begin{array}{c}
a \\
b
\end{array}\right)=H\left(\begin{array}{l}
a \\
b
\end{array}\right)=\left(M-\frac{i}{2} \Gamma\right)\left(\begin{array}{l}
a \\
b
\end{array}\right) .
$$

If $C P$ is not conserved then the eigenvectors, the mass eigenstates $\left|B_{L}\right\rangle$ and $\left|B_{H}\right\rangle$, are not the CP eigenstates but are

$$
\left|B_{L}\right\rangle=p\left|B^{o}\right\rangle+q\left|\bar{B}^{o}\right\rangle, \quad\left|B_{H}\right\rangle=p\left|B^{o}\right\rangle-q\left|\bar{B}^{o}\right\rangle
$$

where

$$
p=\frac{1}{\sqrt{2}} \frac{1+\epsilon_{B}}{\sqrt{1+\left|\epsilon_{B}\right|^{2}}}, \quad q=\frac{1}{\sqrt{2}} \frac{1-\epsilon_{B}}{\sqrt{1+\left|\epsilon_{B}\right|^{2}}} .
$$

CP is violated if $\epsilon_{B} \neq 0$, which occurs if $|q / p| \neq 1$.

The time dependence of the mass eigenstates is

$$
\begin{aligned}
\left|B_{L}(t)\right\rangle & =e^{-\Gamma_{L} t / 2} e^{-i m_{L} t / 2}\left|B_{L}(0)\right\rangle \\
\left|B_{H}(t)\right\rangle & =e^{-\Gamma_{H} t / 2} e^{-i m_{H} t / 2}\left|B_{H}(0)\right\rangle,
\end{aligned}
$$

leading to the time evolution of the flavor eigenstates as

$$
\begin{aligned}
& \left|B^{o}(t)\right\rangle=e^{-\left(i m+\frac{\Gamma}{2}\right) t}\left(\cos \frac{\Delta m t}{2}\left|B^{o}(0)\right\rangle+i \frac{q}{p} \sin \frac{\Delta m t}{2}\left|\bar{B}^{o}(0)\right\rangle\right) \\
& \left|\bar{B}^{o}(t)\right\rangle=e^{-\left(i m+\frac{\Gamma}{2}\right) t}\left(i \frac{p}{q} \sin \frac{\Delta m t}{2}\left|B^{o}(0)\right\rangle+\cos \frac{\Delta m t}{2}\left|\bar{B}^{o}(0)\right\rangle\right),
\end{aligned}
$$

where $m=\left(m_{L}+m_{H}\right) / 2, \Delta m=m_{H}-m_{L}$ and $\Gamma=\Gamma_{L} \approx \Gamma_{H}$. Note that the fraction of $B^{o}$ remaining at time $t$ is given by $\left\langle B^{o}(t) \mid B^{o}(t)\right\rangle^{*}$, and is a pure exponential, $e^{-\Gamma t}$, in the absence of $\mathrm{CP}$ violation.

Indirect $C P$ violation in the neutral $B$ system

As in the case of $K_{L}$ decay, we can look for the rate asymmetry

$$
\begin{aligned}
a_{s l} & =\frac{\Gamma\left(\bar{B}^{o}(t) \rightarrow X \ell^{+} \nu\right)-\Gamma\left(B^{o}(t) \rightarrow X \ell^{-} \bar{\nu}\right)}{\Gamma\left(\bar{B}^{o}(t) \rightarrow X \ell^{+} \nu\right)+\Gamma\left(B^{o}(t) \rightarrow X \ell^{-} \bar{\nu}\right)} \\
& =\frac{1-\left|\frac{q}{p}\right|^{4}}{1+\left|\frac{q}{p}\right|^{4}} \approx O\left(10^{-3}\right) .
\end{aligned}
$$

These final states occur only through mixing as the direct decay occurs only as $B^{\circ} \rightarrow X \ell^{+} \nu$. To generate CP violation we need an interference between two diagrams. In this case the 
two diagrams are the mixing diagram with the t-quark and the mixing diagram with the $c$-quark. This is identical to what happens in the $K_{L}^{\circ}$ case. This type of CP violation is called "indirect." The small size of the expected asymmetry is caused by the off-diagonal elements of the $\Gamma$ matrix in equation (1.24) being very small compared to the off-diagonal elements of the mass matrix, i.e. $\left|\Gamma_{12} / M_{12}\right| \ll 1$ and $\operatorname{Im}\left(\Gamma_{12} / M_{12}\right) \neq 0$. This results from the nearly equal widths of the $B_{L}^{o}$ and $B_{H}^{o}[20]$.

In the case of the $B_{s}^{o}$ a relatively large, $\approx 15 \%$ component of $B_{s}$ decays is predicted to end up as a $c \bar{c} s \bar{s}$ final state. Since $\bar{B}_{s}$ decays with the same rate into the same final state, it has been predicted [21] [23] [22] that there will be a substantial width difference $\Delta \Gamma=\Gamma_{H}-\Gamma_{L} \approx 15 \% \Gamma$, between $\mathrm{CP}+$ and $\mathrm{CP}$ - eigenstates. BTeV can easily measure this lifetime difference by measuring the lifetime of a mixed CP state such as $D_{s}^{+} \pi^{-}$and comparing with the CP- state $J / \psi \eta^{\prime}$. The CP+ state $K^{+} K^{-}$can also be used [24]. For finite $\Delta \Gamma$, equations 1.29 and 1.30 are modified [25]. See section 1.8.5 for more details.

\section{$C P$ violation for $B$ via interference of mixing and decays}

Here we choose a final state $f$ which is accessible to both $B^{\circ}$ and $\bar{B}^{\circ}$ decays. The second amplitude necessary for interference is provided by mixing. Fig. 1.6 shows the decay into $f$ either directly or indirectly via mixing. It is necessary only that $f$ be accessible from either

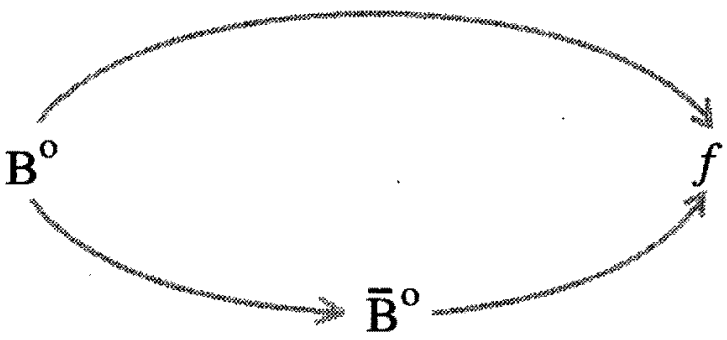

Figure 1.6: Two interfering ways for a $B^{o}$ to decay into a final state $f$.

state. However if $f$ is a CP eigenstate the situation is far simpler. For CP eigenstates

$$
C P\left|f_{C P}\right\rangle= \pm\left|f_{C P}\right\rangle
$$

It is useful to define the amplitudes

$$
A=\left\langle f_{C P}|\mathcal{H}| B^{o}\right\rangle, \quad \bar{A}=\left\langle f_{C P}|\mathcal{H}| \bar{B}^{o}\right\rangle .
$$

If $\left|\frac{A}{A}\right| \neq 1$, then we have "direct" CP violation in the decay amplitude, which was discussed above. Here CP can be violated by having

$$
\lambda=\frac{q}{p} \cdot \frac{\bar{A}}{A} \neq 1,
$$


which requires only that $\lambda^{2}$ acquire a non-zero phase, i.e. $|\lambda|$ could be unity and CP violation can occur.

The asymmetry, in this case, is defined as

$$
a_{f_{C P}}=\frac{\Gamma\left(B^{o}(t) \rightarrow f_{C P}\right)-\Gamma\left(\bar{B}^{o}(t) \rightarrow f_{C P}\right)}{\Gamma\left(B^{o}(t) \rightarrow f_{C P}\right)+\Gamma\left(\bar{B}^{o}(t) \rightarrow f_{C P}\right)}
$$

which for $|q / p|=1$ gives

$$
a_{f_{C P}}=\frac{\left(1-|\lambda|^{2}\right) \cos (\Delta m t)-2 \operatorname{Im} \lambda \sin (\Delta m t)}{1+|\lambda|^{2}} .
$$

For the cases where there is only one decay amplitude $A,|\lambda|$ equals 1 , and we have

$$
a_{f C P}=-\operatorname{Im} \lambda \sin (\Delta m t) \text {. }
$$

Only the amplitude, $-\operatorname{Im} \lambda$ contains information about the level of $\mathrm{CP}$ violation, the sine term is determined only by $B^{o}$ mixing. In fact, the time integrated asymmetry is given by

$$
a_{f_{C P}}=-\frac{x}{1+x^{2}} \operatorname{Im} \lambda
$$

where $x=\frac{\Delta m}{\Gamma}$. For the case of the $B_{d}^{o}, x /\left(1+x^{2}\right)=0.48$, which is quite lucky as the maximum size of the coefficient is -0.5 .

$\operatorname{Im} \lambda$ is related to the CKM parameters. Recall $\lambda=\frac{q}{p} \cdot \frac{\bar{A}}{A}$. The first term is the part that comes from mixing:

$$
\begin{gathered}
\frac{q}{p}=\frac{\left(V_{t b}^{*} V_{t d}\right)^{2}}{\left|V_{t b} V_{t d}\right|^{2}}=\frac{(1-\rho-i \eta)^{2}}{(1-\rho+i \eta)(1-\rho-i \eta)}=e^{-2 i \rho} \cdot \text { and } \\
\operatorname{Im} \frac{q}{p}=-\frac{2(1-\rho) \eta}{(1-\rho)^{2}+\eta^{2}}=\sin (2 \beta)
\end{gathered}
$$

To evaluate the decay part we need to consider specific final states. For example, consider $f \equiv \pi^{+} \pi^{-}$. The simple spectator decay diagram is shown in Fig. 1.7. For the moment we will assume that this is the only diagram which contributes. Later we will show why this is not true. For this $b \rightarrow u \bar{u} d$ process we have.

$$
\frac{\bar{A}}{A}=\frac{\left(V_{u d}^{*} V_{u b}\right)^{2}}{\left|V_{u d} V_{u b}\right|^{2}}=\frac{(\rho-i \eta)^{2}}{(\rho-i \eta)(\rho+i \eta)}=e^{-2 i \gamma},
$$

and

$$
\operatorname{Im}(\lambda)=\operatorname{Im}\left(e^{-2 i \beta} e^{-2 i \gamma}\right)=\operatorname{Im}\left(e^{2 i \alpha}\right)=\sin (2 \alpha)
$$

\footnotetext{
${ }^{2} \lambda$ here is not the same variable that occurs in the Wolfenstein representation of the CKM matrix.
} 


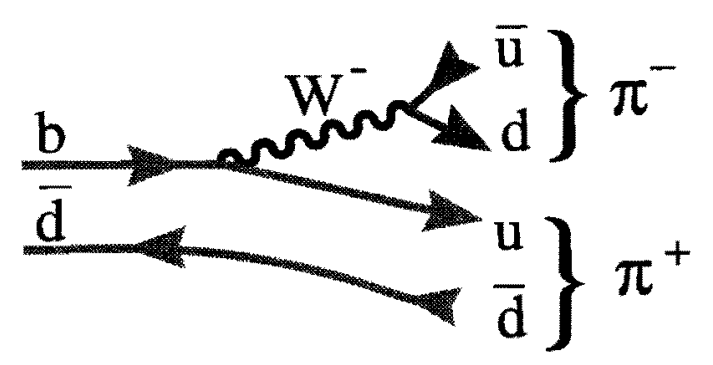

Figure 1.7: Decay diagram at the tree level for $B^{\circ} \rightarrow \pi^{+} \pi^{-}$.

The final state $J / \psi K_{S}$ plays an especially important role in the study of CP violation. It is a CP eigenstate and its decay is dominated by only one diagram, shown in Fig. 1.8. In this case we do not get a phase from the decay part because

$$
\frac{\bar{A}}{A}=\frac{\left(V_{c b} V_{c s}^{*}\right)^{2}}{\left|V_{c b} V_{c s}\right|^{2}}
$$

is real. In this case the final state is a state of negative $C P$, i.e. $C P\left|J / \psi K_{S}\right\rangle=-\left|J / \psi K_{S}\right\rangle$.

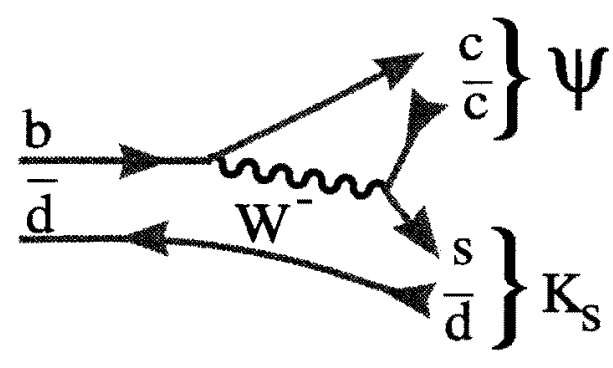

Figure 1.8: Decay diagram at the tree level for $B^{o} \rightarrow J / \psi K_{S}$.

This introduces an additional minus sign in the result for $\operatorname{Im} \lambda$. Before finishing discussion of this final state we need to consider in more detail the presence of the $K_{S}$ in the final state. Since neutral kaons can mix, we pick up another mixing phase. This term creates a phase given by

$$
\left(\frac{q}{p}\right)_{K}=\frac{\left(V_{c d}^{*} V_{c s}\right)^{2}}{\left|V_{c d} V_{c s}\right|^{2}}
$$

which is zero. It is necessary to include this term, however, since there are other formulations of the CKM matrix than Wolfenstein, which have the phase in a different location. It is important that the physics predictions not depend on the CKM convention. ${ }^{3}$

In summary, for the case of $f=J / \psi K_{S}, \operatorname{Im} \lambda=-\sin (2 \beta)$.

\footnotetext{
${ }^{3}$ Here we don't include CP violation in the neutral kaon since it is much smaller than what is expected in the $B$ decay.
} 


\subsection{Techniques for Determining $\beta$}

The decay $B^{o} \rightarrow J / \psi K_{S}$ is the primary source for measurements of $\sin (2 \beta)$. In the common phase convention, $C P$ violation is expected to arise mostly from the mixing, driven by $\operatorname{Im}(q / p)$, while the decay amplitude, $\operatorname{Im}(\bar{A} / A)$, is expected to contribute only a small part (see.Fig. 1.8). This decay is expected to have only a small Penguin contribution, but even if the Penguin contribution is significant it does not change the contribution of the mixing phase or the decay phase. ${ }^{4}$

While we expect that $\sin (2 \beta)$ will have been measured before $\mathrm{BTeV}$, we do aim to improve significantly on the precision of the measurement. Furthermore, we intend to be able to remove "ambiguities." When we measure $\sin (2 \phi)$, where $\phi$ is any angle, we have a four-fold ambiguity in $\phi$, namely $\phi, \pi / 2-\phi, \phi+\pi$ and $3 \pi / 2-\phi$. These ambiguities can mask the effects of new physics. Our task is to remove as many of the ambiguities as possible.

\subsubsection{Removal of Two of the $\beta$ Ambiguities}

The decay $B \rightarrow J / \psi K^{*}(890)$, where $K^{*} \rightarrow K_{S} \pi^{0}$ can be used to get information about the sign of $\cos (2 \beta)$, which would remove two of the ambiguities [26]. This decay is described by three complex decay amplitudes. Following a suggestion of Dighe, Dunietz, and Fleischer [27, 28], we write the decay amplitudes $A_{0}=-\sqrt{1 / 3} S+\sqrt{2 / 3} D, A_{\|}=\sqrt{2 / 3} S+\sqrt{1 / 3} D$, and $A_{\perp}=P$, where $S, P$, and $D$ denote $\mathrm{S}, \mathrm{P}$, and $\mathrm{D}$ wave amplitudes, respectively. Normalizing the decay amplitudes to $\left|A_{0}\right|^{2}+\left|A_{\|}\right|^{2}+\left|A_{\perp}\right|^{2}=1$ and eliminating one overall phase leaves four independent parameters.

The full angular distribution of a $B$ meson decaying into two vector particles is specified by three angles. The helicity angle basis [29] has been used for angular analyses of $B \rightarrow J / \psi K^{*}$ decays. An alternative basis, called the transversity basis is more suitable for extracting parity information [28].

In the transversity basis, the direction of the $K^{*}$ in the $J / \psi$ rest frame defines the $\mathrm{x}$-axis of a right-handed coordinate system. The $K \pi$ plane fixes the y-axis with $p_{y}(K)>0$ and the normal to this plane defines the z-axis. The transversity angles $\theta_{\mathrm{tr}}$ and $\phi_{\mathrm{tr}}$ are then defined as polar and azimuth angles of the $l^{+}$in the $J / \psi$ rest frame. The third angle, the $K^{*}$ decay angle $\theta_{K^{*}}$, is defined as that of the $K$ in the $K^{*}$ rest frame relative to the negative of the $J / \psi$ direction in that frame. Using these definitions the full angular distribution of the $B \rightarrow J / \psi K^{*}$ decay is [28]:

$$
\begin{aligned}
\frac{1}{\Gamma} \frac{\mathrm{d}^{3} \Gamma}{\mathrm{d} \cos \theta_{\mathrm{tr}} \mathrm{d} \cos \theta_{K^{*}} \mathrm{~d} \phi_{\mathrm{tr}}} \\
=\frac{9}{32 \pi}\left\{2\left|A_{0}\right|^{2} \cos ^{2} \theta_{K^{*}}\left(1-\sin ^{2} \theta_{\mathrm{tr}} \cos ^{2} \phi_{\mathrm{tr}}\right)\right. \\
\quad+\left|A_{\|}\right|^{2} \sin ^{2} \theta_{K^{*}}\left(1-\sin ^{2} \theta_{\mathrm{tr}} \sin ^{2} \phi_{\mathrm{tr}}\right) \\
\quad+\left|A_{\perp}\right|^{2} \sin ^{2} \theta_{K^{*}} \sin ^{2} \theta_{\mathrm{tr}} \sin ^{2} \phi_{\mathrm{tr}}
\end{aligned}
$$

\footnotetext{
${ }^{4}$ Actually the only phase that has physical meaning is the product of $q / p \cdot \bar{A} / A$.
} 


$$
\begin{aligned}
& -\operatorname{Im}\left(A_{\|}^{*} A_{\perp}\right) \sin ^{2} \theta_{K^{*}} \sin 2 \theta_{\mathrm{tr}} \sin \phi_{\mathrm{tr}} \\
& +\frac{1}{\sqrt{2}} \operatorname{Re}\left(A_{0}^{*} A_{\|}\right) \sin 2 \theta_{K^{*}} \sin ^{2} \theta_{\mathrm{tr}} \sin 2 \phi_{\mathrm{tr}} \\
& \left.+\frac{1}{\sqrt{2}} \operatorname{Im}\left(A_{0}^{*} A_{\perp}\right) \sin 2 \theta_{K^{*}} \sin 2 \theta_{\mathrm{tr}} \cos \phi_{\mathrm{tr}}\right\} .
\end{aligned}
$$

For $\bar{B}$ decays the interference terms containing $A_{\perp}$ switch sign while all other terms remain unchanged.

Results shown in Table 1.1 have been obtained from CLEO and CDF using the decay $\bar{K}^{* o} \rightarrow K^{-} \pi^{+}$.

\begin{tabular}{ccc}
\hline \hline Parameter & CLEO [30] & CDF [31] \\
\hline$\left|A_{0}\right|^{2}=\Gamma_{\mathrm{L}} / \Gamma$ & $0.52 \pm 0.07 \pm 0.04$ & $0.59 \pm 0.06 \pm 0.02$ \\
$\left|A_{\perp}\right|^{2}=|P|^{2}$ & $0.16 \pm 0.08 \pm 0.04$ & $0.13_{-0.06}^{+0.12} \pm 0.03$ \\
\hline \hline
\end{tabular}

Table 1.1: Resulting decay amplitudes from the fit to the transversity angles. The first error is statistical and the second is the estimated systematic uncertainty.

The parity odd component, $\left|A_{\perp}\right|^{2}$, is three standard deviations from zero in the average of the two experiments, and is $\approx 25 \%$ of the rate of the parity even component. This is likely large enough to allow the determination of the sign of the interference terms using the tagged $K^{* o} \rightarrow K_{S} \pi^{o}$ decays; that, in turn, allows a determination of the sign of the product of $\cos (2 \beta)$ with a strong phase-shift. The sign of this phase-shift can either be obtained from factorization, which is a dangerous procedure, or using the much weaker assumption of $\mathrm{SU}(3)$ symmetry, and analyzing the time-dependent oscillations in the decay $B_{s} \rightarrow J / \psi \phi$ [26], where the mixing phase is expected to be small.

Another independent method of removing two of the ambiguities is to measure the sign of the $\cos (2 \beta)$ term in the decay $B^{o} \rightarrow J / \psi K^{o}, K^{o} \rightarrow \pi^{ \pm} \ell^{\mp} \nu$. This idea developed by Kayser [32], works because of the interference between $K_{L}$ and $K_{S}$ in the decay, where the decay amplitudes are equal. The time evolution of the decay width can be expressed in terms of the $B^{o}$ decay time $\left(t_{B}\right)$ and the $K^{o}$ decay time $\left(t_{K}\right)$ as

$$
\begin{aligned}
& \Gamma\left(t_{B}, t_{K}\right) \propto \\
& e^{-\Gamma_{B} t_{B}}\left\{e^{-\gamma_{s} t_{K}}\left[1 \mp \sin (2 \beta) \sin \left(\Delta m_{B} t_{B}\right)\right]\right. \\
& +e^{-\gamma_{L} t_{K}}\left[1 \pm \sin (2 \beta) \sin \left(\Delta m_{B} t_{B}\right)\right] \\
& \pm(\mp) 2 e^{-\frac{1}{2}\left(\gamma_{s}+\gamma_{L}\right) t_{K}}\left[\cos \left(\Delta m_{B} t_{B}\right) \cos \left(\Delta m_{K} t_{K}\right)\right. \\
& \left.\left.+\cos (2 \beta) \sin \left(\Delta m_{B} t_{B}\right) \sin \left(\Delta m_{K} t_{K}\right)\right]\right\},
\end{aligned}
$$

where the top sign of each pair is for $B^{o}$, and the bottom for $\bar{B}^{o}$. The first pair of signs in the third line refers to the kaon decay mode $\pi^{-} \ell^{+} \nu(K)$, while the second pair is for $\pi^{+} \ell^{-} \bar{\nu}$ $(\bar{K})$. 
To get an idea of the predicted asymmetries, we integrate this equation over $t_{B}$. There are four different rates that can be denoted as combinations of $B$ and $\bar{B}$ with $K$ and $\bar{K}$. In Fig. 1.9 we show the four rates as solid lines if $\cos (2 \beta)$ were positive and the four rates as dashed lines if $\cos (2 \beta)$ were negative. These were done for $\sin (2 \beta)=0.7$. If $\sin (2 \beta)$ were smaller the rate differences would be larger and vice-versa.

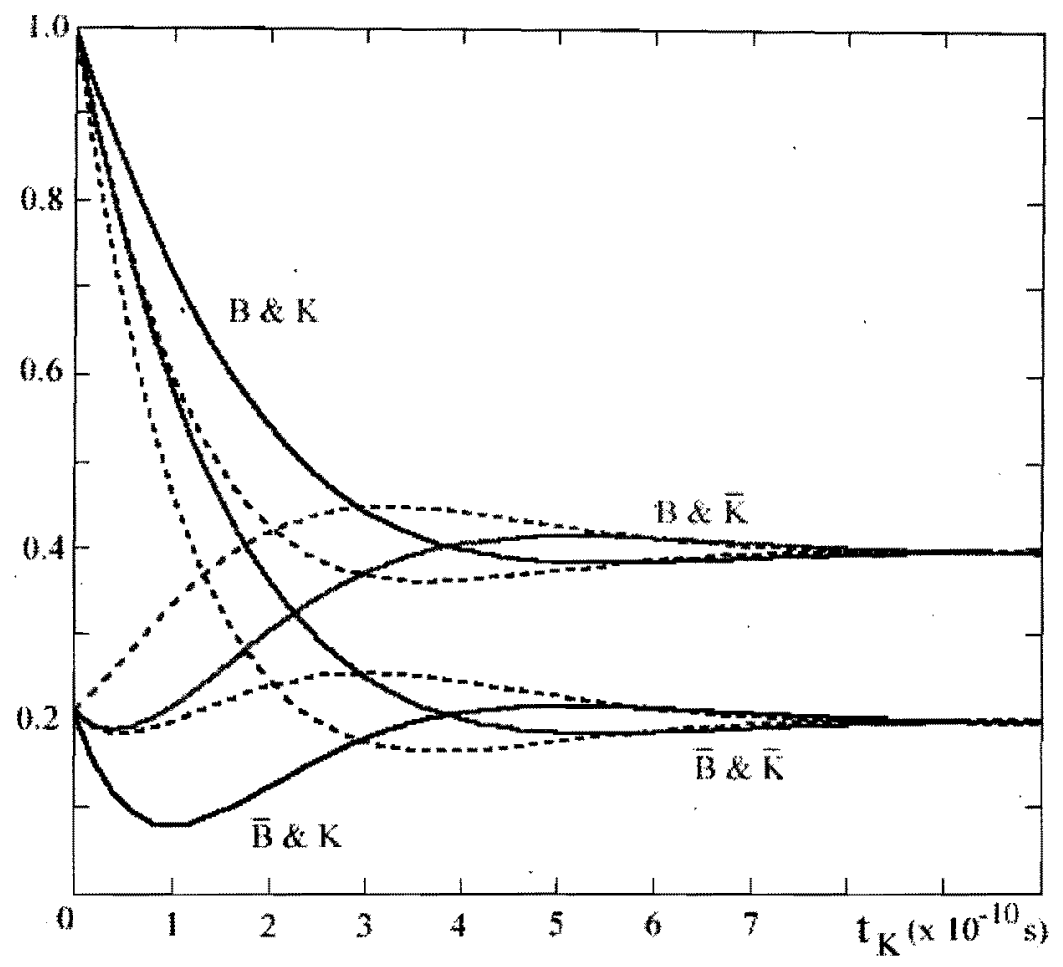

Figure 1.9: The decay rates for $B^{o} \rightarrow J / \psi K^{o}, K^{o} \rightarrow \pi \ell \nu$, as a function of $K^{o}$ decay time, integrated over the $B^{\circ}$ decay time. The solid lines have the sign of the $\cos (2 \beta)$ term as positive, while the corresponding dashed lines have negative values. The absolute normalization is arbitrary, and $\sin (2 \beta)$ was fixed at 0.7 .

The differences are large over about five $K_{S}$ lifetimes. Since only the sign of the $\cos (2 \beta)$ term needs to be found, all other parameters, including $\sin (2 \beta)$ are specified. Unfortunately, the event rate is rather small, since $\mathcal{B}\left(K_{S} \rightarrow \pi \ell \nu\right)=1.4 \times 10^{-3}$ and although $\mathcal{B}\left(K_{L} \rightarrow\right.$ $\pi \ell \nu)=0.66$, only $1 \%$ of the $K_{L}$ decay soon enough to be of use. Roughly, we have about 100 times fewer events than in $J / \psi K_{S}$. However, if the backgrounds are not too large, it will only take on the order of a hundred events to successfully determine the sign of $\cos (2 \beta)$ using this technique.

It is interesting to note that measuring this combination of $B^{o}$ and $K^{o}$ decay modes can lead to measurements of CPT violation [33]. 


\subsection{Comment on Penguin Amplitude}

Many processes can have penguin components. The diagram for $B^{o} \rightarrow \pi^{+} \pi^{-}$is shown in Fig. 1.10. The $\pi^{+} \pi^{-}$final state is expected to have a rather large penguin amplitude $\sim 20 \%$ of the tree amplitude. Then $|\lambda| \neq 1$ and $a_{f_{C P}}$, equation 1.37 , develops a $\cos (\Delta m t)$ term. In the $J / \psi K_{S}$ case, the penguin amplitude is expected to be small since a $c \bar{c}$ pair must be

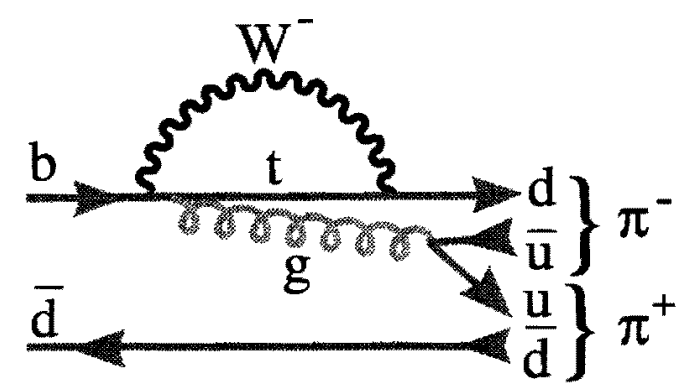

Figure 1.10: Penguin diagram for $B^{o} \rightarrow \pi^{+} \pi^{-}$.

"popped" from the vacuum. Even if the penguin decay amplitude were of significant size, the decay phase, $\operatorname{Im}(\bar{A} / A)$ is the same as the tree level process, and quite small.

\subsection{Techniques for Determining $\alpha$}

Measuring $\alpha$ is more difficult than measuring $\beta$ in several respects. First of all, the decay amplitudes are modulated by $V_{u b}$ rather than $V_{c b}$, making the overall rates small. Secondly, the gluonic Penguin rates are of the same order causing well known difficulties in extracting the weak phase angle (see section 1.6 above). The Penguin diagrams add a third amplitude to the tree level and mixing amplitudes. It turns out, however, that this complication can be a blessing in disguise. The interference generates $\cos (2 \alpha)$ terms in the decay rate, that can be used to remove discrete ambiguities.

The decay $B^{\circ} \rightarrow \pi^{+} \pi^{-}$has oft been cited as a way to measure $\sin (2 \alpha)$. However, the Penguin pollution mentioned above, makes it difficult to extract the angle. Current data from CLEO gives $\mathcal{B}\left(B^{o} \rightarrow K^{\mp} \pi^{ \pm}\right)=\left(1.72_{-0.24}^{+0.25} \pm 0.12\right) \times 10^{-5}$ and $\mathcal{B}\left(B^{o} \rightarrow \pi^{+} \pi^{-}\right)=$ $\left(0.43_{-0.14}^{+0.16} \pm 0.05\right) \times 10^{-5}[34]$, showing a relatively large Penguin amplitude which cannot be ignored. Gronau and London [35] have shown that an isospin analysis using the additional decays $B^{-} \rightarrow \pi^{-} \pi^{o}$ and $B^{o} \rightarrow \pi^{o} \pi^{o}$ can be used to extract $\alpha[36]$, but the $\pi^{o} \pi^{o}$ final state is extremely difficult to detect in any existing or proposed experiment. Other authors have suggested different methods [37], but they all have theoretical assumptions. Thus, measurement of the CP asymmetry in $B^{o} \rightarrow \pi^{+} \pi^{-}$cannot, in our view, provide an accurate determination of $\sin (2 \alpha)$ unless some new breakthrough in theory occurs.

There is however, a theoretically clean method to determine $\alpha$. The interference between Tree and Penguin diagrams can be exploited by measuring the time dependent CP violating 
effects in the decays $B^{\circ} \rightarrow \rho \pi$ as shown by Snyder and Quinn [38]. There are three such neutral decay modes, listed in Table 1.2 with their respective Penguin and Tree amplitudes, denoted by $T^{i j}$, where $i$ lists charge of the $\rho$ and $j$ the charge of the $\pi$. For the $\rho^{o} \pi^{o}$ mode, isospin constraints are used to eliminate $T^{o o}$. The amplitudes for the charged decays are also given.

Table 1.2: $B^{\circ} \rightarrow \rho \pi$ Decay Modes

\begin{tabular}{ll}
\hline & \\
Decay Mode & Decay Amplitudes \\
\hline$\sqrt{2} A\left(B^{+} \rightarrow \rho^{+} \pi^{o}\right)$ & $=S_{1}=T^{+o}+2 P_{1}$ \\
$\sqrt{2} A\left(B^{+} \rightarrow \rho^{o} \pi^{+}\right)$ & $=S_{2}=T^{o+}-2 P_{1}$ \\
$A\left(B^{o} \rightarrow \rho^{+} \pi^{-}\right)$ & $=S_{3}=T^{+-}+P_{1}+P_{o}$ \\
$A\left(B^{o} \rightarrow \rho^{-} \pi^{+}\right)$ & $=S_{4}=T^{-+}-P_{1}+P_{o}$ \\
$2 A\left(B^{o} \rightarrow \rho^{o} \pi^{o}\right)$ & $=S_{5}=T^{+-}+T^{+-}-T^{+o}-T^{o+}-2 P_{o}$ \\
\hline
\end{tabular}

For the $\rho \pi$ final state, the $\rho$ decay amplitude can be parameterized as

$$
f(m, \theta)=\frac{\cos (\theta) \Gamma_{\rho}}{2\left(m_{\rho}-m-i 0.5 \Gamma_{\rho}\right)},
$$

where $m_{\rho}$ is the $\rho$ mass of $0.77 \mathrm{GeV}$ and $\Gamma_{\rho}$, the width of $0.15 \mathrm{GeV} . \theta$ is the helicity decay angle and the $\cos (\theta)$ dependence arises because the $\rho$ must be fully polarized in this decay which starts with a spin- $0 B$ and ends with a spin-1 $\rho$ and spin- $0 \pi$.

The full decay amplitudes for $B^{o} \rightarrow \rho \pi \rightarrow \pi^{+} \pi^{-} \pi^{o}$ and the corresponding $\bar{B}^{o}$ decay are given by

$$
\begin{aligned}
& A\left(B^{o}\right)=f^{+} S_{3}+f^{-} S_{4}+f^{o} S_{5} / 2 \\
& A\left(\bar{B}^{o}\right)=f^{+} \bar{S}_{3}+f^{-} \bar{S}_{4}+f^{o} \bar{S}_{5} / 2
\end{aligned}
$$

where the superscript on the $f$ indicates the charge of the $\rho$. The sum over the three neutral $B$ decay amplitudes involves only tree amplitudes; the Penguins vanish. The angle between this sum for $B^{o}$ decays ( $\equiv T$ ) and the sum for $\bar{B}^{o}(\equiv \bar{T}$ ) is precisely $\alpha$. Computing the amplitudes gives a series of terms which have both $\sin (\Delta m t)$ and $\cos (\Delta m t)$ time dependences and coefficients which depend on both $\sin (2 \alpha)$ and $\cos (2 \alpha)$.

To extract $\alpha$ only the neutral modes need be measured. Further constraints and information about Penguin phases can be extracted if the charged $B$ 's are also measured. But this is difficult because there are two $\pi^{\circ}$ 's in the $\rho^{+} \pi^{o}$ decay mode.

The $\rho \pi$ final state has many advantages. First of all, it has a relatively large branching ratio. The latest CLEO measurement for the $\rho^{\circ} \pi^{+}$final state is $(1.0 \pm 0.3 \pm 0.2) \times 10^{-5}$ [34]. The rate for the neutral $B$ final state $\rho^{ \pm} \pi^{\mp}$ is $\left(2.8_{-0.7}^{+0.8} \pm 0.4\right) \times 10^{-5}$; while the $\rho^{o} \pi^{o}$ final state is limited at $90 \%$ confidence level to $<5.1 \times 10^{-6}$ [39]. These measurements are consistent with theoretical expectations [40]. Secondly, since the $\rho$ is fully polarized in the 
$(1,0)$ configuration, it decays as $\cos ^{2} \theta$, where $\theta$ is the angle of one of the $\rho$ decay products with the $\pi$ in the $\rho$ rest frame. This causes the periphery of the Dalitz plot to be heavily populated, especially the corners. A sample Dalitz plot is shown in Fig. 1.11. This kind of distribution is good for maximizing the interferences, which helps minimize the error. Furthermore, little information is lost by excluding the Dalitz plot interior, a good way to reduce backgrounds.

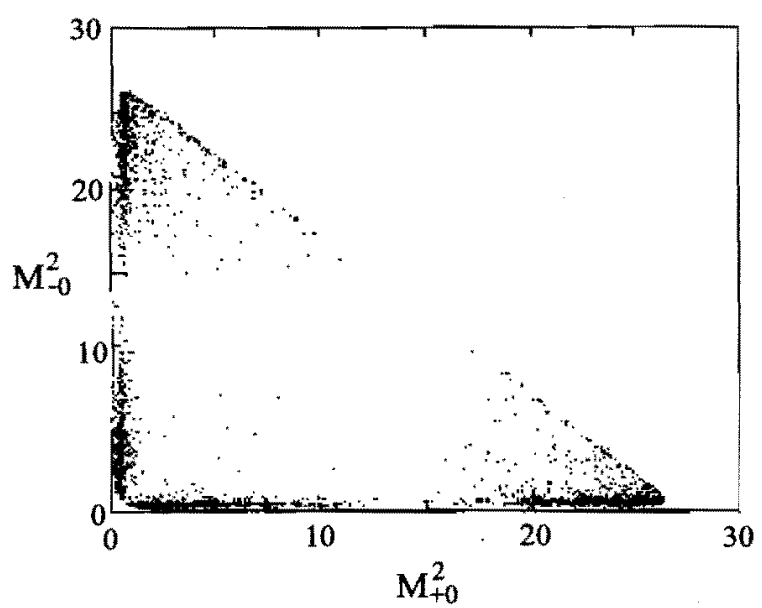

Figure 1.11: The Dalitz plot for $B^{o} \rightarrow \rho \pi \rightarrow \pi^{+} \pi^{-} \pi^{o}$ from Snyder and Quinn.

Snyder and Quinn have performed an idealized analysis that uses 1000 or 2000 flavor tagged background free events. The 1000 event sample usually yields good results for $\alpha$, but sometimes does not resolve the ambiguity. With the 2000 event sample, however, they always succeed.

Recently Quinn and Silva have pointed out ways of using time integrated untagged data to specify some of the parameters with larger data samples [41]. Some concern for the effect of the $B^{*}$ pole on the data has been expressed by Deandrea et al [42].

The decay $B^{o} \rightarrow \pi^{+} \pi^{-}$can be used with some theoretical input to resolve the remaining ambiguity in $\sin (2 \alpha)$. The difference in CP asymmetries between $\pi \pi$ and $\rho \pi$ is given by

$$
a(\pi \pi)-a(\rho \pi)=-2\left(A_{P} / A_{T}\right) \cos \left(\delta_{P}-\delta_{T}\right)[\cos (2 \alpha) \sin (\alpha)],
$$

where $A_{P}$ and $A_{T}$ denote the Penguin and Tree amplitudes, respectively, and the $\delta$ 's represent their strong phase shifts. Factorization can be used to get the sign of $A_{P} / A_{T}$ and the strong phase shifts are believed to be small enough that $\cos \left(\delta_{P}-\delta_{T}\right)$ is positive [43].

\subsection{Techniques for Determining $\gamma$}

The angle $\gamma$ could in principle be measured using a $\mathrm{CP}$ eigenstate of $B_{s}$ decay that was dominated by the $b \rightarrow u$ transition. One such decay that has been suggested is $B_{s} \rightarrow \rho^{o} K_{S}$. 
However, there are the same "Penguin pollution" problems as in $B^{o} \rightarrow \pi^{+} \pi^{-}$, but they are more difficult to resolve in the vector-pseudoscalar final state. (Note, the pseudoscalarpseudoscalar final state here is $\pi^{o} K_{S}$, which does not have a measurable decay vertex.)

Fortunately, there are other ways of measuring $\gamma$. CP eigenstates are not used, which introduces discrete ambiguities. However, combining several methods should remove these. We have studied three methods of measuring $\gamma$.

\subsubsection{Measurement of $\gamma$ Using Time-Dependent CP violation in $B_{s}$ Decays}

The first method uses the decays $B_{s} \rightarrow D_{s}^{ \pm} K^{\mp}$ where a time-dependent $C P$ violation can result from the interference between the direct decay and the mixing-induced decay [44]. Fig. 1.12 shows the two direct decay processes for $\bar{B}_{s}^{o}$.
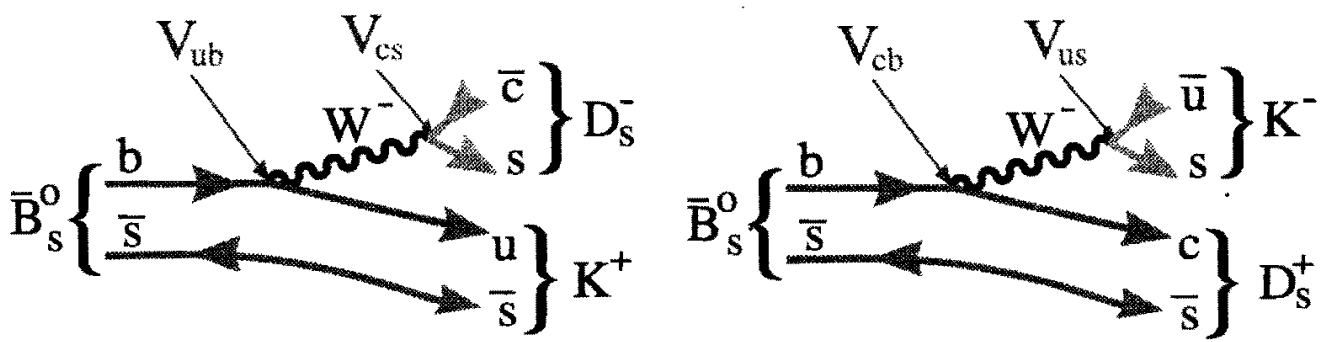

Figure 1.12: Two diagrams for $\bar{B}_{s}^{o} \rightarrow \dot{D}_{s}^{ \pm} K^{\mp}$.

Consider the following time-dependent rates that can be separately measured using flavor tagging of the other $b$ :

$$
\begin{array}{r}
\Gamma\left(B_{s} \rightarrow f\right)=|M|^{2} e^{-t}\left\{\cos ^{2}(x t / 2)+\rho^{2} \sin ^{2}(x t / 2)-\rho \sin (\phi+\delta) \sin (x t)\right\} \\
\Gamma\left(\bar{B}_{s} \rightarrow \bar{f}\right)=|M|^{2} e^{-t}\left\{\cos ^{2}(x t / 2)+\rho^{2} \sin ^{2}(x t / 2)+\rho \sin (\phi-\delta) \sin (x t)\right\} \\
\Gamma\left(B_{s} \rightarrow \bar{f}\right)=|M|^{2} e^{-t}\left\{\rho^{2} \cos ^{2}(x t / 2)+\sin ^{2}(x t / 2)-\rho \sin (\phi-\delta) \sin (x t)\right\} \\
\Gamma\left(\bar{B}_{s} \rightarrow f\right)=|M|^{2} e^{-t}\left\{\rho^{2} \cos ^{2}(x t / 2)+\sin ^{2}(x t / 2)+\rho \sin (\phi+\delta) \sin (x t)\right\},
\end{array}
$$

where $M=\langle f \mid B\rangle, \rho=\frac{\langle f \mid \bar{B}\rangle}{\langle f \mid B\rangle}, \phi$ is the weak phase between the 2 amplitudes and $\delta$ is the strong phase between the 2 amplitudes. The three parameters $\rho, \sin (\phi+\delta), \sin (\phi-\delta)$ can be extracted from a time-dependent study. If $\rho=O(1)$ the fewest number of events are required.

In the case of $B_{s}$ decays where $f=D_{s}^{+} K^{-}$and $\vec{f}=D_{s}^{-} K^{+}$, the weak phase is $\gamma^{5}$ Using this technique $\sin (\gamma)$ is determined with a four-fold ambiguity. If $\Delta \Gamma\left(B_{s}\right)$ is of the order of $10 \%$, then the ambiguities can be resolved.

\footnotetext{
${ }^{5}$ This is an approximation. The phase is precisely $\gamma-2 \chi+\chi^{i}$, see section 1.9 .
} 


\subsubsection{Measurement of $\gamma$ Using Charged $B$ Decay Rates}

Another method for extracting $\gamma$ has been proposed by Atwood, Dunietz and Soni [45], who refined a suggestion by Gronau and Wyler [46]. A large CP asymmetry can result from the interference of the decays $B^{-} \rightarrow K^{-} D^{o}, D^{o} \rightarrow f$ and $B^{-} \rightarrow K^{-} \bar{D}^{o}, \bar{D}^{o} \rightarrow f$, where $f$ is a doubly-Cabibbo suppressed decay of the $D^{o}$ (for example $f=K^{+} \pi^{-}, K \pi \pi$, etc.). The overall amplitudes for the two decays are expected to be approximately equal in magnitude. (Note that $B^{-} \rightarrow K^{-} \bar{D}^{\circ}$ is color-suppressed and $B^{-} \rightarrow K^{-} D^{0}$ is color-allowed.) The weak phase difference between them is $\gamma$. To observe a CP asymmetry there must also be a nonzero strong phase between the two amplitudes. It is necessary to measure the branching ratio $\mathcal{B}\left(B^{-} \rightarrow K^{-} f\right)$ for at least 2 different states $f$ in order to determine $\gamma$ up to discrete ambiguities. Three-body $D^{\circ}$ decays are not suggested since the strong $D$ decay phase shifts can vary over the Dalitz plot. Even in quasi-two body decays, such as $K^{*} \pi$ there may be residual interference effects which could lead to false results. Therefore, the modes that can best be used are $D^{o} \rightarrow K^{-} \pi^{+}$and $K^{+} K^{-}\left(\pi^{+} \pi^{-}\right)$final states.

We now discuss this method in more detail. Consider a two-body $B^{-}$decay into a neutral charmed meson, either a $D^{o}$ or a $\bar{D}^{\circ}$ and a $K^{-}$. Let us further take the final state of the charmed meson to be a $K^{+} \pi^{-}$. There are two sequential decay processes that can lead to this situation, shown in Fig. 1.13. One is where the $B^{o}$ decays into a $D^{o}$, that decays in a doubly-Cabibbo suppressed process. The other is where the $B^{\circ}$ decays via a $b \rightarrow u$ transition to a $D^{\circ}$, that decays via a Cabibbo allowed process.
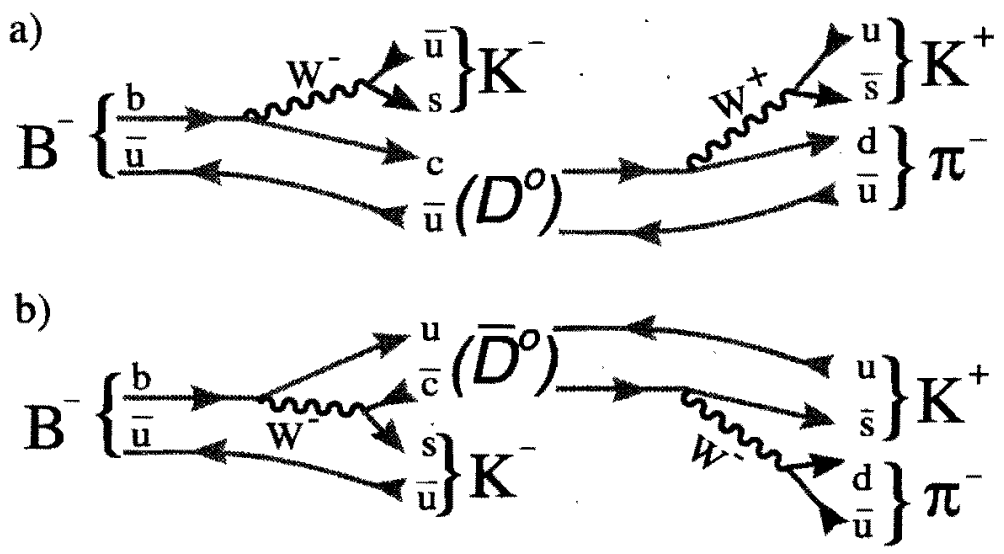

Figure 1.13: Diagrams for the two interfering processes, (a) $B^{-} \rightarrow D^{\circ} K^{-}$(color allowed) followed by $D^{o} \rightarrow K^{+} \pi^{-}$(doubly-Cabibbo suppressed), and (b) $B^{-} \rightarrow \bar{D}^{o} K^{-}$(color suppressed) followed by $D^{o} \rightarrow K^{-} \pi^{+}$(Cabibbo allowed).

Remarkably, the decay rate for these two processes is quite similar leading to the possibility of large interference effects. Even if the interference effects are not large it is possible to use this method to determine $\gamma$, with some ambiguities. To see how this works, let us define the decay amplitudes and phases in Table 1.3 for two processes, one as described above and 
the other where the $D^{o}$ or $\bar{D}^{o}$ decays into a CP eigenstate. (To be specific, we will take the $K^{+} K^{-}$final state.)

Table 1.3: Amplitudes and Phases for $B^{-} \rightarrow D^{\circ} / \bar{D}^{\circ} K^{-}$

\begin{tabular}{lcccc}
\hline \hline Decay Mode & $B$ & $D$ & Strong. & Weak \\
& Amplitude & Amplitude & Phase & Phase \\
\hline$B^{-} \rightarrow D^{o} K^{-}, D^{o} \rightarrow K^{+} \pi^{-}$ & $\sqrt{a}$ & $\sqrt{c_{d}}$ & $\delta_{B 1}+\delta_{C d}$ & 0 \\
$B^{-} \rightarrow \bar{D}^{o} K^{-}, \bar{D}^{o} \rightarrow K^{+} \pi^{-}$ & $\sqrt{b}$ & $\sqrt{c}$ & $\delta_{B 2}+\delta_{C}$ & $\gamma$ \\
$B^{-} \rightarrow D^{o} K^{-}, D^{o} \rightarrow K^{+} K^{-}$ & $\sqrt{a}$ & $\sqrt{c_{C P}}$ & $\delta_{B 1}+\delta_{C P}$ & 0 \\
$B^{-} \rightarrow \bar{D}^{\circ} K^{-}, \bar{D}^{\circ} \rightarrow K^{-} K^{+}$ & $\sqrt{b}$ & $\sqrt{c_{C P}}$ & $\delta_{B 2}+\delta_{C P}$ & $\gamma$ \\
\hline \hline
\end{tabular}

All quantities remain the same for the $B^{+}$decays, except that the phase $\gamma$ changes sign. The observed decay rates for the four processes can now be calculated by adding and squaring the amplitudes for the same final state. For example, the decay rate for $B^{-} \rightarrow\left[K^{+} \pi^{-}\right] K^{-}$ (where $\left[K^{+} \pi^{-}\right]$denotes a $K^{+} \pi^{-}$pair at the $D^{o}$ mass), is given by

$$
\Gamma\left(B^{-} \rightarrow\left[K^{+} \pi^{-}\right] K^{-}\right)=a c_{d}+b c+2 \sqrt{a c_{d} b c} \cos \left(\xi_{1}+\gamma\right),
$$

where $\xi_{1}$ is a combination of $B$ and $D$ phase shifts, $\delta_{B 2}-\delta_{B 1}+\delta_{C}-\delta_{C d}$ and is unknown. Similarly, the decay rates for the other processes are

$$
\begin{aligned}
\Gamma\left(B^{+} \rightarrow\left[K^{-} \pi^{+}\right] K^{+}\right) & =a c_{d}+b c+2 \sqrt{a c_{d} b c} \cos \left(\xi_{1}-\gamma\right) \\
\Gamma\left(B^{-} \rightarrow\left[K^{+} K^{-}\right] K^{-}\right) & =a c_{C P}+b c_{C P}+2 \sqrt{a b c_{C P}^{2}} \cos \left(\delta_{B}-\gamma\right) \\
\Gamma\left(B^{+} \rightarrow\left[K^{+} K^{-}\right] K^{+}\right) & =a c_{C P}+b c_{C P}+2 \sqrt{a b c_{C P}^{2}} \cos \left(\delta_{B}+\gamma\right)
\end{aligned}
$$

where $\delta_{B}=\delta_{B 1}-\delta_{B 2}$.

In these four equations, the quantities which are known, or will be precisely known before this measurement is attempted are the decay widths $a, c_{d}, c$ and $c_{C P}$. The unknowns are the decay width $b$, two strong phase shifts $\xi_{1}$ and $\delta_{B}$ and the weak phase shift $\gamma$. Thus the four equations may be solved for the four unknowns. We can find $\sin \gamma$ with a two-fold ambiguity. If more decay modes are added the ambiguity can be removed. The $B^{-}$decay mode can be changed from a $K^{-}$to a $K^{*-}$, which could change the strong $B$ decay phase shift, or a different $D^{\circ}$ decay mode can be used, such as $K^{-} \pi^{+} \pi^{+} \pi^{-}$, which would change the strong $D$ decay phase shift. In the latter case, we have to worry about differences in strong phase shifts between $D^{\circ}$ and $\bar{D}^{\circ}$ due to resonant structure, but use of this mode can shed some information on ambiguity removal.

Comparison of the solutions found here and using $B_{s} \rightarrow D_{s}^{ \pm} K^{\mp}$ as described in the previous section are likely to remove the ambiguities. 


\subsubsection{Measurement of $\gamma$ Using $B \rightarrow K \pi$ and $B \rightarrow \pi \pi$ Decay Rates and Asymmetries}

CLEO has observed the pseudoscalar-pseudoscalar decays $B^{o} \rightarrow K^{\mp} \pi^{ \pm}, B^{-} \rightarrow K^{-} \pi^{o}, B^{-} \rightarrow$ $K^{\circ} \pi^{-}$, all with branching ratios around $1.5 \times 10^{-5}$ and observed or set upper limits on the dipion final states [34]. Therefore, the Penguin and Tree contributions for $B \rightarrow K \pi$ probably do not differ by more than a factor of five, so they can produce observable CP violating effects.

Proposals for extracting information on $\gamma$ have been made using the following experimental ratios:

$$
\begin{aligned}
R & =\frac{\tau\left(B^{+}\right)}{\tau\left(B^{0}\right)} \frac{\mathcal{B}\left(B^{0} \rightarrow \pi^{-} K^{+}\right)+\mathcal{B}\left(\bar{B}^{0} \rightarrow \pi^{+} K^{-}\right)}{\mathcal{B}\left(B^{+} \rightarrow \pi^{+} K^{0}\right)+\mathcal{B}\left(B^{-} \rightarrow \pi^{-} \bar{K}^{0}\right)}, \\
R_{*} & =\frac{\mathcal{B}\left(B^{+} \rightarrow \pi^{+} K^{0}\right)+\mathcal{B}\left(B^{-} \rightarrow \pi^{-} \bar{K}^{0}\right)}{2\left[\mathcal{B}\left(B^{+} \rightarrow \pi^{0} K^{+}\right)+\mathcal{B}\left(B^{-} \rightarrow \pi^{0} K^{-}\right)\right]},
\end{aligned}
$$

The first, $R$, is by Fleischer and Mannel [48], and the second $R_{*}$, is by Neubert and Rosner [49], who updated an older suggestion of Gronau and Rosner [50]. The latter paper prompted much theoretical discussion about the effects of isospin conservation and rescattering $[51,52$, $53,54]$. A recent paper of Neubert [55] takes into account these criticisms and provides a framework to limit $\gamma$.

More information is obtainable if the CP averaged $\pi^{ \pm} \pi^{o}$ branching ratios are also measured, and a $\mathrm{CP}$ violating observable defined as

$$
\widetilde{A} \equiv \frac{A_{\mathrm{CP}}\left(\pi^{0} K^{+}\right)}{R_{*}}-A_{\mathrm{CP}}\left(\pi^{+} K^{0}\right)
$$

where for example

$$
A_{\mathrm{CP}}\left(\pi^{0} K^{+}\right)=\frac{\Gamma\left(B^{+} \rightarrow \pi^{o} K^{+}\right)-\Gamma\left(B^{-} \rightarrow \pi^{o} K^{-}\right)}{\Gamma\left(B^{+} \rightarrow \pi^{o} K^{+}\right)+\Gamma\left(B^{-} \rightarrow \pi^{o} K^{-}\right)} .
$$

To summarize Neubert's strategy for determining $\gamma$ : From measurements of the CPaveraged branching ratio for the decays $B^{ \pm} \rightarrow \pi^{ \pm} \pi^{0}, B^{ \pm} \rightarrow \pi^{ \pm} K^{0}$ and $B^{ \pm} \rightarrow \pi^{0} K^{ \pm}$, the ratio $R_{*}$ and a parameter $\bar{\varepsilon}_{3 / 2}$ are determined. Next, from measurements of the rate asymmetries in the decays $B^{ \pm} \rightarrow \pi^{ \pm} K^{0}$ and $B^{ \pm} \rightarrow \pi^{0} K^{ \pm}$the quantity $\widetilde{A}$ is determined.

In Fig. 1.14, we show the contour bands as given by Neubert in the $\phi-\gamma$ plane. Here $\phi$ is a strong interaction phase-shift. Assuming that $\sin \gamma>0$ as suggested by the global analysis of the unitarity triangle, the sign of $A$ determines the sign of $\sin \phi$. In the plot, we assume here that $0^{\circ} \leq \phi \leq 180^{\circ}$. For instance, if $R_{*}=0.7$ and $\widetilde{A}=0.2$, then the two solutions are $(\gamma, \phi) \approx\left(98^{\circ}, 25^{\circ}\right)$ and $(\gamma, \phi) \approx\left(153^{\circ}, 67^{\circ}\right)$, only the first of which is allowed by the upper bound $\gamma<105^{\circ}$ following from the global analysis of the unitarity triangle

shown here (section 1.2 or in [15]). It is evident that the contours are rather insensitive to the rescattering effects. According to Neubert, the combined theoretical uncertainty is of order $\pm 10^{\circ}$ on the extracted value of $\gamma$. 


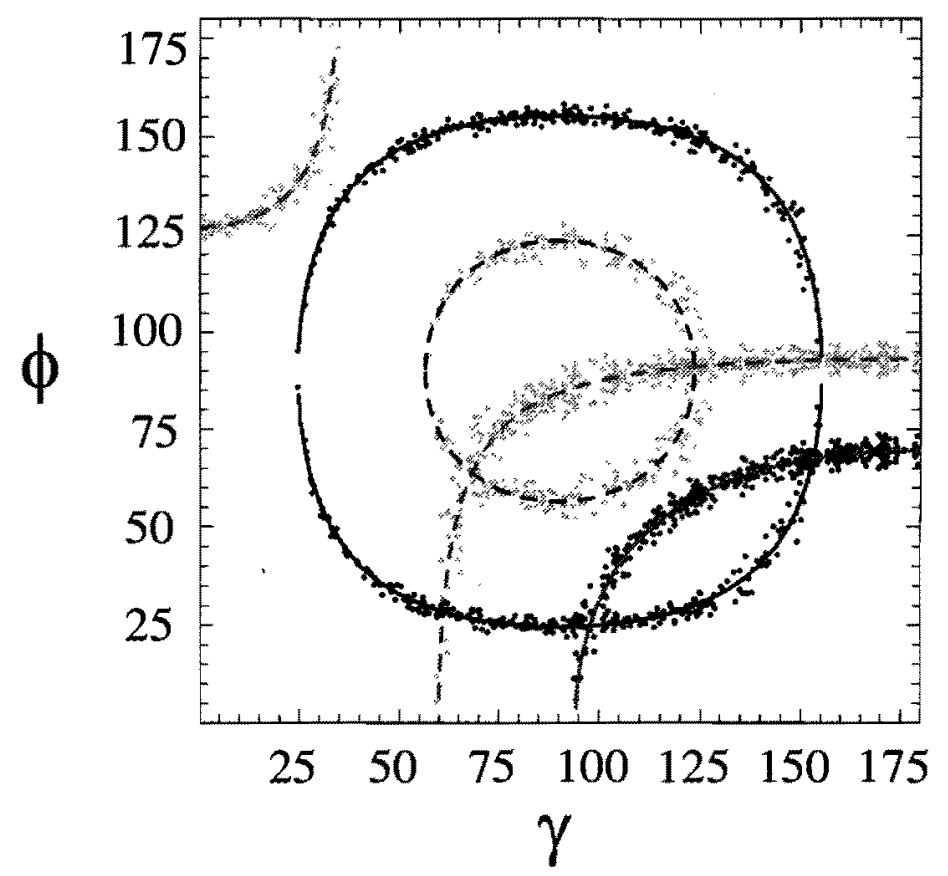

Figure 1.14: Contour plots from Neubert [55] for the quantities $R_{*}$ ("hyperbolas") and $\widetilde{A}$ ("circles") plotted in the $\phi-\gamma$ plane. The units are degrees. The scatter plots show the results including rescattering effects, while the lines refer to $\varepsilon_{a}=0$. The solid curves correspond to the contours for $R_{*}=0.7$ and $\widetilde{A}=0.2$, the dashed ones to $R_{*}=0.9$ and $\widetilde{A}=0.4$.

From the contour plots for the quantities $R_{*}$ and $\widetilde{A}$ the phases $\gamma$ and $\phi$ can then be extracted up to discrete ambiguities. There are also errors in theoretical parameters that must be accounted for.

\subsubsection{Measurement of $\gamma$ Using CP Asymmetries in $B^{o} \rightarrow \pi^{+} \pi^{-}$and $\boldsymbol{B}_{s}^{o} \rightarrow \boldsymbol{K}^{+} \boldsymbol{K}^{-}$}

Yet another interesting method for determining $\gamma$ has been suggested by Fleischer [56]. The decays $B^{o} \rightarrow \pi^{+} \pi^{-}$and $B_{s}^{o} \rightarrow K^{+} K^{-}$are related to each other by interchanging all down and strange quarks, which is called $U$-spin flavor symmetry [57]. Both channels can occur via Penguin or singly-Cabibbo suppressed tree levels diagrams, shown in Fig. 1.15.

For $B^{o} \rightarrow \pi^{+} \pi^{-}$the transition amplitude is given by

$$
A\left(B_{d}^{0} \rightarrow \pi^{+} \pi^{-}\right)=\lambda_{u}^{(d)}\left(A_{\mathrm{cc}}^{u}+A_{\mathrm{pen}}^{u}\right)+\lambda_{c}^{(d)} A_{\mathrm{pen}}^{c}+\lambda_{t}^{(d)} A_{\mathrm{pen}}^{t},
$$

where $A_{\mathrm{cc}}^{u}$ is due to the tree contributions, and the amplitudes $A_{\mathrm{pen}}^{j}$ describe penguin topologies with internal $j$ quarks $(j \in\{u, c, t\})$. These penguin amplitudes take into account both QCD and electroweak penguin contributions. The quantities

$$
\lambda_{j}^{(d)} \equiv V_{j d} V_{j b}^{*}
$$



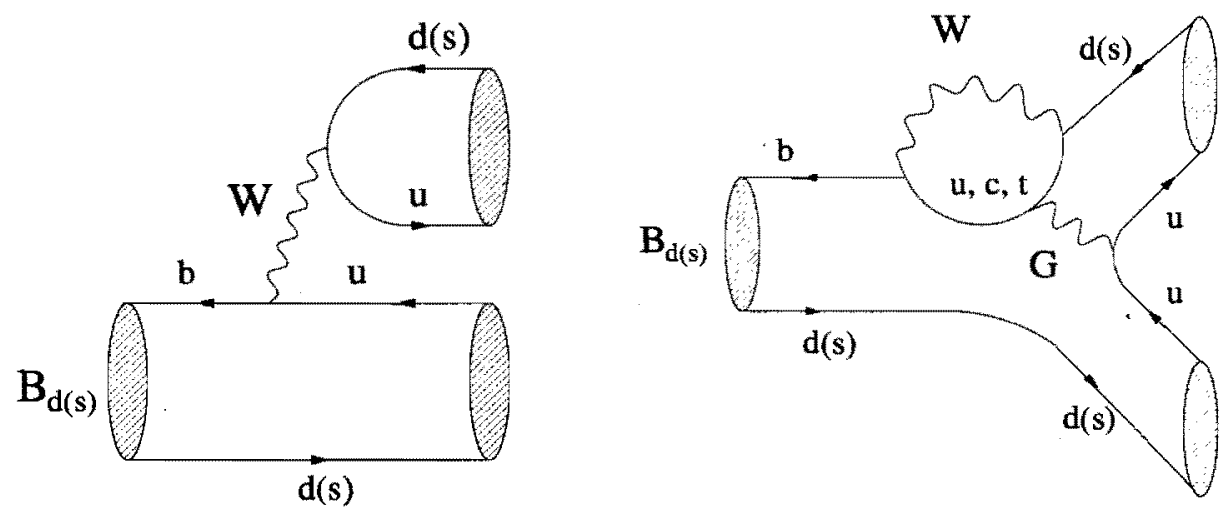

Figure 1.15: Feynman diagrams contributing to $B_{d} \rightarrow \pi^{+} \pi^{-}$and $B_{s} \rightarrow K^{+} K^{-}$(from Fleischer).

are the usual CKM factors. If we make use of the unitarity of the CKM matrix and use the Wolfenstein parameterization, we have

$$
A\left(B_{d}^{0} \rightarrow \pi^{+} \pi^{-}\right)=e^{i \gamma}\left(1-\frac{\lambda^{2}}{2}\right) \mathcal{C}\left[1-d e^{i \theta} e^{-i \gamma}\right]
$$

where

$$
\mathcal{C} \equiv \lambda^{3} A R_{b}\left(A_{\mathrm{cc}}^{u}+A_{\text {pen }}^{u t}\right)
$$

with $A_{\text {pen }}^{u t} \equiv A_{\text {pen }}^{u t}-A_{\text {pen }}^{t}$, and

$$
d e^{i \theta} \equiv \frac{1}{\left(1-\lambda^{2} / 2\right) R_{b}}\left(\frac{A_{\mathrm{pen}}^{c t}}{A_{\mathrm{cc}}^{u}+A_{\mathrm{pen}}^{u t}}\right)
$$

The quantity $A_{\text {pen }}^{c t}$ is defined in analogy to $A_{\text {pen }}^{u t}$, and the CKM factors are given by

$$
\lambda \equiv\left|V_{u s}\right|=0.22, \quad A \equiv \frac{1}{\lambda^{2}}\left|V_{c b}\right| \sim 0.8, \quad R_{b} \equiv \frac{1}{\lambda}\left|\frac{V_{u b}}{V_{c b}}\right| \sim 0.4
$$

For the following considerations, time-dependent CP asymmetries play a key role. In the case of a general $B_{d}$ decay into a final CP eigenstate $|f\rangle$, satisfying

$$
(\mathcal{C P})|f\rangle=\eta|f\rangle
$$

where $\eta$ here is not the Wolfenstein parameter, we have (see equation 1.37)

$$
\begin{aligned}
& a_{\mathrm{CP}}\left(B_{d}(t) \rightarrow f\right) \equiv \frac{\Gamma\left(B_{d}^{0}(t) \rightarrow f\right)-\Gamma\left(\overline{B_{d}^{0}}(t) \rightarrow f\right)}{\Gamma\left(B_{d}^{0}(t) \rightarrow f\right)+\Gamma\left(\overline{B_{d}^{0}}(t) \rightarrow f\right)} \\
& \quad=\mathcal{A}_{\mathrm{CP}}^{\mathrm{dir}}\left(B_{d} \rightarrow f\right) \cos \left(\Delta m_{d} t\right)+\mathcal{A}_{\mathrm{CP}}^{\mathrm{mix}}\left(B_{d} \rightarrow f\right) \sin \left(\Delta m_{d} t\right) .
\end{aligned}
$$


For the case of $B^{\circ} \rightarrow \pi^{+} \pi^{-}$, the decay amplitude takes the same form as (1.59), and we obtain the following expressions for the "direct" and "mixing-induced" CP-violating observables:

$$
\begin{aligned}
& \mathcal{A}_{\mathrm{CP}}^{\mathrm{dir}}\left(B_{d} \rightarrow f\right)=-\left[\frac{2 d \sin \theta \sin \gamma}{1-2 d \cos \theta \cos \gamma+d^{2}}\right] \\
& \mathcal{A}_{\mathrm{CP}}^{\mathrm{mix}}\left(B_{d} \rightarrow f\right)=\eta\left[\frac{\sin (2 \beta+2 \gamma)-2 d \cos \theta \sin (2 \beta+\gamma)+d^{2} \sin 2 \beta}{1-2 d \cos \theta \cos \gamma+d^{2}}\right],
\end{aligned}
$$

where $\eta$ is equal to +1 ; for negligible values of the "penguin parameter" $d$, we have $\mathcal{A}_{C P}^{\mathrm{mix}}\left(B_{d} \rightarrow\right.$ $\left.\pi^{+} \pi^{-}\right)=\sin (2 \beta+2 \gamma)=-\sin (2 \alpha)$. However, the penguin contributions are expected to play an important role.

Consider now the decay $B_{s}^{0} \rightarrow K^{+} K^{-}$. It originates from $\bar{b} \rightarrow \bar{u} u \bar{s}$ quark-level processes, as can be seen in Fig. 1.15. Using a notation similar to that in (1.59), we obtain

$$
A\left(B_{s}^{0} \rightarrow K^{+} K^{-}\right)=e^{i \gamma} \lambda \mathcal{C}^{\prime}\left[1+\left(\frac{1-\lambda^{2}}{\lambda^{2}}\right) d^{\prime} e^{i \theta^{\prime}} e^{-i \gamma}\right]
$$

where

$$
\mathcal{C}^{\prime} \equiv \lambda^{3} A R_{b}\left(A_{\mathrm{cc}}^{u^{\prime}}+A_{\mathrm{pen}}^{u t^{\prime}}\right)
$$

and

$$
d^{\prime} e^{i \theta^{\prime}} \equiv \frac{1}{\left(1-\lambda^{2} / 2\right) R_{b}}\left(\frac{A_{\mathrm{pen}}^{c t^{\prime}}}{A_{\mathrm{cc}}^{u^{\prime}}+A_{\mathrm{pen}}^{u t^{\prime}}}\right)
$$

correspond to (1.60) and (1.61), respectively. The primes remind us that we are dealing with a $\bar{b} \rightarrow \bar{s}$ transition. It should be emphasized that (1.59) and (1.67) are completely general parameterizations of the $B_{d}^{0} \rightarrow \pi^{+} \pi^{-}$and $B_{s}^{0} \rightarrow K^{+} K^{-}$decay amplitudes within the Standard Model, relying only on the unitarity of the CKM matrix. In particular, these expressions take into account also final-state interaction effects, which can be considered as long-distance penguin topologies with internal up- and charm-quark exchanges.

There may be a sizeable width difference $\Delta \Gamma_{s} \equiv \Gamma_{\mathrm{H}}^{(s)}-\Gamma_{\mathrm{L}}^{(s)}$ between the $B_{s}$ mass eigenstates [58], which may allow studies of CP violation with "untagged" $B_{s}$ data samples [23]. Such untagged rates take the following form:

$$
\Gamma\left(B_{s}^{0}(t) \rightarrow f\right)+\Gamma\left(\overline{B_{s}^{0}}(t) \rightarrow f\right) \propto R_{\mathrm{H}} e^{-\Gamma_{\mathrm{H}}^{(s)} t}+R_{\mathrm{L}} e^{-\Gamma_{\mathrm{L}}^{(s)} t}
$$

whereas the time-dependent $\mathrm{CP}$ asymmetry is given by

$$
\begin{aligned}
& a_{\mathrm{CP}}\left(B_{s}(t) \rightarrow f\right) \equiv \frac{\Gamma\left(B_{s}^{0}(t) \rightarrow f\right)-\Gamma\left(\overline{B_{s}^{0}}(t) \rightarrow f\right)}{\Gamma\left(B_{s}^{0}(t) \rightarrow f\right)+\Gamma\left(\overline{B_{s}^{0}}(t) \rightarrow f\right)} \\
& =2 e^{-\Gamma_{s} t}\left[\frac{\mathcal{A}_{C P}^{\mathrm{dir}}\left(B_{s} \rightarrow f\right) \cos \left(\Delta m_{s} t\right)+\mathcal{A}_{\mathrm{CP}}^{\mathrm{mix}}\left(B_{s} \rightarrow f\right) \sin \left(\Delta m_{s} t\right)}{e^{-\Gamma_{\mathrm{H}}^{(s)} t}+e^{-\Gamma_{\mathrm{L}}^{(s)} t}+\mathcal{A}_{\Delta \Gamma}\left(B_{s} \rightarrow f\right)\left(e^{-\Gamma_{\mathrm{H}}^{(s)} t}-e^{-\Gamma_{\mathrm{L}}^{(s)} t}\right)}\right]
\end{aligned}
$$


with $\mathcal{A}_{\Delta \Gamma}\left(B_{s} \rightarrow f\right)=\left(R_{\mathrm{H}}-R_{\mathrm{L}}\right) /\left(R_{\mathrm{H}}+R_{\mathrm{L}}\right)$. If the $B_{s}^{0} \rightarrow f$ decay amplitude takes the same form as (1.67), we have

$$
\begin{gathered}
\mathcal{A}_{\mathrm{CP}}^{\mathrm{dir}}\left(B_{s} \rightarrow f\right)=+\left[\frac{2 \tilde{d}^{\prime} \sin \theta^{\prime} \sin \gamma}{1+2 \tilde{d}^{\prime} \cos \theta^{\prime} \cos \gamma+\tilde{d}^{\prime 2}}\right] \\
\mathcal{A}_{\mathrm{CP}}^{\mathrm{mix}}\left(B_{s} \rightarrow f\right)=+\eta\left[\frac{\sin (2 \chi+2 \gamma)+2 \tilde{d}^{\prime} \cos \theta^{\prime} \sin (2 \chi+\gamma)+\tilde{d}^{\prime 2} \sin 2 \chi}{1+2 \tilde{d}^{\prime} \cos \theta^{\prime} \cos \gamma+\tilde{d}^{\prime 2}}\right] \\
\mathcal{A}_{\Delta \Gamma}\left(B_{s} \rightarrow f\right)=-\eta\left[\frac{\cos (2 \chi+2 \gamma)+2 \tilde{d}^{\prime} \cos \theta^{\prime} \cos (2 \chi+\gamma)+\tilde{d}^{\prime 2} \cos 2 \chi}{1+2 \tilde{d}^{\prime} \cos \theta^{\prime} \cos \gamma+\tilde{d}^{\prime 2}}\right] .
\end{gathered}
$$

These observables are not independent quantities, and satisfy the relation

$$
\left[\mathcal{A}_{\mathrm{CP}}^{\mathrm{dir}}\left(B_{s} \rightarrow f\right)\right]^{2}+\left[\mathcal{A}_{\mathrm{CP}}^{\mathrm{mix}}\left(B_{s} \rightarrow f\right)\right]^{2}+\left[\mathcal{A}_{\Delta \Gamma}\left(B_{s} \rightarrow f\right)\right]^{2}=1
$$

In the general expressions (1.72)-(1.74), we have introduced the abbreviation

$$
\tilde{d}^{\prime} \equiv\left(\frac{1-\lambda^{2}}{\lambda^{2}}\right) d^{\prime}
$$

and $2 \chi=2 \arg \left(V_{t s}^{*} V_{t b}\right)$ denotes the $B_{s}^{o}-\overline{B_{s}^{0}}$ mixing phase. Within the Standard Model, we have $2 \chi \approx 0.03$ due to a Cabibbo suppression of $\mathcal{O}\left(\lambda^{2}\right)$, implying that $2 \chi$ is very small. This phase can be determined using $B_{s} \rightarrow J / \psi \eta^{\prime}$ decays (see section 1.9).

Since the decays $B_{d} \rightarrow \pi^{+} \pi^{-}$and $B_{s} \rightarrow K^{+} K^{-}$are related to each other by interchanging all strange and down quarks, the $U$-spin flavor symmetry of strong interactions implies

$$
\begin{aligned}
& d^{\prime}=d \\
& \theta^{\prime}=\theta .
\end{aligned}
$$

In contrast to certain isospin relations, electroweak penguins do not lead to any problems in the $U$-spin relations (1.77) and (1.78), according to Fleischer.

In general we have five physics quantities of interest, $2 \chi, d, \theta, 2 \beta$ and $\gamma$. Let us now assume that $\sin (2 \beta)$ will be measured and $\sin (2 \chi)$ either measured or tightly limited. Only $d, \theta$ and $\gamma$ then need to be determined.

We have four possible measured quantities provided by the time-dependent CP asymmetries of the modes $B_{d} \rightarrow \pi^{+} \pi^{-}$and $B_{s} \rightarrow K^{+} K^{-}$. These four quantities are $\mathcal{A}_{\mathrm{CP}}^{\text {mix }}\left(B_{s} \rightarrow\right.$ $\left.K^{+} K^{-}\right), \mathcal{A}_{\mathrm{CP}}^{\mathrm{mix}}\left(B_{d} \rightarrow \pi^{+} \pi^{-}\right), \mathcal{A}_{\mathrm{CP}}^{\mathrm{dir}}\left(B_{s} \rightarrow K^{+} K^{-}\right)$and $\mathcal{A}_{\mathrm{CP}}^{\mathrm{dir}}\left(B_{d} \rightarrow \pi^{+} \pi^{-}\right)$. To implement this plan we need measure only 3 of these four quantities, or combinations of them. For example, it may be difficult to independently determine $\mathcal{A}_{\mathrm{CP}}^{\mathrm{mix}}\left(B_{d} \rightarrow \pi^{+} \pi^{-}\right)$and $\mathcal{A}_{\mathrm{CP}}^{\mathrm{dir}}\left(B_{d} \rightarrow \pi^{+} \pi^{-}\right)$, because of the small number of observable $B^{o}$ oscillations before the exponential decay reduces the number of events too much. However, the sum

$$
\begin{aligned}
a_{C P}^{\pi^{+} \pi^{-}} & =\int_{0}^{\infty} \mathcal{A}_{\mathrm{CP}}^{\mathrm{dir}} \cos \left(\Delta m_{d} t\right)+\mathcal{A}_{\mathrm{CP}}^{\mathrm{mix}} \sin \left(\Delta m_{d} t\right) \\
& =\frac{1}{1+x^{2}} \mathcal{A}_{\mathrm{CP}}^{\mathrm{dir}}+\frac{x}{1+x^{2}} \mathcal{A}_{\mathrm{CP}}^{\mathrm{mix}}
\end{aligned}
$$


can be determined and used with the other two measurements from $B_{s}^{o} \rightarrow K^{+} K^{-}$. Clearly other scenarios are possible.

\subsubsection{Opportunities with $B_{s}$ Mesons if $\Delta \Gamma$ is $\sim \mathbf{1 0 \%}$}

Measurement of $\Delta \Gamma$ can be used to estimate in an interesting but model dependent manner the value of $\Delta m_{s}$ and thus provides a redundant check on $B_{s}$ mixing measurements [23].

Should a large enough $\Delta \Gamma$ be determined there exist other possible ways to determine some of the interesting physics quantities discussed above. Some of these studies can be done without flavor tagging. In fact, the time evolution of untagged observables for a $B_{s}$ decay into a vector-vector final state is proportional to

$$
\left(e^{-\Gamma_{H} t}-e^{-\Gamma_{L} t}\right) \sin \phi_{C K M}
$$

where $\phi_{C K M}$ is a CP violating angle from the CKM matrix and depends on the specific decay mode.

In general the angular distribution for $B_{s} \rightarrow V V$ is expressed in terms of transversity in a manner similar to equation 1.46 , with the major difference being that the angular variables are time dependent. The time evolution of the decay $B_{s} \rightarrow J / \psi \phi$ is given in Table 1.4 [59].

\begin{tabular}{cc}
\hline \hline Observable & Time evolution \\
\hline$\left|A_{0}(t)\right|^{2}$ & $\left|A_{0}(0)\right|^{2}\left[e^{-\Gamma_{L} t}-e^{-\bar{\Gamma} t} \sin (\Delta m t) \sin (2 \chi)\right]$ \\
$\left|A_{\|}(t)\right|^{2}$ & $\left|A_{\|}(0)\right|^{2}\left[e^{-\Gamma_{L} t}-e^{-\bar{\Gamma} t} \sin (\Delta m t) \sin (2 \chi)\right]$ \\
$\left|A_{\perp}(t)\right|^{2}$ & $\left|A_{\perp}(0)\right|^{2}\left[e^{-\Gamma_{H} t}+e^{-\bar{\Gamma} t} \sin (\Delta m t) \sin (2 \chi)\right]$ \\
\hline $\operatorname{Re}\left(A_{0}^{*}(t) A_{\|}(t)\right)$ & $\left|A_{0}(0) \| A_{\|}(0)\right| \cos \left(\delta_{2}-\delta_{1}\right)\left[e^{-\Gamma_{L} t}-e^{-\bar{\Gamma} t} \sin (\Delta m t) \sin (2 \chi)\right]$ \\
$\operatorname{Im}\left(A_{\|}^{*}(t) A_{\perp}(t)\right)$ & $\left|A_{\|}(0) \| A_{\perp}(0)\right|\left[e^{-\bar{\Gamma} t} \sin \left(\delta_{1}-\Delta m t\right)\right.$ \\
& $\left.+\frac{1}{2}\left(e^{-\Gamma_{H} t}-e^{-\Gamma_{L} t}\right) \cos \left(\delta_{1}\right) \sin (2 \chi)\right]$ \\
$\operatorname{Im}\left(A_{0}^{*}(t) A_{\perp}(t)\right)$ & $\left|A_{0}(0) \| A_{\perp}(0)\right|\left[e^{-\bar{\Gamma} t} \sin \left(\delta_{2}-\Delta m t\right)\right.$ \\
& $\left.+\frac{1}{2}\left(e^{-\Gamma_{H} t}-e^{-\Gamma_{L} t}\right) \cos \left(\delta_{2}\right) \sin (2 \chi)\right]$ \\
\hline \hline
\end{tabular}

Table 1.4: Time evolution of the decay $B_{s} \rightarrow J / \psi\left(\rightarrow l^{+} l^{-}\right) \phi\left(\rightarrow K^{+} K^{-}\right)$of an initially (i.e. at $t=0)$ pure $B_{s}$ meson. $\delta_{1,2}$ are strong phase shifts. by

Combining with the decay of the $\bar{B}_{s}$ the time evolution of the untagged sample is given

$$
\frac{d^{3} \Gamma\left(J / \psi\left(\rightarrow l^{+} l^{-}\right) \phi\left(\rightarrow K^{+} K^{-}\right)\right)}{d \cos \theta d \varphi d \cos \psi} \propto \frac{9}{16 \pi}\left[2\left|A_{0}(0)\right|^{2} e^{-\Gamma_{L} t} \cos ^{2} \psi\left(1-\sin ^{2} \theta \cos ^{2} \varphi\right)\right.
$$




$$
\begin{gathered}
+\sin ^{2} \psi\left\{\left|A_{\|}(0)\right|^{2} e^{-\Gamma_{L} t}\left(1-\sin ^{2} \theta \sin ^{2} \varphi\right)+\left|A_{\perp}(0)\right|^{2} e^{-\Gamma_{H} t} \sin ^{2} \theta\right\} \\
+\frac{1}{\sqrt{2}} \sin 2 \psi\left\{\left|A_{0}(0) \| A_{\|}(0)\right| \cos \left(\delta_{2}-\delta_{1}\right) e^{-\Gamma_{L} t} \sin ^{2} \theta \sin 2 \varphi\right\} \\
+\left\{\frac{1}{\sqrt{2}}\left|A_{0}(0) \| A_{\perp}(0)\right| \cos \delta_{2} \sin 2 \psi \sin 2 \theta \cos \varphi\right. \\
\left.\left.-\left|A_{\|}(0) \| A_{\perp}(0)\right| \cos \delta_{1} \sin ^{2} \psi \sin 2 \theta \sin \varphi\right\} \frac{1}{2}\left(e^{-\Gamma_{H} t}-e^{-\Gamma_{L} t}\right) \delta \phi\right] .
\end{gathered}
$$

Thus a study of the time dependent angular distributions can lead to a measurement of $\sin (2 \chi)$, especially if $\Delta \Gamma$ is determined before hand. It is also possible to integrate over two of the angles if statistics is limited. The distribution in $J / \psi$ decay angle can be written as

$$
\frac{d \Gamma(t)}{d \cos \theta} \propto\left(\left|A_{0}(t)\right|^{2}+\left|A_{\|}(t)\right|^{2}\right) \frac{3}{8}\left(1+\cos ^{2} \theta\right)+\left|A_{\perp}(t)\right|^{2} \cdot \frac{3}{4} \sin ^{2} \theta
$$

where the CP violating angle originates from the imaginary parts of the interference terms in the $A$ 's.

Other final states have been suggested that provide a measurement of $\gamma$ using the above ideas. One particularly interesting set of decays is $B_{s} \rightarrow K^{*+} K^{*-}$ and $B_{s} \rightarrow K^{* o} \bar{K}^{* a}[60]$.

Finally, it is important to realize that determination of a non-zero $\Delta \Gamma$ allows the mea-

surement of $\operatorname{Re}\left(\frac{q}{p} \cdot \frac{\bar{A}}{A}\right)$, that in turn allows the removal of the ambiguities in the CKM angle of interest [23]. For the $B_{s}$ decays mentioned here this could be $\gamma$ or $\chi$.

\subsection{Summary of CKM Tests}

Our goal is to measure separately the four angles $\alpha, \beta, \gamma$ and $\chi$. To extract the angles we will measure usually either the $\sin (2 \phi)$ or the $\sin \phi$ which leads to discrete ambiguities in the determination of $\phi$.

For example, any determination of $\sin (2 \phi)$, where $\phi$ is any angle found using the interference of a single decay amplitude with a mixing amplitude has a four-fold ambiguity, where $\phi, \pi / 2-\phi, \pi+\phi, 3 \pi / 2-\phi$ are all allowed solutions. One may take the point of view that we know that $\eta$ is a positive quantity and thus we can eliminate two of the four possibilities. However, this would be dangerous in that it could lead to our missing new physics. The only evidence that $\eta$ is positive arises from the measurements of $\epsilon$ and $\epsilon^{\prime}$ and the fact that theoretical calculations give $B_{K}>0$ for $\epsilon$. Even accepting that $K_{L}$ decays give $\eta>0$, it would be foolhardy to miss new physics just because we now assume that $\eta$ must be positive rather than insisting on a clean resolution of the ambiguities that could show a contradiction.

Each of these four angles can be measured most easily using the modes listed in Table 1.5 Other modes which also may turn out to be useful include $B^{o} \rightarrow D^{*+} \pi^{-}$and its charge- 
Table 1.5: Primary modes useful for measuring CP asymmetries for different CKM angles

\begin{tabular}{lc}
\hline \hline Decay Mode & Angle \\
\hline$B^{o} \rightarrow J / \psi K_{S}$ & $\sin (2 \beta)$ \\
$B^{o} \rightarrow \rho \pi \rightarrow \pi^{+} \pi^{-} \pi^{o}$ & $\sin (2 \alpha)$ \\
$B_{s}^{o} \rightarrow D_{s}^{\mp} K^{ \pm}$ & $\sin \gamma$ \\
$B^{-} \rightarrow \overline{D^{o}} K^{-}$, and c.c. & $\sin \gamma$ \\
$B_{s} \rightarrow J / \psi \eta^{\prime}$ & $\sin (2 \chi)$ \\
\hline \hline
\end{tabular}

conjugate [61], which measures $\sin (-2 \beta-\gamma)$ albeit with a small $\approx 1 \%$ predicted asymmetry, ${ }^{6}$ and $B \rightarrow K \pi$ modes which can be used to find $\gamma$ albeit with theoretical uncertainties.

There are three alternative ways to measure $\gamma$, discussed in section 1.8, which serve both to remove ambiguities and perform checks. It will be much more difficult to find other modes to check $\alpha$, however. One approach is to measure the CP asymmetry in $B^{\circ} \rightarrow \pi^{+} \pi^{-}$and use theoretical models to estimate the effects of Penguin pollution. Minimally, a great deal would be learned about the models. It also turns out that the third ambiguity in $\alpha$ can be removed by comparing the CP violating asymmetry in $\pi^{+} \pi^{-}$with that found in $\rho \pi$ and using some mild theoretical assumptions [43]. After the three angles $\alpha, \beta$ and $\gamma$ have been measured, we need to check if they add up to $180^{\circ}$. A discrepancy here would show new physics. To be sure, this check is not complete if ambiguities have not been removed. (Even if the angles sum to $180^{\circ}$, new physics could hide.)

We also want to measure as precisely as possible the side of the bd triangle (see Fig. 1.4) that requires a precise measurement of $B_{s}$ mixing [63]. The other side is proportional to the magnitude of $V_{u b}$. This will no doubt be measured by $e^{+} e^{-} b$-factories and the precision will be limited by theoretical concerns if form-factors in the exclusive decays and $q^{2}$ distributions in the inclusive decays have been decisively measured.

New physics can add differently to the phases in different decay modes if it contributes differently to the relative decay amplitudes $A / \bar{A}$. Therefore it is interesting to measure $\mathrm{CP}$ violation in redundant modes. For example, the decay $B^{o} \rightarrow \phi K_{S}$ should also measure $\sin (2 \beta)$. If it is different than that obtained by $B^{o} \rightarrow J / \psi K_{S}$, that would be a strong indication of new physics [64]. We list in Table 1.6 other interesting modes to check $\sin (2 \beta)$. The branching ratios listed with errors have been measured $[65,66]$, while those without are theoretical estimates.

Silva and Wolfenstein [67] following Aleksan, Kayser and London [8] argue that the best way to reveal the presence of new physics is to measure $\chi$ and two of $\alpha, \beta$ and $\gamma$ and compare with magnitudes of some CKM elements. Recall, in the six CKM triangles in Fig. 1.1, there are four and only four independent phases. Since the angles $\alpha, \beta$ and $\gamma$, when properly measured must add to $180^{\circ}$, Silva and Wolfenstein take only two of them as independent. The angles $\beta$ and $\gamma$ are expected to be large in the Standard Model. $\chi$ is expected to be of

\footnotetext{
${ }^{6}$ To measure a CP asymmetry this way requires using equations 1.51 , and extracting the strong phase, amplitude ratio, and a small asymmetry: a very difficult task.
} 
Table 1.6: Other modes useful for cross-checking $\sin (2 \beta)$

\begin{tabular}{lc}
\hline & \\
Decay Mode & Branching Ratio \\
\hline$B^{o} \rightarrow \phi K_{S}$ & $(0.04-1.5) \times 10^{-5}$ \\
$B^{o} \rightarrow D^{+} D^{-}$ & $\approx 10^{-3}$ \\
$B^{o} \rightarrow D^{*+} D^{-}$ & $\approx 10^{-3}$ \\
$B^{o} \rightarrow \eta^{\prime} K^{o}$, & $(5.9 \pm 1.9) \times 10^{-5}$ \\
$B^{o} \rightarrow J / \psi\left(\pi^{o}, \eta\right.$ or $\left.\eta^{\prime}\right)$ & $(3.4 \pm 1.6) \times 10^{-5}$ \\
\hline \hline
\end{tabular}

the order $2 \lambda^{2} \eta \sim 0.02$ and $\chi^{\prime}$ of the order 0.003 .

There is no direct way of measuring the small angle $\chi^{\prime}$. However the angle $\chi$ can be measured by using $B_{s}$ decays. The most direct way is to measure the time dependent $\mathrm{CP}$ violation caused by the mixing of interference and decay in the modes $B_{s} \rightarrow J / \psi \eta, \eta \rightarrow \gamma \gamma$, or $B_{s} \rightarrow J / \psi \eta^{\prime}, \eta^{\prime} \rightarrow \rho^{o} \gamma$. The time resolution has to be excellent in order to resolve the fast $B_{s}$ oscillations. The all-charged mode $B_{s} \rightarrow J / \psi \phi$ can also be used [59], but this requires a complicated angular analysis and consequently a great deal of data.

Let us try and get some idea of the sizes of the angles $\alpha, \beta$ and $\gamma$. The expected range of angles derived from Fig. 1.4 is shown in Fig. 1.16. These plots show only the most likely

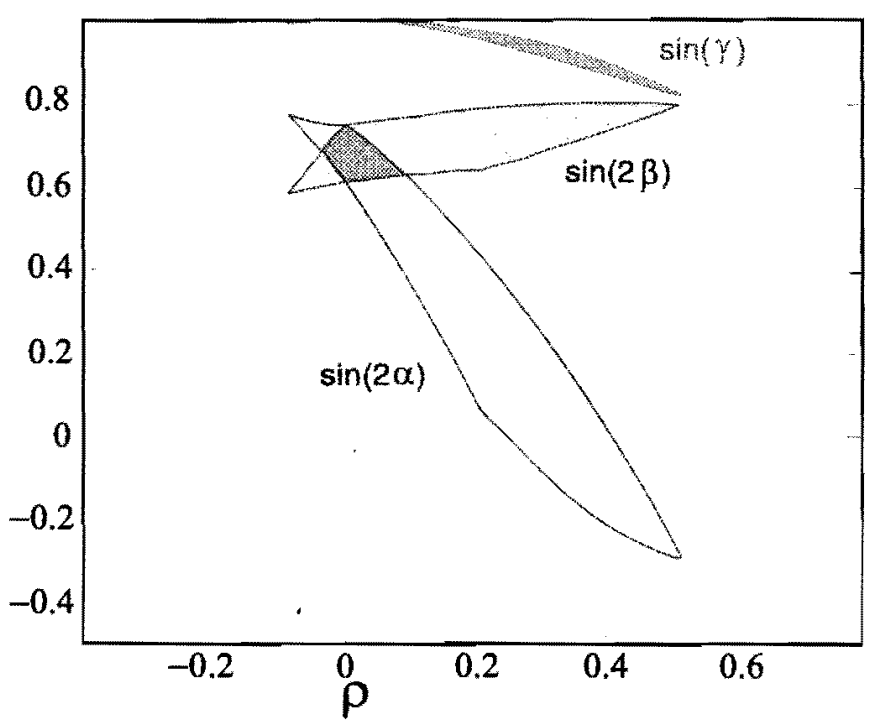

Figure 1.16: The "allowed" values for the three angles of the CKM triangle derived from the allowed, i.e. $1 \sigma$ overlap, region of Fig. 1.4.

values in the Standard Model. Recall that they are based on the overlap of $\pm 1 \sigma$ bands from constraints on $V_{u b} / V_{c b}, \epsilon$, and $B_{d}$ mixing. However, this gives us a good indication on where 
we should target our measuring potential. The angle $\chi$ as mentioned previously is expected to be small $\approx 2 \lambda^{2} \eta$.

Measurements of the magnitudes of CKM matrix elements all come with theoretical errors. Some of these are hard to estimate. We now try and view realistically how to combine $\mathrm{CP}$ violating phase measurements with the magnitude measurements to best test the Standard Model.

The best measured magnitude is that of $\left|V_{u s} / V_{u d}\right|=\lambda=0.2205 \pm 0.0018$, where the quoted error has approximately equal experimental and theoretical contributions [11]. Silva and Wolfeustein [67], along with Aleksan, Kayser and London [8] show that the Standard Model can be checked in a profound manner by seeing if:

$$
\sin \chi=\left|\frac{V_{u s}}{V_{u d}}\right|^{2} \frac{\sin \beta \sin \gamma}{\sin (\beta+\gamma)} .
$$

Here the precision of the check is limited by the measurement of $\sin \chi$, not of $\lambda$. This check is likely to reveal if new physics is present, even if other checks have not shown new physics. The example given by Silva and Wolfenstein [67] is a new phase added to the Standard Model mixing phase, which is missed in the measurement of $\alpha$ plus $\beta$ since it cancels in the sum. Two other checks can be performed, which have similar structures.

$$
\begin{aligned}
& \sin \chi=\left|\frac{V_{u b}}{V_{c b}}\right|^{2} \frac{\sin \gamma \sin (\beta+\gamma)}{\sin \beta}, \\
& \sin \chi=\left|\frac{V_{t d}}{V_{t s}}\right|^{2} \frac{\sin \beta \sin (\beta+\gamma)}{\sin \gamma} .
\end{aligned}
$$

Note that it is, in principle, possible to determine the magnitudes of $\left|V_{u b} / V_{c b}\right|$ and $\left|V_{t d} / V_{t s}\right|$, just by measuring the angles precisely enough. This is true because of the unitarity of the CKM matrix.

Before these precision tests can be carried out, it will be useful to check the consistency of the measurements of $\epsilon$ in $K_{L}$ decay, $\left|V_{u b} / V_{c b}\right|$ from semileptonic $b$ decay and the ratio of $x_{s} / x_{d}, B_{s} / B_{d}$ mixing parameters. The accuracy of the $\rho-\eta$ constraint from $\epsilon$ is currently determined mostly by $V_{c b}$ and the parameter $B_{K}$, which can only be calculated not measured. While the determination of $V_{c b}$ will improve in the next several years before it is limited by theoretical errors, the accuracy of $B_{K}$ will probably not improve, and it will remain difficult to assign a well defined error. Thus, the allowed band in $\epsilon$ will shrink somewhat, but it will remain relatively broad.

$B_{s}$ mixing may as yet be measured by SLD or CDF. If the oscillations prove to be fast enough, $\mathrm{BTeV}$ will make the first measurement. In either case, the ultimate utility of the measurement in restricting the region of the $\rho-\eta$ plane will be determined by the accuracy of the calculation of the ratio $f_{B_{d}}^{2} B_{B_{d}} / f_{B_{s}}^{2} B_{B_{s}}$, after the measurement of $x_{s}$ becomes accurate enough. These parameters have been and are being calculated on the lattice. The accuracy in the above ratio is estimated not to be better than about $10 \%$ [68]. 
The ultimate error on $\left|V_{u b}\right|$ will also be theoretical in origin. Experimental measurements from $e^{+} e^{-} B$ factories will perform extensive measurements of inclusive semileptonic $b \rightarrow u \ell \nu$ decays as well as exclusive decays to the $\pi \ell \nu$ and $\rho \ell \nu$ decays. The form-factors as well as the branching ratios will be measured and should help to restrict the models. However, at this time there isn't a compelling theoretical framework such as HQET for $b \rightarrow c \ell \nu$. Thus, we estimate the theoretical error to be currently on the order of $\pm 14 \%$, and hope for further improvements.

Thus the intersection of the mixing ratio band with the $V_{u b}$ band should provide a crossing point with reasonable errors. The measurement of each of the individual angles, $\alpha, \beta$ and $\gamma$ will need to be consistent within the error band, otherwise the Standard Model will be violated.

\subsection{Rare Decays as Probes beyond the Standard Model}

Rare decays have loops in the decay diagrams which makes them sensitive to high mass gauge bosons and fermions. Thus, they are sensitive to new physics. However, it must be kept in mind that any new effect must be consistent with already measured phenomena such as $B_{d}^{o}$ mixing and $b \rightarrow s \gamma$.

These processes are often called "Penguin" processes, for unscientific reasons [69]. A Feynman loop diagram is shown in Fig. 1.17 that describes the transition of a $b$ quark into a charged $-1 / 3 s$ or $d$ quark, which is effectively a neutral current transition. The dominant charged current decays change the $b$ quark into a charged $+2 / 3$ quark, either $c$ or $u$.

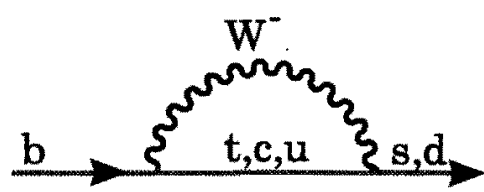

Figure 1.17: Loop or "Penguin" diagram for a $b \rightarrow s$ or $b \rightarrow d$ transition.

The intermediate quark inside the loop can be any charge $+2 / 3$ quark. The relative size of the different contributions arises from different quark masses and CKM elements. For $b \rightarrow s$, in terms of the Cabibbo angle $(\lambda=0.22)$, we have for $t: c: u-\lambda^{2}: \lambda^{2}: \lambda^{4}$. The mass dependence favors the $t$ loop, but the amplitude for $c$ processes can be quite large $\approx 30 \%$. Moreover, as pointed out by Bander, Silverman and Soni [70], interference can occur between $t, c$ and $u$ diagrams and lead to CP violation. In the Standard Model it is not expected to occur when $b \rightarrow s$, due to the lack of a CKM phase difference, but could occur when $b \rightarrow d$. In any case, it is always worth looking for this effect; all that needs to be done, for example, is to compare the number of $K^{*-} \gamma$ events with the number of $K^{*+} \gamma$ events.

There are other possibilities for physics beyond the Standard Model to appear. For example, the $W^{-}$in the loop can be replaced by some other charged object such as a Higgs; it is also possible for a new object to replace the $t$. 


\subsection{1 $b \rightarrow s \gamma$}

This process occurs when any of the charged particles in Fig. 1.17 emits a photon. CLEO first measured the inclusive rate [71] as well as the exclusive rate into $K^{*}(890) \gamma$ [72]. There is an updated CLEO measurement [73] using 1.5 times the original data sample and a new measurement from ALEPH [74].

To remove background CLEO used two techniques originally, one based on "event shapes" and the other on summing exclusively reconstructed $B$ samples. CLEO uses eight different shape variables [71], and defines a variable $r$ using a neural network to distinguish signal from background. The idea of the $B$ reconstruction analysis is to find the inclusive branching ratio by summing over exclusive modes. The allowed hadronic system is composed of either a $K_{S} \rightarrow \pi^{+} \pi^{-}$candidate or a $K^{\mp}$ combined with 1-4 pions, only one of which can be neutral. The restriction on the number and kind of pions maximizes efficiency while minimizing background. It does however lead to a model dependent error. Then both analysis techniques are combined. Currently, most of the statistical power of the analysis $(\sim 80 \%)$ comes from summing over the exclusive modes.

Fig. 1.18 shows the photon energy spectrum of the inclusive signal, compared with the model of Ali and Greub [75]. A fit to the model over the photon energy range from 2.1 to 2.7 $\mathrm{GeV} / \mathrm{c}$ gives the branching ratio result shown in Table 1.7, where the first error is statistical and the second systematic.

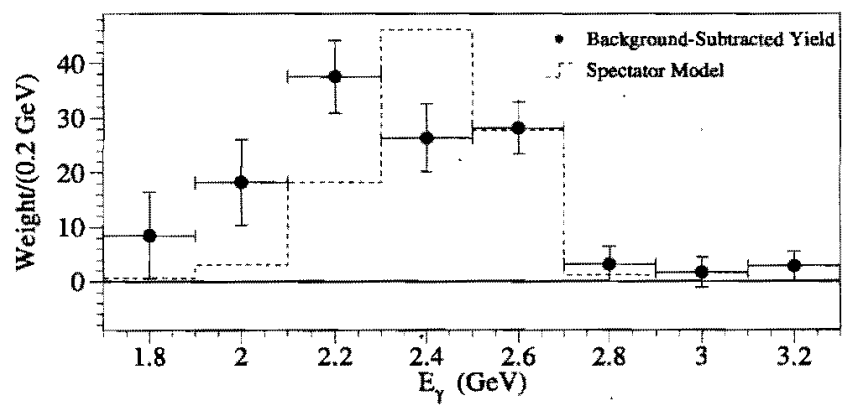

Figure 1.18: The background subtracted photon energy spectrum from CLEO. The dashed curve is a spectator model prediction from Ali and Greub.

Table 1.7: Experimental results for $b \rightarrow s \gamma$

\begin{tabular}{|lc|}
\hline Sample & branching ratio \\
\hline CLEO & $(3.15 \pm 0.35 \pm 0.41) \times 10^{-4}$ \\
ALEPH & $(3.11 \pm 0.80 \pm 0.72) \times 10^{-4}$ \\
Average & $(3.14 \pm 0.48) \times 10^{-4}$ \\
Theory[76] & $(3.28 \pm 0.30) \times 10^{-4}$ \\
\hline
\end{tabular}

ALEPH reduces the backgrounds by weighting candidate decay tracks in a $b \rightarrow s \gamma$ event 
by a combination of their momentum, impact parameter with respect to the main vertex and rapidity with respect to the $b$-hadron direction [74]. Their result is shown in Table 1.7. The world average experimental value is also given, as well as the theoretical prediction.

The Standard Model prediction is in good agreement with the data.

Hewett has given a good review of the many minimal supergravity models which are excluded by the data [77]. Improved experimental and theoretical accuracy are required to move beyond the Standard Model here. A measurement of $b \rightarrow d \gamma$ would be most interesting.

Triple gauge boson couplings are of great interest in checking the standard model. If there were an anomalous $W W \gamma$ coupling it would serve to change the Standard Model rate. $p \bar{p}$ collider experiments have also published results limiting such couplings [78]. In a twodimensional space defined by $\Delta \kappa$ and $\lambda$, the D0 constraint appears as a tilted ellipse and the $b \rightarrow s \gamma$ as nearly vertical bands. In the standard model both parameters are zero.

\subsubsection{The Exclusive Decays $K^{*} \gamma$ and $\rho \gamma$}

The exclusive branching ratio is far more difficult to predict than the inclusive. CLEO measures $\mathcal{B}\left(B \rightarrow K^{*}(890) \gamma\right)=(4.2 \pm 0.8 \pm 0.6) \times 10^{-5}$, with this exclusive final state comprising $(18 \pm 7) \%$ of the total $b \rightarrow s \gamma$ rate $[79]$.

CLEO also limits $\mathcal{B}(B \rightarrow \rho \gamma)<1.2 \times 10^{-5}$ at $90 \%$ confidence level [79]. This leads to a model dependent limit on $\left|V_{t d} / V_{t s}\right|^{2}<0.45-0.56$, which is not very significant. It may be possible that improved measurements can find a meaningful limit, although that has been disputed [80].

\subsection{3 $b \rightarrow s \ell^{+} \ell^{-}$}

The diagrams that contribute to $b \rightarrow s \ell^{+} \ell^{-}$, where $\ell$ refers to either an electron or muon are shown in Fig. 1.19.
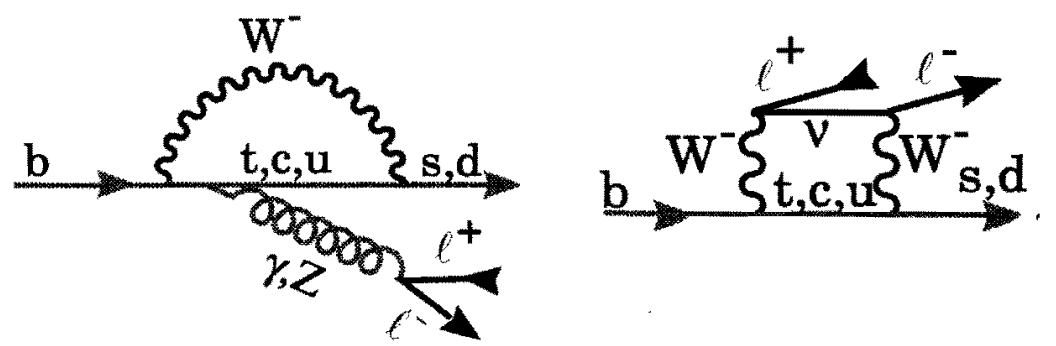

Figure 1.19: Loop or "Penguin" diagram for a $b \rightarrow s \ell^{+} \ell^{-}$transition.

Since more diagrams contribute here than in $b \rightarrow s \gamma$, different physics can be probed. CP violation can be looked at in both the branching ratios and the polarization of the lepton pair [81]. No signals have been seen as yet in any inclusive or exclusive modes. When searching for such decays, care must be taken to eliminate the mass region in the vicinity of the $J / \psi$ 
Table 1.8: Searches for $b \rightarrow s \ell^{+} \ell^{-}$decays

\begin{tabular}{lclc}
\hline \hline$b$ decay mode & $90 \%$ c.l. upper limit & Group & Ali et al Prediction [82] \\
\hline$s \mu^{+} \mu^{-}$ & $50 \times 10^{-6}$ & UA1 [83] & $(8 \pm 2) \times 10^{-6}$ \\
$K^{* o} \mu^{+} \mu^{-}$ & $5.7 \times 10^{-6}$ & CLEO [84] & \\
& $4.0 \times 10^{-6}$ & CDF [85] & $2.9 \times 10^{-6}$ \\
& $23 \times 10^{-6}$ & UA1 [83] & \\
$K^{* o} e^{+} e^{-}$ & $9.5 \times 10^{-6}$ & CLEO [84] & \\
$K^{-} \mu^{+} \mu^{-}$ & $13 \times 10^{-6}$ & CLEO [84] & $5.6 \times 10^{-6}$ \\
& $9.7 \times 10^{-6}$ & CLEO [84] & $0.6 \times 10^{-6}$ \\
$K^{-} e^{+} e^{-}$ & $5.2 \times 10^{-6}$ & CDF [85] & \\
\hline \hline
\end{tabular}

or $\psi^{\prime}$ resonances, lest these more prolific processes, that are not rare decays, contaminate the sample. The results of searches are shown in Table 1.8.

$\mathrm{BTeV}$ has the ability to search for both exclusive and inclusive dilepton final states. The inclusive measurement can be done following the techniques used by CLEO to discover inclusive $b \rightarrow s \gamma$ and set upper limits on $b \rightarrow s \ell^{+} \ell^{-}$. CLEO doesn't have vertex information, so they choose track combinations assigning a kaon hypothesis to one track and pion hypotheses to the other charged tracks. They allow up to four pions, only one of which can be neutral and proceed to reconstruct each combination as if it were an exclusive decay mode. If any combination succeeds, they keep it. BTeV can improve on this procedure in two ways. First of all $\mathrm{BTeV}$ will have $\mathrm{RICH} K \pi$ separation. Secondly we can insist that the charged particles are consistent with coming from a $b$ decay vertex. Of course, we lose the power of the beam energy constraint that is so efficient at rejecting background at the $\Upsilon(4 S)$. However, it is a detailed question as to whether or not we more than make up the rejection power by using our advantages.

$B$ 's can also decay into dilepton final states. The Standard Model diagrams are shown in Fig. 1.20. In (a) the decay rate is proportional to $\left|V_{u b}\right|^{2} f_{B}^{2}$. The diagram in (b) is much larger for $B_{s}$ than $B_{d}$, again the factor of $\left|V_{t s} / V_{t d}\right|^{2}$. Results of searches are given in Table 1.9 .
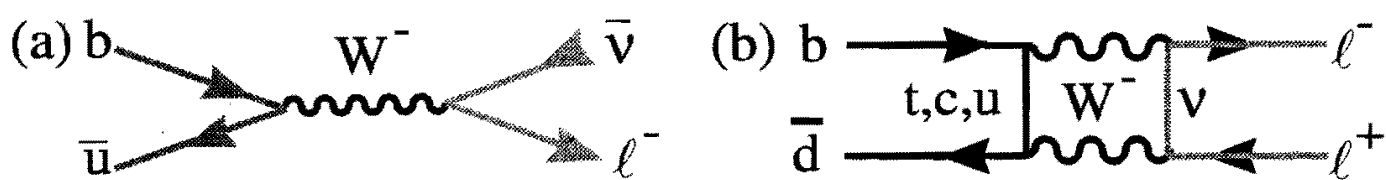

Figure 1.20: Decay diagrams resulting in dilepton final states. (a) is an annihilation diagram, and (b) is a box diagram.

Searches for rare decays modes make up an important part of the BTeV physics program. 
Table 1.9: Upper limits on $b \rightarrow$ dilepton decays $(@ 90 \%$ c.l.)

\begin{tabular}{|c|c|c|c|c|c|c|}
\hline & \multicolumn{2}{|c|}{$\mathcal{B}\left(B^{\circ} \rightarrow \ell^{+} \ell^{-}\right)$} & \multirow{2}{*}{$\begin{array}{c}\mathcal{B}\left(B_{s} \rightarrow \ell^{+} \ell^{-}\right) \\
\mu^{+} \mu^{-}\end{array}$} & \multicolumn{3}{|c|}{$\mathcal{B}\left(B^{-} \rightarrow \ell^{-} \bar{\nu}\right)$} \\
\hline & $e^{+} e^{-}$ & $\mu^{+} \mu^{-}$ & & $e^{-\pi}$ & $\mu-\bar{\nu}$ & $\tau^{-} \ddot{\nu}$ \\
\hline $\mathrm{SM}^{\dagger}$ & $2 \times 10^{-15}$ & $8 \times 10^{-11}$ & $2 \times 10^{-9}$ & $10^{-15}$ & $10^{-8}$ & $10^{-5}$ \\
\hline UA1 [83] & & $8.3 \times 10^{-6}$ & & & & \\
\hline CLEO [86] & $5.9 \times 10^{-6}$ & $5.9 \times 10^{-6}$ & & $1.5 \times 10^{-5}$ & $2.1 \times 10^{-5}$ & $2.2 \times 10^{-3}$ \\
\hline CDF [89] & & $2.0 \times 10^{-6}$ & $6.8 \times 10^{-6}$ & & & \\
\hline ALEPH [87] & & & & & & $1.8 \times 10^{-3}$ \\
\hline L3 $[90]$ & & & & & & $5.7 \times 10^{-4}$ \\
\hline
\end{tabular}

\subsection{The Search for Mixing and CP Violation in Charm Decays}

Predictions of the Standard Model contribution to mixing and CP violation in charm decay are small. Thus, this provides a good place to search for new physics.

The current experimental limit on charm mixing [91] is

$$
r_{D}=\frac{1}{2}\left[\left(\frac{\Delta m_{D}}{\Gamma}\right)^{2}+\left(\frac{\Delta \Gamma}{2 \Gamma}\right)^{2}\right]<5 \times 10^{-3} .
$$

while the Standard Model expectation is $\sim 10^{-6}$ [92] [93].

For CP violation the current limit is $\sim 10 \%$ [11], while the Standard Model expectation is $\sim 10^{-3}[92][94]$. BTeV can probably reach the Standard Model level of CP violation in charm decays. (The $D^{*+}$ provides a wonderful flavor tag.) 


\section{Bibliography}

[1] P. Langacker, "CP Violation and Cosmology" in CP Violation, ed. C. Jarlskog, World Scientific, Singapore p 552 (1989).

[2] So far CP violation has only been measured in decays of the $K^{o}$ meson. Results for $\epsilon$ are from 1964. Recent results from $\mathrm{KTeV}$ and $\mathrm{NA48}$ are in agreement with earlier measurements from NA31 for a value of $\epsilon^{\prime} / \epsilon$ on the order of $1-22 \times 10^{-4}$. In addition, CDF has seen an indication in $B^{o}$ decays. T. Affolder et al, Phys. Rev. D61 072005 (2000), hep-ex/9909003.

[3] A. D. Sakharov, JETP Lett. 6, 24 (1967).

[4] N. Cabibbo, Phys. Rev. Lett. 10, 531 (1963); M. Kobayashi and K. Maskawa, Prog. Theor. Phys. 49, 652 (1973).

[5]" L. Wolfenstein, Phys. Rev. Lett. 51, 1945 (1983).

[6] S. Stone, "Prospects For B-Physics In The Next Decade," in Techniques and Concepts of High-Energy Physics IX, ed. by T. Ferbel, NATO ASI Series, Plenum, NY (1996).

[7] M. Artuso, "Flavour Physics: The Questions, The Clues and the Challenges," Plenary talk at EPS HEP '99, Tampere, Finland, to appear in proceedings, hep-ph/9911347.

[8] R. Aleksan, B. Kayser and D. London, Phys. Rev. Lett. 73 (1994) 18 (hep-ph/9403341).

[9] M. Gaillard and B. Lee, Phys. Rev. D10, 897, (1974); J. Hagelin, Phys. Rev. D20, 2893, (1979); A. Ali and A. Aydin, Nucl. Phys. B148, 165 (1979); T. Brown and S. Pakvasa, Phys. Rev. D31, 1661, (1985); S. Pakvasa, Phys. Rev. D28, 2915, (1985); I. Bigi and A. Sanda, Phys. Rev. D29, 1393, (1984).

[10] J. Rosner, "The Cabibbo-Kobayashi-Maskawa Matrix," in B Decays, Revised 2nd Edition, ed. S. Stone, World Scientific, Singapore (1994), p470.

[11] Particle Data Group, C. Caso et al, The European Physical Journal C3 (1998) 1.

[12] L. Lellouch and C.-J. D. Lin, CERN-TH/99-344 (hep-ph/9912322); D. Becirevic et al, ROMA 1285/00 (hep-lat/0002025) 
[13] A. J. Buras, "Theoretical Review of B-physics," in BEAUTY '95 ed. N. Harnew and P. E. Schlein, Nucl. Instrum. Methods A368, 1 (1995).

[14] S. Stone, "Future of Heavy Flavour Physics: Experimental Perspective," presented at Heavy Flavours 8, June 1999, Southampton, UK to appear in proceedings.

[15] J.L. Rosner, Nucl. Phys. Proc. Suppl. 73 29, (1999) (hep-ph/9809545).

[16] Private communication from A. Buras.

[17] S. Plaszczynski and M. H. Schune, "Overall Determination of the CKM Matrix," presented at "Heavy Flavours 8," Southampton, UK, July, 1999, to appear in proceedings, hep-ph/9911280.

[18] F. Caravaglios et al, "Determination of the CKM Unitarity Triangle Parameters by End of 1999," hep-ph/0002171.

[19] The first papers explaining the physics of mixing and CP violation in $B$ decays were A. Carter and A. I. Sanda, Phys. Rev. Lett. 45, 952 (1980); Phys. Rev. D23, 1567 (1981); I. I. Bigi and A. I. Sanda, Nucl. Phys. B193, 85 (1981); ibid B281, 41 (1987).

[20] I. Bigi, V. Khoze, N. Uraltsev, in CP Violation, ed. C. Jarlskog, World Scientific, Singapore 175 (1989).

[21] A. S. Dighe, I. Dunietz, H. J. Lipkin, J. L. Rosner Phys. Lett. B369 144 (1996); R. Fleischer and I. Dunietz, Phys. Lett. B387 361 (1996); Y. Azimov and I. Dunietz Phys. Lett. B395 334 (1997);

[22] M. Beneke, G. Buchalla, I. Dunietz, Phys. Rev. D 54, 4419 (1996).

[23] I. Dunietz, Phys. Rev. D52, 3048 (1995).

[24] If $\Delta \Gamma$ is non-zero and there is large Penguin amplitude contributing, the lifetime distribution is not a simple exponential. However, using a $\Delta \Gamma$ of $15 \%$ and a ratio of Penguin to Tree rates of 4 to 1 , we find only a $1 \%$ effect on the lifetime.

[25] For the exact formulae see I. Bigi and A. Sanda, "CP Violation," Cambridge (1999), p 183.

[26] A. Dighe, I. Dunietz, and R. Fleischer, Phys. Lett. B433(1998) 147-149 (hep$\mathrm{ph} / 9804254)$.

[27] I. Dunietz, H. Quinn, A. Snyder, W. Toki, and H.J. Lipkin, Phys. Rev. D 43 (1991) 2193.

[28] A.S. Dighe, I. Dunietz, H.J. Lipkin and J.L. Rosner, Phys. Lett. B 369 (1996) 144.

[29] See, for example, M. Jacob and G.C. Wick, Ann. Phys. (N.Y.) 7 (1959) 404. 
[30] C. P. Jessop, et al. (CLEO). Phys. Rev. Lett. 79, 4533 (1997).

[31] A. Ribon, "B-Physics at the Tevatron Collider," presented at Les Rencontres de Physique de la Valle d'Aoste, La Thuile, Italy, Feb. 28-March 6, 1999.

[32] B. Kayser, "Cascade Mixing and the CP-Violating Angle Beta," in Les Arcs 1997, Electroweak Interactions and Unified Theories, p389 (hep-ph/9709382). Previous work in this area was done by Y. Aimov, Phys. Rev. D42, 3705 (1990).

[33] D-S. Du and Z-T. Wei, "Test of CPT Symmetry in Cascade Decays," hep-ph/9904403 (1999).

[34] A. Gritsan "Charmless Hadronic B Meson Decays with CLEO," presented at Lake Louise Winter Institute 2000, to appear in proceedings.

[35] M. Gronau, Phys. Rev. Lett. 63, 1451 (1989); M. Gronau and D. London, Phys. Rev. Lett. 65, 3381 (1990).

[36] The theoretical accuracy of this approach is limited by electroweak penguins, that are expected to be rather small in this case. In principle, they can be taken into account, as pointed out by Buras and Fleischer in hep-ph/9810260, and also by Gronau, Pirjol and Yan in hep-ph/9810482.

[37] N. G. Deshpande, X. G. He, and S. Oh, Phys. Lett. B384 (1996) 283-287 (hepph/9604336), and references therein.

[38] A. E. Snyder and H. R. Quinn, Phys. Rev. D. 48, 2139 (1993).

[39] Y. Gao and F. Wurthwein, "Charmless Hadronic $B$ Decays at CLEO," in DPF99 proceedings, hep-ex/9904008.

[40] A. Ali, G. Kramer, and C.D. Lu, Phys. Rev. D59 (1999) 014005 (hep-ph/9805403).

[41] H. R. Quinn and J. P. Silva, "The Use of Early Data on $B \rightarrow \rho \pi$ Decays," hep$\mathrm{ph} / 0001290$.

[42] A. Deandrea et al, "Measuing $B \rightarrow \rho \pi$ Decays and the Unitarity Angle Alpha," hep$\mathrm{ph} / 0002038$.

[43] Y. Grossman and H. R. Quinn, "Removing Discrete Ambiguities in CP Asymmetry Measurments," hep-ph/9705356 (1997).

[44] D. Du, I. Dunietz and Dan-di Wu, Phys. Rev D34, 3414 (1986). R. Aleksan, I Dunietz, and B. Kayser, Z. Phys. C54, 653 (1992). R. Aleksan, A. Le Yaouanc, L. Oliver, O. Pène and J.-C. Raynal, Z. Phys. C67 (1995) 251 (hep-ph/9407406).

[45] D. Atwood, I. Dunietz and A. Soni, Phys. Rev. Lett. 78, 3257 (1997). 
[46] M. Gronau and D. Wyler, Phys. Lett. B 265, 172 (1991).

[47] Y. Kwon et al (CLEO), "Study of Charmless Hadronic $B$ Decays into the Final States $K \pi, \pi \pi$ and $K K$, with the First Observations of $B^{o} \rightarrow \pi^{+} \pi^{-}$and $B^{o} \rightarrow K^{o} \pi^{o}$," Conf 99-14, hep-ex/9908039 (1999).

[48] R. Fleischer, and T. Mannel, Phys. Rev. D57 (1998) 2752-2759 (hep-ph/9704423).

[49] M. Neubert and J. L. Rosner, Phys. Rev. Lett. 81 (1998) 5076-5079 (hep-ph/9809311).

[50] M. Gronau and J. L. Rosner, Phys. Rev. D57 (1998) 6843-6850 (hep-ph/9711246); M. Gronau, and D. Pirjol, "A Critical Look at Rescattering Effects on $\gamma$ from $B^{+} \rightarrow K \pi$, hep-ph/9902482 (1999); M. Gronau and J. L. Rosner, "Combining CP Asymmetries in $B \rightarrow K \pi$ Decays, hep-ph/9809384 (1998).

[51] J.-M. Gerard and J. Weyers, Eur. Phys. J. C7 1(1999) (hep-ph/9711469).

[52] A. Falk, A. Kagan, Y. Nir and A. Petrov, Phys. Rev. D57 4290 (1998) (hep$\mathrm{ph} / 9712225)$.

[53] M. Neubert, Phys. Lett. B424 152 (1998) (hep-ph/9712224).

[54] D. Atwood and A. Soni, Phys. Rev. D58 036005 (1998) (hep-ph/9712287).

[55] JHEP 9902:014 (1999) (hep-ph/9812396).

[56] R. Fleischer, Phys. Lett. B459 306 (1999) (hep-ph/9903456).

[57] I. Dunietz, "Extracting CKM Parameters from B Decays," in Proceedings of the Workshop on $B$ Physics at Hadron Accelerators, ed. P. McBride and S. Mishra, Snowmass, Co, June (1993), Fermilab-Conf-93/90-T.

[58] For a recent calculation of $\Delta \Gamma_{s}$, see M. Beneke, G. Buchalla, C. Greub, A. Lenz and U. Nierste, Phys. Lett. 459631 (1999) (hep-ph/9808385).

[59] A. Dighe, I. Dunietz, and R. Fleischer, Eur. Phys. J. C6 (1999) 647-662 (hep$\mathrm{ph} / 9804253)$.

[60] R. Fleischer and I. Dunietz, Phys. Rev. D55 259 (1997); R. Fleischer, "Extracting CKM Phases from Angular Distributions of $B_{d, s}$ Decays into Admixtures of CP Eigenstates hep-ph/9903540 (1999).

[61] I. Dunietz, Phys. Lett. B427 (1998) 179-182 (hep-ph/97124).

[62] D. Atwood, B. Blok and A. Soni, Int. J. Mod. Phys. A11, 3743 (1996); see also N. Deshpande, X. He \& J. Trampetic, Preprint OITS-564-REV (1994); see also J. M. Soares, Phys. Rev. D53, 241 (1996). G. Eilam, A. Ioannissian R. R. Mendel and P. Singer, Phys. Rev. D53, 3629 (1996). 
[63] Another method of measuring $\left|V_{t d}\right|$ is to measure the branching ratio of $K^{+} \rightarrow \pi^{+} \nu \bar{\nu}$. A precise measurement would still be subject to theoretical uncertainties mostly arising from the uncertainty in the charmed quark mass and $\left|V_{c b}\right|$ See G. Buchalla, A. J. Buras, and M. E. Lautenbacher, Rev. Mod. Phys., 68, 1125 (1996) (hep-ph/9512380). A Brookhaven experiment, E787, has claimed a signal of one event and hopes to obtain substantially more data. See S. Adler, et al (E787), Phys. Rev. Lett 79, 2204 (1997).

[64] Y. Grossman, G. Isidori, M. Worah, Phys. Rev. D58 057504 (1998).

[65] S. J. Richichi et al (CLEO) "Two-Body $B$ Meson Decays to $\eta$ and $\eta$ ' Observation of $B \rightarrow \eta K^{*} "$ Conf 99-12 (hep-ex/9908019) (1999).

[66] S. Kopp, "Studies of $B^{\circ}$ Decays for Measuring $\sin (2 \beta)$ " presented at DPF'99, to appear in proceedings.

[67] J. P. Silva and L. Wolfenstein, Phys. Rev. D55 5331 (1997) (hep-ph/9610208),

[68] Private communication from J. Rosner, A. Buras, C. Bernard and others.

[69] K. Lingel, T. Skwarnicki and J. G. Smith, Ann. Rev. Nucl. Part. Sci. 48169 (1998) (hep-ex/9804015).

[70] M. Bander, D. Silverman and A. Soni, Phys. Rev. Lett. 43, 242 (1979).

[71] M. S. Alam et al (CLEO), Phys. Rev. Lett. 74, 2885 (1995).

[72] R. Ammar et al (CLEO), Phys. Rev. Lett. 71, 674 (1993).

[73] S. Glenn et al (CLEO), "Improved Measurement of $\mathcal{B}(b \rightarrow s \gamma)$," submitted to XXIX Int. Conf. on High Energy Physics, Vancouver, Canada, July 1998 paper ICHEP98 1011 (1998).

[74] B. Barate et al (ALEPH), "A Measurement of the Inclusive $b \rightarrow s \gamma$, Branching Ratio," CERN-EP/98-044 (1998).

[75] A. Ali and C. Greub, Phys. Lett. B 259, 182 (1991). The parameters for this fit are $<m_{b}>=4.88 \mathrm{GeV}$ and $P_{F}=250 \mathrm{MeV} / \mathrm{c}$.

[76] A. Czarnecki and W. J. Marciano, "Electroweak Radiative Corrections to $b \rightarrow s \gamma$," submitted to XXIX Int. Conf. on High Energy Physics, Vancouver, Canada, July 1998 paper ICHEP98 714 (1998); ibid Phys. Rev. Lett. 81, 277 (1998); see also see also M. Neubert, "Theoretical Status of $b \rightarrow X_{s} \gamma$ Decays," hep-ph/9809377 (1998); A. Ali, "Theory of Rare B Decays," hep-ph/9709507 DESY 97-192 (1997); N. G. Deshpande, "Theory of Penguins in $B$ Decays," in $B$ Decays Revised 2 nd Edition, ed. by S. Stone, World Scientific, Singapore, (1994).

[77] J. L. Hewett, " $B$ Physics Beyond the Standard Model," hep-ph/9803370 (1998). 
[78] S. Abachi et al (D0), Phys. Rev. D 56, 6742 (1997); F. Abe et al (CDF), Phys. Rev. Lett. 78, 4536 (1997).

[79] R. Ammar et al, "Radiative Penguin Decays of the B Meson," CLEO-CONF 96-6 (1996).

[80] D. Atwood, B. Blok \& A. Soni, Int. J. Mod. Phys. A11, 3743 (1994) and Nuovo Cimento 109A, 873 (1994); N. Deshpande, X. He \& J. Trampetic, Phys. Lett. B 362, 1996 (;) see also J. M. Soares, Phys. Rev. D 53, 241 (1996); G. Eilam, A. Ioannissian \& R. R. Mendel, Z. Phys. C71, 95 (1995).

[81] S. Fukae, C.S. Kim , T. Morozumi, and T. Yoshikawa, "A Model Independent Analysis of the Rare $B$ Decay $B \rightarrow X_{s} \ell^{+} \ell^{-}$, KEK-TH578, hep-ph/9807254 (1998), and references cited therein.

[82] A. Ali, C. Greub and T. Mannel, "Rare B Decays in the Standard Model," in Hamburg 1992, Proceedings, ECFA Workshop on a European B-meson Factory, Eds. R. Aleksan and A. Ali, p155 (1993).

[83] C. Albajar et al, Phys. Lett. B262, 163 (1991).

[84] R. Godang et al, "Search for Electroweak Penguin Decays $B \rightarrow K \ell^{+} \ell^{-}$and $B \rightarrow K^{*} \ell^{+} \ell^{-}$ at CLEO," CLEO-CONF 98-22 (1998).

[85] T. Affolder et al (CDF), Phys. Rev. Lett. 83, 3378 (1999).

[86] R. Ammar et al, Phys. Rev. D49, 5701 (1994); M. Artuso, et al, Phys. Rev. Lett. 75, 785 (1995).

[87] D. Buskulic et al, Phys. Lett. B343, 444 (1995).

[88] A. Ali and T. Mannel, Phys. Lett. B264, 447 (1991). Erratum, ibid, B274, 526 (1992).

[89] F. Abe et al (CDF), Phys. Rev. D 57, R3811 (1998).

[90] M. Acciarri et al. (L3), Phys. Lett. B 396 (1997) 327-337.

[91] E. M. Aitala et al, Phys. Rev. Lett. 77, 2384 (1996).

[92] G. Burdman, "Potential for Discoveries in Charm Meson Physics," hep-ph/9508349.

[93] H. Georgi, Phys. Lett. B297, 353 (1992); T. Ohl et al, Nucl. Phys. B 403, 603 (1993).

[94] I. I. Bigi and H. Yamamoto, Phys. Lett. B 349, 363 (1995). 


\section{Part II}

\section{Detector Description}




\section{Chapter 2}

\section{Key Design Considerations for the BTeV Detector}

In Part II of the proposal, we present the design of the BTeV detector.

We begin with a discussion of the key "drivers" of the detector design: the physics of $B$ production at the Tevatron and the characteristics of the machine itself. These, taken together with the physics goals outlined in Part I, determine the main requirements for the detector design.

After explaining the requirements of the design, we describe the baseline detector for $\mathrm{BTeV}$, which can achieve our currently stated physics goals, and is designed to have the capability to perform measurements of new interesting final states that may be recognized as important in the future. A schematic of the experiment is shown in Fig. 2.1.

The key design features of BTeV include:

- A dipole located on the IR, which gives BTeV an effective 'two arm' acceptance;

- A precision vertex detector based on planar pixel arrays;

- A detached vertex trigger at Level 1 which makes BTeV efficient for most final states, including purely hadronic modes;

- Excellent particle identification using a Ring Imaging Cherenkov Detector (RICH);

- A high quality $\mathrm{PbWO}_{4}$ electromagnetic calorimeter capable of reconstructing final states with single photons, $\pi^{o}$ 's, $\eta$ 's or $\eta^{\prime \prime}$, , and identifying electrons;

- Precision tracking using straw tubes and silicon microstrip detectors, which provide excellent momentum and mass resolution;

- Excellent identification of muons using a dedicated detector with the ability to supply a dimuon trigger; and

- A very high speed and high throughput data acquisition system which eliminates the need to tune the experiment to specific final states. 

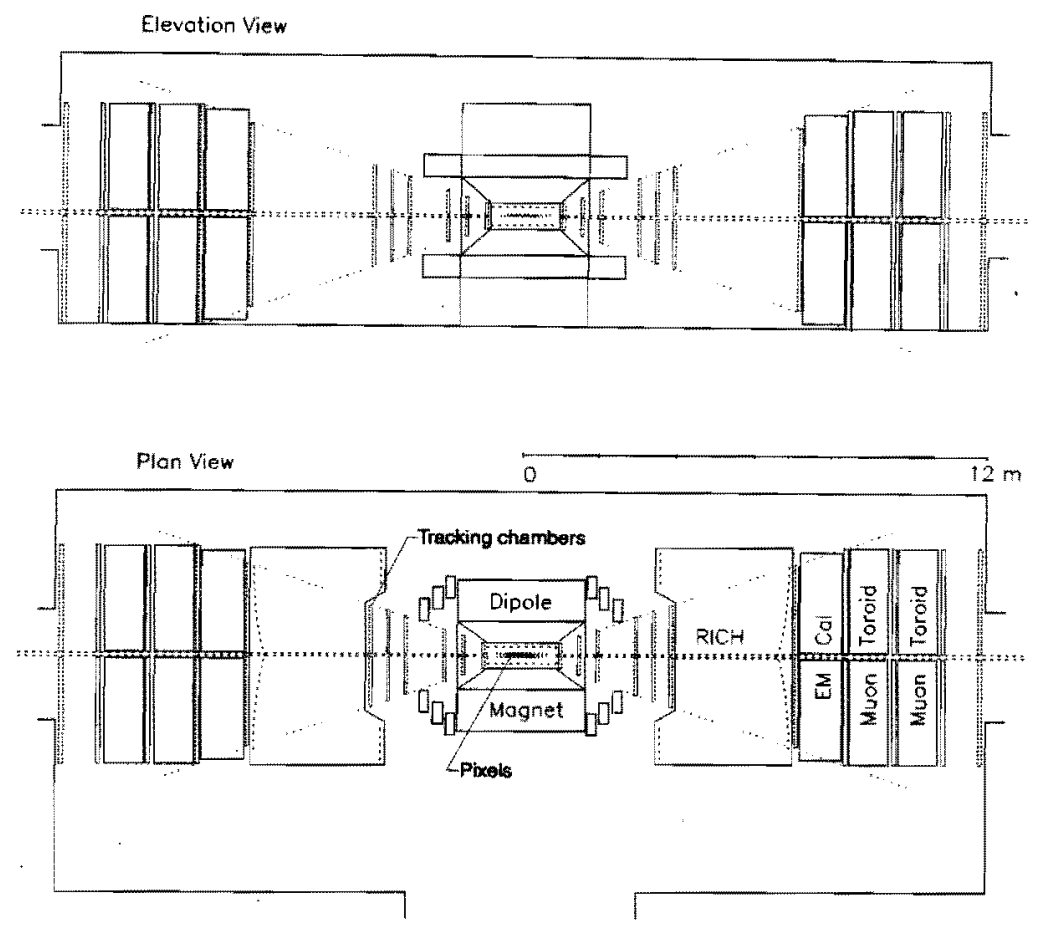

Figure 2.1: Layout of BTeV/C0 Spectrometer

The level of detail in this Part is sufficient to provide the reader with a good overview of the experimental apparatus and a reasonable understanding of the solution to all the various problems associated with carrying out our ambitious program studying $B$ decays. However, many details which an expert might need to understand the detailed implementation and status of development of each technology are not presented here but are placed in appendices, which are referenced in the text.

\subsection{Rationale for a Forward Detector}

The kinematics of hadronic beauty and charm production play a major role in the design of $\mathrm{BTeV}$. We review the most important features here. In hadron colliders all $B$ species, $B^{o}$, $B^{+}, B_{s}^{o}, b$-baryons, and even $B_{c}$ mesons, are produced at the same time.

\subsubsection{The $b \bar{b}$ Production Cross-Section}

It is customary to characterize heavy quark production in hadron collisions with two variables, the momentum transverse to the beams, $p_{t}$, and the rapidity,

$$
y=\frac{1}{2} \ln \left(\frac{E+p_{\|}}{E-p_{\|}}\right),
$$


where $E$ is the particle's energy and $p_{\|}$is its longitudinal momentum. Sometimes, the pseudorapidity $\eta$ is used, where

$$
\eta=-\ln (\tan (\theta / 2))
$$

where $\theta$ is the angle of the particle with respect to the beam direction. This latter variable was first invented by those who studied high energy cosmic rays, and did not necessarily know the masses of the particles in their detectors.

The $p \bar{p}$ production of $b$ quarks has been measured in the Tevatron at a center-of-mass energy of $1.8 \mathrm{TeV}$ in the central rapidity region $|\eta|<1$ by CDF [1] and D0 [2], and in the forward region $3.2>y>2.4$ by DO [3]. Both CDF and D0 find that the $b \bar{b}$ production cross-section in the central region is underestimated by the Mangano, Nason and Ridolfi (MNR) next-to-leading order QCD calculation [7] by a factor of approximately two. Since the QCD calculation predicts a cross-section of $50 \mu \mathrm{b}$, when integrated over $\eta$ and $p_{t}$, using the data in the central regions leads to a total $b \bar{b}$ production cross-section of $100 \mu \mathrm{b}$. The D0 central and forward data are shown in Fig. 2.2.

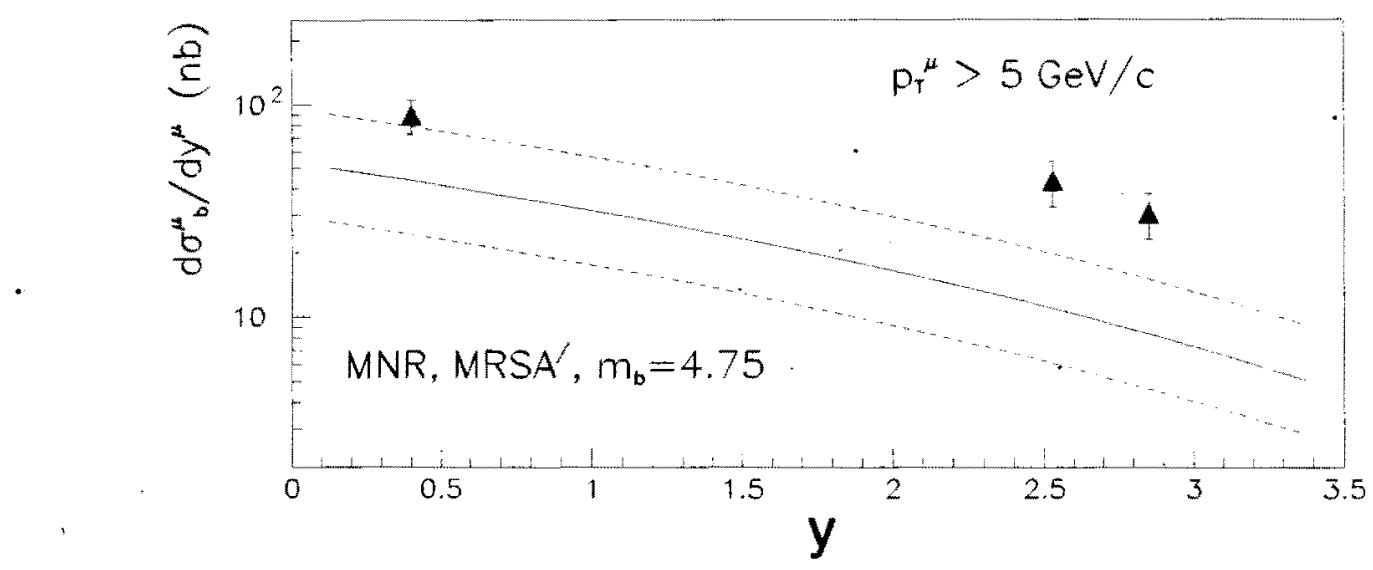

Figure 2.2: The $b \bar{b}$ cross-section as a function of the rapidity of muons from $b$ decay, $y^{\mu}$, measured by $\mathrm{D} 0$ for both the forward and central rapidity regions, using muons from $b$ decays with $p_{t}>5 \mathrm{GeV} / \mathrm{c}$. The solid curve is the prediction of the next-to-leading order QCD calculation for a $b$-quark mass of $4.75 \mathrm{GeV}$. The dashed curves represent the estimated theoretical $1 \sigma$ error band.

The measured cross-section in the higher $y^{\mu}$ region is $3.6 \pm 0.8$ times higher than the QCD calculation, leading to a total estimated $b \bar{b}$ production cross-section of $180 \mu \mathrm{b}$. BTeV will operate in the range $1.9>\eta>4.5$. While we have no reason to dispute the D0 measurement, we will conservatively normalize our estimates to a $b \bar{b}$ production cross-section of $100 \mu \mathrm{b}$.

There is some evidence from HERA that the fragmentation of charmed particles is influenced by the leading quarks in the beam so that the fragmentation produces, in some cases, faster $D$ 's than the parent $c$-quarks [4]. This effect is expected to be smaller for $b$ quarks at the center-of-mass Tevatron. If such an effect were present it would increase BTeV's 


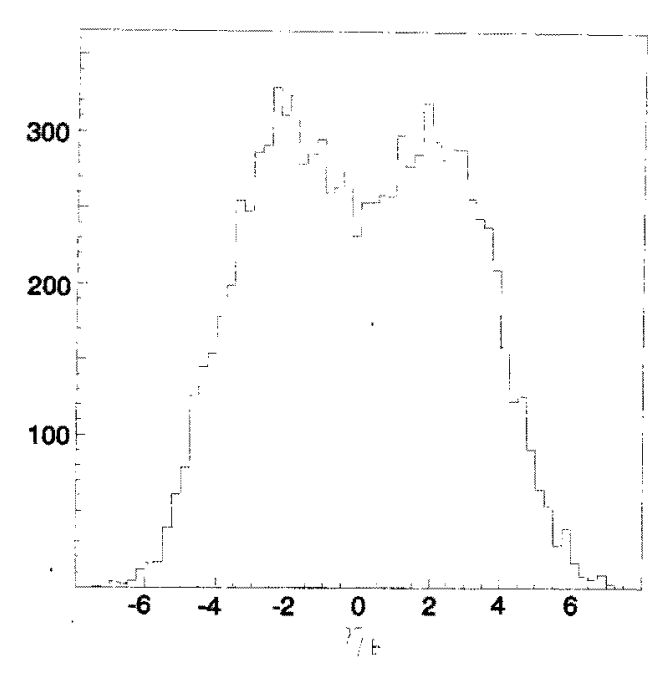

Figure 2.3: The $B$ yield versus $\eta$.

acceptance in the forward direction and it would also generate a small asymmetry between $B$ mesons and $\bar{B}$ mesons in the proton direction (opposite in the antiproton direction). All possible production and instrumental asymmetries have to be checked in any case, to obtain accurate measurements of $\mathrm{CP}$ violation.

\subsubsection{Characteristics of Hadronic $b$ Production}

According to QCD calculations of $b$ quark production, the $b$ 's are produced "uniformly" in $\eta$ and have a truncated transverse momentum, $p_{t}$, spectrum, characterized by a mean value approximately equal to the $B$ mass [5]. The distribution in $\eta$ is shown in Fig. 2.3.

There is a strong correlation between the $B$ momentum and $\eta$. Shown in Fig. 2.4 is the $\beta \gamma$ of the $B$ hadron versus $\eta$ from the Monte Carlo physics generator Pythia at $\sqrt{s}=2$ $\mathrm{TeV}$. It can clearly be seen that near $\eta$ of zero, $\beta \gamma \approx 1$, while at larger values of $|\eta|, \beta \gamma$ can easily reach values of 6 . This is important because the observed decay length varies with $\beta \gamma$ and, furthermore, the absolute momenta of the decay products are larger allowing for a suppression of the multiple scattering error.

Since the detector design is somewhat dependent on the Monte Carlo generated $b$ production distributions, it is important to check that the correlations between the $b$ and the $\bar{b}$ are adequately reproduced. Fig. 2.5 shows the azimuthal opening angle distribution between a muon from a $b$ quark decay and the $\bar{b}$ jet as measured by CDF [6] and compares it with the MNR next-to-leading order QCD predictions [7].

The MNR model does a good job representing the shape, which shows a strong backto-back correlation. The normalization is about a factor of two higher in the data than the theory, which is generally true of CDF $b$ cross-section measurements.

The "flat" $\eta$ distribution hides an important correlation of $b \bar{b}$ production at hadronic colliders. In Fig. 2.6 the production angle of the hadron containing the $b$ quark is plotted versus 


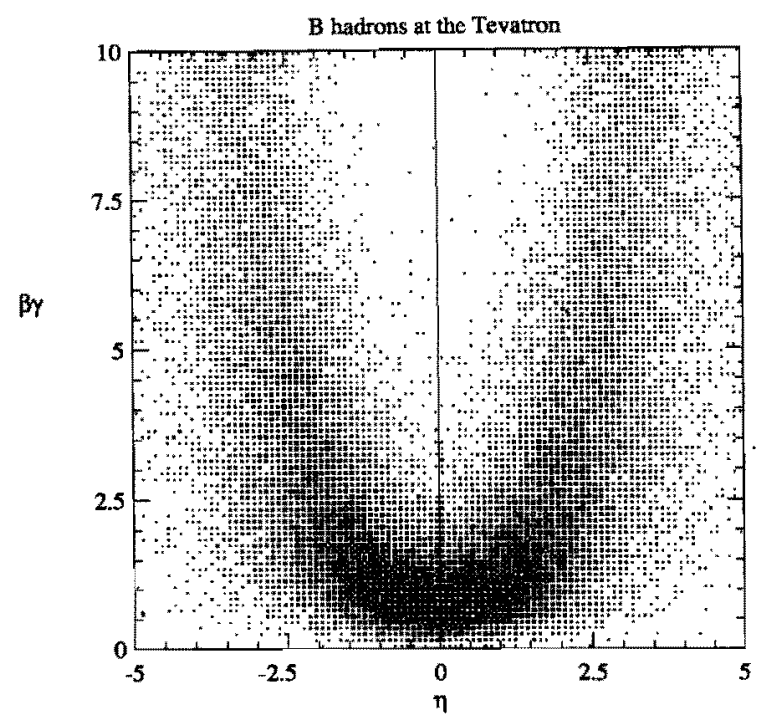

Figure 2.4: $\beta \gamma$ of the $B$ versus $\eta$.

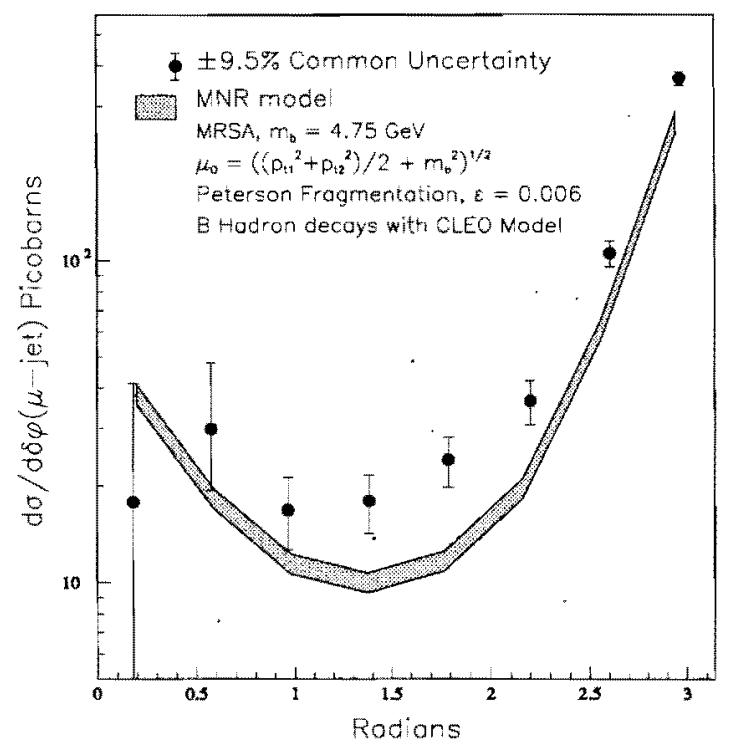

Figure 2.5: The differential $\delta \phi$ cross-sections for $p_{T}^{\mu}>9 \mathrm{GeV} / \mathrm{c},\left|\eta^{\mu}\right|<0.6, \mathrm{E}_{T}^{\bar{b}}>10 \mathrm{GeV}$, $\left|\eta^{\bar{b}}\right|<1.5$ compared with theoretical predictions. The data points have a common systematic uncertainty of $\pm 9.5 \%$. The uncertainty in the theory curve arises from the error on the muonic branching ratio and the uncertainty in the fragmentation model.

the production angle of the hadron containing the $\bar{b}$ quark. Here zero degrees represents the direction of the incident proton and 180 degrees, the incident anti-proton. There is a very strong correlation in the proton or the anti-proton directions: when the $B$ is forward the $\bar{B}$ is also forward. (We call both the proton and anti-proton directions forward.) This correlation 
between $B$ and $\bar{B}$ production is not present in the central region (near 90 degrees).

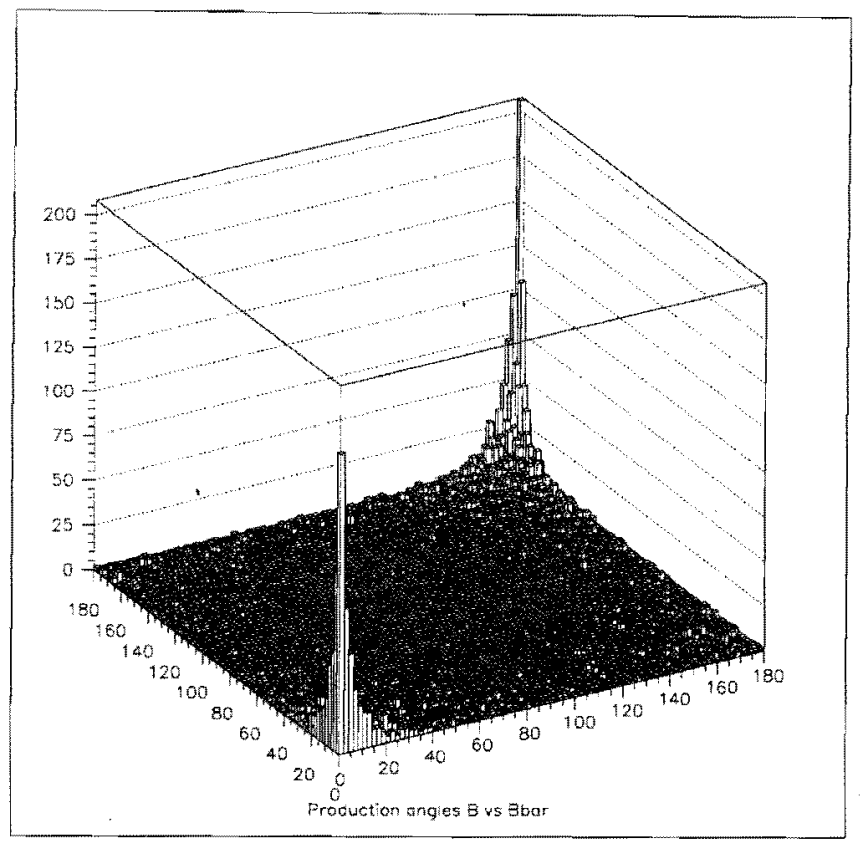

Figure 2.6: The production angle (in degrees) for the hadron containing a $b$ quark plotted versus the production angle for a hadron containing $\bar{b}$ quark, from the Pythia Monte Carlo generator.

Thus, the forward direction at the Tevatron presents us with a number of striking advantages. First of all, there is a large cross-section for the production of correlated $b \bar{b}$ pairs. Secondly, the $B$ hadrons that are formed have relatively large momenta, on average 30 $\mathrm{GeV} / \mathrm{c}$, and their decay products are not multiply scattered by large amounts. This allows us to make precision measurements of their spatial origins; so we can determine if they arise from $B$ hadrons that traveled on the order of several $\mathrm{mm}$ prior to their decay. Furthermore the geometry is very natural for certain aspects of detector technology that significantly enhance the physics performance. For example, a Ring Imaging Cherenkov detector using a gas radiator matches the $3-70 \mathrm{GeV} / \mathrm{c}$ momentum range for $B$ decay products. The Cherenkov photons can be detected using a relatively small area array of photomultiplier tubes or HPD's. Powerful particle identification is essential for high sensitivity $b$ experiments. Another example is the ability to put the silicon pixel vertex detector inside the main beam vacuum. Precision detection of the $B$ decay vertices is crucial for the trigger and in rejecting backgrounds. For these reasons, we have designed a detector with "forward coverage."

Charm production is similar to $b$ production but has a much larger cross section. Current theoretical estimates are that charm is $1-2 \%$ of the total $p \bar{p}$ cross-section. The cross section 
is even more strongly peaked in the forward direction because the average transverse momentum is of the order of only $1.5 \mathrm{GeV} / \mathrm{c}$. The charm cross section has never been measured because experiments with good acceptance in the central region have very low efficiency for triggering and reconstructing charm. The favorable kinematics in the forward direction gives $\mathrm{BTeV}$ a very high efficiency for reconstructing charm.

Table 2.1 gives the Tevatron parameters which are especially relevant to BTeV design and physics reach.. We expect to start serious data taking with a luminosity of about $5 \times 10^{31} \mathrm{~cm}^{-2} \mathrm{~s}^{-1}$; our ultimate luminosity goal is $2 \times 10^{32} \mathrm{~cm}^{-2} \mathrm{~s}^{-1}$. At the higher luminosity we expect an average of 2 total interactions per crossing, 1.3 of which are not elastic or quasi-elastic.

Table 2.1: The Tevatron as a $b$ and $c$ source for $\mathrm{BTeV}$

\begin{tabular}{|l|c|}
\hline Luminosity (BTeV design) & $2 \times 10^{32} \mathrm{~cm}^{-2} \mathrm{~s}^{-1}$ \\
$b \bar{b}$ cross-section & $100 \mu \mathrm{b}$ \\
$\#$ of $b$ 's per $10^{7} \mathrm{sec}$ & $4 \times 10^{11}$ \\
$\frac{\sigma(b \bar{b})}{\sigma(\text { total) }}$ & $\sim 0.15 \%$ \\
$c \bar{c}$ cross-section & $>500 \mu \mathrm{b}$ \\
Bunch spacing & $132 \mathrm{~ns}$ \\
Luminous region length & $\sigma_{z}=30 \mathrm{~cm}$ \\
Luminous region width & $\sigma_{x}, \sigma_{y} \approx 50 \mu \mathrm{m}$ \\
Interactions/crossing & $<2.0>$ \\
\hline
\end{tabular}




\section{Bibliography}

[1] F. Abe et al., "Measurement of the $B$ Meson Differential Cross-Section in $p \bar{p}$ collisions at $\sqrt{s}=1.8 \mathrm{TeV}$," CDF/PUB/BOTTOM/PUBLIC/3759 submitted to ICHEP '96 and references theirin; F. Abe et al., Phys. Rev. Lett. 75, 1451 (1995).

[2] R. Abbott et al., "The $b \bar{b}$ Production Cross Section and Angular Correlations in $p \bar{p}$ collisions at $\sqrt{s}=1.8 \mathrm{TeV}$," FERMILAB-Pub-99/144-E; S. Abachi et al, Phys. Rev. Lett. 74, 3548 (1995).

[3] D. Fein, "Tevatron Results on $b$-Quark Cross Sections and Correlations," presented at Hadron Collider Physics (HCP99) (Bombay), January 1999.

[4] I. Redondo, "ZEUS Results on Charm in DIS As AN Indication of Beam Drag Effects," and E. Norrbin and T. Sjostrand, "Drag Effects in Charm Photoproduction," both in

- Proceedings of the workshop for Monte Carlo Generators for HERA Physics, 1998/99, ed. by A. T. Doyle et al., http://www.desy.de/ heramc/proceedings/wg60.

[5] M. Artuso, "Experimental Facilities for b-Quark Physics," in $B$ Decays revised 2nd Edition, Ed. S. Stone, World Scientific, Singapore (1994).

[6] F. Abe et al., Phys. Rev. D 53, 1051 (1996).

[7] M. Mangano, P. Nason and G. Ridolfi, Nucl. Phys. B 373, 295 (1992). 


\section{Chapter 3}

\section{Co Experimental Area and Analysis Magnet}

\subsection{The Co Experimental Area}

The experiment will be carried out in the newly constructed $\mathrm{C} 0$ collision hall, shown in Figure 3.1. The hall is a $216 \mathrm{~m}^{2}$ (9 m wide by $24 \mathrm{~m}$ long) enclosure centered along the $\mathrm{C} 0$ straight section of the Tevatron. The hall is asymmetric in the transverse direction, as best seen in the plan view, Figure 3.3, extending $3.5 \mathrm{~m}$ from the beam on the west side and $5.5 \mathrm{~m}$ from the beam on the east side. The Tevatron beams are $2.5 \mathrm{~m}$ above the floor slab and $4.25 \mathrm{~m}$ below the roof of the hall. There are flared transition enclosures both upstream and downstream. The enclosure specifications and dimensions are compatible with the detector described in this document.

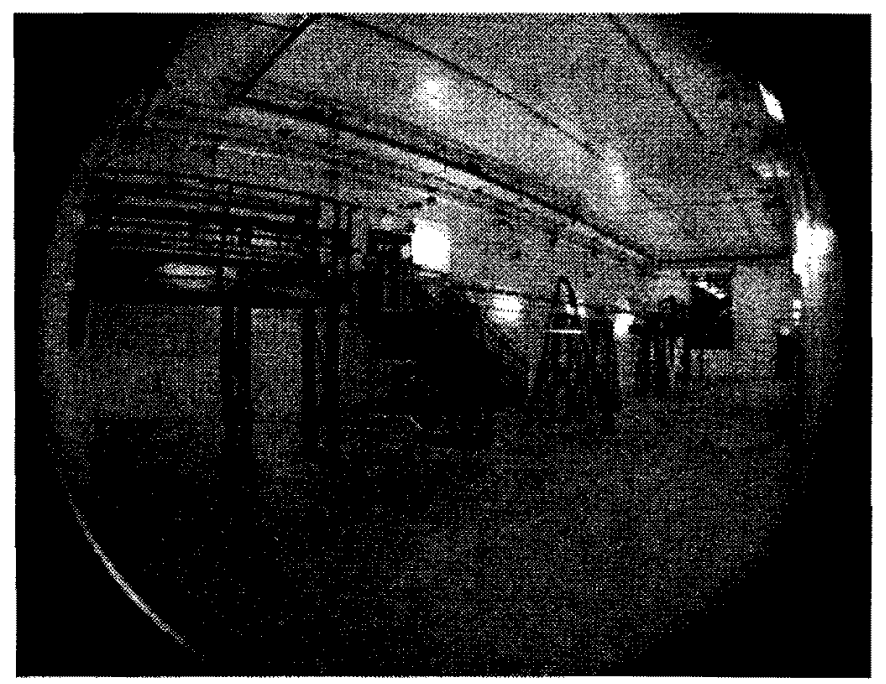

Figure 3.1: C0 Collision Hall 


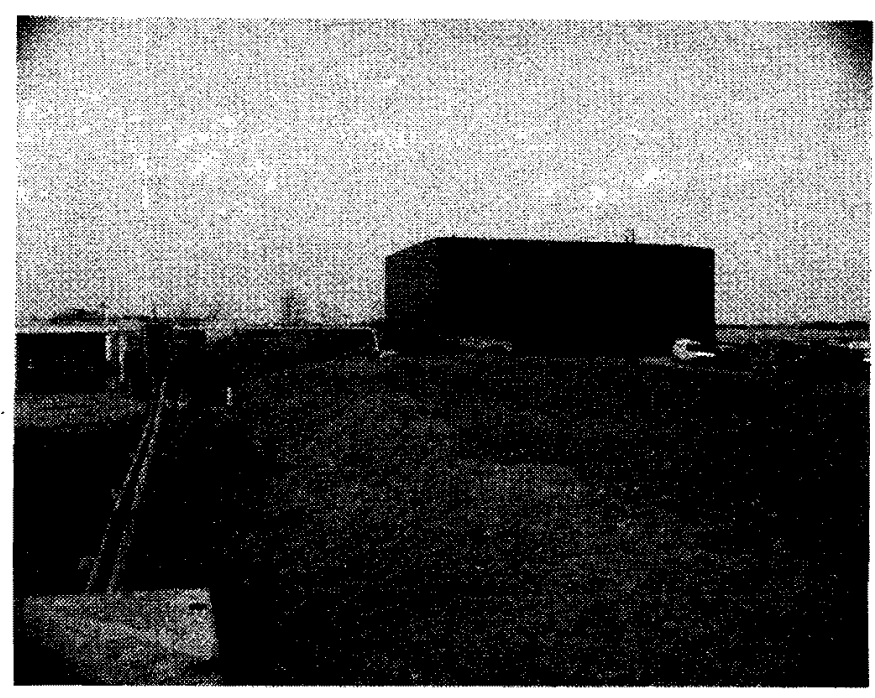

Figure 3.2: Tevatron berm and C0 Assembly Building

To the east of the collision hall is the $\mathrm{C} 0$ assembly building (see Figure 3.2) a steel framed, industrial type structure containing a $150 \mathrm{~m}^{2}$ assembly hall at the collision hall elevation. Detector components will be constructed in the assembly hall and moved into the collision hall through a $6 \mathrm{~m} \times 6 \mathrm{~m}$ "shield door." The $\mathrm{C} 0$ assembly building also has space on various levels for the electrical, water and air handling systems needed for the $\mathrm{BTeV}$ detector. Figure 3.3 shows a layout of the assembly building and collision hall with the vertex magnet and the 4 muon toroids of the proposed BTeV detector superimposed.

Detector elements are brought into the area at a ground level loading dock and lowered to the assembly floor using the 30 ton crane which covers the loading dock and assembly hall. There is no crane in the beam enclosure; all detector elements must be designed so that they can be constructed or staged in the assembly area and moved through the shielding door into the enclosure. During operation the shielding door is sealed with a concrete door for radiation protection. The concrete door moves on rollers and is stored in the south end of the assembly area in an alcove when the door is open.

There are cable ducts from the experiment enclosure to a $150 \mathrm{~m}^{2}$ equipment room at grade level on the north end of the assembly building. Preliminary engineering has been done on converting this equipment room into a two or three level electronics/counting room and office area for experimenters. Electric power, air handling, other utilities, as well as an elevator, lavatories, etc., although not yet installed, have been designed to accommodate $\mathrm{BTeV}$ needs in these areas.

\subsection{The BTeV/C0 Spectrometer Magnet}

The vertex magnet in the proposed $\mathrm{BTeV}$ spectrometer is based on an existing magnet, the SM3 magnet, which is currently part of the decommissioned Fermilab MEast Spectrometer. 


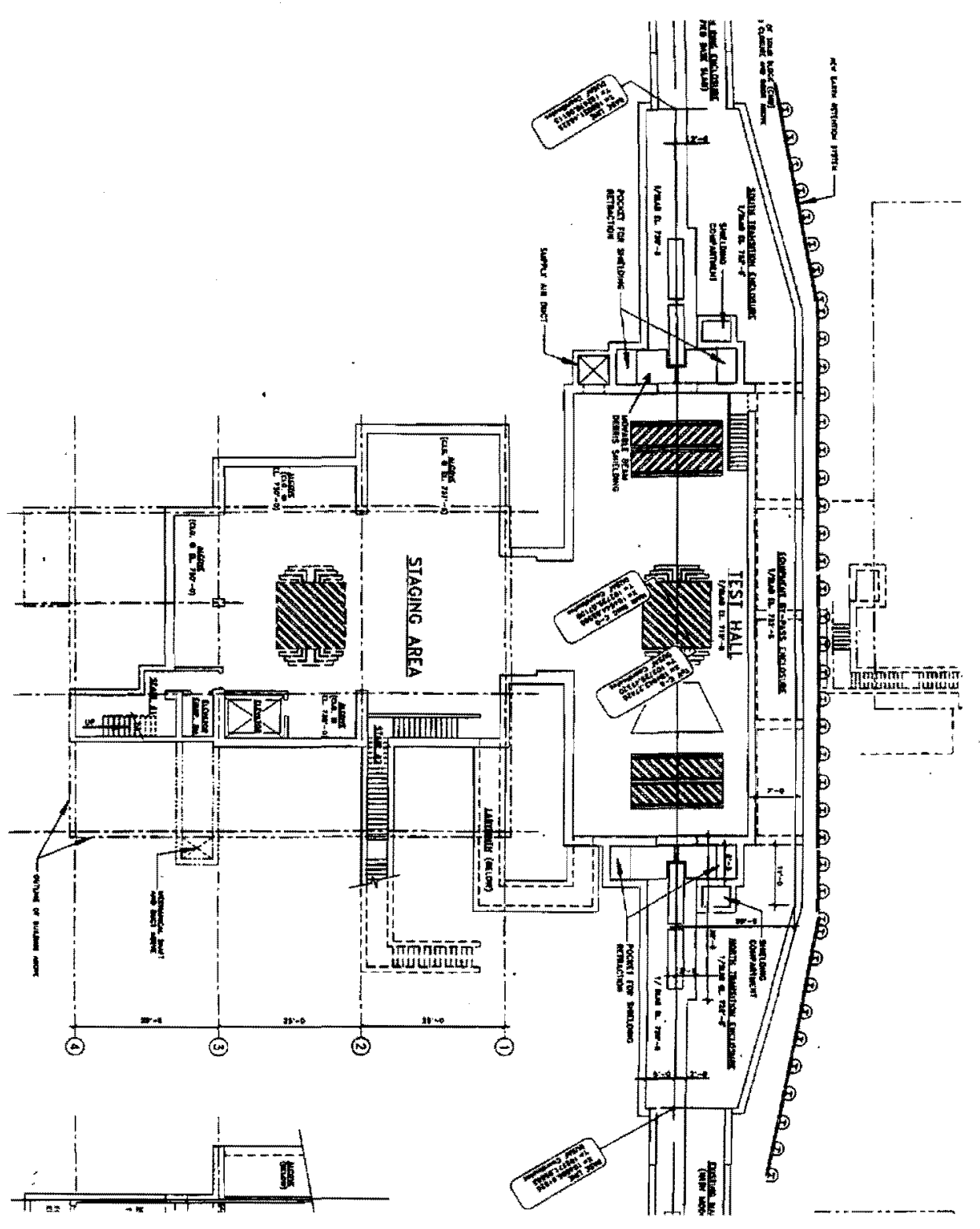

Figure 3.3: Layout of C0 Collision Area 
It is shown, after modification, on the proposed layout, Figure 2.1 , of the $\mathrm{BTeV} / \mathrm{C} 0$ spectrometer. The SM3 magnet was assembled in 1981 from soft iron blocks that were recovered from the decommissioned Nevis Cyclotron. The coils for the magnet were built of $5 \mathrm{~cm}$ square aluminum conductor by the Sumitomo Corporation under the aegis of the US-Japan Agreement on High Energy Physics. The magnet operated in MEast from 1982 until 1997, at a central field of about 0.8 Tesla, serving experiments E605, E772, E789, and E866.

The SM3 magnet was assembled by welding together, in place, various blocks of the Nevis iron. It has a total weight of 500 metric tons. The construction and assembly drawings for this magnet have been located and an engineering procedure for disassembling the magnet has been developed. A test disassembly of two of the 20 large iron pieces on the magnet occurred in 1999. The test allows us to estimate the full disassembly costs with more certainty. After transportation to $\mathrm{C} 0$, the modified magnet will be reassembled in the $\mathrm{C} 0$ assembly hall and rolled into the $\mathrm{C} 0$ collision hall, as shown in Figure 3.3.

The central field specified for the $\mathrm{BTeV} / \mathrm{C} 0$ spectrometer is much higher than the operating excitation of the existing SM3 magnet. Studies with the magnetostatic modeling programs POISSON and OPERA have led to a design for a new pole-piece for SM3. This pole-piece, indicated in Figure 3.4, yields a central field of 1.6 Tesla, and an integrated dipole field of $5.2 \mathrm{~T}-\mathrm{m}$.

The design has an integrated sextupole field component on the symmetry axis of 0.002 $\mathrm{T} / \mathrm{m}$. This is small compared to the natural sextupole moment of the Tevatron dipoles and is acceptable for insertion into the Tevatron lattice. In this design the magnet would draw 650 $\mathrm{kW}$ of power at $4200 \mathrm{amps}$, similar to its previous operating power levels (it was previously powered by two $500 \mathrm{~kW}$ Transrex power supplies). Note that the magnet will be oriented so that charged particles are deflected in the vertical plane. The properties of the magnet, with the pole faces shimmed to the $\mathrm{BTeV}$ requirements, are shown in Table 3.1.

Table 3.1: BTeV/C0 Vertex Dipole Properties

\begin{tabular}{|l|l|l|}
\hline Property & Value & Comment \\
\hline $\int B \times d l$ & $5.2 \mathrm{~T}-\mathrm{m}$ & $2.6 \mathrm{~T}$-m on each \\
& & side of center of IR \\
Central Field & $1.6 \mathrm{Tesla}$ & \\
Steel Length & $3.2 \mathrm{~m}$ & \\
Overall length & $5.3 \mathrm{~m}$ & \\
Magnet Vert. aperture & $\pm 0.3 \mathrm{rad}$ & \\
Magnet Horz. aperture & $\pm 0.3 \mathrm{rad}$ & \\
\hline
\end{tabular}

The magnet is centered on the interaction region in $\mathrm{Z}$ thus creating two forward spectrometers. In quark-antiquark production at $2 \mathrm{TeV}$, the bottom quark and antiquark are usually either both boosted in the proton beam direction, or both boosted in the antiproton beam direction. Thus, having two spectrometers doubles the acceptance of the experiment 


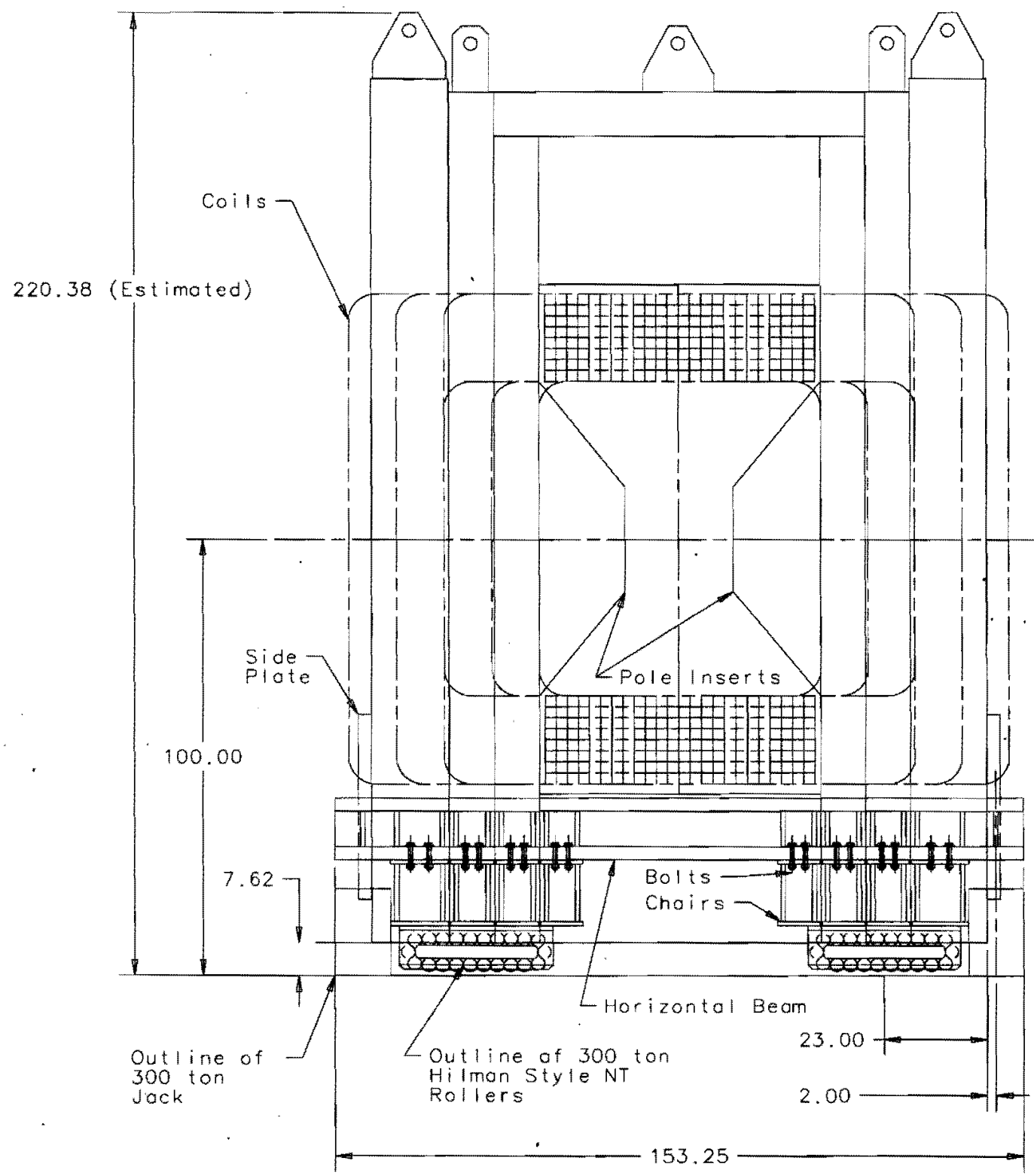

Figure 3.4: Cross section of the modified SM3 dipole with rollers and pole piece inserts. All dimensions are in inches. 


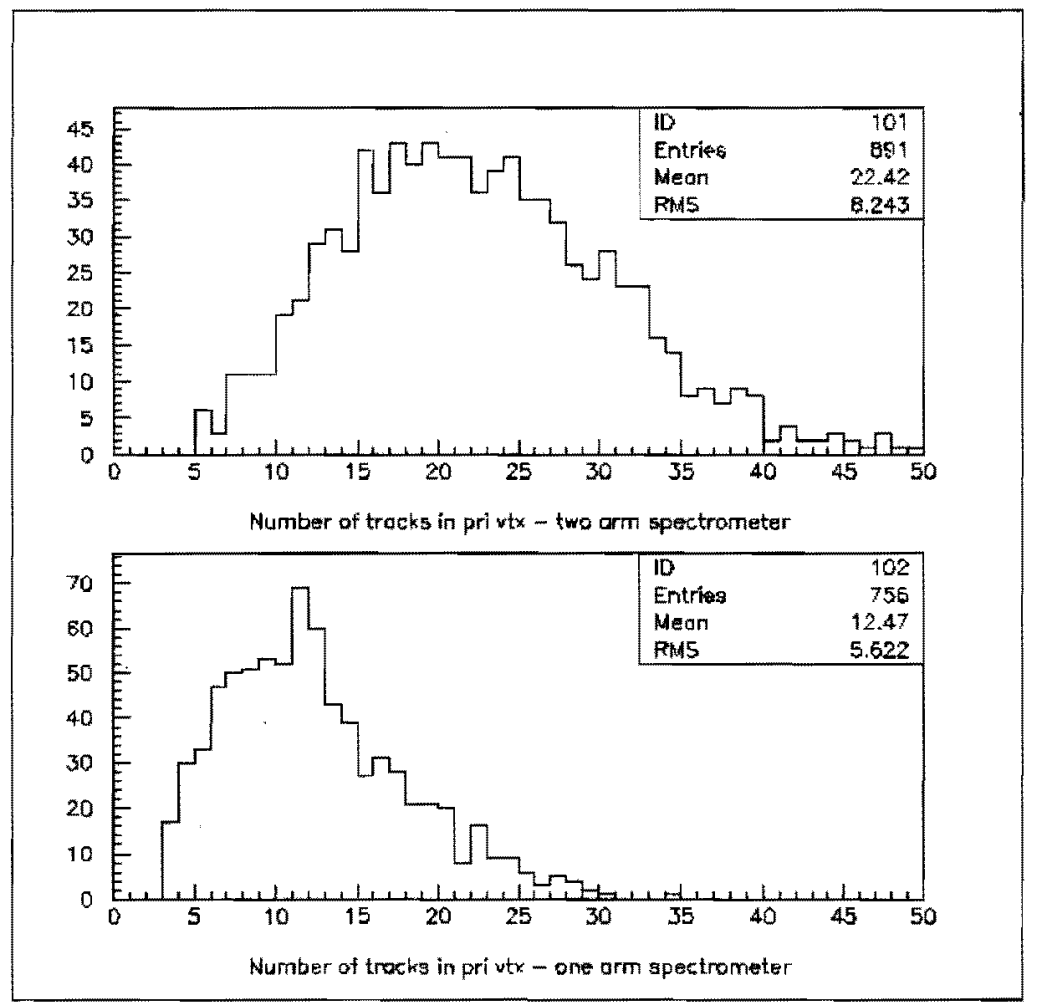

Figure 3.5: Fraction of events with $\mathrm{N}$ primary tracks above $1 \mathrm{GeV} / \mathrm{c}$ momentum for a) a dipole centered on the IR giving an effective two-arm acceptance and b) a single forward spectrometer arm.

for tagged decays. Having two spectrometers further improves the experiment because the extended coverage increases the number of tracks from the underlying eyent that can be used to determine the primary vertex. Figure 3.5 shows the number of high energy primary tracks (low multiple scattering) that contribute to locating the primary vertex for single arm coverage and for two arm coverage. If we require three such tracks to determine the primary vertex, then 2.2 times more events survive with two arms than with one, and the primary vertex resolution is enhanced.

In this central dipole geometry, there is a strong magnetic field at the vertex detector. Because of the excellent spatial resolution of the vertex detector, it is possible to get a crude measurement of the track momentum using the vertex detector alone. This measurement allows the first level trigger to properly weight tracks in vertex fits, and to impose separation cuts based on normalized miss distances. 


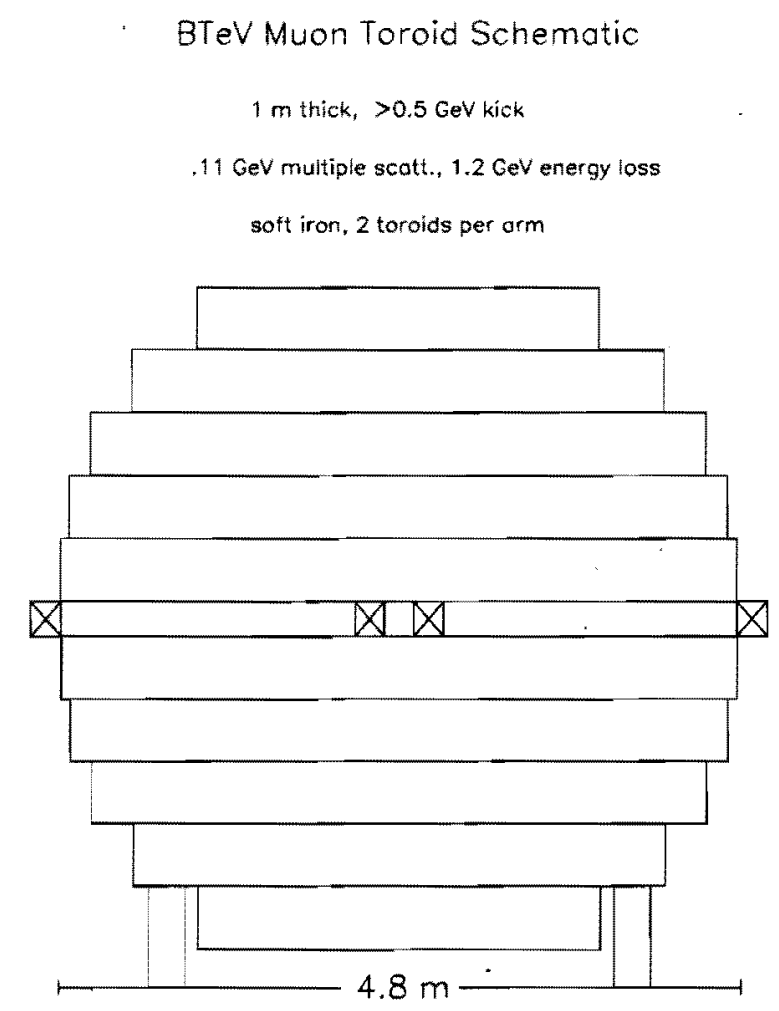

Figure 3.6: Schematic of Muon toroid.

The second spectrometer arm will also be useful during the R\&D and early data-taking phases of the experiment. It is possible, given the lab's budget, that only one arm will be fully instrumented initially. The other side can be used to test new detector concepts or to test prototypes of production components under actual beam conditions until it becomes possible to instrument it fully.

\subsection{The Muon Toroid Magnets}

In order to absorb hadrons and simultaneously deflect muons (thus allowing momentum discrimination in the muon trigger), each spectrometer arm contains two $1 \mathrm{~m}$ thick magnetized iron absorber walls. Each of the 4 toroids is $4.8 \mathrm{~m}$ in diameter, weighs 200 tons, and has 2 excitation coils as shown in Fig. 3.6. Each toroid will be energized by 64 turns of $1000 \mathrm{Amp}$ water-cooled conductor yielding a toroidal magnetic field varying from $19 \mathrm{kGauss}$ at small radius to $16 \mathrm{kGauss}$ at larger radius. This implies a $\mathrm{p}_{T}$ kick of $>0.5 \mathrm{GeV} / \mathrm{c}$ for each toroid, or $>1 \mathrm{GeV} / \mathrm{c}$ total for each muon. 


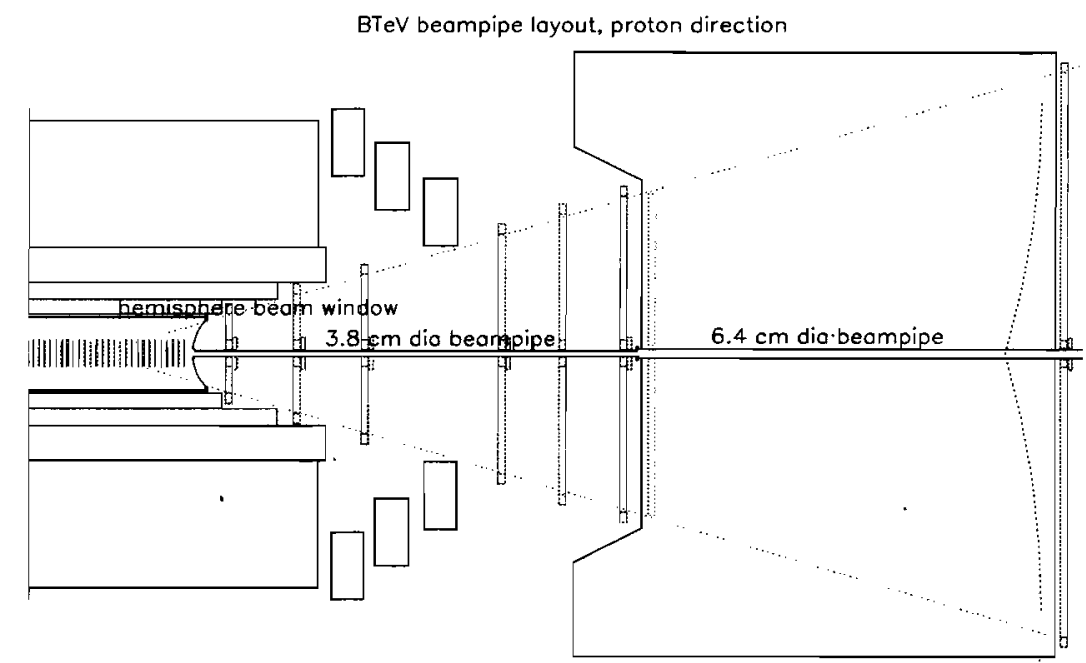

Figure 3.7: Schematic of the tracking detectors and beampipe.

\subsection{The Spectrometer Beampipes}

The beampipes in the regions from the end of the silicon pixel vacuum tank to the first Tevatron accelerator elements downstream of the muon detectors will be constructed of thin-walled aluminum. The beampipe will transition from the pixel vacuum box to the $3.8 \mathrm{~cm}$ diameter beampipe in the tracking detector region via a mushroom shaped concave dome formed from 0.030" aluminum as shown in Figure 3.7. The $3.8 \mathrm{~cm}$ diameter cylindrical beampipes in the tracking detector region, and the $6.4 \mathrm{~cm}$ diameter cylindrical beampipes in the RICH, calorimeter, and muon detector regions will also be formed from 0.030 " aluminum. A special low-mass flange coupling near the upstream face of the RICH detector, coupled with a bellows section downstream of the muon detector, will allow for thermal motions and will facilitate beampipe assembly and removal. 


\section{Chapter 4}

\section{Pixel Vertex Detector}

\subsection{Introduction}

The pixel vertex detector provides high resolution space points near the interaction, which are used both online and offline to reconstruct tracks and associate them with their parent vertices. We have chosen to use silicon pixel detectors because they provide high precision space points with very few noise hits, and they are quite radiation hard. Radiation hardness enables us to place detector elements very close to the beam (in vacuum, separated from the beam only by a thin RF shield), minimizing track extrapolation errors. The measurement of space points, with very little noise, provides superior pattern recognition, allowing us to reconstruct tracks and vertices in real time, and trigger on events containing reconstructable heavy flavor decays.

The pixel detector contains nearly thirty million rectangular pixels, each $50 \mu \mathrm{m} \times 400 \mu \mathrm{m}$. Each sensor pixel is read out by a dedicated electronics cell. The sensor pixel and the readout cell are connected by a "bump bond." The basic building block of the detector is a hybrid assembly consisting of a sensor, a number of readout chips, and a flexible printed circuit which carries I/O signals and power. The sensors are $\sim 5 \mathrm{~cm} \times 1 \mathrm{~cm}$, contain $\sim 25000$ pixels, and are mated to $\sim 5$ readout chips in a process called "flip chip assembly." These hybrid modules are supported by a moveable carbon structure that allows the pixel sensors to be positioned a safe distance away from the beamline until stable conditions have been established in the Tevatron, at which point they are moved as close to the beamline as radiation damage considerations will allow. This structure also provides cooling for the readout electronics.

\subsection{Detector Specifications}

The baseline vertex detector consists of a regular array of 31 "stations" of "planar" pixel detectors distributed along the interaction region (see Figure 4.1). Each station contains one plane with the narrow pixel dimension vertical, and one with the narrow dimension 


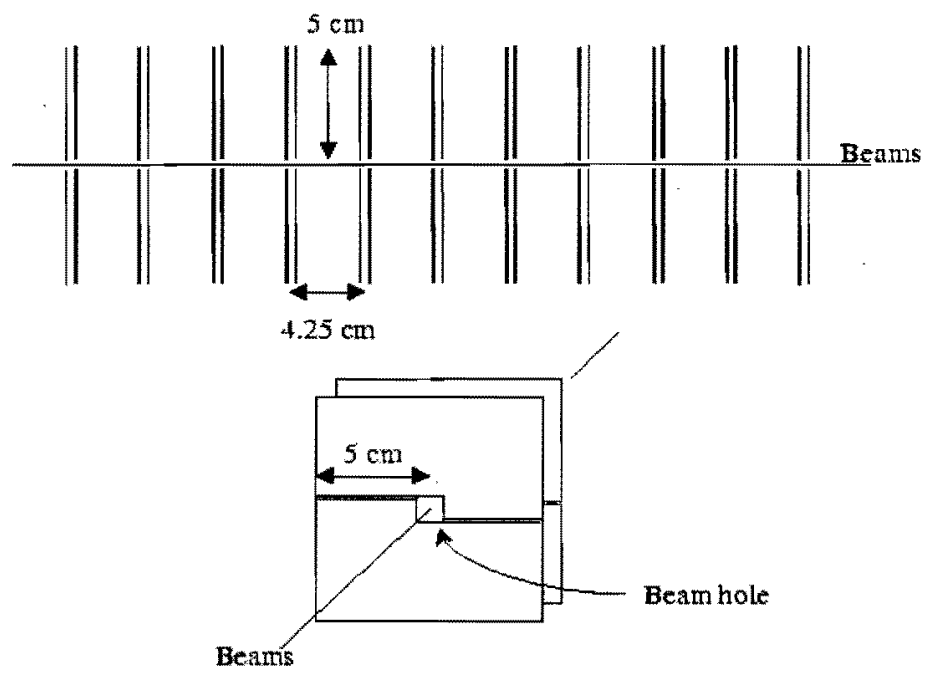

Figure 4.1: Schematic drawing of part of the pixel detector.

horizontal, and is composed of two $5 \mathrm{~cm} \times 10 \mathrm{~cm}$ halves. The half stations are mounted above and below the beam and are arranged so that a small square hole is left for the beams to pass through. Table 4.1 summarizes the properties of the pixel detector.

\subsection{Sensor, Readout-Chips, and Their Bonding}

With the pixel sensors positioned to leave a $12 \mathrm{~mm} \times 12 \mathrm{~mm}$ square beam hole, the edges of the sensors $6 \mathrm{~mm}$ from the beam will be exposed to a fluence of $\sim 10^{14}$ particles $/ \mathrm{cm}^{2}$ per year, given a luminosity of $2 \times 10^{32} \mathrm{~cm}^{-2} \mathrm{sec}^{-1}$. This is similar to the fluence expected in the CMS and ATLAS pixel detectors. The issues affecting the radiation hardness of sensors are material and implant type, guard-ring design for high voltage operation, and operating temperature. The choices made by $\mathrm{BTeV}$ are to use $\mathbf{n}^{+}-\mathrm{on}-\mathrm{n}$ sensors with relatively low resistivity bulk material, probably with p-spray (as opposed to p-stop) $\mathbf{n}$-side electrode separation, with multiple p-side guard rings, operated at about $-5^{\circ} \mathrm{C}$. These choices, and the choice of pixel dimensions and signal processing requirements (e.g. the number of ADC bits required) have been made based on input from four sources. These are (1) simulation (see Appendix A), (2) BTeV experience with ATLAS sensors in beam tests at Fermilab, (3) tests on devices made in collaboration with BNL and CMS, and (4) close communication with groups world-wide working on these issues for LHC experiments. The silicon may be oxygen-diffused as prototyped by the RD42 (ROSE) Collaboration at CERN[1], depending on $\mathrm{BTeV}$ tests with recently received SINTEF detectors of normal and oxygen-diffused types. 
Table 4.1: Pixel Vertex Detector

\begin{tabular}{|l|l|}
\hline Property & Value \\
\hline Pixel size & rectangular: $50 \mu \mathrm{m} \times 400 \mu \mathrm{m}$ \\
Plane Dimensions & $10 \mathrm{~cm} \times 10 \mathrm{~cm}$ \\
Central Square Hole Dimensions (adjustable) & nominal setting: $12 \mathrm{~mm} \times 12 \mathrm{~mm}$ \\
Total Planes & 62 \\
Total Stations & 31 \\
Pixel Orientations (per station) & one with narrow pixel dimension \\
& vertical \& the other with \\
Separation of Stations & narrow dimension horizontal \\
x-Plane to y-Plane Separation (within station) & $4.25 \mathrm{~cm}$ \\
Total Station Depth (incl cooling, supports) & $5.0 \mathrm{~mm}$ \\
Sensor Thickness & $6.5 \mathrm{~mm}$ \\
Readout Chip Thickness & $250 \mu \mathrm{m}$ \\
Total Station Radiation Length (incl RF shielding) & $200 \mu \mathrm{m}$ \\
Total Pixels & $2 \%$ \\
Total Silicon Area & $3 \times 10^{7}$ \\
Readout & $\approx 0.6 \mathrm{~m}{ }^{2}$ \\
Trigger & analog readout $(3 \mathrm{bits})$ \\
Rate Requirements & signals are used in Level 1 trigger \\
Noise Requirement & time between beam crossings is $132 \mathrm{~ns}$. \\
& desired: $<10^{-6}$ per channel/crossing \\
Resolution & required: $<10^{-5}$ per channel/crossing \\
Radiation Tolerance & better than $9 \mu \mathrm{m}$ \\
Power per Pixel & $>6 \times 10^{14}$ particles $/ \mathrm{cm}^{2}$ \\
Operating Temperature & $<60 \mu$ Watt \\
\hline \hline
\end{tabular}

The pixel sensors will be read out by an Application Specific Integrated Circuit (ASIC) designed at Fermilab, called FPIX2. This chip will differ from pixel readout chips under development for LHC experiments in two significant ways. First, its analog front end has been optimized for the $132 \mathrm{~ns}$ time between beam crossings planned for the Tevatron. Second, its output bandwidth is much greater than any other chip under development; high enough bandwidth to easily read out all hit data.from every beam crossing and provide it to the Level1 trigger hardware. FPIX2 is being designed for fabrication in an $0.25 \mu \mathrm{m}$ CMOS process using guard rings and enclosed-geometry transistors for radiation hardness. Recent studies by RD49[2] have shown that, given these design practices, standard deep submicron CMOS integrated circuits are at least as radiation hard as circuits implemented in military processes developed specifically for radiation hardness. In addition, the standard deep submicron CMOS is more readily available, has better yield, faster delivery times, and is much less expensive. 
FPIX2 is the product of an R\&D effort which started in early 1997. Two generations of prototype readout devices have firmly established the basic front-end design and readout architecture. The first generation prototype (FPIX0) contained a 12 column $\times 64$ row array of $50 \mu \mathrm{m} \times 400 \mu \mathrm{m}$ pixels. The second generation prototype (FPIX1) contained 18 columns $\times 160$ rows of pixels of the same size. We have not yet finalized the specification for the size of FPIX2, but it may be as large as 32 columns $\times 256$ rows $(12.8 \mathrm{~mm} \times 12.8 \mathrm{~mm}$ active area).

The silicon sensors will be connected to the readout chips using bump-bonding technology. We have had experience with indium bonding down to $30 \mu \mathrm{m}$ pitch, and both fluxed and fluxless lead-tin solder bonding at $50 \mu \mathrm{m}$ pitch. Both indium and fluxless solder yield acceptable results. We believe that either of these two technologies will yield a failure rate of $\sim 2 \times 10^{-4}$ or better. This belief is based on the results of our own large-scale tests with dummy circuits, and on the results obtained by other pixel R\&D groups.

\subsection{Multichip Assemblies and High Density Interconnec- tions}

Multichip assemblies will be made by bonding $\sim$ five readout chips (the number of chips in a module will depend on the final readout chip size) to a single sensor. Each multichip assembly will have a multilayer kapton "high density interconnect" (HDI) mounted on it. The HDI will provide connections to bias the sensor, control the readout chips, and receive output data.

One edge of each readout chip will have wire-bond pads for connection to the HDI. We have demonstrated this capability with FPIX1 chips and a prototype five-chip HDI made by Fujitsu. This prototype multilayer flexible circuit has $20 \mu \mathrm{m}$ lines, and $20 \mu \mathrm{m}$ line-to-line separation. Vias from an outer layer to an inner layer in the flex circuit have a pad size of 108 $\mu \mathrm{m}$ (diameter) and a hole size of $25 \mu \mathrm{m}$. Vias between the inner layers have a $350 \mu \mathrm{m}$ pad size and a $25 \mu \mathrm{m}$ hole size. Electrical tests of the five-chip assemblies have demonstrated that the prototype five-chip module performs at least as well as our previous single chip assemblies.

In our baseline design, the HDI will carry signals between the FPIX2's and the control and readout circuitry located at one end of the HDI. This circuitry will include a radiation hard data serializer, a VCSEL optical driver, a PIN diode optical receiver, and a control, monitoring, and timing ASIC. The VCSEL and the optical receiver are commercial radiation hard parts. We plan to use a radiation hard data serializer ASIC (CHFET) developed for CMS, which we have tested. The control, monitoring, and timing ASIC will be developed at Fermilab. We are also pursuing the possibility that the FPIX2 chips can drive data directly (using high speed LVDS over copper) to commercial FPGA's located far enough from the beamline so that they need not be radiation hard. In this scenario, the control and monitoring functions would also be performed by commercial devices located outside the high radiation area. 


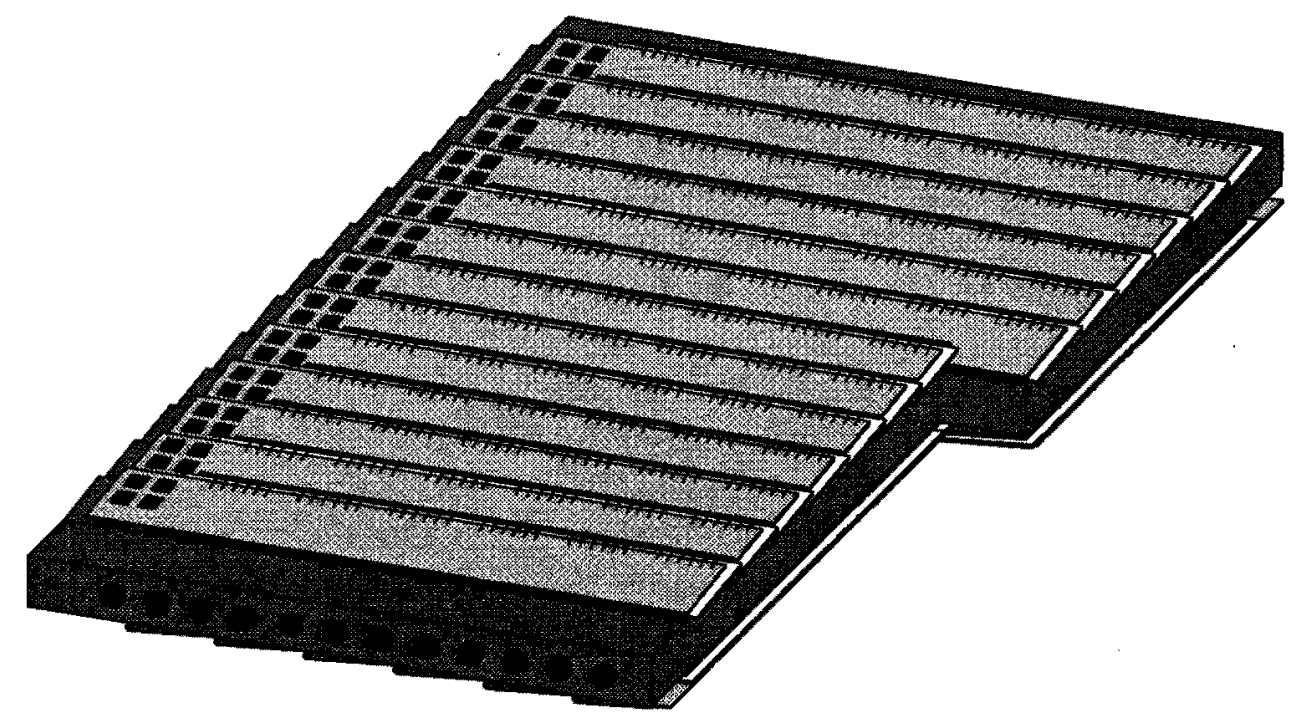

Figure 4.2: Pixels on the half plane.

Figure 4.2 shows a sketch of a half-plane assembly.

\subsection{Cooling Support, Shielding, and Housing}

The pixel half-planes will be "L-shaped," with one frame above and one below the beam. Each frame will be movable such that the alignment with respect to the beam is adjustable. In that way, the size of the beam hole will also be adjustable, separately both horizontally and vertically. This will allow us to optimize the balance between radiation damage and physics reach. Smaller hole size gives better vertex resolution, acceptance, tagging efficiency, and thus physics reach. The experiment performance can be optimized as experience is gained, both in operating conditions and physics analysis issues.

The mechanical supports will be carbon composite structures which will include integrated cooling channels (tubes). These structures will be manufactured by Energy Science Laboratories, Inc. (ESLI) by sandwiching nonporous carbon tubes between two sheets of carbon "flocking," which consists of many individual carbon fibers and looks like velvet material. Additional carbon will then be vapor deposited into the structure, making a rigid composite. The "fuzzy carbon" surfaces of this structure will be machined to provide a "shingled" surface on which the multichip assemblies will be mounted. This construction allows sensors on one side of the cooling support to overlap, and therefore allows two pixel measurement planes to be mounted on a single cooling support. Heat is transferred through the thickness of the readout chips to the carbon fibers of the fuzzy surface. These carbon fibers efficiently transfer the heat to the coolant flowing in the cooling tubes. ESLI has delivered a first prototype for thermal and mechanical testing (see Figure 4.3). 

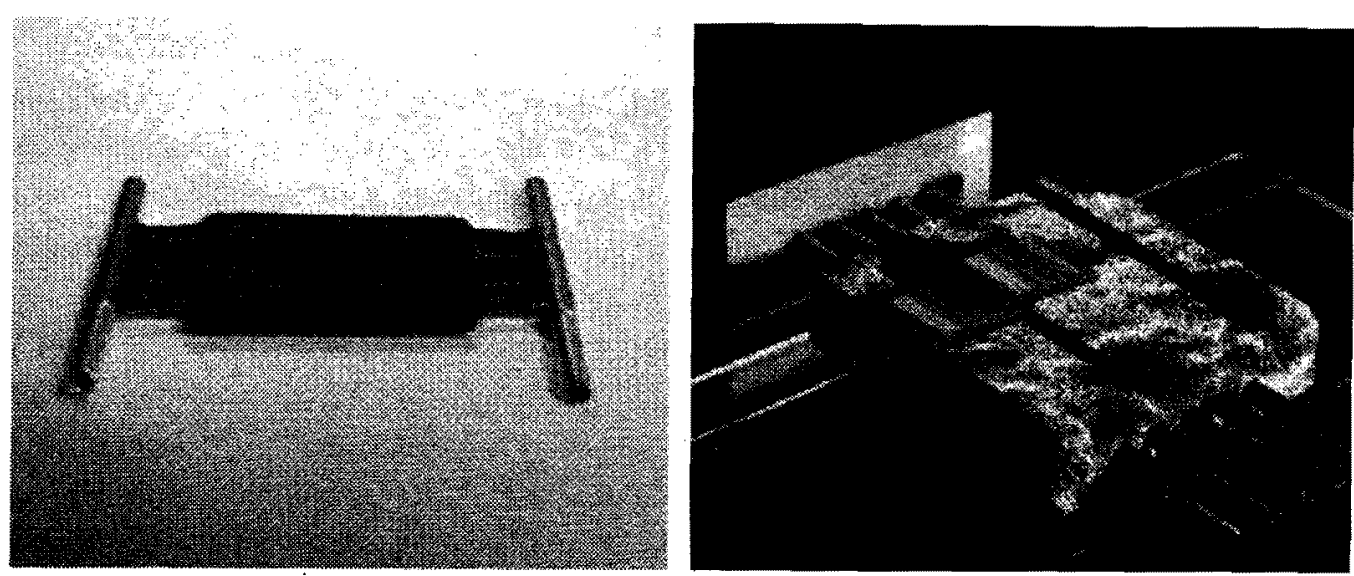

Figure 4.3: Sample fuzzy carbon composites; note the shingling in the picture on the right.

It is likely that the pixel detector and readout hybrid assemblies will need to be electrically shielded from the circulating beams. The HERA-B vertex detector currently operates successfully with a $150 \mu \mathrm{m}$ thick aluminum RF shield[3]. We have built, and successfully operated, a test facility to simulate the Tevatron beam (using an RF generator and a wire inside a stainless steel pipe) to allow us to understand the detailed needs of the $\mathrm{BTeV}$. pixel system (see Appendix A).

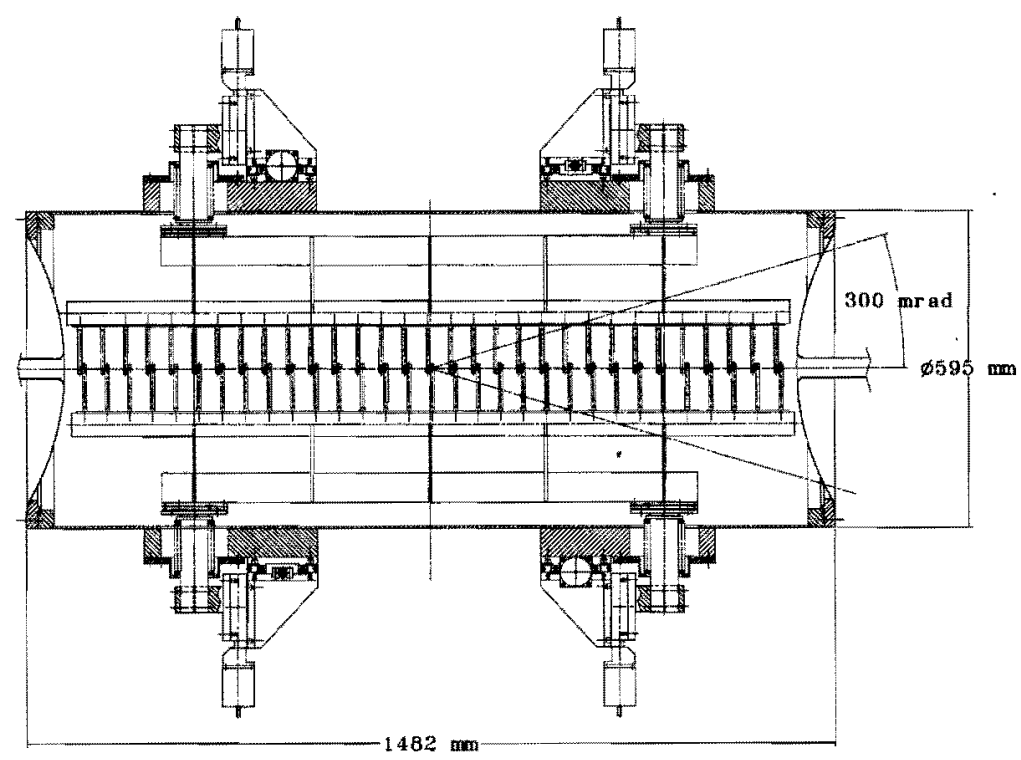

Figure 4.4: Side view of the vacuum vessel and support structure for the pixel detector.

Figure 4.4 shows a conceptual design for the aluminum vacuum vessel and carbon support structure for the pixel detector. The vessel is cylindrical with a length of $\sim 150 \mathrm{~cm}$ and a 
diameter of $59.5 \mathrm{~cm}$. Particles within the $300 \mathrm{mrad}$ acceptance of the spectrometer traverse only the pixel stations and the $0.75 \mathrm{~mm}$ thick exit window. The carbon support and cooling planes will be held in position by a structural cooling manifold, whose position will be controlled by motors located just outside the vacuum vessel. The design and orientation of these motors will allow for operation in the $\sim 1 \mathrm{~T}$ field of the spectrometer magnet at the motor locations.

The expected alignment precision of the pixel assembly will be $\sim 1 \mu \mathrm{m}$, a level which has been achieved by HERA-B[4]. Final alignment, of course, will be done with particle tracks, with a rapid-turnaround computing system dedicated to obtaining and checking the alignment parameters for the trigger at the start of each fill. Note, however, that the interaction vertex is determined event-by-event in the same coordinate system as the secondary vertices. Thus, the exact position of the pixel array with respect to the beam need not be reproduced fill to fill.

\subsection{Total Material}

\begin{tabular}{|c||c|c|c|c|}
\hline Item & Thickness $(\mathrm{mm})$ & $X_{0}(\mathrm{~mm})$ & Coverage & $X / X_{0}(\%)$ \\
\hline \hline Sensor & 0.25 & 93.6 & 1.20 & 0.32 \\
\hline Readout chip & 0.20 & 93.6 & 1.15 & 0.25 \\
\hline \hline Bumps and wire bond & 0.02 & 10.0 & 0.02 & 0.004 \\
\hline \hline HDI & 0.224 & 284 & 1.00 & 0.08 \\
\hline \hline Components on HDI & & & & 0.04 \\
\hline \hline Glue & & & & 0.02 \\
\hline \hline Coolant $($ water $/$ alcohol $)$ & 0.35 & 400 & 0.10 & 0.01 \\
\hline \hline Substrate $(\mathrm{C}-C)$ & 0.5 & 427 & 0.50 & 0.06 \\
\hline \hline Shielding $($ Al $)$ & 0.1 & 89.0 & 1.00 & 0.11 \\
\hline \hline Total & & & & 0.89 \\
\hline \hline
\end{tabular}

Table 4.2: Material budget of a BTeV pixel plane. The column labelled "coverage" shows the factor applied to account for overlaps of the sensors and readout chips, and for geometric coverage (e.g. area covered by bump bonds/total area). The numbers given for components on the HDI and for glue are derived from the ATLAS Pixel Detector TDR [5].

Table 4.2 summarizes the material budget for one pixel plane. The numbers for the support and cooling structure for a full station, and for the coolant, have been divided by two (coverage column), since two planes are held on one support. To account for the nonsensitive area in each module (required for guard rings and scribe edge for the sensor, and for the periphery and wire bond pads of the readout chip), a $20 \%$ overlap of sensors and a $15 \%$ overlap of readout chips are included. 


\subsection{Test Beam Results}

During the 1999 Fermilab fixed target run, tests were performed using both FPIX0 and FPIX1 readout chips bonded to ATLAS prototype pixel sensors (see Appendix A). Data was taken with as many as four pixel devices in a telescope configuration. These four pixel planes, and smaller subsets of them, were placed between upstream and downstream siliconmicrostrip telescope elements. Thus, the pixel devices could be separately studied as they might be used in an experiment, as well as compared to beam-track projections from the microstrip system. Resolution was studied as a function of the angle between the beam and the pixel plane. In a short test, a diamond target was placed upstream of the four plane pixel telescope, and multi-particle interactions were recorded and analyzed (see Figure 4.5). In addition, tests were done with a pixel plane in a magnetic field while beam particles traversed the pixel plane and upstream telescopes. Each pixel configuration was tested with a variety of applied sensor biases and readout thresholds to study the sensitivity of the results to these parameters.

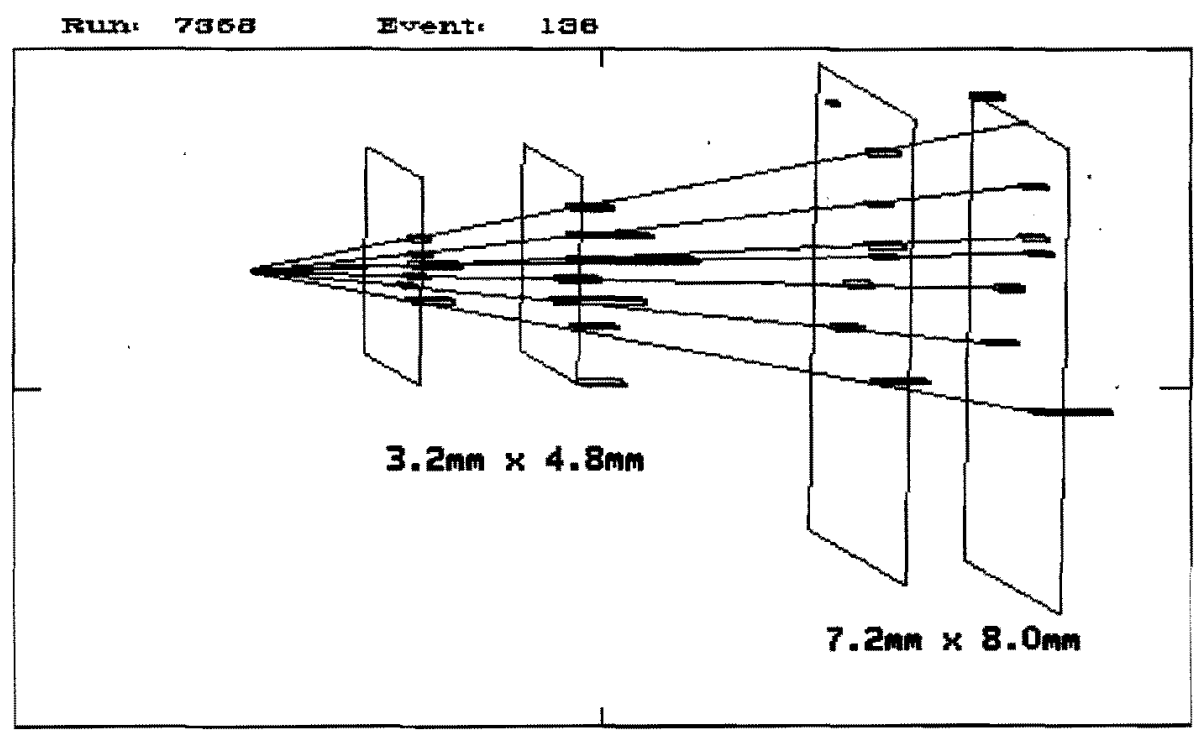

Figure 4.5: Multiparticle interaction observed in Fermilab beam test. This density of tracks is much higher than expected in the $\mathrm{BT}$ eV pixel detector.

Figure 4.6 shows the resolution (in the coordinate measured by the $50 \mu \mathrm{m}$ pixel pitch) as a function of the incident beam angle for an FPIX0-instrumented detector. The solid line in the plot is the (Gaussian) resolution function used for the Monte Carlo studies presented in this proposal. This line is a piecewise linear fit to the resolution obtained in $\mathrm{BTeV}$ pixel simulations. FPIX0 provided an analog output which was digitized by an off-chip 8-bit FADC. The plot shows both the resolution obtained using this 8-bit information directly, 


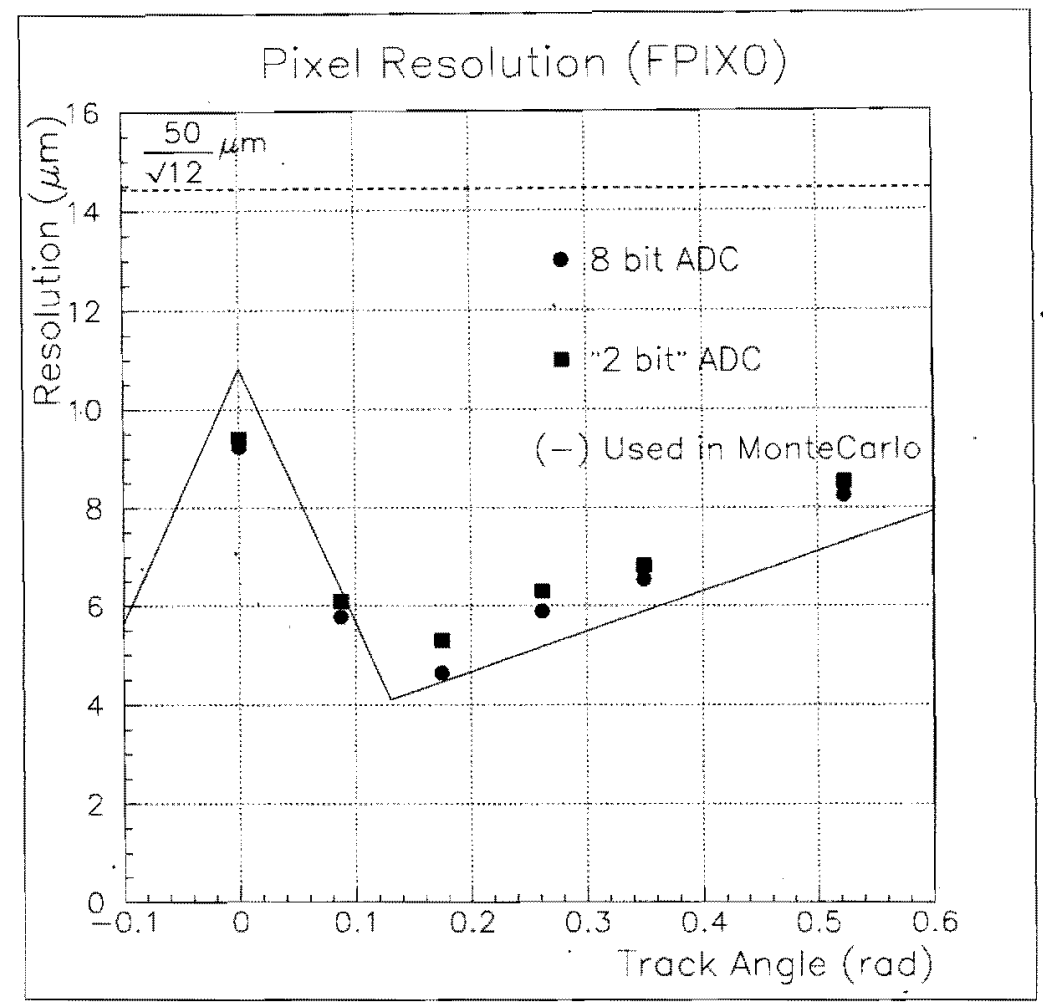

Figure 4.6: Resolution as a function of the angle of the incident beam.

and also the resolution obtained by degrading the pulse height to 2-bits of information. This result confirms our simulation result that very good resolution can be obtained using charge sharing even with very coarse digitization. Based on these results it has been decided that FPIX2 will have a 3 -bit FADC in each pixel cell. This will provide excellent spatial resolution. In addition, since the ADC will be implemented using eight independent comparators, some of the thresholds may be used to indicate the presence of delta rays and photon conversions.

We have also used test beam data to parameterize the non-Gaussian tails (due to low energy delta rays) in the pixel detector resolution function. This parameterization has been used in Monte Carlo simulations of the vertex trigger.

These test beam results show several important things:

- ATLAS prototype sensors coupled to FPIX chips meet BTeV resolution requirements

- The FPIX-type front-end performs as needed

- The BTeV multiplicity is easily handled by $50 \mu \mathrm{m} \times 400 \mu \mathrm{m}$ pixels

- Alignment and support stability are important to meet the resolution goals 


\subsection{Summary}

Over the last three years, BTeV collaborators have been working vigorously to establish a pixel detector capability for Fermilab, tuned to the unique features of the Tevatron crossing time and BTeV trigger needs. Since the earliest concerns were related to sensors, readout, and bump bonding, the primary focus has been on those issues. Progress has been gratifyingly rapid, both at Fermilab and for the LHC. This progress is evident in the success of the test beam effort at Fermilab, the results of which validate the ideas used for this BTeV proposal. The proposal to use a "shingled" fuzzy carbon support and cooling structure appeared recently, and preliminary cooling capacity tests are underway on a prototype. This is the necessary first step needed to validate the material estimate presented here. Yields of all pixel-related components have been much higher than anticipated, giving hope that a focus on simplicity within our aggressive technical approach will succeed. This approach, combined with the early implementation of a significant subsample $(\sim 10 \%)$ of final-design detectors, should allow reliable planning and achievable goals for the experiment. 


\section{Bibliography}

[1] F. Lemeilleur, et al., "3 $3^{\text {rd }}$ RD48 Status Report", CERN/LHC 2000-009 (31 December 1999). Available at http://rd48.web.cern.ch/RD48/status-reports/RD48-3rd-statusreport.doc (requires MS Word)

[2] P. Jarron, et al., " $3^{\text {rd }}$ RD49 Status Report Study of the Radiation Tolerance of ICs for LHC"; CERN/LHCC 2000-03 (13 January 2000). Available at http://www.cern.ch/RD49/RD49News/RD49StatusReport3.pdf (requires Acrobat Reader 4)

[3] C. Bauer et al., "First Experience and Results from the HERA-B Vertex Detector System"; Nucl. Instrum. Meth. A 418, (1998) 65.

[4] Iris Abt, private communication.

[5] "ATLAS Pixel Detector Technical Design Report", CERN/LHCC 98-13, available at http://atlasinfo.cern.ch/Atlas/GROUPS/INNER_DETECTOR/PIXELS/tdr.html 


\section{Chapter 5}

\section{Forward Tracking System}

\subsection{Introduction}

The major functions of the forward charged particle tracking system are to provide high precision momentum measurements for tracks found in the pixel system, to reconstruct and measure all parameters for tracks which do not pass through the vertex detector (such as $K_{s}$ and $\Lambda^{0}$ daughter tracks), and to project tracks into the RICH counters, EM calorimeters, and Muon detectors. Measurements from the forward tracking system are also used online in the Level 2 trigger, as explained in Chapter 8.

\subsection{General Description}

The baseline forward tracking system consists of 14 stations, 7 in each arm, placed transversely to the beam at various distances from the interaction point. Three stations are placed in the fringe-field region of the dipole magnet, three stations in the field-free region just upstream of the RICH, and one station just downstream of the RICH. The entire system extends over a distance of $\sim \pm 7 \mathrm{~m}$ and provides $\theta$-angle coverage from $\sim \pm 10 \mathrm{mrad}$ up to $\pm 300 \mathrm{mrad}$.

The design of the forward tracking system has been driven by the high density of tracks produced in the forward direction, especially with multiple interactions per crossing. Two different types of detectors are used. Most of the solid angle is instrumented using straw tube drift chambers. Straws have been chosen because they can be used to make large chambers with small cell size, and because they can be built to surround the beam pipe without requiring a heavy frame near the beam. The track density very close to the beam requires detectors with even higher granularity; we have chosen to instrument the central section of each station with silicon microstrip detectors.

Tables 5.1 and 5.2 list all the geometric parameters and the main characteristics of the forward tracker.

This forward tracking system configuration has sufficient segmentation to handle the 
Table 5.1: Properties of the baseline forward straw tracker

\begin{tabular}{|l|l|}
\hline \hline Property & Value \\
\hline Straw size & $4 \mathrm{~mm}$ diameter \\
Central hole & $24 \mathrm{~cm} \times 24 \mathrm{~cm}$ \\
Total Stations & $14(7$ per arm $)$ \\
Z positions $(\mathrm{cm})$ & $96,146,196,296,341,386,706$ \\
Half size $(\mathrm{cm})$ & $30,45,60,90,105,118,210$ \\
Views per station & $3(\mathrm{X}, \mathrm{U}, \mathrm{V})$ \\
Layers per view & 3 \\
Total number of straws & 66,780 \\
Total station thickness & $0.6 \% X_{0}$ \\
Total channels & 118,440 \\
Readout & ASD + timing chip (6 bits $),$ sparsified \\
\hline \hline
\end{tabular}

Table 5.2: Properties of the baseline forward silicon tracker

\begin{tabular}{|l|l|}
\hline \hline Property & Value \\
\hline Si-sensors & $6^{\prime \prime}$ wafers, $p$-on- $n$ type \\
Pitch & $100 \mu \mathrm{m}$ \\
Thickness & $200 \mu \mathrm{m}$ \\
Sensor configuration & 4 ladders of 2 sensors +2 single sensors \\
Coverage & $24 \mathrm{~cm} \times 24 \mathrm{~cm}$ \\
Central hole & $5.4 \mathrm{~cm} \times 5.4 \mathrm{~cm}(7 \mathrm{~cm} \times 7 \mathrm{~cm}$ in last station $)$ \\
Total stations & $12(6$ per arm $)$ \\
Z positions $(\mathrm{cm})$ & $100,150,200,300,390,710$ \\
Views per station & $3(\mathrm{X}, \mathrm{U}, \mathrm{V})$ \\
Channels per view & 3,000 \\
Total channels & 108,000 \\
Readout & sparsified binary \\
\hline \hline
\end{tabular}

high hit multiplicities that are expected when $b \bar{b}$ events are produced in the forward region. Fig. 5.1 shows occupancies in the straw tracker predicted by BTeVGeant for the case in which a $b \bar{b}$ event is produced at the design luminosity of $2 \times 10^{32} \mathrm{~cm}^{-2} \mathrm{~s}^{-1}$. It is worth noting that these occupancies are almost a factor of two higher than those expected on average at this luminosity. The maximum occupancy in the silicon strip detectors, which have 40 times finer pitch than the straw chambers, is everywhere less than $\sim 4 \%$ for the conditions of Fig. 5.1. 


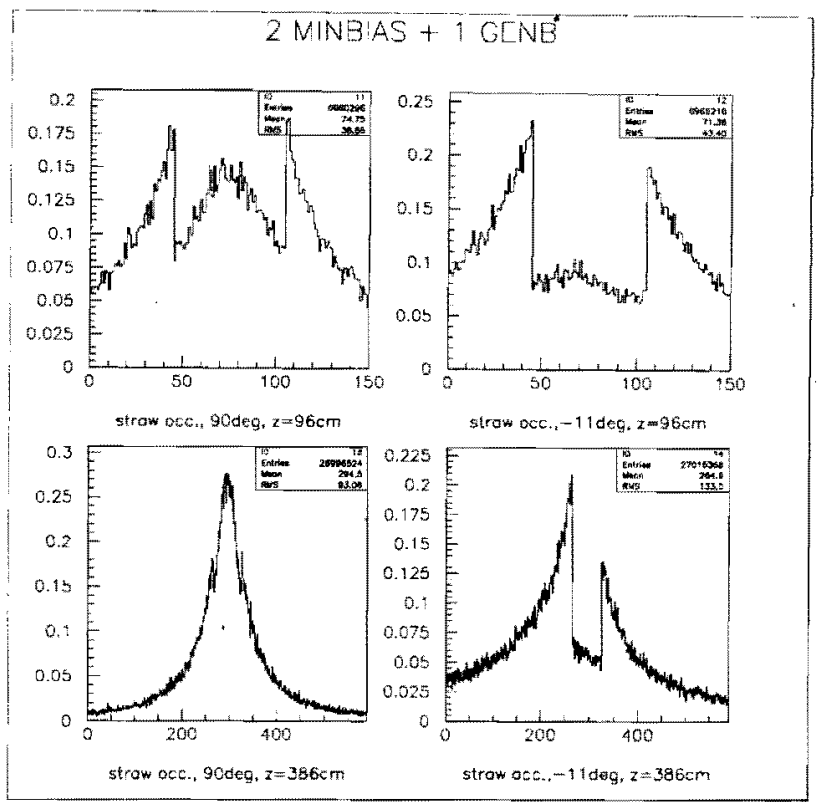

Figure 5.1: Occupancies in the first station of straws, and the station just upstream of the RICH counter, when a $b \bar{b}$ event is produced at the design luminosity of $2 \times 10^{32} \mathrm{~cm}^{-2} \mathrm{~s}^{-1}$. The two histograms on the left are for $\mathrm{X}$-view straws, while those on the right are for $\mathrm{U}$-view straws. The $\mathrm{V}$-views have identical occupancies (mirrored about zero) to the $\mathrm{U}$-views.

\subsection{Forward Straw Tracker}

\subsubsection{Detector Description and Layout}

The forward straw tube tracker consists of stations that provide 3 coordinate measurements, $X, U$ and $V$, where the two stereo views, $U$ and $V$, are at $\pm 11.3^{\circ}$ around the $Y$ bend coordinate. With three layers per view, this configuration provides excellent resolution in the bend plane while maintaining a robust ability to reject ghost combinations of hits. It has sufficient redundancy to achieve a high detection efficiency and to resolve the left/right ambiguity a very large fraction of the time. The construction is modular, following a technique developed for the SDC straw tracker[1].

All the sense wires for the straw cells that do not terminate at the central hole are divided electrically using a small glass capillary bead following the technique used for the ATLAS TRT [2]. This cuts the occupancy rates in half. The sense wires in straws that span more than $80 \mathrm{~cm}$ have additional supports, which are realized following the helical design developed for the ATLAS TRT. 


\subsubsection{Front End Electronics and Drift Time Measurement}

The straw tube chambers will be instrumented using electronics developed by the University of Pennsylvania [3], initially for the SDC straw chambers, and more recently for the ATLAS TRT. These radiation hard integrated circuits include high gain preamplifiers, pole-zero networks for pulse shaping and ion-tail cancellation, and leading edge discriminators.

The drift time will be measured using digital TDC's. The information from the straw tracker, like all information from every subsystem in the $\mathrm{BTeV}$ spectrometer, must be digitized and read out for every crossing. This means that a new TDC must be designed for $\mathrm{BTeV}$. The small diameter of the straws makes the specifications of this TDC easy to achieve. A six-bit single-hit TDC, with $1.5 \mathrm{~ns}$ wide bins covering $96 \mathrm{~ns}$, is sufficient to provide a drift distance measurement precision better than $100 \mu \mathrm{m}$.

\subsubsection{Technical issues}

We are developing a prototype straw tube which places an aluminum conduction layer between two Kapton films, the inner one next to the gas volume being a carbon loaded, low resistivity film. The idea is that the Kapton forms a protective barrier, similar to the graphite layers deposited on the inner surface of the ATLAS TRT straws. Without this protective barrier, there is a danger that the aluminum layer may be etched away, limiting the lifetime of the straw. We measured the surface resistivity of the aluminum coated, carbon loaded Kapton film of our prototype to be $6.5 \pm 1.0 \Omega$ /square, which is comparable to the specified value for the TRT straw tube. The details of the prototype straw material are listed in Table 5.3. The 1 mil thickness of each film is chosen as a compromise between the $0.5 \mathrm{mil}$ thick films used for SDC straw tubes (which had very little mechanical rigidity). and the thicker and reinforced TRT straws. Our final design will likely have the straws supporting some of their own weight. If this turns out to not to be the case, we may use instead 0.5 mil Kapton films which have been shown to work and would provide a reduction of material in the detector volume.

\begin{tabular}{|l|l|}
\hline Description & BTeV Straw Prototype \\
\hline \hline Kapton film & Inner: Polyimide type XC \\
& $25 \pm 2.5 \mu m$ thickness \\
& Outer: Polyimide type $100 \mathrm{VN}$ \\
& $25 \pm 2.5 \mu m$ thickness \\
\hline Density & $1.42 \mathrm{~g} / \mathrm{cm}^{3}$ \\
\hline Aluminum layer & $(0.2 \pm 0.08) \mu m$ thickness \\
\hline Resistivity of inner Kapton layer & $6.5 \pm 1.0 \Omega /$ square \\
\hline \hline
\end{tabular}

Table 5.3: Summary of material specifications for the BTeV prototype straw tubes 


\subsection{Forward Silicon Tracker}

\subsubsection{Detector Description and Layout}

Our design consists of stations with three planes of $200 \mu \mathrm{m}$ thick single-sided silicon microstrip detectors with $100 \mu \mathrm{m}$ pitch. On each plane, ten silicon detector wafers are mounted on low mass carbon fiber support and are arranged, wherever possible, in ladders of two daisychained Si-sensors to minimize the number of readout channels. The resulting configuration is depicted in Fig. 5.2.

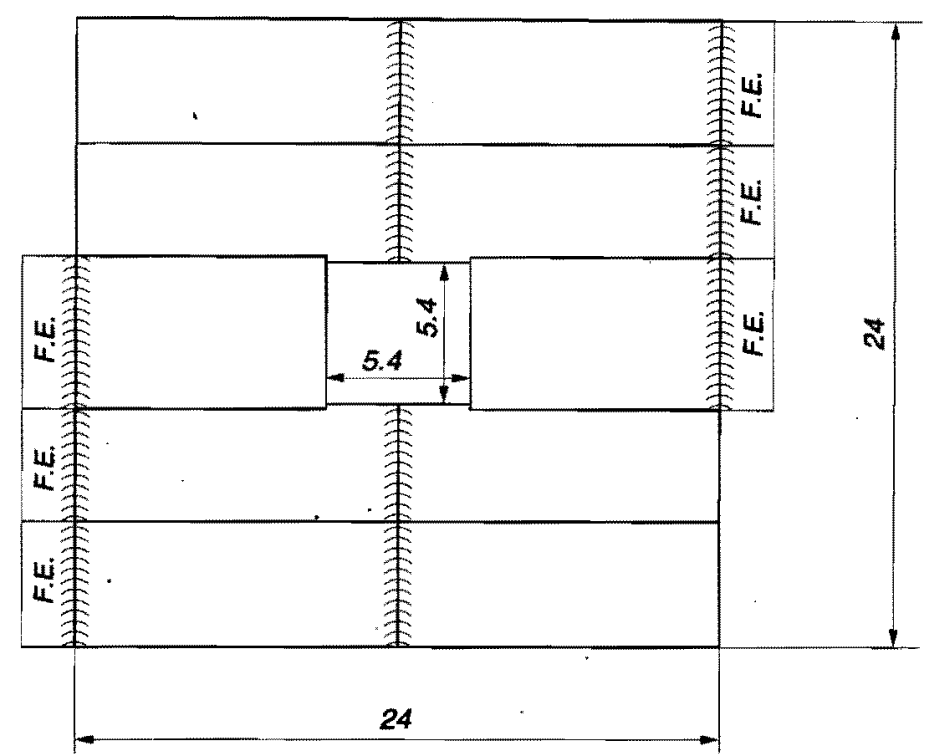

Figure 5.2: Sketch of a silicon detector plane. It consists of 4 ladders of two daisy-chained Si-sensors plus 2 single sensors. One strip overlap between contiguous detector elements ensures good efficiency over the entire plane. Dimensions are in centimeters and $F$.E. stands for Front-End read out electronics.

Three views are provided by rotating the planes by the appropriate angles: $X, U$ and $V$, where the two stereo views, $U$ and $V$, are at $\pm 11.3^{\circ}$ around the $Y$ bend coordinate. Each plane consists of about 3,000 channels; the entire system of 12 stations, 6 in each arm, has about 108,000 channels in total.

The Si-sensors, having a length up to $12 \mathrm{~cm}$, are of the standard $p$-on- $n$ type, with multiple n-side guard rings to allow high voltage operation, and are produced using 6 " wafer technology.

The front-end electronics is distributed along the two opposite sides of each plane where it is cooled by a fluid circulating in a duct embedded in the support structure all around the periphery of the plane. This is also enough to keep the sensors at low temperature, provided that the sensors and the support are in close thermal contact. In our structure, we ensure good thermal conductivity all across the planes by employing long sensors. 
The preamplifiers are $\mathrm{AC}$ coupled to the strips by means of capacitors directly integrated on the sensors. Each channel is read out in binary mode providing a $\sigma=100 \mu \mathrm{m} / \sqrt{12}=29$ $\mu \mathrm{m}$ resolution, adequate for our physics goals.

We do not foresee any major problems in building these detectors since we can profit from the enormous experience accumulated in CDF, as well as in other experiments, and that coming from the ongoing R\&D programs for LHC experiments. Nevertheless, we anticipate a possible minor concern that requires a proper backup solution. If the production of 200 $\mu \mathrm{m}$ thick 6 " wafers would be problematic, we will use ladders of shorter sensors wherever necessary.

\subsubsection{Radiation Issues}

It is well known that the exposure of silicon detectors to high radiation doses causes damage that limits their useful lifetime. Thanks to the enormous progress accomplished during the last few years, we can now build detectors that can be operated after exposure to fluences in excess of $10^{14}$ particles $/ \mathrm{cm}^{2}[4]$.

In $\mathrm{BTeV}$, we expect a radiation level at the silicon detectors that decreases rapidly with increasing distance from the beam. Important radiation damage effects, if any, will be confined to a small region around the central hole of the stations.

The highest levels of radiation occur at the station closest to the interaction region in the two symmetric arms of the apparatus. As shown in Fig. 5.3, the maximum value of the fluence is expected to be $\sim 1.6 \times 10^{13}$ particles $/ \mathrm{cm}^{2} /$ year, given a luminosity of $2 \times 10^{32}$ $\mathrm{cm}^{-2} \mathrm{~s}^{-1}$. This is slightly less than the dose expected for Layer 0 of the CDF silicon tracker at the same luminosity [5]. With a proper choice of detectors, and by keeping them at low temperature, such as $0^{\circ} \mathrm{C}$, we will operate the detectors with a safety margin superior to that of CDF and those of LHC experiments. In the worst case scenario, we can expect serious radiation damage effects only on a minor portion of our detectors close to the beam after several years of operation.

We are starting an $R \& D$ program on silicon sensors to investigate other possibilities to further extend the useful lifetime of the forward silicon tracker. In particular, oxygenated sensors look very promising.

\subsubsection{Readout Electronics}

Even given the low occupancy expected in the Forward Silicon Tracker, the output bandwidth required to read out all hit information from every crossing is higher than is provided by any SSD chip, either already fabricated or being developed for another experiment. For this reason we have decided to develop a new readout chip with very high readout bandwidth. We will also take the opportunity to design a continuous-time-filter preamplifier capable of exploiting all the advantages offered by the relatively long bunch-crossing period of the Tevatron collider $(T=132 \mathrm{~ns})$. We are considering a new preamplifier that is derived from the $\mathrm{BaBar}$ silicon strip front-end. We can anticipate an $\mathrm{ENC} \sim 1000 e^{-}$for semi-Gaussian 


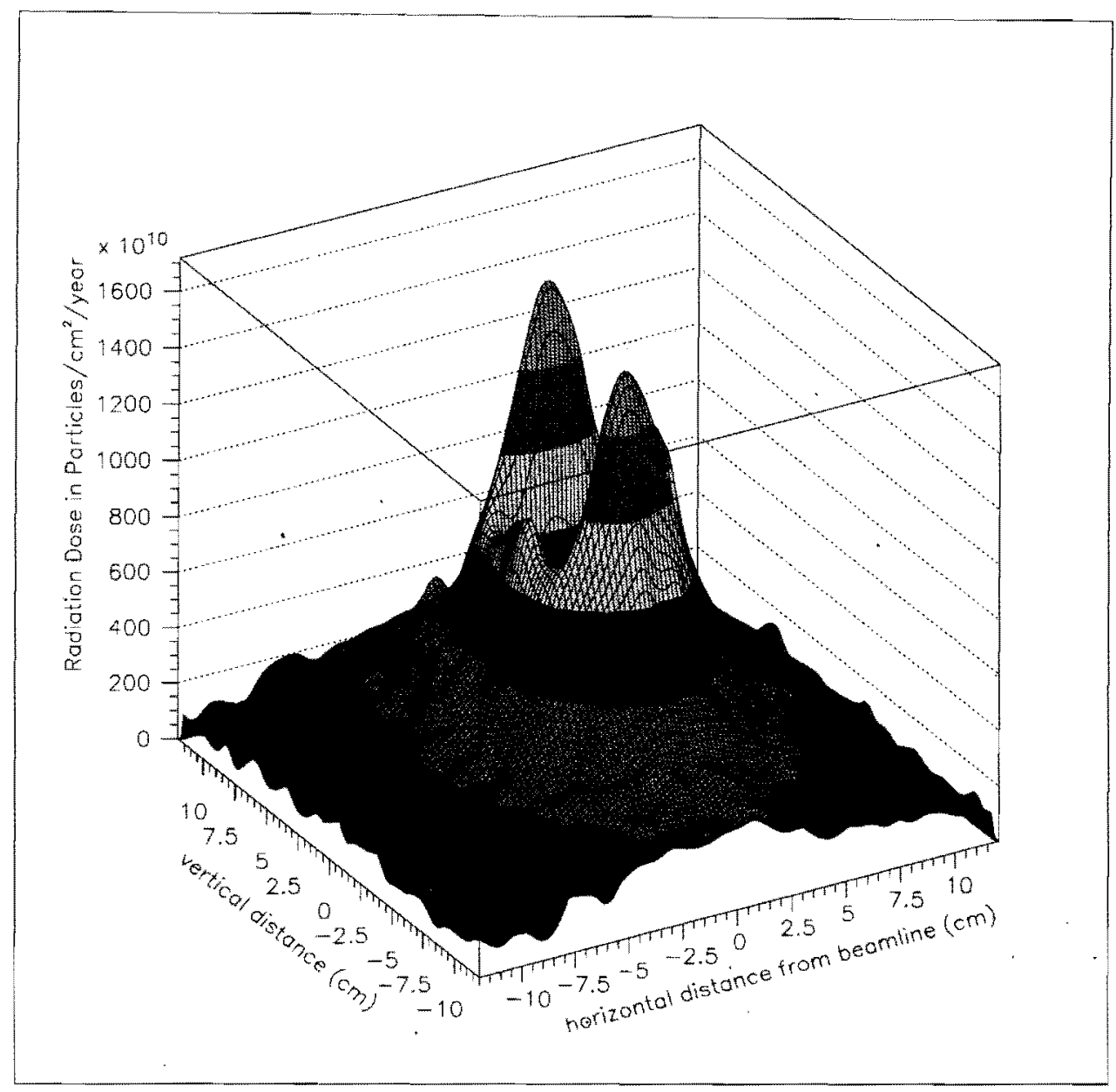

Figure 5.3: Radiation dose as a function of position in Forward Silicon Tracker Station \# 1. The horizontal magnetic field concentrates more particles above and below the square central beam hole than on either side.

shaping with $100 \mathrm{~ns}$ peaking time and a capacitive load at the input of $\sim 30 \mathrm{pF}$, as expected for our longest strips. This noise performance represents in our view "the state of the art" for silicon strip preamplifiers.

The binary readout we are presently considering is a simplified version of the readout scheme implemented in the FPIX2 pixel readout chip. It is very fast and employs a flexible scheme for zero-suppression and readout, that can be easily adapted to strips. The SSD readout chips will be designed to interface to the same electronics we will employ to read out pixel chips.

We have initiated an $R \& D$ program to design this chip, which will be implemented using $0.25 \mu \mathrm{m}$ CMOS, following the radiation tolerant design rules developed for the FPIX 2 design. 


\subsection{Forward Tracker Performance}

The system just described ensures excellent tracking performance over the full acceptance of the forward spectrometer. Figures 5.4 and 5.5 show the expected average fractional momentum resolution for $b$ decay products as a function of track momentum and of the track production angle with respect to the beam axis. For these histograms, an effective position resolution of $\sigma_{X, U, V}=150 \mu \mathrm{m}$ was assumed for each view of the straws and a resolution of $\sigma_{X, U, V}=29 \mu \mathrm{m}$ assumed for the silicon strip detectors.

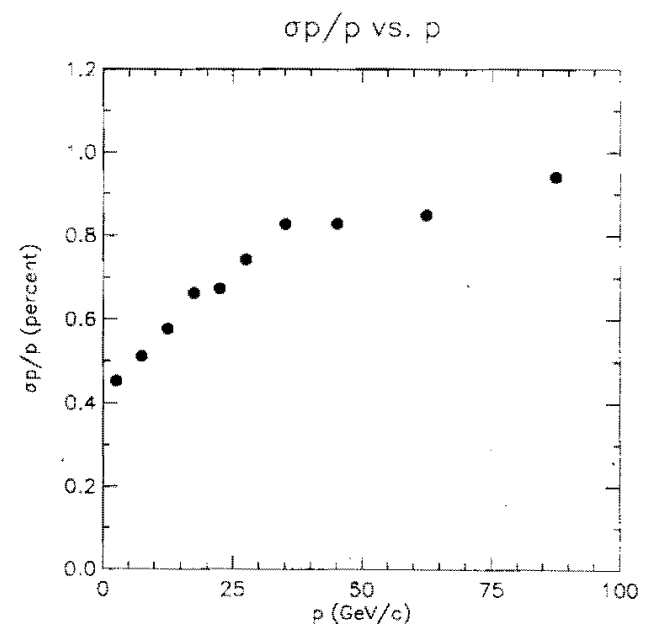

Figure 5.4: Momentum resolution as a function of track momentum for $b$ decay products.

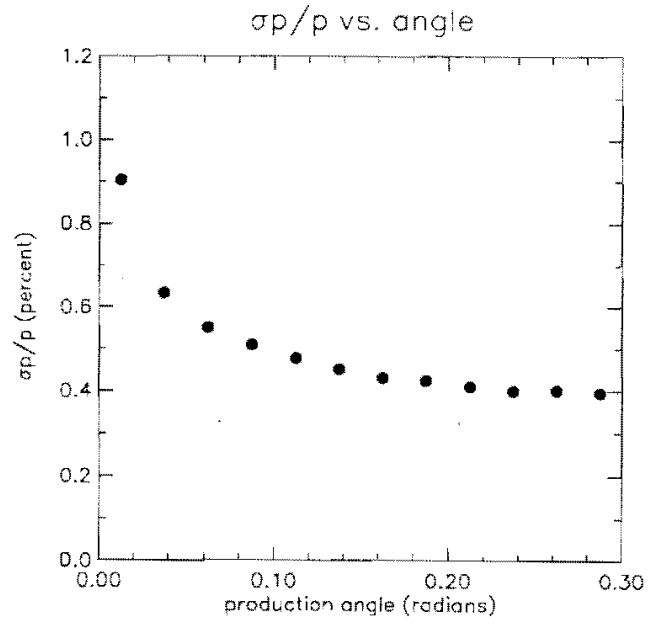

Figure 5.5: Momentum resolution as a function of polar production angle for $b$ decay products. 


\section{Bibliography}

[1] Y. Arai, et al., "A modular straw drift tube tracking system for the Solenoidal Detector Collaboration experiment, Part I: Design"; Nucl. Instrum. Meth. A 381 (1996) 355.

[2] ATLAS Inner Detector Technical Design Report, CERN/LHCC/97-16,17.

[3] F.M. Newcomer, R. Van Berg, J. Van der Spiegel and H.H. Williams, Nucl. Instrum. Meth. A 283 (1989) 806.

[4] G. Tonelli, et al., "The R\&D program for silicon detectors in CMS"; Nucl. Instrum. Meth. A 435 (1999) 109.

[5] M.A. Frautschi, "Radiation Damage Issues for the SVX II Detector"; CDF/DOC/SEC_VTX/PUBLIC/2368. 


\section{Chapter 6}

\section{Charged Particle Identification System}

Excellent charged hadron particle identification is a critical component of a heavy quark experiment. Even for a spectrometer with the excellent mass resolution of $\mathrm{BTeV}$, there are kinematic regions where signals from one final state will overlap those of another final state. For example, $B_{s} \rightarrow D_{s} K^{-}$signal must be distinguished from $B_{s} \rightarrow D_{s} \pi^{-}$background in order to measure the CKM phase $\gamma$. These ambiguities can be eliminated almost entirely by an effective particle identifier. In addition, many physics investigations involving neutral $B$-mesons require "tagging" of the flavor of the signal particle by examining the properties of the "away-side." Our studies show that kaon tagging is a very effective means of doing this. "Same-side" kaon tagging is also very effective for $B_{s}$ mesons.

\subsection{Requirements}

In the design of any particle identification system, the dominant consideration is the momentum range over which efficient separation of the various charged hadron types $-\pi, K$, and $p$ - must be provided. In BTeV, the physics goal which sets the upper end of the momentum requirement is the desire to cleanly separate $B_{d}^{o} \rightarrow \pi^{+} \pi^{-}$from $B_{d}^{o} \rightarrow K^{+} \pi^{-}$and $B_{s}^{o} \rightarrow K^{+} K^{-}$. These two-body decays produce reasonably high momentum pions and kaons. Fig. 6.1 shows the momentum distribution of pions from the decay $B_{d}^{o} \rightarrow \pi^{+} \pi^{-}$for the case where the two particles are within the spectrometer's acceptance. The low momentum requirement is defined by having high efficiency for "tagging" kaons from generic $B$ decays. Since these kaons come mainly from daughter $D$-mesons in multibody final state $B$-decays, they typically have much lower momentum than the particles in two body decays. Fig. 6.2 shows the momentum distribution of tagging kaons for the case where the signal particles are within the geometric acceptance of the spectrometer. About $1 / 5$ of the tagging kaons never exit the end of the spectrometer dipole. Almost all kaons exiting the dipole have momenta above $3 \mathrm{GeV}$. Based on these plots, we have set the momentum range requirement for the particle identification system to be

$$
3 \mathrm{GeV} / \mathrm{c}<P_{\text {particle id }}<\sim 70 \mathrm{GeV} / \mathrm{c}
$$


Finally, kaons and pions from directly produced charm decays have momenta which are not very different from the kaons from $B$-decays. The range set by the $B$-physics requirements is a reasonable, if not optimal, choice also for charm physics.
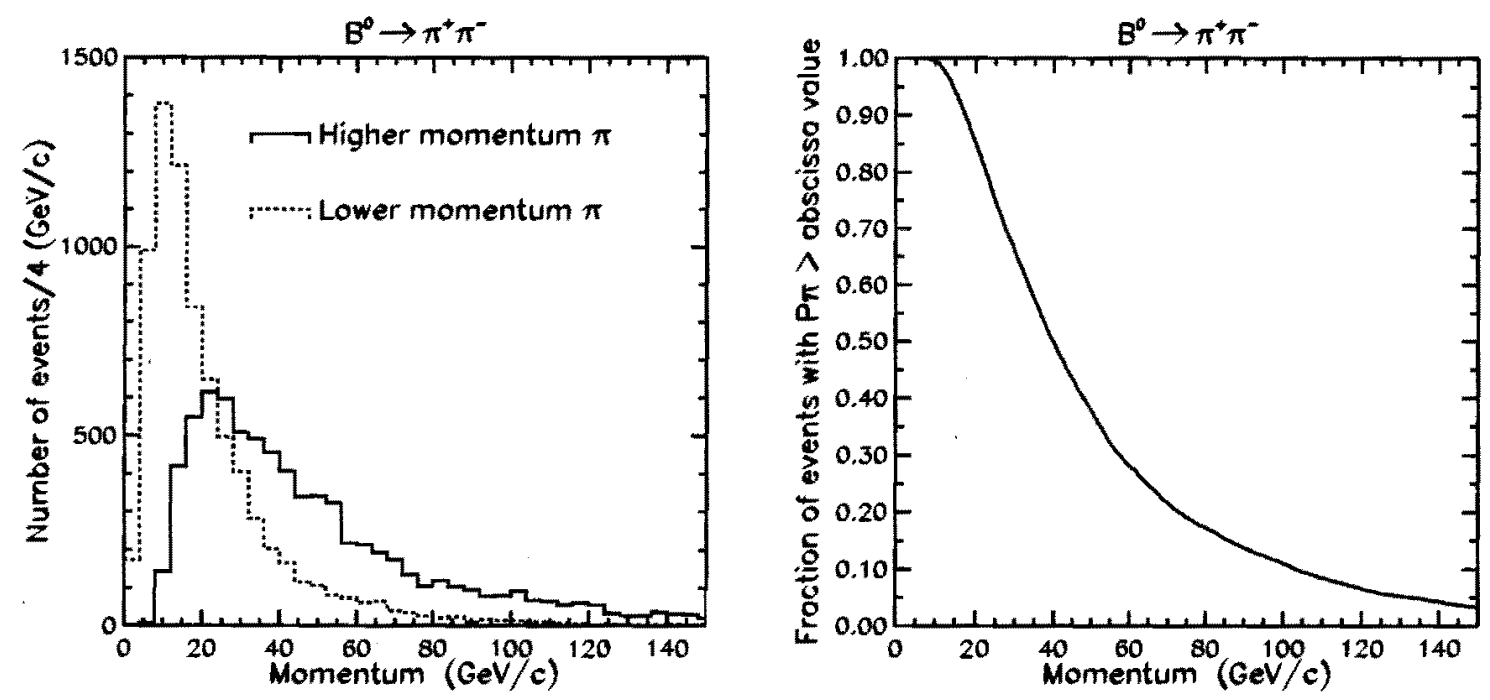

Figure 6.1: The momentum distribution of pions in $B_{d} \rightarrow \pi^{+} \pi^{-}$decays. The left plot shows distributions for the lower (dashed line) and higher (solid line) momentum pion in this decay. The right plot presents the latter distribution in integral form, which gives loss of efficiency as a function of the high momentum cut-off of the particle ID device.

\section{2 $\mathrm{RICH}$ radiators}

Because of the large momentum range and limited longitudinal space available for a particle identification system in the $\mathrm{C} 0$ enclosure, there is really only one choice of detector technology - a gaseous ring-imaging Cherenkov counter. Pions and kaons can be separated in this momentum region with a single gas radiator. We choose $C_{4} F_{10}$ which has an index of refraction of 1.00138 in the visible range. The momentum dependence of the Cherenkov angle for pions, kaons and protons in this gas is shown in Fig. 6.3. Many other experiments use this gas, including DELPHI (endcap) [1], HERA-B [2] and HERMES [3]. It was also chosen for one of the LHC-b RICH detectors [4].

Note that below about $9 \mathrm{GeV}$, no gas can provide $K / p$ separation and that, since kaons are below threshold, the $\mathrm{RICH}$ operates in a threshold mode for $K / \pi$ separation (except that it has much better noise discrimination than a normal threshold counter because it still measures a Cherenkov ring for pions). In order to improve identification of low momentum particles, one interesting possibility is to insert a thin $(\sim 4 \mathrm{~cm})$ piece of aerogel at the entrance to the gas RICH as proposed by LHC-b [5] and already implemented by HERMES [3]. For example, aerogel with a refractive index of $n=1.03$ would lower the $\pi, K, p$ 

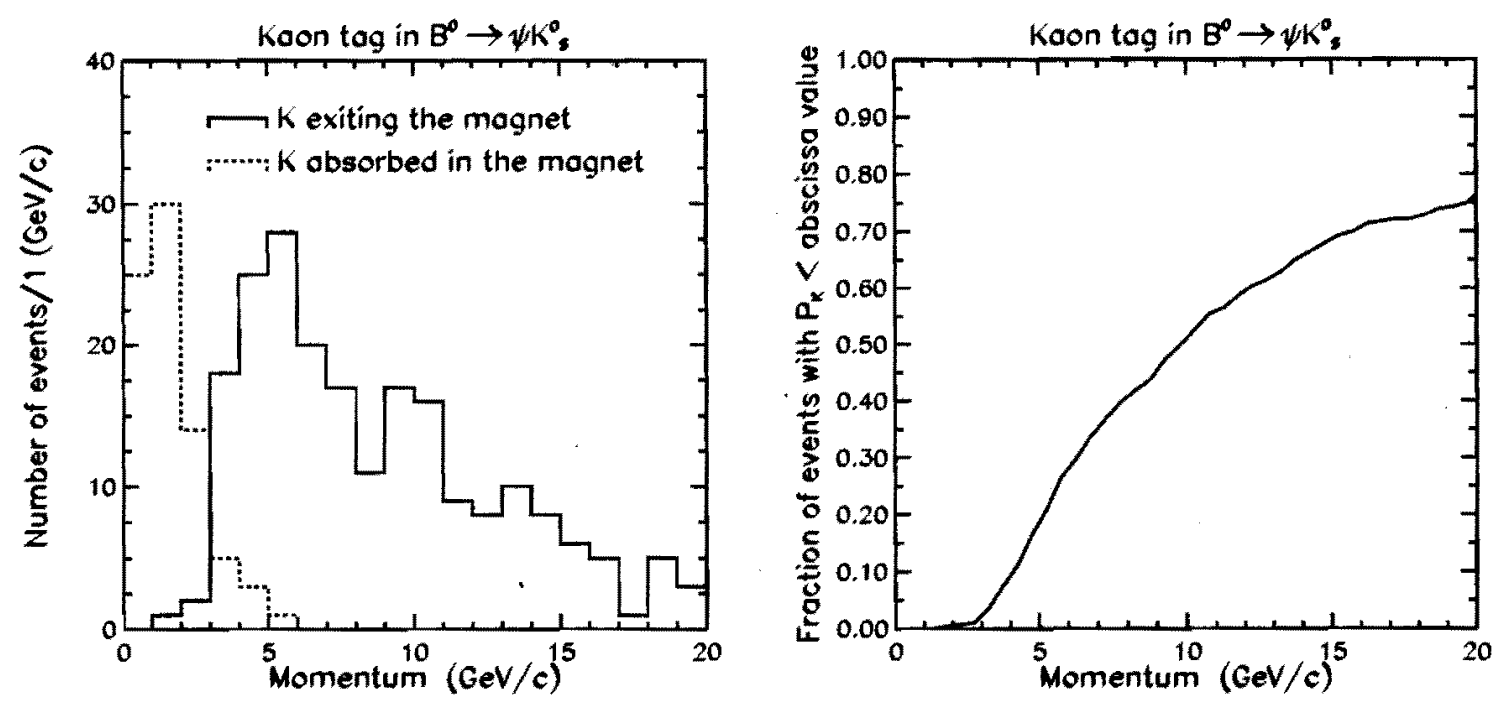

Figure 6.2: The momentum distribution of "tagging" kaons for the case where the signal particles $\left(\psi K_{S}^{0}\right)$ are within the geometric acceptance of the spectrometer. The left plot shows distributions for kaons absorbed in (dashed line) and exiting from (solid line) the magnet. The right plot presents the latter distribution in integral form, which gives loss of efficiency as a function of the low momentum cut-off of the particle ID device.

momentum thresholds from $2.5,9.0,17.1 \mathrm{GeV} / \mathrm{c}$ to $0.6,2.0,3.8 \mathrm{GeV} / \mathrm{c}$ respectively as illustrated in Fig. 6.3. Shorter wavelength Cherenkov photons undergo Rayleigh scattering inside the aerogel itself. They are absorbed in the radiator or exit at random angles. A thin window between the aerogel and the gas radiator passes photons only in the visible range, diminishing the scattered component.

The same photo-detection system can detect Cherenkov rings produced in both the gaseous and the aerogel radiators if it operates in visible wavelengths.

\subsection{RICH dimensions}

The RICH detector can be located behind the tracking chambers just outside the central dipole magnet, about 4 meters away from the interaction point. The length of the RICH detector must be less than 3 meters to allow sufficient space for the EM calorimeter and the muon system. The aerogel radiator with thickness of about $4 \mathrm{~cm}$ will be mounted at the entrance to the RICH vessel. The gaseous $C_{4} F_{10}$ radiator will fill the rest of the RICH volume. The transverse dimensions of the $\mathrm{RICH}$ entrance window are set by the aperture of the dipole magnet to be about $2.7 \mathrm{~m} \times 2.7 \mathrm{~m}$.

Spherical mirrors at the end of the gas volume reflect Cherenkov photons back and focus them into rings at the photo-detection surface. The photo-detectors should be located away from the flux of particles exiting the magnet, therefore the mirrors are tilted. In order to minimize geometric aberrations from an off-axis mirror configuration, the mirrors will be 


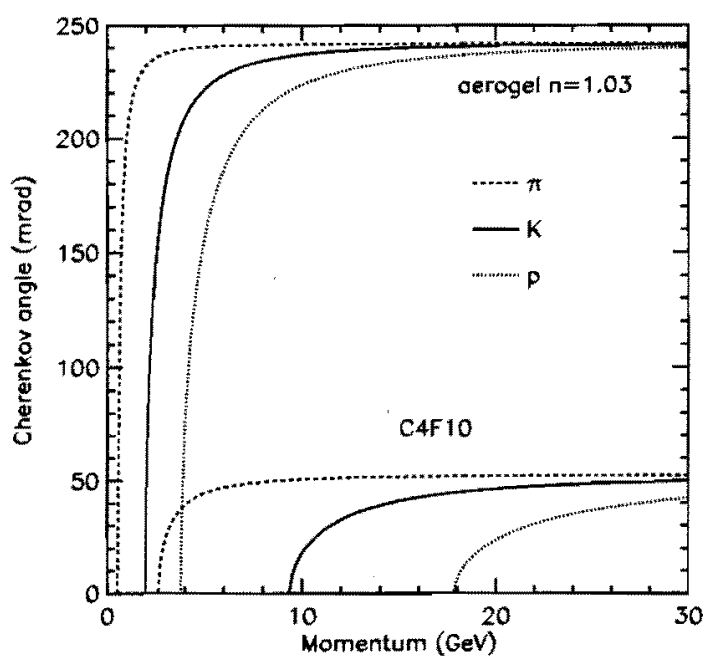

Figure 6.3: Cherenkov angles for various particle species as a function of particle momentum for $C_{4} F_{10}$ and aerogel $(n=1.03)$ radiators.

split along the mid-line of the detector, reflecting photons to photo-detectors located on each side of the vessel in the non-bend view ( $x-z$ plane). The longer the RICH detector the smaller the tilt angle. Since the geometric aberrations due to the mirror tilt are significant for the gas radiator, we plan on the longest $\mathrm{RICH}$ detector we can accommodate within the space limitations. This also maximizes the photon yield from the gaseous radiator, again improving the resolution of the device. Thus, the gas radiator will be approximately 3 meters long and the mirror radius will be 7 meters. The mirror tilt angle will be $240 \mathrm{mrad}$. Note that the mirror tilt angle cannot be further reduced by use of additional flat mirrors at the other end of the detector, like in the designs of HERA-B and downstream LHC-b RICH detectors, since the front of the gas volume must stay transparent to the aerogel photons. Such a scheme would be difficult even without aerogel radiator since there is not enough lateral space in the experimental hall. The transverse size of the mirror will be about $4 \mathrm{~m}$ $\times 4 \mathrm{~m}$. A possible configuration for the mirrors is to make them from an array of individual hexagons. Each mirror half would consist of 18 full hexagons $(76.2 \mathrm{~cm}$ tip-to-tip) and 6 half hexagons. A reflectance efficiency of about $90 \%$ has been achieved in HERA-B detector with $7 \mathrm{~mm}$ thick Pyrex coated with $200 \mathrm{~nm} \mathrm{Al}$ and $30 \mathrm{~nm} \mathrm{MgF} \mathrm{m}_{2}$.

To find the size and optimal position and orientation of the photo-detection surface, we have used a ray tracing Monte Carlo. Even though the true focal plane of a spherical mirror is not planar, non-planar surfaces do not improve resolution significantly and are difficult to realize in practice. Thus, we have assumed that the Cherenkov rings are focused on a plane. Photo-detection systems considered (see the next section) work the best for normal light incidence. Thus the photo-detection plane must be tilted in the $x-z$ plane to follow the mirror tilt. The simulation indicates that tilt by $400 \mathrm{mrad}$ produces a normal incidence on average. 
Since the actual emission point along the track for Cherenkov photon is unknown, the Cherenkov angle reconstruction assumes emission at the track mid-point. The emission point error contributing to Cherenkov angle resolution is magnified by mirror tilt from $0.2 \mathrm{mrad}$ to $0.51 \mathrm{mrad}$. This error imposed by geometrical considerations sets the scale for the other two major contributions to Cherenkov angle resolution: chromatic error and photo-detector segmentation error (called also photon position error) which can be controlled by parameters of the photo-detection devices.

The geometry of the proposed RICH detector is shown in Fig. 6.4.
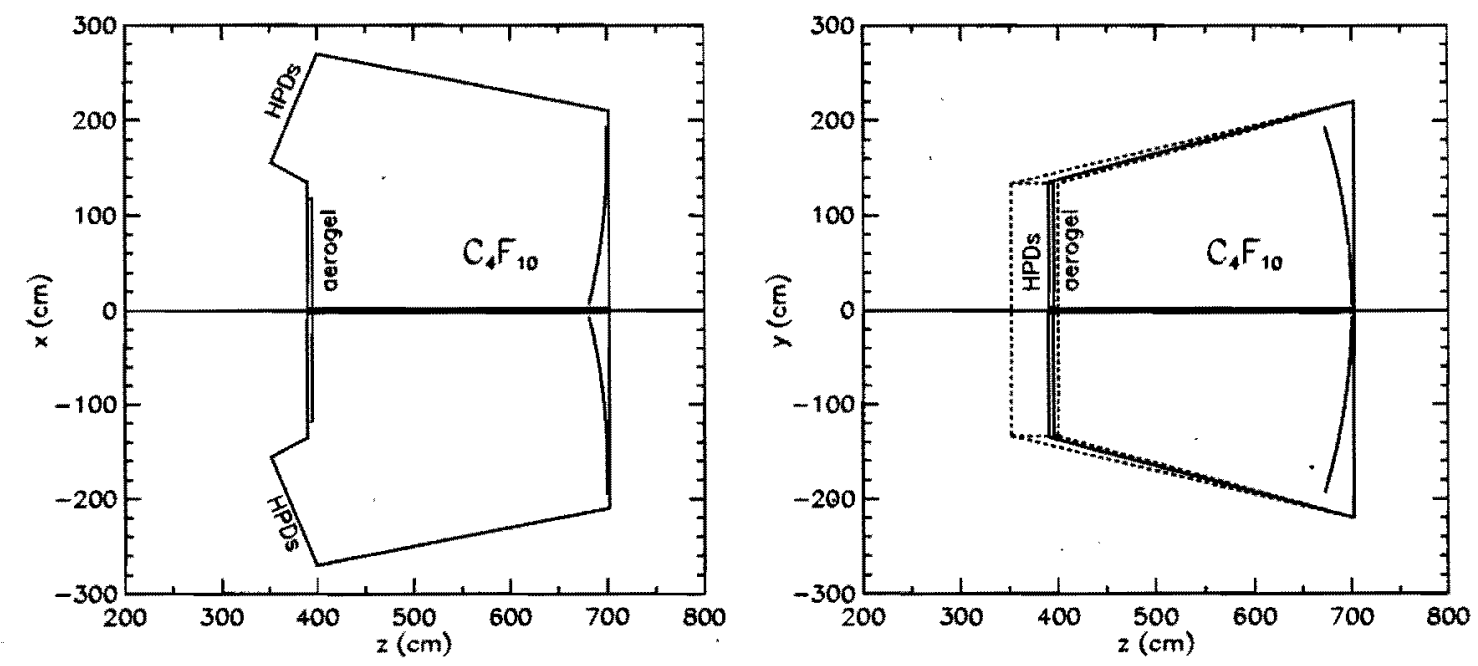

Figure 6.4: BTeV RICH geometry outline.

\subsection{Photo-detectors}

We choose to work in the "visible wavelength" regime, above $280 \mathrm{~nm}$ to minimize chromatic aberrations. Because of the open geometry of the forward spectrometer and the absence of a magnetic field around the RICH detector, arrays of photo-multipliers (PMT) or hybrid photo-diodes (HPD) can be used. We choose to use HPDs which offer a cheaper solution. Multi-anode PMTs are considered a back-up option. In this section we describe the HPD based system. The alternative PMT system is described and compared to our baseline choice in Appendix A.

HPDs are commercially available from DEP (Delft Electronic Products B.V.) in the Netherlands. DEP was founded in the late 1960s and is Europe's leading producer of a wide range of image intensifiers and photon counters (HPDs and MCPs) with markets in defense, medical and industrial sectors.

In an HPD, a photo-electron emitted by the photo-cathode is accelerated onto a segmented silicon diode by a very high voltage. Segmentation of the diode can be adjusted to 
match specific needs for spatial resolution. Initial HPDs from DEP suffered from a large dead area around the diode. This has been greatly reduced by an electrostatic focusing system. DEP can now produce HPDs as large as $8.3 \mathrm{~cm}$ in diameter. These tubes were developed by DEP in collaboration with the LHC-b group. A drawing of a PP0380V tube from DEP is shown in Fig. 6.5. The tubes have circular cross-section.

A photon incident at a quartz window refracts and reaches a photo-cathode deposited on the inner window surface. The window has a spherical shape to provide some optical focusing of light onto the photo-cathode. About $80 \%$ of the tube area is active. Closely packed tubes will cover $91 \%$ of the area they occupy, thus overall geometrical light collection efficiency is $73 \%$. Therefore, there is no need to use any additional demagnification systems to recover the geometric inefficiency.

In the HPD, a photo-electron emitted by a photo-cathode is directed and accelerated towards the diode. The photo-cathode is operated at a voltage of $-20 \mathrm{kV}$ with respect to the diode to provide enough energy to the electron for sizable charge generation in the silicon diode. The gain of the device is about 5000 and is proportional to the accelerating voltage. We expect to detect such signals with a signal-to-noise ratio of about 7:1. The charge collection from the silicon depends on the bias voltage and can be easily accomplished in a single $132 \mathrm{~ns}$ accelerator cycle. The present version PP0380V tubes contain a diode with 61 hexagonal pixels. The diode chip is bump-bonded to another chip with conductive traces arranging pixel outputs into a regular series appropriate for use with standard connectors. Pins attached to this chip take the signals out of the vacuum enclosure, where front-end electronics can be attached. Tests of the PP0380V tube by the LHC-b group showed that the electrostatic focusing works well and that good signal-to-noise can be obtained [6].

In the LHC-b RICH detectors, fine segmentation is required. ${ }^{1}$ They plan to have 2048 pixels in each HPD tube, which makes it impossible to use the present DEP design in which individual pixel signals are taken to the outside of the tube. Therefore, the LHC-b group is developing their own silicon chip in which the diode is integrated with the front-end read-out and resides inside the tube.

The diode segmentation needed in $\mathrm{BTeV}$ is 163 pixels per tube. We can either follow the LHC-b path and develop our own integrated diode-readout chip to be put inside HPD by DEP, or we can rely on DEP and their subcontractors to modify their present scheme to accommodate more pixels per tube. Our current plan is the second solution that decouples development of read-out electronics from the manufacturing of the HPDs.

It appears that the IDE company from Norway already has a chip which can be easily modified to our read-out needs. Thus, no in-house chip development will be needed for the front-end processing. In the preliminary design we will have 163 hexagonal pixels per tube as illustrated in Fig. 6.5. The corresponding photon position error is 0.51 mrad. More

\footnotetext{
${ }^{1}$ The upstream LHC-b RICH detector is placed very close to the interaction point and therefore has small mirror radius, and consequently very compact Cherenkov rings. The downstream LHC-b RICH detector with large mirror radius is geared to identify high momentum tracks (BTeV does not need to cover these momenta because of the lower beam energy) and therefore a gas with low refractive index is used. This makes Cherenkov images compact again.
} 

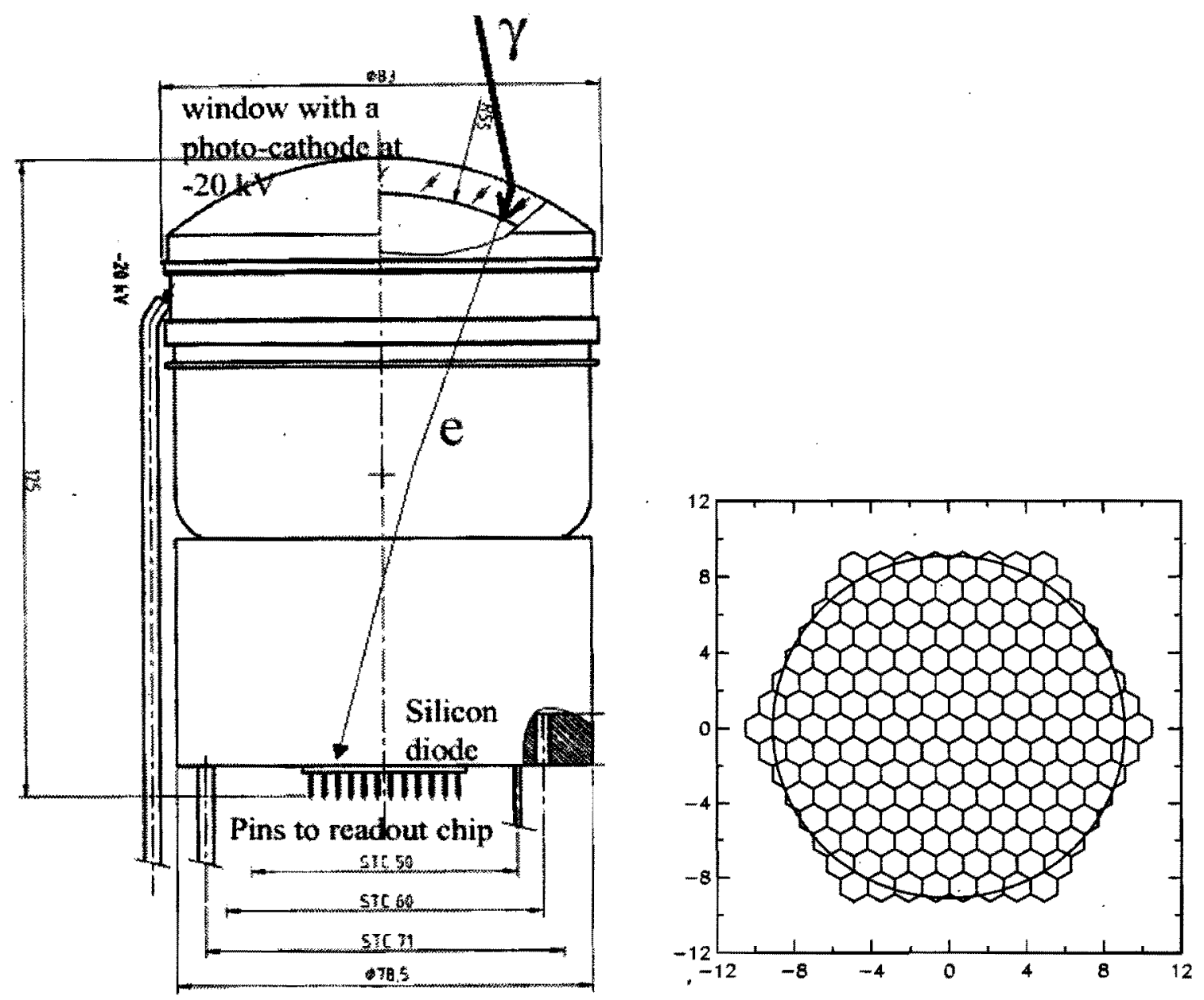

Figure 6.5: PP0380V HPD from DEP (on the left) and possible pixel structure on HPD silicon diode (on the right). Only parts inside the circular area would be actually implemented. Dimensions are expressed in mm. 
information on the RICH read-out can be found in Appendix A.

The last major factor impacting the RICH performance is the wavelength coverage determined by the photo-cathode and window material. The wavelength sensitivity determines chromatic error (arising from wavelength dependence of the radiator refraction index) and is the major factor in the number of Cherenkov photons detected per track.

Quartz windows are a standard feature in the HPD tubes as they can easily sustain the large high voltage on the photo-cathode. High quality quartz extends the wavelength coverage from the visible range down to $160 \mathrm{~nm}$. Such a large wavelength coverage results in a large chromatic error of $1.2 \mathrm{mrad}$ per photon and in a large number of photons radiated per track $(\sim 235)$. When the wavelength coverage is limited, the photon yield drops but the chromatic error per photon improves. These two effects offset each other. The simulations show that a shallow optimum in Cherenkov resolution per track is reached when the wavelengths are limited to $>280 \mathrm{~nm}$. This results in a chromatic error of $0.37 \mathrm{mrad}$ per photon with a photon yield of 80 photons per track. Perhaps such wavelength coverage can be achieved with appropriate coating of the quartz window. Some R\&D will be needed to resolve this issue. In the present simulations we assumed a bialkali photo-cathode with the $280 \mathrm{~nm}$ cut-off. The total Cherenkov angle resolution is $0.81 \mathrm{mrad}$ per photon and $0.09 \mathrm{mrad}$ per track.

To determine the number of HPDs needed, we plot in Fig 6.6 the $\pi^{+} \pi^{-}$efficiency, for the requirement of $4 \sigma \mathrm{K} / \pi$ separation for both tracks, as a function of tube cost. For comparison, simulation of the PMT system is also shown. The system with 1888 HPDs (indicated by the vertical line in Fig 6.6) approaches the full geometrical coverage limit. We plan to order 192 spare HPD tubes which can be deployed and instrumented on the outside perimeter of the detector. With 163 pixels per tube, the entire detector (two identical RICH detectors) will have up to 339,040 electronic channels.

\subsection{Expected resolution for the aerogel radiator}

The chromatic error for aerogel photons is expected to be about $1.8 \mathrm{mrad}$. This is likely to be an overestimate since we did not simulate attenuation of shorter wavelengths in the radiator itself. The effect of the lucite filter between the aerogel and gas radiators was simulated as a sharp cut-off at $300 \mathrm{~nm}$.

HERMES reports [3] an additional $0.7 \mathrm{mrad}$ error, mostly due to aerogel density variation. The emission point error is expected to be small $(0.2 \mathrm{mrad})$ because the radiator in only $4 \mathrm{~cm}$ thick. The detector granularity error is $0.6 \mathrm{mrad}$. Putting all these factors together we predict the Cherenkov resolution per photon to be around 2 mrad.

We assume 10 detected photo-electrons per track based on the recent reports from HERMES. This photon yield cannot be increased by using a thicker radiator because of light scattering and absorption in the aerogel itself. Further improvements in aerogel transparency would increase the number of photons per Cherenkov ring.

The expected Cherenkov resolution per track is about $0.6 \mathrm{mrad}$. Various contributions to

the Cherenkov angle resolution for $C_{4} F_{10}$ and aerogel radiators are summarized in Table 6.1. 


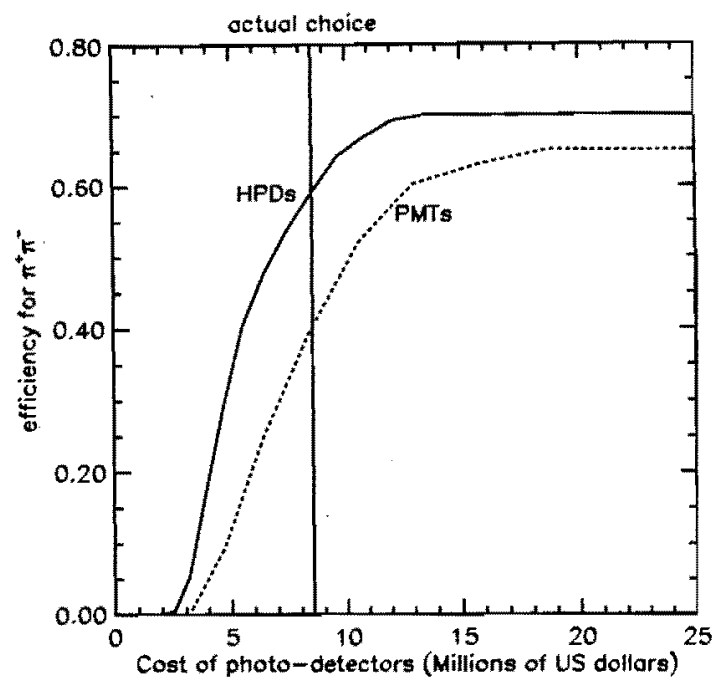

Figure 6.6: Efficiency for $B_{d} \rightarrow \pi^{+} \pi^{-}$as a function of the cost of the photo-detectors. Efficiency is defined relative to the reconstructed events with both tracks crossing the $\mathrm{RICH}$ detector. Each track was required to have enough photons for $4 \sigma \mathrm{K} / \pi$ separation (from the gaseous radiator alone). Effects due to Cherenkov ring overlaps are not taken into account. The actual choice of a size of the HPD system is shown by vertical line.

More details related to the aerogel radiator can be found in Appendix A.

\subsection{Expected physics performance}

In a real environment, Cherenkov rings from different tracks overlap in the detector. This is illustrated in Fig. 6.7. A realistic simulation of efficiency and fake rates must take into account ambiguities in track-photon assignment.

We have analyzed simulated data with an algorithm which could be applied to real data. The reconstruction is performed in two steps. In the first pass, all hits within $\pm 3 \sigma$ of a mass hypothesis are included in the per track average, excluding those hits which are within $\pm 3 \sigma$ of the pion hypothesis for any other track. The second pass is essentially the same except that instead of assuming that all tracks are pions in the hit exclusion, the most likely mass hypothesis based on the first-pass results is used. To discriminate between two mass hypotheses for the same track (e.g. $K$ or $\pi$ ) we cut on the modified $\chi^{2}$ difference: $\Delta \chi_{K \pi}^{2}=\chi_{\pi}^{2}-\chi_{K}^{2}$, with $\chi_{h}^{2}=\left(\theta_{t r k h}-\theta_{h}\right)^{2} / \sigma^{2}\left(\theta_{t r k h}\right)-\log \left(N_{h}\right)$. Here, $\theta_{t r k h}\left(\theta_{h}\right)$ is the measured (expected) Cherenkov angle per track for given mass hypothesis $h$, and $N_{h}$ is the number of photons found within $\pm 3 \sigma$ of this hypothesis. If no photons are found for a given hypothesis above the Cherenkov radiation threshold, a large $\chi^{2}$ value is set. The best discrimination can be obtained by combining the $\chi^{2}$ difference from the gaseous and aerogel radiators: $\Delta \chi_{K \pi}^{2}=\Delta \chi_{K \pi \text { gas }}^{2}+\Delta \chi_{K \pi \text { aerogel }}^{2}$. For a given cut value on the $\Delta \chi_{K \pi}^{2}$ we obtain a value for efficiency and fake rate. 
Table 6.1: Expected performance of BTeV RICH system. The photon yield and the resolution per track given here do not take into account any reconstruction losses due to overlap of Cherenkov rings from different tracks in the same event.

\begin{tabular}{||l|l|l||}
\hline \hline & $C_{4} F_{10}$ & Aerogel $n=1.03$ \\
\hline emission point error & $0.51 \mathrm{mrad}$ & $0.2 \mathrm{mrad}$ \\
segmentation & $0.51 \mathrm{mrad}$ & $0.6 \mathrm{mrad}$ \\
chromatic error & $0.37 \mathrm{mrad}$ & $1.9 \mathrm{mrad}$ \\
\hline total error per photon & $0.81 \mathrm{mrad}$ & $2.0 \mathrm{mrad}$ \\
\hline number of photons & 80 & 10 \\
\hline total error per track & $0.09 \mathrm{mrad}$ & $0.6 \mathrm{mrad}$. \\
\hline \hline
\end{tabular}

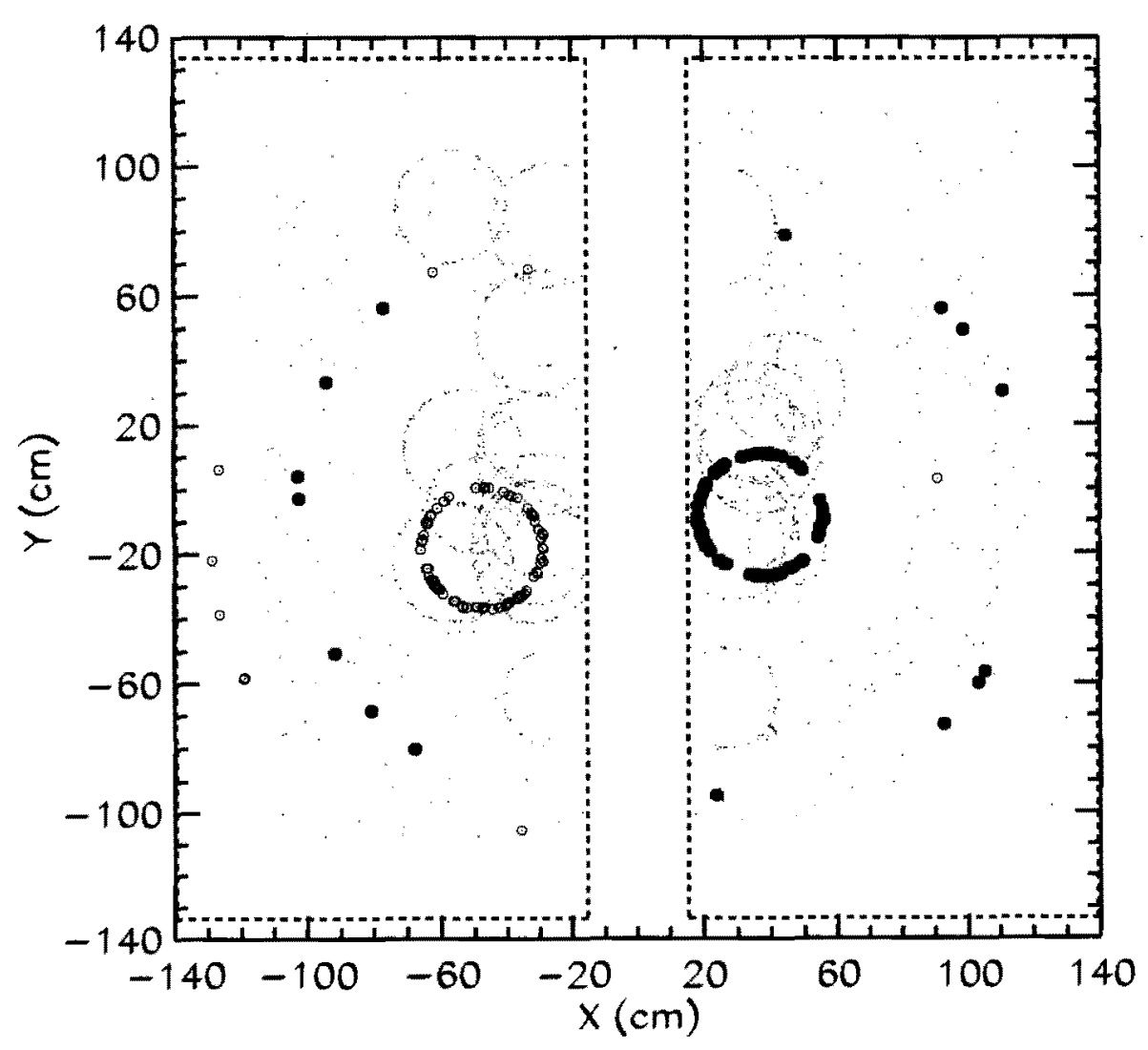

Figure 6.7: A typical reconstructed $B_{d} \rightarrow \pi^{+} \pi^{-}$event. Gaseous and aerogel Cherenkov photons radiated by the signal pions are highlighted. 


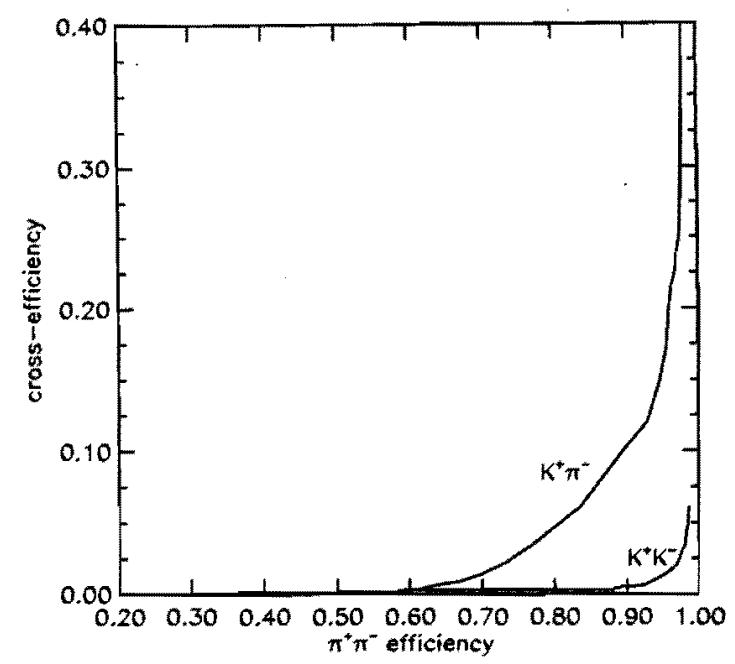

Figure 6.8: Cross-efficiency of particle identification system for $B_{d} \rightarrow K^{ \pm} \pi^{\mp}$ and $B_{s} \rightarrow$ $K^{+} K^{-}$as a function $B_{d} \rightarrow \pi^{+} \pi^{-}$particle identification efficiency. The efficiencies are defined relative to the number of events with both tracks entering the RICH detector. The Monte Carlo simulation included an average of two minimum bias interactions in addition to the $b \bar{b}$ production.

We have analyzed Monte Carlo samples of reconstructed $B_{d} \rightarrow \pi^{+} \pi^{-}$(signal), $B_{d} \rightarrow$ $K^{ \pm} \pi^{\mp}$ (background) and $B_{s} \rightarrow K^{+} K^{-}$(background) events. Background cross-efficiency is plotted as a function of $B_{d} \rightarrow \pi^{+} \pi^{-}$efficiency in Fig. 6.8. Separation of $\pi^{+} \pi^{-}$from $K^{+} K^{-}$is easier than from $K^{ \pm} \pi^{\mp}$ as there are two tracks to discriminate on. The plot shows that the cross-efficiency can be made essentially zero for still sizable signal efficiency. A higher signal yield can be obtained if some background can be tolerated. The analysis method presented here can be improved by using the likelihood ratio for the two mass hypotheses, with the likelihood combining the $\chi^{2}$ defined above with a probability for the observed number of photons found on the ring. Also, instead of removing photons from the ring overlaps, a global event fit of all mass assignments can be performed. 


\section{Bibliography}

[1] W. Adam et al., Nucl. Inst. \& Meth. A343, 60 (1994).

[2] J.L. Rosen, Proceedings of The Fifth International Workshop on $B$-Physics at Hadron Machines, Los Angeles, USA, Oct. 13-17, 1997, published in Nucl. Inst. and Meth. A408 (1998) 191; S. Kopar, Proceedings of the The 3rd International Workshop on Ring Imaging Cherenkov Detectors Weizmann Institute of Science Ein-Gedi, Dead-Sea, Israel, Nov. 15-20, 1998.

[3] P. Carter, Proceedings of the The 3rd International Workshop on Ring Imaging Cherenkov Detectors Weizmann Institute of Science Ein-Gedi, Dead-Sea, Israel, Nov. 15-20, 1998. "Proposal for Dual Radiator RICH for HERMES", E. Cisbani et al., Hermes Internal Note 97-005.

[4] LHC-B, Technical Proposal, CERN LHCC 98-4, LHCC/P4, 1998.

[5] R. Forty, CERN-PPE/96-176, Sept. 1996 published in Proc. of the $4^{\text {th }}$ Int. Workshop on $B$-physics at Hadron Machines, Rome, Italy, June 1996, F. Ferroni, P. Schlein (Eds.), North-Holland, 1996.

[6] E. Albrecht et al., "Performance of Hybrid Photon Detector Prototypes With $80 \%$ Active Area For The RICH Counters of LHCb", submitted to Nucl. Inst. \& Meth. A, See also GLAS-PPE-1999-22 (hep-ex/0001053) and Nucl. Inst. \& Meth. Phys. Res. A433, 159 (1999). 


\section{Chapter 7}

\section{Electromagnetic Calorimetry}

\section{$7.1 \quad$ Introduction}

A thorough investigation of $B$ decays requires the ability to study decay modes containing single photons, $\pi^{o}$ 's, and $\eta$ 's. The addition of a high quality crystal calorimeter to the CLEO detector was a first in coupling excellent photon detection to charged particle detection and demonstrated its importance in $B$ decay studies [1]. Furthermore, the identification of electrons is most useful. Some of the important decay modes for BTeV include: $B^{o} \rightarrow$ $(\rho \pi)^{o} \rightarrow \pi^{+} \pi^{-} \pi^{o}, B_{s}^{o} \rightarrow \psi \eta$, and $\psi \eta^{\prime}$, and $B^{o} \rightarrow K^{* o} \gamma$ and $\rho^{o} \gamma$

\subsection{Choice of An Electromagnetic Calorimeter Based on $\mathrm{PbWO}_{4}$ Crystals}

Several technologies were considered that were radiation tolerant, had excellent energy and spatial resolution, were affordable and available. These included:

- A sampling calorimeter with lead and either scintillator or liquid argon

- A total ionizing calorimeter based on liquid krypton

- A calorimeter based on $\mathrm{PbWO}_{4}$ crystals.

Lead and scintillator was rejected because of poor resolution. Fermilab is reluctant to deal with the numerous safety issues related to operating liquid argon or liquid krypton in the $\mathrm{C} 0$ enclosure. These devices also have long charge collection times which can, however, be overcome by careful pulse shaping. Liquid krypton, in particular, looked like an attractive possibility. Liquid detectors are radiation hard. However, the constant term in the energy resolution may be considerably larger due to the relatively large amount of material before the active sampling material [2].

We selected for our baseline a calorimeter made of $\mathrm{PbWO}_{4}$ crystals. This technology has been developed by CMS. Our choice is based on several considerations: 
- It satisfies our requirements on energy and spatial resolution. Blocks of size roughly $2.6 \mathrm{~cm}$ can be made. CMS expects to achieve a stochastic term of $2.7 \%$ and a constant term of $0.55 \%$ using Avalanche PhotoDiodes (APDs) for readout. We plan to use photomultiplier tubes (PMTs) and expect to have a smaller stochastic term, $\sim 1.6 \%$.

- This material is very resistant to radiation damage, especially when doped with either $\mathrm{Nb}$ or La. CMS expects their crystals to survive a dose of $\sim 100 \mathrm{kGy}$. This should be sufficient for $\mathrm{BTeV}$.

- They are fast - they deliver $99 \%$ of their light output within $100 \mathrm{~ns}$, which is safely less than the bunch crossing time of 132 ns at the Tevatron.

The properties of $\mathrm{PbWO}_{4}$ which are important for the calorimeter are given in Table 7.1.

Table 7.1: Properties of $\mathrm{PbWO}_{4}$

\begin{tabular}{|l|l|}
\hline Property & Value \\
\hline Density $\left(\mathrm{g} / \mathrm{cm}^{3}\right)$ & 8.28 \\
Radiation Length $(\mathrm{cm})$ & 0.89 \\
Interaction Length $(\mathrm{cm})$ & 22.4 \\
Light Decay Time (ns): & $5(39 \%)$ \\
& $15(60 \%)$ \\
& $100(1 \%)$ \\
Refractive Index & 2.30 \\
Maximum of emission $(\mathrm{nm})$ & 440 \\
Temperature Coefficient $\left(\% /{ }^{\circ} \mathrm{C}\right)$ & -2 \\
Light output/NaI(Tl) (\%) & 1.3 \\
Light output (pe/MeV into a 2" PMT) & 10 \\
\hline \hline
\end{tabular}

\subsection{Description of the BTeV Calorimeter}

Our detector uses crystals very similar in size and shape to CMS. The CMS crystals are tapered to point at the interaction region. The crystals, which follow a 5 radiation length preradiator, are $220 \mathrm{~mm}$ long, and $26 \mathrm{~mm}$ square in the back [3]. Our crystals are the same length and virtually the same width. We also will use a projective geometry, but the taper is smaller.

The outer radius was chosen by weighting the efficiency of a larger calorimeter, up to the spectrometer acceptance of $300 \mathrm{mr}$, with the cost. In Fig. 7.1 we show the efficiency of detecting the reaction $\bar{B}^{o} \rightarrow D^{*+} \rho^{-}, D^{*+} \rightarrow \pi^{+} D^{o}, D^{o} \rightarrow K^{-} \pi^{+}$and $\rho^{-} \rightarrow \pi^{-} \pi^{o}$ as a function of calorimeter radius.

The calorimeter cost can be parameterized roughly as

$$
T(\$)=700(\$) \times N_{c}+3,400,000(\$),
$$


Table 7.2: Properties of the BTeV electromagnetic Calorimeter

\begin{tabular}{|l|l|}
\hline Property & Value \\
\hline transverse block size, back & $26.0 \mathrm{~mm} \times 26.0 \mathrm{~mm}$ \\
tapered, smaller in front & $25.4 \mathrm{~mm} \times 25.4 \mathrm{~mm}$ \\
Block length & $22 \mathrm{~cm}$ \\
Radiation Lengths & 25 \\
Front end electronics & PMT \\
Digitization/readout & QIE (FNAL-KTEV) \\
Inner Dimension & $\pm 9.88 \mathrm{~cm} \times \pm 9.88 \mathrm{~cm}$ \\
Outer Radius & $160 \mathrm{~cm}$ \\
Total blocks per arm & 11850 \\
\hline \hline
\end{tabular}

where $N_{c}$ is the number of crystals and $T$ the total cost. The fixed costs represent mainly startup of crystal production, the crystal container, the light calibration pulsar and electronics development. Weighing the efficiency versus total cost, we adopted an outer radius of $160 \mathrm{~cm}$, corresponding to 23,700 crystals for both arms.

One end of the calorimeter is shown in Fig. 7.2 with the crystal hits displayed for an event generated with GEANT containing a $B^{o} \rightarrow \rho^{o} \pi^{o}$ decay. The two photons from the $\pi^{o}$ decay are indicated by large circles. One photon has $19.3 \mathrm{GeV}$ of energy; while the other has $2.4 \mathrm{GeV}$. The minimum energy displayed is $10 \mathrm{MeV}$. This corresponds to the minimum energy crystal we use in measuring the shower energy. It can be seen even from this one event that there is much more activity near the beam line than further out in radius. We will return to this point later.

CMS will use Avalanche Photodiodes (APD) to read out the barrel and Vacuum Phototriodes to read out the endcap. These devices are necessary because of the high magnetic field in which the crystals are positioned. In $\mathrm{BTeV}$ we are far from any magnetic fields, so we can use photomultiplier tubes. This provides less noise and better resolution as will be described below, in a well understood and tested technology. In fact, $\mathrm{BTeV}$ will use modified Hamamatsu R5380 tubes equipped with quartz windows to withstand radiation. These are similar to the ones used by $\mathrm{KTeV}$.

As part of its acceptance criteria, CMS requires that all of their crystals emit more than 10 photoelectrons per $\mathrm{MeV}$ into a XP2262B photomultiplier tube covering the back face of the crystal. Using a smaller phototube, we expect to collect in excess of 7 photoelectrons per $\mathrm{MeV}$. CMS actually collects $\approx 4$ photoelectrons per $\mathrm{MeV}$ into two $25 \mathrm{~mm}^{2}$ APD's on each crystal.

There are identical detectors in each arm of the spectrometer. Each detector is housed in a temperature and humidity controlled hut. There will be a dry nitrogen environment. Temperature stabilization is necessary because of the thermal coefficient of the $\mathrm{PbWO}_{4}$ light output. In addition, the gains are monitored with a laser pulsing system.

Detectors are read out with PMTs. The light emitted by the crystal peaks at $440 \mathrm{~nm}$. The output of the PMT will go to a modified QIE chip located near the photomultiplier 


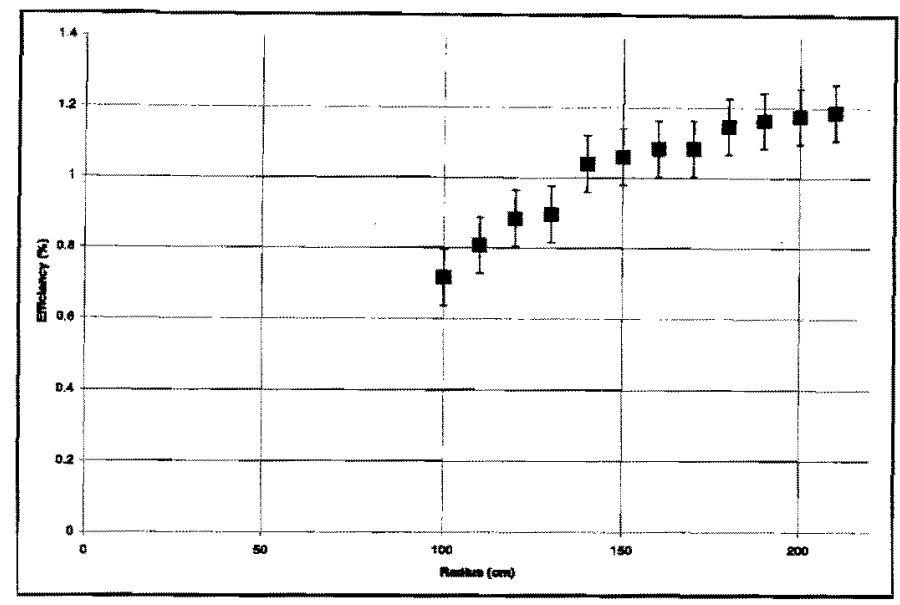

Figure 7.1: The efficiency as a function of calorimeter radius for the reaction $\bar{B}^{o} \rightarrow D^{*+} \rho^{-}$.

base [4], but outside the region of intense radiation. This chip provides a pulse height for each beam crossing. The expected light output is 7000 photoelectrons at $1 \mathrm{GeV}$. The detector is far enough away from the $\mathrm{BTeV}$ dipole so that there should be no need for special magnetic shielding from that source. Since we will magnetize the iron of the muon filter just downstream of the detector, we will put iron shield plates between the calorimeter's PMTs and the muon filter.

\subsection{Effects of Radiation}

The radiation levels at the crystals and phototubes are discussed in Appendix A. The maximum radiation levels occur close to the beam. There is also a relatively narrow vertical band of higher than average dose caused by the sweeping action of the BTeV dipole.

The simulations shown here were done for a smaller calorimeter of about 10,000 crystals. The integrated dose rates for most crystals are quite modest. In Fig. 7:3 (left) we show the dose distribution in the crystals (here dose means the maximum dose in any part of the crystals), for 1 year of running at a luminosity of $2 \times 10^{32} \mathrm{~cm}^{-2} \mathrm{~s}^{-1}$. We also show (right) the cumulative fraction of crystals with doses less than that shown on the horizontal axis. We see that $\sim 90 \%$ of the crystals have a yearly accumulated dose of less than $1000 \mathrm{~Gy}(0.1$ Mrad). 


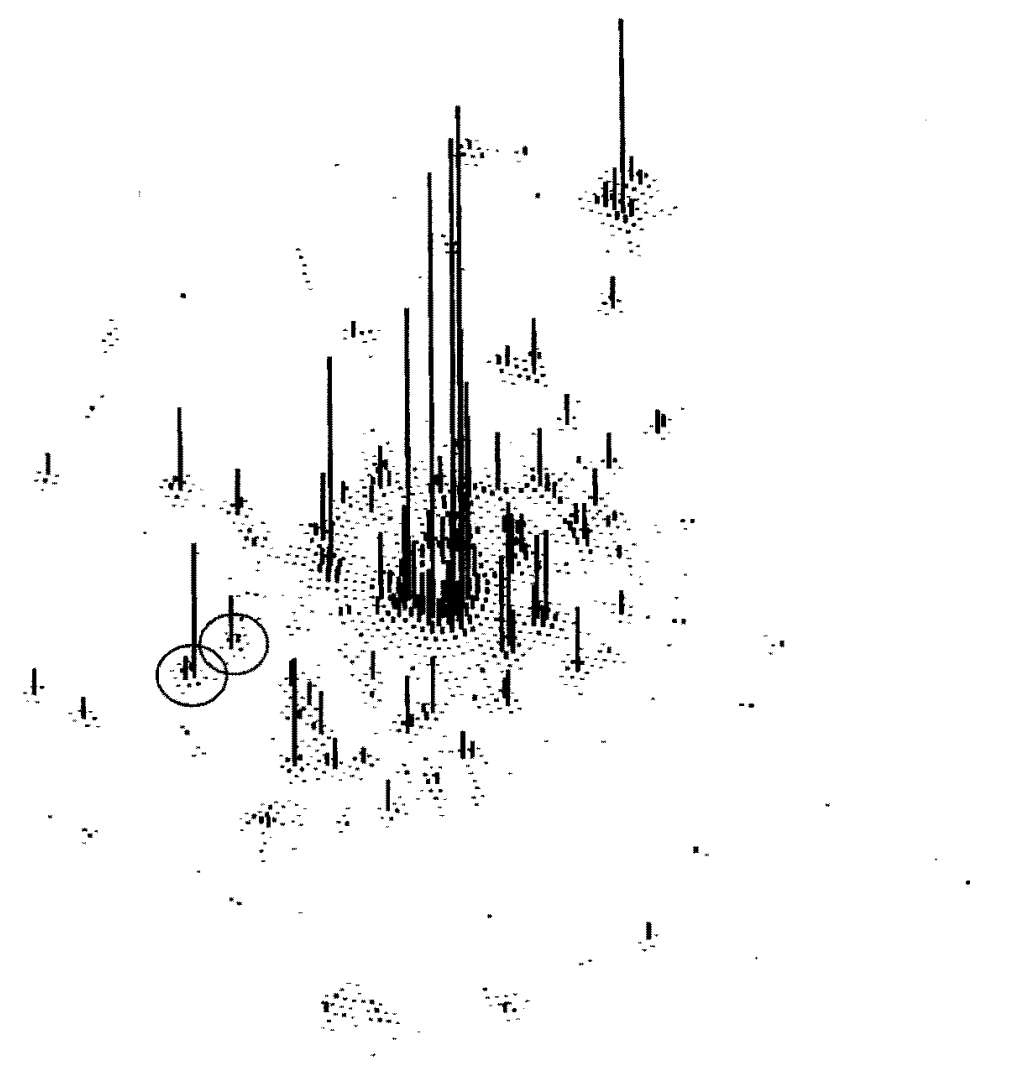

Figure 7.2: The energies in the $\mathrm{PbWO}_{4}$ calorimeter (one end) for an event containing two photons from the decay sequence $B^{\circ} \rightarrow \rho^{o} \pi^{o}, \pi^{o} \rightarrow \gamma \gamma$. The photons of energies 19.3 and 2.4 $\mathrm{GeV}$ are surrounded by large circles. All energies above $10 \mathrm{MeV}$ are shown, with the height of line proportional to the energy.

\subsection{Expected Resolution}

The detailed estimates of the expected resolution are given in Appendix A. Briefly, $22 \mathrm{~cm}$ long crystals were selected after a GEANT study determined that length to be optimal. The transverse size was chosen to be essentially the same as CMS is already using, though making the crystals one to two mm larger, if possible, would sacrifice little performance and cut the costs by $4-8 \%$, due to a reduced number of photomultiplier tubes and electronics channels.

Using 7 photoelectrons/ $\mathrm{MeV}$ we find contributions to the stochastic term in the energy resolution to be $1.2 \% / \sqrt{E}$ from photon statistics and $1.08 \% / \sqrt{E}$ due to crystal size and clustering (transverse and longitudinal leakage) where $E$ is in units of $\mathrm{GeV}$. For the constant term we use the CMS estimate of $0.55 \%$. We note that $\mathrm{KTeV}$ has achieved a constant term 

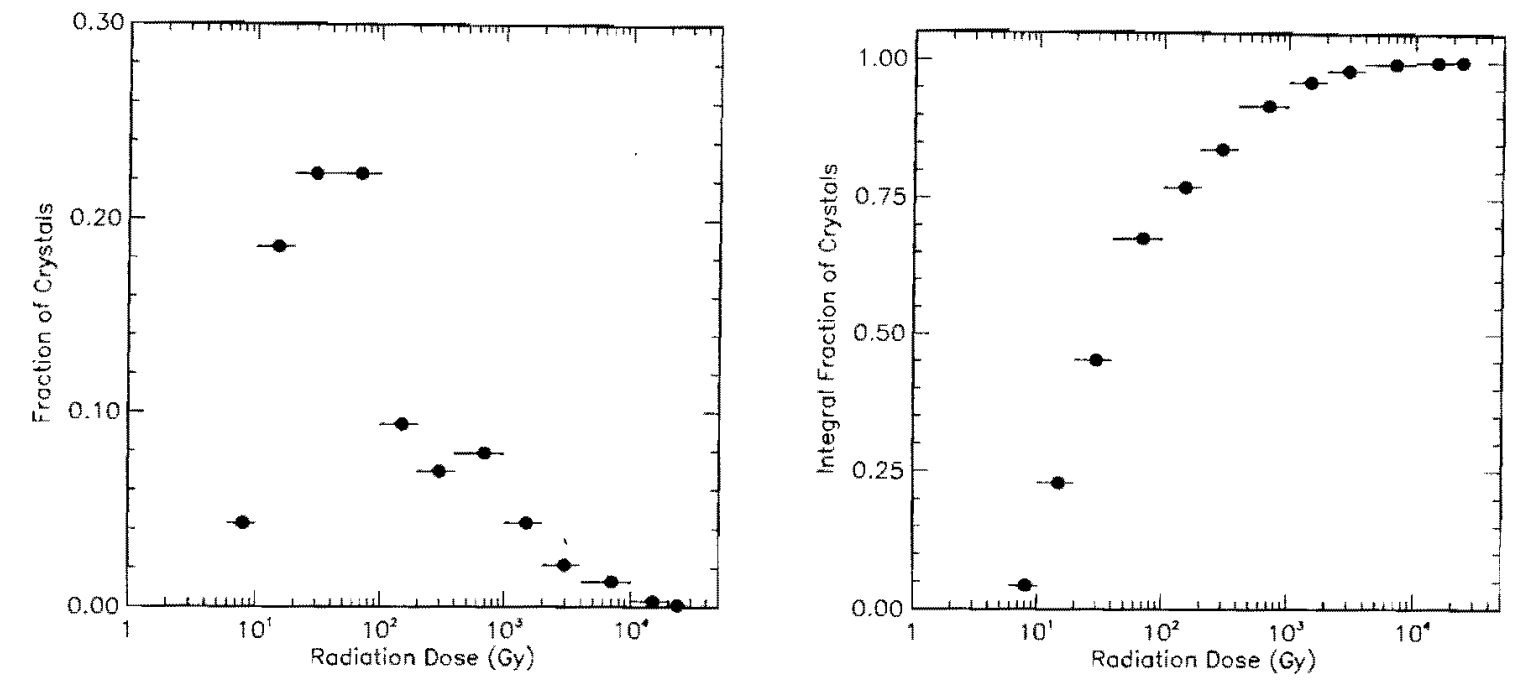

Figure 7.3: (left) The maximum dose distribution in the crystals for for 1 year of running at a luminosity of $2 \times 10^{32} \mathrm{~cm}^{-2} \mathrm{~s}^{-1}$. (right) The integral of the maximum dose distribution.

of $0.45 \%$ [5]. Overall we expect the energy resolution to be

$$
\frac{\sigma_{E}}{E}=\sqrt{\frac{a^{2}}{E}+b^{2}}=\frac{a}{\sqrt{E}} \oplus b,=\frac{1.6 \%}{\sqrt{E}} \oplus 0.55 \%
$$

- The spatial resolution in both directions transverse to the crystal axis is expected to be

$$
\sigma_{x}=\frac{3500 \mu m}{\sqrt{E}} \oplus 200 \mu m
$$

With these resolutions, the mass resolution is excellent. Fig. 7.4 shows the invariant $\gamma \gamma$ mass for $\pi^{o}$ 's of $10 \mathrm{GeV}$ energy incident on the calorimeter where the energies and positions are simulated by GEANT and our cluster finder is used to reconstruct the photons. The mass resolution is $2.6 \mathrm{MeV}$, which compares favorably to that found in CLEO (5 MeV) [6].

\subsection{Examples: Photon Efficiencies in $B^{o} \rightarrow K^{*} \gamma$ and $B \rightarrow \rho \pi$}

In the hadron environment, there is great concern that there is so much activity from charged track interactions in the calorimeter and additional photons that the signal photons will be totally obfuscated. As a test case we consider the decay $B^{\circ} \rightarrow K^{*} \gamma$. Although we are only concerned with the gamma reconstruction here, we require that the two charged tracks from the $K^{*}$ decay reach the $\mathrm{RICH}$ detector, in order to insure that the $B^{o}$ 's were in the 


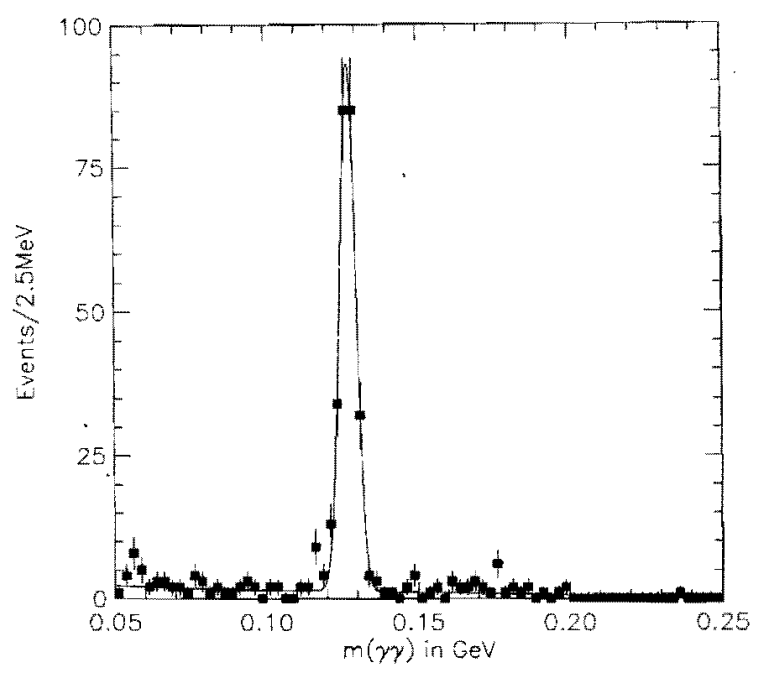

Figure 7.4: The $\gamma \gamma$ invariant mass for $10 \mathrm{GeV} \pi^{o}$ 's incident on the calorimeter. The fit is to a Gaussian signal function plus a polynomial background.

acceptance of the spectrometer. The decay was simulated by GEANT at a mean interaction rate of two per crossing.

To identify photons we find clusters of energy whose shape is consistent with an electromagnetic shower and we require that no charge track extrapolate close to the cluster. In Fig. 7.5 we show the difference between measured and generated photon energy divided by the generated energy.

The BTeV resolution is about a factor of two better than CLEO. The CLEO spectrum is absolutely clean; there is a small background in the BTeV distribution. Our concern is a large overlap with fragments from other particles that would cause the photon energies to be shifted high and out of the peak, but this apparently is not the case. In Fig. 7.6 we show radial distribution of generated photons from $K^{*} \gamma$, and accepted ones and the efficiency, where we define accepted photons as ones with energies within $3 \sigma$ of the peak that pass the shower shape and isolation cuts. We note that the same number for the CLEO barrel calorimeter calculated in the same manner using GEANT is $89 \%$. While our efficiences start out considerably lower than CLEO, they increase rapidly and demonstrate the usefulness of the calorimeter.

We also looked at the $\pi^{\circ}$ efficiency as a function of the radius of the $\pi^{\circ}$ at the $z$ position of the calorimeter for $B \rightarrow \rho^{\circ} \pi^{\circ}$ and $B \rightarrow \rho^{+} \pi^{-}$decays. The efficiency, shown in Fig. 7.7 plateaus at a radial distance of about $75 \mathrm{~cm}$. This simulation was run in a larger than proposed calorimeter so we could view the dependence on radius more easily. 

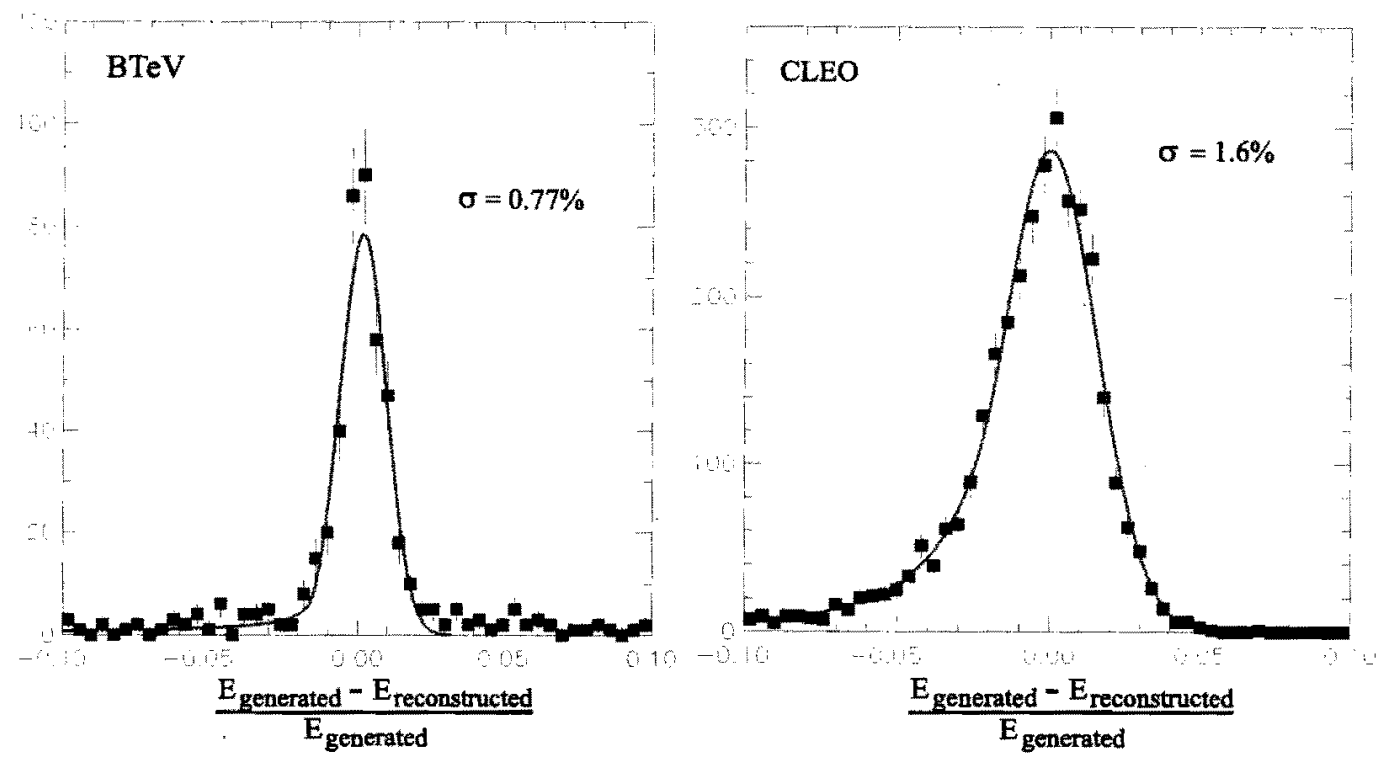

Figure 7.5: The difference between the measured and generated energies, divided by the generated energy, for reconstructed photons as simulated by GEANT for BTeV (left) and CLEO (right). The photons candidate clusters were required to have shower energy shapes consistent with that expected for photons and to be isolated from charged tracks. The BTeV simulation was run at 2 interactions/crossing.

\subsection{Crystal Production}

There are two producers of $\mathrm{PbWO}_{4}$ crystals working for CMS. One of these companies is in Russia, the Bogoroditsk Techno Chemical Plant, and the other is. in China at two sites, Beijing and Shanghai. CMS crystal production is scheduled to finish in 2005 . We have visited the crystal production facilities in Russia and in China. Our Russian and Chinese collaborators have been most helpful in setting up these visits. The Russians already have been producing production crystals $(>6000)$ and are eager to have our business. They have supplied prices and possible schedules. The Chinese have not started production as of this writing, but they are very close to doing so. They are also very interested in BTeV production. Our Chinese collaborators at Shandong University are also interested and capable of producing $\mathrm{PbWO}_{4}$ crystals. We would like to initiate a startup program with them as soon as possible. Generally we think it important to have more than one supplier of crystals. Because of the open nature of the $\mathrm{BTeV}$ detector, crystal installation can proceed in place over a long period of time. 

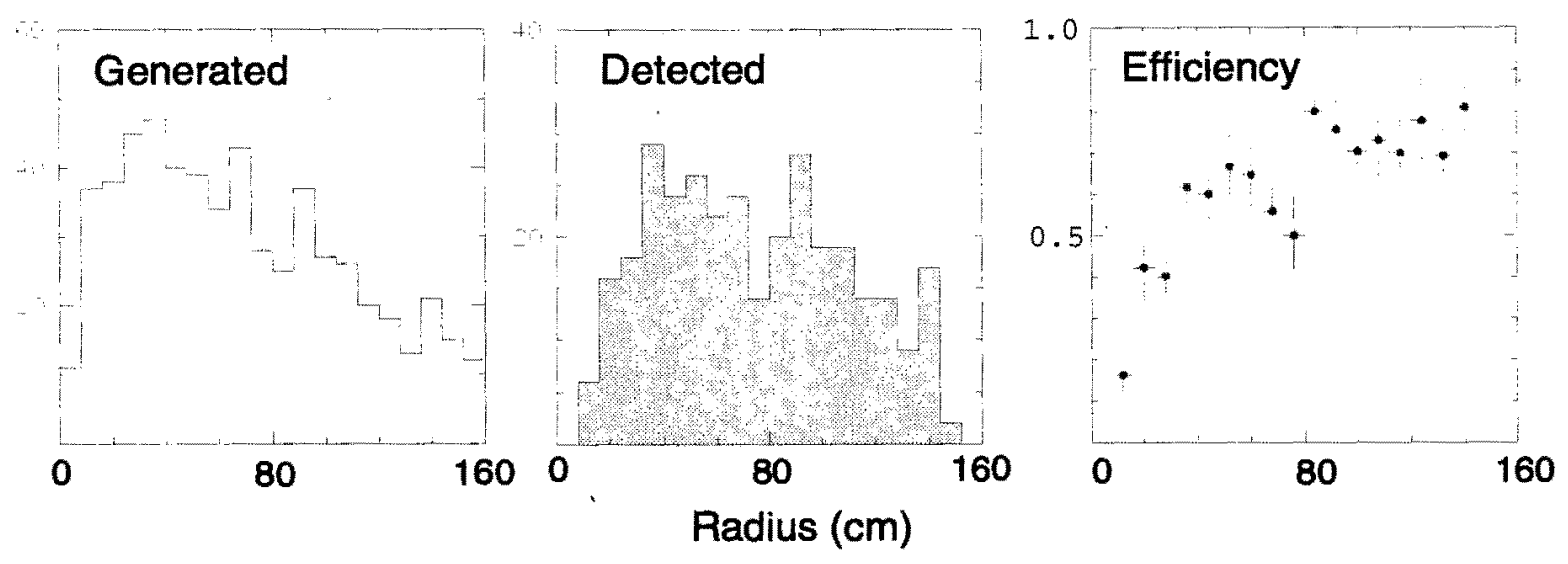

Figure 7.6: The radial distribution of generated and detected photons from $B^{o} \rightarrow K^{*} \gamma$ and the resulting efficiency. The detector was simulated by GEANT and the resulting crystal energies were clustered by our software. The charged tracks from the $K^{*}$ were required to hit the RICH. The simulation was run at 2 interactions/crossing.

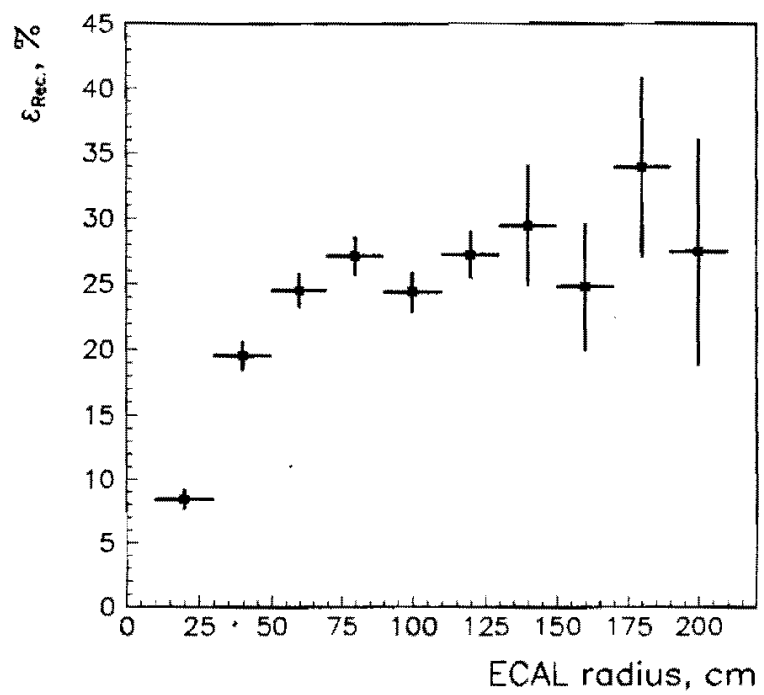

Figure 7.7: The efficiency as a function of the radial distance from the beam line of $\pi^{o} \mathrm{~s}$ from $B \rightarrow \rho \pi$ decays at the position of the calorimeter. The simulation was run at 2 interactions/crossing in the "large" calorimeter. 


\section{Bibliography}

[1] E. Blucher, B. Gittelman, B. K. Heltsley, J. Kandaswamy, R. Kowalewski, Y. Kubota, N. Mistry, A. Bean, and S. Stone, "Tests of Cesium Iodide Crystals for an Electromagnetic Calorimeter," Nuclear Instruments \& Methods A249, 201 (1986).

[2] We note that a past favorite, CsI (undoped), was attractive from many points of view. However, it is not radiation hard enough. Furthermore, it is not quite fast enough to completely produce all its charge in under one crossing. Lead glass has been rejected because it is not likely to survive the yearly radiation dose.

[3] CMS, "The Electromagnetic Calorimeter Project Technical Design Report," CERN/LHCC 97-33, CMS TDR 4 (1997).

[4] R. J. Yarema et al., A Fast Wide Range Charge Integrator and Encoder ASIC For Photomultiplier Tubes," FEMILAB-PUB-92-311 (1992). This chip was developed for KTEV.

[5] Private communication from Ed Blucher. The dominant cause of the constant term seems to be a differing crystal response depending on where on the face of the crystal the incident photon strikes. This effect was not observed in the CLEO CsI calorimeter (see ref. 1).

[6] Y. Kubota et al., "The CLEO II Detector," Nuclear Instruments \& Methods A320, 66 (1991). 


\section{Chapter 8}

\section{Muon Detector}

The $\mathrm{BTeV}$ muon system has two primary functions:

- $J / \psi$ and Prompt Muon Trigger: Besides providing interesting physics (including $J / \psi$ final states of $B$ decays, direct $J / \psi$ production, and semileptonic decays), this trigger performs an important service role by selecting (bottom) events on which the more aggressive and technically challenging vertex trigger can be debugged and evaluated.

- Muon Identification: Many of the experiment's physics goals (rare decay searches, CP violation studies which require tagging, studies of beauty mixing, searches for charm mixing, etc.) rely on efficient muon identification with excellent background rejection.

We have selected a toroidal magnet design combined with fine-grained tracking elements. This design permits a "stand-alone" trigger: i.e. a muon trigger based solely on information from the muon detector. In addition, improved background rejection is possible by comparing this measurement with momentum and tracking information from the rest of the spectrometer. The system design has been chosen to reduce and uniformly distribute occupancies and to minimize confusion in pattern recognition. To provide a viable trigger, the system must obtain a rejection rate at Level 1 of a few hundred. The goal for muon misidentification is $10^{-3}$.

Given the objective of a stand-alone trigger and the size limitations set by the experimental hall, one can make fairly general calculations that place specific (and restrictive) constraints on the design of the system. We first describe these calculations and use them to motivate the overall design of the muon system. We then describe the trigger efficiency and rejection studies we have performed.

\subsection{General Design Considerations}

The fractional momentum resolution in a magnetic spectrometer can be parameterized as $\sigma_{p} / p=\sqrt{a^{2}+(b p)^{2}}$ where the $a$ term depends on the bending power and multiple scattering environment of the detectors and the $b$ term depends on the bending power and the detector 

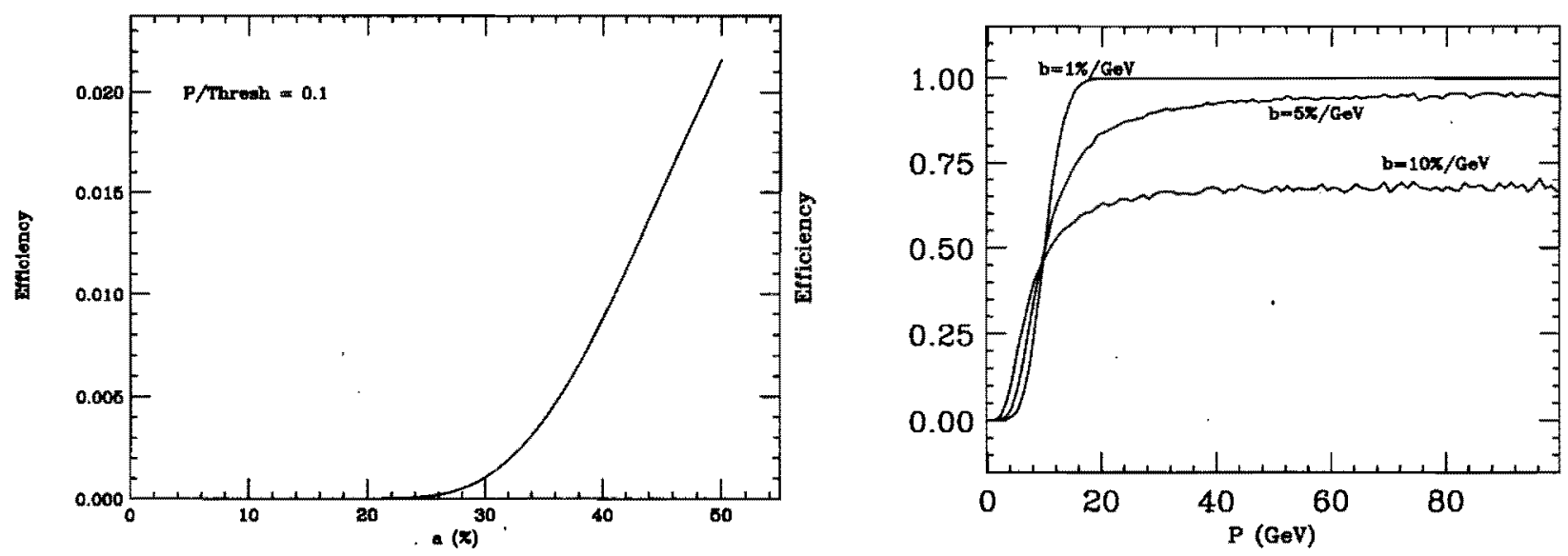

Figure 8.1: (left) Efficiency, calculated in a simple Gaussian model, for a muon with 1/10 the threshold momentum to pass a momentum threshold as a function of $a$, the MCS term in the resolution formula. (right) Efficiency as a function of muon momentum for a trigger designed to fire with a $50 \%$ efficiency at $10 \mathrm{GeV}$. The multiple scattering dominated term is fixed $(a=25 \%)$ while the measurement dominated term varies from $1 \% / \mathrm{GeV}<b<10 \% / \mathrm{GeV}$.

layout and spatial resolution. Figure 8.1a shows that the potential rejection provided by a trigger for low momentum muons significantly degrades once the low momentum fractional resolution exceeds $25 \%$. At $a=25 \%$, the trigger rejects very soft muons at roughly the $4 \sigma$ level. At higher momentum, where multiple scattering is less important, one becomes sensitive to the $b$ term. The high momentum resolution influences how sharp a momentum threshold one can make in a stand-alone muon trigger. Figure $8.1 \mathrm{~b}$ illustrates this point by showing the trigger efficiency as a function of momentum for several $b$ values. These considerations suggest minimum performance criteria of $a<25 \%$ and $b<1 \% / \mathrm{GeV}$.

\subsection{Baseline Muon System}

Several measurement and shielding scenarios were studied before reaching the baseline design. In assessing possible layouts, we compute the momentum resolution using an error matrix which incorporates Gaussian models for the detector resolution and multiple Coulomb scattering. The interaction region is modeled as a Gaussian beam spot with $\sigma_{x}=\sigma_{y}=1 \mathrm{~mm}$ and $\sigma_{z}=30 \mathrm{~cm}$. The result of these studies gives us the baseline geometry shown in Fig. 8.2.

\subsubsection{Baseline Geometry}

Two toroids, $1 \mathrm{~m}$ long with $1.5 \mathrm{~T}$ fields, provide the bending power. The muon detectors will be set up in three stations, one between the toroids and two behind the toroids. The momentum can be measured using the two, well shielded, downstream stations and the nominal beam constraint. The station between the two toroids provides a powerful confirming hit to eliminate fake tracks. 


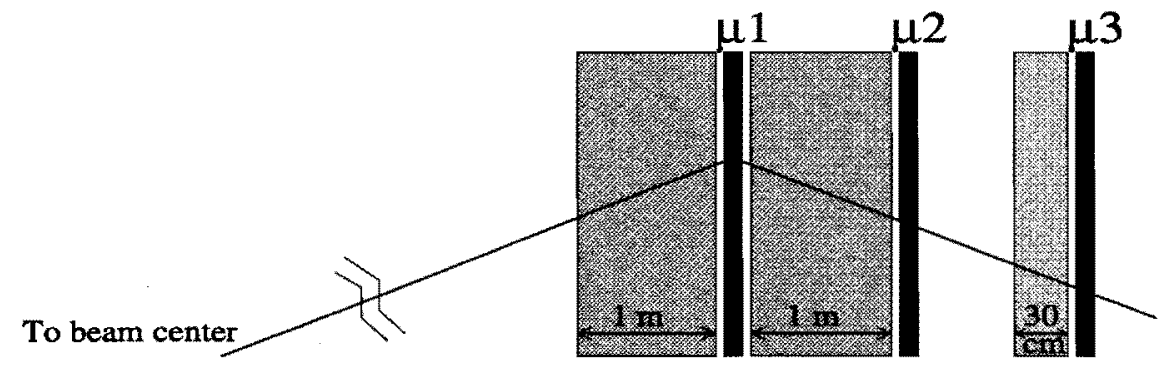

Figure 8.2: Layout of the baseline geometry, shown in elevation view. The three dark boxes, labelled $\mu_{1}, \mu_{2}$, and $\mu_{3}$, represent detector stations with 4 measurement views per station. The two lighter boxes with lengths of $1 \mathrm{~m}$ represent magnetized steel toroids, which provide bending power for the muon momentum measurement and which also serve as hadron absorbers. The $30 \mathrm{~cm}$ long lighter box is an unmagnetized iron shield. The downstream trajectory is measured by $\mu_{2}$ and $\mu_{3}$. The upstream trajectory is measured using the nominal beam center with possible help from $\mu_{1}$. To obtain sufficient bending power, both $1 \mathrm{~m}$ steel sections must be magnetized.

\subsubsection{Baseline Detector}

The basic building block in the construction of a detector station is a "plank" of $3 / 8$ " diameter stainless steel proportional tubes as shown in Fig. 8.3. Thirty-two tubes are arranged in a double layer with an offset of half a tube ("picket fence" geometry) and will be soldered at each end to a brass gas manifold and supported in the middle by soldering to brass support rib piece(s). This provides a sturdy, self-supporting building block which acts as an excellent Faraday cage. Proportional tubes have been selected as the detector technology because they are robust and have the necessary rate capability. We intend to use a fast gas (e.g. $88 \% \mathrm{Ar}, 10 \% \mathrm{CF}_{4}, 2 \% \mathrm{CO}_{2}\left(v_{d} \approx 9 \mathrm{~cm} / \mu \mathrm{s}\right)$ [1]) so the maximum collection time (drift plus charge integration) for a signal should be less than $60 \mathrm{~ns}$, allowing us to gate off hits due to incoming beams which arrive 70 ns before particles from the interaction region. We will use thin walled $(0.01$ ") stainless steel tubes. This is a proven technology - it has been used successfully by the CDF collaboration where similar tubes [2] had a long lifetime with low failure rate. The tubes will be strung with $30 \mu \mathrm{m}$ gold-plated tungsten wire.

The $0.5 \mathrm{~cm}$ wire spacing of this design has no dead regions and has an effective spatial resolution of $5 \mathrm{~mm} / \sqrt{12}=1.4 \mathrm{~mm}$ which meets our requirements outlined in Section 8.1. Figure 8.4 shows the momentum resolution for various muon system configurations assuming a $2.5 \mathrm{~mm}$ resolution and incorporating the magnetic fields and multiple scattering. The top curves show the result for only one magnetized toroid which is clearly ineffective. The bottom curves show results when both steel filters are magnetized. The case where only a beam constraint and $\mu_{2}-\mu_{3}$ are used is illustrated by the dashed red curves. Including information from $\mu_{1}$ produces the solid magenta curves. Curves are shown for three different azimuths. The chosen geometry exhibits good performance relative to our criteria on $a$ and $b$. The importance of $\mu_{1}$ lies in providing redundant information to eliminate fake tracks and for matching tracks with the inner tracker at higher trigger levels and offline. Its effect on 


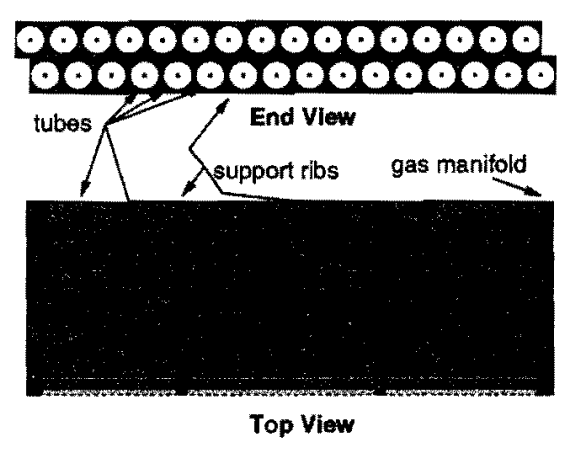

Figure 8.3: End and top views of one "plank" of proportional tubes.

Table 8.1: Parameters of the baseline BTeV Muon System.

\begin{tabular}{|l|c|}
\hline Radial coverage & $16-240 \mathrm{~cm}$ \\
Toroid Z-location (center) & $\pm 830,960 \mathrm{~cm}$ \\
Average Station Z-location & $\pm 890,1020,1150 \mathrm{~cm}$ \\
Total Length & $3.3 \mathrm{~m}$ \\
Toroid Length (each) & $1 \mathrm{~m}$ \\
Toroidal Field & $1.5 \mathrm{~T}$ \\
Tube cell size & $1 \mathrm{~cm}$ (diameter) \\
Wire spacing: & $0.5 \mathrm{~cm}$ (staggered) \\
Spatial resolution & $1.5 \mathrm{~mm}$ \\
Total channels & 39,936 per arm \\
Momentum resolution & $\sigma_{p} / p=19 \% \oplus 0.6 \% \times p$ \\
\hline
\end{tabular}

the momentum resolution is less important.

To minimize occupancy at small radii and to minimize pattern recognition confusion, each detector station will consist of eight overlapping pie shaped "octants," as shown in Fig. 8.5a. The four views $(r, u, v$, and $r)$ in each octant are shown in Fig. 8.5b. The $r$ (radial) view is repeated to provide redundancy for the most important view and to help reject fake tracks in the trigger. The $u$ and $v$ views are rotated $\pm 22.5^{\circ}$ from the $r$ view and are used to measure $\phi$ to resolve hit ambiguities, thereby reducing the misidentification rate. The views stack on top of each other and are built from the planks described above. There will be 13 planks in each view of an octant. Pairs of octants will be combined into quads which will be the structure moved in and out of the BTeV detector.

A summary of the baseline BTeV muon system is given in Table 8.1. Further details may be found in Appendix A.

\subsubsection{Front-end Electronics}

Although we will only be reading out a latch bit and not using TDC's, we are considering gating the system to exclude hits coming from the incoming beam. Therefore the front-end 


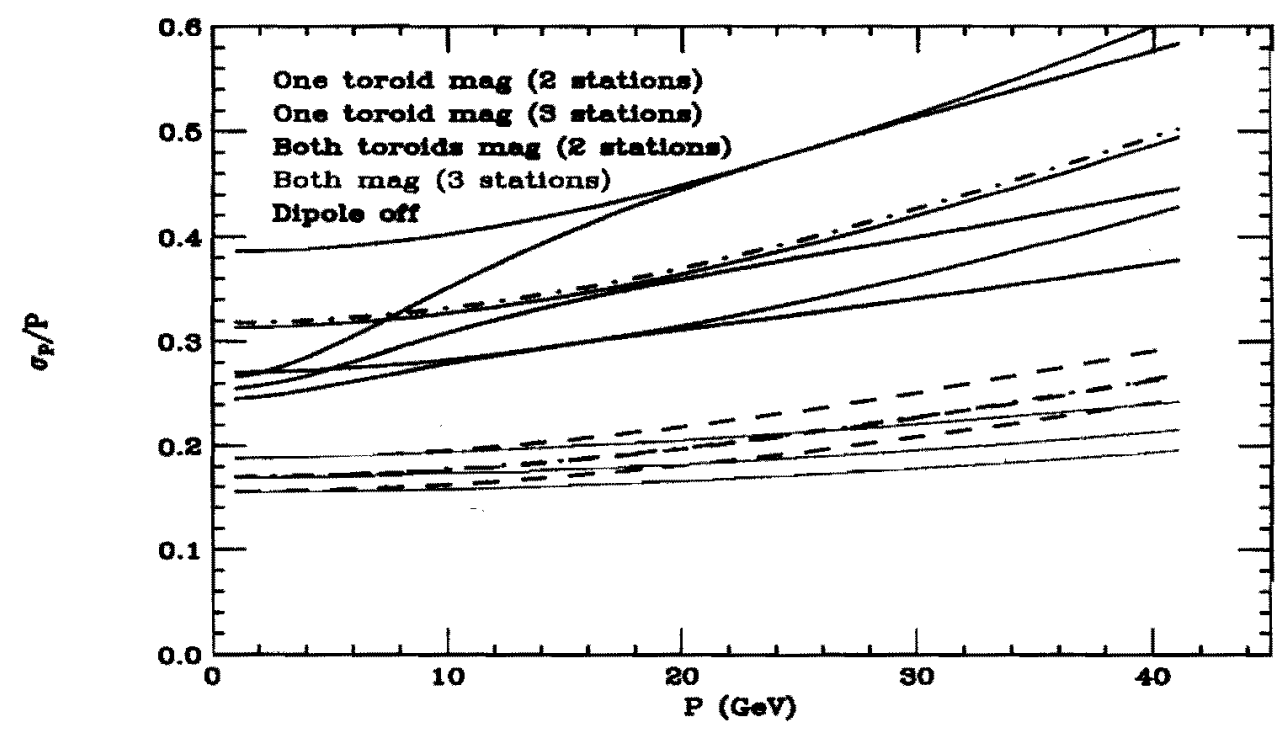

Figure 8.4: Plots of the fractional momentum resolution versus momentum for various muon system configurations. All plots assume $2.5 \mathrm{~mm}$ RMS spatial resolution. The plots appear in groups of 3 corresponding to $\phi=270^{\circ}$ (best) $0^{\circ}$ and $90^{\circ}$ (worst). The $\phi$ dependence illustrates interference between the central dipole and muon toroid(s). The top (bottom) curves show the resolution when one (two) toroids are magnetized. The black and red curves illustrate the case where only the two stations after the second toroid are used. In this case the trajectory upstream of the toroid comes entirely from the beam constraint. The blue and magenta curves illustrate the case where the station between the two toroids is used to help determine the trajectory prior to the toroids. The green curves assume only the final two stations are used, and the dipole is turned off.
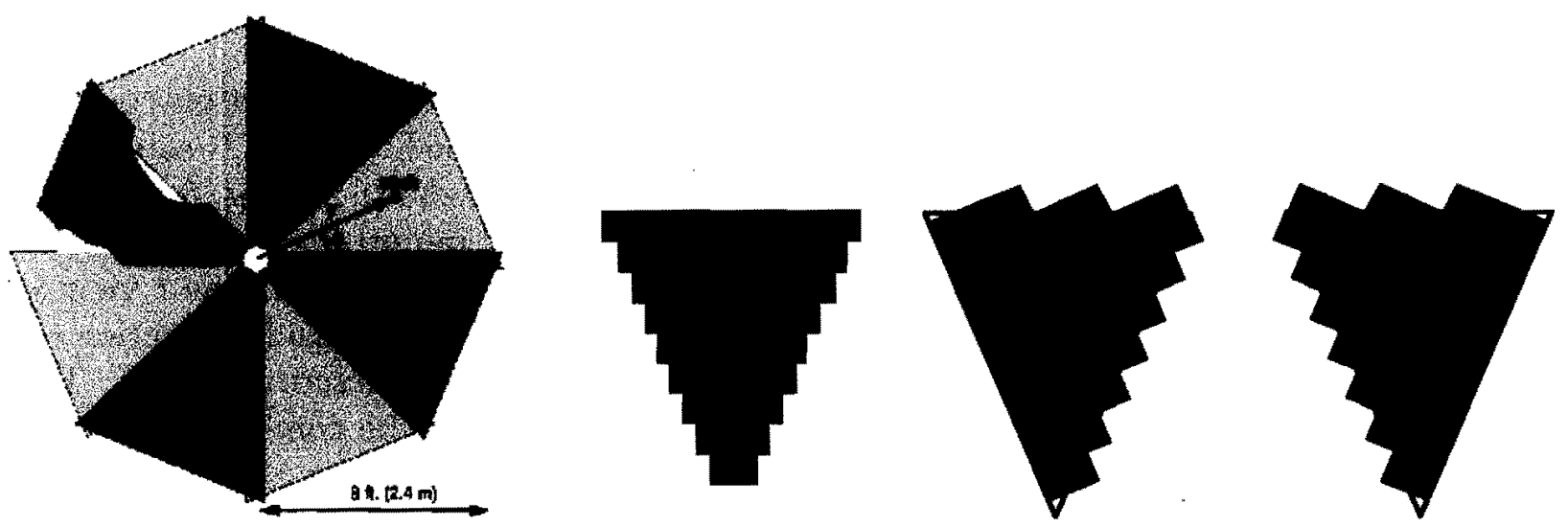

Figure 8.5: (left) Beams-eye view of one muon detector station, which consists of eight overlapping octants arranged in two layers. One octant is cut away in places to show the overlap between adjacent octants. (right) Arrangement of planks to form the four views in an octant ( $r$ view is repeated). There will be 13 planks per view (more than shown). 
electronics need a double pulse resolution $<30$ ns. The electronics should also be low cost and have sufficient gain. We intend to mimic the setup used for the CDF COT. There will be a PC board to deliver high voltage to each proportional tube, and a PC board with electronics to amplify and digitize the tube signal. Both boards will be located directly at the end of a plank.

We plan to utilize the ASDQ integrated circuit developed at the University of Pennsylvania to amplify and digitize the signals coming from the proportional tubes. This chip will be used in the Run-II CDF central outer tracker for a similar purpose. The ASDQ amplifies the first $\sim 8-10 \mathrm{~ns}$ of the signal and outputs an LVDS (equivalent) differential logic signal. This chip, when mounted on a PC board, has a low effective threshold of about $2 \mathrm{fC}$ and features a double pulse resolution of $\sim 20$ ns. The chip seems to be an ideal choice for the $\mathrm{BTeV}$ muon system. Beam tests of the predecessor to the ASDQ, the ASD8B, indicate that it is suitable for our use. Noise problems encountered during the beam test with the ASD8B required creating a Faraday cage out of Aluminum, copper plated G10 and copper tape. We will address this problem by completely enclosing the electronics and proportional tube connections in a Faraday enclosure. This is the reason that the gas manifolds (Fig. 8.3) will be made of brass and soldered to the proportional tubes.

The ASDQ digital signals will be sparsified, serialized, and read out using a standard Fermilab readout protocol. Fiber optic cables will transfer the data from the plank to a buffer memory. Slow control and monitoring functions will be performed via fiber optic link as well.

Additional details regarding the front-end electronics and readout can be found in Appendix A.

\subsection{Trigger Studies}

We have studied the triggering performance of our baseline muon system using both MCFast and full GEANT simulations. We used minimum bias events to study rejection rates and $B^{0} \rightarrow J / \psi K_{s}^{0}$ and $B_{s}^{0} \rightarrow J / \psi K^{* 0}$ where $J / \psi \rightarrow \mu \mu$, to investigate trigger efficiency. The minimum bias events were generated with PYTHIA and include elastic scattering, single and double diffractive, low $p_{T}$ scattering and semi-hard QCD $2 \rightarrow 2$ processes. The number of events per crossing is generated from a Poisson distribution with average two. In generating the signal events, a Poisson distributed number of minimum bias events (average of 2) were added to the signal event.

We studied the performance of the baseline muon system using several levels of simulation. The first of these simulation studies was designed to study the acceptance for $B^{0} \rightarrow J / \psi K_{s}^{0}$ events and rejection of minimum bias events using the MCFast simulation package. This study allowed us to make detailed studies of the kinematic cuts required to create an effective trigger. The MCFast simulations were based on the parameterized Gaussian momenta resolutions which were obtained using the semi-analytic calculations shown in Fig. 8.4.

While the MCFast simulations were quite useful for initial testing of muon triggers, to have confidence in our ability to reject minimum bias background we require more realistic 
simulations. This was achieved using a full GEANT simulation which simulates additional hits in the muon system due to non-prompt sources, $\delta$-rays, electromagnetic shower debris and hadronic shower leakage. After describing this simulation we discuss properties of the "noise hits" and conclude by describing the performance of several (stand-alone) muon triggers within this realistic environment.

\subsubsection{GEANT simulation description}

The geometry used in the GEANT simulation is similar to our baseline geometry. There are two $1 \mathrm{~m}$ long cylindrical toroids with inner radius of $14 \mathrm{~cm}$ and outer radius $240 \mathrm{~cm}$. One muon station is placed between the two toroids and two behind the toroids. As described in Section 8.3.2 it was found that additional shielding in front of the third station and around the beam pipe dramatically improved the noise levels so this was added. The aluminum support structure was also simulated. The tube walls were simulated in a simplified form using a planar sheet and in an exact form using cylindrical tubes. In this simulation, each octant had one $r$-view, one $u$-view, and one $v$-view. We expect that the extra downstream $r$-view can only improve the results. Therefore, this simulation is a pessimistic prediction.

All default processes plus $\delta$-rays were simulated. Photons and electrons were traced down to an energy of $0.1 \mathrm{MeV}$ while hadrons and muons were traced down to $10 \mathrm{MeV}$.

The digitization was performed in two, alternate, ways. In the approximate tube wall simulation (with planar sheets) a sensor volume composed of a large sheet was used. When this sensor volume was reached, the coordinates were used to determine the tube which should be fired. The efficiency for this method was $100 \%$ for tracks within $95 \%$ of the tube radius. In the simulation which used cylindrical tube walls, an individual sensor volume equal to $85 \%$ of the radius of the tube was used inside each tube. Therefore, knowing which sensor volume a particle was in gave the tube number directly. The information which was output for the trigger included the type, momentum, and parent of each particle which left a hit in the muon system as well as a list of all hits associated with the particle.

Using computer farms at the University of Puerto Rico-Mayaguez and Vanderbilt University we generated over 100,000 minimum bias events and more than $10,000 B$ to $J / \psi$ final state signal events. Events were simulated assuming two minimum bias interactions/crossing.

\subsubsection{Properties of additional "noise" hits}

Figure 8.6 shows the characteristics of the hits in the muon detector for $B_{s}^{0} \rightarrow J / \psi K_{s}^{0}$ events with the original geometry. The noise hits are dominated by low momentum secondaries coming from interactions with the walls of the beam hole in the muon filters as is evident from the radial position distributions at the downstream faces of the filters. Secondaries spray out of the downstream ends of the holes in both filters. Figure 8.7a documents this problem. The hit distribution in the muon planes can be understood by considering that the percentage of such tracks striking a given plane will increase with the lever arm between

the nearest filter and the plane. (All planes go down to the same radial position.) The 
effect is noticeable in stations 1 and 2 which are just downstream of filters but is largest for station 3. All planes in station 3 receive a large flux due to the long lever arm between this station and the second filter. To reduce this effect we added a $30 \mathrm{~cm}$ thick filter with the same radial coverage as the toroids just in front of the third station. The improvement is presented in Fig. $8.7 \mathrm{~b}$. Interactions with the beam pipe as well as $\delta$-ray production also contribute significantly to the noise. Additional shielding $(8 \mathrm{~cm})$ around the beam pipe is also proposed and the resulting improvement is shown in Fig. 8.7c and $8.7 \mathrm{~d}$.
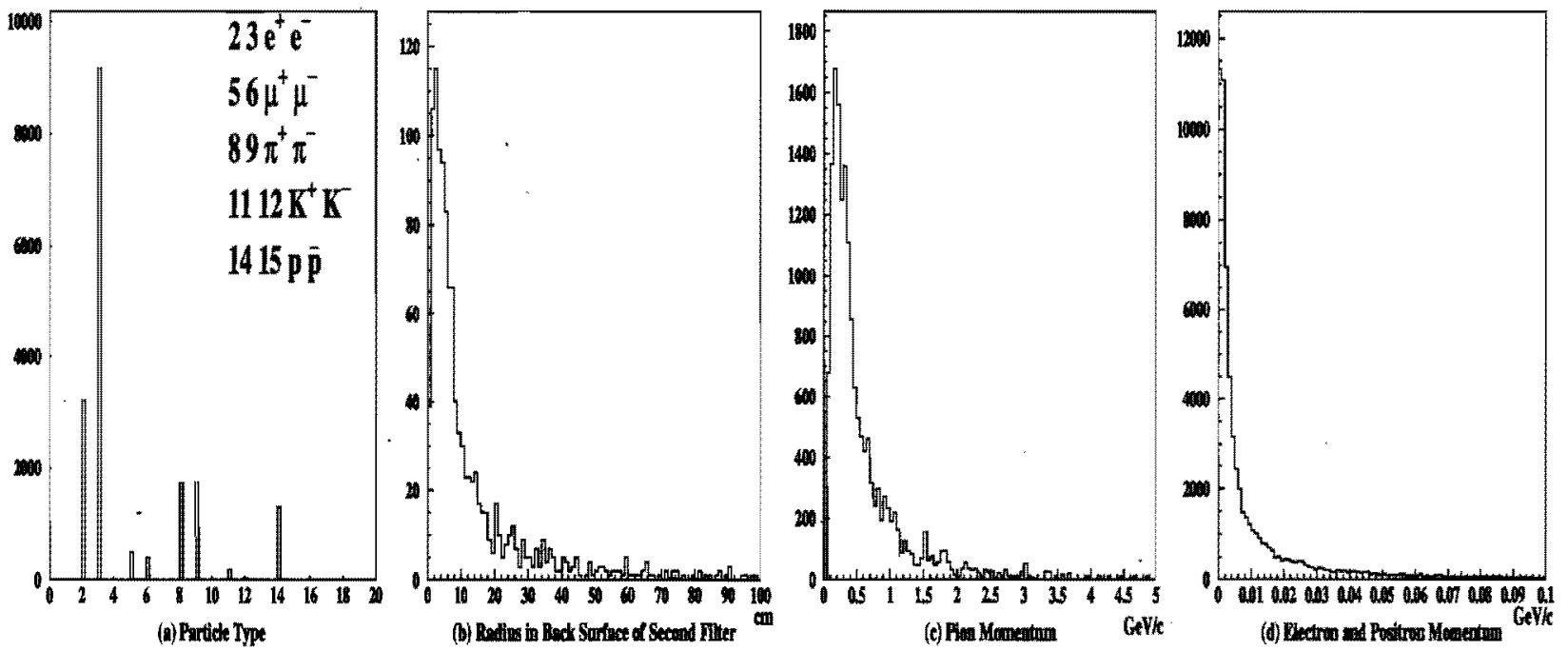

Figure 8.6: Characteristics of muon detector hits for $J / \psi K_{s}^{0}$ events. (a) Particle type. Each particle type is assigned an integer code, shown in the legend. For example, $\pi^{+}$is given the code 8. The electromagnetic and hadronic noise is larger than the muon signal. The excess of electrons over positrons is due to $\delta$-ray production. (b) Projected radial position at the downstream face of the second filter for tracks that hit station 3 . The large percentage of tracks emanating from the vicinity of the hole in the filter is quite evident. (c) and (d) Momentum of pion and $e^{+} / e^{--}$noise. Noise secondaries have much lower momentum than $J / \psi$ muons.

\subsubsection{A muon "tracking" trigger}

To establish an "upper" limit on muon trigger performance in the presence of the GEANT generated noise we studied the performance of a muon "tracking" trigger. This trigger loops over all hits within a given octant sector to choose the set of hits which forms the best muon track with the nominal beam center. The best set of hits is defined using a $\chi^{2}$ test to the hypothesis that the muon system hits form a track which emanates from the nominal beam origin with production angles $x_{o}^{\prime}, y_{o}^{\prime}$, and momentum $p$. It is important to emphasize that this is a simulation of a "stand-alone" trigger. There is no use of any tracking information from the inner tracker; all information comes from the muon system. 

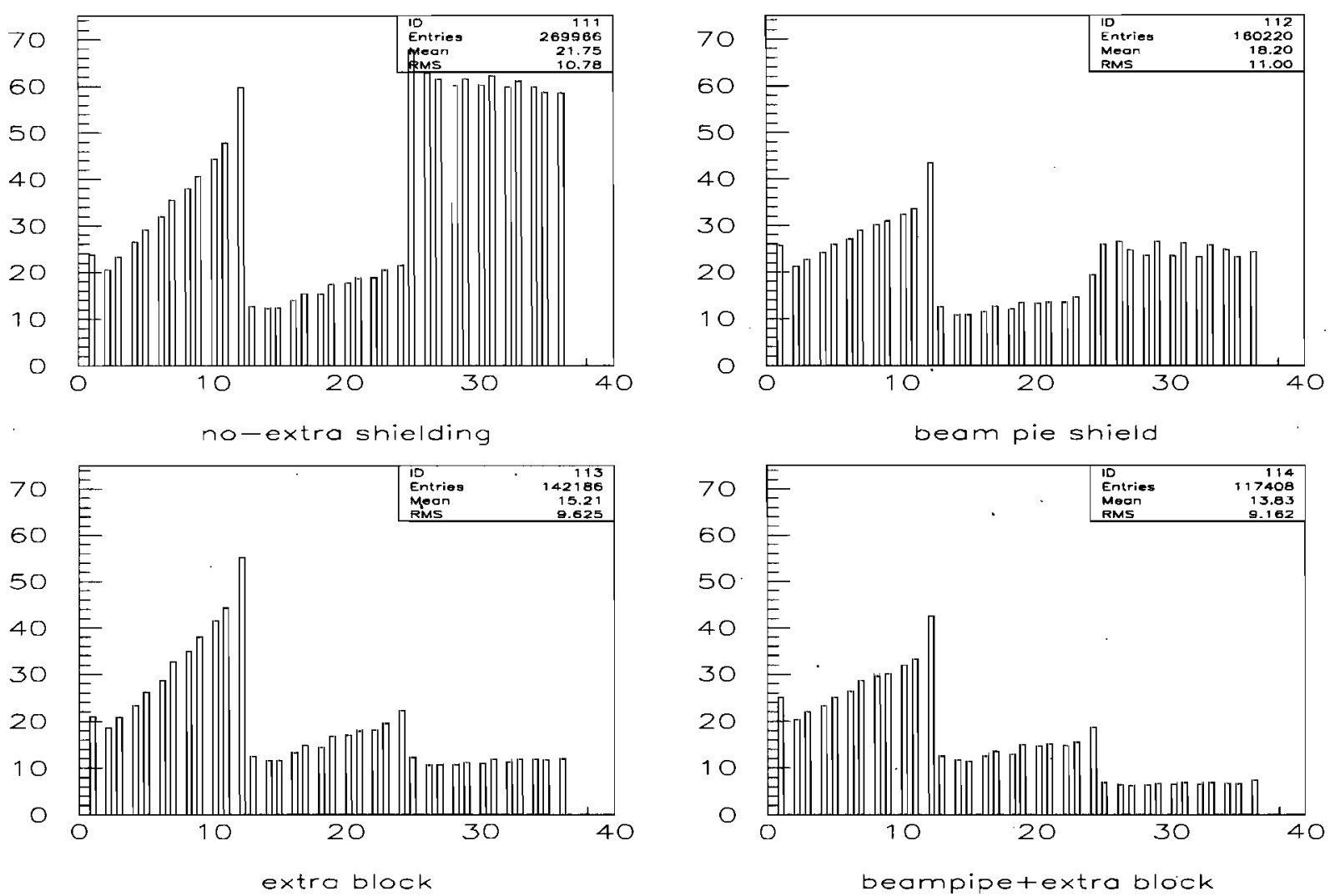

Figure 8.7: Distribution of hits among muon detector planes for different shielding configurations. There are twelve planes per station. (a: upper left) Original design; no extra shielding. (b: lower left) Extra shielding block in front of the third station. (c: Upper right) Extra shielding around the beam pipe. (d: Lower right) Both beam pipe and third station shielded.

For expediency we make several simplifications. We use a least-squares $\chi^{2}-e . g$. there is no attempt to build the $\chi^{2}$ including the correlated covariance matrices due to multiple Coulomb scattering. We also use a significantly simplified magnetic description. A sudden bend approximation is used for the central dipole field. The toroid field in each octant is modeled by a constant field pointing perpendicular to the octant sector bisector and is thus an approximation to a circular field. Particles are traced through the toroids according to the sudden bend approximation. The tracks are assumed to pass exactly through the nominal beam center and no attempt is made to incorporate the smearing of the nominal beam center in constructing the $\chi^{2}$. There is no attempt to take into account $d E / d x$ loss. All equations are linearized in the 3 fit parameters $x_{o}^{\prime}, y_{o}^{\prime}$, and $q / P$ which means that the fit is a classic, non-iterative linear fit.

It is important to note that although the fitting process has been simplified, the GEANT modeling of the muon system has not. A complete magnetic trace is used throughout. Appropriate multiple scattering and $d E / d x$ losses are incorporated. A realistic luminous 
region is used and non-prompt muons are generated from detached vertices.

Figure 8.8 quantifies the rejection power and relative efficiency of this tracking dimuon trigger. The $J / \psi$ efficiency is plotted versus the minimum bias rejection ratio. The $J / \psi$ efficiency is normalized to events where both muons from the $J / \psi$ have momentum greater than $5 \mathrm{GeV} / c$ and both muons also leave hits in all three stations of the muon system. All reconstructed muons are required to have $\chi^{2}<25.2$ and the two muons must have opposite reconstructed charge and appear in two different octants. The main branch of the "cut tree" is a requirement on the minimum radius of all the muon hits. Branching off is a set of increasingly tighter cuts on the maximum $\chi^{2}$. The third branch is a set of cuts on the minimum reconstructed $P_{T}$.

We are able to achieve a rejection in excess of 500 to 1 with an efficiency of nearly $50 \%$ with the principal cuts being a radius greater than $32 \mathrm{~cm}$, a $\chi^{2}<14.4$, and a minimum $P_{T}>0.4 \mathrm{GeV} / c$, although several different cut selections give essentially identical results.

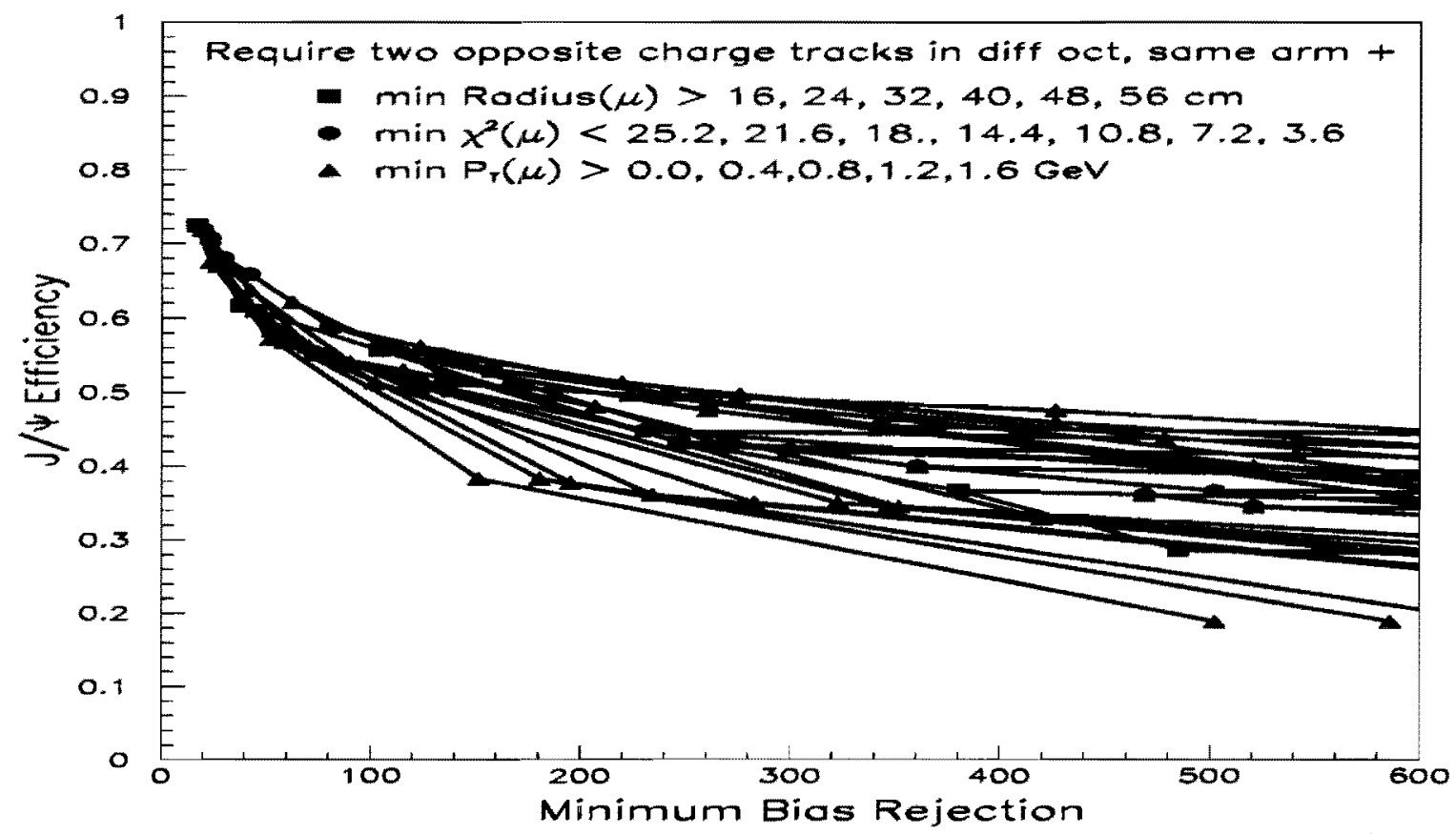

Figure 8.8: $J / \psi$ efficiency versus minimum bias rejection rate for cuts on the minimum radius, maximum $\chi^{2}$, and the minimum muon $P_{T}$. Two oppositely charged tracks in different octants in the same arm must pass the relevant cut. Black lines connect the squares which show the variation as the minimum radius is increased. From each black square a red line connecting the circles shows the change as the maximum $\chi^{2}$ cut is decreased. From each red circle a green line connecting the triangles shows the variation as the minimum reconstructed muon $P_{T}$ is increased. All information in this stand-alone trigger comes from hits in the muon system alone. 


\subsubsection{Simplified Tracking Trigger}

We have also studied a simpler trigger which only uses the $r$-view hits and ignores the dipole contribution. It uses a more efficient algorithm that only fits likely candidate hits. Results for this trigger are shown in Fig. 8.9 where the $J / \psi$ efficiency is plotted versus the minimum bias rejection ratio. The cut variables include the reconstructed momentum and $p_{T}$. In addition, the minimum radius cut requires that all hits associated with the track are at a radius greater than the radius cut. This shows that a very simple stand-alone dimuon trigger can provide rejection rates up to 600:1 while retaining an efficiency of $25 \%$.

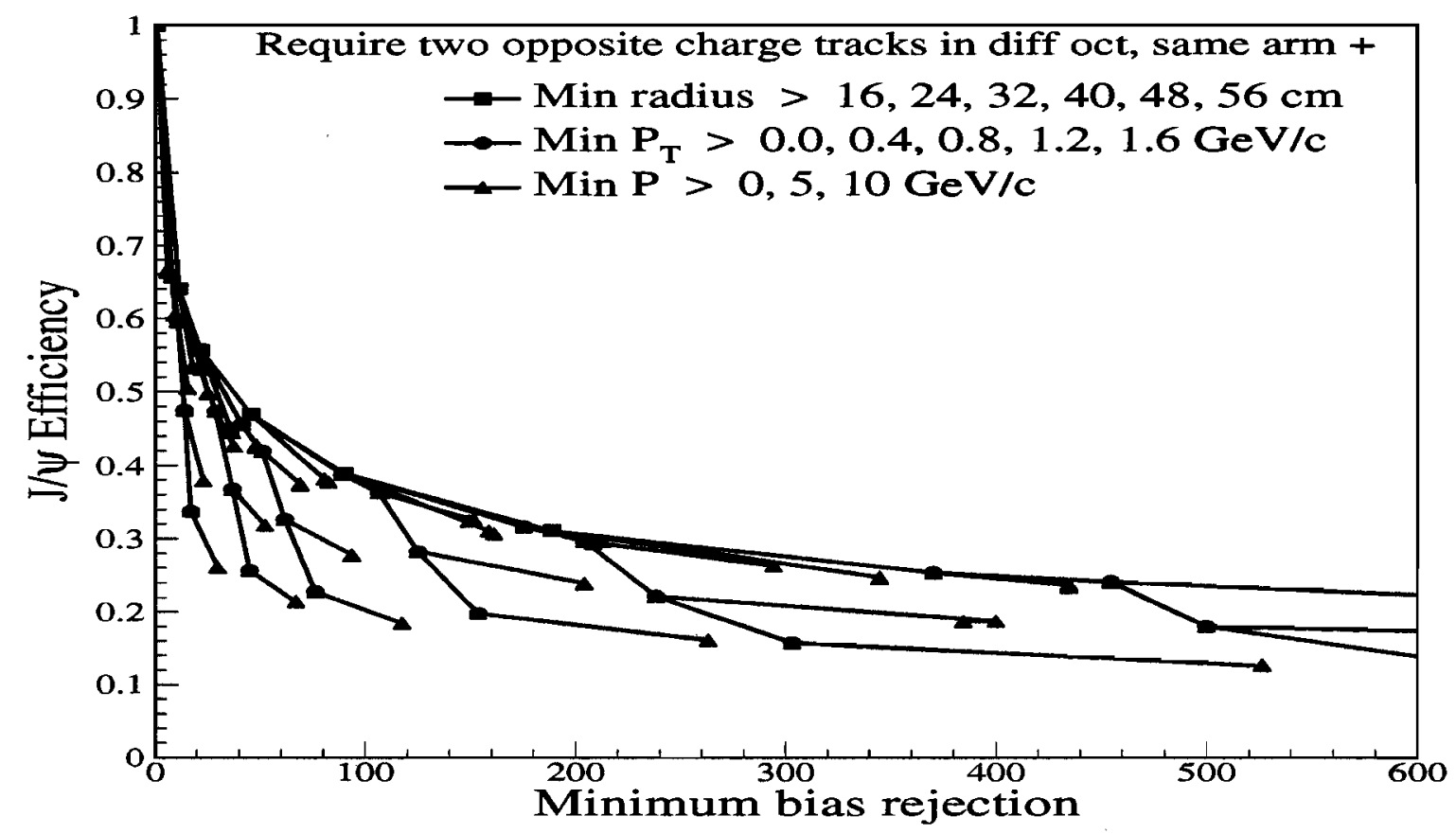

Figure 8.9: $J / \psi$ efficiency versus minimum bias rejection rate, using a simplified tracking trigger, for cuts on the minimum radius, minimum muon $p_{T}$, and the minimum muon momentum. Two oppositely charged tracks in different octants in the same arm must pass the relevant cut. Black lines connect the squares which show the variation as the minimum radius is increased.. From each black square a red line connecting the circles shows the change as the minimum muon $p_{T}$ cut is increased. From each red circle a green line connecting the triangles shows the variation as the minimum muon momentum is increased.

\subsubsection{Trigger Summary}

Our original MCFast simulation only included muon hits due to real muons. Therefore, the entire minimum bias background came from pion and kaon decays-in-flight. This produced an average of 1.2 muon tracks in a minimum bias event with a resulting average occupancy of $0.015 \%$. From this we were able to obtain rejection ratios of 600 to 1 with efficiencies of $85 \%$. Using a much more realistic GEANT simulation (described in Section 8.3.1) brings 
the average occupancy up to $1.2 \%$ for minimum bias events ( 2 per crossing) and $2.1 \%$ for $B$ to $J / \psi$ events (plus 2 minimum bias events). Using a straightforward tracking algorithm described in Section 8.3.3 we are still able to retain a 600:1 rejection ratio with efficiency of nearly $50 \%$ even though our occupancy has increased by a factor of 100 . This trigger will be implemented using a modification of the silicon pixel vertex trigger. If this proves too difficult, we have the option to use the simpler trigger described in Section 8.3.4 which also achieves a 600:1 rejection ratio at a still acceptable efficiency of $25 \%$. These efficiencies and rejection rates were calculated using a simulation which did not include the second $r$-view. Therefore, we expect that these results will only get better. The dimuon trigger, which rejects at 600:1, uses a small fraction of the Level 1 bandwidth which is dominated by the vertex trigger which rejects at 100:1. Therefore, this trigger is suitable for both calibrating the vertex trigger and taking physics data. Following the Level 1 trigger (at either Level 2 or Level 3), we can gain further rejection by requiring the tracks which are found by the muon system to correspond to tracks found by the silicon pixels and forward trackers. After requiring a link we can also cut on the more accurate forward-tracker momentum, require the track be detached from the primary vertex, and/or make an invariant mass cut. 


\section{Bibliography}

[1] J.M. Butler et al., "Study Of Fast Gases, Resolutions And Contaminants In The D0 Muon System," Nucl. Instrum. Meth. A290, 122 (1990).

[2] S. Bhadra, S. Errede, L. Fishback, H. Keutelian and P. Schlabach, "The Design And Construction of the CDF Central Drift Tube Array," Nucl. Instr. Meth. A268, 92 (1988). 


\section{Chapter 9}

\section{The BTeV Trigger}

In this chapter, we begin with a description of the trigger requirements for $\mathrm{BTeV}$. We then discuss the strategy used to achieve these goals and provide some details of the hardware implementation and associated trigger algorithms. We conclude with a summary of the trigger performance. Additional details of the trigger algorithm and performance are presented in the simulation section, and a more detailed discussion of the trigger hardware is provided in Appendix A.

\subsection{Goals and Requirements for the BTeV Trigger Sys- tem}

BTeV plans to study a broad range of $B$ decays and to employ many different $B$ tagging techniques. Other experiments [1] include a fairly simple "first level" of triggering which, while significantly reducing the number of events so that the higher trigger levels have more time to deal with the survivors, also restricts the types of final states that are accepted. Trigger strategies that require the presence of specific final-state particles, such as muons, or demand the presence of a few high- $p_{t}$ hadrons, are examples of this. BTeV, instead, will trigger on the key property that differentiates $B$ (and charm) particles from other particles, namely their characteristic lifetimes. This allows our trigger to reject light-quark background events at the necessary $\sim 10^{-3}$ level and still be efficient for beauty and charm decays containing only hadrons in the final state (e.g. $B_{s} \rightarrow D_{s} K, B^{o} \rightarrow \rho \pi$ and charm states such as $D^{o} \rightarrow K^{+} K^{-}$), as well as those containing leptons (such as $B^{o} \rightarrow \psi K_{s}$ or $B_{s} \rightarrow \psi \eta$ ).

This strategy requires the $\mathrm{BTeV}$ trigger to do track and vertex reconstruction on every beam crossing and to search for evidence of an interaction followed by the decay of a particle within a few hundred microns to a few millimeters away from the primary vertex. In practice, this is done by reconstructing all primary vertices and selecting events that have additional tracks with large impact parameters with respect to the nearest primary vertex. 


\subsubsection{Rejection Requirement}

The goal is to reduce the full interaction rate of $15.2 \mathrm{MHz}$ at a luminosity of $2 \times 10^{32} \mathrm{~cm}^{-2} 2 \mathrm{~s}^{-1}$ (7.6 MHz beam-crossing rate with an average of 2 interactions per crossing) to about $4 \mathrm{kHz}$ of crossings written to permanent storage. The rate of events containing $B$ decays with daughter particles that all fall inside the $\mathrm{BTeV}$ acceptance is about $1 \mathrm{kHz}$. We expect that the trigger will not be perfect, since some $B$ events will have only some of their tracks within the acceptance but will still satisfy the trigger, and since some charm events will also satisfy the trigger. Therefore, we allow a safety factor of 4 . It follows that the trigger must accept no more than approximately one out of every 2000 beam crossings.

\subsubsection{Efficiency Requirement}

To obtain clean samples of heavy-quark events, decay-vertex cuts must be made in the offline analysis. A vertex-based trigger maximizes our trigger efficiency, because the events we reject with online vertex cuts have a strong correlation to those that would eventually be rejected during analysis. The efficiency requirement for the trigger is that it must accept $\approx 50 \%$ of the events that would have survived in an ideal world in which the full offline analysis could have been run on every beam crossing.

\subsubsection{Rate and Deadtime Requirement}

Since the beam crossing rate is $7.6 \mathrm{MHz}$, a trigger decision must be made on average every $132 \mathrm{~ns}$. We want the deadtime from all sources to be practically zero: deadtime $\ll 10 \%$.

\subsection{BTeV Trigger Strategy}

In order to make a trigger decision on average in $132 \mathrm{~ns}$, we plan to employ a three-level trigger scheme (Fig. 9.1). Each level has the same goal: to select events with detached vertices typical of heavy-quark decays. The levels differ in the parts of the BTeV detector whose data they have access to, the amount of time on average they have to make their decision, and the type of hardware used to implement them.

Level 1 uses information from the pixel detector only. It finds and fits tracks, constructs primary vertices, and looks for tracks with large impact parameters signifying a $B$ decay. To speed up the calculation, Level 1 finds tracks only as they enter the pixel detector and as they exit it. Momentum matching is used in linking the entering and exiting track segments together into tracks. Since the pixel detector is embedded in a $1.6 \mathrm{~T}$ magnetic field and has spatial resolution better than $10 \mu \mathrm{m}$, the track momentum can be crudely measured even within a track segment that spans only three pixel stations. After segment linking, an improved momentum estimate is used to weight tracks in vertex fits, and in the calculation of normalized miss distances. 


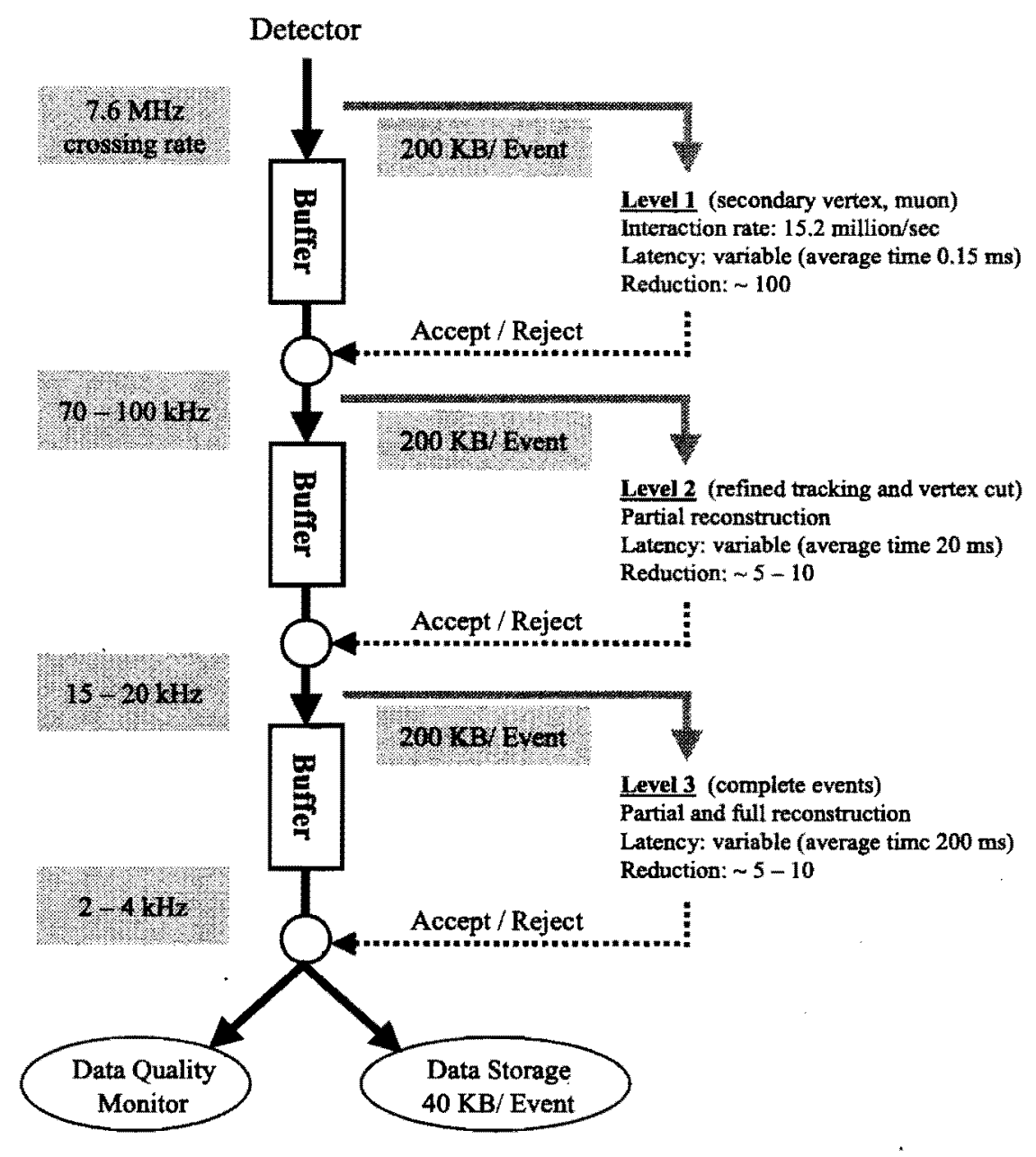

Figure 9.1: Overview of the three-level trigger system.

The Level 1 trigger operates at the full beam-crossing rate and is the most demanding part of the trigger. It is able to carry out track reconstruction at this high rate because of the very high-quality, low-noise, three-dimensional tracking information provided by the pixel detector. Our specifications for the maximum allowable data-transfer bandwidth out of Level 1 and into Level 2, and for the maximum computing power available at Level 2, require that Level 1 reject $\sim 99 \%$ of all beam crossings that do not contain $B$ events. The trigger efficiency, as defined above, should be at least $60 \%$.

Level 2 redoes the pixel-detector reconstruction, but this time finds all the hits associated with each track found at Level 1. It then combines information from the pixel detector with information from the first three stations of the forward tracker. The extra information allows Level 2 to reject fake or poorly-measured tracks, and to calculate the momentum of each track with much better precision. This enables it to do fits with a good estimate of the 
covariance matrix for the tracks. It then searches for tracks with large impact parameters with respect to the primary vertex. Since Level 2 operates on the $1 \%$ of all beam crossings that pass Level 1 , on average it must make a trigger decision every $13.2 \mu \mathrm{s}$. We expect that Level 2 will reject $90 \%$ of all crossings that pass Level 1, while retaining more than $90 \%$ of crossings containing a $B$ event.

Level 3 provides the last factor of 2 or 3 of rejection. It does full pattern recognition using the pixel detector and all stations of the forward tracker. It then performs a full threedimensional vertex reconstruction to find primary, secondary, and tertiary vertices. Based on this vertex information (plus some specialized cuts to rescue a few difficult-to-trigger topologies such as single-prong secondaries), events are selected for permanent storage. Level 3 is expected to be nearly $100 \%$ efficient on crossings containing $B$ events. Since it only needs to inspect one crossing out of 1000; it has on average $132 \mu$ s to make a trigger decision.

To reduce the amount of data recorded, Level 3 can also summarize the raw event to some extent. Especially at the highest luminosities, only part of the information of the event will be written out. For example, the calorimeter, RICH, and muon information on the side away from the $B$ candidate will be discarded.

The computations required by the Level 1 algorithm take much longer than 132 ns even with the very fast processors that will be available when the experiment runs. We deal with this by implementing parallel pipelines, so that the computations are done in parallel by many processors. There is thus a long latency between the time the event occurs and the times of the various trigger decisions. The parallelism entails handling many crossings at once, as well as analyzing the data from each crossing with some degree of sub-event parallelism. The system uses a very large number of advanced commercial components mainly field-programmable gate arrays (FPGAs) and digital signal processors (DSPs) - to achieve the required throughput.

Data must be retained for the time it takes to make the trigger decision. This is done in a very large buffer memory. Since this memory does not need to be in the radiation field near the detector, inexpensive mass-produced computer memory can be used. To reduce the 'amount of data that needs to be stored while the trigger decision is being made, the data for each crossing are "sparsified" or "zero-suppressed" as they come off the detector (via the front-end electronics) into the buffer memory. We estimate that an average crossing will have 200-300 kbytes of sparsified data. We plan to have total buffer memory of $\approx 0.5-1.0$ terabyte, which is significantly more than required to handle this and all other levels of buffering.

Since computations will be occurring for many (actually a few thousand) crossings simultaneously, and the computations for a single crossing will be carried out on more than one processor, it is a challenge to keep all processors fully occupied and not waiting for information from other processors. To achieve the best pipeline operation, we do not require trigger decisions to be time ordered. It is "normal" for trigger decisions to be made for some crossings while processors are still working on much earlier crossings. We have tried to reduce the number of synchronization points, where idle time could occur, to a minimum. We have provided buffering wherever such a problem could appear. As soon as a Level 1 
decision is made, a Level 1 accept or reject is sent back to the buffer-memory managers so that the buffers can be freed for new events (in the case of a reject) or retained for the Level 2 trigger (in the case of an accept). The Level 2 trigger can reject an event at any of several points in its algorithm, as can Level 3. As soon as the trigger decision is known, the answer, either a reject or an accept, is sent back to the buffer-memory managers. Time-outs will be employed in various places throughout the system to prevent "thrashing" on pathological events and to handle certain failures, for example the "death" of a processing node.

\subsection{Trigger System Implementation}

\subsubsection{Trigger System Overview}

Fig. 9.1 shows the conceptual scheme we envision, with Level 1 running at the full crossing rate. The Level 1 vertex trigger is the main physics trigger, but the ancillary dimuon triggers are simple to implement and (by allowing a sample of heavy-quark events to be recorded with no vertex-reconstruction bias) will provide a cross-check of the efficiency of the vertex trigger. Events satisfying Level 1 will be sent to a farm of Level 2 processors, which will be general-purpose CPUs. The Level 2 processors will request event data from additional detectors, mainly from the forward tracker and the muon detector, in order to refine the trigger decision, with still more event data requested as successive cuts are passed. Once a complete event has been built, the event is by definition at Level 3. At Level 3, a strippeddown version of the offline analysis can be performed, and events can be compressed into a summary format to reduce the needed bandwidth to record events for permanent storage.

Although separate buffers are shown in Fig. 9.1, the Level 1, 2, and 3 buffers will most likely be implemented as a single physical buffer, i.e., the pieces of the event will reside in the Level 1 buffers for the individual detector subsystems until called forth by each level of the trigger algorithm. This arrangement (also used by CMS [2]) serves to reduce the event-building bandwidth, since the event is built up gradually as it progresses to higher and higher trigger levels, with complete events assembled only at the Level 2 output rate. Fig. 9.2 is a more detailed block diagram showing these features.

For definiteness we have based our design studies on hardware that is now (or soon to be) available. We recognize that rapid progress in the electronics and computing industries means that when the actual design choices are made $(\approx 2004)$, many of these parts microprocessors, digital signal processors (DSPs), field-programmable gate arrays (FPGAs), random-access memories (RAMs), and so forth - will have been superseded by devices having greater performance per dollar.

It is important to recognize that although the trigger is a technical challenge, it is based on chips, processors, memories, and network components that are commercially available, with speed and capabilities that are steadily growing, and with prices steadily dropping. 


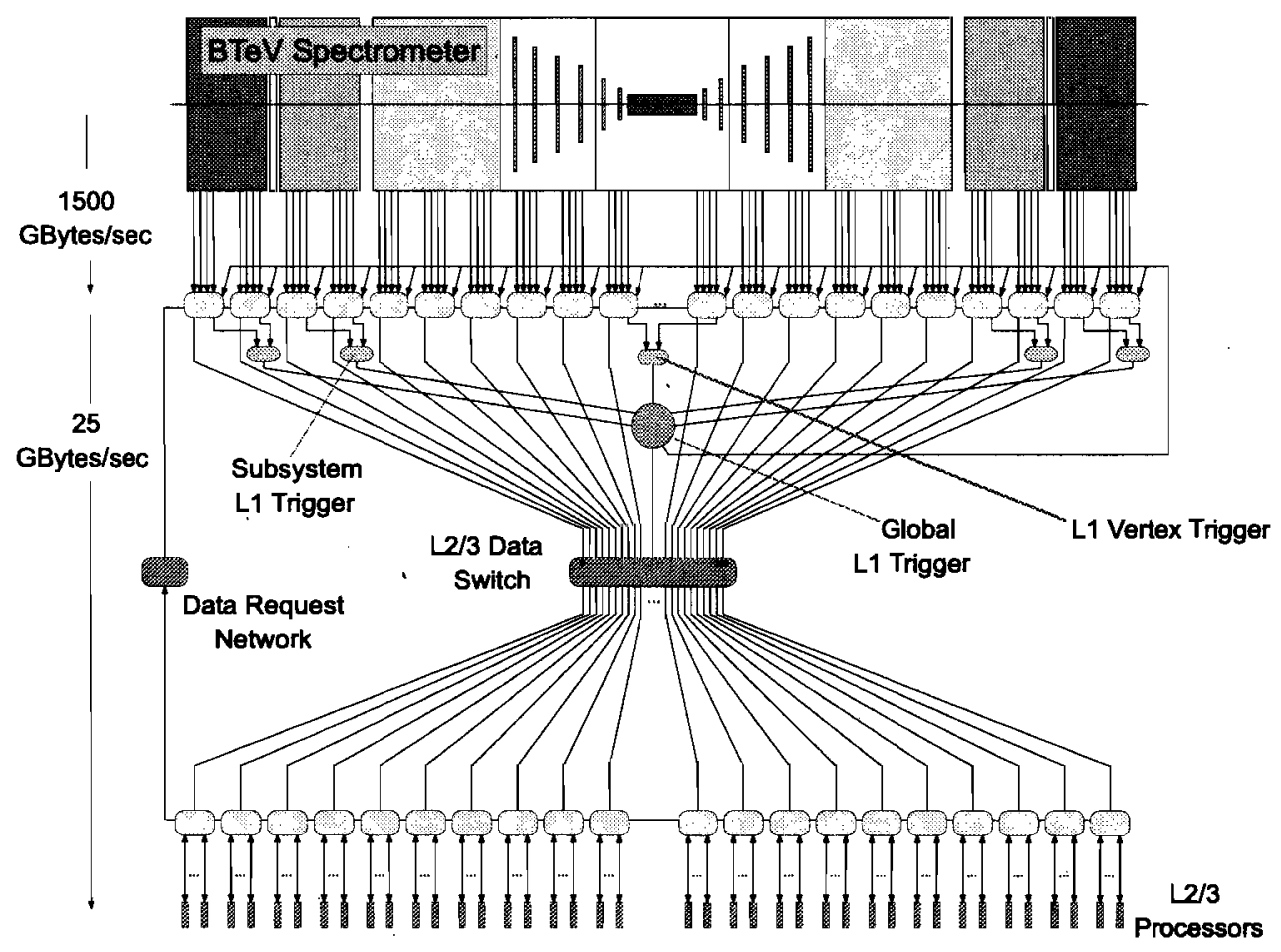

Figure 9.2: Block diagram of proposed DAQ system indicating design bandwidths before and after the Level 1 trigger: up to 1500 GBytes/s of data are digitized from detector subsystems and stored in Level 1 buffers, and up to 25 GBytes/s are passed to Levels 2 and 3.

\subsubsection{Level 1 Vertex Trigger}

The "centerpiece" of the BTeV trigger system is the Level 1 detached-vertex trigger (Fig. 9.3). We have considered various track-finding algorithms, and while the one we discuss here (called "BB33") already meets our requirements, we may yet revise it to lower costs or improve performance. The BB33 algorithm seeks to reduce combinatorial processing time by 1) use of track-finding hardware featuring massive parallelism and 2) careful choice of the region of interest for track "seeds."

The Level 1 algorithm has three phases:

- Track "segment finding";

- Segment matching and track fitting; and

- Vertex finding.

The most demanding part of the baseline algorithm (in terms of both computing power and data-transfer bandwidth) is identifying track segments in each set of three adjacent stations ("segment finding"). This is done in a custom hardware processor using FPGAs. The track segments are then linked into tracks using farms of DSPs. Because of the criticality of the segment finder, we provide a rather detailed discussion below. 


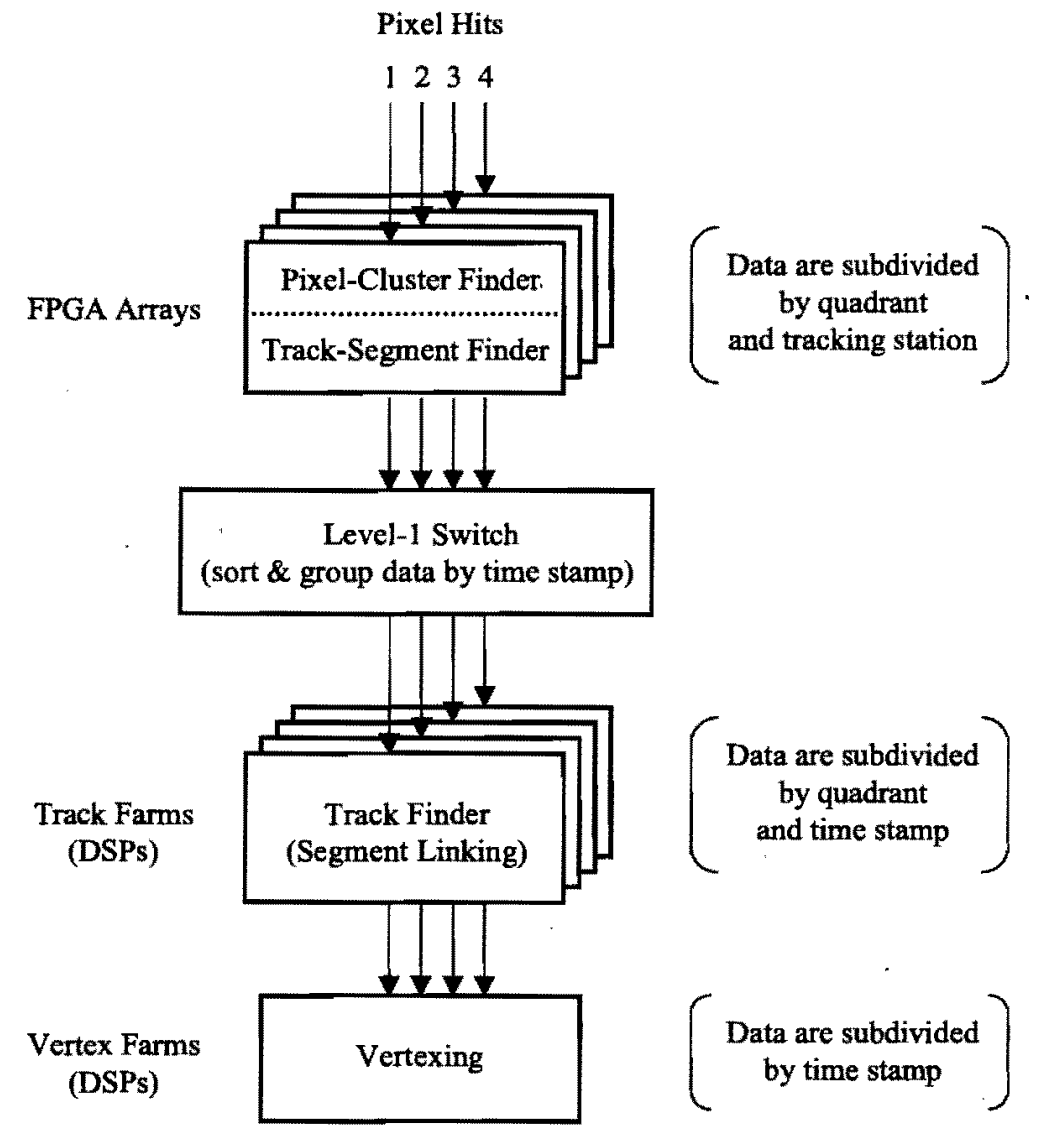

Figure 9.3: Block diagram of the baseline Level 1 trigger.

\subsubsection{Segment-finding algorithm}

As described in Chapter 3, the baseline vertex detector consists of 31 stations each containing two pixel planes, with pixel size $50 \times 400 \mu \mathrm{m}^{2}$. The pixel planes are arranged in doublets, within which the pixel narrow dimension of one plane measures the "bend" $(y)$ view, and that of the other plane measures the "nonbend" $(x)$ view. Track segments are first found in the precision- $y$ view and then confirmed in the precision- $x$ view. For each set of three adjacent stations $(N-1, N, N+1)$, the steps are as follows:

1. For each precision- $y$ "seed" pixel cluster in station $N-1$, form doublets using precision$y$ pixel clusters in station $N$.

2. Form triplets by requiring a confirming precision-y pixel cluster in station $N+1$.

3. Require at least two confirming precision- $x$ clusters in stations $N-1, N, N+1$.

Since magnetic bending is small over these short distances $(\Delta z \leq 8.5 \mathrm{~cm})$, linear extrapolation is used in projecting from one plane to the next, which is straightforward in FPGAs 


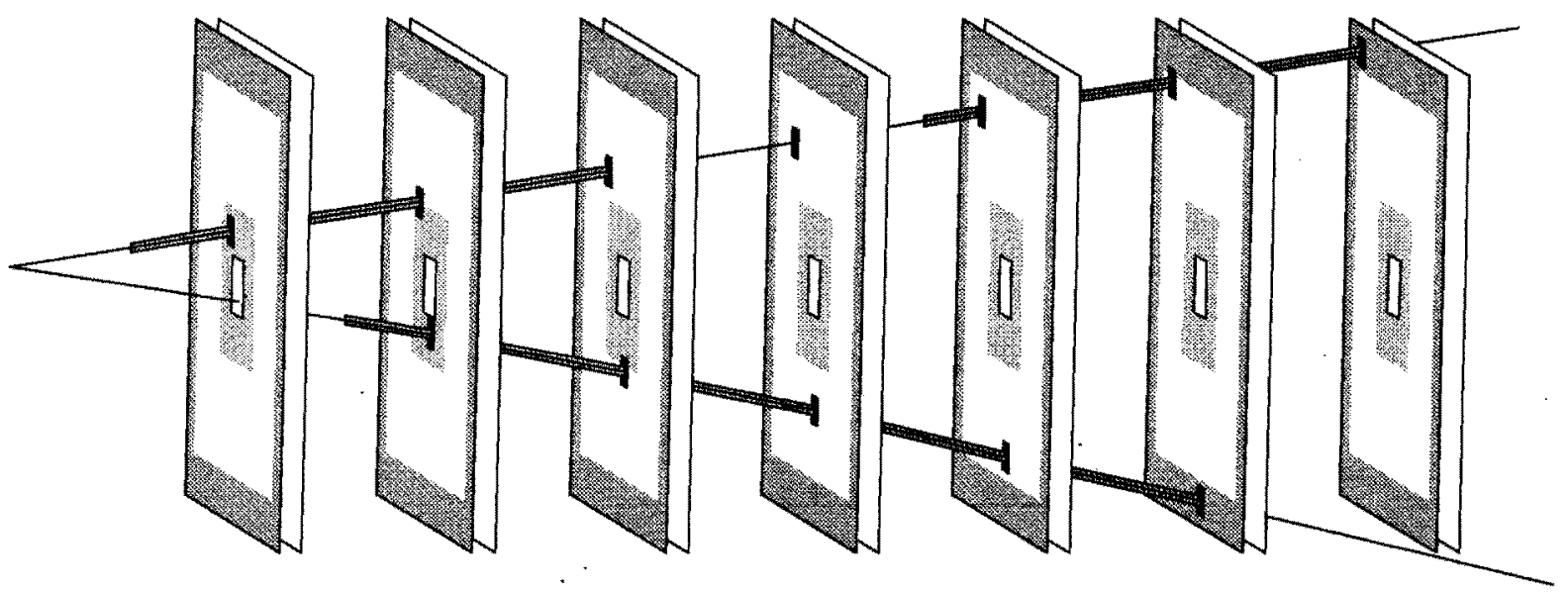

Figure 9.4: Sketch of pixel planes showing (shaded) "inner" and "outer" frames within which track seeds are sought. In the figure, track seeds for the track near the top of the figure are found in the first and last tracking stations. For the lower track the seeds are found in the second and second-to-last stations.

using fixed-point arithmetic. As discussed below, to limit the combinatorics, only "inner" and "outer" pixel clusters are used as seeds. In our baseline studies the inner pixels are taken to be those within $1 \mathrm{~cm}$ of the outer edge of the beam hole, and the outer pixels those with $|x|$ or $|y|>4 \mathrm{~cm}$ (Fig. 9.4).

\subsubsection{Coping with combinatorics}

Since in principle every pixel-cluster doublet could be part of a track, at $\approx 15$ clusters/plane it could potentially be necessary to test a few hundred such hypotheses per station per beam crossing. Sub-event parallelism is achieved by subdividing each pixel plane into quadrants and by looking for track segments simultaneously in all four quadrants of all 31 stations in parallel. This 124-fold parallelism enables the segment finding to keep up with the crossing rate.

With $\approx 3.5$ pixel clusters per quadrant at 2 interactions per crossing, the number of hypotheses to be examined per quadrant is still $\approx 12$, implying a large and expensive installation if all track segments are to be found within one crossing period (i.e., every $132 \mathrm{~ns}$ ). To cope with this, our baseline "BB33" algorithm is restricted to finding tracks near their production point, where they should project back into the beam hole, and near their exit point from the vertex detector, where they should project beyond the detector fiducial volume. This means that only those pixel clusters within a frame of "inner" pixels (i.e. , pixels within a specified distance of the inner edge of each pixel plane) and a frame of "outer" pixels (within a specified distance from the outer edge) need to be used as "seeds" for track finding (see Fig. 9.4). These geometric restrictions substantially reduce the number of hypotheses that must be examined and the amount of hardware needed. A further rationale for this choice is that the pixel clusters near the production point give the best resolution for 
pixel-plane hit data

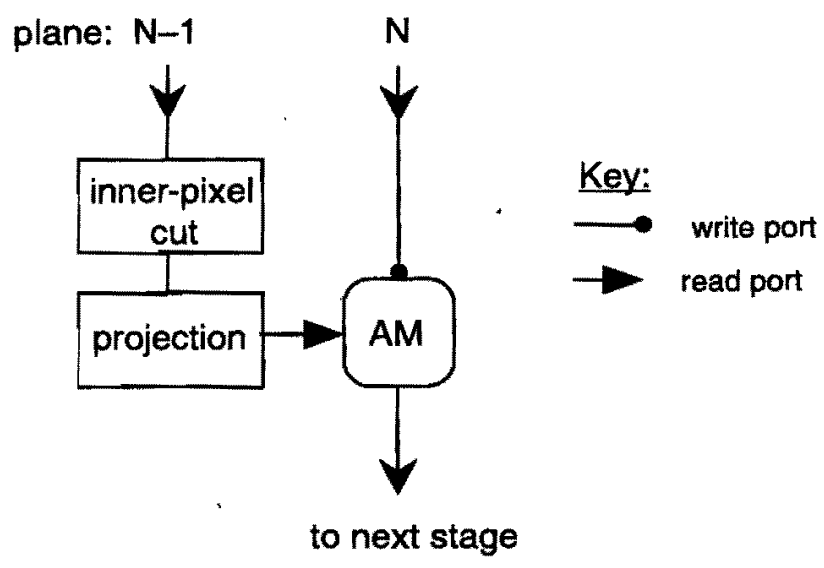

Figure 9.5: Segment-finding "kernel" consisting of an associative memory (AM) with cut and projection operators.

projection to the vertex, while combining them with the clusters near the track exit gives good momentum resolution (needed for an accurate extrapolation to the vertex and proper weighting in vertex fits). Also, the pixel clusters near the track exit provide the best link to the downstream tracking detectors at Level 2.

\subsubsection{Segment finding with the FPGA tracker}

Segment finding is performed for all stations in parallel in "station-quadrant" processors using associative memories [3]. The associative memories are implemented in large FPGAs, for example, the Xilinx Virtex XCV400E, with a cycle time of 20 ns. Details of the implementation are discussed in Appendix A [4]. The key point is that associative memories "unroll" a sequential search algorithm by testing an input value from a "query" list against all the elements of a stored list in parallel. They can thus perform in $n$ cycles a search operation that in typical FORTRAN track-finding code would require a nested DO loop and take $n \times m$ cycles (for example, to search for matches between a list of length $n$ and another list of length $m$ ).

To show how it is possible to carry out segment finding with simple hardware in the time available, we must discuss the segment-finding algorithm of section 9.3.2.1 in somewhat more detail. Step 1 of the segment-finding algorithm is carried out as follows by the simple data-processing structure shown in Fig. 9.5:

a) The pixel clusters from plane $N$ are stored in an associative memory.

b) The pixel clusters from plane $N-1$ pass through a cut operator that compares the position of each cluster to preset limits. Clusters that are in the "inner-pixel" region (Fig. 9.4) are passed to the projection operator. 


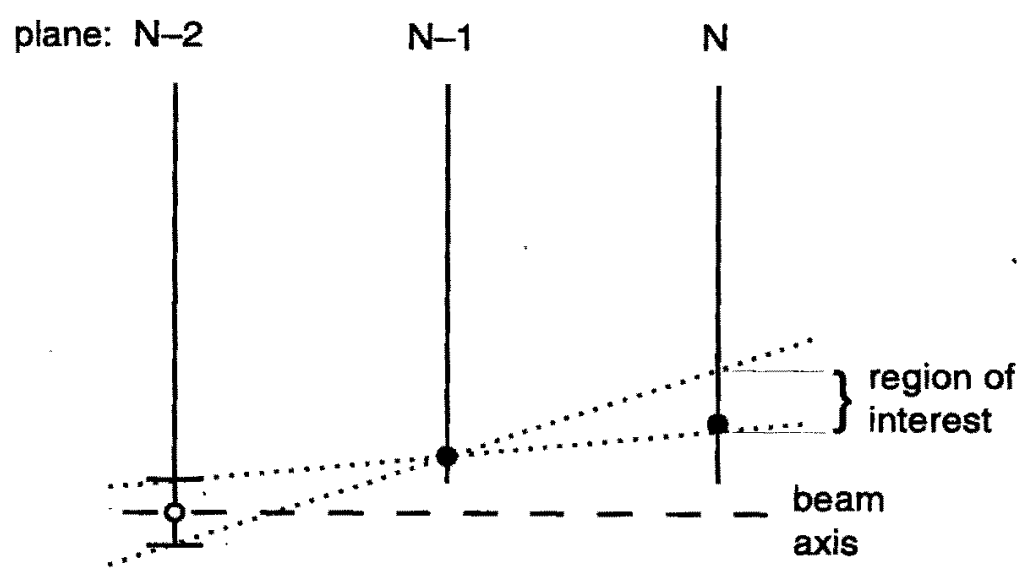

Figure 9.6: Use of a "virtual hit" in finding track segments. The open point with error bars is the virtual hit, while the full points are pixel clusters in planes $N$ and $N-1$.

c) The projection operator projects each inner pixel cluster from plane $N-1$ to plane $N$ by using a "virtual hit" that is defined to be on the beam axis at a location that corresponds to the position of plane $N-2$ (see Fig. 9.6). The goal is to find track segments that are in the beam hole at plane $N-2$. By projecting a straight line from this virtual hit through a given inner pixel cluster in plane $N-1$, we select a small region of interest in plane $N$. Valid track segments must have a confirming pixel cluster within this region of interest. The location of the region of interest is passed to the read port of the associative memory.

d) The associative memory tests whether there is a confirming pixel cluster in the region of interest in plane $N$. If a confirming cluster is found, its position together with enough additional information to specify the track segment is passed on to the next stage of the segment finding.

We have just described the first step for finding "interior" track segments, i.e. those at the track's point of entry into the vertex detector. As shown in Fig. 9.7, the segment-finding "kernel" just described is replicated to carry out the remaining steps of the algorithm. To find "exterior" segments (at the point of track exit), the same operations just described are carried out using outer pixel clusters in station $N-1$ and a virtual hit located beyond the outer edge of the pixel plane at station $N-2$. The hardware of Fig. 9.7 performs the complete segment-finding algorithm for both interior and exterior segments.

The time required to process the data from a station-quadrant is equal to the time required to fill the hit lists, at 1 cycle per pixel cluster, plus the time required to query them. Since there is double buffering in the associative memories, the filling time can overlap the processing time for the previous event. The average number of FPGA cycles per event can then be estimated as the average number of pixel clusters per station-quadrant, which in our 
BB33 Associative implementation for 1 station

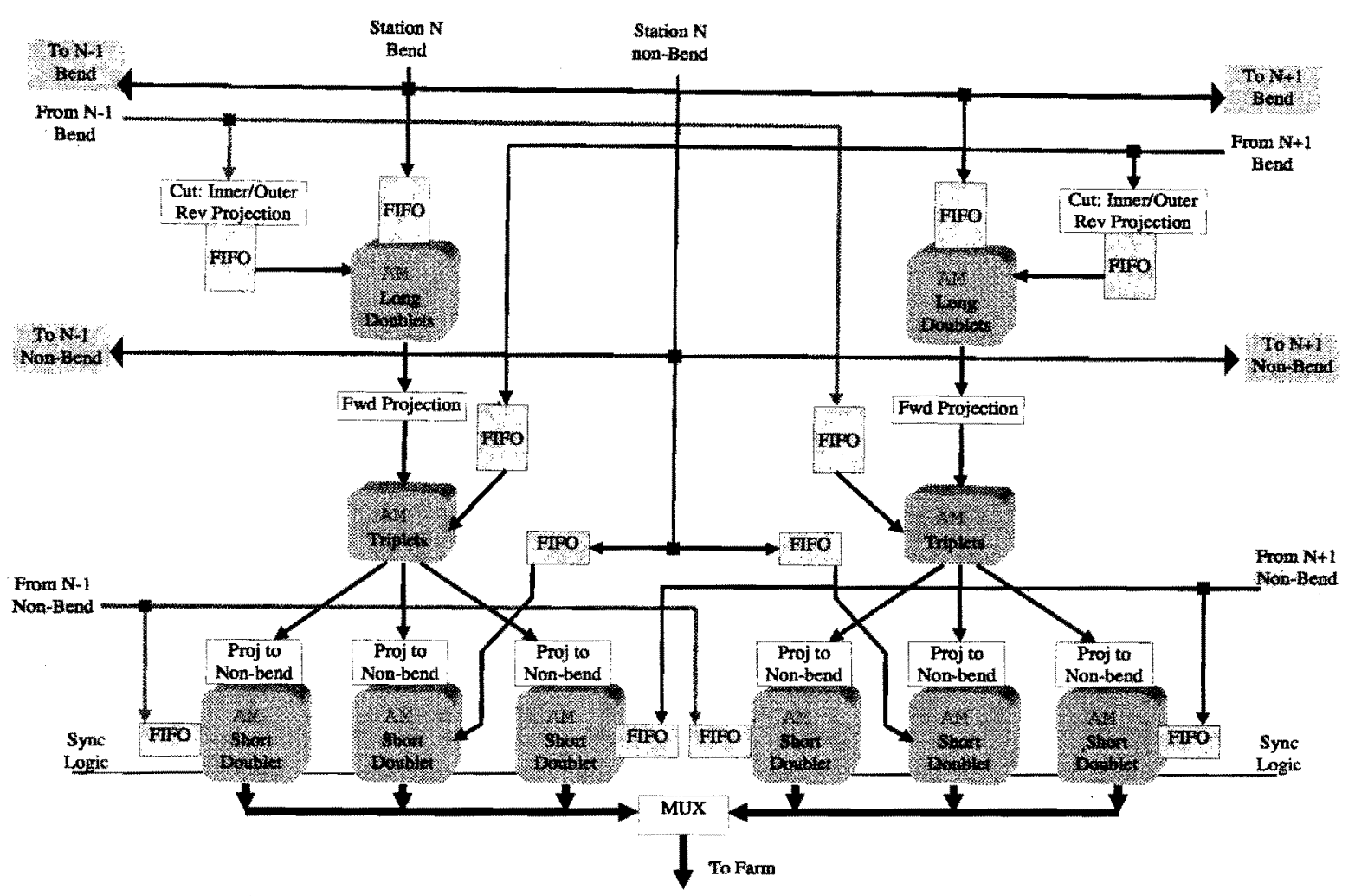

Figure 9.7: Schematic of a "unit cell" of the segment-finding hardware. Note that the drawing is left-right symmetric: the five associative memories in the left half of the drawing are configured to find interior track segments with polar angle $\theta<90^{\circ}$ (i.e. , segments directed at one arm of the BTeV spectrometer) and exterior segments with $\theta>90^{\circ}$, and those in the right half of the drawing find interior track segments with $\theta>90^{\circ}$ (directed at the other spectrometer arm) and exterior segments with $\theta<90^{\circ}$.

Geant simulation of minimum-bias interactions is 3.5 at an average of 2 interactions/crossing. The average time per event is then $70 \mathrm{~ns}$, well within the $132 \mathrm{~ns}$ available.

Note that the operators that perform these operations form a parallel-pipelined dataflow computational structure [5]. Once the pipelines are full, all operators are simultaneously busy, each working on a different piece of the calculation. To smooth out stochastic variations in the dataflow in each pipeline, the operators are provided with first-in/first-out (FIFO) input buffers (see Fig. 9.7). Each of the components in the structure is implemented using a small fraction of an FPGA, so that 16 FPGAs per station suffice to carry out the calculation at the crossing rate without falling behind. 


\subsubsection{Track and vertex farms}

The track segments for each quadrant are brought together into track farms, comprised of TI TMS320C67X floating-point DSPs ${ }^{1}$ in our design study. In the track farms, the segments within each quadrant are linked together into tracks, and all tracks from all quadrants are then brought together and sent to a vertex farm to be associated into primary vertices. To reduce the complexity of the switch needed for the Level 1 trigger, events are parceled out to eight sets of farms on a round-robin basis according to the beam-crossing time stamp (see Fig. 9.3). There are thus a total of 32 track farms ( 8 per quadrant) and 8 vertex farms.

The numbers of DSPs required in the track and vertex farms are estimated based on timing studies of the segment-linking and vertex-finding code. Timing analyses show that the optimizing C compiler for the TMS320C67X is not effective at generating efficient machine code for our trigger calculations. The reason is that the trigger code performs numerous sequential and scalar calculations (such as divisions and operations with transcendental functions). We find that for the complicated architecture of the DSP, with its eight pipelined processing units, expert-optimized assembly-language code is considerably more effective, and we have studied code optimizations with the goal of keeping all processing units busy for as large a fraction of the time as possible. This often requires interleaving calculations and rearranging the order of calculations compared to that in the $\mathrm{C}$ code. By carefully tailoring the calculations to the specific architecture of the TMS320C67X we have achieved speedup factors of 20 to 50 , which lead to a significant reduction in the total number of DSPs required for Level 1. The resulting timings, and the number of processors required to carry out the Level 1 trigger calculations in an average of $132 \mathrm{~ns} / \mathrm{crossing}$, are detailed in Appendix A and summarized in Table 9.1.

Table 9.1: DSP processing times and numbers of DSPs required for the track- and vertexprocessing farms. The numbers of DSPs are based on the time required to complete the track and vertex calculations in $132 \mathrm{~ns}$, on average.

\begin{tabular}{lcc}
\hline \hline Task & estimated time/crossing & \# DSPs \\
\hline Track processing & $55 \mu \mathrm{s} /$ quadrant & 1,668 \\
Vertex processing & $77 \mu \mathrm{s}$ & 585 \\
\hline Total DSP count & & 2,253 \\
\hline \hline
\end{tabular}

\subsubsection{Performance of the Level 1 algorithm}

The studies detailed in the trigger chapter of the simulation section (all carried out at a luminosity corresponding to an average of 2 interactions per crossing) show that the Level 1 algorithm described above finds about $95 \%$ of tracks with momenta above $5 \mathrm{GeV} / \mathrm{c}$ (Fig. 9.8). (Below this momentum the efficiency drops due to neglect of track curvature in the segment

\footnotetext{
${ }^{1}$ Rated at 1200 MFLOPS with $200 \mathrm{MHz}$ clock.
} 


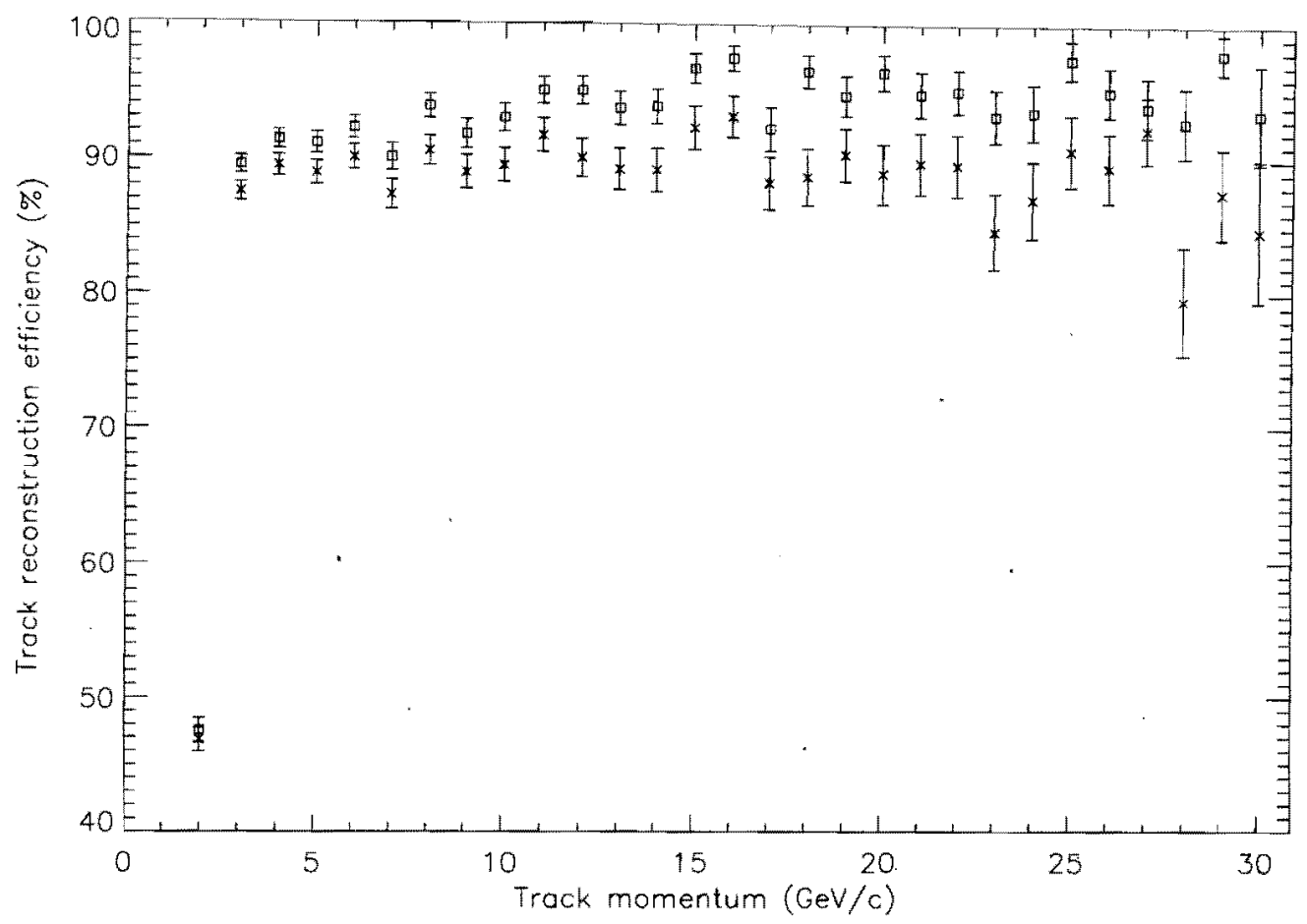

Figure 9.8: Track reconstruction efficiency vs. momentum for tracks that are contained within the fiducial volume of the vertex detector. Tracks are required to enter the detector from the beam region, traverse at least four tracking stations, and exit the pixel detector. Data points with squares show the tracking efficiency, which is about $95 \%$ above $5 \mathrm{GeV} / \mathrm{c}$, prior to clean-up cuts. Data points with asterisks show the efficiency after clean-up cuts.

finding.) After finding the tracks, we impose simple (and draconian) cuts to remove all tracks that share pixel clusters with any other track. (We may decide to revise these cuts in the future to implement an arbitration scheme that increases the Level 1 track-reconstruction efficiency.)

The Level 1 trigger decision is based on a requirement that at least $n$ tracks (all directed at one arm of the $\mathrm{BTeV}$ spectrometer) miss a primary vertex by at least $m \sigma$. This requirement imposes a minimum miss distance for the tracks. However, the miss distance is also required to be less than $2 \mathrm{~mm}$ to exclude tracks that may be associated with other primary vertices. Table 9.2 shows the Level 1 efficiency for various processes of interest requiring at least 2 tracks to miss a primary vertex by $6 \sigma$, and Fig. 9.9 shows how efficiencies vary with $n$ and $m$. In addition to detachment requirements, to improve the point-back resolution [7], displacedvertex track candidates are required to exceed a transverse-momentum threshold of $0.5 \mathrm{GeV}$. Efficiencies for typical beauty decays are $\gtrsim 50 \%$ while $99 \%$ of light-quark events are rejected. Given the order-of-magnitude-higher charm cross section and the larger branching ratios, BTeV's large samples of beauty decays will also be accompanied by comparable samples 

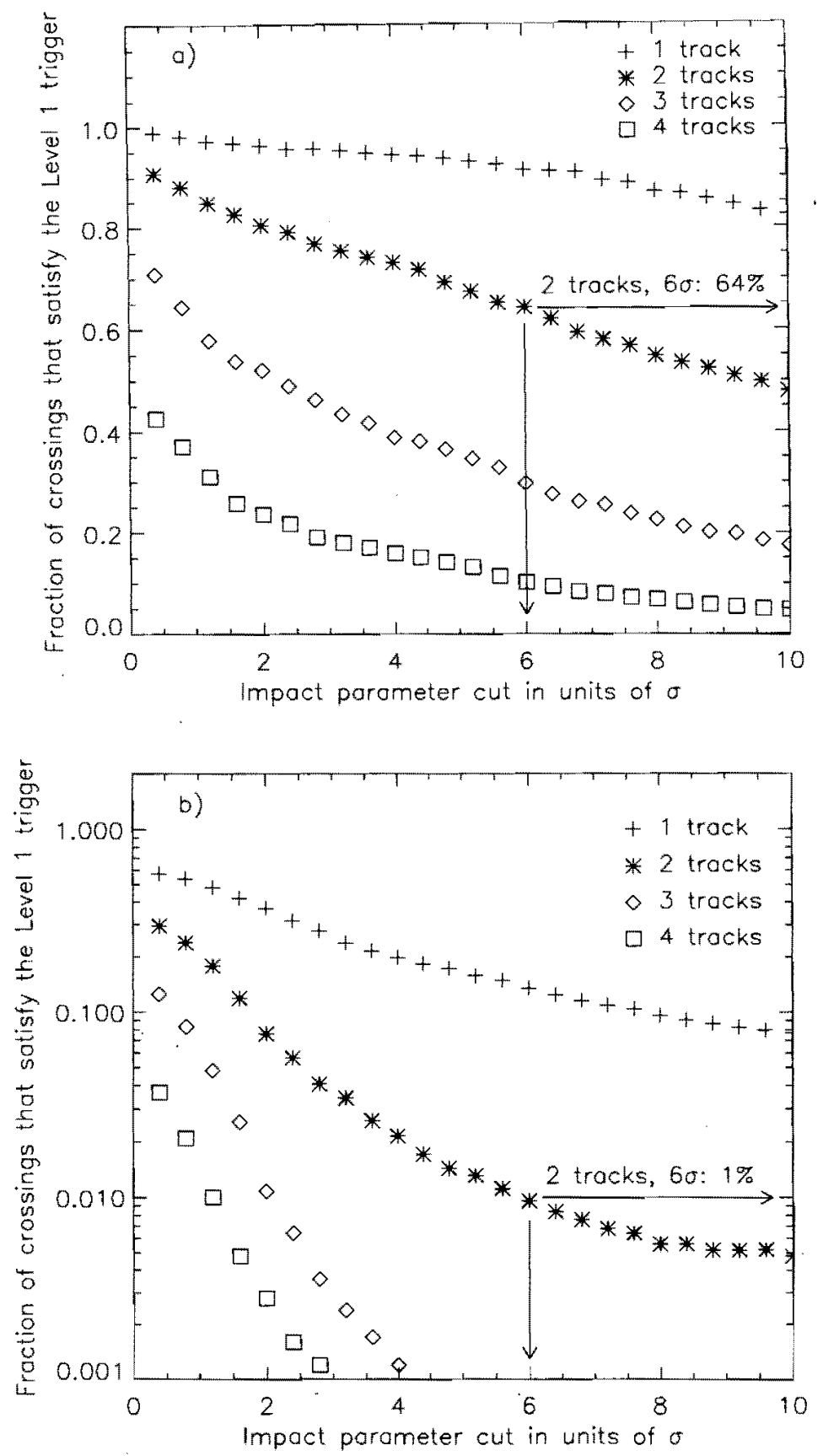

Figure 9.9: a) trigger efficiencies for $B^{o} \rightarrow D^{*+} \rho^{-}, D^{*+} \rightarrow \pi^{+} D^{o}, D^{o} \rightarrow K^{-} \pi^{+}$for various detachment requirements for an average of two interactions/crossing, and b) the trigger response for minimum-bias crossings with the same detachment requirements. Trigger efficiencies are plotted $v s$. normalized impact parameter $m$ for $n=1,2,3$, and 4 detached tracks: The arrows indicate the Level 1 cut requiring $n=2$ and $m>6$, which gives a minimum-bias rejection of $99 \%$. 
Table 9.2: Level 1 trigger efficiencies for minimum-bias events and various processes of interest that are required to pass off-line analysis cuts. All trigger efficiencies are determined for the Level 1 vertex trigger for beam crossings with an average of two interactions per crossing using the Monte Carlo code shown in the table.

\begin{tabular}{lcc}
\hline \hline Process & Eff. (\%) & Monte Carlo \\
\hline \hline Minimum bias & 1 & BTeVGeant \\
\hline$B_{s} \rightarrow D_{s}^{+} K^{-}$ & 74 & BTeVGeant \\
$B^{0} \rightarrow D^{*+} \rho^{-}$ & 64 & BTeVGeant \\
$B^{0} \rightarrow \rho^{0} \pi^{0}$ & 56 & BTeVGeant \\
$B^{0} \rightarrow J / \psi K_{s}$ & 50 & BTeVGeant \\
$B_{s} \rightarrow J / \psi K^{* 0}$ & 68 & MCFast \\
$B^{-} \rightarrow D^{0} K^{-}$ & 70 & MCFast \\
$B^{-} \rightarrow K_{s} \pi^{-}$ & 27 & MCFast \\
$B^{0} \rightarrow 2-$ body modes & 63 & MCFast \\
$\left(\pi^{+} \pi^{-}, K^{+} \pi^{-}, K^{+} K^{-}\right)$ & & \\
\hline \hline
\end{tabular}

of decays from directly-produced charm, giving access to possible new physics via charm mixing, $C P$ violation, and rare decays [8].

\subsubsection{Pixel processors}

The pixel processors are the interface between the pixel-detector readout electronics, which provides lists of pixel hits with pulse heights and time stamps, and the Level 1 vertex-trigger hardware. Tracks that pass through the pixel detector typically produce a measurable pulse height distributed over a few adjacent pixels. The measurements in these pixels are combined into pixel clusters that provide the position measurement. These pixel clusters are used for pattern recognition by the Level 1 vertex trigger. The pixel processors also gather all of the data for a given beam crossing for input to the vertex trigger, since the pixel hits do not necessarily emerge from the readout electronics in time order. The pixel processors are designed to be part of the station-quadrant processor boards, each with a total of six large FPGAs. Two of the FPGAs are the pixel processors (one for each pixel plane in a tracking station), while the remaining four FPGAs are used for the track-segment finding. The pixel processors are designed to process hits at a $50 \mathrm{MHz}$ maximum rate to keep up with the interaction rate of $15.2 \mathrm{MHz}$ (beam crossing rate of $7.6 \mathrm{MHz}$ ).

\subsubsection{Global Level 1 trigger}

The picture of the Level 1 trigger that we have presented focuses on the Level 1 vertex trigger, which is based on the pixel detector. The actual Level 1 trigger will be more complex, since it includes several different triggers. This complexity is managed by the Global Level 1 Trigger, GLV1. 
First, there will be several different vertex-related triggers. We have described a final selection that requires a minimum number of tracks to miss the primary vertex by a given number of standard deviations.. In fact, there will be a variety of such triggers, some accepting events with a few tracks with large detachments and others accepting events with more tracks that have smaller detachments. We also want to record a sample of events that would otherwise have failed the trigger requirements in various ways in order to understand how the efficiency of the trigger "turns on." This last group of triggers may be prescaled.

Second, there will be triggers that involve other detectors. We have previously mentioned the dimuon trigger. In addition to improving the overall efficiency for triggering on states containing $J / \psi$ 's, this trigger will permit an independent cross-check on the efficiency of the vertex triggers.

Third, there will probably be triggers that combine information from more than one detector at GLV1. For example, a single-high- $p_{t}$-muon trigger would be interesting but may have too high a rate. However, the global Level 1 trigger could accept a high- $p_{t}$ singlemuon trigger if the event also satisfies a relaxed vertex requirement - perhaps one track with a large impact parameter. (At Level 1, one would not know that the high- $p_{t}$ muon corresponded to the single high-impact-parameter track).

Fourth, there will be special triggers. One example will be a variety of minimum- and lowbias triggers that will be heavily prescaled. Another example will be special alignment and calibration triggers. We will certainly collect special triggers or use minimum-bias triggers to do the quasi-real-time alignment of the pixel detector.

The main physics trigger will not be prescaled. Global Level 1 must have the ability to prescale other less important physics triggers and calibration triggers. We also need the ability at Level 1 to adjust the prescale factors dynamically. For example, we want to be able to reduce the prescale factors on some of the triggers as the luminosity falls. We also want the ability to increase the number of alignment triggers taken at the beginning of a store and then reduce them once enough events have been collected to establish initial alignment constants for the store.

The operation of the Global Level 1 trigger is as follows. The GLV1 receives "trigger packets" from each trigger processor. Each packet contains a header which has "trigger primitives" with a format known to the GLV1. These packets arrive asynchronously. The GLV1 buffers these packets until it receives all the packets it is supposed to receive for a crossing, or until a timeout occurs for that crossing. In normal operation, as soon as all packets are received the GLV1 inspects the headers and generates all the various triggers from truth tables that have been downloaded. It then applies the appropriate prescale to each trigger and takes the OR of the result. If any of the triggers is satisfied, it issues a Level 1 accept. This results in the event data being transferred to the so-called "switch buffer" for transfer to the Level 2 trigger. If no trigger is satisfied, the GLV1 issues a Level 1 reject. This results in the Level 1 event buffers being freed to be used for other events. In general, the trigger processors, which are exchanging messages with GLV1, will have timeouts that are less than the GLV1 timeout. If the allowed time for the arrival of a Level 1 trigger primitive expires before a trigger packet from a processor arrives, an error flag will be generated. A 
prescaled sample of these events can then be recorded for further analysis and diagnostics. The rest of the GLV1 decision proceeds as normal.

The fact that the GLV1 receives data packets from the trigger processors allows the processors to send additional data with the trigger primitive information. This could include diagnostic information. It could even include "event information." It is quite likely that we will want to write some of the results of the Level 1 trigger calculations out as part of the event going to the Level 2 trigger. The data may be used for diagnostic purposes or may even be used to "prompt" the Level 2 trigger, for example by directing it to process particular tracks. There are several ways within this architecture to write such data to Level 2 . Sending it as part of the trigger-processor packet to GLV1 is only one method.

Since it inspects every crossing, the Level 1 trigger system will be the primary luminosity monitor for the experiment. The GLV1 will keep luminosity statistics and send them to various data logging and monitoring systems.

To minimize the number of designs that must be developed and maintained, the Global Level 1 Trigger will be implemented as a DSP "farm box" identical to the ones used for track and vertex processing.

\subsection{Levels $2 / 3$}

The Level 2 algorithm refines the tracks found at Level 1 by adding pixel clusters from the planes located between the "inner" and "outer" segments of each track, and (optionally) by adding hits from the first three stations of the forward tracking system (straw tubes and silicon strips). It then performs a Kalman-filter track fit. This improves the momentum resolution from $\approx 6 \%$ to $\approx 3 \%$, and the vertex-fit Gaussian core to $\sigma \approx 185 \mu \mathrm{m}$. With a requirement on the presence of a secondary vertex or detached tracks having a minimum detached $P_{t}$, the result is a joint light-quark rejection of $\approx 500$-to- 1 per crossing for Level 1 and Level 2 combined, with $\approx 50 \%$ overall efficiency for most $B$ decays of interest. The execution time extrapolates to about 15 milliseconds per event on the processor described below. This performance is sufficient for BTeV operation at design luminosity. The Level 2 trigger continues to be studied and refined, both to improve its efficiency and rejection and to decrease its execution time.

The Level $2 / 3$ trigger is implemented as a farm of commercial processors. These could, for example, be INTEL or ALPHA processors running the LINUX operating system. We expect that by the time BTeV runs, processor clock speeds will be at least $2.5 \mathrm{GHz}$. (This is a very conservative assumption, since $800 \mathrm{MHz}$ processors are already available in upper-end personal computers.) Given an average decision time of $15 \mathrm{~ms}$ per Level 2 node, we will need about 2000 of these CPUs.

Assuming existing magnetic-tape technology, the $\approx 10-\mathrm{kHz}$ output rate of Level 2 is about an order of magnitude too high (for media costs) for recording 200-kByte events. Level 3 thus needs to provide a factor $\approx 10$ in bandwidth reduction. This can be achieved in various ways, for example by imposing tighter vertex cuts, use of particle-ID information, event topology selection, or event compression (summarizing the raw data). The scenario of 
Fig. 9.1 assumes factors of about 4 from the trigger selection and a factor of 4 from event compression (200-300 kbytes/event to 50-75 kbytes/event).

When the Level 2 trigger is satisfied, a Level 2 accept is issued and all remaining data are transferred to the CPU. The buffer memories are freed at this time and the data for that crossing reside only in that CPU's memory. The Level 3 trigger has access to the results of the Level 2 calculations and has an average of about $100-200 \mathrm{~ms} /$ event to make its decision. If the Level 3 trigger decides that the crossing should be recorded, it compresses and reformats the event and sends it to a small "data acquisition" farm for eventual archiving to a permanent medium for offline analysis. The output rate to tape is 4000 events $/ \mathrm{s}$ of an average size of $50 \mathrm{kbytes}$, which gives a total output rate of $200 \mathrm{Mbytes} / \mathrm{s}$. This can be handled by a farm of about $20-40$ processors, with staging disks and two high-performance tape drives attached to each processor. 


\section{Bibliography}

[1] For example, HERA-B and LHC- $b$; see talks by E. Gerndt and O. Schneider, Beauty '99 Conference, Bled, Slovenia.

[2] S. Cittolin et al, in Workshop on Recent Developments in High Energy Physics, NCSR Demokritos, 9-11 April, 1998, p. 87.

[3] Associative memories (data-storage structures designed for rapid parallel search operations) are finding increasing use in industry; see for example the "content-addressable" memories made by MOSAID Technologies, http://www.mosaid.com/networking/index.html.

[4] See also D. Husby, "Systolic Associative Arrays for Track Finding" http://wwwese.fnal.gov/eseproj/trigger/asmem/asmem.pdf, and "Associative Memory for Track Finding," http://www-ese.fnal.gov/eseproj/trigger/asmem/.

[5] See for example M. H. Schub et al., Nucl. Instr. Meth. A376, 49 (1996); W. Sippach, G. Benenson, and B. Knapp, IEEE Trans. Nucl. Sci. NS-27, 578 (1980); R. G. Cooper, IEEE Trans. Comp. C-26, 1123 (1977).

[6] D. Husby et al., Nucl. Instrum. Meth. A383 (1996) 193.

[7] W. Selove, in Proceedings of the Workshop on $B$ Physics at Hadron Accelerators, P. McBride and C. S. Mishra, eds., Fermilab-CONF-93/267 (1993), p. 617.

[8] D. M. Kaplan and V. Papavassiliou, in CP Violation, X.-H. Guo, M. Sevior, and A. W.Thomas, eds. (World Scientific, Singapore, 2000), p. 116; D. M. Kaplan, in Proc. Symposium on Flavor Changing Neutral Currents: Present and Future Studies, Santa Monica, CA, 19-21 Feb 1997, D. B. Cline, ed. (World Scientific, Singapore, 1997), p. 81. 


\section{Chapter 10}

\section{Data Acquisition System}

In $\mathrm{BTeV}$, the Data Acquisition System and the trigger are intimately connected. In the preceding chapter, we focused on trigger requirements and algorithms. In this chapter, we describe the BTeV Data Acquisition System - the DAQ, concentrating on the data movement from the detector, through the various stages of the trigger, and finally onto mass storage.

\subsection{Data Acquisition}

The BTeV system will digitize, sparsify, and transmit data at the beam crossing rate of 7.6 $\mathrm{MHz}$ into off-detector buffer memories. This approach is taken because a large subset of the data is used in the first level trigger, the data must be digitized in any case, and a very sophisticated first level trigger is planned. With this approach applied to all subdetectors, the first level buffers can hold many more beam crossings of data than those found in typical front-end integrated circuits designed to have (typically small) on-chip buffering. This means that the decision time of the Level 1 trigger can be extended by as much as two orders of magnitude, if desired, allowing for much more sophisticated trigger processing than would be possible given a short (typically a few $\mu \mathrm{s}$ ) Level 1 latency.

Three distinct logical trigger levels are described in section 9.2. From an engineering perspective, there is little difference between Level 2 and Level 3; these levels are both executed in general purpose processors. The only distinction between these levels is that the start of the Level 3 processing presupposes that ALL data have been transferred to the processor, whereas the Level 2 processing operates only on a subset of the total data. Thus, triggers in the BTeV system are separated into two physical levels, referred as L1 and L2/3. This is typical of most proposed large scale data acquisition systems and acknowledges the greatly increased performance and decreased cost of general purpose processors. In effect, much of the early and intermediate processing in previous trigger architectures has been moved into the first level hardware, Level 1 in our case, while the remaining processing occurs in a greatly expanded array of general purpose processors.

Following the Level 1 trigger, the remainder of the system is sized for a minimum L1 rejection of $75: 1$, which represents an $\mathrm{L} 1$ accept rate of $\approx 100 \mathrm{kHz}$. This rate must be 
directed to the $\mathrm{L} 2 / 3$ processors though a switch. Moreover, the L2/L3 processors must be able to make decisions at this rate or data will be lost. Studies based on a preliminary Level 2 trigger algorithm and an estimate of the Level 3 processing time based on experience with our own offline analysis and from other experiments indicate that this can be achieved with $\approx 2500$ general purpose processors of about 2500 MIPS each in the L2/3 processor array. The use of general purpose processors does not preclude the use of dedicated L 2 trigger logic in the form of attached coprocessors.

The specifications shown in Table 10.1 are used as the baseline for the BTeV data acquisition. These numbers represent both arms of the detector.

\begin{tabular}{|l|l|}
\hline \hline event size & 100 kBytes \\
number of detector data links & 5000 \\
number of L1 data buffers & 400 \\
number of L2/3 data links & 64 \\
number of L2/3 processors & 2500 \\
\hline
\end{tabular}

Table 10.1: Estimates of Hardware for BTeV Trigger and Data Acquisition System

The total system buffer memory, assuming $400 \mathrm{L1} /$ switch input buffers and 64 switch output buffers, is almost 100 Gigabytes. Buffers in the $\mathrm{L} 1 / 2 / 3$ processors will push total system memory to approximately 400 Gigabytes. A block diagram of the trigger and data acquisition system is shown in Fig. 9.1.

\subsubsection{Detector Multiplexing}

To efficiently balance data rates on the detector data links, a relatively large number of detector channels will be multiplexed into each high speed link. In the case of the RICH subsystem, for example, it is expected that approximately 1200 single bit signals will be multiplexed and encoded into one link. The input to the modules driving these links will depend in detail on the detector subsystem, but the use of programmable logic will allow for some variation in signal widths and sparsification algorithms while using common hardware. The detector multiplexer drives the detector data link and receives the coded clock signal for fanout to the front-end components.

\subsubsection{Detector Data Links.}

Each detector data link must transfer approximately $100 \mathrm{MBytes} / \mathrm{sec}$. This rate can be accommodated by a single 1-2 Gbps fiber. The choice of technology and link speed depends on the physical placement of the first level buffers. The total bandwidth of the detector data links is about 500 GBytes/sec. 


\subsubsection{L1 and Switch Input Buffers}

Each Level 1 Buffer must accept data from a subsection of the detector at a $1200 \mathrm{MByte} / \mathrm{sec}$ average rate and the data must be held in this buffer pending a Level 1 trigger decision. The BTeV L1 trigger is based in large part on track reconstruction in the vertex detector and decision times may vary over a wide range depending on event complexity. For this reason, it is desirable that the $\mathrm{L} 1$ buffer controller support receipt of both asynchronous and out-of-order L1 accepts and rejects. Following each L1 accept, the data are transferred to a switch input buffer. The function of the switch input buffer is to hold data until an L2/3 processor assignment is made, the data have been requested by the processor, and the switch rotation allows transmission of the data. Data requests to the switch input buffer also arrive asynchronously and in arbiträry order.

As a practical matter, the L1 buffer and switch input buffer may use the same physical memory, with the data "transferred" between buffers by reassignment of pointers. The size of an individual data buffer is expected to be approximately 128 MBytes.

Data from some detector data links will also be used in the L1 trigger. In this case, the $\mathrm{L} 1$ buffers receive data from the L1 trigger instead of the detector.

\subsubsection{Ring Buffer Interconnects}

Our design approach is sufficiently flexible to accommodate different options for data transmission to the $\mathrm{L} 2 / 3$ processors. If all data is transmitted to the $\mathrm{L} 2 / 3$ processors following an L1 accept, the most natural arrangement of L1 buffers is to place an equal number on each switch input link. If the data is transmitted in steps (staged readout), placement of the L1 buffers should be optimized for the frequency of readout. Using a ring architecture achieves the same multiplexing functionality as a fixed backplane bus, while also allowing reconfiguration of the L1 buffers to match changes in trigger rate, switch size, and access frequency of the buffers.

The ring and switch interconnects utilize 1 Gbps serial links based on the same physical layer standard as Gigabit Ethernet, Fiber Channel, and "Infiniband". All data are transferred in fixed length packets, similar in size to an ATM cell.

\subsubsection{Staged Readout}

To reduce the size of the data switch, the Level 2 trigger may operate in stages. In each stage, it would then request only enough information from the switch input buffers to make a decision to continue to the next stage of processing. Events may be then rejected at any stage in the Level 2 analysis, with the remaining data cleared from the input buffers without being transmitted through the data switch.

The reduction in data switch bandwidth requirement is offset by the increase in bandwidth required for the data request messages flowing in the reverse direction. In addition, the input buffer depth must be increased significantly to provide greater latency for event data used in later stages of the L2 algorithm. Buffer management logic also becomes more 
complicated. The cost reduction comes from the potential decrease in the number of required output buffers. An analysis of the minimum number of required output buffers and the expected fraction of data needed in the average L 2 calculation will be performed to determine if the staged readout approach is cost-effective.

\subsubsection{Data Switch}

A simple, unidirectional, packet synchronous, TDM switch has been shown to be the most efficient for event building applications. The switch configuration follows a standard "barrel shift" rotation and can be expanded to any size in $N \log N$, where $\mathrm{N}$ is approximately the total data rate required by the Level $2 / 3$ trigger divided by $100 \mathrm{Mbytes} / \mathrm{sec}$ (switch maximum throughput) times a safety factor. We expect to eliminate $80-90 \%$ of the events at level 2 based on inspecting a very small amount of the data from each event. Under rather conservative assumptions, the $\mathrm{BTeV}$ data switch is expected to require a maximum of 64 channels, which can be implemented using two stages of $16 \times 16$ switch modules.

The advantage of this switch is that it requires no complex internal control. Source and destination addresses contained in the data packets are used only to specify buffers on a common ring and are not needed by the switch for data routing. The switch is unidirectional, but packets are recirculated by connecting the last output buffer to the first input buffer in each ring. This provides a mechanism for input-to-input message transfers (throttle requests to the global trigger), output-to-output message transfers (L3 accepted events to logging processors) and output-to-input messages (event requests to the global trigger/event supèrvisor).

Because the switch is easily expanded, full implementation can be delayed until the accept rates of the L1 and L2/3 triggers are determined, and the switch sized accordingly. The cost of the switch is not a large factor in the overall system cost.

\subsubsection{Control Network}

A broadcast network is needed to distribute the accept/reject messages to the switch input buffers. This network is implemented as a second ring which follows the same physical path as the ring buffer interconnects.

\subsubsection{Switch Output Buffers}

The switch output buffers are similar in function to the switch input buffers. This is the point where all the requested data from a single event is received from the switch and forwarded to a processor. Connection to processors is by way of Fast Ethernet links, with 64 links per switch output buffer. Up to 4096 processors can be connected to 64 switch output buffers, and additional buffers can be added to each ring if the processor array is expanded. 


\subsubsection{Data Logging}

Events accepted by the Level 3 trigger are sent to data logging processors. These processors are attached to switch output buffers in the same way as any other L2/3 processor. Accepted events are placed back on the ring and recirculate through the data switch to the appropriate output port. Given the high trigger rejection, this utilizes approximately $1 \%$ of the data switch bandwidth while avoiding the need for a separate data logging network.

\subsubsection{Detector Control Links}

All detector subsystems transmit data at the beam crossing rate. This simplifies the delivery of clock and control signals to the detector front-end components because L1 and L2 accept/reject signals are not required at this level. It is assumed that a $53 \mathrm{MHz}$ coded clock will be sufficient to provide crossing and synchronization information. Clock and data recovery should be provided by the detector multiplexing module and then delivered independently to the front-end.

\subsubsection{Diagnostics}

All system buffers have a control link for the purpose of sending or receiving data request messages. This provides a convenient path for system diagnostics at little additional cost. We expect to make widespread use of embedded processing and embedded servers in these modules to allow remote test and status monitoring. An additional low speed interface (USB) is available for stand-alone diagnostics.

\subsubsection{General Operation}

At startup, buffers can be initialized through the control link. The data switch is initialized by filling the rings with empty data packets, after which it operates in a packet synchronous mode. The front-end systems are synchronized through the clock distribution system.

Event fragments are timestamped with the crossing number which is used for all Level 1 trigger accept/reject messages. Events accepted at L1 are tracked through the remainder of the system by the L1 event number. An L1 accept causes the buffer control logic to move a memory pointer from the crossing number list to the L1 event number list. At the same time, the L1 event number is added to the list of events available for L2/3 processing.

An event request from an L2/3 processor is sent to the Event Supervisor (see below). The Event Supervisor returns a packet containing an L1 event number, along with any trigger information considered useful to the processor. At this point the processor is responsible for disposition of the event. It may request some or all of the data from switch input buffers for use in the L2 decision. Following an L2 accept or reject the processor makes a final data request to all remaining switch input buffers. Buffers then delete or transmit/delete the event data. Each switch input buffer receives exactly one data request message per event. 


\subsubsection{Component Placement}

The detector data links and L1 buffers represent a large part of the overall data acquisition system cost. Placement of the buffers influences the technology and cost of the links. The buffers will occupy the equivalent of $256 \mathrm{U}$ subracks. The $\mathrm{L} 1$ subsystem triggers should be located in the same area as the L1 buffers. These components would be placed outside of the collision hall to facilitate maintenance and to allow reconfiguration of rings based on changes in trigger rate and buffer access frequency. This requires the use of optical links. Placement of all other data acquisition components (switch, output buffers and processors) is not critical, since there are a relatively small number of interconnects.

\subsection{Event Supervisor and Monitor}

As our design has developed, the Global Level 1 Trigger system began to acquire many of the functions of an 'event supervisor and monitoring system'. In this section, we will describe this functionality. We will refer to the hardware that does these tasks as the Event Supervisor and Monitor, ESAM. Whether, in the end, it is provided by the same hardware responsible for the Global Level 1 Trigger or it assumes a separate identity is an implementation detail.

The ESAM will maintain a list of events which have satisfied the Level 1 trigger. When a $L 2 / 3$ processor becomes available to process a new event, it sends a request for an event to the ESAM. The ESAM then sends a data packet to the processor which contains the event number to analyze. It also includes the status of all the triggers for that event so that the processor can execute different code depending on how the event triggered. It may also send more extensive event data from the trigger processors. Once the ESAM has sent an event number and associated data to a Level $2 / 3$ processor, it is done with that event.

ESAM is the natural location for accumulating statistics on the performance of the Level 1 trigger. It will periodically collect statistics from GLV1 and send this data up to online analysis computers.

One interesting application of this is in luminosity monitoring. Since the BTeV Level 1 trigger inspects every crossing, it will be possible to count the number of crossings with no interaction as well as the number of interactions in each crossing that has at least one. Given the large acceptance of the pixel detector for inelastic collisions, we believe that the Level 1 trigger system will function as a highly effective, real time luminosity monitor. We will set up a separate data path, using fast Ethernet, from ESAM to a small dedicated processor to accumulate statistics from Level 1 luminosity information, to archive the information, and to run various status displays for operations.

ESAM will also play a role in data throttling - that is trying to reduce the rate of data sent to the upper trigger levels if they begin to fall behind and the switch buffer memory approaches its capacity. The 'switch buffer memory' which holds the event data while waiting for a L2/3 processor to become free will be able to issue a 'full warning' to ESAM when it becomes $90 \%$ full. ESAM will send this to Global Level 1 which can attempt to undertake some action to alleviate the problem. For example, it could begin to adjust prescales to 
reduce the total trigger rate. 'It could also stop sending events. When the 'switch buffer memory' is completely full, it will issue a 'full' indication. ESAM will send this to the GLV1, which will stop issuing Level 1 accepts until this condition is cleared. This will result in deadtime and ESAM will have to keep track of how much deadtime there is for purposes of normalization and for diagnostic purposes. It is, of course, a BTeV design goal for this to almost never occur.

We are also discussing 'partitioning.' Partitioning refers to the ability to divide the Trigger/DA system into more than one semi-independent piece, each of which approximates a completely independent trigger/DA system. This capability is especially useful in the debugging stage of an experiment. In this stage, some subsystems may not be installed, some may not yet be operational, some may be down for maintenance, and some may be hanging or crashing. It is important to be able to work on one or a collection of subsystems independently of the state of the others. The BTeV architecture permits a reasonable level of partitioning. The ability of Level 2 processors to request subevents rather than whole events lends itself to partitioning. With the appropriate software, we can extend this capability to Level 3 so a process can proceed when it gets just the subset of data it needs. On the other hand, the requirement that an event be given to one and only one processor creates some difficulties for partitioning.

In our current plan, the ESAM will maintain separate trigger lists for different 'event' types. It will also maintain separate lists of event numbers for each triggering event type. It will have an algorithm for assigning events satisfying triggers in more than one list. The algorithm will probably be based on the desired event rates and priorities for each event type. Level 2 processors will request an event of a specific type. ESAM will return an event number from the list for that type. The Level 2 processor can then request the subevents that it wants to analyze. If the 'switch buffer' is configured so that it waits a certain minimum interval before it sends an event (so that it is sure that it has the whole event), and if it can send a message saying that it doesn't have a requested subevent, then the fact that some (presumably) unneeded subevents are missing does not cause a problem. With this scheme, subgroups can have their own triggers and select the subset of the data they want to read independently of each other. However, since each event will go to only one processor, ESAM will have to decide how events satisfying the requirements of multiple trigger lists should be apportioned.

\subsection{Level 2/3 Trigger}

As discussed above, the Level 2/3 Trigger is a processor farm. The baseline design of the farm calls for 2000-4000 general purpose processors, such as INTEL/Pentium PCs, running Linux. Each processor runs the same analysis code. Requests for new data are sent to the Global Level 1 Trigger, GLV1, which coordinates the initial data requests for each processor in the Level $2 / 3$ Trigger. The Level $2 / 3$ analysis software for BTeV performs data analysis that is usually part of the "offline analysis" in other experiments. Consequently, the Level $2 / 3$ Trigger can select events using cuts that are usually reserved for offline.analyses, and can 
transform event data into a condensed format (usually referred to as a DST format) before sending the data to event-logging processors.

An important aspect of the Level 2/3 Trigger architecture is that each processor is capable of making trigger decisions at both Level 2 and Level 3 . The distinction between Level 2 and Level 3 depends on the memory buffers in which the data for an event reside, and will be described below. A second aspect of the trigger architecture is that each event is assigned to a single processor in the $\mathrm{PC}$ farm, so that a single processor selects or rejects an event at Level 2 and, if it passes Level 2, at Level 3.

The Level 2 analysis for an event begins when an idle processor sends a request to the Event Supervisor for a new event. The ESAM, which maintains a list of events that have satisfied the Level 1 Trigger, responds by sending a data packet to the processor. The data packet contains the event number, and Level 1 trigger information. The processor begins the Level 2 analysis of the event based on the Level 1 trigger information, which summarizes all of the trigger conditions that were satisfied at Level 1. In addition to the trigger information, the processor usually requires data from various detectors to complete the analysis and arrive at a trigger decision. The data reside in switch buffers. The processor requests data from these switch buffers directly. For example, the processor may send a request for data recorded by the two forward tracking stations that are closest to the dipole magnet. The requested data are sent from the switch buffer to the processor's memory and the memory it occupied in the switch buffer is freed. At this point, part of the event is in the processor memory and the remainder is in the switch buffer. In this example, the added data blocks are used to improve the momentum determination of tracks found by the vertex detector, so that the processor can calculate more precise impact parameters to select tracks coming from $B$ decays. After the data are received and processed, the processor may request additional data (such as from the other forward trackers or from the muon detectors, in the case of a Level 1 muon trigger), or complete the Level 2 analysis by sending a trigger decision to the switch buffer.

A processor that has completed the Level 2 analysis for an event sends one of two trigger decisions to the switch buffer. If the event is rejected, a "L2 reject" is sent and any remaining data blocks for that event are deleted from the switch buffers, freeing that memory for new events. The processor is ready to process a new event. If the event is accepted, a "L2 accept" is sent, and the switch buffer sends the rest of the data data blocks for that event to the processor for subsequent Level 3 analysis, and removes that data from the switch buffers. This marks the beginning of the Level 3 trigger. At this stage, the data for an event reside entirely in the memory of one processor (the same processor that performed the Level 2 analysis for the event), and nowhere else.

A processor that performs the Level 3 analysis for an event can perform a complete analysis using all of the data that are associated with that event. The data analysis is comparable to "offline" analyses in other experiments, and includes track reconstruction, vertex reconstruction, muon identification, and (possibly) charged hadron identification. Events that are rejected by a processor at Level 3 are simply terminated by that processor. Events that are accepted are sent to data-logging processors. 


\subsection{Data Logging and Online Event Analysis}

Each event is handled by only one Level $2 / 3$ processor. (i.e., an event is not scattered across processors nor is it copied to more than one processor). All accepted events need to be moved out of the L2/3 processors and onto tape for long term data storage, and a fraction of the events need to be made available for online monitoring (by "consumer processes").

Events coming out of the Level 3 processors will not be raw data, but already processed data (except for a highly prescaled sample used for monitoring the data reduction algorithm itself). This should shrink the event size from $200 \mathrm{kBytes}$ to $50 \mathrm{kBytes}$. Assuming an event size of $50 \mathrm{kBytes}$, and a $4 \mathrm{kHz}$ event rate to the loggers, the data rate to tape is on the order of $200 \mathrm{MBytes} / \mathrm{sec}$. The consumer processes will also add an additional 5-10\% of throughput.

Since the data logging rate out of each Level 3 processor is small, it is less cost effective to attach logging media to these individual nodes than to provide a small number of separate logger nodes. The necessary bandwidth to the logger nodes is also small (2-3\%) compared to the raw data coming up the Level $2 / 3$ farms, so the same switch could be used to pass the event to the logger nodes as well. Alternatively, a dedicated network could be provided between the Level 3 processors and the logging nodes. In the current plan, a single event is routed to the next free logging node but buffering several events in the Level 3 processors and sending them out together is possible if it turns out to be beneficial.

The number of logging nodes themselves is clearly a function of the data rate. It is assumed that data will first be buffered to disk before being written to tape. This serves a dual purpose; buffering enough data before a transfer to keep the tape drives streaming, and protecting against tape media errors and tape drive failures. With current market technology, a single processor node can handle $10 \mathrm{Mbytes} / \mathrm{sec}$ to tape including the initial disk write. We will therefore require a minimum of 20 data logging nodes. We are planning for twice this number to have a safety factor and to be able to handle failures.

Consumer events should not interfere with data logging rates and additionally may contain samples of rejected or flawed events that we may want to look at but not to write out to permanent storage. The consumer processes will therefore run on separate nodes from the logger processes.

\subsection{Front End Electronics}

In this section, we discuss those aspects of our front end electronics which are common among systems. Detailed information on the readout of each detector can be found in the chapters describing the individual detectors. We conclude with a brief discussion of software support for electronics development.

\subsubsection{Infrastructure and Other Support Systems}

A goal of the $\mathrm{BTeV}$ experiment is to minimize hardware and software development while meeting all the requirements of the experiment. This goal can be accomplished by: 
- Choosing cost-effective, commercially-available hardware and software whenever possible;

- Using hardware and software developed elsewhere whenever possible; and

- When hardware and software need to be developed for BTeV, developing them in such a way that they can be used in the maximum number of BTeV front-end, trigger, and data acquisition systems.

Commonality in hardware and/or software when possible in BTeV's front-end, trigger, and data acquisition electronics will save not only substantial amounts of money but valuable personnel resources. A BTeV workshop was held to understand

- what already-developed electronics and software can be used in BTeV and

- where hardware and/or software; and commonality is possible in BTeV's front-end, trigger, and data acquisition systems.

The following subsections describe our choices for some infrastructure items and other support systems.

\subsubsection{Electronics Packaging - Subracks and Related Infrastructure}

Using a mechanical packaging and bus protocol standard that is commonly used internationally offers several advantages over the lifetime of an experiment. Cost and personnel time savings are the two most important advantages. Standards are well-documented and stable. Various electronics and mechanical components supporting the standard are commerciallyavailable from industry. For example, subracks, processor modules, (module size) adapters, extenders, subrack power supplies and interface ICs are commercially available items not requiring development. This leaves us free to concentrate only on the development of specific front-end, trigger, data acquisition, controls and monitoring electronics. By choosing a commonly-used packaging and bus protocol standard, implementers, as well as people who maintain the electronics over the lifetime of the experiment, have a mature development and testing hardware and software environment. If the standard has been used in previous experiments at Fermilab, we can realize considerable cost and personnel time savings because local expertise is already available and infrastructure is in place.

We have chosen the VME64 Extensions (VME64x) subrack mechanical packaging and bus protocol standard as the baseline implementation for the $\mathrm{BTeV}$ front-end, trigger and data acquisition electronics. This is an American National Standards Institute (ANSI) standard, ANSI/VITA 1.1-1997. This standard and its physics community VME64xP extension are being used for all of CDF's and a majority of D0's Run II electronics. Over 250 VME64xP subracks have been purchased by CDF and D0. Other standards used in conjunction with this standard are:

1. IEEE1101.10 - the subrack module mechanical standard for VME64x; 
2. IEEE1101.11 - the subrack rear I/O board (transition module) mechanical standard for VME64x;

3. ANSI/VITA 1.3-1997 - the VME64x 9U x 400mm module format standard;

4. ANSI/VITA 25 - VISION, a software VME API (subroutine calls) standard; and

5. ANSI/VITA 23 - VME64 Extensions for Physics (VME64xP), a VME64x-compatible standard which extends VME64x by, for example, specifying $7 \mathrm{U}$ and $10 \mathrm{U}$ subrack implementations and specifying mandatory control and status registers.

These standards offer many advantages for $\mathrm{BTeV}$, which will take data for long periods of time in a challenging environment. Attention to cooling, power, electronic noise rejection and ease of maintenance are all requirements which are addressed by the standards.

An alternative method of packaging $\mathrm{BTeV}$ electronics has been proposed. This method draws on products developed in the world-wide, very price-competitive, $\mathrm{PC}$ packaging industry. No communications busses would be used. Slow controls for component initialization, downloading and monitoring would be done by interconnecting Ethernet between PC packages. Electronics would thus not be modularized but, for example, each set of front-end electronics would be housed in its own PC package. Power to that package would be via a $110 \mathrm{VAC}$ line cord and power supplies within the package. This option potentially offers cost savings over bussed systems but would require the development of a new infrastructure for hardware testing and software development. This option would only be adopted if it resulted in substantial cost savings over the life of the experiment taking into account all development, support, and maintenance issues.

\subsubsection{Data and Clock/Timing Links and Link Interfaces}

Possible and probable areas of hardware commonality in data and control/timing links and other components include:

- Data links from front-end electronics to Level 1 buffers;

- Data links from the Level 1 buffers to the event building switch;

- Data links from the event building switch to event buffers preceding online processors;

- High-speed front-end data links from on-detector electronics, possibly rad-hard, to local electronics;

- Low-noise data links from on-detector electronics, possibly rad-hard, to local electronics;

- Data links to Level 1 trigger subsystems and from those subsystems to the Global Level 1 trigger; 
- Control/timing links to front-end electronics and trigger subsystems; and.

- Level 1 buffer electronics.

Two ongoing developments and one existing Hewlett Packard (HP) data link series of products should satisfy most if not all of the data and control/timing link applications given above. HP's G-links are transmitters and receivers which operate up to 1.5 Gigabits per second over fiber or copper. CERN is developing a G-link compatible radiation-hard driver for use in its LHC CMS (HCal and ECal) and ATLAS experiments. Fermilab is assisting that development, and we plan to use it for pixel data readout links, and possibly other subsystem data readout links. CDF SVX Run II electronics required the development of a radiationhard, low-noise transceiver. This transceiver will go into production soon. It is compatible with the IEEE-1596 LVDS (Low-Voltage Differential Signalling) standard developed for the Scalable Coherent Interface standard. This is a differential current standard intended to provide low-noise transmission of digital signals at very high speeds for a few tens of meters maximum.

We will adopt a minimum set of "BTeV data link" standards and, hopefully, a single "BTeV control/timing link" standard. The types of "BTeV links" will include:

- Serial optical G-link 1.3 Gigabit per second data link transmitting up to 20 bits every $1 / 53 \mathrm{MHz}$ (radiation-hard and non radiation-hard); and

- Parallel copper LVDS-like to LVDS 53MHz data link (radiation-hard and non radiationhard).

The parallel copper LVDS-like to LVDS data link would be used to move data a short distance from the detector to an area near the detector where electronics is more accessible. It provides for relatively low-power, low-noise data transmission off the detector. An alternative for this application is an array of Vertical Cavity Surface Emitting Lasers (VCSELs) and an optical ribbon cable link. VCSELs are inherently radiation hard. If this approach is cost effective and does not add more mass than desired, only minimal electronics need be on or near the detector. All the other electronics (e.g., data multiplexers, Level 1 buffers, trigger electronics, etc.) can be in the counting room and thus very accessible.

Our front-end and trigger subsystem implementers will easily be able to interface their electronics to $\mathrm{BTeV}$ standard readout and data acquisition electronics and control/timing signals. This will be accomplished by providing them with data and control/timing link daughterboards. Along with the daughterboards, the implementers will be provided with mechanical and electrical specifications for the daughterboards and component and artwork specifications for circuitry on their boards used to interface to the daughterboards. The following daughterboards are under consideration:

- Serial optical G-link or G-link compatible data link transmitter daughter-board;

- Parallel copper LVDS-like data link transmitter daughter-board; and

- Serial optical G-link data receiver control/timing link daughter-board 


\subsubsection{Slow Controls and Monitoring}

In an experiment the size of $\mathrm{BTeV}$, several hundred devices need to be controlled (e.g., high-voltage systems, laser pulsers, interlock systems, etc.). Several thousand 'points' (e.g., power supply voltages, temperatures, gas mixes, interlocks) need to be monitored at regular intervals. Alarm or caution situations need to be established via hard-wiring or in software for equipment and/or personnel safety. Past experiments at Fermilab have chosen to use commercial or in-house-designed hardware and in-house- developed software for slow controls and monitoring systems despite the fact that total process automation solutions (hardware, software and user interfaces) are provided by a multitude of companies worldwide. The beamline cryogenics controls and monitoring system was the first system at Fermilab to use an industry total process automation system. Their hardware controllers and input devices were purchased from Moore Products and compatible software and graphical user interfaces from Intellution. The success of this system was remarkable. Mechanical engineers bought it and implemented it with no Fermilab professional software or electronics engineering help. Significantly fewer people were required to operate the system. As a result CDF will use an identical system for its Run II slow controls and monitoring system. BTeV's baseline slow controls and monitoring system is based on this system.

\subsubsection{Clock and Timing Distribution}

The $\mathrm{BTeV}$ experiment is different from all other current and past Fermilab experiments in that a) tracking (pixels) will be used in the lowest level trigger and b) all front-end subsystems will be read out into Level 1 buffers at the 132 nanosecond bunch crossing rate. Most frontend subsystems will require only a bunch crossing clock and a $53 \mathrm{MHz}$ accelerator clock. Some will operate asynchronously not needing a bunch crossing clock. For Run II, CDF has used a modular subsystem for distributing clock and timing information to its front-end and trigger subsystems. Differential ECL signals over high-quality cables are used for longdistance runs from the clock/timing sources to the collision hall. Low-current differential signals (LVDS drivers and receivers) are used to transport clock and timing signals to ondetector front-end electronics and counting room electronics. We will try to use as much of the work done by CDF as practical for its clock and timing distribution subsystem.

An alternative approach to sending clock and timing information to front-end and trigger subsystems is used in the Run II CDF SVX system. A conditioned accelerator clock (with jitter essentially removed) is used to clock timing data (e.g., bunch crossing) into HP Glinks via optical cables. Optical splitters can be used to distribute the clock and timing information. At the output of the G-link receiver, the clock is rederived from the output data strobes of the G-link receiver. The bunch crossing timing signal is received as a G-link receiver data bit. 


\subsubsection{Electronics Cooling and Rack Protection}

Several systems for cooling and protecting electronics racks exist at Fermilab. Our cooling system will be designed so that the exhaust air from a VME subrack will not exceed 95 degrees Fahrenheit. The fans will minimally provide 400 linear feet per minute of air flow. The air/water heat exchangers will reduce the temperature of 95 degrees Fahrenheit input air to 85 degrees Fahrenheit at the above air flow. There is no reason we can't use either CDF's or D0's Run II electronics cooling and rack protection system. This system will be connected to the slow controls and monitoring system described above.

\subsubsection{Software for Module and Subsystem Development and the Online Data Acquisition and Readout System}

$\mathrm{BTeV}$ front-end and trigger subsystem developers can use existing software for developing modules and subsystems. An infrastructure for testing VME-based boards has been developed for CDF, and is the foundation for testing CDF's Run II SVX, Muon and Calorimetry system hardware. This framework, called CDFVME, is Java-based code that communicates to the test subrack(s) via CORBA, a powerful object request broker, which here is used to provide the functionality of a remote procedure-like software tool. The CORBA implementation is ROBIN, and requires a VME processor in the subrack running the VxWorks operating system. VxWorks is the real-time operating system that will be supported at Fermilab throughout Run II for both CDF and D0.

The CDFVME software consists of a series of Java classes to interface to generic VME boards (e.g., read and write register commands), plus templates that users can customize for their specific board. CDFVME also contains a framework in which to run a series of tests in batch mode, and to cycle the readout code. Error logging is also provided.

CDFVME uses FISION, the Fermilab supported implementation of the ANSI standard software (ANSI/VITA 25) called VISION to communicate across the VME backplane. FISION is currently supported on Motorola MV16x and PowerPC microprocessor VME CPU modules. CDFVME was jointly developed by Yale, the Online and Database Support Department of the Computing Division, and CDF.

The choice of downstream $\mathrm{L} 2 / 3$ processors in $\mathrm{BTeV}$ will be driven by price/performance issues. Current market trends indicate that the processors will be some type of $\mathrm{PC}$ processor running Linux. Linux PCs will be used for both CDF and D0 Level 3 online processor farms.

Licensing costs and various support issues are generating interest by software implementers in evaluating real time operating system alternatives to VxWorks. Real-time Linux is a possible contender for post Collider Run II data acquisition systems.

Development code for data acquisition should move in the direction of modern programming standards. Run II software has already been designed for object oriented languages such as $\mathrm{C}++$ and Java. CORBA is the standard protocol for communicating between objects on remote nodes. 


\section{Chapter 11}

\section{Plan for Deployment in C0}

\subsection{Detector Installation}

The proposed $\mathrm{BTeV}$ detector is not a monolithic detector like CDF or D0; it is similar in layout and construction to a traditional fixed-target detector. Most of the detector elements are individually mounted and can be separately removed from the detector for repairs. This attribute makes the initial construction and testing of the various components of the $\mathrm{BTeV}$ spectrometer much less dependent on the schedule of Tevatron shutdowns and maintenance periods.

The five large physical components, the vertex magnet and the four muon toroids, will be deployed first. This is important for two reasons. First, they fill most of the assembly hall during their assembly; their installation in the collision hall frees up needed space for the assembly and testing of the remaining detector elements. Second, they will form the backbone of an overhead rail and suspension system that allows the other detector elements to be inserted and removed without an overhead crane. Once installed, they are essentially passive, need never be moved, and do not affect Tevatron operations.

The beampipe in the region of the toroids and EM calorimeter does not need to be removed in order to insert or remove any detector elements. Also, both the straw tube and silicon tracking chambers clam-shell around the beampipe. The vacuum vessel containing the pixel detector can be extracted from the vertex magnet in either the upstream or downstream direction if the tracking chambers between it and the RICH tank are first rolled sideways. The crystals and photomultiplier tubes of the electromagnetic calorimeter can be serviced in place or by moving submodules from the support structure. The RICH sensors can also be serviced in place without removing the counters. This flexibility allows the RICH counters, EM calorimeter, tracking chambers, and pixel detector to be installed, tested, and repaired if necessary, in any arbitrary order in relatively brief periods of access. 


\section{$11.2 \quad$ Staging}

Although the proposed detector is modular, installing it in the Tevatron will of course be a complicated and time consuming process. We realize test beam studies can go a long way in checking out modular components before their installation. We do envisage an extensive test beam program for the individual detector components. The trigger, on the other hand, is difficult to test in external beams. For Level 1 hardware trigger tests, it would be most helpful to install a substantial segment of the pixel detector inside the vertex magnet, in the Tevatron. Subsequently, for Level 2 hardware tests, it would also be necessary to install a portion of the forward tracking system.

Subsequently, the remaining components in one arm would be installed and detector operation could begin using relatively short periods at the end of stores or whenever luminosity became available. Installation of the second arm could occur as time and money permitted. The physics program can begin as soon as the first arm is completed.

\subsection{Commissioning Plan and Goals}

BTeV will be in a position to use colliding beams during the latter phases of CDF and D0 operation in Run $2 \mathrm{~B}$. The lab program during this period will have to carefully balance the needs of CDF and D0 for integrating luminosity with BTeV's needs for commissioning.

$\mathrm{BTeV}$ 's goals during this period are to commission all the detector components of the first arm, and then the second. We start by getting the Level 1 and Level 2 triggers to work and proceed with the rest of the components. Our minimum goal is to have enough colliding beam exposure to carry out some of the more straight-forward measurements, those which require only a small amount of integrated luminosity. Possible first measurements would include $\sin 2 \beta$ via $\psi K_{s}, B_{s}$ mixing, the study of $K^{*} \gamma$, and the measurement of the charm cross section in the forward direction. These will allow BTeV to debug and explore all aspects of its detector and triggers and to test its analysis programs and procedures. In addition, we believe some of these measurements will be competitive with the then-current state of the art.

We anticipate being able to check the detector out initially with collisions from a wire target and expect that this will have no impact on CDF and D0 operations. Some initial studies of the trigger may also be possible with a wire target or perhaps multiple wire targets. Low luminosity running, perhaps by using some time at the end of stores, is the next logical step and permits BTeV to check out its detector and trigger initially with one interaction per crossing. Operation at high luminosity for occasional stores to study the detector performance when the average number of interactions per crossings is high would follow. Finally, some amount of dedicated high luminosity running to accomplish the initial limited physics program above or some variant of it would be scheduled. Only this last phase of the startup is expected to have any significant impact on CDF and D0 operation. If all areas were running at once, the degradation of luminosity in CDF and DO is expected to be no worse than $33 \%$, during such brief periods. New ideas or circumstances may help reduce 
the impact even more. BTeV believes that the value of even small amounts of running will have a huge effect on its ability to commission the detector and believes that the impact on CDF and D0 can be kept low so that their physics reach is not noticeably affected. 


\section{Part III}

\section{Physics Simulations}




\section{Chapter 12}

\section{Description of Simulation and Analysis Tools}

Detailed simulation is the key technique for developing a detector design which achieves the physics objectives of BTeV. To accompany the simulations we have also developed analysis tools which approach those that we will use to analyze data from the experiment.

In this part of the BTeV proposal, we first discuss the simulation and analysis tools we have used to design the detector and to establish its physics reach. Next we present the results of studies of triggering and tagging, which are important to our physics sensitivity, followed by results on a variety of final states that are currently major goals of our physics program. The studies demonstrate, in addition to the excellent capabilities on these particular final states, the breadth and power of the BTeV spectrometer. The results presented here are only a small part of the simulation effort, which is used to study every aspect of detector design. Results of these studies appear throughout Part II and the appendices.

We have used two complementary simulation packages. The first, MCFast [1], is a fast parameterized simulation and analysis environment which allows the user to quickly change the detector design from a geometry file without having to do any coding. It is very useful in developing and refining the detector design. All early work on $\mathrm{BTeV}$ was done with $\mathrm{MC}$ Fast, including the simulations done for the Preliminary Technical Design Report[2] (PTDR), which was submitted in the spring of 1999 . The second package, BTeVGeant, is a rather complete simulation of the detector based on the GEANT 3 simulation framework[3]. It has a more complete implementation of all of the physics processes that operate on particles, charged and neutral, as they traverse the detector. Unlike MCFast, the BTeVGeant detector model is implemented by code and, therefore, recoding is required to change the detector model; so BTeVGeant is most appropriate for use only after the design has stabilized. We continue to use both systems: MCFast is used for studies of design changes and optimizations and for fast turnaround on issues for which a detailed simulation is unnecessary; BTeVGeant is used to study subtle backgrounds, tails of distributions, etc. We have especially relied on BTeVGeant for studies of final states that contain electromagnetic showers. BTeVGeant has a more complete and realistic description of the detector, including beampipes, supports, ma- 
terial from readout electronics, etc. In order to facilitate comparisons between BTeVGeant and MCFast, BTeVGeant writes a file containing a good approximation of its detector specification in a form that can be input to MCFast; in this way we ensure that that studies done with either system are modeling as much as possible, given the intrinsic limitations of MCFast, the same detector. These comparisons provide new validation of MCFast in the areas which are of most interest to $\mathrm{BTeV}$. Below, we describe both systems, starting with BTeVGeant.

Analysis involves: fitting of charged tracks and calculation of their position, angles and momenta; reconstruction of photons and neutral pions; identification of charged particle types $-\pi, \mathrm{K}$, or $\mathrm{p}$ - based on signals from the Ring Imaging Cherenkov counters; identification of muons and electrons; reconstruction of the primary interaction vertex, or vertices; and reconstruction of secondary and tertiary decay vertices.

We carry this out using a framework which can operate on the output of either BTeVGeant or MCFast (although many calculations using MCFast have the analysis embedded in the simulation program itself, as a user routine). Track reconstruction is done at the trigger level by programs which are optimized specifically for triggering. For analysis, track pattern recognition is not yet done; instead the Monte Carlo truth information is used to collect hits which belong on each track and each track is fitted using a Kalman Filter. This algorithm gives a full covariance matrix for use in vertex fitting. The trigger-level track pattern recognition, which has much fewer resources at its disposal than will the ultimate offline pattern recognition, has been demonstrated to have high efficiency and few false tracks; therefore the absence of true pattern recognition in the analysis package does not represent a serious overestimate of our efficiency. Shower reconstruction is performed using a package which is derived from the CLEO offline analysis package, which in turn was derived from the Crystal Ball offline analysis package. Particle identification and muon reconstruction are also done at this time. A fitting package is also provided in the analysis framework; this package can perform both mass-constraint fits and vertex fits, with the option of including the beam envelope as a constraint in the vertex fit. Below, we describe three of these packages - the Kalman filter, the shower reconstruction, and the particle identification program. The reconstruction programs used by the trigger are described in chapter 9 .

\subsection{BTeVGeant}

A detector simulation package based on GEANT 3, BTeVGeant, has been developed for the physics studies presented in this proposial. The full detector geometry has been implemented within BTeVGeant, which writes out a simplified geometry description in the format of an MCFast geometry file. This facilitates comparisons between BTeVGeant and MCFast. BTeVGeant is, of necessity, written in mixed languages: Fortran $77, \mathrm{C}, \mathrm{C}^{++}$. This allows us to take advantage of both the GEANT 3 tracing package, which is written in Fortran, and the existing $\mathrm{BTeV}$ trigger code, which is written in $\mathrm{C}$. The $\mathrm{BTeV}$ vertex fitting package is written in Fortran, while the reconstruction code for the electromagnetic calorimeter, our newest package, is written in $\mathrm{C}^{++}$. This strategy will allow us to migrate to the GEANT 4 
package[4], which is written in $\mathrm{C}^{++}$, with relative ease. We have done performance studies of BTeVGeant and have used it for many of the Monte Carlo simulations described in this proposal.

\subsubsection{Design Considerations for BTeVGeant}

We began development of a detailed Monte Carlo with a complete and up-to-date detector geometry description and a full simulation of the detector response about one year ago. Previously, we had presented results based on a parametric version of the electromagnetic calorimeter and MCFast tracing through the detector. Many physics processes are included in the MCFast modeling of the detector, but some are treated in an incomplete manner. For example, the MCFast model of multiple scattering does not include non-Gaussian tails, which is sufficient for many detector design studies but not for detailed physics simulations.

The GEANT based simulation studies and analyses have been aimed primarily at the following three final states of b-meson decays:

$$
\begin{gathered}
B_{s} \rightarrow D_{s} K, D_{s} \rightarrow \phi \pi, K^{*} K \\
B^{0} \rightarrow D^{*-}(2010) \rho^{+} \\
B^{0} \rightarrow \dot{\rho} \pi
\end{gathered}
$$

We chose GEANT 3 for our detailed simulations for several reasons. We felt that GEANT 4, the more modern simulation tool kit in $\mathrm{C}^{++}$designed to support LHC experiments, had not been sufficiently tested, nor was it supported at Fermilab. Since we made our decision, however, numerous and very promising benchmarks have been done on GEANT 4 and we plan to incorporate GEANT 4 into future simulation developments. ${ }^{1}$

The critical specifications for BTeVGeant are:

- A "hit level" simulation, leading ultimately to a complete digitization of all detector components. By "hit level", we mean a simulation of the interaction of the track with the detector element, giving for instance a wire position and drift time, or the energy deposition in a block of the electromagnetic calorimeter. A "digitization" is a simulation of the TDC or ADC response, followed by an exact simulation of the data stream. While digitization is crucial for benchmarking the final trigger or reconstruction code, the digitization rarely has a significant effect on the resolution or hit occupancy and, for many studies, the simulated hits need not be fully digitized.

- Generation and tracking of all secondaries and decay products occurring within a beam crossing; secondaries may be generated in the tracking system, in the electromagnetic calorimeter, in the beampipe and in their mechanical support systems.

\footnotetext{
${ }^{1}$ For instance, a member of the Muon Collider and Neutrino Factory group has successfully tested the design of a muon cooling channel in GEANT 4.
} 
- A realistic implementation of the current detector geometry, including the magnets, beam pipes and supports for the king chambers and pixel detector. From the GEANT 3-based geometry, a simplitied version of it must be generated so that the comparisons and cross-checks with the results obtained with MCFast can be done in a straightforward and convenient manner.

- Simulation of the charged particle reconstruction code and generation of Kalman filtered tracks, allowing tracking and vertexing resolution studies. We use a track fitting technique that is similar to the one in MCFast and which is described in section 12.1.8.

- Production of an output stream of simulated hits (or digitizations) so that trigger algorithms and prototypes of reconstruction code can be studied. The Level 1 and Level 2 trigger algorithms do full pattern recognition using the hits.

\subsubsection{The BTeV Detector Geometry in BTeVGeant}

We chose to implement the detector geometry by coding it as a GEANT 3 application in which the sizes and positions of the GEANT 3 volumes are declared via Fortran data statements. The disadvantage of this coding style is obvious: in order to understand the geometry the user must read source code and, in order to modify the geometry, the user incurs compilation overhead. MCFast, on the other hand, works from an ASCII geometry file which is much easier to read and to modify.

In addition to filling the GEANT 3 geometry information, each geometry subroutine also fills $\mathrm{BTeV}$ specific data structures, which were designed with the event reconstruction code and the trigger simulation code in mind. The trigger and analysis codes do not use the internal GEANT 3 geometry representation. These BTeV specific data structures are serialized and written to the output stream, allowing the offline analysis packages to have access to the same geometry information as was used for the simulation.

We now describe the features of the GEANT 3 implementation of the major subsystems of the BTeV detector; an overview of these subsystems is found in Part II of this proposal and Figure 12.1 shows GEANT 3 drawings of the full detector.

- The spectrometer dipole magnet. The horizontal gap of $\approx 20$ inches has been sized to maximize the magnetic field at the interaction point and to leave enough space for the pixel detector and its support system. The yoke inserts, or shim plates, and the main magnet yoke are entered as a set of iron plates. The coils, assumed to be made of solid copper, are described by a set of rectangular boxes.

- The pixel detector: The pixel detector region, shown in Figures 12.2 and 12.3, consists of a cylindrical vacuum tank, the support and cooling structures and the silicon wafers. The pixel tank has, on each end, a thin hemispherical aluminum window, implemented in GEANT 3 as a section of a sphere. The glassy-carbon manifold and the coolant liquid (water + alcohol) are implemented as tubes and small rectangular boxes. There are two pixel planes per station. Each pixel plane is divided longitudinally (along the beam 
line) into two regions, one simulating the sensor and readout wafers (pure silicon) and the other simulating the sum of the readout infrastructure, the FLEX-circuit, cooling and the mechanical support (a mixture of copper and plastic). The detector planes are subdivided into a $9 \times 9$ checker-board array, with each cell representing one wafer. In order to allow for a hole for the beam, the center wafer in this array is not present. In reality, the wafers will be slightly tilted, $\approx 7$ degrees away from perpendicular to the beam, such that the sensitive area of one chip overlaps the dead readout periphery of its neighbor. This detail, known as "shingling", does not have a significant impact on resolutions, occupancies or pattern recognition complexity and it has been omitted from the description.

- The beam pipe: The pixel tank window and the beam pipe up to the entrance of the Cherenkov counter will be installed in one piece for each arm. The forward tracking devices will be installed around this beam pipe. However, in order to facilitate the simulation of the forward tracking stations, the GEANT 3 description of the beam pipe is subdivided into shorter longitudinal segments; this ensures that the GEANT 3 volumes in different sub-volume hierarchies do not overlap. The flanges connecting the beam pipe to the inner Cherenkov beam pipes have also been included in the description.

- The forward tracking system (FT): A detail of two forward tracking stations is shown in Figure 12.4. There are two distinct sets of forward tracking devices: planes of straw tubes and silicon strip planes. Each have their own container volumes and support structures. For instance, the silicon strip planes are supported from both sides, and over their entire height, by a $5 \mathrm{~mm}$ thick carbon plate.

- The RICH detectors: The radiators, gas and aerogel, have been implemented as trapezoidal and rectangular boxes respectively. A track, emitted from the luminous region, will encounter, in order, the Cherenkov entrance window, a negligible amount of Cherenkov gas, the aerogel volume, the main gas volume, the mirror and the exit window. Other details included in the simulation are the readout panel, located outside of the fiducial tracking volume, the beam pipe and the beam vacuum. The full simulation of the response of the RICH detector is done at analysis time and is described in detail in Appendix A.

- The Electromagnetic Calorimeters (ECAL): The BTeV detector has two electromagnetic calorimeters, one in each arm. BTeVGeant can simulate two different options for the ECAL geometry: 1) A planar box-shaped calorimeter built of rectangular lead tungstate, $\mathrm{PbWO}_{4}$, crystals, $2.6 \mathrm{~cm}$ on side and $22 \mathrm{~cm}$ long; and 2) a projective calorimeter built of tapered crystals, $2.6 \times 2.6 \mathrm{~cm}^{2}$ at the front face and $22 \mathrm{~cm}$ long, installed to point at the I.P. While the box-shaped calorimeter was the first to be coded and is still used for the validation of MCFast, the projective geometry is now the BTeV baseline calorimeter. Users of BTeVGeant choose between the two geometry options with a runtime switch. 
- The Muon detector. The muon detector, shown in Figures 12.5 and 12.6 , consists of hadron filters, beam pipe shielding and detector stations. The hadron filters are implemented as GEANT 3 boxes, filled with iron and with a hole for the beam. Each detector station consists of eight overlapping pie shaped octants. There are 3 views $(r, u, v)$; the $u$ and $v$ views are strung at $\pm 22.5^{\circ}$ with respect to the $r$ view.

\subsubsection{The Magnetic Field Model}

The magnetic field which will be produced by the main $\mathrm{BTeV}$ dipole magnet has been computed using the Tosca package[5]. In order to simplify and to speed up the simulation and analysis codes, two approximations are made in the model of the magnetic field used by BTeVGeant. First, only the main component of the magnetic field, $B_{x}$, is used. Second, $B_{x}$ is assumed to be piecewise constant with discontinuous jumps occurring at fixed values of $z$. The sum of $B_{x} \Delta z$, summed over the region of non-zero field, is constrained to be equal to the integral $\int B_{x} d z$ from Tosca. The motivation for the second approximation is that it allowed us to use the Kalman filter from MCFast without re-writing the transport routines. Although the simulated hits obtained using this approximate model of the field will not match the real hits, this approximation affects neither the tracking resolution nor the eventual pattern recognition problems. In particular, a constant field is a good model of the field within the volume of the pixel detectors.

\subsubsection{Tracking Accuracy}

The GEANT 3 propagation routines are coded in single precision, which was found to be insufficient to model the combination of high precision devices and long lever arms which are present in the $\mathrm{BTeV}$ spectrometer. We reached this conclusion after benchmarking the position accuracy of the GEANT 3 propagation routines against the MCFast propagation routines, which are native double precision. The tests were performed in the absence of any stochastic processes. When we replaced the single precision GEANT 3 Runge-Kutta integrator with a double precision version of the same code, GEANT 3 produced acceptable results. Although multiple scattering will usually be larger than the inaccuracies in the single precision tracing, we chose to run using the double precision version because it allows us to run the verification suite for the track and vertex fitters. This verification suite includes running with no multiple scattering and with reduced multiple scattering.

\subsubsection{Calorimetry Cuts}

GEANT 3 permits the user to control the thresholds and cutoffs of the many physics processes which it simulates. Low thresholds give a more realistic and detailed simulation but can require large amounts of computer time. This, in turn, can make it difficult to produce large enough samples of simulated events to make precise statements about efficiency and background rejection. Therefore it is important to tune wisely the cutoffs and thresholds so 


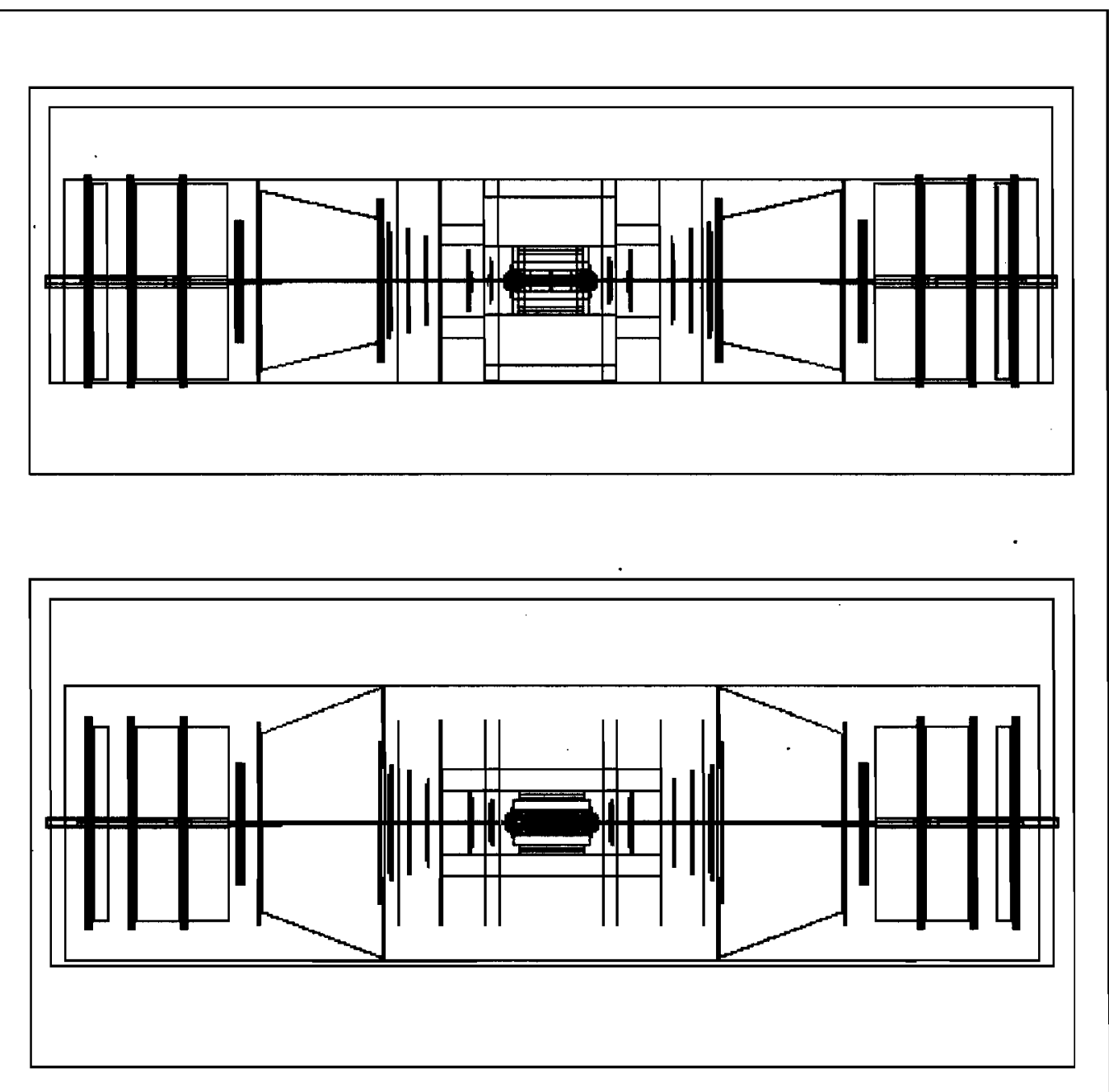

Figure 12.1: The $\mathrm{BTeV}$ spectrometer as drawn by BTeVGeant in elevation view (upper figure) and plan view (lower figure). The total length of this representation of the C0 Hall is about $26 \mathrm{~m}$. 


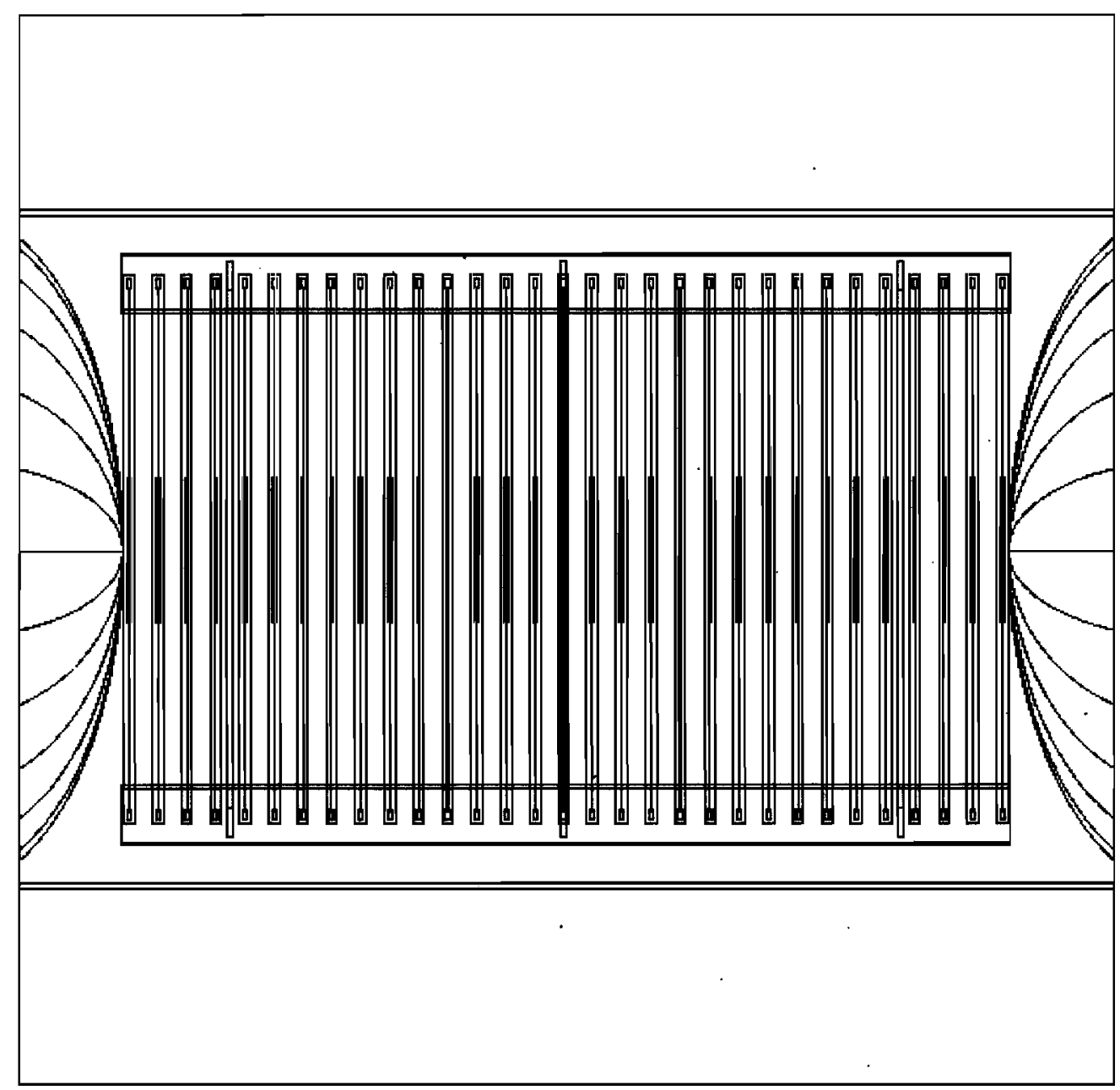

Figure 12.2: The Pixel detector and the hemispherical window, elevation view. The pixel region is $128 \mathrm{~cm}$ long. The 31 stations are clearly visible, except the central one which is masked by a structural support. The lines surrounding the planes within a station are fiducial, or buffer volumes, to ease the tracking debugging and do not correspond to real material. The medium inside the tank is vacuum. 


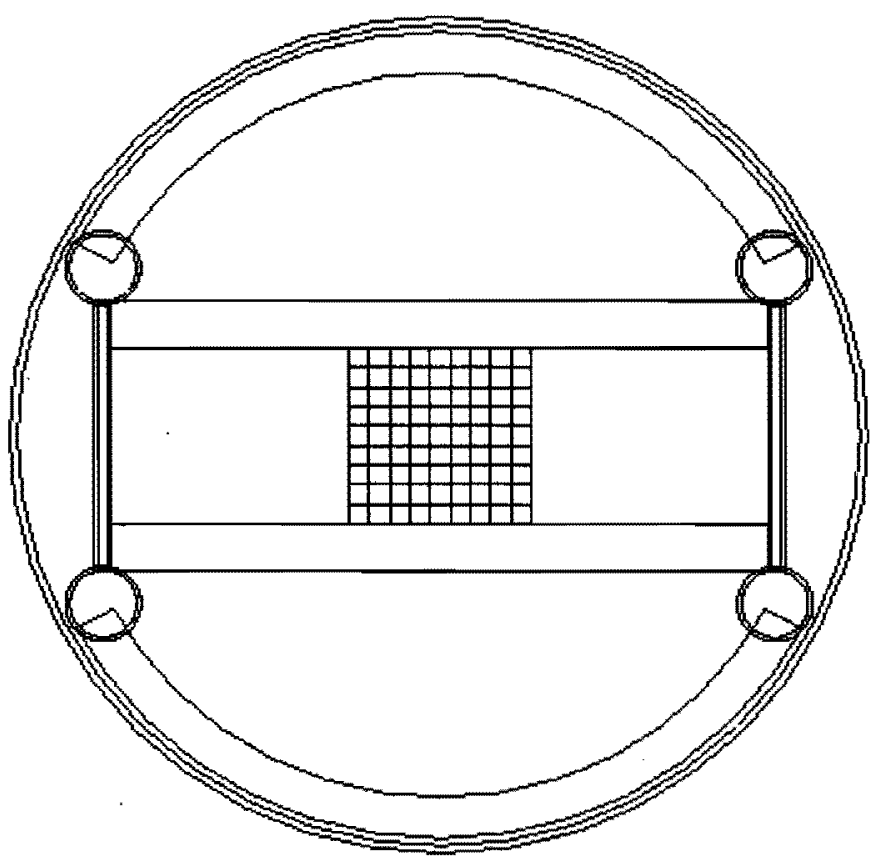

Figure 12.3: The Pixel detector, beam's eye view, showing the layout of the pixel wafers (center), the cooling tube manifolds (left and right, thick lines), the main cooling tube (circle in each corner), the support structure (section of the arc, top and bottom) and the vacuum tank walls. The vacuum tank is 20 inches in diameter. In order to provide the beam hole, the center cell of the $9 \times 9$ array of pixel wafers is not present. 


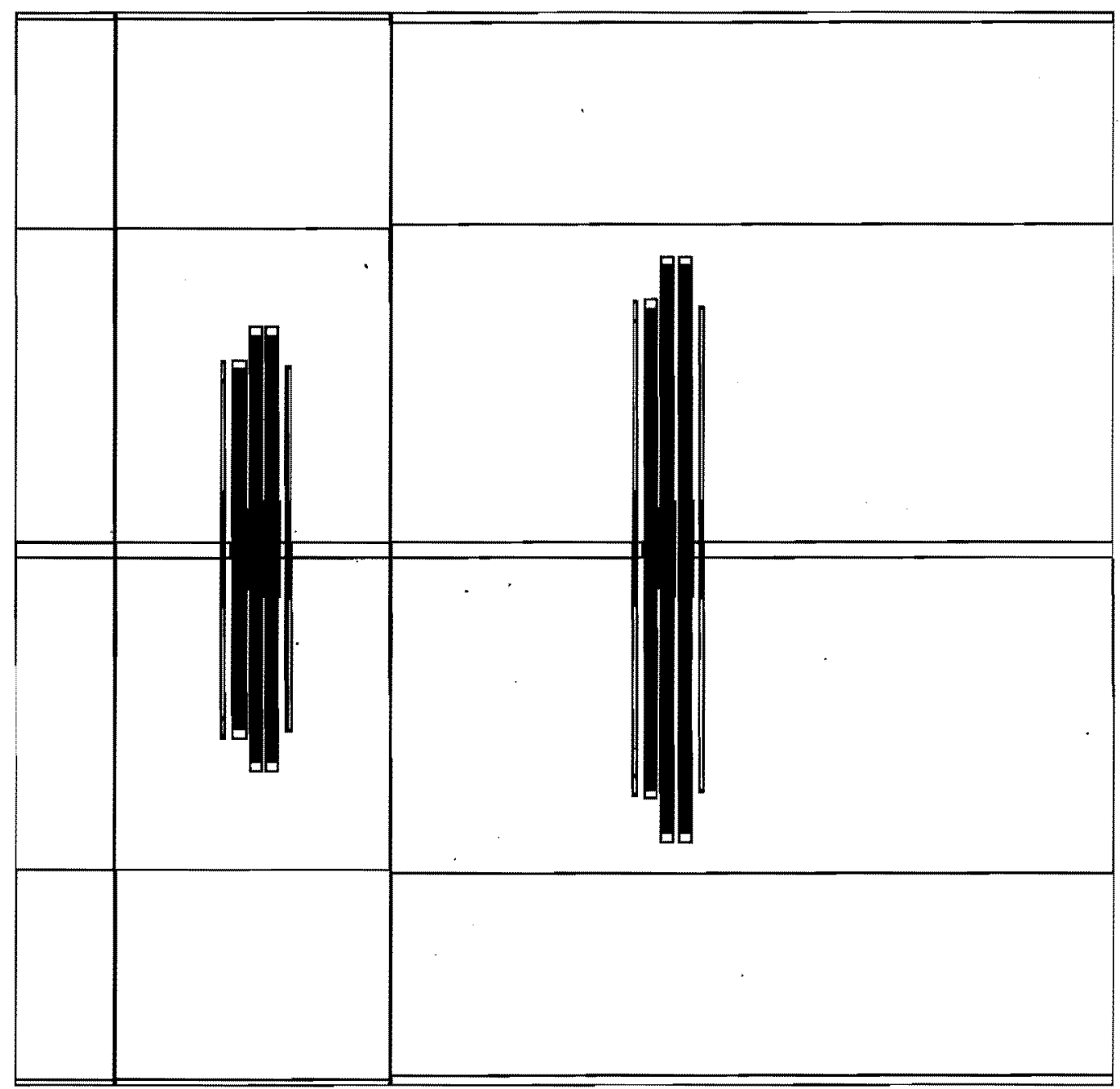

Figure 12.4: An elevation view of the region centered around $\mathrm{z}=+1.6 \mathrm{~m}$, showing straw tube stations 2 and 3 ; each station consists of three views and three planes per view for a total of 9 planes per station. Upstream and downstream of each straw tube station are forward silicon strip planes and their support structure. 


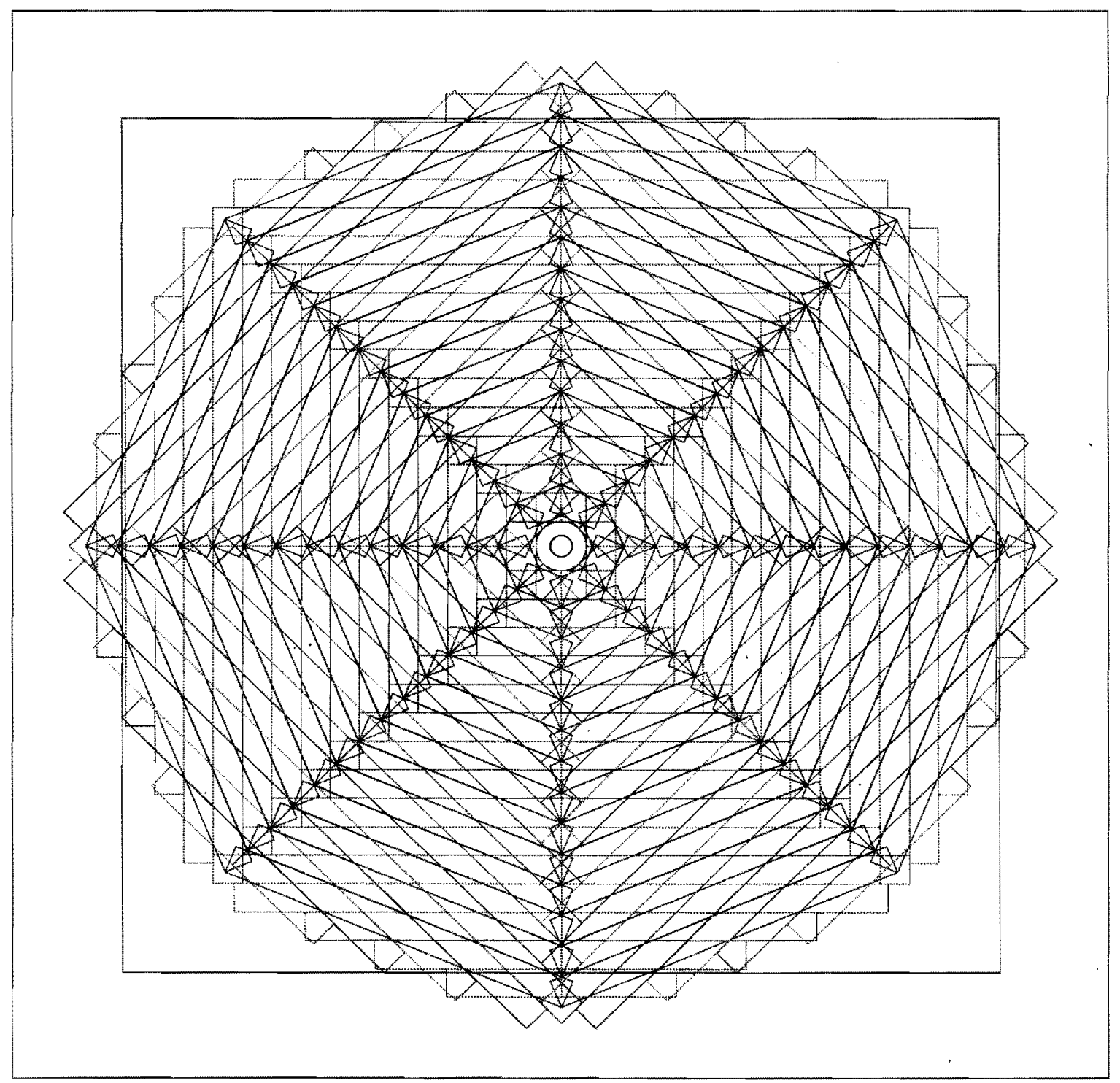

Figure 12.5: A beam's eye view of the muon detector, showing the beampipe shielding, the hadron filter and the layout of the muon planks. 


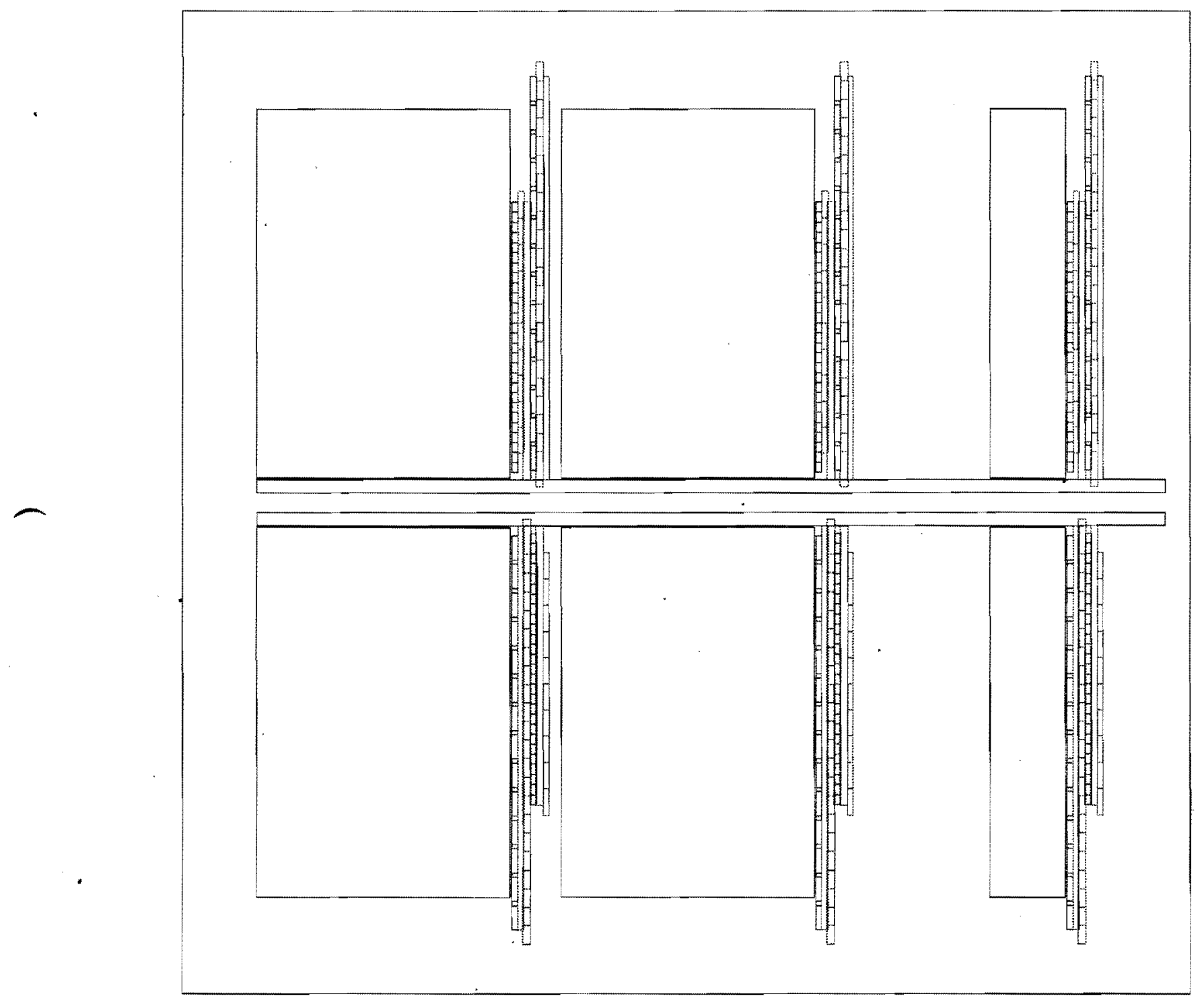

Figure 12.6: An elevation view of the region from $7.8 \mathrm{~m}$ to $11.4 \mathrm{~m}$, showing muon stations 1,2 and 3 . Also shown are the hadron filters and the beam pipe shielding. 
as to minimize the CPU time without creating an artificially rosy picture of the detector response. For BTeVGeant, most of the CPU time is spent in the simulation of the electromagnetic calorimeter so the proper tuning of the ECAL cutoffs has the largest impact on performance. We describe below how we chose values for the two most significant cutoff parameters, "cutele" and "cutgam". These are, respectively, the energies below which the tracking of electron and photons is stopped.

We generated 1500 signal events in the channel $D^{*-} \rho^{+}$and passed these events through $\mathrm{BTeVGeant}$. This was repeated for several different values of cutele and cutgam. The output files from these runs were analyzed using the BTeV ECAL cluster finding code (Section 12.4) to produce photon candidates; a photon candidate was rejected if any reconstructed charged track was too closely associated with it. From each event, pairs of photon candidates were selected and their invariant mass calculated. Figure 12.7 shows the $\gamma \gamma$ invariant mass spectra for the various runs; in each case a clear $\pi^{0}$ signal is present. The spectra correspond to the following choices of cutele and cutgam:

- a) $[$ left upper corner] cutele $=500 \mathrm{keV}$ cutgam $=65 \mathrm{keV}$

- b) [right upper corner] cutele $=1 \mathrm{MeV}$ cutgam $=65 \mathrm{keV}$

- c) $[$ left bottom corner $]$ cutele $=1 \mathrm{MeV}$ cutgam $=1 \mathrm{MeV}$

- d) $[$ right bottom corner] cutele $=1 \mathrm{MeV}$ cutgam $=3 \mathrm{MeV}$

Each mass spectrum in Figure 12.7 was fitted using a Gaussian to parameterize the $\pi^{0}$ line shape plus a 3rd order polynomial to parameterize the background. The two figures of merit which come from each fit are the resolution on the $\pi^{0}$ mass $(\sigma)$ and the ratio of signal/background for the $\pi^{0}$ peak. Figure 12.8 summarizes these figures of merit for the four runs. Neither figure of merit shows a significant dependence on either cutele or cutgam.

The investigation of the cutoff parameters was completed by studying the ECAL response for events containing only a single $10 \mathrm{GeV}$ photon, generated at the face of the calorimeter. For this study the two figures of merit were the energy resolution of the reconstructed shower $(\sigma(E) / E)$ and the width of the reconstructed shower. These were examined as a function of cutele and cutgam and the results are summarized in Figure 12.9. We see that the width of the showers depends only slowly on cutele but that showers become dramatically narrower as cutgam increases. The energy resolution is not a strong function of cutele nor does it depend strongly on cutgam, for cutgam less than $1 \mathrm{MeV}$. At cutgam of $3 \mathrm{MeV}$ the energy resolution is artificially lowered due to the decrease in the shower width.

Figure 12.10 shows the CPU time required to run BTeVGeant on the $D^{*} \rho$ events for the different sets of the cutoff parameters. We see that cut set c) consumes significantly less CPU time than either a) or b) while little further improvement is achieved by raising cutgam to $3 \mathrm{MeV}$, point d). From these studies, we conclude that the appropriate choices are cutgam $=1 \mathrm{MeV}$ and cutele $=1 \mathrm{MeV}$. 

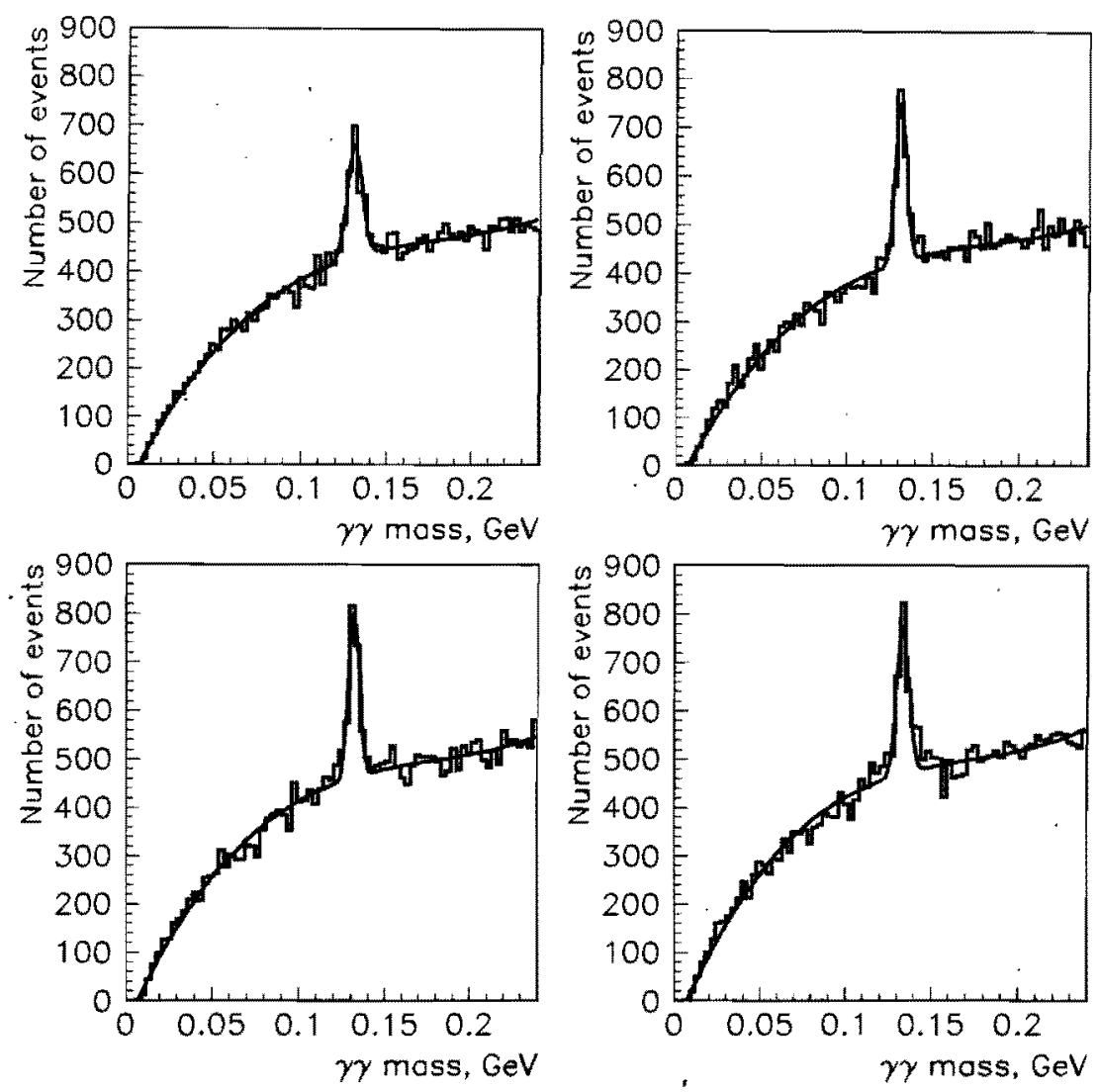

Figure 12.7: Histograms of $m_{\gamma \gamma}$ for the four different sets of cuts: (upper left) cutele $=500$ $\mathrm{keV}$, cutgam $=65 \mathrm{keV}$; (upper right) cutele $=1 \mathrm{MeV}$, cutgam $=65 \mathrm{keV}$; (lower left) cutele $=1 \mathrm{MeV}$, cutgam = $1 \mathrm{MeV}$; (lower right) cutele $=1 \mathrm{MeV}$, cutgam $=3 \mathrm{MeV}$. 

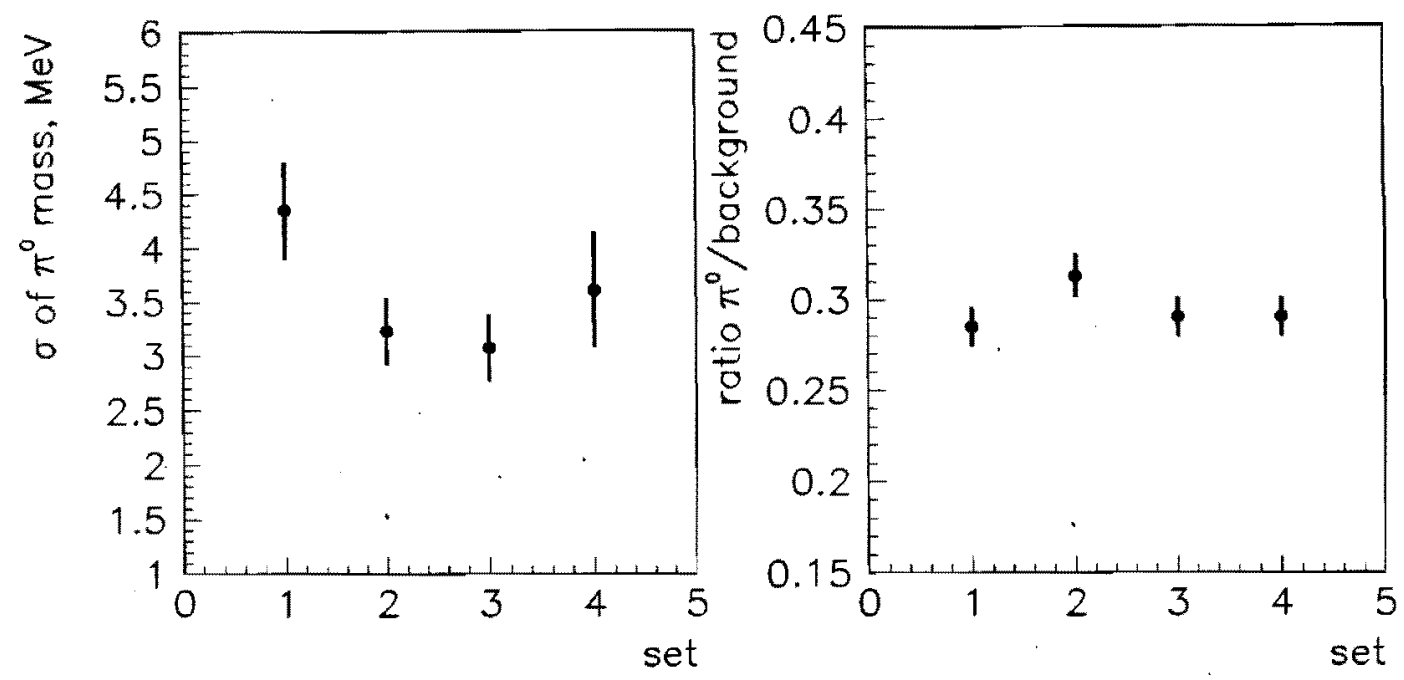

Figure 12.8: The $\pi^{0}$ mass resolution ( $\sigma=$ width of the mass peak) and the ratio of $\pi^{0}$ s/background for the four different sets of gamma and electron cutoffs. The horizontal axis refers to the 4 sets of cuts shown in Figure 12.7.
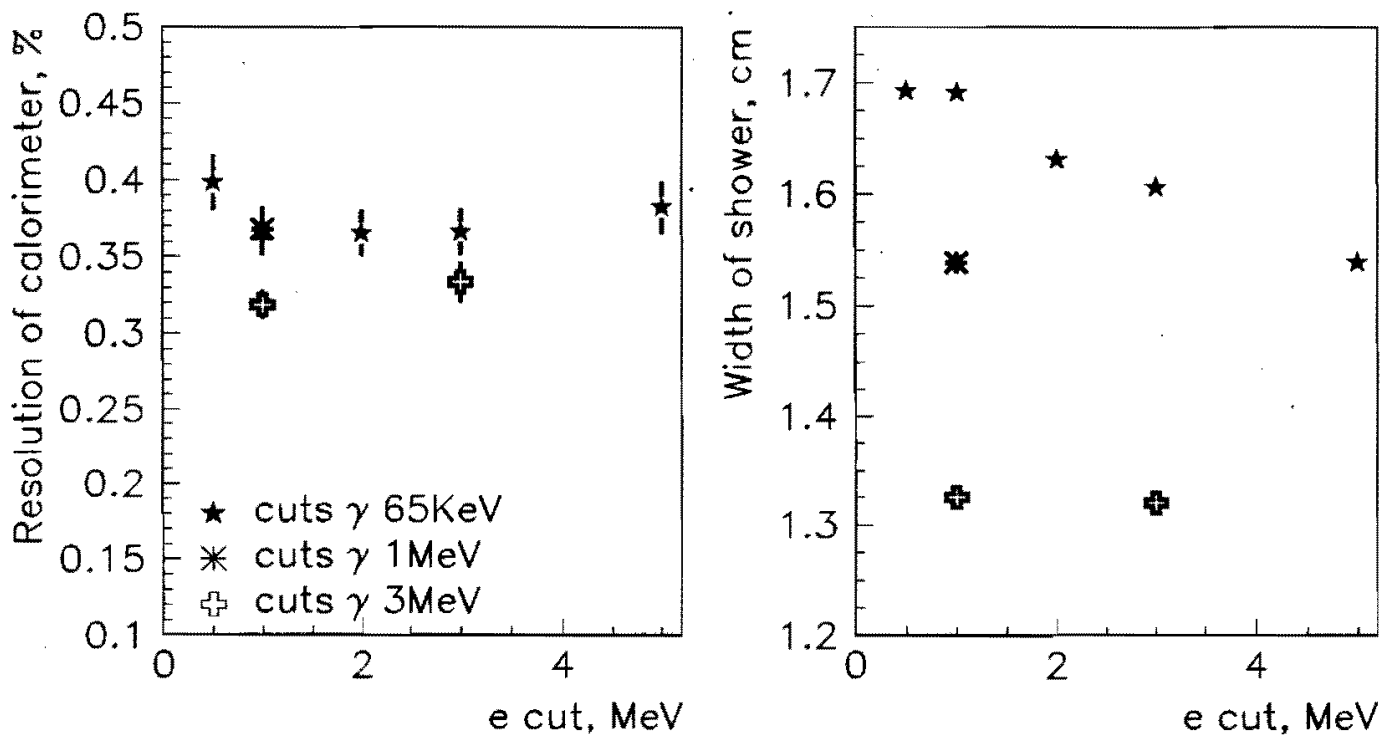

Figure 12.9: The energy resolution $\left(\frac{\sigma(E)}{E}\right)$ and the width of the showers for different gamma and electron cutoff values 


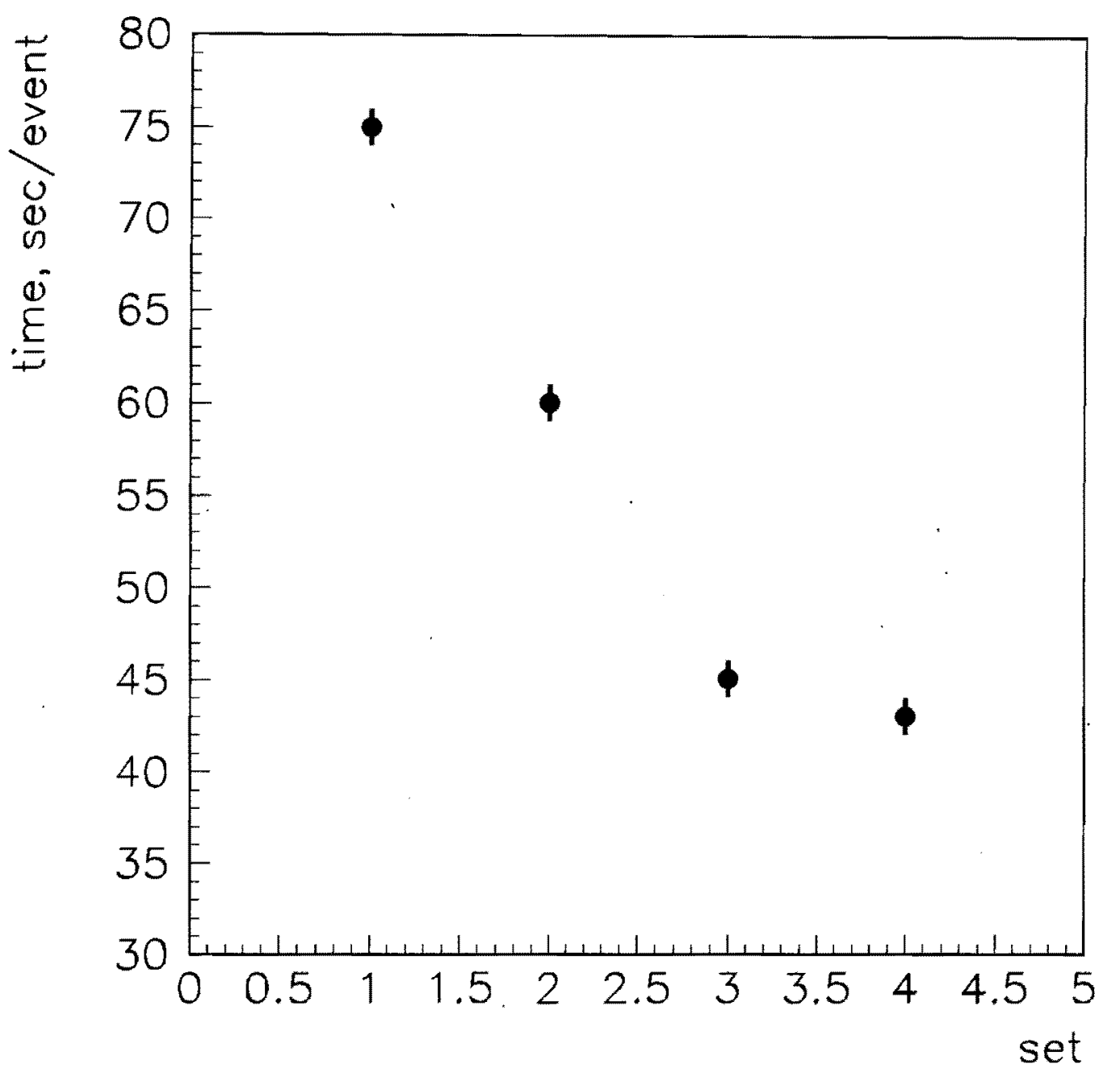

Figure 12.10: The BTeVGeant simulation time for $D^{*} \rho$ events for the four different sets of cuts. The horizontal axis refers to the 4 sets of cuts shown in Figure 12.7. 


\subsubsection{Hits and Digitizations}

In the language of GEANT 3 a "hit" refers to the intersection of a track with a sensitive volume while a "digitization" refers to the set of ADC and/or TDC values which would be produced by that hit. In a hit level simulation, the appropriate measured quantity, a drift time or position in a plane, is computed from the hit information and is smeared by the nominal resolution of the device; it is not digitized. BTeVGeant currently does a hit level simulation for all detector components and it has hooks in place for a digitization level simulation. Because a hit level simulation is sufficient for most purposes, digitization code exists only for the pixel detector. This choice saves computer time.

For the forward tracking system the plane and wire numbers, or plane and strip numbers, are generated for each hit. The drift times for the straws are not yet digitized but the position resolution is taken into account in the hit generation. This hit level information is used by a prototype of the Level 2 trigger code, and will later be used in the offline tracking pattern recognition code.

Hits in the RICH detector are not yet simulated in BTeVGeant. The full simulation of the response of the $\mathrm{RICH}$ detector is done at analysis time and is described in detail in Appendix A.

A hit in a lead tungstate crystal represents the sum of the energy deposited in that crystal by one or more electromagnetic or hadronic showers. The energy stored in the hit is the true energy which would be measured by a perfect device. Effects such as light collection efficiency, readout specifications and imperfections are applied in a parametric way at analysis time. Therefore these can be varied without rerunning BTeVGeant. This approach has been used by CMS and has been shown to be reliable. The data structure for ECAL hits also stores information about which particles or showers contributed to the energy sum in that crystal. This data structure has the same format as that for MCFast ECAL hits and it is described in detail in the MCFast manual [6].

\subsubsection{The I/O layer}

BTeVGeant reads its input data from StdHep format files using the StdHep package [7]. The code which builds events, or beam crossings, can read events from two separate files, a file of signal events and a file of background events. The mixing of events from these two files to form one beam crossing is discussed in section 12.3 .

BTeVGeant writes its output data using the MCFIO I/O package [8], which has been used extensively in the StdHep package [7] and in MCFast [1]. MCFIO can serialize arbitrary data structures, via user-written XDR filters, into machine independent IEEE standard streams. An MCFIO file, or stream, is structured into HEP events, or beam crossings, and each event is viewed as a container of data blocks. In addition to the event data, the first event contains the geometry information ( one block for each subdetector ), the global volume dictionary and the set of Kalman surfaces. The blocks which comprise the event data are:

- A copy of the original StdHep event, containing the input signal interaction plus zero 
or more background interactions (the truth table);

- The pixel, silicon and straw hits;

- Hits in the electromagnetic calorimeter;

- The truth table for tracks produced by decays or interactions in the detector;

- The Kalman-filtered tracks.

\subsubsection{Track Pattern Recognition and The Kalman Filter}

We regard the performance of the Level 1 and Level 2 trigger simulations which include full pattern recognition as proof that there are practical algorithms which have high efficiency, few false tracks, and reasonable execution time. We have not yet had time to move these algorithms into the analysis package. Therefore, a pseudo pattern recognition is done by using the Monte Carlo truth table to assign hits to tracks; this is the same level of pattern recognition which is done in MCFast.

Two other features of the pseudo pattern recognition simulate the behavior of a real pattern recognition program. If a track curls in the detector (ie. $p_{z}$ changes sign), the pseudo pattern recognition code only considers hits on the first arc of the track; hits on subsequent arcs are created and are present to confuse real pattern recognition programs. A small fraction of the tracks clip the edge of the magnet yoke or coil and continue downstream to make hits in the forward tracking system. Hits downstream of the encounter with the yoke or coil are ignored by the pseudo pattern recognition.

Tracks with fewer than 10 measurements in the sum of the pixel, straw and forward silicon detectors are not Kalman filtered.

Once hits have been assembled into a track, the track is fitted using a Kalman filter [9, $10,11,12]$. Its required input is a list of surfaces, or "nodes", ordered in $z$. One node is required to represent each hit on the track, each scattering surface and each magnetic field boundary. The notion of a fixed magnetic field boundary arises from the piecewise constant field model used by BTeVGeant; this was discussed in section 12.1.3.

Since most of the GEANT volumes are boxes with faces perpendicular to the beam axis, the scattering surfaces encountered by tracks can be described as surfaces' at fixed $z .^{2}$ The magnetic field boundaries also occur at fixed $z$. These surfaces at fixed values of $z$ are referred to as the "Kalman surfaces".

In BTeVGeant, assembling the node lists and executing the Kalman filter is done in three distinct phases:

\footnotetext{
${ }^{2}$ Two exceptions to this are the hemispherical window of the pixel tank and the beam pipe beyond the pixel tank. The window can be adequately approximated as being at fixed $z$ and the few tracks which cross the beam pipe can be dealt with as follows: the track can be fitted without the beam pipe in the node list; the intersection of the fitted track and the beam pipe can be used to compute the correct location for a node to represent the scattering in the beam pipe; the track can then be refitted, including the scatter in the beam pipe.
} 
- At geometry initialization time, the list of "Kalman surfaces" is defined.

- While Geant is tracing a track through the detector, hit-level information is created. At this time the associated Kalman node is also created.

- When Geant has finished tracing a track, nodes representing the Kalman surfaces are merged into the hit list and the track is Kalman filtered.

At the end of the each event the Kalman filtered tracks are written out as event data. Since the Kalman surfaces, the magnetic fields and the raw hits are also stored in the output stream, we are able to refit tracks in a subsequent analysis program.

\subsubsection{Optimization for Production}

All of the material in the $\mathrm{C} 0$ hall, including walls, supports and the magnet yoke are represented in BTeVGeant. While showers occurring in volumes located far away from the sensitive detectors are very unlikely to produce background hits, they take as much CPU time as showers occurring in the calorimeter. These rare backgrounds will be studied in dedicated runs while, in normal runs, particles will be stopped before they have a chance to initiate showers outside of the region of interest. In order to implement this, particles are stopped whenever they find themselves in a GEANT volume which is on a list of "stop points". Stop-points are located at the dipole yoke or coils, the walls of the C0 pit, the electromagnetic calorimeter and the muon steel. The stop-point at the ECAL is useful for analyses which consider only charged tracks. Enabling of the stop-points is controlled via the input command file.

Another utility allows us to save considerable CPU time when studying background processes for channels in which a single $\pi^{0}$ or photon is included in an otherwise all charged final state, for example $B^{0} \rightarrow \rho \pi$. Such events are simulated in two phases. During the first phase, all of the particles are simulated up to the electromagnetic calorimeter and are then held in temporary storage. The charged tracks are then Kalman filtered and a final-state specific analysis is run. This analysis will accept or reject the event based on the results of the charged particle tracking, the vertex topology and the tracking based triggers. If the analysis determines that this event fails then there is no need to simulate the response of the calorimeter; BTeVGeant will then discard the event and begin processing the next event. If, on the other hand, the event passes the analysis, then it must be fully simulated and BTeVGeant continues with its second phase. In this phase, the particles which were stored during the first phase are popped off their stack and BTeVGeant tracks them through the remainder of the detector, subject to the stop-points. If only a small fraction of background events pass the tracking cuts, then, because the majority of the CPU time is spent in the ECAL simulation, this method results in large savings in CPU time. For the $B^{0} \rightarrow \rho \pi$ simulation and analysis, we save a factor of 3 in computing time. 


\subsubsection{BTeVGeant Production Using a PC Farm}

To run as many simulations as possible using BTeVGeant, most BTeVGeant simulations were run on the Fermilab Computing Division PC Farm. This farm consists of 50 worker nodes, each with two $500 \mathrm{MHz}$ Pentium III CPUs. Most BTeVGeant simulation samples used for physics studies were run on $\sim 60$ CPUs over a period of three months. For decay modes where the electromagnetic calorimeter (ECAL) is not simulated, the simulation time is 10 seconds per event on a single CPU. Including the ECAL increases this to 60 seconds per event. ${ }^{3}$ Output file sizes are about $150 \mathrm{kBytes}$ per event without the ECAL, and $240 \mathrm{kB}$ with the ECAL. Output file sizes for background files are reduced by not including some of the raw hits in the output. The quoted output file sizes are after compression using the compression program gzip which reduces the total data size from $2.5 \mathrm{~TB}$ to $1.2 \mathrm{~TB}$.

The events generated using BTeVGeant are summarized in Table 12.1. Care was taken to properly seed the event generation process. Signal interactions were generated sequentially so as to always use the random number seed from the previous event. The minimum bias interactions embedded in signal interactions were separately generated for each production "run" and seeded by the time-of-day. The total CPU time used for this production is equivalent to that used by running on a single $500 \mathrm{MHz}$ Pentium III CPU for 9.6 years. The output data are stored on the Fermilab Mass Storage System.

Table 12.1: Summary of BTeVGeant simulations run on the Fermilab PC Farm. The data output sizes shown are for the compressed output.

\begin{tabular}{lrrl}
\hline \hline Decay Mode & \#Events & Size (GB) & Comments \\
\hline$B_{s} \rightarrow D_{s}^{-} K^{+}$ & $100 \mathrm{~K}$ & 9 & $D_{s}^{-} \rightarrow \phi \pi^{-}$ \\
& $55 \mathrm{~K}$ & 8 & $D_{s}^{-} \rightarrow K^{* 0} K^{-}$ \\
background from $b \bar{b}$ & $1000 \mathrm{~K}$ & 90 & $b \rightarrow D_{s}^{-} X$ \\
background from $b \bar{b}$ & $1000 \mathrm{~K}$ & 90 & $\bar{b} \rightarrow D_{s}^{-} X$ \\
\hline$B^{0} \rightarrow D^{*-} \rho^{+}$ & $125 \mathrm{~K}$ & 35 & $D^{*-} \rightarrow \overline{D^{0}} \pi^{-}, \bar{D}^{0} \rightarrow K^{+} \pi^{-}$ \\
background from $b \bar{b}$ & $1000 \mathrm{~K}$ & 175 & $b, \bar{b} \rightarrow D^{*-} X$ \\
background from $c \bar{c}$ & $100 \mathrm{~K}$ & 4 & $\bar{c} \rightarrow D^{*-} X$ with embedded cuts \\
\hline$B^{0} \rightarrow \rho \pi$ & $125 \mathrm{~K}$ & 35 & $\rho^{0} \pi^{0}$ \\
$\cdot$ & $125 \mathrm{~K}$ & 35 & $\rho^{+} \pi^{-}$ \\
background from $b \bar{b}$ & $5000 \mathrm{~K}$ & 400 & embed cuts in GEANT for speed up \\
\hline$B^{0} \rightarrow J / \psi K_{s}^{0}$ & $150 \mathrm{~K}$ & 3 & embed cuts in GEANT for speed up \\
\hline background from $b \bar{b}$ & $40 \mathrm{~K}$ & 10 & $b, b \rightarrow J / \psi X$ with embedded cuts \\
\hline$b b$ & $2000 \mathrm{~K}$ & 260 & 1 million is without ECAL \\
$c \bar{c}$ & $200 \mathrm{~K}$ & 4 & L1 Trigger applied \\
Minimum Bias (uds) & $1000 \mathrm{~K}$ & 6 & L1 Trigger applied \\
\hline \hline
\end{tabular}

\footnotetext{
${ }^{3}$ The lower CPU time of $44 \mathrm{~s}$ shown in Fig. 12.10 was for simulating the calorimeter in only one arm.
} 


\subsection{MCFast Simulation Package}

Early design studies to explore the detector layout, and many of the physics studies for this proposal, were prepared using the MCFast simulation package [1]. MCFast was developed by the Fermilab Computing Division to provide a general framework for simulation and analysis of a variety of detector geometries and detector options. The primary goal of MCFast is fast and accurate detector simulation with the speed and flexibility achieved through parameterization. The program allows the user to trace particles through simple geometrical shapes, does track fitting and provides a fast showering algorithm which can be used to generate hits in calorimeters. User analysis hooks are provided so that the detector simulation and analysis can be unified in one job. Events can be written out using an MCFIO [8] interface similar to that described for BTeVGeant in Section 12.1.7.

MCFast treats tracking devices and scattering surfaces as thin planes. It recognizes detector elements that are either planes perpendicular to the beam (Z-planes) axis or thin cylinders (R-planes) centered on the beam axis. In MCFast v5_0, rectangular planes perpendicular to the $x$ and $y$ axes (X-planes and Y-planes) are also recognized. Another recent refinement is the treatment of pixel detectors in which the wafers overlap. These additions have some cost in execution time but bring added flexibility and functionality to the package. Calorimeters and large volume absorbers (magnet yokes, muon filter) are handled in a different fashion. The normal tracing through the detector list is interrupted and tracing through large volumes is done in a series of small steps. The step size, which depends on the physics process, can also be set by the user.

In the preparation of the PTDR[2], we used MCFast v4_2, which included multiple scattering, $\mathrm{dE} / \mathrm{dx}$, and bremsstrahlung in the tracing of charged particles. Decays in flight, pair conversions and secondary hadronic interactions were also included. Hits in sensitive detector elements were generated during the tracing step and then passed to the track fitting package which was based on the same Kalman filter technique that is described for BTeVGeant in Section 12.1.8. Realistic hit generation for the pixel detectors was included as follows: for the narrow dimension of the pixel, the resolution function was modeled as a Gaussian whose width is dependent on the angle of incidence of the track on the pixel plane. Delta rays were not included. The hits could also be fed to the trigger simulation package which did complete pattern recognition and which had its own track fitter and vertex finder. The main limitation of MCFast v4_2 was that the tracing step could not correctly account for multiple scattering in a detector which contained both R-planes and Z-planes. Therefore, the simulations for the PTDR were done without a realistic model for beampipe; this was only possible because the pixel detectors are inside the beampipe.

MCFast v5_0 has been used for many of the physics analyses presented in this proposal. This version contains the same basic features as MCFast $\mathrm{v} 4.2$, but in addition there is an integrated tracing package which properly unifies the tracing steps for Z-planes, R-planes, $\mathrm{X}$-planes and $\mathrm{Y}$-planes; this package is implemented in $\mathrm{C}^{++}$. The integrated tracing improvements permits tracing and scattering of particles in both the planar elements, such as forward tracking detectors, and the radial elements, such as beampipes, in a consistent 
manner. In addition the showering algorithms have been updated and coupled to a more efficient hit generation package for the calorimeters. The Kalman filter track fitter has been separated from the underlying MCFast specific internal structures and has been adapted for use in both MCFast and BTeVGeant. Other improvements to MCFast were included to support operation on the Linux farms.

\subsubsection{Charged Particle Tracking in MCFast}

MCFast tracking is a two step process. In the first step, tracks are followed from their production point along their trajectory and at each detector surface that is encountered one or more operations takes place. These operations include multiple scattering, energy loss, elastic and inelastic hadronic scattering, bremsstrahlung and pair conversion. If the surface represents a sensitive element then a hit may also be created. In addition, a decay in flight may occur at any point along the track or a shower may start when a particle passes through dense material such as a magnet yoke, calorimeter or hadron filter. The collection of all points on one track which are recorded in this step is referred to as the trace list of the track. In the second step, the trace list of each track is used as the input to a Kalman filter. The output of the Kalman filter has been validated over the full range of momentum and angles which are important for $\mathrm{BTeV}$.

Several approximations are made in the MCFast tracking. Multiple scattering is approximated by a Gaussian distribution. More importantly, the input to the Kalman filter does not contain any pattern recognition errors.

In general, the location of a hit in a tracking detector is taken as the intersection of the particle trace with the detector plane. This is then smeared by the nominal resolution of the device, which is taken from the geometry file: MCFast has an internal parameterization of the resolution of each device and the writer of the geometry file can supply the values of its parameters. Most devices have a default option which will be selected if the user does not supply values for the parameters; an example of such a default is pitch $/ \sqrt{12}$ for silicon strip detectors. For pixel detectors only, there is another option which can be selected via the geometry file: the resolution can be returned, on a hit by hit basis, from a user supplied function. For the $\mathrm{BTeV}$ pixel detector several different models of the spatial resolution were used in MCFast simulation studies: the constant $\sigma$ model, the variable $\sigma$ model, fast clustering and slow clustering.

The constant $\sigma$ model was the model used in the simulations for the BTeV EOI. In this model, the spatial resolution is a Gaussian. with a constant width which is equal to the pitch $/ \sqrt{12}$. This model is used for both the short and the long measurement directions of the pixel. The default model, the one used for most calculations presented in this document and the one was used in the PTDR, is the variable $\sigma$ model.

In the variable $\sigma$ model, the spatial resolution remains Gaussian but the width of the Gaussian for the short dimension of the pixel is a function of the angle of incidence of the track on the pixel plane. The width of the Gaussian for the long dimension of the pixel remains at the pitch divided by $\sqrt{12}$. For reference, the distribution of the $x$ and $y$ angles of 
incidence are shown in Figure 12.11 parts a) and b). These figures were made using typical $b \bar{b}$ events and they only include hits from tracks with at least 20 hits. The width of the distribution for $\tan \theta_{y}$, is greater than that for $\tan \theta_{x}$ because of the bending of the tracks in the magnetic field of the analyzing dipole. Parts c) and d) of Figure 12.11 show the value of $\sigma$, as a function of angle of incidence, for, respectively, pixels which measure $x$ and pixels which measure $y$. The minimum of the $y$ resolution is offset from $\theta_{y}=0$ because of Lorentz force effects.

In the fast clustering model a Monte Carlo simulation is run for each intersection of a track with a pixel plane. This simulation explicitly generates the digitized pulse heights recorded on nearby pixels. A 3-bit linear ADC and a threshold of $2000 e^{-}$were assumed. Once each cluster has been generated, the pulse heights are used to obtain an estimator of the position of the hit; this estimator uses the knowledge of the angle of incidence.

In addition to returning an estimator of the $(x, y)$ position of the hit, the fast clustering model also returns an estimate for the error on that position. This error is used by the Kalman filter and is similar to the error used in the variable $\sigma$ model. In the long dimension the nominal resolution is the pitch divided by $\sqrt{12}$ and, because almost all clusters are only one pixel wide, the shape of the resolution function is box-like.

The important improvement achieved through the fast clustering method is that its resolution functions are closer to reality, having a box-like component and noticeable tails. Using the fast clustering model, however, adds about $70 \%$ to the execution time of a typical physics analysis job. This is a sufficient degradation that this model is not routinely used for physics analyses. The principal weakness of the fast clustering model is that the overlapping of two or more clusters is not considered.

The slow clustering model takes the overlaps into account but at the expense of large inerease in CPU time. For this proposal BTeVGeant has been used for these more detailed studies.

\subsubsection{Vertexing Package}

The MCFast package contains a set of vertex fitting routines which can be used during the analysis phase to combine sets of fitted tracks into vertices. The package can work in the presence of a solenoidal magnetic field, in a dipole field in either the $x$ or the $y$ direction or in a field-free region. The control of the vertex fitting is left to the user who decides which tracks should be grouped to form a candidate vertex. The package also contains utilities to build new "particles" from a list of fitted tracks and the package is able to use these new "particles" in subsequent fits.

The fitting algorithm and its implementation are based on code written for CLEO [13], which has a solenoidal field. The extension of the package work in a dipole field ( $x$ or $y$ direction only) is accomplished by rotating the tracks to a new coordinate system in which the magnetic field points along the $z$ direction; the output of the vertex fitter is rotated back to the normal MCFast coordinate system.

The base operation of the package is to perform a vertex fit, returning a vertex position, 

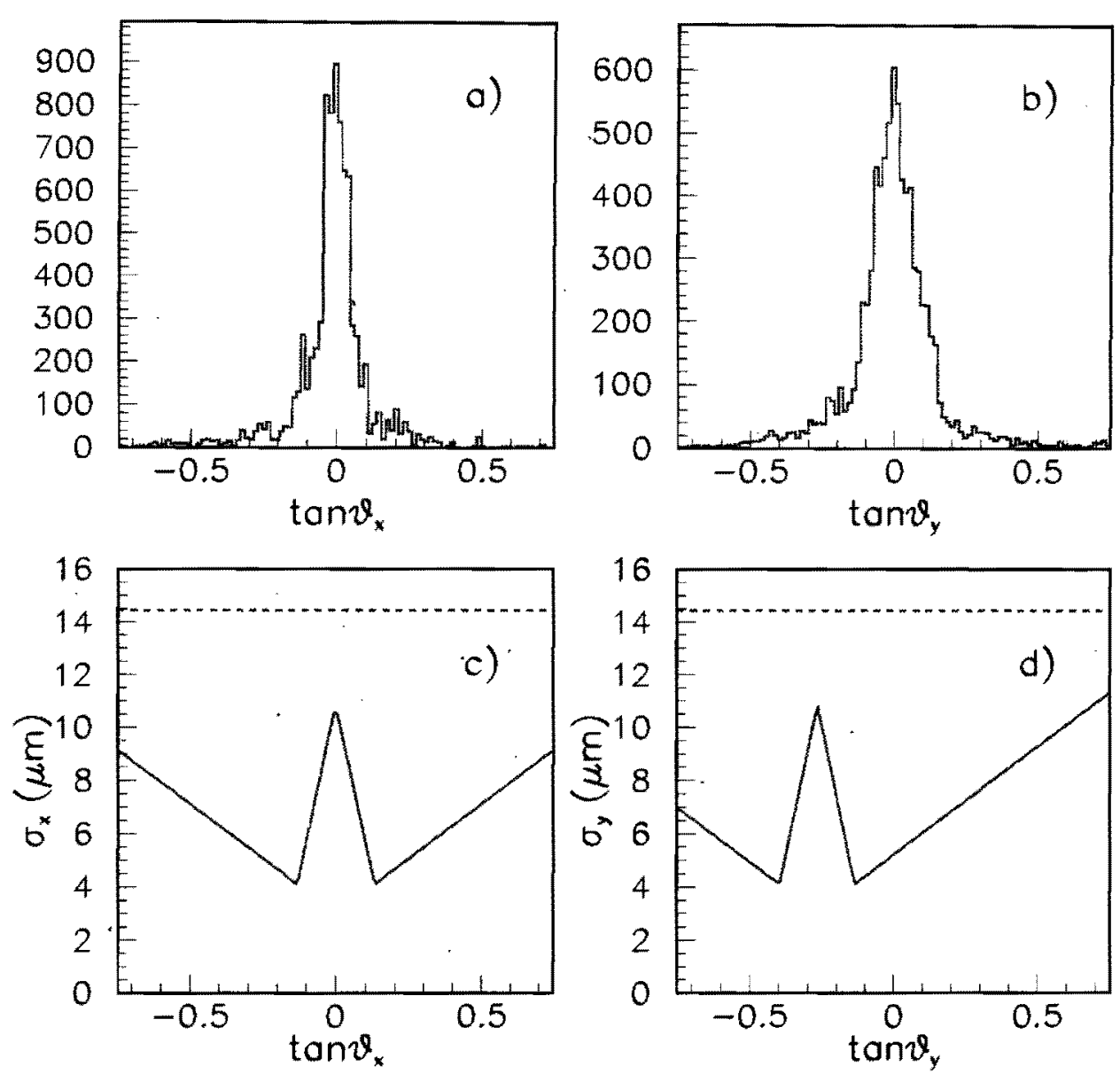

Figure 12.11: The distributions of a) $\tan \theta_{x}$ and b) $\tan \theta_{y}$ for hits on tracks, in $b \bar{b}$ events, with at least 20 hits. Here $\theta_{x}$ is the angle of incidence, in the $x z$ plane, of a track which intersects a pixel detector. Similarly for $\theta_{y}$. For most studies reported in this document, the pixel detectors are modeled as having a spatial resolution function which is Gaussian. The dependence of the $\sigma$ of the Gaussian on angle of incidence is shown in c) for pixels which measure $x$ and in d) for pixels which measure $y$. In both cases the solid line shows the value of $\sigma$ for the short dimension while the dashed line shows the value for the pitch divided by $\sqrt{12}$. 
its covariance matrix and the $\chi^{2}$. There are options in the package to update the tracks after the fit and to include a beam constraint when fitting the vertex. The package also contains a utility to perform fits with a mass constraint. This vertexing package has been used with both MCFast and BTeVGeant based analyses.

\subsubsection{Simulation of Secondary Hadronic Interactions}

The approach to secondary hadronic interactions in MCFast is purely phenomenological. No attempt is made to model the underlying hard scattering process. The probability of an interaction occurring is determined by the collision length and absorption length of the material. Energy dependent cross-sections are not used inside MCFast.

The number of charged particles produced in a secondary interaction of a charged pion on a proton target is a function of the center-of-mass energy of the interaction $s$. The mean number of charged particles $n_{\text {ave }}$ is given by,

$$
n_{\text {ave }}=a+b \log s+c(\log s)^{2}
$$

where $a=-0.35, b=1.21$ and $c=0.03$ as given by Dumarchez et al. for pion-proton interactions [14]. The distribution is characterized by KNO scaling which is modeled as a truncated Gaussian. Figure 12.12 can be compared with Figure 5 in Firestone et al. [15]. For a heavier target the mean increases as a function of the atomic number $A$ [16]. The average number of neutrals is assumed to be $50 \%$ of the charged multiplicity. The number of secondary particles from an interaction is limited to a maximum of 25 to avoid overflowing arrays.

The momentum distribution of secondary particles is approximately flat in rapidity and approximately Gaussian in $p_{T}$ with respect to the direction of the interacting particle [17]. The simulated distributions for a particle with momentum $50 \mathrm{GeV} / \mathrm{c}$ are shown in Figure 12.13. These can be compared with Figure 30 in Brenner et al. [17] and Fig 69 in [16]. The momentum of each outgoing particle is selected from these distributions and then energy is conserved by rescaling the momenta. The momenta are then rotated into the lab frame and the new particles are added to the StdHep list.

Elastic scattering is characterized by the distribution of four-momentum transfer $\operatorname{squared}\left(-t=p_{T}^{2}\right)$. The distribution is given by

$$
d \sigma / d t=e^{(b t)}
$$

where $b \approx 8[18]$. The simulated distribution is shown in Fig 12.14 .

\subsubsection{The MCFast showering subpackage}

The MCFast showering works by depositing energy in detector elements using an algorithm based on parameterized shower shape distributions. This procedure produces showers distributed over detector elements which can be processed by realistic shower reconstruction 


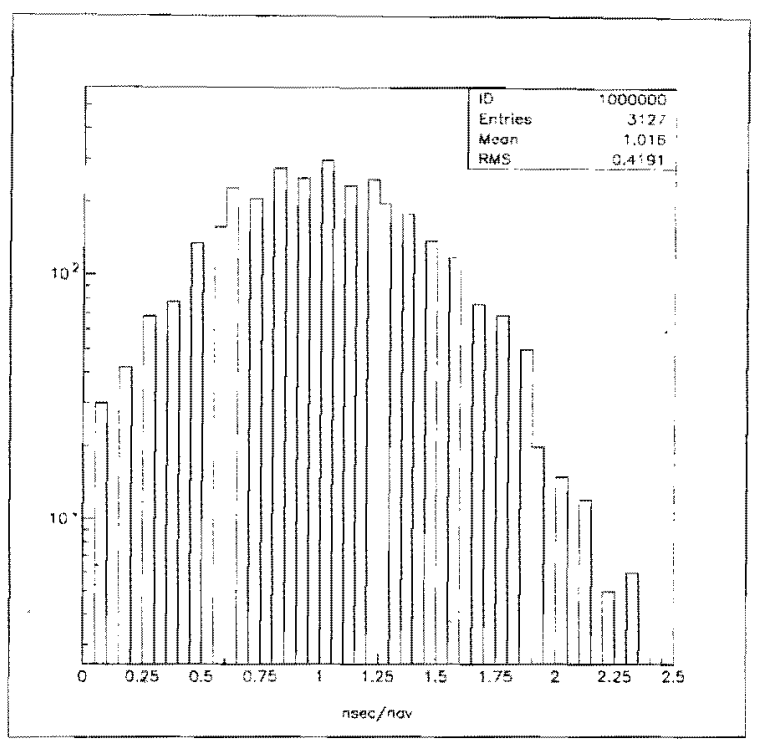

Figure 12.12: KNO scaling in MCFast: The scaled number of secondaries, $n_{\text {sec }} / n_{\text {ave }}$, as generated in MCFast.
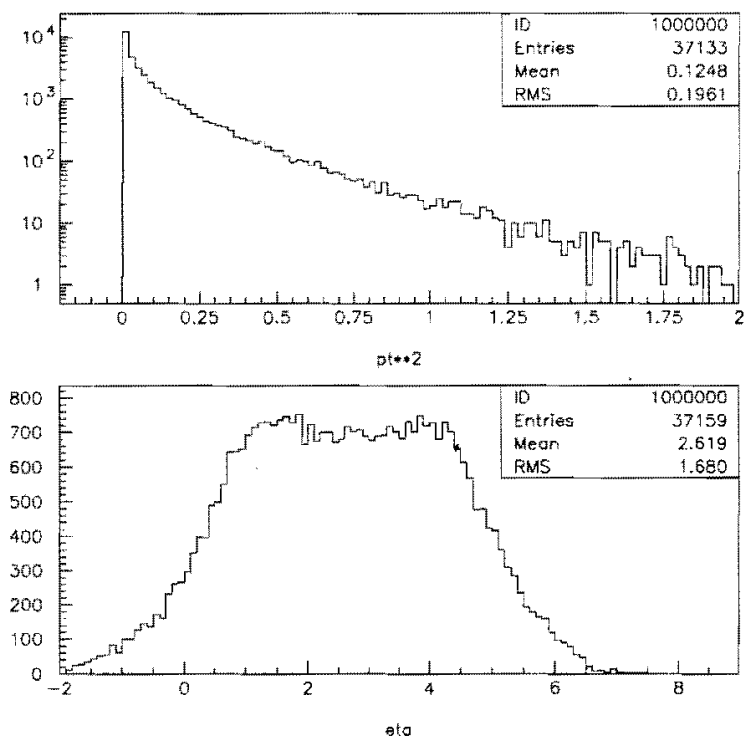

Figure 12.13: Distributions, produced by MCFast, of kinematic variables of secondary particles generated by an incident particle of $50 \mathrm{GeV} / \mathrm{c}$ : (a) $p_{T}$ distribution, (b) $\eta$ distribution 


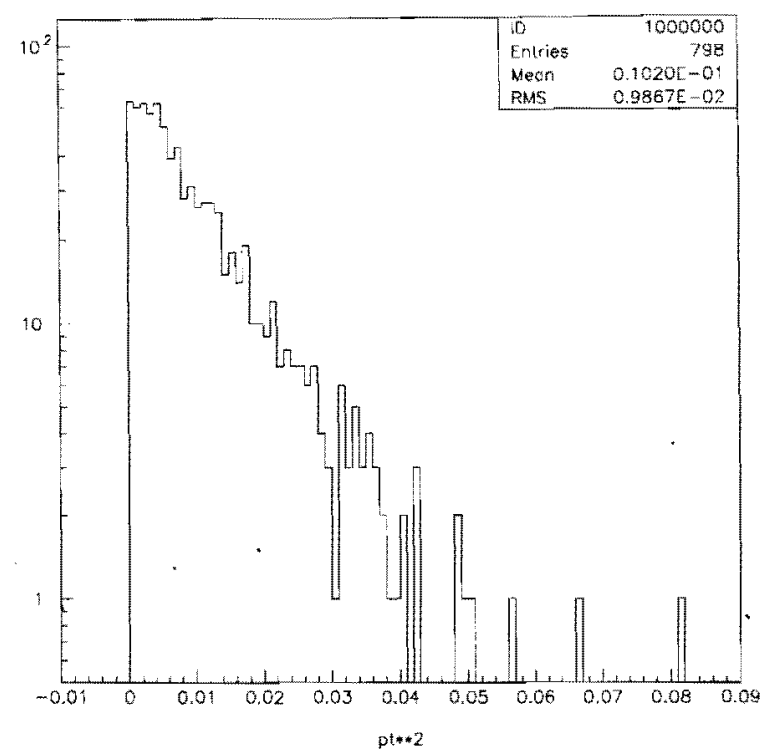

Figure 12.14: Distribution of $p_{t}^{2}$ for elastic scattering from MCFast.

programs. The program executes faster than a full GEANT simulation and represents the energy deposition in detectors quite well. It has been shown to be consistent with GEANT over the range of energies relevant to $\mathrm{BTeV}$.

The MCFast showering subpackage is completely integrated into the MCFast MonteCarlo package. The user defines tracking systems, calorimeters and absorbers through the detector description file and MCFast takes care of converting particles to showers, through an interrupt to the normal tracing processes at the calculated shower conversion point. In addition, energy losses and multiple scattering in the bulk material of the calorimeters and absorbers are taken into account.

The showering simulation is based on an extensive parameterization scheme. There is no detailed, particle by particle, description of an electromagnetic or hadronic shower. Instead, the energy densities due to the high energy showering processes within volumes are parameterized, and shower fluctuations are simulated globally rather than locally. In the calorimeters, these energy densities are mapped onto a user-controlled readout grid, and calorimetry hits are generated.

Unlike particle tracing in MCFast, the simulation of the shower propagation is intrinsically 3-dimensional. To keep the program simple, only three volume types are recognized: BOX, TUBE and CONE. These shapes must be oriented in such a way that the symmetry axis is along the $\mathrm{Z}$-axis.

At the initialization stage, MCFast computes the surface boundaries for these volumes. At the tracing stage, once such a surface is reached, the calorimetry subpackage returns an estimate of the mean free path length for the particle. For electrons/positrons and photons, 
it is simulated according to the formula $f(x)=e^{-x / X_{0}}$, where $X_{0}$ is related to the radiation length. For hadrons $f(x)=e^{-x / \lambda}$, where $\lambda$ is the absorption length (the mean free path for inelastic interactions). Based on this information and a random number, the location of the interaction inside the calorimeter is computed. Should an interaction occur, the tracing code switches from "particle" tracing to "shower" tracing and the core of the shower is propagated through the remaining geometry elements. This enables the propagation of the shower from one calorimeter to the next.

Upstream of the conversion point, the particle behaves as a minimum ionizing particle (MIP), sensitive to magnetic fields, if any, and undergoing energy loss and multiple scattering. The corresponding energy deposition is recorded as hits in the calorimeter.

Showering starts at the shower conversion point. Unlike normal charged particles, the core of the shower propagates in a straight line. There is no provision for the propagation shower to be sensitive to the magnetic field in the region.

Downstream of the conversion point, the deposited energy is calculated on a step by step basis, according to the shower longitudinal profile formulae. In most cases the step size is one radiation length but this will be reduced if the particle is close to a boundary or it is close to ranging out. After each step, the shower core position, energy and momentum are calculated and the control is given to the hit generation, if requested. There are two options for hit generation either of which can be selected in the command file: all of the energy for this step may be deposited in the current cell or it may be spread over neighboring cells according to a model of transverse shower profiles. The former is a much faster model while the latter is much more accurate.

For electromagnetic showers, the longitudinal profile is described as,

$$
d E / d x=E_{0} b \frac{(b t)^{a-1} e^{-b t}}{\Gamma(a)}
$$

where

$$
\begin{gathered}
t=x / X_{0}, \quad b \doteq 0.5, \quad a=1+b t_{\text {max }}, \\
t_{\text {max }}=1.0 \times\left(\ln (y)+C_{i}\right), \quad i=e, \gamma, \quad\left(C_{\gamma}=+0.5, \quad C_{e}=-0.5\right) \\
y=E / E_{c}, \quad E_{c}=\frac{800 \mathrm{MeV}}{Z+1.2},
\end{gathered}
$$

where $Z$ is the atomic number and where $E_{0}$ is the energy of incident particle.

For hadronic showers, the longitudinal profile is described as,

$$
d E / d x=E_{0}\left(w b \frac{(b t)^{a-1} e^{-b t}}{\Gamma(a)}+(1-w) d \frac{(d u)^{c-1} e^{-d u}}{\Gamma(c)}\right)
$$

where,

$$
\begin{aligned}
t & =x / X_{0} \\
u & =x / \lambda
\end{aligned}
$$




$$
\begin{aligned}
a & =0.6165+0.3183 \ln \left(E_{0}\right) \\
b & =0.2198 \\
c & =a \\
d & =0.9099-0.0237 \ln \left(E_{0}\right) \\
w & =0.4634 .
\end{aligned}
$$

When the shower transverse profile option is enabled, the energy deposited at each step is distributed around the shower axis according to a calculated shower transverse profile. The transverse profile becomes wider as the shower moves deeper into a calorimeter.

The transverse profile for both electromagnetic and hadronic showers is described as

$$
\frac{d E}{d R}=e^{\left(-R / A_{1}\right)}+A_{2} e^{\left(-R / A_{3}\right)}
$$

In this formula, parameters $A_{1}, A_{2}, A_{3}$ depend on the material properties and on the current shower depth. These dependencies differ for electromagnetic and hadronic showers.

The transverse distribution of deposited energy during each step occurs in a plane perpendicular to the direction of motion of the shower core. Since showers may have crossed calorimeter boundaries, energy deposition is also computed in the corresponding cells of those neighboring calorimeters. Thus, even if a shower develops in an absorber, it still may produce hits in nearby calorimeters.

The total deposited energy is smeared during the process of showering simulation according to the energy resolution formula

$$
\frac{\sigma_{E}}{E}=\frac{a}{\sqrt{ } E} \oplus b
$$

where $a$ and $b$ are input parameters for the code. For a sampling calorimeter, a visible fraction of energy is calculated.

The analysis of calorimeter hits can be performed through the use of an interface to the cluster finding routines that are described in Section 12.4. The shower shapes are similar though not identical to those produced by BTeVGeant. A comparison between MCFast generated showers and GEANT generated showers is given in Section 13.1.

\subsubsection{BTeV Geometry Description for MCFast}

The geometry description for our MCFast simulations is derived from the geometry used for BTeVGeant simulations. This MCFast geometry description is used for the MCFast based physics studies presented in this proposal and it includes:

- A dipole magnetic field described by 11 regions of piecewise constant field extending to $\pm 320 \mathrm{~cm}$, including the fringe field. The description includes a yoke and coils which act as absorbers. The central field region around the vertex detector has a horizontal field of $1.6 \mathrm{~T}$. 
- A 20 inch diameter central vacuum vessel with thin planar end walls, surrounding the vertex detector.

- A 1.5 inch diameter Aluminum beam pipe extending from the end walls of the central vacuum vessel, through the forward tracking detector to a flange at $\mathrm{z}=380 \mathrm{~cm}$. The diameter of pipe is increased to 2.5 inches on the far side of the flange.

- A silicon pixel vertex detector with 31 stations of 2 planes each. The silicon sensor plus its electronics and shielding is assumed to have the same amount of scattering as would 900 microns of silicon. The pixel dimensions are $50 \times 400$ microns. The resolution of the pixel detectors is parameterized as a function of the angle of the track.

- A forward silicon system with 3 stations of strip detectors per arm. For these simulations, the strip pitch is 100 microns and there are 4 views per station.

- A set of forward tracking chambers with 7 stations in each arm. In each arm the three stations at smallest $|z|$, have large holes to accommodate the forward silicon tracker. There are 9 planes of straws in each station, arranged as 3 views ( $Y, U, V)$ with 3 planes per view. The straw chamber resolution is assumed to be 150 microns.

- A RICH detector with aerogel, $\mathrm{C}_{4} \mathrm{~F}_{10}$ gas and a Pyrex mirror. The RICH performance is normally parameterized during the analysis phase. A more complete RICH simulation is outlined in Appendix A.

- A box-like electromagnetic calorimeter with $\mathrm{PbWO}_{4}$ crystals which are $2.6 \mathrm{~cm}$ on a side and $22.00 \mathrm{~cm}$ in length.

- A muon system with 3 muon detectors and 2 muon absorbers in each arm. 


\subsection{Physics Event Generators}

Pythia 6.129 [19] was used to generate physics processes for the BTeV simulation studies presented in this proposal. For these studies, Pythia was told not to decay hadrons containing heavy quarks; the decays of hadrons containing heavy quarks were modeled through the CLEO decay Monte Carlo QQ 9.2b [20]. Both BTeVGeant and MCFast use StdHep v4_09 [7] as an interface to the generator packages. The Evgen framework that is provided with the MCFast package provides a uniform command interface to the standard particle physics event generators; other generators such as Herwig [21] and Isajet [22] can be easily used for physics studies.

The size of the interaction region was taken as $\sigma_{x}=\sigma_{y}=50 \mu \mathrm{m}$ and $\sigma_{z}=30 \mathrm{~cm}$ corresponding to our current understanding of the projected running conditions in the $\mathrm{C} 0$ interaction region. The number of interactions per crossing at a beam crossing frequency of $132 \mathrm{~ns}$ is expected to be on average 2 at a luminosity of $2 \times 10^{32} \mathrm{~cm}^{-2} \mathrm{~s}^{-1}$, which means that there will often be more than one interaction per crossing. The effects of multiple interactions per crossing have been accounted for in these simulation studies. Simulation events include the signal interactions, containing primary $b$ or $c$ quarks, and the so called minimum bias events, which include elastic $p \bar{p}$ scattering, diffractive $p \bar{p}$ scattering and various QCD parton scattering processes producing quarks and gluons. Approximately $1.6 \%$ of the minimum bias interactions produce a $c \bar{c}$ pair and approximately $0.13 \%$ of the interactions produce a $b \bar{b}$ pair.

In both BTeVGeant and MCFast, each simulated beam crossing is built up from one or more interactions between beam particles. The construction of events utilizes the relationship between multiple, independent Poisson processes: the sum of $N$ Poisson processes, each with mean $n_{i}$, is itself a Poisson process with a mean of $n$, where $n=\sum_{i=1}^{N} n_{i}$. For the study of signal processes involving the decay of a $b$ or $c$ quark, a beam crossing is simulated by selecting exactly one $b \bar{b}$ (or $c \bar{c}$ ) event and a Poisson distributed number of minimum bias events; the mean of the Poisson distribution is 2 interactions per crossing. 


\subsection{ECAL Cluster Finder Package}

\subsubsection{Event Information from the ECAL Detector}

$\mathrm{BTeV}$ plans to use two electromagnetic calorimeters, each about $7.5 \mathrm{~m}$ away from the interaction region, one in the forward direction - positive $z$ - and the other in the backward direction - negative $z$. They are made from $\mathrm{PbWO}_{4}$ crystals and, in the baseline simulation, each ECAL wall contains 11,850 crystals, each connected to a phototube. In the baseline design for the ECAL, the crystals are arranged in a projective geometry such that each crystal points back to the interaction. See also Chapter 7 .

The light generated in each crystal from electromagnetic showers or other charged particles is detected and converted into a digitized signal that is proportional to the number of photons incident on the photocathode. After a calibration step these numbers represent the total amount of energy deposited in each crystal for each event. From the array of energies for each calorimeter, we extract the following information:

- Shower information that includes a total energy and a position measurement.

- Matching information that connects particle tracks and showers.

- Shape information, specifically tuned to recognize overlapping showers and the case of two close by photons coming from the same $\pi^{0}$.

The two programs employed to simulate BTeV, BTeVGeant and MCFast, generate the same output structures to describe the hit pattern in the calorimeter crystals. It is a general description of a two dimensional array, but the shape of the segmentation into single crystals, also called cells, is not restricted to rectangles alone. We exploit this feature to simulate two different geometries, one made from parallel blocks of crystals, and the other made from an array of crystals each of which points to the interaction region. The second variant is referred to as the pointing or projective geometry.

Both MCFast and GEANT have been used to simulate an ECAL made from a stack of parallel crystals. In this geometry, each crystal has the same dimensions of $2.6 \times 2.6 \times 22 \mathrm{~cm}^{3}$. The inner surface of each crystal is placed $\pm 739 \mathrm{~cm}$ in $z$, and a hole for the beam is simulated by leaving out the central $6 \times 6$ crystal square.

Only GEANT has been used to simulate the projective ECAL. It is simulated as crystals that are segments made from a spherical shell around the center of the detector. Its inner radius is $739 \mathrm{~cm}$, and its thickness is $22 \mathrm{~cm}$, which corresponds to the length of each $\mathrm{PbWO}_{4}$ crystal. Each segment represents a single crystal and is made from a slice in $\theta$, the angle between a point and the beam line, and $\phi$, the azimuthal angle around the beam. The area of each segment is kept close to the area of a $2.6 \times 2.6 \mathrm{~cm}^{2}$ real crystal, but the shape of the simulated segment is slightly curved. While this does not exactly describe the crystals which will be manufactured, we believe that this model simulates the response of a projective geometry well enough for the purposes of this proposal. 


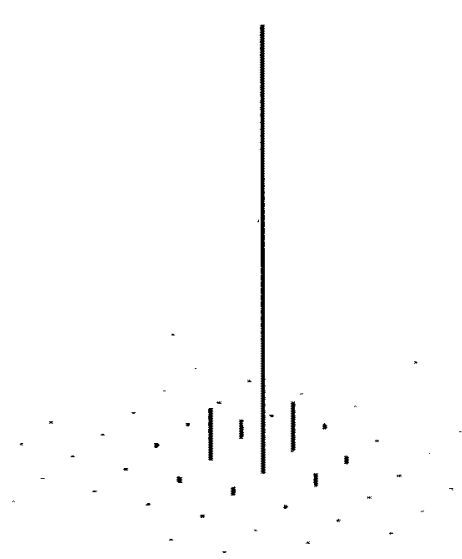

Figure 12.15: Hit pattern in a photon cluster in GEANT; The length of each line represents the energy in a single crystal. The simulation uses an internal energy cutoff to restrict the shower propagation. In this picture GEANT used a value of $100 \mathrm{keV}$.

\subsubsection{Definition of the Cluster Finder Output Data .}

The cluster finder algorithm is designed to identify specific patterns in the calorimeter hit data. The two defined patterns are:

- Cluster - a region of crystals with connected topology; and

- Bump - a local maximum in the energy response within a cluster.

Figure 12.15 shows the result of the showering simulation in GEANT for a single $20 \mathrm{GeV}$ photon. Our cluster finder algorithm will employ threshold cuts so we can balance the quality of the energy estimate for a shower, which improves with the inclusion of many crystals, and the probability of adding energy from nearby showers, which is minimized by using fewer crystals. We expect a readout related threshold of $\simeq 2.5 \mathrm{MeV}$, which removes some of the hits in the shower shown above, and which reduces the data volume produced by the ECAL electronics. However, in this algorithm we added another energy threshold, at the level of $10 \mathrm{MeV}$, to ensure that the energy estimates use only the core of the shower pattern. In this way we can reduce the likelihood that overlapping showers will contaminate the true energy of each other.

A cluster is the basis of the algorithm. It represents all connected crystals that have an energy higher than the cluster threshold. The shape of a cluster can vary, since it only requires the crystals to be neighbors when it adds them to the same cluster. However, a crystal can only be part of one cluster.

A bump is centered around a crystal cell in a cluster that has the maximum energy deposited into it compared to its adjacent crystals, and thus represents a local maximum. 

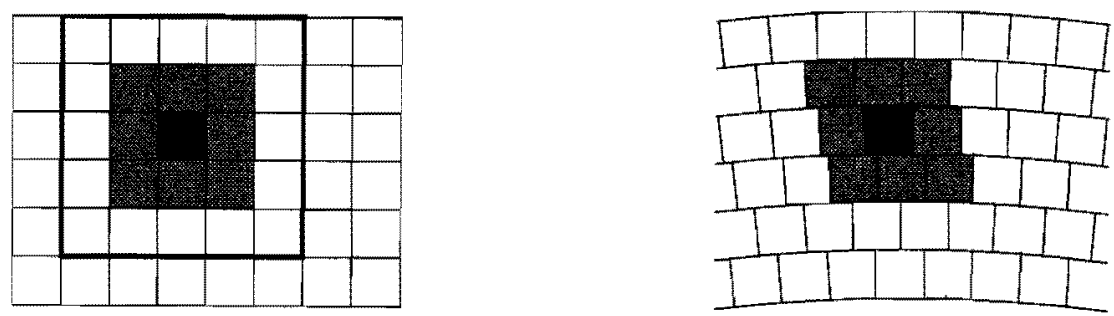

Figure 12.16: Geometrical placement of cells in the parallel (left) and pointing (right) ECAL simulations.

It has a fixed shape made from rings of neighboring cells. Figure 12.16 shows parts of the crystal arrangement from the parallel and projective ECAL geometries. The gray colored squares are considered neighbors of the cell filled with black color. All these cells belong to the bump, even if they are also in another bump or even in a different cluster. They only need to have an energy larger than the energy threshold. If a cell is part of more than one bump, an unfolding algorithm will partition the energy of that cell assigning some fraction of its energy to each bump to which the cell belongs.

\subsubsection{General Algorithm}

The first step in the cluster finder algorithm is to create connected regions of crystals with an energy higher than a tuneable threshold, known as the clustering threshold. The program uses a table of neighbor coordinates per cell to grow a cluster outwards from a starting cell. Thus, the same code is used for all supported geometries. The starting cell is selected from the list of cells that are sorted by energy and contain energies higher than the pedestal threshold. Any energy lower than this threshold is always ignored; therefore, a cell with a low energy response is not added to any cluster. A cell can only be assigned to one cluster, and after it has been added to a growing cluster, it is no longer considered when the code starts a new cluster.

After all cells are used to build clusters, each cluster is searched for local maxima. The same table of neighbors is used to compare a candidate cell's energy with each of its adjacent cells' energy. If it is found to be a local maximum, the cell is used as the center of a bump. Again, we use a tunable threshold to control the minimum energy of a center cell in a bump.

Each object, i.e. cluster or bump, that is generated contains data recording its energy, position, and shape. A program using the output of the cluster finder can efficiently access those items from memory. Also, a list of crystals assigned to each object is stored, in case an analysis requires additional information.

The most important data generated for clusters and bumps are the total energy and the position. These algorithms impose the previously mentioned energy threshold cut on the energy of a cell before it is used. By imposing this cut we can improve the resolution of these measurements using a calibration function to correct for the expected difference between the energy sum and the real energy in the shower. The total energy of a cluster is measured 


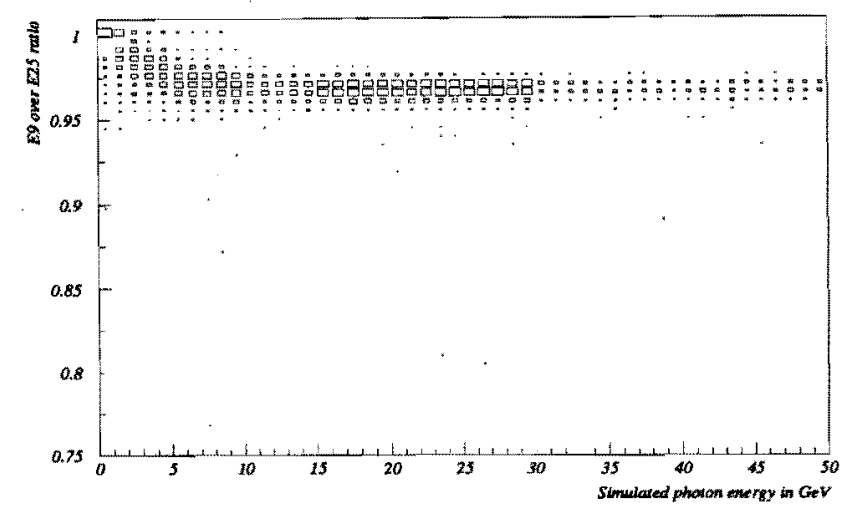

Figure 12.17: Measured ratio of E9 over E25 versus simulated photon energy.

using the overall energy sum for all crystal cells with a response that crosses the energy threshold. If the clustering threshold is higher than the energy threshold, then the cluster threshold will truncate the energy summation at the edge of the cluster.

The energy algorithm for a bump is different from that for a cluster. It is assumed that a bump represents an electromagnetic shower, initiated by a photon or electron. Due to the shape of the shower propagation, most of the energy is contained in the center cell and its nearest neighbors (for square segments there are 8 neighbors), with small leakage into further away neighbors. The sum of the energy in these cells is referred to as E9, since it contains contributions from up to 9 cells. To estimate the total energy of such a shower, we chose to use all adjacent cells and all cells of the next closest ring. This energy sum is called E25, since we add 25 cells in total, if they all cross the energy threshold. We use the ratio E9/E25 to test the hypothesis that the bump represents an electromagnetic shower. Figure 12.17 shows the distribution of this ratio for single photon events simulated by GEANT.

We use the second moment and the derived value of the "second moment mass" for a cluster to identify showers that contain two photons from the same $\pi^{0}$ but which only have one local maximum. The second moment is defined as

$$
M(2)^{2}=\sum_{\text {cells }} E_{\text {cell }}\left(\vec{C}-\vec{x}_{\text {cell }}\right)^{2} / \sum_{\text {cells }} E_{\text {cell }}
$$

where $\vec{C}$ is the unit vector to the center-of-gravity of a cluster, $E_{\text {cell }}$ is the energy response of each cell in a cluster, and $\vec{x}_{\text {cell }}$ is the unit vector to that cell. We find the values of this variable for single photon showers, $(M(2, \gamma))$, by using GEANT simulations and then obtain the "second moment mass" using,

$$
m^{2}=\left(M(2, \text { cluster })^{2}-M(2, \gamma)^{2}\right) E_{\text {cluster }}^{2}
$$

This variable will peak at the invariant mass of the $\pi^{0}$ if the cluster contains the two photons from the decay of one $\pi^{0}$. Figure 12.18 shows the distribution of $m^{2}$ for $\gamma$ and $\pi^{0}$ particles with energies of $40 \mathrm{GeV}$ and $70 \mathrm{GeV}$. At $40 \mathrm{GeV}$ a clear separation between merged $\pi^{0}$ 

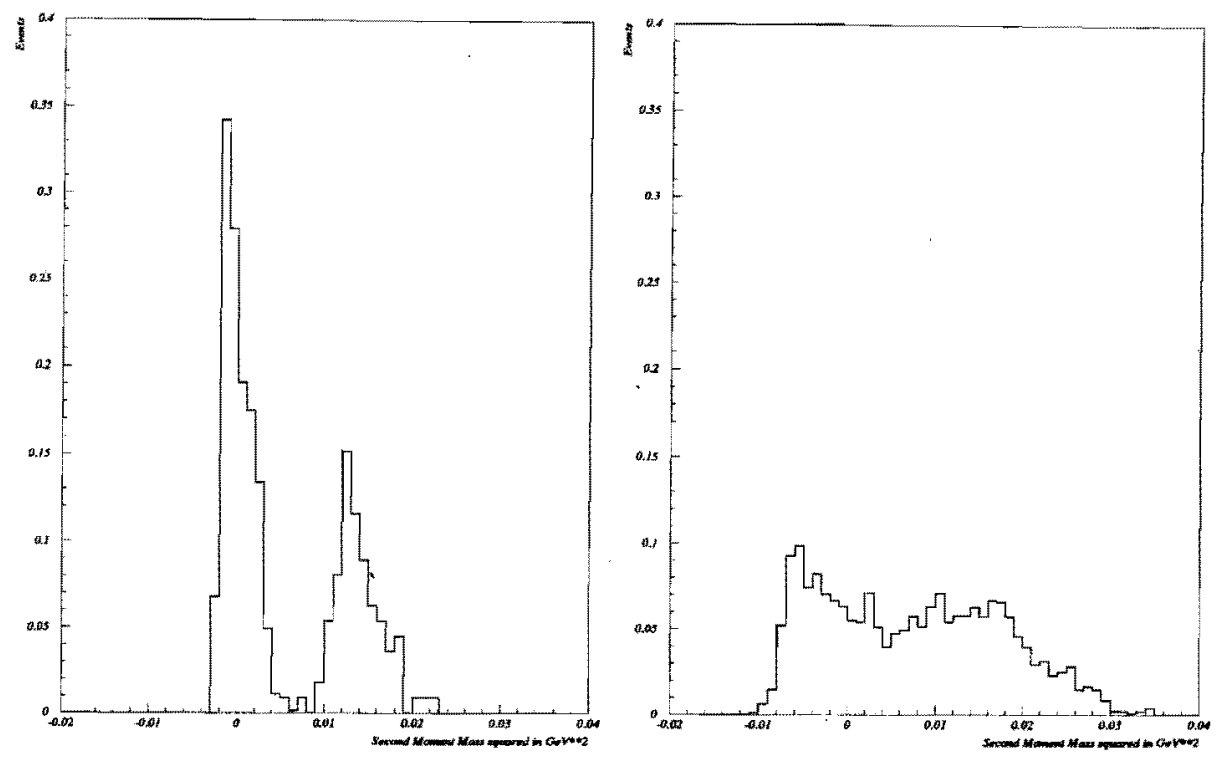

Figure 12.18: Second moment mass squared distribution for $40 \mathrm{GeV}$ (left) and $70 \mathrm{GeV}$ clusters (right). The simulated events contain either a single $\gamma$ or a single $\pi^{0}$. Each contribution due to one of the particle species is normalized to 1 .

showers and photon clusters is visible. When the energy of the cluster reaches $70 \mathrm{GeV}$ it becomes more difficult to distinguish the two sources for clusters. Still, a cut to suppress $99 \%$ of all photons will have an efficiency of about $64 \%$ for finding a $\pi^{0}$ cluster with that energy.

\subsubsection{Important Corrections}

Various effects introduce biases into the raw results obtained using the algorithms described above. There are basic geometric effects and effects due to limitations on the measurements. These effects can change event by event, and differ from photon to photon in the same event. The clustering code corrects for these effects either directly, as for the impact angle of the photon, or on a statistical basis as in the case of the expected average loss in the energy measurement. Here is the list of corrections put into the clustering code:

1. Average energy measurement residual, which depends on the total energy found in the bump.

2. Position bias correction for the center-of-gravity of the bump, "S curve correction."

3. Unfolding of overlapping showers that share the energy response from the same cell.

4. Correction of shape and position measurements for the primary vertex position. 
5. Correction of the impact position for the non-projective geometry, " $z$-depth correction," which is used in the MCFast simulation.

The described algorithm and corrections can be improved with further study.

\subsubsection{Implementation and Performance}

The core of the clustering algorithm was written as a library that accepts user and simulation data input, generates the above described objects, and gives access to the information stored in them. The described calibration and correction parameters were found using single photon and $\pi^{0}$ event data.

We then tested the performance of the calorimeter and the clustering code by analyzing simulations of two different classes of events: the first class of events was artificial events in which only a single $\gamma$ or a single $\pi^{0}$ was produced; the second class was realistic events, which included selected $B$ meson decay chains plus a Poisson distributed number of background interactions per event. Some of these results are discussed in Chapter 7 of this proposal.

Next, we show the general performance of the cluster finder algorithm by plotting the finding efficiency for events which contain only a single photon. The finding efficiency for a photon was measured by,

- analyzing the simulated events using the cluster finder library,

- looking for the neutral shower that gives the closest match with the momentum vector from the generator level,

- and then accepting only those showers where the measured energy agrees with the generated photon's energy within $3 \sigma$.

In all analyses, we impose standard cuts to select neutral shower candidates, using the ratio of E9 over E25 to select showers consistent with the shower shape of an isolated photon, and demanding that the shower be well isolated from charged tracks which project onto the ECAL. The latter cut is only relevant when analyzing $B$ events, not single photon events. Figure 12.19 shows the obtained efficiencies for events containing only a single photon. The finding efficiency is not $100 \%$, because it includes effects from the material, such as $e^{+} e^{-}$pair creation, between the creation point and the impact point in the ECAL. As expected, there is no change in the efficiency with the energy of the photon, however we do see a change with the radial position in the ECAL. The reason is the change in the total radiation length seen by the photon when crossing various detector components. Also, at the physical edge of the ECAL we lose efficiency due to leakage of parts of the shower into a region with no crystals. This result should be compared to the radial finding efficiency when the photon is embedded in realistic events, as shown in Chapter 7.6, which includes the effects of masking due to tracks and nearby showers. 

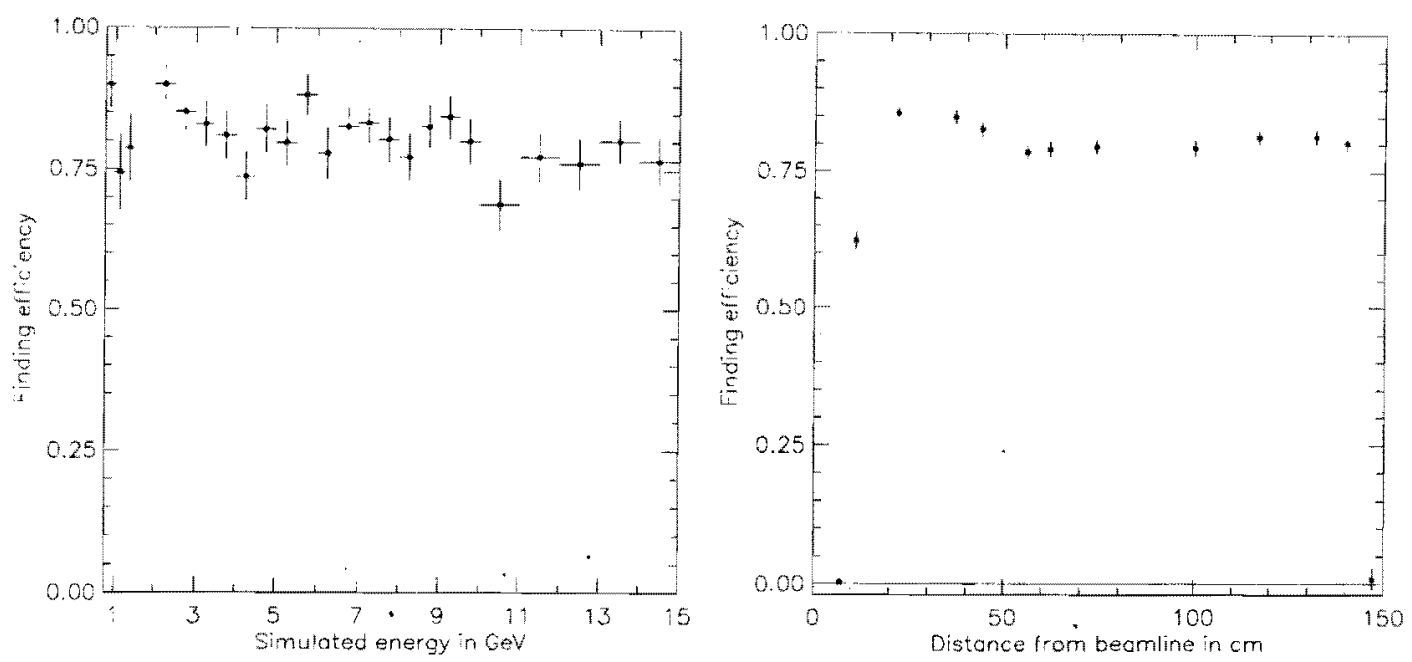

Figure 12.19: Fraction of correctly found photons in the calorimeter. (a) versus simulated energy, at fixed radius $(100 \mathrm{~cm})$, (b) versus radius of impact point in ECAL, at fixed energy $(10 \mathrm{GeV})$. See the text for the selection criteria.

\subsubsection{Electron Identification using the ECAL Cluster Finder}

The clustering algorithm was used to measure the performance of the calorimeter for identifying electrons and positrons. A sample of $B^{0} \rightarrow D^{*-} \rho^{+}$events simulated using BTeVGeant was used in this study, this sample contains a mean of 2 , Poisson distributed, non-beauty background interactions per event.

For reconstructed (charged) tracks that enter into the calorimeter acceptance, Fig. 12.20(a) shows the distance of the closest reconstructed bump for both electrons, (positrons implied also), and hadrons. Pions, kaons and protons are taken together for this study. About $85 \%$ of all electrons with momentum greater than $1 \mathrm{GeV} / \mathrm{c}$ have a matching bump within $2.5 \mathrm{~cm}$. For these tracks with matching bumps, Fig. 12.20(b) shows the ratio of the reconstructed bump energy to the track momentum $(E / p)$. As expected, the $E / p$ values for electrons are sharply peaked near 1 whereas hadrons have low values of $E / p$. The dotted line in Fig. 12.20 (b) shows the $E / p$ values for the approximately $15 \%$ of the hadrons which deposit energy in essentially a single crystal. These are either non-interacting hadrons or ones that have decayed to a muon before entering the calorimeter. Figure 12.20(c) shows the shower shape variable E9/E25 for tracks with matching bumps, for both electrons and hadrons.

Electron identification using the calorimeter information can be provided for tracks that enter into the acceptance of the calorimeter. The simple criteria used for this study in identifying a track are:

- The track must have a matching bump in the calorimeter within $2.5 \mathrm{~cm}$.

- $0.90<E / p<1.05$. 


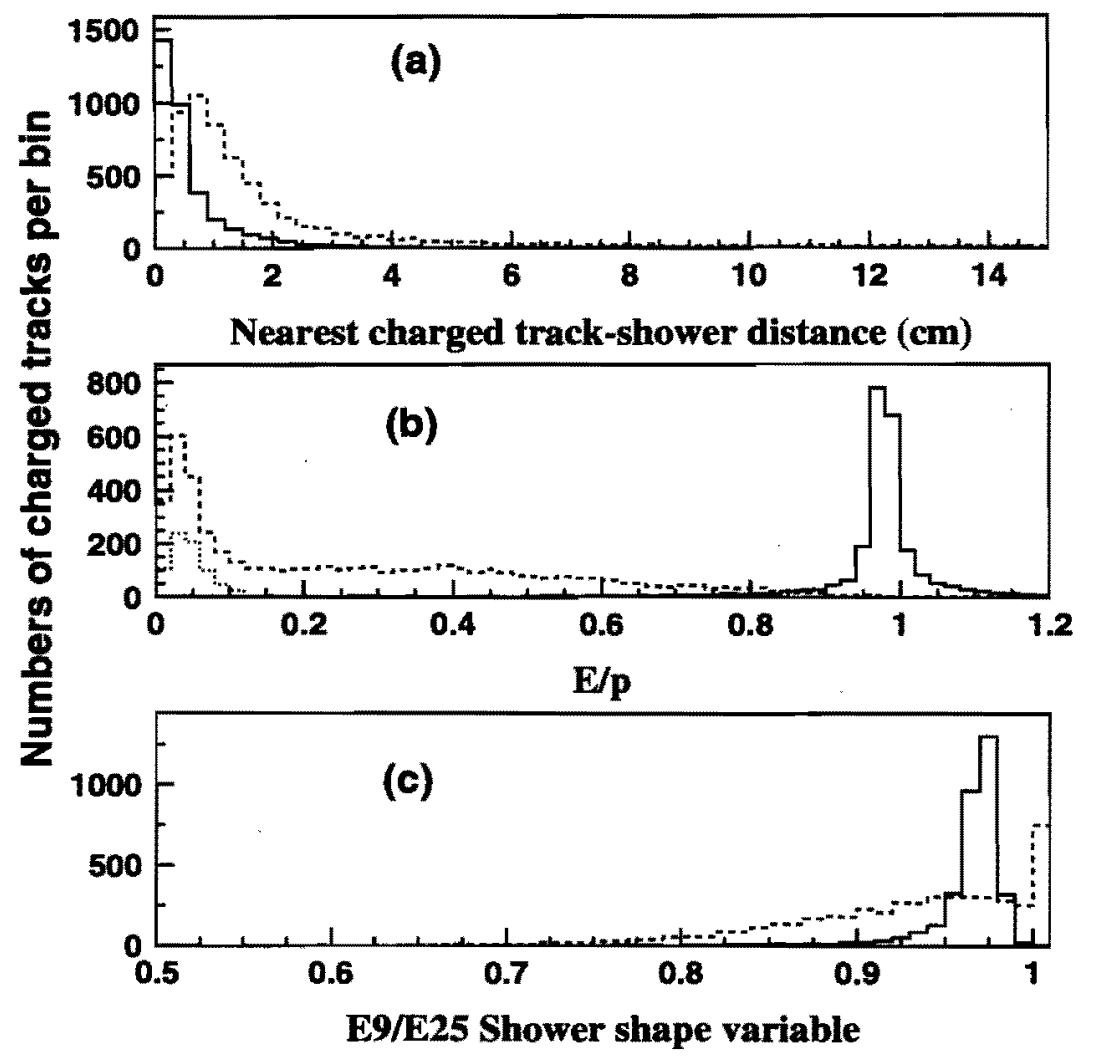

Figure 12.20: (a) Distance of charged tracks to the closest reconstructed bump in the calorimeter for electrons/positrons (solid line) and for $\pi / \mathrm{K} / \mathrm{p}$ hadrons (dashed line); (b) $E / p$ for reconstructed charged tracks for electrons (solid line), all hadrons (dashed line), and hadrons with E9/E25=1 (dotted line); (c) E9/E25 shower shape variable for electrons (solid line) and hadrons (dashed line). 

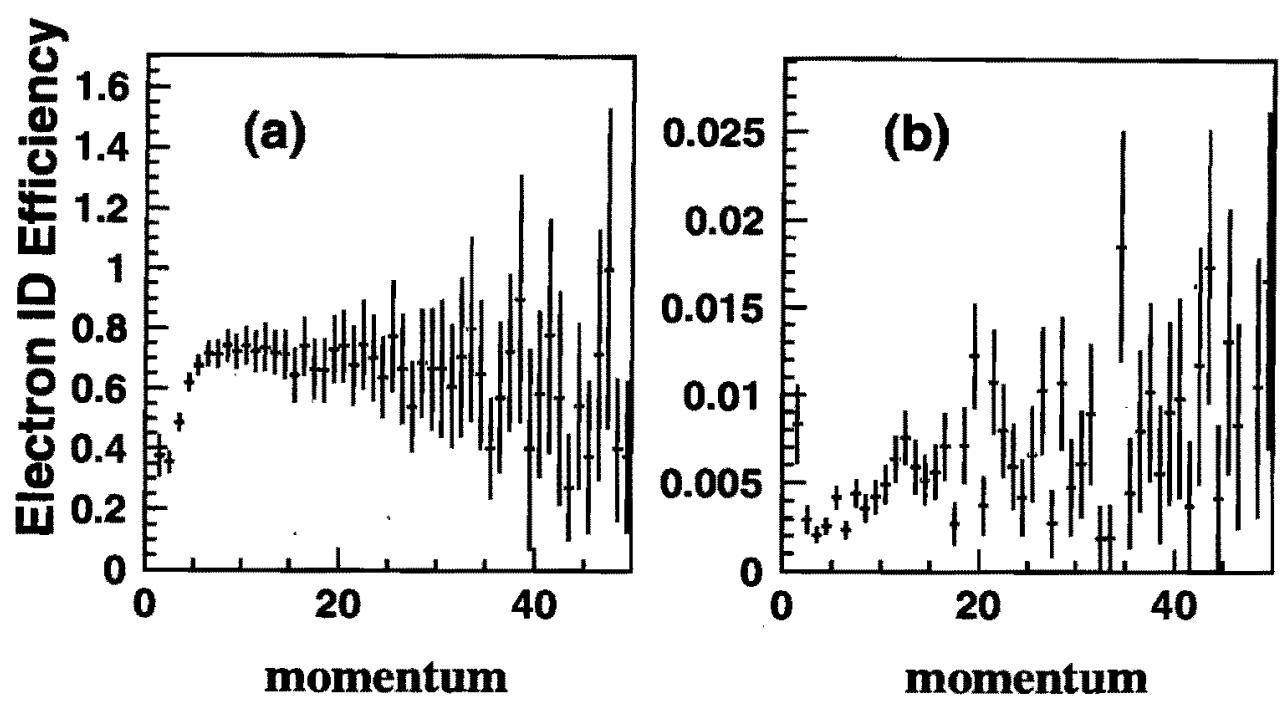

Figure 12.21: Electron. identification efficiency as a function of track momentum in $\mathrm{GeV} / \mathrm{c}$ for (a) electrons; and (b) hadrons.

- $E 9 / E 25>0.90$.

Figure 12.21(a) shows the efficiency for correctly identifying an electron as a function of its momentum using these criteria. The level of misidentification of a hadron as an electron is shown in Figure 12.21(b) and is less than about 1\%. By tightening the selection criteria on $E / p$ and $E 9 / E 25$ this misidentification rate can be significantly reduced with little loss in the efficiency for identifying real electrons. 


\section{Bibliography}

[1] P. Avery et al., "MCFast: A Fast Simulation Package for Detector Design Studies", in the Proceedings of the International Conference on Computing in High Energy Physics, Berlin, (1997). Documentation can be found at http://www-pat.fnal.gov/mcfast.html .

[2] The BTeV Collaboration, http://www-btev.fnal.gov/public_documents/ptdr/ptdr.html .

[3] GEANT: CERN Program Library Long Writeup W5013, http://wwwinfo.cern.ch/asdoc/geant_html3/geantall.html .

[4] A description of GEANT4 is available at http://wwwinfo.cern.ch/asd/geant4/geant4.html .

[5] TOSCA is a Finite Element Analysis package for electromagnetic calculations and is distributed by Vector Field.

[6] The MCFast calorimetry package is described in, http://www-pat.fnal.gov/mcfast/version_doc/v5_0/simulator/calor_user_guide.ps .

[7] A description of StdHep is available at http://www-pat.fnal.gov/stdhep.html .

[8] A description of MCFIO is available at http://www-pat.fnal.gov/mcfio.html .

[9] P. Billoir, Nucl. Instrum. Meth. A225, 352 (1984).

[10] Paul Avery, http://www.phys.ufl.edu/ avery/fitting.html, Fitting Theory V: Track Fitting Using the Kalman Filter.

[11] M.Regler and R. Fruwirth, "Reconstruction of Charged Particle Trajectories", Plenum Publishing Company (1989).

[12] R. Bock et al., "Data Analysis Techniques for High-Energy Physics Experiments", Cambridge University Press (1990).

[13] Paul Avery, http://www.phys.ufledu/ avery/fitting.html, "Applied Fitting Theory VII: Building Virtual Particles" and "The Data Analysis and Kinematic Fitting With the KWFIT Library." 
[14] J. Dumarchez et al., "Average Multiplicity and KNO Scaling in Hadron-Hadron Interactions", Il Nuovo Cimento 66A, 114 (1981).

[15] A. Firestone et al., " $\pi^{-} p$ interactions at $360 \mathrm{GeV} / \mathrm{c}$ : Measurement of the total and elastic cross sections and the charged-particle multiplicity distribution", Phys. Rev. D14, 2902 (1976).

[16] H. Fesefeldt, "GHEISHA: The simulation of Hadronic Showers - Physics and Applications", PITHA 85/02.

[17] A. Brenner et al., "Experimental Study of single-particle inclusive hadron scattering and associated multiplicities", Phys. Rev. D26, 1497 (1982).

[18] C. W. Akerlof et al., "Hadron-proton elastic scattering at 50, 100, and $200 \mathrm{GeV} / \mathrm{c}$ momentum", Phys. Rev. D 142864 (1976).

[19] H. U. Bengtsson and T. Sjostrand, Comput. Phys. Comun. 46, 43 (1987).

[20] QQ is developed and maintained by the CLEO Collaboration. See http://www.Ins.cornell.edu/public/CLEO/soft/QQ/ For the studies reported here, it is used for its model of the decays of hadrons containing heavy flavors.

[21] G. Marchesini and B.R. Webber, Nucl. Phys. B310, 461 (1988); Nucl. Phys. B330, 261 (1988);

I. G. Knowles, Nucl. Phys. B310, 571 (1988);

G. Abbiendi, et al., Comp. Phys. Comm. 67, 465 (1992).

[22] F. Paige and S. Protopopescu, Brookhaven National Laboratory Report BNL-37066 (1985) (unpublished). 


\section{Chapter 13}

\section{Comparison of MCFast and BTeVGeant for the Calorimeter and Tracker}

This chapter describes studies which were done to compare various quantities computed using BTeVGeant with the same quantities computed using MCFast. The two programs were described in Chapter 12. The purpose of these studies was two-fold, to catch errors in the representation of the detector inside the programs and to demonstrate the validity of the approximations and parameterizations made in MCFast. The first section of this chapter compares the simulation of the electromagnetic calorimeter and the second section compares the simulation of the tracking system.

\subsection{MCFast vs. BTeVGeant showering}

This section describes the comparison of the showering results generated by the two simulation packages, MCFast and GEANT. The showering in MCFast is parameterized, but gives a reliable representation of the longitudinal and transverse profiles of electromagnetic showers which can be used for many physics and trigger studies. In fact, many of our early studies of photon detection efficiency done with MCFast have been confirmed by BTeVGeant. Studies which involve examining the details of the shower shape, such as merged $\pi^{0}$ reconstruction, can also be done with MCFast, but we chose to use the more detailed simulation provided by BTeVGeant for the analyses presented in this proposal.

The clustering algorithm used for reconstructing showers is exactly the same for BTeVGeant and MCFast. The output of the clustering step can be used to illustrate some of the differences between the two showering models. For this study, we have used events containing only a single photon with a fixed energy of $20 \mathrm{GeV}$ originating at the center of the $\mathrm{BTeV}$ detector and at an angle of about $100 \mathrm{mrad}$ from the beam line so that the shower will be well contained in the calorimeter. The details of the upstream detector differ slightly in the two simulation packages; the MCFast geometry description is missing some of material in the support structures. The size, composition and layout of the two calorimeters is the same in MCFast and BTeVGeant. We reconstructed clusters from hit patterns generated by 

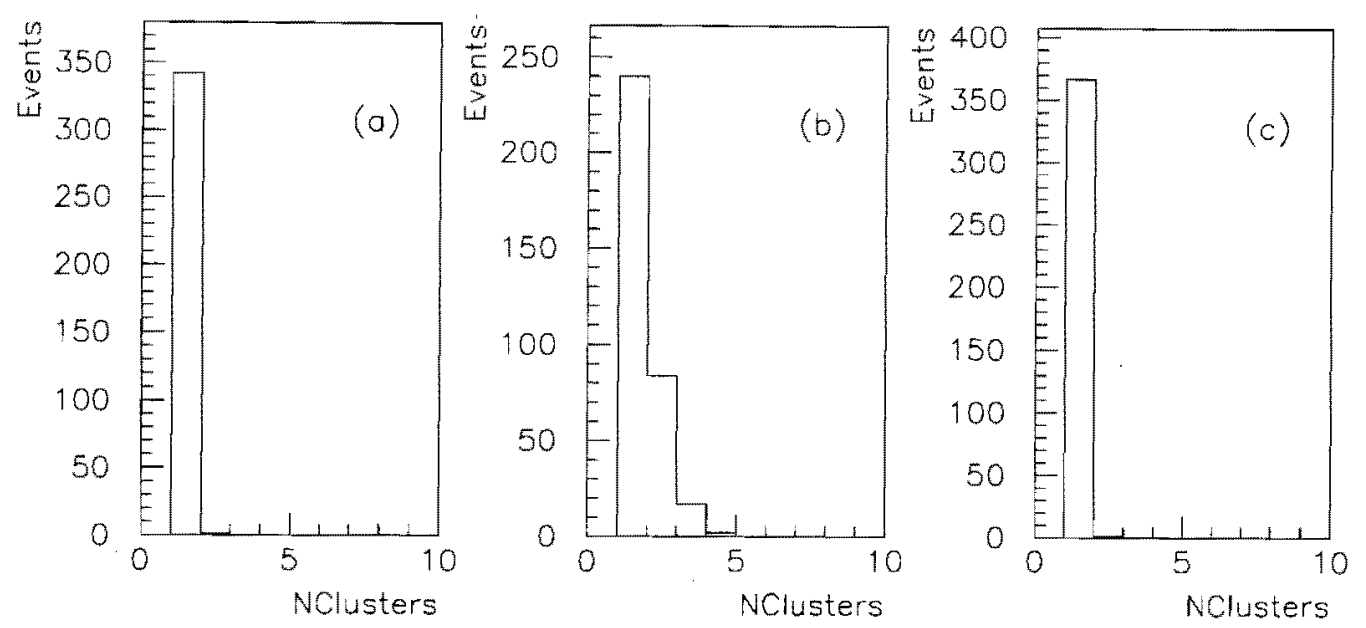

Figure 13.1: Comparison of the number of found clusters in the BTeV calorimeter for single photons of $20 \mathrm{GeV}$; (a) GEANT with a $40 \mathrm{MeV}$ cluster threshold, (b) GEANT with a $10 \mathrm{MeV}$ cluster threshold, (c) MCFast with a $40 \mathrm{MeV}$ cluster threshold. In each case, the primary cluster contains most of the energy. The secondary clusters are made from only one or two crystals, each containing a small amount of energy.

the two packages in the crystal calorimeter and compared the resulting reconstructed cluster information. A cluster is a set of crystals that form a connected region. Each crystal in a cluster must have an energy greater than the cluster threshold in order to be included in the cluster. The default cluster threshold is $40 \mathrm{MeV}$ for both BTeVGeant and MCFast shower reconstruction.

A shower generated by a single photon should make one cluster in the calorimeter. To ensure that the original photon actually initiated the shower in the electromagnetic calorimeter (ECAL), we excluded photons that pair converted in the detector elements in front of the ECAL. MCFast sometimes creates two clusters because it randomly deposits discrete amounts of energy in its modeling of the transverse shower spread. The energy deposition is calculated for each trace step and then subdivided into a number of discrete energy packets called subhits. Each subhit is distributed randomly around the shower axis according to the formulae given in Section 12.2.4. An electromagnetic shower in MCFast is truncated at 4.5 Moliere radii from the shower axis.

During the reconstruction of a cluster, we impose a clustering threshold, or a minimum energy for a cell to be included in a cluster. The total number of crystals in the reconstructed cluster can vary with the cluster threshold. In MCFast, the default smallest subhit produced for a $20 \mathrm{GeV}$ photon shower is normally higher than the default $40 \mathrm{MeV}$ cluster threshold, so the number of hits in the reconstructed cluster is not sensitive to reducing the cluster threshold. GEANT showering produces hits in a large number of crystals but many contain subthreshold energies, so the number of hits per cluster can be very sensitive to the cluster energy threshold.

Figure 13.1 shows a plot of the number of clusters found in single $20 \mathrm{GeV}$ photon events. 

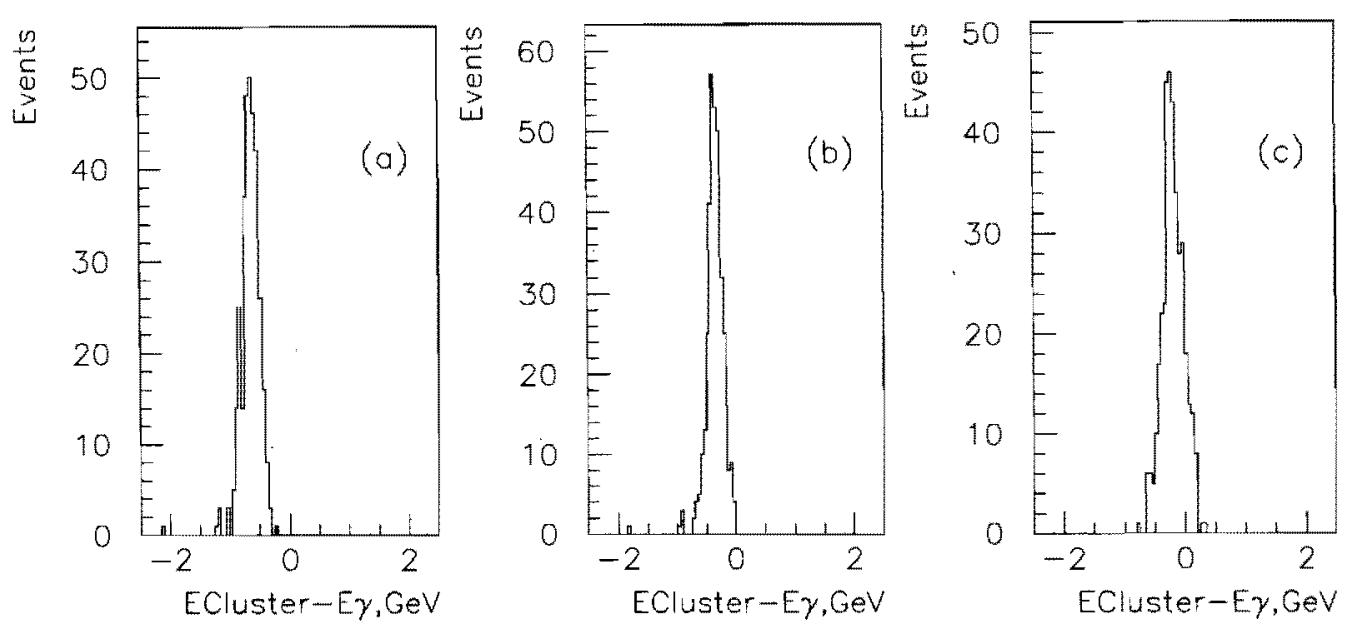

Figure 13.2: Comparison of the relative difference between the cluster energy and total energy from a $20 \mathrm{GeV}$ single photon; (a) BTeVGeant reconstructed with a $40 \mathrm{MeV}$ cluster threshold per crystal, (b) BTeVGeant with a $10 \mathrm{MeV}$ cluster threshold per crystal, (c) MCFast with a $40 \mathrm{MeV}$ cluster threshold per crystal

As expected, both MCFast and BTeVGeant normally create one cluster of crystals in each event. The MCFast shower model occasionally produces an extra cluster containing a small fraction of the total energy. The second cluster is not produced when the number of subhits in the modeling of the transverse shower spread is increased. GEANT produces disconnected clusters when the cluster threshold is decreased to $10 \mathrm{MeV}$. These secondary clusters contain only a small fraction of the total energy.

- Figure 13.2 shows the difference between the sum of crystal energies in the largest reconstructed cluster and the total energy of the photon. The width of the distribution is largely due to the smearing expected from photo-statistics. Showers generated by BTeVGeant and reconstructed with a cluster threshold of $40 \mathrm{MeV}$ (a) have a small tail on the negative side of the distribution, which indicates that the shower simulation produced a significant number of hits with energies less than the clustering threshold and thus the overall energy in the shower was underestimated in reconstruction. Reducing the cluster threshold improves the situation, both shifting and sharpening the peak, as it adds in energy from the outer edges of the shower. MCFast (c) does not have a large tail since most hits are above the cluster threshold, but the distribution is broader. Note that leakage out of the back of the crystals would result in an overall shift of the distribution.

For electromagnetic showers, the shower energy is normally determined during analysis from the bump energy (E25), which sums the energy in the 25 blocks surrounding and including the seed crystal which is the crystal with a locally maximum amount of energy (see Section 12.4). The bump energy E25 is not as sensitive to the detailed simulation of the shower shape as the reconstructed cluster energy since most of the energy from a photon is mostly contained within 25 crystals in the BTeV calorimeter. Figures 13.3 and 13.4 show the reconstructed bump energies from the sum of 25 and 49 crystals surrounding the center of 

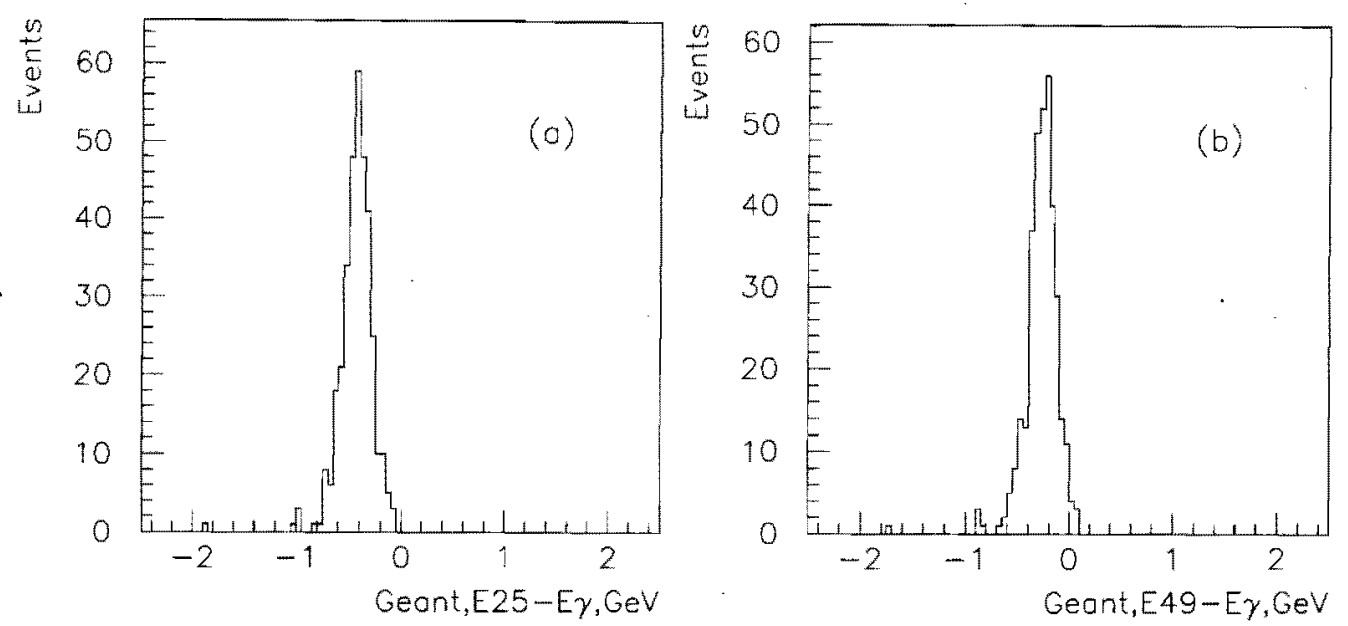

Figure 13.3: The difference between the photon energy and the reconstructed bump energies for $20 \mathrm{GeV}$ photons as simulated by BTeVGeant. The bump energy is determined from the sum of crystal energies (a) E25 - summing 25 crystals around the peak; and (b) E49 summing 49 crystals.
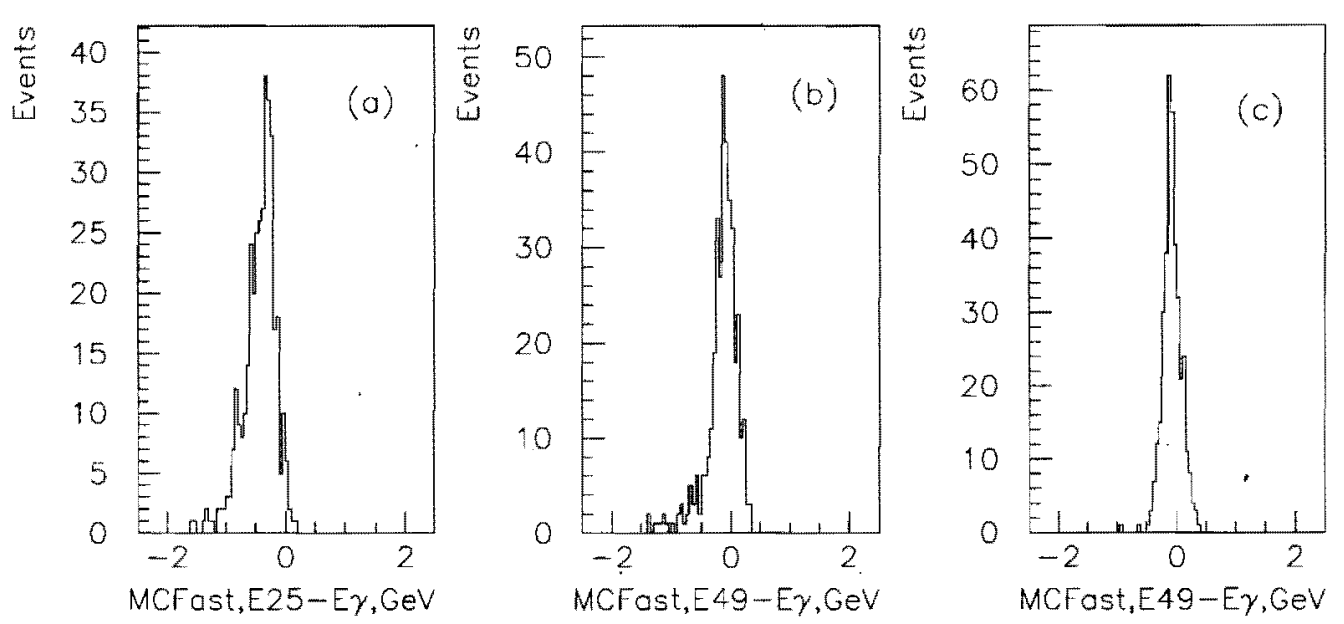

Figure 13.4: The difference between the photon energy and the reconstructed bump energies for $20 \mathrm{GeV}$ photons as simulated by MCFast. The bump energy is determined from the sum of crystal energies (a) E25 for default MCFast; (b) E49 for default MCFast showers with 25 subhits per step in the transverse shower spread step; and (c) E49 for MCFast showers with 100 subhits per step. 


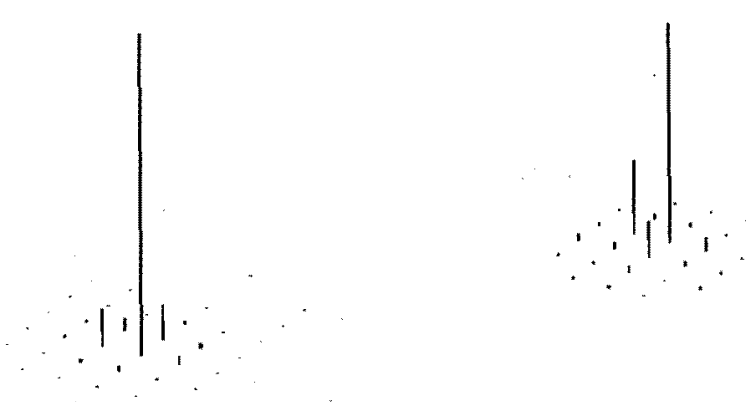

Figure 13.5: Comparison of the hit pattern in a $20 \mathrm{GeV}$ photon cluster as generated by (left) BTeVGeant, (right) MCFast. The length of each line represents the energy in a single crystal. Both plots use the same scale. The minimum energy per crystal is $40 \mathrm{MeV}$.

the bump. There is slightly more leakage to the outer row of crystals in MCFast. The energy resolution improves when 100 subhits are created during each transverse shower spread step.

In general, the BTeVGeant electromagnetic showering model distributes the energy from a shower over more crystals than MCFast and each crystal at the edge of the cluster contains less energy. BTeVGeant deposits more of the energy close to the shower core and small amounts of energy over a large region transversely. MCFast generates a somewhat wider shower core with truncated tails. Figure 13.5 shows the energy pattern in a cluster from BTeVGeant and MCFast for a cluster threshold of $40 \mathrm{MeV}$ per crystal.

The difference in the showering models can also be seen by looking at the number of crystals above the clustering threshold. The total number of crystals will be less if the bulk of the deposited energy stays close to the shower core. Figure 13.6 shows the number of crystals that were used to form the reconstructed cluster. As discussed previously, many crystals in the BTeVGeant simulation do not cross the default threshold of $40 \mathrm{MeV}$, and therefore are not added to the cluster. MCFast and BTeVGeant with a $10 \mathrm{MeV}$ cluster threshold produce nearly the same number of hit crystals per primary cluster.

During reconstruction the details of the shower shape are important for distinguishing between electromagnetic and hadronic showers. The showers in MCFast are similar enough that it is possible to develop efficient selection criteria for photons and electrons in both cases. The hadronic shower shape in MCFast is also parameterized. Full event simulation including electromagnetic and hadronic showers using MCFast has given us a reasonable prediction of the photon efficiency as compared to the more detailed BTeVGeant. Test beam data with photomultiplier readout are not readily available to us, so a complete detailed data vs. Monte Carlo comparison has not yet been done. 

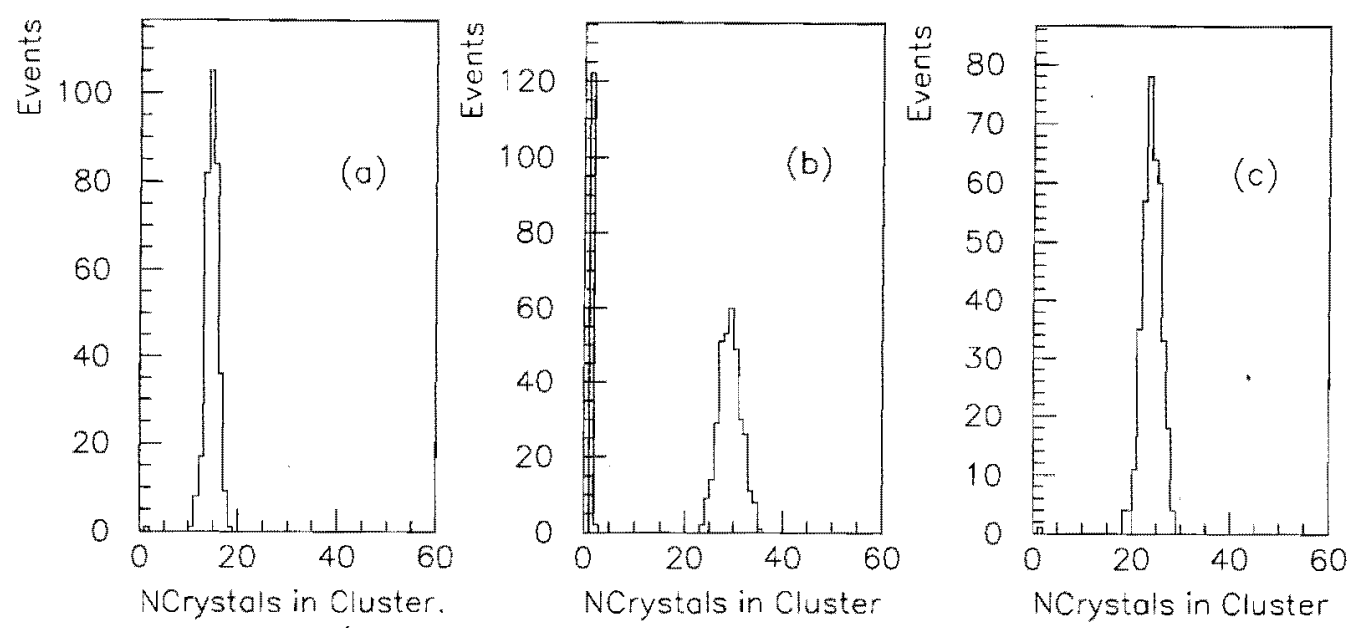

Figure 13.6: Comparison of number of crystals per cluster; (a) BTeVGeant reconstructed with a $40 \mathrm{MeV}$ cluster threshold per crystal, (b) BTeVGeant with a $10 \mathrm{MeV}$ cluster threshold per crystal, (c) and MCFast with a $40 \mathrm{MeV}$ cluster threshold per crystal

\subsection{MCFast vs. GEANT tracking}

\subsubsection{Introduction}

This section-describes comparisons between the BTeVGeant model of the $\mathrm{BTeV}$ tracking system and the MCFast model of the same system. The comparison is made both in low level quantities, such as occupancies and multiplicities, and in high level quantities, such as mass and vertex resolutions.

In order to ensure that the same detector was simulated by the two programs, the MCFast geometry file was generated automatically by BTeVGeant. Because BTeVGeant allows a more sophisticated model of detector elements, some of the finer details of the BTeVGeant model are simplified or are missing from the MCFast model.

$\mathrm{BTeVGeant}$ and MCFast read the same input files but differences in the detector response arise as the input tracks are propagated through the detector. Most prominently, BTeVGeant has a highly developed and complete model of hadronic interactions between particles and the detector material, while MCFast uses a greatly simplified model. In addition, MCFast uses a Gaussian model of multiple scattering while BTeVGeant uses the Moliere model, which has non-Gaussian tails. BTeVGeant explicitly models $\delta$-ray production but MCFast does not. MCFast has simplified models of other processes such as pair creation and bremsstrahlung. MCFast has vacuum, rather than air, between major detector elements - this is most significant in the model of the forward tracking system. In MCFast, showers which develop in one medium, such as the muon shield or the calorimeter, cannot create hits in neighboring tracking systems ${ }^{1}$ in BTeVGeant, showers do create hits in neighboring tracking volumes.

\footnotetext{
${ }^{1}$ They do deposit energy in neighboring calorimeters and that energy is recorded in the calorimeter hit structure.
} 
There is one other subtle effect: as a particle passes through a diffuse medium, such as the RICH gas, BTeVGeant models the scattering as occurring at many places along the track; MCFast models the scattering as occurring at a thin plane at the center of the gas volume. The net effect of these differences is that the two programs produce different estimates for device occupancy, track multiplicity and track parameter resolution. These, in turn, imply different trigger efficiencies, different efficiencies for analysis cuts and different mass resolutions.

The following sections will demonstrate that, although the two programs produce very different estimates of occupancies, they produce very similar estimates for most quantities of physics interest.

\subsubsection{Occupancies .}

Figure 13.7 shows the multiplicity of pixel clusters and the multiplicity of various trigger primitives for $B_{s}^{0} \rightarrow D_{s}^{ \pm} K^{\mp}$ events which were processed using BTeVGeant and which passed analysis cuts similar to those described in section 16.4.1. Figure 13.8 shows the same information except that the events were simulated using MCFast. These figures are similar to Figure 14.1, except they were prepared using events from a different channel; otherwise the information in the caption to Figure 14.1 applies. From these figures one sees that BTeVGeant produces about $20 \%$ more pixel hits than does MCFast. This causes a small, barely noticeable, increase in the doublet yield but no significant change in the yields of triplets or tracks.

The major impact of higher multiplicity is the possibility that it might confuse pattern recognition algorithms. Of the systems discussed in this proposal, the Level-1 trigger has the most potential to be confused by this increased multiplicity. Because the absolute occupancy remains small, the impact of the increase in occupancy is expected to be negligible. This was confirmed by studying the trigger efficiency for the $B_{s}^{0} \rightarrow D_{s}^{ \pm} K^{\mp}$ events used to make Figures 13.7 and 13.8. The trigger efficiencies were measured to be $69 \pm 1 \%$ and $68 \pm 2 \%$, for BTeVGeant and MCFast, respectively. These numbers differ from those presented in Chapter 14 because different cuts were used to define the events for which the trigger efficiency was measured.

In MCFast, the response of the muon system is also simplified; most importantly hadronic showers in the shielding material do not leak out of the back of the shielding block to create hits in the muon counters. Section 8.3.2 describes a BTeVGeant based study of the sources of noise in the muon system; none of the dominant noise sources are modeled in MCFast. An equivalent statement is that, in MCFast, all hits in the muon counters are produced by real muons, including muons from decay in flight of $\mathrm{B}$ mesons, $\mathrm{D}$ mesons, kaons and pions. A figure of merit to compare the two programs is the average occupancy of the muon counters for beam crossings which contain a Poisson distributed number of minimum bias interactions, with a mean of two interactions per crossing. In BTeVGeant the average occupancy was determined to be $1.2 \%$ while in MCFast it was determined to $0.015 \%$.

On the other hand, both MCFast and BTeVGeant properly range out muons as they 

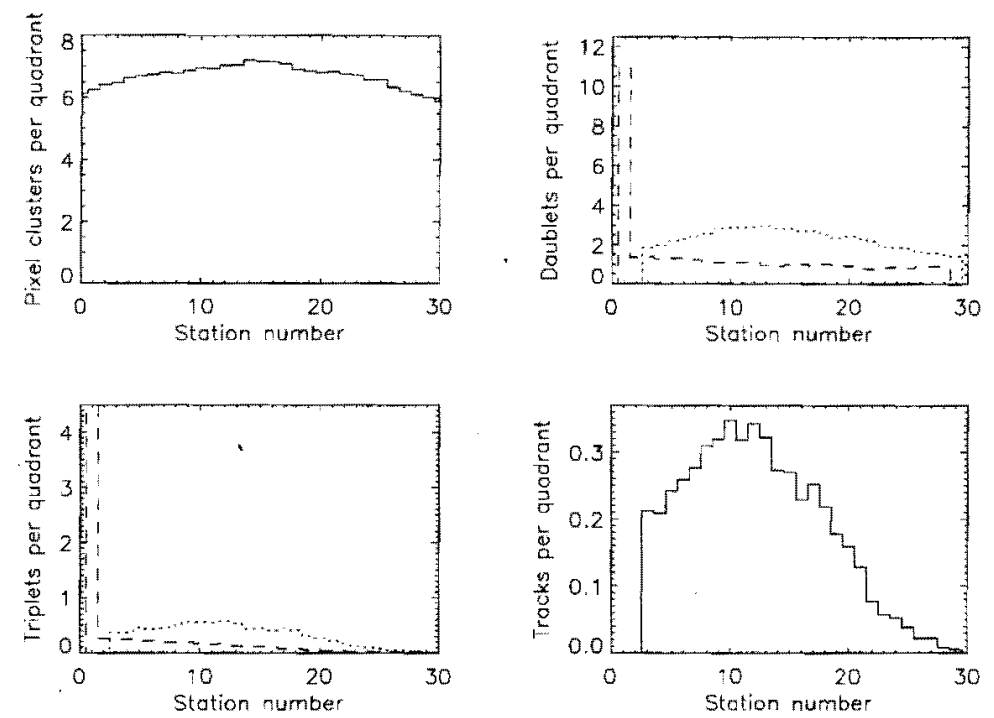

Figure 13.7: Multiplicities of pixel hits and trigger primitives for events generated with BTeVGeant. See the text for details.
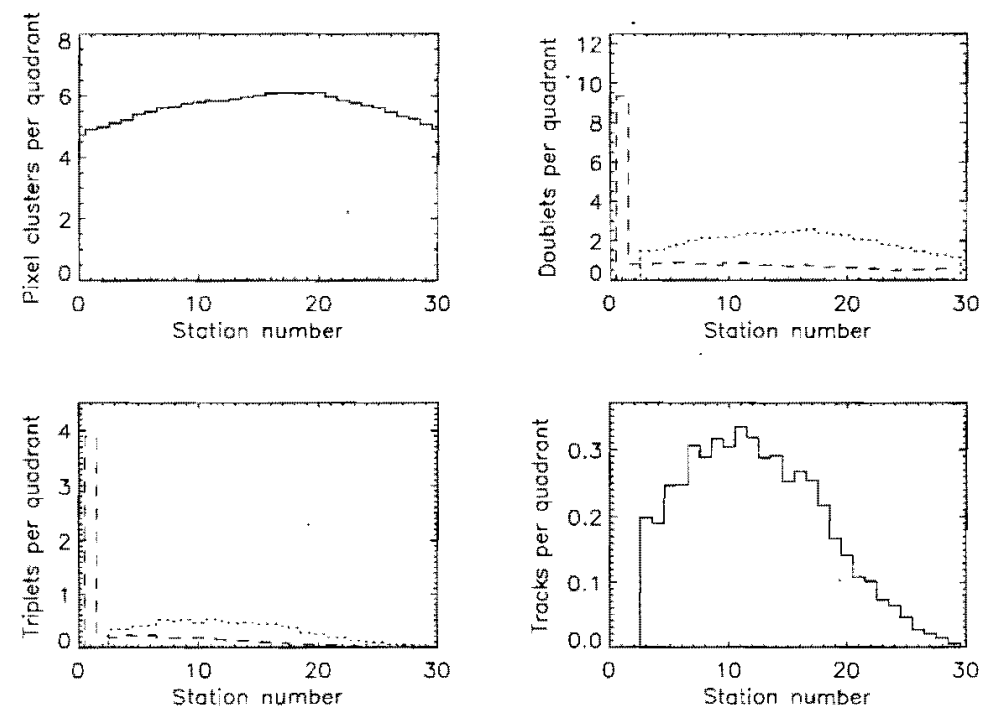

Figure 13.8: Multiplicities of pixel hits and trigger primitives for events generated with MCFast. See the text for details. 
Strow Occupancies, 2 Minimum Bias Events
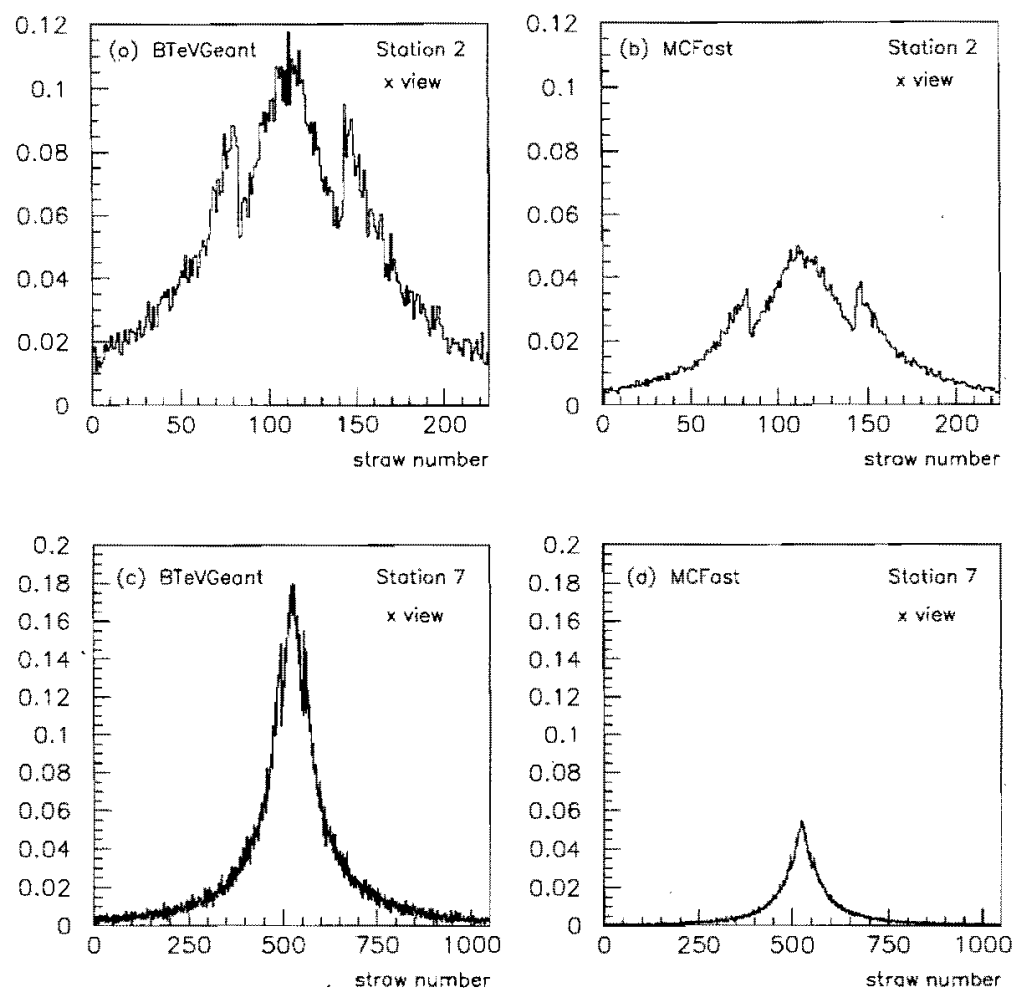

Figure 13.9: Comparison of straw chamber occupancies as computed by BTeVGeant (left) and MCFast (right). Station 2 (top) is the station which is second most downstream and station 7 (bottom) is the station which is closest to the interaction region. For all parts of the figure, the vertical axis the probability per beam crossing that the straw will have a hit.

suffer ionization energy loss in the shielding material. Therefore both programs can be used for determining the efficiency for fiducial cuts on muons. The bottom line is that MCFast cannot be used for determining the rates at which hadrons fake muons but it can make use of an externally supplied parameterization of fake rates.

The occupancies in the forward tracking system are also underestimated in MCFast; the reasons for this are similar to those discussed above for the muon system. Figure 13.9 compares the occupancies of several straw planes, as computed in BTeVGeant, with those computed by MCFast. The gross features of the distributions are the same but MCFast underestimates the rates by a factor which varies from about 2 far from the interaction region to about 3 close to the interaction region. The same general behavior is seen in the strip detectors: MCFast underestimates the occupancies by a factor of about 2 to 3 . For the strip detectors BTeVGeant predicts an occupancy which is at most $\simeq 1 \%$ in the hottest 


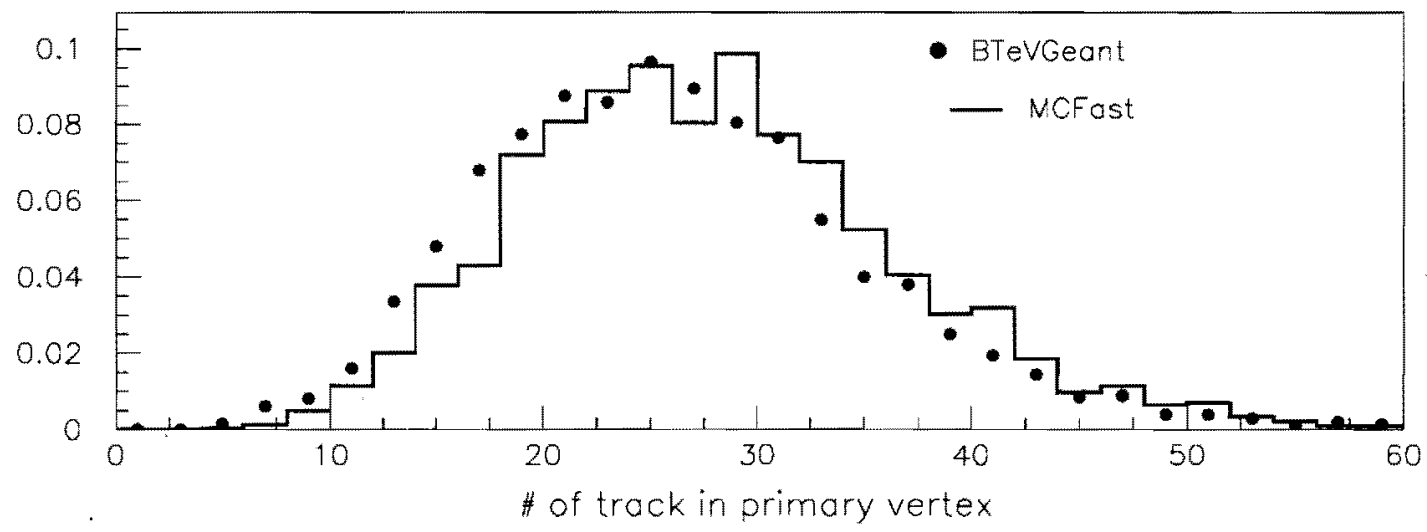

Figure 13.10: Primary vertex multiplicity for $B^{0} \rightarrow \pi^{+} \pi^{-}$candidates which pass the analysis cuts described in Section 16.1.

regions. This study shows that it is important to use BTeVGeant for any study which is sensitive to occupancies in the forward tracking system; for other studies, such as questions about resolutions, both packages will give reasonable answers.

\subsubsection{Efficiency and Resolution for $B^{0} \rightarrow \pi^{+} \pi^{-}$}

The comparison of high level tracking quantities was done using the decay mode $B^{0} \rightarrow \pi^{+} \pi^{-}$ which was analyzed as described in Section 16.1. These studies were performed using approximately 23,000 generated $B^{0} \rightarrow \pi^{+} \pi^{-}$events. The efficiency for the $B^{0}$ candidates to pass all analysis cuts was determined to be $8.0 \pm 0.2 \%$ using BTeVGeant and $8.8 \pm 0.2 \%$ using MCFast; these numbers exclude the trigger efficiency. Other results from the study are shown in Figures 13.10 and 13.11. Each of the histograms in Figure 13.11 has been fitted to a Gaussian and the results of those fits are reported in Table 13.1.

One of the reasons for moving from MCFast to BTeVGeant was the concern that MCFast would seriously underestimate the amount of tails in the distributions of important physics quantities. It is clear from the figure that this is not a serious problem in any of the distributions. For example, in the mass resolution plot, Figure 13.11e), the BTeVGeant result has $1.7 \%$ of the signal more than $\pm 3 \sigma$ from the mean, while the MCFast result has $0.9 \%$ of the signal more than $\pm 3 \sigma$ from the mean.

Inspection of the figures shows that the agreement between the two packages is excellent for $\Delta_{\mathrm{DCA}}$ and reasonably good for both $\Delta L$ and the primary vertex multiplicity. The agreement on the mass resolution is not as good. The disagreement in the mass resolution, $29 \mathrm{MeV} / c^{2}$ for BTeVGeant vs $22 \mathrm{MeV} / c^{2}$ for MCFast, is not yet understood in detail and is being investigated. The quantities $\Delta L$ and $\Delta_{\mathrm{DCA}}$ are sensitive mostly to the behavior of the pixel detector, but the mass resolution is sensitive to all of the details in the model of the tracking system, including the details of multiple scattering throughout the downstream 

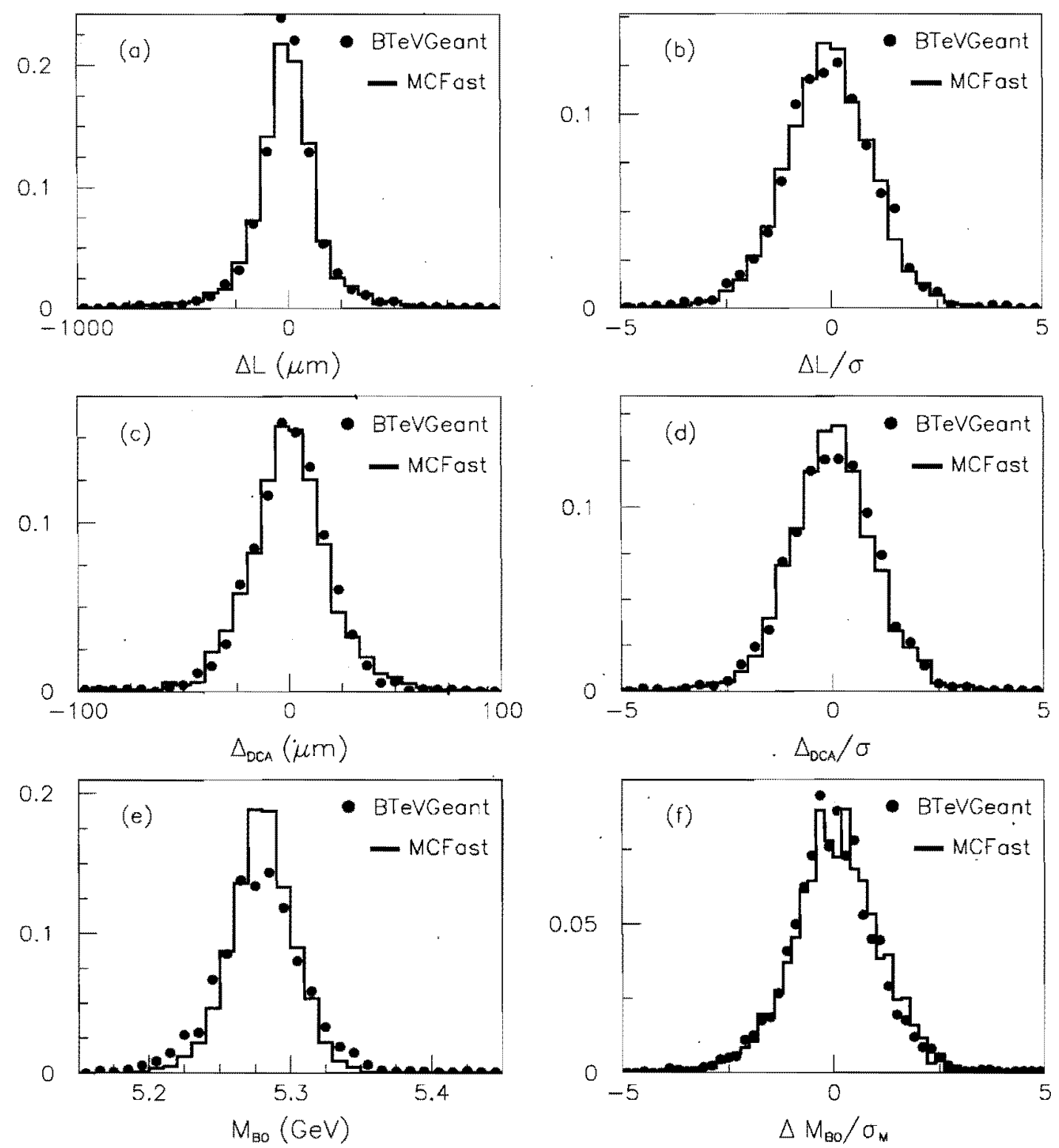

Figure 13.11: Comparisons between MCFast and BTeVGeant using $B^{0} \rightarrow \pi^{+} \dot{\pi}^{-}$candidates which pass the analysis cuts described in Section 16.1: a) the measured decay length minus the generated decay length, $\Delta L$, for the $B^{0}$ candidate; b) $\Delta L / \sigma$, where $\sigma$ is the error on $\Delta L ; \mathrm{c})$ the measured DCA minus the generated DCA, $\Delta_{\mathrm{DCA}}$, where DCA is the distance of closest approach of the $B^{0}$ candidate to the primary vertex; d) $\Delta_{\mathrm{DCA}} / \sigma$, where $\sigma$ is the error on $\Delta_{\mathrm{DCA}}$; e) the measured $\pi^{+} \pi^{-}$invariant mass; f) $\Delta M / \sigma$, where $\Delta M$ is the measured $\pi^{+} \pi^{-}$invariant mass minus the generated $B^{0}$ mass and where $\sigma$ is the error on $\Delta M$. Each figure has about 1850 entries for BTeVGeant and about 2030 entries for MCFast. 


\begin{tabular}{cccccc}
\hline & \multicolumn{2}{c}{ MCFAST } & \multicolumn{2}{c}{ GEANT } \\
Quantity & Units & Mean & $\sigma$ & Mean & $\sigma$ \\
\hline \hline$\Delta L$ & $(\mu)$ & $-8 . \pm 2$. & $119 . \pm 2$. & $-6 . \pm 3$. & $114 . \pm 2$. \\
$\Delta L / \sigma$ & & $-0.05 \pm .02$ & $1.00 \pm 0.02$ & $0.04 \pm .02$ & $1.03 \pm 0.02$ \\
$\Delta_{\mathrm{DCA}}$ & $(\mu \mathrm{m})$ & $-0.3 \pm 0.3$ & $17.6 \pm 0.3$ & $0.3 \pm 0.4$ & $17.0 \pm 0.3$ \\
$\Delta_{\mathrm{DCA}} / \sigma$ & & $-0.00 \pm 0.02$ & $0.963 \pm 0.014$ & $0.00 \pm 0.02$ & $0.959 \pm 0.015$ \\
$M_{\pi \pi}$ & $\left(\mathrm{GeV} / c^{2}\right)$ & $5.2800 \pm 0.0005$ & $0.0219 \pm 0.0003$ & $5.2790 \pm 0.0007$ & $0.0289 \pm 0.0005$ \\
$\Delta M / \sigma$ & & $0.04 \pm 0.02$ & $0.989 \pm 0.015$ & $-0.09 \pm 0.02$ & $0.982 \pm 0.015$ \\
\hline
\end{tabular}

Table 13.1: Results obtained by fitting each histogram in Figure 13.11 to a Gaussian. The fits were performed over an interval of approximately $\pm 3 \sigma$ about the mean.

spectrometer. In any case, most of the results presented in this proposal have been computed using the poorer, BTeVGeant, mass resolution.

Finally, the histograms in the right-hand column of Figure 13.11 indicate that, for both programs, the Kalman filter correctly determines the covariance matrices of the tracks and that the mass and vertex fitters correctly propagate those errors. 


\section{Chapter 14}

\section{Trigger Simulations for Levels 1 and 2 and a brief discussion of Level 3}

\subsection{Introduction}

The vertex trigger is the primary physics trigger for $\mathrm{BTeV}$. We trigger on $B$ events by taking advantage of the main difference between heavy quark events and typical hadronic eventsthe presence of detached beauty or charm vertices. The vertex trigger finds these vertices by utilizing the superior pattern recognition capabilities of the pixel detector to reconstruct tracks and vertices in the first stage of the trigger, Level 1, and in all subsequent stages of the trigger.

The baseline design of the vertex trigger consists of three levels. Each level contributes to the reconstruction of events, and successive levels impose more and more refined selection criteria to select $B$ events and reject light-quark background events. At Level 1 the vertex trigger reduces the trigger rate by a factor of 100 while maintaining high efficiency for $B$ decays that can be successfully reconstructed in the spectrometer. The tracks and vertices found by the Level 1 trigger are passed to Level 2. At Level 2 the reconstruction of tracks and vertices is improved by reviewing the pixel data used at Level 1, by including additional pixel hits in the tracks, and by introducing data from the forward tracking system. At Level 3, all of the data for a beam crossing are available and can be used to impose the selection criteria for the final trigger decision. The trigger rate is reduced by an additional factor of 20 by Levels 2 and 3 .

In this chapter we focus primarily on simulations of the Level 1 vertex trigger, with simulation results for Levels 2 and 3 presented at the end of the chapter. We present results for a Level 1 trigger that has been extensively modified compared to the trigger scheme that was presented in the BTeV Preliminary Technical Design Report (PTDR). The new Level 1 trigger is designed to work with the redesigned pixel system, which has less material than the PTDR pixel system due to a reduction in the number of pixel planes and substrates (see Chapter 4). Eliminating one of three pixel planes per tracking station has significantly reduced the amount of material in the pixel detector; however, the new 
vertex trigger must achieve a level of performance comparable to the PTDR trigger using only two-thirds of the pixel measurements. Our simulations show that this goal has been accomplished, and additional improvements in the trigger performance relative to the PTDR have been achieved.

Moreover, our simulations of the vertex trigger have improved compared to simulations performed for the PTDR. As before, our simulation results are based on a genuine reconstruction package that processes pixel-hit clusters generated by a Monte Carlo program. The Monte Carlo track information is only used to monitor the performance of the trigger algorithms; it is never used to prompt the pattern recognition or reconstruction algorithms.

There are two significant changes that improve the simulation of the vertex trigger. The first change is that our results are now based on simulations of pixel clusters generated by a GEANT Monte Carlo program. We use GEANT to simulate particle trajectories in heavyand light-quark events, and to generate hits from hadronic interactions, photon conversions, decays in flight, and delta rays. The second change in our trigger simulation is the way in which we simulate pixel clusters. We introduce non-Gaussian tails to generate realistic pixel hits that include the effects of low-energy delta rays. These non-Gaussian tails are based on parameterizations of $1999 \mathrm{BTeV}$ test beam data (see Appendix A). Together with the GEANT simulation, these changes represent a significant improvement in the level of realism used to simulate the Level 1 vertex trigger.

\subsection{Level 1 Trigger}

As described in Chapter 9, the Level 1 trigger is a detached-vertex trigger that receives data from the pixel detector, reconstructs tracks and vertices, selects $B$ events, and reduces the overall event rate by a factor of 100 . $B$ events are found by looking for $B$-decay tracks that have a large impact parameter with respect to a primary vertex. The trigger reconstructs primary vertices for every beam crossing, and counts the number of tracks with an impact parameter that is in an acceptable range. Tracks with very large impact parameters are not counted to exclude tracks associated with other primary vertices, and strange particle decay daughters. The decision to select an event is based on a cut that requires a minimum number of tracks with acceptable impact parameters.

The design of the Level 1 trigger is tied closely to the design of the pixel vertex detector. Compared to the BTeV PTDR, the pixel vertex detector now has two pixel planes per tracking station instead of three. With only two planes per station, the PTDR strategy of finding track mini-vectors in a single three-plane station had to be abandoned. Instead, the new Level 1 trigger finds track segments that span three tracking stations. A potential problem with this approach is that an overwhelming number of pixel cluster combinations may need to be considered for pattern recognition, since track segments are now found over a much larger region of the pixel detector compared to the very localized regions used to find mini-vectors. The solution is to restrict the pattern recognition to a subset of pixel clusters in order to reduce the number of combinations that must be considered by the trigger hardware.

We impose several restrictions to reduce combinatorics in the Level 1 trigger. These 
restrictions are implemented so that they do not degrade the track reconstruction efficiency for tracks that are needed to implement an effective trigger. Details of the pattern recognition algorithm are presented in Chapter 9; here we provide a description of three important features that account for the performance of the Level 1 trigger algorithm.

The first key feature of the algorithm is that it considers only a fraction of the available pixel data to initiate pattern recognition, while maintaining high efficiency for tracks from primary vertices and $B$-decay vertices. Pixel clusters that are used to "seed" the pattern recognition are restricted to an inner region (i.e., pixel clusters close to the beam region, within $1 \mathrm{~cm}$ of the inner edge of each pixel plane) or an outer region (clusters within $1 \mathrm{~cm}$ of the outer edge of a pixel plane or in the two outermost stations). This geometric restriction (see Fig. 9.4 in Chapter 9) provides an effective means to limit the number of pixel cluster combinations that must be considered for pattern recognition. Fig. 14.1a shows simulation results for the average number of pixel clusters for one quadrant of a pixel plane (solid histogram) for all 31 tracking stations for minimum bias events with an average of two interactions per beam crossing. Dotted and dashed histograms represent the number of clusters in the inner and outer regions, respectively. These histograms show the fraction of hits used to "seed" the pattern recognition.

The second important feature of the Level 1 algorithm is that it uses pixel clusters in a manner that further limits the number of pixel cluster combinations that must be considered for pattern recognition. The inner pixel clusters are used to find the beginning of a track, while the outer pixel clusters are used to find the end of a track in the pixel detector. Furthermore, the pattern recognition imposes cuts that require that tracks must enter the pixel detector at the inner edge of the detector (i.e., the tracks must come from the beam region), and tracks must exit the pixel detector at the outer edge of the detector or at the two outermost tracking stations (the first and last stations in the detector). These requirements eliminate many of the tracks associated with hadronic interactions or photon conversions that originate in the pixel detector, and may cause false triggers if they were to be reconstructed as tracks having a large impact parameter with respect to a primary vertex. The requirements also eliminate beam-halo tracks that do not cross an inner edge of the pixel detector.

The third key feature of the Level 1 algorithm is that each stage in the algorithm reduces the number of trigger primitives that must be considered by subsequent stages. This is an important feature, since it reduces the amount of data that must be passed through successive stages of the trigger hardware. For example, the first stage of the Level 1 algorithm considers pairs of pixel clusters (called doublets) from adjacent tracking stations. Ideally these doublets represent the first two pixel clusters that define the beginning of a track (these are called interior doublets), or the last two clusters at the end of a track (exterior doublets). Usually the two clusters that make up a doublet come from two different tracks, and it is conceivable that the total number of doublets could exceed the total number of pixel clusters and could overwhelm the next trigger stage. However, our simulations show that the number of doublets found by the Level 1 algorithm is less than the number of pixel clusters, so the algorithm is able to reduce the number of trigger primitives that must be examined by 

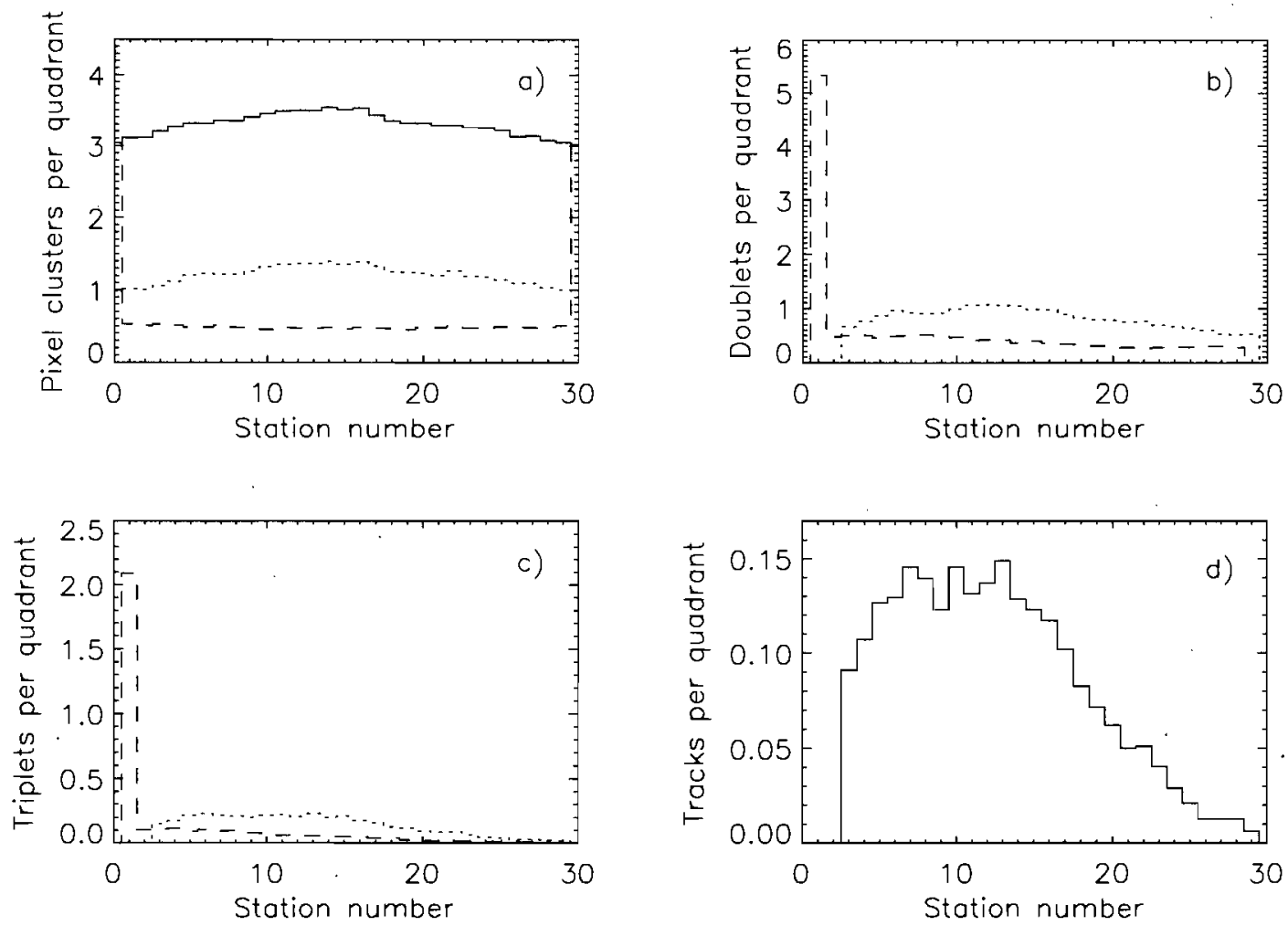

Figure 14.1: a) the average number of pixel clusters per quadrant (solid line) for 31 tracking stations for minimum bias events with an average of two interactions per crossing. The dotted and dashed histograms show the number of pixel clusters in the inner and outer regions, respectively. Histograms in b) and c) show the average number of interior (dotted) and exterior (dashed) doublets and triplets per quadrant directed at one arm of the two-arm $\mathrm{BTeV}$ spectrometer, and d) shows the average number of tracks found for the same arm of the spectrometer. Histograms of doublets, triplets, and tracks directed at the other arm of the spectrometer are mirror images of histograms in b), c), and d). 
the next stage of the trigger. This is evident in Fig. 14.1b, which shows the average number of interior doublets (dotted histogram) and exterior doublets (dashed histogram) directed at one arm of the two-arm BTeV spectrometer. The number of doublets is less than the number of pixel clusters for all tracking stations, except for Station 1. Here the number of exterior doublets exceeds the number of pixel clusters (note the histogram bin with more than 5 entries in Fig. 14.1b), since Station 1 is an outer station where tracks are exiting the pixel detector. The larger number of exterior doublets in this station means that additional hardware is needed to provide more data paths, which introduces additional parallelism (the same thing occurs for Station 30 for exterior doublets directed towards the other arm of the spectrometer). This additional parallelism also reduces the number of histogram entries for Station 1 in Fig. 14.1c. The figure shows the average number of interior and exterior triplets found by the Level 1 algorithm. At this stage in the trigger, the doublets are combined with pixel clusters in the neighboring, third, tracking station to produce triplets. The algorithm finds fewer triplets compared to the number of doublets found by the previous trigger stage. Finally, Fig. 14.1d shows that the number of tracks found by the last stage of the algorithm is less than the number of triplets found by the previous stage. In this case the tracks are found by combining an interior triplet (the beginning of a track) with an exterior triplet (the end of a track), and once again the algorithm is able to reduce the number of trigger primitives.

\subsubsection{Level 1 Trigger Studies}

All studies of the Level 1 trigger are performed using GEANT. We generate pixel clusters for the vertex-detector geometry described in Chapter 12, and include hadronic reinteractions, photon conversions, decays in flight, and delta rays. As mentioned above, we introduce nonGaussian tails that include the effects of low-energy delta rays. All studies are performed with an average of two interactions per beam crossing, except when we vary the number of interactions to study the trigger response for more extreme running conditions.

We study the performance of trigger algorithms using minimum bias events and different types of $B$-events. We have studied a variety of cuts, implemented at various stages in the trigger, and have chosen to use a few cuts in addition to the final vertexing cuts to help reject minimum bias interactions. For example, the Level 1 pattern recognition eliminates low momentum tracks (tracks with $p<3 \mathrm{GeV} / \mathrm{c}$ ) to avoid tracks that may suffer from excessive multiple scattering and could easily be reconstructed as having a large impact parameter with respect to a primary vertex. Moreover, all tracks are required to pass through at least four tracking stations to remove erroneous combinations of pixel clusters that can mimic what appear to be acceptable 3-station tracks (in these cases the interior and exterior triplets are usually constructed from identical pixel clusters). Lastly, a clean-up step removes all tracks that share pixel clusters with any other tracks. This method of removing fake tracks is simple, and perhaps overly severe, but it is effective in eliminating fake tracks at an early stage in Level 1. We have not performed an exhaustive study of possible trigger cuts, so we anticipate additional improvements resulting from future studies of the Level 1 trigger. 


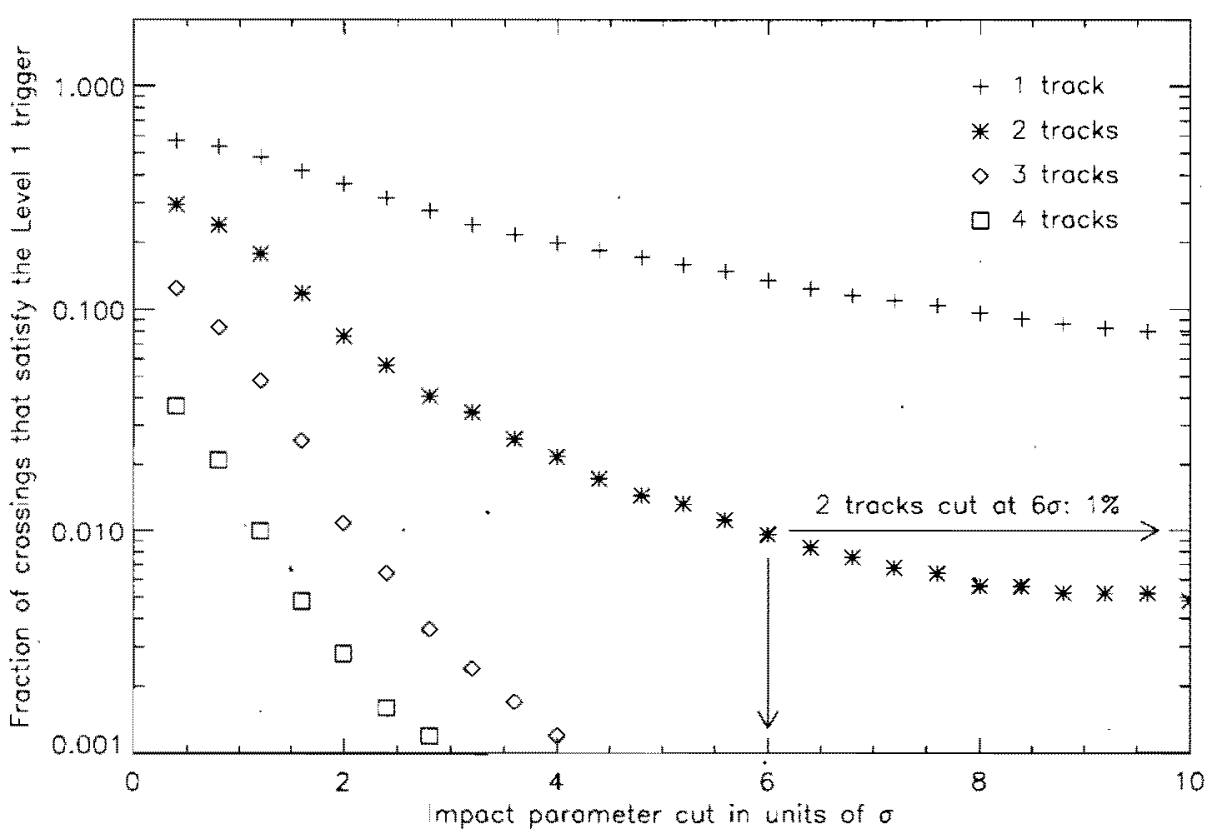

Figure 14.2: Trigger response for minimum bias events with an average of two interactions per beam crossing. The figure shows four sets of points requiring at least $1,2,3$, or 4 detached tracks. The arrows show a cut that requires at least 2 detached tracks with an impact parameter that exceeds $6 \sigma$, and achieves $99 \%$ rejection.

The Level 1 vertexing cuts are the final cuts that determine the Level 1 efficiency for $B$-events and the rejection of minimum bias events. These cuts are selected to provide $99 \%$ rejection for minimum bias crossings. The vertexing cuts require that at least $n$ tracks (all directed at one arm of the BTeV spectrometer) miss a primary vertex by at least $m \sigma$. The impact parameter is required to be less than $2 \mathrm{~mm}$ to exclude tracks that may be associated with other primary vertices in crossings with more than one interaction. The $2 \mathrm{~mm}$ cut also rejects daughter tracks from strange-particle decays. Fig. 14.2 shows the trigger response for minimum bias crossings for a range of vertexing cuts. There are four sets of points corresponding to the requirement of $n=1,2,3$, or 4 detached tracks. The horizontal scale specifies the minimum impact parameter for detached tracks. For purposes of this proposal, the requirement of 2 tracks at $6 \sigma$ is used. This provides a Level 1 rejection for minimum bias events of a factor of 100 . For the actual experiment we would likely run with a mix of prescaled triggers

With the vertexing cuts set to achieve the desired minimum bias rejection, we can study the trigger efficiency for different types of $B$-events. For $B_{s} \rightarrow D_{s}^{+} K^{-}$we obtain the trigger efficiencies shown in Fig. 14.3. Our cut, requiring at least 2 tracks with a minimum impact parameter of $6 \sigma$, gives us a trigger efficiency of $74 \%$ for this decay mode. Trigger efficiencies 
Table 14.1: Level-1 trigger efficiencies for minimum-bias events and various processes of interest that are required to pass off-line analysis cuts. All trigger efficiencies are determined for beam crossings with an average of two interactions per crossing using the Monte Carlo code shown in the table.

\begin{tabular}{lcc}
\hline \hline Process & Eff. (\%) & Monte Carlo \\
\hline \hline Minimum bias & 1 & BTeVGeant \\
\hline$B_{s} \rightarrow D_{s}^{+} K^{-}$ & 74 & BTeVGeant \\
$B^{0} \rightarrow D^{*+} \rho^{-}$ & 64 & BTeVGeant \\
$B^{0} \rightarrow \rho^{0} \pi^{0}$ & 56 & BTeVGeant \\
$B^{0} \rightarrow J / \psi K_{s}$ & 50 & BTeVGeant \\
$B_{s} \rightarrow J / \psi K^{* o}$ & 68 & MCFast \\
$B^{-} \rightarrow D^{0} K^{-}$ & 70 & MCFast \\
$B^{-} \rightarrow K_{s} \pi^{-}$ & 27 & MCFast \\
$B^{0} \rightarrow 2-$ body modes & 63 & MCFast \\
$\left(\pi^{+} \pi^{-}, K^{+} \pi^{-}, K^{+} K^{-}\right)$ & & \\
\hline \hline
\end{tabular}

for other $B$-decay modes are shown in Table 14.1.

It is important to realize that the Level 1 trigger, which requires at least two detached tracks, is able to trigger on $B$ events that involve decay modes with fewer than two charged tracks at the $B$-decay vertex. An example of this is the $B^{-} \rightarrow K_{s} \pi^{-}$mode listed in Table 14.1. Since this mode has only one track associated with the $B^{-}$decay vertex, the majority of triggers come from detached tracks associated with the other $B$ decay in the event.

Most of our studies of the Level 1 trigger efficiency are based on the decay mode $B_{s} \rightarrow D_{s}^{+} K^{-}$. These include studies of pixel noise and inefficiencies, described in the next section. Although we have not intentionally optimized cuts for this particular decay mode, it is conceivable that the current set of Level $1 \mathrm{cuts}$ are more favorable for $B_{s} \rightarrow D_{s}^{+} K^{-}$ than for other decay modes (such as the other modes listed in Table 14.1). Fig. 14.4 shows the trigger efficiency for this $B_{s}$ decay mode versus the rejection for minimum bias crossings. The arrows indicate our current Level 1 vertex cut. The figure shows that we have an effective cut that maintains high efficiency for $B_{s} \rightarrow D_{s}^{+} K^{-}$, and achieves $99 \%$ rejection for minimum bias crossings.

\subsubsection{Pixel Noise and Inefficiency Studies}

The Level 1 pattern recognition is exceptionally robust with respect to pixel inefficiencies and noise hits in the vertex detector. Fig. 14.5, which shows the trigger response for $B_{s} \rightarrow D_{s}^{+} K^{-}$and minimum bias crossings versus the number of noise hits in each pixel plane, summarizes the results from our noise and inefficiency studies. There are three sets of points that correspond to three different pixel efficiencies. There is a noticeable decrease in 


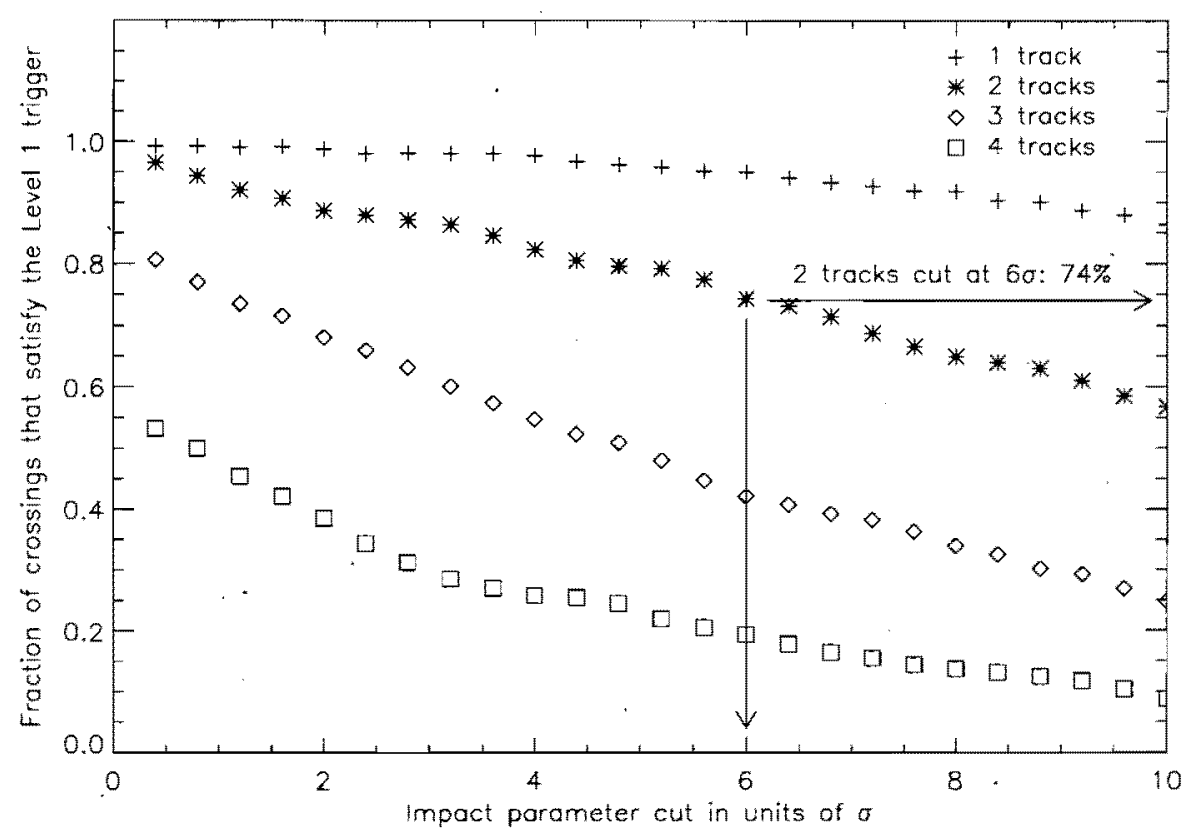

Figure 14.3: Trigger efficiency for $B_{s} \rightarrow D_{s}^{+} K^{-}$events with an average of two interactions per beam crossing. The figure shows four sets of points requiring at least $1,2,3$, or 4 detached tracks. The arrows show a cut that requires at least 2 detached tracks with an impact parameter that exceeds $6 \sigma$, and gives a trigger efficiency of $74 \%$. 


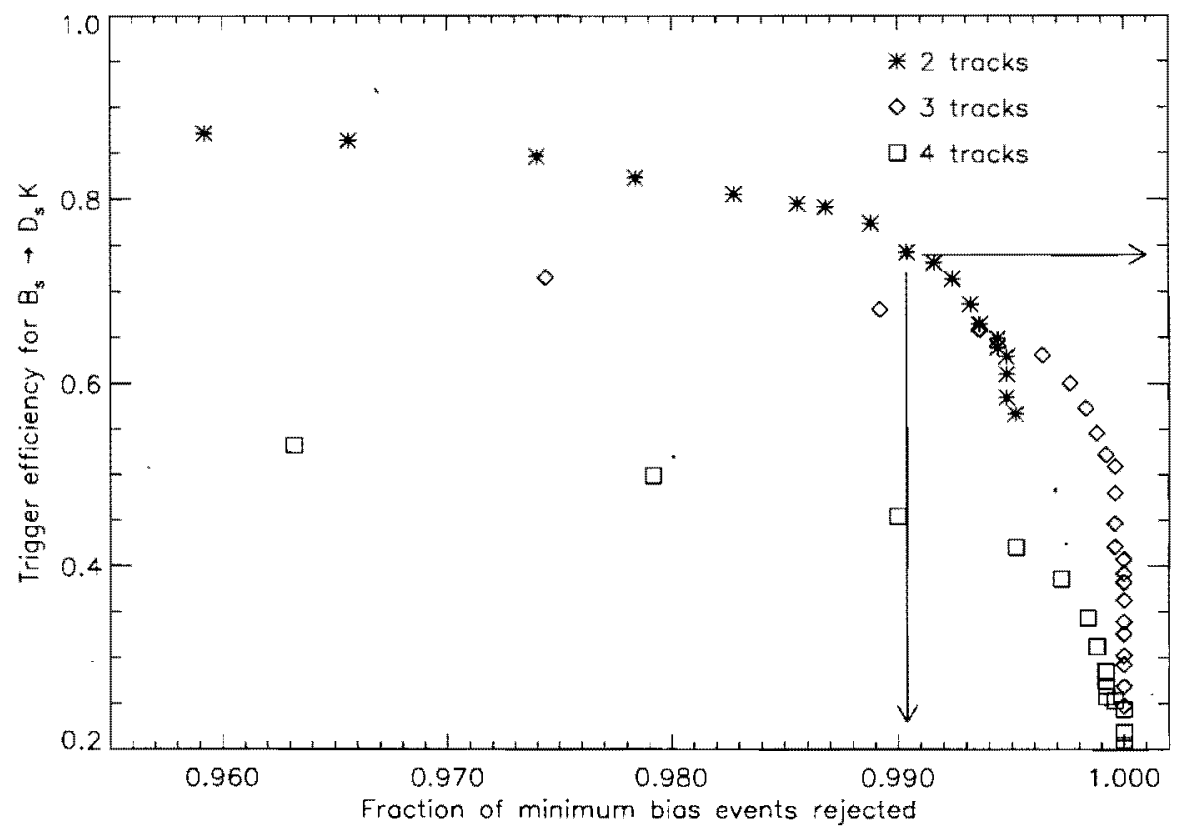

Figure 14.4: Trigger efficiency for $B_{s} \rightarrow D_{s}^{+} K^{-}$vs. rejection for minimum bias events. The figure shows three sets of cuts requiring a minimum impact parameter for 2,3 , or 4 tracks. Increasing the impact parameter cut increases the fraction of minimum bias events that are rejected, and the arrows indicate the cut $(2$ tracks at $6 \sigma)$ that we use to achieve $99 \%$ rejection. 


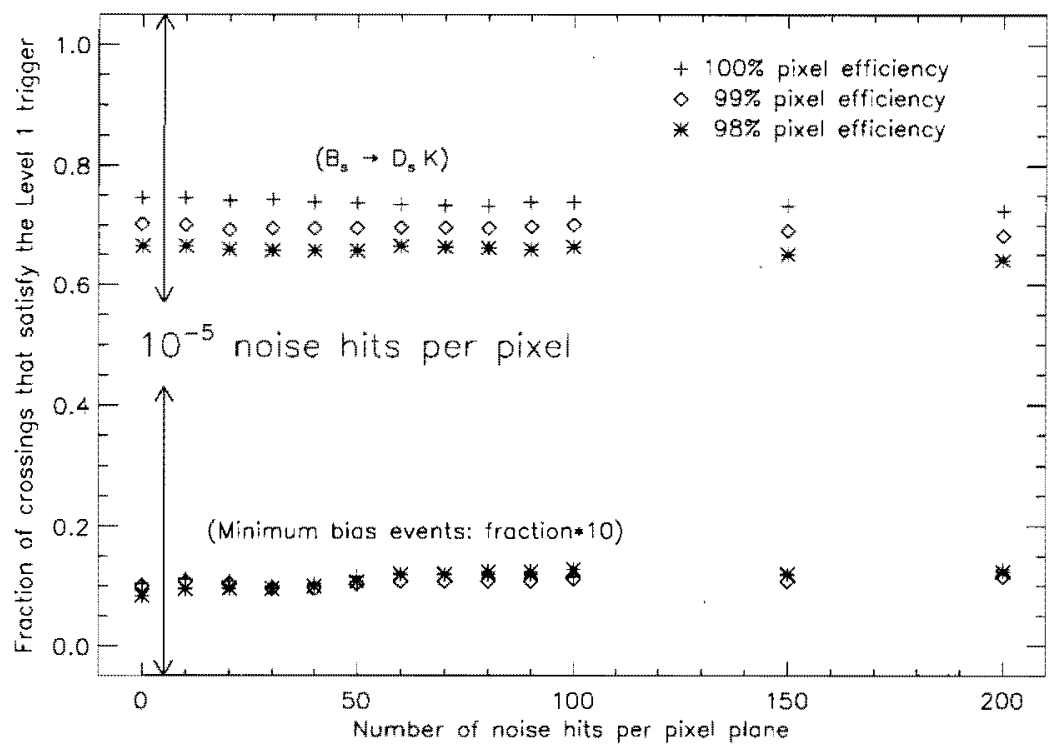

Figure 14.5: Fraction of $B_{s} \rightarrow D_{s}^{+} K^{-}$and minimum bias crossings, with an average of two interactions per crossing, that satisfy the Level 1 trigger for three different pixel efficiencies (three sets of points) vs. the number of noise hits that have been added to each pixel plane in the vertex detector. Results for minimum bias crossings have been multiplied by 10 .

the trigger efficiency for $B_{s} \rightarrow D_{s}^{+} K^{-}$with decreasing pixel efficiency (we observe a slightly different behavior for minimum bias crossings, described below). We expect to achieve a pixel efficiency that exceeds $99 \%$, so the trigger efficiency for $B_{s} \rightarrow D_{s}^{+} K^{-}$should exceed $70 \%$ - a trigger efficiency that is less than the first set of points (plus signs) and greater than the second set of points (diamonds).

We add noise hits to the trigger simulation to study how sensitive the pattern recognition is to spurious pixel clusters. We have studied two types of noise distributions without observing any significant difference. In the first study we generate a uniform distribution of noise over an entire pixel plane. In the second study (results are presented in Fig. 14.5) we generate pixel hits that mimic the distribution of GEANT hits distributed over a pixel plane. This may be a more realistic representation of correlated noise in the pixel detector. We expect the noise level in the detector to be less than $10^{-5}$ noise hits per pixel, which corresponds to about 5 additional pixel hits per plane. In our studies we add up to 200 pixel hits per plane, and observe a slight (few percent) decrease in the trigger efficiency for $B_{s} \rightarrow D_{s}^{+} K^{-}$. This decrease probably results from the clean-up step in the Level 1 trigger that removes tracks with shared hits.

For minimum bias crossings we get results that are similar to the results obtained for $B_{s} \rightarrow D_{s}^{+} K^{-}$. At low noise levels we observe a decrease in the "trigger efficiency" with decreasing pixel efficiency. However, in contrast to the $B_{s} \rightarrow D_{s}^{+} K^{-}$results, we observe a 


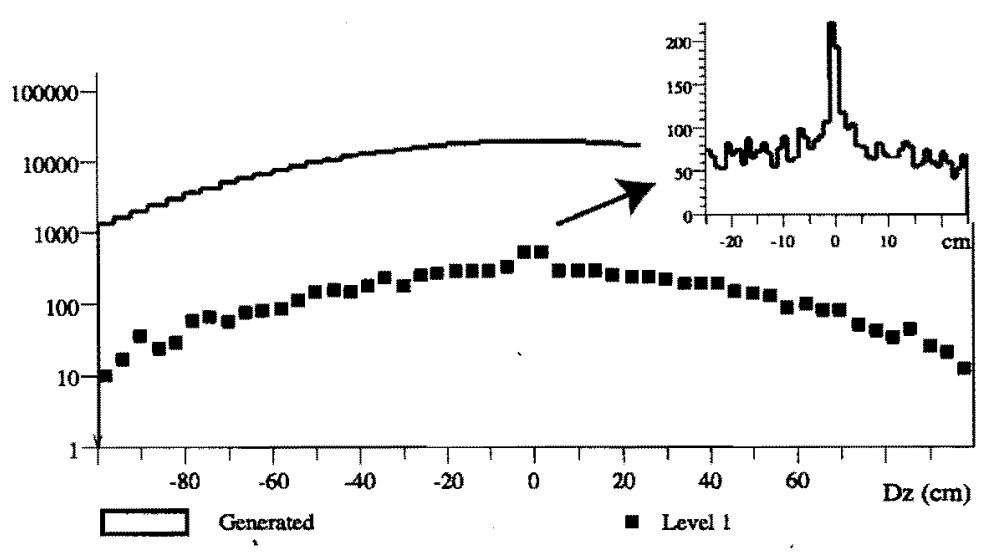

Figure 14.6: Distance between primary vertices

very slight increase in the fraction of minimum bias crossings that satisfy the Level 1 trigger as the number of noise hits increases. We believe that the pattern recognition is substituting noise hits for the "correct" hits that belong to a track, which tends to increase impact parameters and cause false triggers. This behavior appears to be even more noticeable (the effect is exaggerated in Fig. 14.5 by multiplying the minimum bias results by 10) for lower pixel efficiencies. Here the noise hits are used in place of pixel clusters that are lost due to pixel inefficiencies. Needless to say, Level 1 is exceptionally stable with respect to noise, and we do not expect any noticeable deterioration in the trigger performance for the amount of noise expected to occur in the pixel detector.

\subsubsection{Multiple-Interaction Studies}

At the design luminosity of $2 \times 10^{32} \mathrm{~cm}^{-2} \mathrm{~s}^{-1}$ there will be an average of two interactions per crossing. As long as primary vertices are relatively far apart, the Level 1 vertex trigger can identify individual vertices and assign each track to the correct primary vertex. A key feature of Level 1 (and subsequent trigger levels) is that detached tracks are required to have an impact parameter that is less than $2 \mathrm{~mm}$ to exclude tracks associated with other primary vertices. As soon as the distance between two primary vertices becomes fairly small, the trigger begins to "see" a primary vertex with detached tracks, which are associated with the other primary. Fig. 14.6 compares the distance between primary vertices for all generated events and those accepted by the Level 1 trigger. It can be seen that multiple interactions cause false triggers only when the distance between vertices is less than a few centimeters.

Another way of observing the level of confusion that is introduced by having multiple interactions in a single beam crossing is to look at the fraction of minimum bias crossings that satisfy the Level 1 trigger as a function of the number of interactions per crossing. Since we desire to trigger on a fixed fraction of the interactions, a linear response would be ideal. As can be seen in Fig. 14.7 the trigger response does deviate from a linear response for 3 


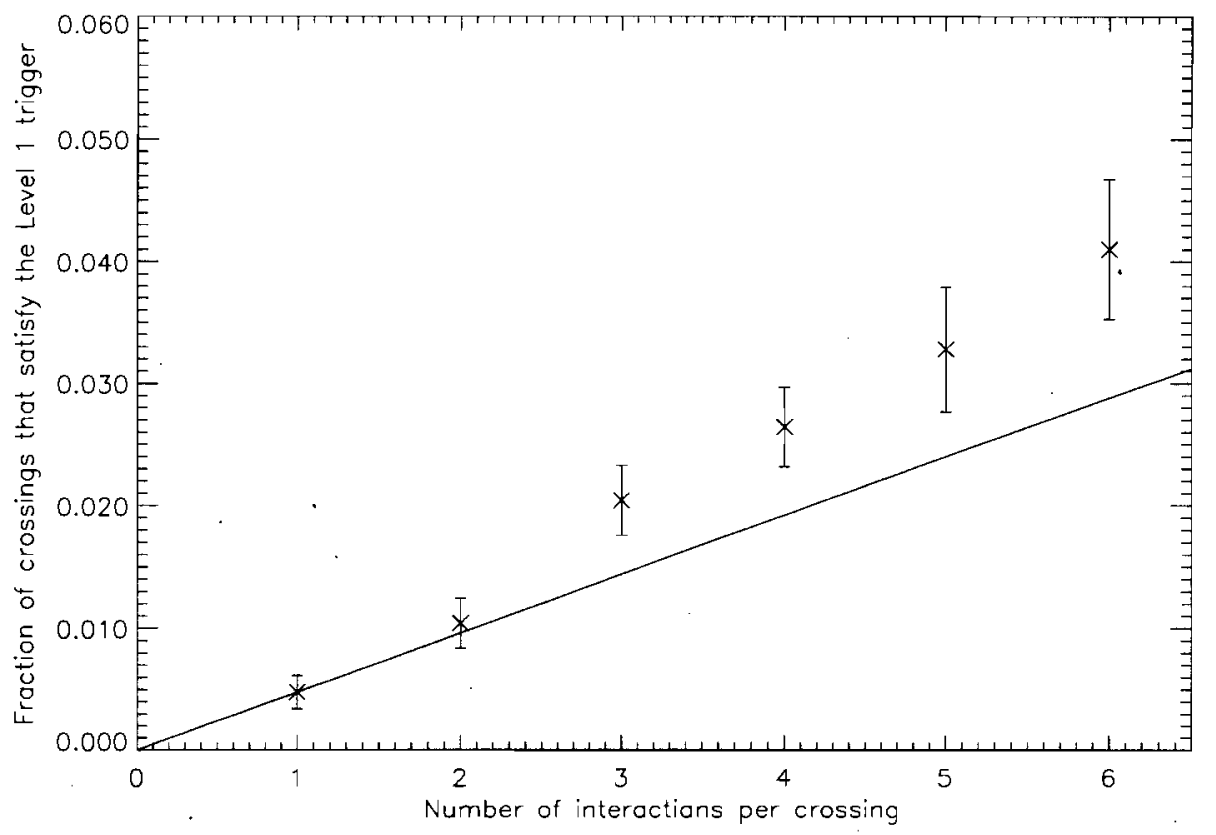

Figure 14.7: Fraction of minimum bias crossings that satisfy the Level 1 trigger vs. the number of interactions per crossing.

interactions/crossing and above. However the deviation is quite tolerable considering that we are planning to run at a mean of 2 interactions/crossing.

\subsection{Status of the Level 2 Trigger}

The Level 2 trigger algorithm is in its initial stage of development. Work on it could begin only after the Level 1 algorithm, which was rewritten to handle the pixel doublet scheme, was completed. Here, we discuss what we have achieved in the short time we have been studying this and project where we expect to wind up.

\subsubsection{Brief description of the Level 2 Algorithm}

The Level 2 trigger starts from the information obtained at Level 1, namely the pixel tracks and primary vertices. Only the tracks associated with the Level 1 primary vertex that had the required number of detached tracks-and therefore fired the Level 1 trigger - are considered. The first step is to add pixel hits to these tracks. By design, the Level 1 algorithm makes no attempt at finding all the hits associated with a given track in order to speed up the pattern recognition. At Level 2, the majority of hits that are added are between the inner and outer Level 1 track segments. The average number of additional hits is approximately 
10 per track. By adding these missing hits and using a Kalman filter to fit these tracks, the momentum resolution improves by roughly a factor of two (from $\left\langle\sigma_{P} / P\right\rangle \approx 6.5 \%$ to $\left\langle\sigma_{P} / P\right\rangle \approx 3.5 \%$ ). The pointing accuracy to the vertex region is also improved.

The second phase of Level 2 consists of a complete vertex pattern recognition using the Kalman fitted tracks. First, primary vertices are reconstructed by assembling pairs of tracks that make an acceptable 3-dimensional vertex. More than one such primary vertex is allowed. Second, secondary vertices are sought using tracks that are not part of any of the primary vertices. These secondary vertices are presumably heavy quark decays. While a primary vertex must occur in the luminous region (within a radius of $150 \mu \mathrm{m}$ ), the secondary vertex must (i) have relatively few tracks, (ii) occur within $5 \mathrm{~cm}$ of a primary, and (iii) must point away from the primary. Finally, the remaining "bachelor" tracks are assigned to one of the primary vertices, one by one, based on the distance of closest approach (DCA) of the track to the vertex. If no secondary vertex and no bachelor tracks are found, the crossing is rejected. Bachelor tracks which pass within a radius of $2 \mathrm{~mm}$ from the beam and with a DCA of $5 \sigma$, as well as tracks from secondary vertices, are considered "detached."

The third phase consists of confirming and improving the detached tracks by matching hits from the inner two forward tracking stations to these tracks and refitting them. This improves the momentum resolution by a factor of $\approx 2$, reaching $\left\langle\sigma_{P} / P\right\rangle \approx 2 \%$. In addition, new Level 2 tracks are sought in the pixel detector. Such tracks must come from the relatively small part of the luminous region surrounding the most populated primary vertex. The first hit of these types of tracks must be found within 5 pixel stations from the primary. The goal is to find a few more detached tracks, possibly improving the position accuracy of secondary vertices. The cuts used in this track reconstruction phase are tuned for low momentum tracks (down to $\approx 1 \mathrm{GeV} / \mathrm{c}$ ), where the Level 1 track reconstruction code (by design) is relatively inefficient. These tracks are added to the appropriate existing vertices, based on their DCA to these vertices, or kept as detached bachelor tracks (DCA > $5 \sigma$ ).

Various criteria based on vertex topology and the transverse momenta of the detached tracks have been considered. We find that the scalar sum of the transverse momentum of all detached tracks ${ }^{1} \Sigma\left|p_{t}\right|$, is relatively robust against variation in the total charged track multiplicity in $B$ decays. This is shown in Fig. 14.8.

\subsubsection{Timing}

A complete prototype of the preliminary Level 2 code has been written and its timing has been benchmarked on a Pentium III $400 \mathrm{MHz}$ processor. The Level 2 code takes roughly $115 \mathrm{~ms}$ per crossing, at the nominal luminosity of $2 \times 10^{32} \mathrm{~cm}^{-2} \mathrm{~s}^{-1}$. The goal of $\approx 50 \mathrm{~ms}$ per crossing on a $2.5 \mathrm{GHz}$ processor has therefore already been reached. Although some effort has been made to organize the data structures and tune the algorithm for speed, particularly the vertex finding, this is by no means fully optimized code. Thus, we still have the possibility to increase the rejection and/or the efficiency of signal events for this Level 2

\footnotetext{
${ }^{1}$ Once again, tracks from secondary vertices are considered detached.
} 


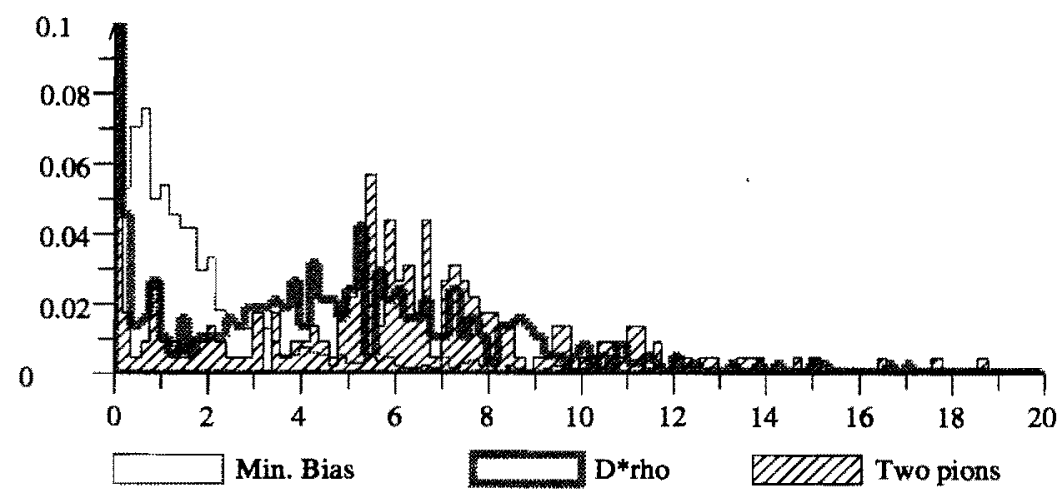

Figure 14.8: The histogram of the detached $p_{t}$ sum, $\Sigma\left|p_{t}\right|$, for two final states of interest and minimum bias events. The first bin corresponds to cases where no Level 2 detached track has been found. The probability for such occurrence (per crossing) is $32 \%$ for minimum bias and $\approx 15 \%$ for $B$ events.

trigger by adding more code; for instance, improved forward tracker reconstruction and/or better vertex pattern recognition.

\subsubsection{Signal efficiency and background rejection}

The efficiency of this Level 2 trigger has been measured on the $B^{0} \rightarrow D^{*+} \rho^{-}$and $B^{0} \rightarrow \pi^{+} \pi^{-}$ final states as well as on minimum bias events. For signal events, only events passing detailed analysis cuts discussed in the following chapter are considered. That is, as for Level 1, we are measuring our triggering efficiency on the $B$ sample of interest. Based on the integral of the distributions shown in Fig. 14.8, the efficiency for signal and background is shown in Fig. 14.9. Requiring $\Sigma\left|p_{t}\right|>2.2 \mathrm{GeV} / \mathrm{c}$ gives a rejection factor of 5 with efficiencies of $70 \%$ for $B^{0} \rightarrow D^{*+} \rho^{-}$and $75 \%$ for $B^{0} \rightarrow \pi^{+} \pi^{-}$. We expect to be able to improve the performance in the next iteration based on our analysis presented below.

The major sources of inefficiency for $B$. events have been identified:

- Approximately $15 \%$ of the Level 1 triggers on $B$ events are false triggers. That is, either the primary vertex with the Level 1 detached tracks is not the primary vertex from which the $b \bar{b}$ pair originated, or the detached tracks are not coming from any of the descendants of the $B$ particle. While the latter class of events is partially recovered by the Level 2 algorithm, the former is definitely lost because the Level 2 trigger only considers the "triggering" primary vertex. Note that we would have to spend more computing time to recover this $\approx 10 \%$ inefficiency. We plan to study the benefit of attempting to analyze at Level 2 the complete set of tracks and vertices obtained at Level 1. 


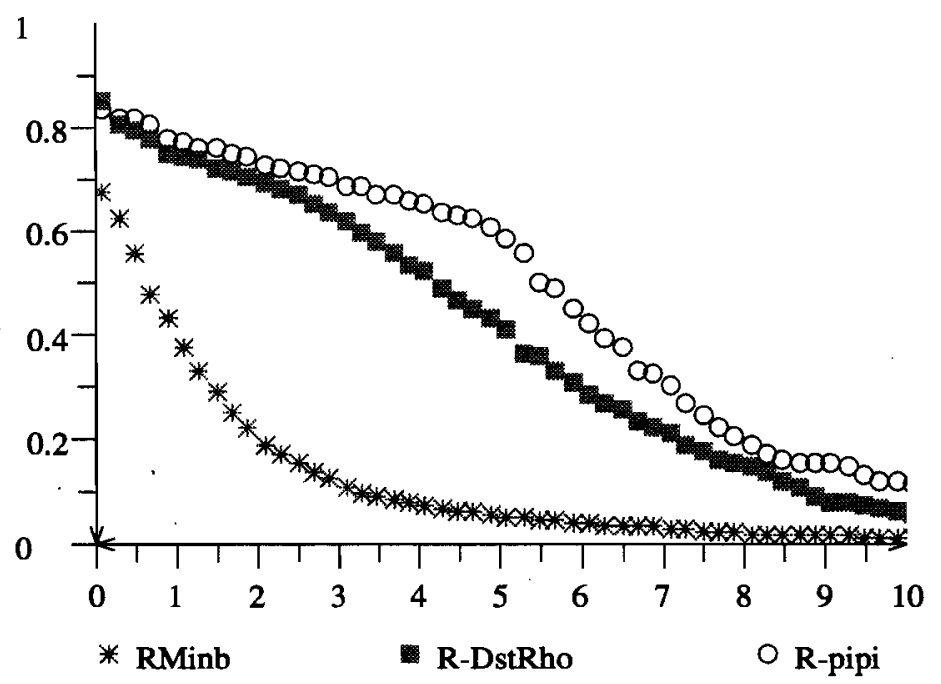

Figure 14.9: The efficiency of the Level 2 trigger vs. detached $P_{t}$ sum, $P t_{D}$, for two final states of interest and minimum bias events. The first bin corresponds to cases where no Level 2 detached tracks have been found. The probability for such occurrences (per crossing) is 32 $\%$ for minimum bias and $\approx 15 \%$ for $B$ events.

- The Level 1 tracking efficiency is about $90 \%$. This is only partly recovered at Level 2 by searching for additional tracks. There is room for improvement here.

As intended, the algorithm triggers mostly on tracks coming from the $b \bar{b}$ system, as shown in Table 14.2. The sources of background events found in the minimum bias sample passing the Level 1 and Level 2 triggers have also been studied:

- About $10 \%$ of the detached tracks are "noise" tracks, due to decays in flight, or, more likely, reinteractions (electromagnetic and hadronic) in the detector. Most of this background is evidently removed by the stringent fiducial cuts placed on the track at the vertices. The remaining sample is not, in fact, a dominant source of false Level 2 triggers.

- The probability of finding one or more pions from a $K_{s}^{0}$ decay in the detached, minimum bias, track sample is about $40 \%$. Approximately $1 / 4$ of these minimum bias events have two such pion tracks. Likewise, the probability of finding at least one charged track from a hyperon decay $(\Lambda, \Sigma)$ is about $20 \%$. This occurs despite the beam proximity fiducial cut and the maximum transverse impact parameter cut of $2 \mathrm{~mm}$. We are studying ways to specifically address these problems.

- In about $63 \%$ of the minimum bias events surviving the Level 2 cuts, there is at least one track originating from a different primary vertex and, in about one half of the 
Table 14.2: The average multiplicity of Level 2 detached tracks $\left\langle N_{\text {sec }}\right\rangle$ from various sources in events passing the analysis cuts and the Level $1+$ Level 2 trigger. A noise track is a track not coming from either a primary vertex or from a heavy quark or strange decay (e.g. secondary interactions). Also listed are the probability $P_{>=1}\left(P_{>=2}\right)$ to find at least one (two) of these tracks in the sample of Level 2 detached tracks.

\begin{tabular}{lccc}
\hline \hline Level 2 Track origin & $\left\langle N_{\text {sec }}\right\rangle$ & $P_{>=1}(\%)$ & $P_{>=2}(\%)$ \\
\hline \hline Noise & 0.06 & 6 & 0 \\
Primary & 1.2 & 47 & 25 \\
From the $D^{*} \rho$ & 1.55 & 95 & 88 \\
From the opposite $B$ decay & 0.95 & 52 & 27 \\
\hline \hline
\end{tabular}

sample, the primary vertex causing the trigger is contaminated by tracks originating from a different primary vertex. Although the primary vertex resolution along the beam axis is around $100-200 \mu \mathrm{m}$, the vertex pattern recognition algorithm can easily be fooled by $p \bar{p}$ collisions occurring with a a few $\mathrm{cm}$ of each other. This, along with strange decays, is the dominant source of the remaining background. We plan to study this problem as well.

We expect to recover the missing tracking efficiency and to mount a successful attack on the $K_{s}$ and hyperon decays. Our goal is to achieve a combined Level 1 and Level 2 trigger rejection of about 1000 to 1 and an overall triggering efficiency for interesting $B$ events of about $50 \%$ for almost all classes of $B$ decays that are of interest to us. At the moment, we are only slightly low in efficiency and about a factor of two low in rejection. However, we have many ideas that we have yet to try, and we are using less computing power than budgeted for the baseline Level 2/3 farm. Finally, we have both the bandwidth and computing power to handle the number of events that are passed by the current algorithm at Level 3 (see below). Because of the uncertainties on this, and to be totally safe we have included a $100 \%$ contingency in the cost of the Level $2 / 3$ farm. In the yield calculations below, we assume that our efficiency goals will be achieved. Based on current Level 1 efficiencies, we use a $90 \%$ Level 2 efficiency estimate in our simulations.

A final remark on charm events. The Level 1 trigger enriches the charm sample by about a factor of two, while the preliminary Level 2 trigger, due to its transverse momentum bias, does not enhance the charm yield. Should BTeV hunt for specific charm decay modes, however, such as two-body final states of the $D^{0}$, the Level 2 mass resolution can be used at little cost in bandwidth.

\subsection{Level 3 Trigger Strategy}

Our objective is to write out about 4000 events per second with an average size of 50 Kbytes. The rejection we require at Level 3 is small - only a factor of 2 to 4 . The data reduction, 
whose purpose is to reduce tape costs, must also be approximately a factor of 4 .

Concerning event rejection, the tasks of the Level 3 trigger are

- to reject the surviving minimum bias events; and

- then to decide which of the remaining events, most of which are $B$ or charm events, to keep.

Because the $B$ and charm rates at the Tevatron are so high, the second task is difficult to achieve because the events that reach this stage are, for the most part, genuine $B$ or charm events. However, many of them are unreconstructable because of their decay mode or because some of their decay products are outside the detector's acceptance. These events will not contribute to our physics reach and can be safely discarded. On the other hand, we do not want to design a trigger that focuses on an overly restrictive list of "fashionable" decay modes since new ideas and new data will cause any such list to change frequently.

Our strategy is to use two complementary approaches:

- We conduct a systematic and exhaustive search through a list of inclusive, and semiinclusive, decay modes. We include all the obvious candidates and various semiinclusive decays to leave the door open for surprises. We propose to use virtually all the relevant information from -most of the subdetectors to test the relevant set of hypotheses, but without doing a complete reconstruction of the event. For instance, a Level 2 detached track from a secondary vertex can bè tentatively identified as a kaon or pion without having to reconstruct or identify all the rings in the Cherenkov detector.

- We use a vertex-topology filter to select $B$-like (also charm-like) vertex topologies without regard to specific decay modes. These selections, because they do not use all the available information, are not as strict and we have to design cuts which balance signal efficiency against rejection.

We plan to take the "OR" of the events that survive these two algorithms. The virtue of this approach is that the first method can be made nearly $100 \%$ efficient on the key modes of interest at the time of data-taking. It is moderately efficient on a wide variety of other modes but can inadvertently exclude something that later becomes interesting. The second method is more inclusive but may have lower efficiency on some key modes of interest. There is, of course, a very large correlation between these two triggers. A prescaled sample of generic Level 2 events satisfying very loose cuts will also be kept to keep the experiment open to new states of interest and to calibrate the cuts used in the two Level 3 algorithms.

The Level 3 trigger must also reduce the size of the events. There are many methods for data reduction, including using standard data compression techniques before the events are actually logged to tape. Since this will probably not achieve the desired reduction, we have to consider additional tactics. Since the direction of the $b \bar{b}$ system is known, there is no point keeping the information from the opposite end of the spectrometer (last straw station, 
RICH, calorimeter, and muon detector). Since the charged particle reconstruction in that hemisphere is only needed to improve the accuracy of the primary vertex, and only the first few stations of the forward tracker are needed to accomplish this, many of the forward tracker hits from that arm can also be dropped. This alone achieves a reduction of nearly a factor of two. If these two methods are not quite enough, there are many other opportunities for data reduction which don't compromise our physics reach. For example, even on the "signal side", we can discard hits and write out only fitted track vectors for charged tracks clearly assoeiated with accompanying minimum bias interactions. (The tracking information may be needed for the particle identification or calorimetry in the offline analysis.) In the same spirit, while considering an exclusive hadronic decay to all charged tracks, the only role of the calorimeter is for electron identification for away side tagging. Only pulse heights from blocks surrounding the detached charged tracks from the other $B$ in the event are needed to test the electron hypothesis. Therefore, the calorimetry data block can be reduced considerably. (This strategy should be used for events which come in under the first trigger algorithm only.)

In order to illustrate the first of the two Level 3 trigger algorithms, we assembled a simple filter for two track $B$ decays. The analysis proceeds by looping on all Level 2 tracks, of opposite signs, requiring that: (i) both tracks are significantly detached from the primary vertex, (ii) the two tracks form a 3-dimensional vertex, and (iii) the vertex is detached from the primary by $5 \sigma$. After a loose mass cut of $\pm 100 \mathrm{MeV} / \mathrm{c}^{2}$, the rejection factor for minimum bias events is $\approx 50,000: 1$ (all trigger levels included). Note that this filter accepts all hadronic, two body mesonic decay modes, as well as $e^{+} e^{-}$and $\mu^{+} \mu^{-}$, of the $B_{0}$ and $B_{s}$. Evidently, the momentum measurement on these two tracks can be improved using the entire forward spectrometer (using little CPU time compared to the full Level 2 task), and additional vertex cuts similar to those used in the analysis described in Section 16.1 will also be used. This will easily reduce the above rate, which is already only a few percent of the total bandwidth allowed to tape, to well below $1 \%$ of the total allowed bandwidth. Similar filters, possibly using data from other detectors, can be constructed for all other decay modes of interest. 


\section{Chapter 15}

\section{Flavor Tagging}

\subsection{Introduction}

For charged $B$ mesons, the flavor of the heavy quark $(b$ or $\bar{b})$ is determined by the charge of the $B$ meson. For neutral $B$ mesons $\left(B_{d}\right.$ and $B_{s}$ ) the quark flavor can usually be determined from the flavor of its decay products, for example by the charge of the kaon in the decay chain $B_{s} \rightarrow \psi \bar{K}^{*}, \bar{K}^{*} \rightarrow K^{-} \pi^{+}$. If the flavor of the $b$ quark at its production point is determined independently, these modes can be used to measure $B_{s} \leftrightarrow \bar{B}_{s}$ and $B_{d} \leftrightarrow \bar{B}_{d}$ oscillations, which are sensitive to $V_{t s}$ and $V_{t d}$, respectively. Decays to CP-eigenstates do not identify the $b$ quark flavor. In fact, amplitudes for these decays interfere with the mixing amplitude producing $\mathrm{CP}$-violating effects. Measurements of CP-asymmetry, resulting e.g. in determination of $\sin 2 \beta$ via $B_{d} \rightarrow \psi K_{S}^{0}$, again require determination of the $b$ quark flavor at the production point - so called "flavor tagging." Every tagging method sometimes produces false identifications and the effectiveness of flavor tagging is characterized by an effective tagging efficiency $\epsilon D^{2}$, where $\epsilon=\left(N_{R}+N_{W}\right) / N, D$ is a "dilution" factor $D=$ $\left(N_{R}-N_{W}\right) /\left(N_{R}+N_{W}\right), N$ is the number of reconstructed signal events, $N_{R}$ the number of right flavor tags in this sample, and $N_{W}$ is the number of wrong flavor tags. Since the measurements mentioned above are among the most important goals of the BTeV program, a high effective tagging efficiency is a crucial design criterion for the experiment.

This section presents the results of a study of the tagging power of several tagging methods which can be used in the BTeV detector. The events studied contain decays in the channel $B_{s} \rightarrow D_{s} K$ which passed the final analysis cuts in the study described in section 16.4.1. For this analysis, each signal event was accompanied by a Poisson distributed number of background events, with a mean of two background interactions per beam crossing. For this study, Pythia was used to generate the physics events and BTeVGeant was used for the detector simulation. Mixing of the opposite side $B$ meson was not switched on in Pythia. 


\subsection{Away Side Tagging}

In $p \bar{p}$ collisions at $2 \mathrm{TeV}$ in the center-of-mass, by far the dominant source of $b$ quark production is via production of $b \bar{b}$ pairs. The away side tagging methods rely on determination of the flavor of the other $b$ quark in the event. The flavor of the away side $b$ can be determined from the charge of the lepton emitted in its semileptonic decay, the charge of vertices found on the away side or from the charge of the kaon produced in its $b \rightarrow c \rightarrow s$ cascade.

The first few steps of the away side algorithm are common to all away side tagging methods. First, all primary interaction vertices in the event were found. Next, a list was built of tracks which were candidates to come from a secondary vertex. To be a candidate, a track must have a momentum greater than $3.0 \mathrm{GeV} / \mathrm{c}$ and must not pass within $3 \sigma$ of any primary vertex. This list served as input for the various tagging methods.

\subsubsection{Lepton Tagging}

While lepton tagging is a very clean tagging method, the branching ratio of $b \rightarrow X \ell^{-} \nu$, about $10 \%$ per light lepton generation, is relatively small compared to the branching ratio into other tagging modes. The lepton tagging algorithm also must deal with possible wrongsign tags which result from the cascade $b \rightarrow c \rightarrow \ell^{+}$. Because leptons from $b \rightarrow \ell^{-}$and $b \rightarrow c \rightarrow \ell^{+}$have quite different transverse momentum $\left(p_{T}\right)$ distributions, good separation can be achieved.

Candidates for muon tags were selected from the secondary track list if they had a momentum greater than $4.0 \mathrm{GeV} / \mathrm{c}$ and were within the geometric acceptance of the muon detector. If there was more than one muon tag candidate in an event, the highest $p_{T}$ muon was chosen to be the tag. A tagging muon with $p_{T}>1.0 \mathrm{GeV} / \mathrm{c}$ was considered to be from the process $b \rightarrow \ell^{-}$, while one with $p_{T}<0.5 \mathrm{GeV} / \mathrm{c}$ was considered to be from the process $b \rightarrow c \rightarrow \ell^{+}$, thereby flipping the sign of the tag. The muon tagging efficiency was found to be $\epsilon=4.5 \%$ with a dilution $D=0.66$, which gives an effective tagging efficiency of $\epsilon D^{2}=2.0 \%$.

Candidates for electron tags were selected using a parametrized electron efficiency and hadron misidentification probability based on the results described in Section 12.4. The tag was required to have $p_{T}>1.0 \mathrm{GeV} / \mathrm{c}$ and assumed to come from the process $b \rightarrow \ell^{-}$. There were not enough events to study electrons from $b \rightarrow c \rightarrow \ell^{+}$. If there was more than one electron tag candidate the highest $p_{T}$ electron was chosen. The electron tagging efficiency was found to be $\epsilon=2.3 \%$ with a dilution $D=0.68$, which gives an effective tagging efficiency of $\epsilon D^{2}=1.0 \%$.

\subsubsection{Kaon Tagging}

Because of the large branching ratio for $b \rightarrow c \rightarrow K^{-} X$, kaon tagging is the most potent tagging method at $e^{+} e^{-} B$ factories. At $\mathrm{BTeV}$, in which the multiplicity of the underlying event is much greater, excellence in both particle identification and vertex resolution is 
required to exploit kaon tagging. Both are strong points of our forward detector geometry.

Candidates for kaon tags were selected from the secondary track list if they were identified as kaons in the RICH detector. If there was more than one kaon tag candidate in an event, the kaon with the largest normalized impact parameter with respect to the primary vertex was selected. In events with multiple primary vertices, the signal $B$ defines which primary is used to calculate the normalized impact parameter. The kaon tagging efficiency was found to be $\epsilon=18 \%$ with a dilution $D=0.52$, resulting in an effective tagging efficiency of $\epsilon D^{2}=4.9 \%$.

\subsubsection{Vertex Charge Tagging}

In this method a search was made for a detached vertex which is consistent with being from the charged decay products of the other $b$. The charge of that vertex determines the charge of the $b$. When the opposite side $b$ hadronizes into a $\bar{B}^{0}$, the tagging vertex has a neutral charge and there is no useful vertex tagging information in the event. However this method has the advantage that it is not affected by mixing of the away side $b$.

The vertex charge tagging algorithm exploits a correlation between the signal $B$ meson and the tagging $b$ hadron: they are produced relatively close in pseudorapidity $(\eta)$ and, therefore, their decay products appear in the same arm of the detector.

Tracks from the secondary list were accepted provided they had $p_{T}>100 \mathrm{MeV} / \mathrm{c}$ and provided they had $\Delta \eta<4$ with respect to the direction of the signal $B^{0}$ candidate. The tracks from the secondary list were sorted into candidate vertices and only vertices with a detachment of at least $1.0 \sigma$ from the primary vertex were accepted. If more than one vertex was found in an event, the one with the highest transverse momentum was selected; if no secondary vertices passed the selection cuts and if there was at least one track with $p_{T}>1.0$ $\mathrm{GeV} / \mathrm{c}$, then the highest $p_{T}$ track was selected. If the charge of the selected track or vertex is non-zero, then it determines the flavor of the away side $b$.

This tagging method is similar to jet charge tagging used by other experiments but we have not yet investigated the possibility of weighting the tracks by their momenta.

The results of this study indicate that we can achieve a tagging efficiency $\epsilon=32 \%$ and a dilution $D=0.36$, which results in an effective efficiency $\epsilon D^{2}=4.1 \%$.

\subsubsection{Combining Away Side Tagging Methods}

In many events, several of the same side tagging methods may give results; moreover it can happen that two methods will give contradictory answers. We have not yet optimized the method of combining all tagging information but have used the following simple algorithm. The methods were polled in decreasing order of dilution and the first method to give an answer was accepted. That is, if lepton tagging gave a result, the result was accepted; if not, and if kaon tagging gave a result, the kaon tag was accepted; if not, and if the vertex charge tagging gave a result, the vertex charge tag was accepted. The results of the away side tagging studies are summarized in Table 15.1. 


\subsection{Same Side Tagging}

The same side tagging method uses the correlations which arise between the signal $B$ meson and the charge of nearby tracks produced either in the fragmentation chain or in $B^{* *}$ decays. For $B_{s}$ mesons, the correlation is with a charged kaon, while for $B_{d}$ decays the correlation is with a charged pion.

Here we present a study of same side tagging for $B_{s}$ mesons. For this study tracks were selected provided they had a momentum greater than $3.0 \mathrm{GeV} / \mathrm{c}$, were identified as kaons in the $\mathrm{RICH}$ and had an impact parameter with respect to the primary vertex less than $2 \sigma$. It was further required that the system comprising the $B_{s}$ candidate plus the candidate tagging track have an invariant mass less than $7.0 \mathrm{GeV} / \mathrm{c}^{2}$. If more than one track passed these cuts, then the track closest in $\phi$ to the $B_{s}$ direction was selected. With this algorithm the same side tagging efficiency was found to be $\epsilon=40 \%$ with a dilution of $D=0.26$, which results in an effective efficiency $\epsilon D^{2}=2.6 \%$.

For same side tagging of $B_{d}$ events we expect to use $B^{* *}$ decays. We have not used the flag in Pythia to turn on $B^{* *}$ production so we have used a sample of $B \rightarrow \psi K_{s}$ decays where the $B$ and the next pion in the StdHep list have an invariant mass in the range 5.6 $5.8 \mathrm{GeV} / \mathrm{c}^{2}$. We assume that $30 \%$ of $B_{d}$ events will come from $B^{* *}$ decays and so weight these events accordingly and assume that the other $70 \%$ of the events will have equal numbers of right sign and wrong sign tags. Very preliminary results indicate we can achieve a tagging efficiency $\epsilon D^{2}=2.2 \%$.

\subsection{Summary of Tagging}

The results from this chapter are summarized in Table 15.1. These results are preliminary and one should be aware that all algorithms have yet to exploit the full power available to them. In particular, the vertexing information has yet to be fully exploited. For example, the $b \rightarrow \ell^{-}$and $b \rightarrow c \rightarrow \ell^{+}$samples differ not only in their $p_{T}$ spectra; they have distinctly different topological properties. Similarly, kaon tagging can be improved if there is evidence that the kaon comes from a tertiary vertex, indicative of the $b \rightarrow c \rightarrow K^{-}$cascade. Finally, the vertex charge algorithm should expect to find two vertices on the away side, the $b$ decay vertex and the $c$ decay vertex; the charge of both vertices provides tagging power. Other tagging methods have yet to be studied such as using a $D^{*}$ from the decay of the opposite side $B$. Finally, we have not yet explored the optimal use of the correlations among all of the methods.

Many of the physics reach studies presented in chapter 16 require values of $\epsilon$ and $D$ as inputs. As argued in the preceding paragraph, the results quoted in Table 15.1 probably underestimate the tagging power of $\mathrm{BTeV}$. Therefore most physics results in chapter 16 are presented using nominal values of $\epsilon=0.7$ and $D=0.37$, giving $\epsilon D^{2}=0.1$. The studies presented in the present chapter should be regarded as evidence that these nominal values lie well within the ultimate reach of the experiment. 


\begin{tabular}{|l|ccc|}
\hline Tag Type & $\epsilon$ & $D$ & $\epsilon D^{2}$ \\
\hline Muon & $4.5 \%$ & 0.66 & $2.0 \%$ \\
Electron & $2.3 \%$ & 0.68 & $1.0 \%$ \\
Kaon & $18 \%$ & 0.52 & $4.9 \%$ \\
Vertex Charge & $32 \%$ & 0.36 & $4.1 \%$ \\
Same Side Kaon & $40 \%$ & 0.26 & $2.6 \%$ \\
Same Side Pion & $88 \%$ & 0.16 & $2.2 \%$ \\
\hline Total for $B_{s}$ & & & $14.6 \%$ \\
Total for $B_{d}$ & & & $14.2 \%$ \\
Total for $B_{s}$ with overlaps & $65 \%$ & 0.37 & $8.9 \%$ \\
\hline
\end{tabular}

Table 15.1: Results, to date, of first generation studies of tagging power. In the text it is discussed that these studies are incomplete and that they likely underestimate the tagging power which can be realized at $\mathrm{BTeV}$. 


\section{Chapter 16}

\section{Specific Physics Final States}

The simulation tools described in the preceding chapters have been used to explore the physics reach of the $\mathrm{BTeV}$ detector. The channels selected for this study include standard $B$ physics benchmarks, channels which illustrate the capabilities of particular detector components and channels which highlight the unique capabilities of BTeV. The standard benchmarks include, $B^{0} \rightarrow \pi^{+} \pi^{-}, B^{0} \rightarrow J / \psi K_{S}^{0}$ and studies of $B_{s}$ mixing using $B_{s} \rightarrow D_{s} \pi$ and $B_{s} \rightarrow J / \psi \bar{K}^{* 0}$. The channel $B^{0} \rightarrow D^{*-} \rho^{+}$has a large branching ratio and it is used to explore the capabilities of the electromagnetic calorimeter; studies with this mode show that $\mathrm{BTeV}$ has the segmentation and resolution required to extract a strong signal, even in the presence of the background events which accompany the signal events. While this result had previously been demonstrated with MCFast based studies, it is reaffirmed here using a BTeVGeant based study, in which noise levels are significantly higher and all resolutions have realistic non-Gaussian tails. The analysis techniques developed during the $B^{0} \rightarrow D^{*-} \rho^{+}$ study were applied both to the study of $B \rightarrow \rho \pi$, an important mode for constraining the CKM angle $\alpha$, and to the study of $B \rightarrow J \psi \eta^{(\prime)}$, the mode which directly measures the CP angle $\chi$. One of the particular strengths of $\mathrm{BTeV}$ is the ability to constrain the CKM angle $\gamma$ using a variety of techniques; results are presented here for the decay modes, $B_{s} \rightarrow D_{s} K^{-}$, $B^{ \pm} \rightarrow D^{\circ} K^{ \pm}$, and $B \rightarrow K \pi$. The chapter concludes with estimates of the sensitivity for rate processes such as $B^{\rightarrow} K^{(*)} \mu^{+} \mu^{-}$and $B^{\rightarrow} X_{s} \mu^{+} \mu^{-}$. Taken together, these studies illustrate the excellent physics reach of $\mathrm{BTeV}$. 


\subsection{A study of $B^{o} \rightarrow \pi^{+} \pi^{-}$(and $B_{s} \rightarrow K^{+} K^{-}$)}

The decay of $B^{o} \rightarrow \pi^{+} \pi^{-}$is the traditional choice for measuring $\sin (2 \alpha)$, but the evidence of large penguin amplitudes in the observation of $B^{o} \rightarrow K^{+} \pi^{-}$by the CLEO collaboration [1] implies that a simple extraction of $\sin (2 \alpha)$ from this mode is no longer likely. However, since this mode has been used to benchmark so many experiments, it is still worthwhile to understand. In addition, it may be useful for the extraction of $\gamma$ when combined with a measurement of $B_{s} \rightarrow K^{+} K^{-}$as explained in Part I.

The data for this study are generated using Pythia while QQ is used to decay the heavy particles. The detector simulation is performed using the BTeVGeant simulation package. We also compare our result with the result obtained using MCFast. Each signal event which is simulated by BTeVGeant (or MCFast) contains one signal interaction $(b \bar{b})$ and $n$ background interactions (minimum bias), where $n$ has a Poisson distribution of mean 2 . This corresponds to our design luminosity of $2 \times 10^{32} \mathrm{~cm}^{-2} \mathrm{~s}^{-1}$.

To find this decay we select two oppositely charged tracks with a displaced vertex and an invariant mass close to the $B_{d}$ mass. Most of the background rejection against random combinations comes from the displaced $B$ vertex and the momentum balance of the $\pi^{+} \pi^{-}$ combination with respect to the direction of the $B$. While particle identification is vital to reject backgrounds from decays like $B^{o} \rightarrow K^{+} \pi^{-}, B_{s} \rightarrow \pi^{+} K^{-}$and $B_{s} \rightarrow K^{+} K^{-}$, it has a small effect on random combinations since most particles are pions.

To start this analysis, we first fit the primary vertices using all tracks which have at least 4 silicon pixel hits. A good primary vertex is required to have $\chi^{2} / N_{p}<4$, where $N_{p}$ is the number of tracks in the primary.

For the two tracks to be considered as $B$-daughter candidates, they must satisfy the following criteria:

- Each track must have $P_{T}>0.5 \mathrm{GeV} / \mathrm{c}$ and at least one track must have $P_{T}>$ $1.5 \mathrm{GeV} / \mathrm{c}$.

- Tracks must have at least eight silicon pixel hits to ensure precise tracking.

- Each track must project into the RICH detector acceptance, because we require particle identification.

- The distance of closest approach (DCA) of the track with respect to the primary vertex must be less than $1 \mathrm{~cm}$; which reduces backgrounds from long lived particles, e.g. $K_{s}$, hyperons. We also require that the normalized DCA (DCA divided by its error) with respect to the primary of each track be $>3$ which removes tracks from the primary.

- Both tracks are in the same arm of detector.

We attempt to fit a secondary vertex with pairs of tracks that satisfy the above criteria. Vertices with $\chi^{2}<4$ are kept for further analysis. For each secondary vertex found, the following selection criteria are applied: 
- The absolute distance between the primary and secondary vertices $(L)$ must be greater than $0.5 \mathrm{~mm}$ and $L / \sigma_{L}>4$.

- Consider all other tracks that do not come from this primary vertex. Form a $\chi^{2}$ with each of these tracks and our selected two tracks for a secondary vertex. Combinations with $\chi^{2}<10$ are rejected, since this might be a many-body $B$ decay.

- The $B^{0}$ direction is calculated from the primary and secondary $B$ vertex positions. The transverse momentum imbalance with respect to that direction must be less than $0.5 \mathrm{GeV} / \mathrm{c}$.

- The invariant mass of the two tracks (assumed to be $\pi^{ \pm}$) must be within $57.5 \mathrm{MeV} / \mathrm{c}^{2}$ $(2 \sigma)$ of $M_{B^{0}}$.

Figure 16.1 shows a comparison of signal and background for several of the variables used above. The background distributions are generated considering all oppositely charged two-track combinations except for the signal $\pi^{+} \pi^{-}$.

Using the selection criteria defined above gives an acceptance and reconstruction efficiency of $8 \%$ for $B^{o} \rightarrow \pi^{+} \pi^{-}$, not including trigger efficiency or particle identification.

It has been shown by the BCD group [2] that the dominant background to $B^{\circ} \rightarrow \pi^{+} \pi^{-}$ comes from random combinations of tracks in events coming from $B$ 's. Tracks from real $B$ 's are already displaced from the primary vertex and have a higher probability of faking a secondary vertex compared to $c \bar{c}$ and minimum bias events.

In addition to background from generic $b \bar{b}$ events, there are several exclusive decay modes of $B$ mesons that can mimic a $B^{o} \rightarrow \pi^{+} \pi^{-}$decay. The decay $B_{s} \rightarrow K^{+} K^{-}$, which is due to a hadronic penguin decay mechanism, is the most important, along with other contributions from $B^{o} \rightarrow K^{+} \pi^{-}$and $B_{s} \rightarrow \pi^{+} K^{-}$. Recent CLEO measurements of some of the $B^{0}$ decay modes give $\mathcal{B}\left(B^{o} \rightarrow \pi^{+} \pi^{-}\right)=0.43 \times 10^{-5}$ and $\mathcal{B}\left(B^{o} \rightarrow K^{+} \pi^{-}\right)=1.7 \times 10^{-5}$ [1]. In order to normalize the $B_{s}$ contribution, we use a $B_{s}$ production rate which is $35 \%$ of the $B^{0}$ rate [3] and assume that the penguin and $b \rightarrow u$ decays of the $B_{s}$ have the same branching ratios as the $B^{0}$. Using these results as input, and without $\pi / K$ discrimination, the two-pion mass plots for the four different two-body decay modes are shown in Fig. 16.2. These plots make it clear that kinematic separation is inadequate to discriminate among these decays.

The $\mathrm{BTeV}$ detector will have an excellent RICH detector for particle identification. We can virtually eliminate two-body backgrounds using the $\mathrm{RICH}$ detector. We passed the simulated background tracks (all tracks including all other interactions in that event), through the RICH simulation code. The efficiency vs background contamination is shown in Fig. 16.3. For an $80 \% \pi^{+} \pi^{-}$signal efficiency, the contamination from $\pi^{ \pm} K^{\mp}\left(K^{+} K^{-}\right)$is $4.0 \%(0.5) \%$.

Since the primary purpose of the Level 1 trigger is to reject light quark backgrounds, there is a strong correlation between triggered events and reconstructed events. The BTeVGeant simulation shows that $64 \%$ of the selected events pass the Level 1 trigger condition. Given a Level 2 efficiency of $90 \%$, this leaves us with 23,700 events per year of running after applying the acceptance, reconstruction efficiency, particle ID efficiency, and trigger efficiency but before flavor tagging. 

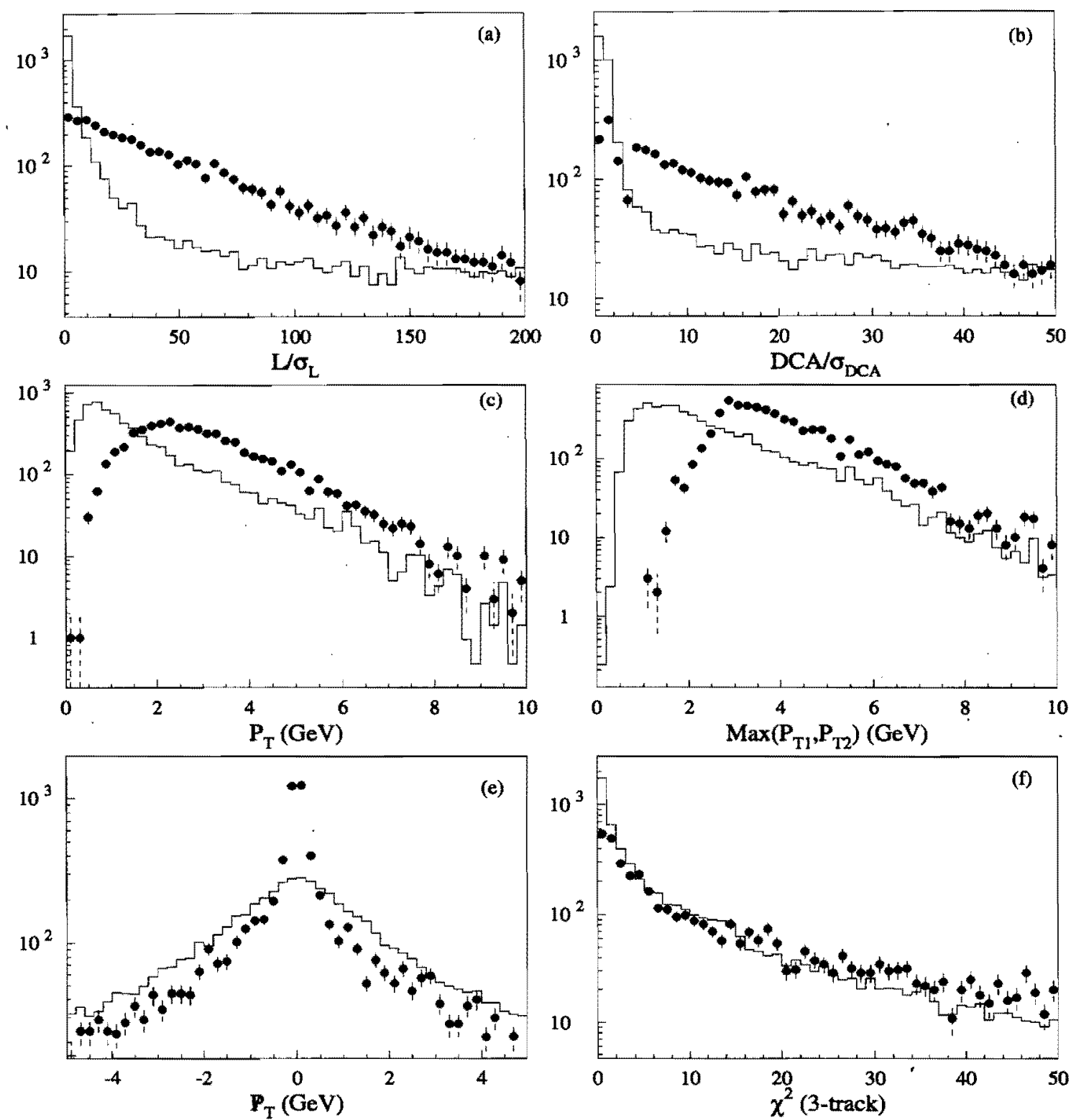

Figure 16.1: Distribution of signal (circles) and background (line) for the most important vertex and kinematic variables. (a) Normalized distance between primary and secondary vertex, $L / \sigma_{L}$, (b) Normalized DCA of track with respect to the primary vertex, $D C A / \sigma_{D C A}$ (c) Transverse momentum of a track, (d) Maximum value of transverse momentum of two tracks, (e) $P_{T}$ imbalance of $\pi^{+} \pi^{-}$with respect to the $B^{\circ}$ direction, and (f) $\chi^{2}$ of secondary vertex using the $\pi^{+} \pi^{-}$with an additional track candidate. 


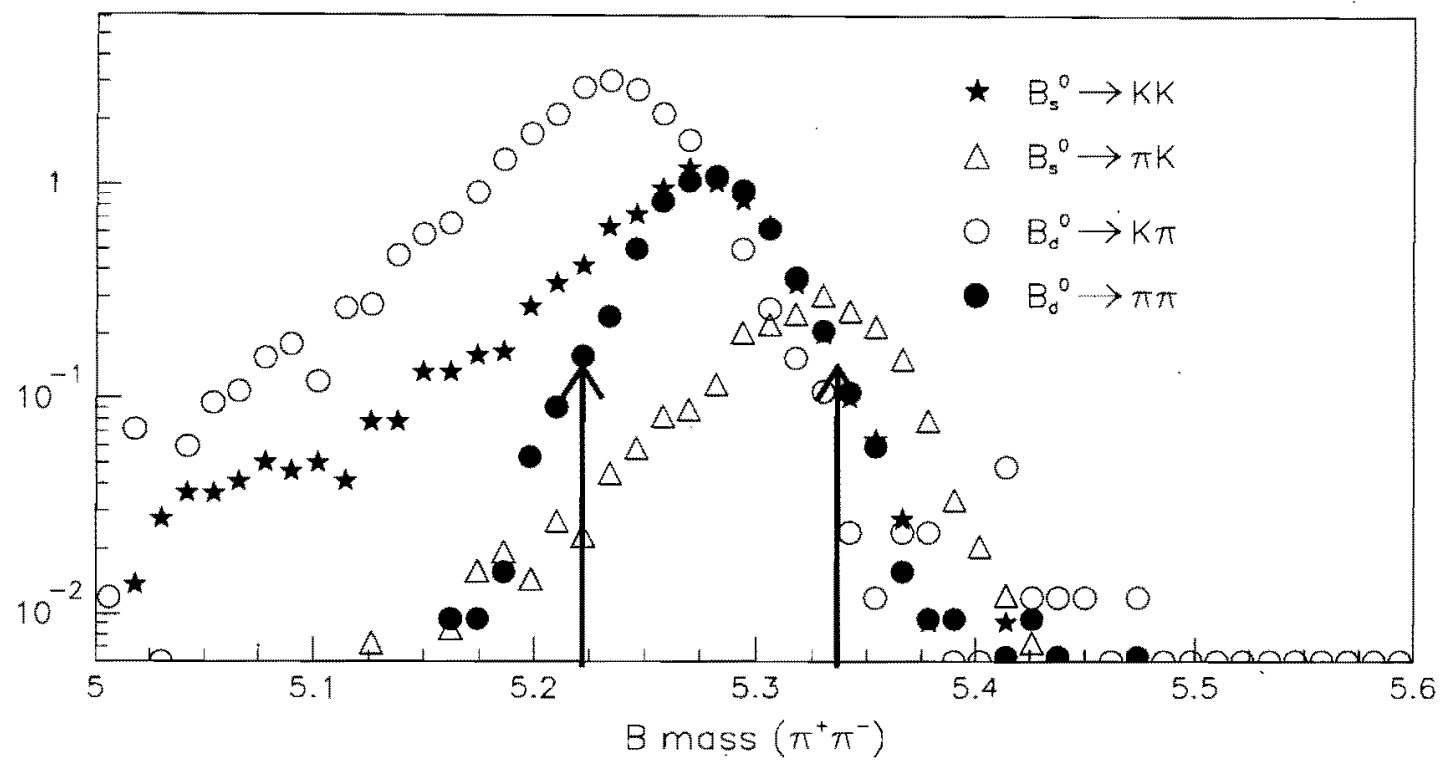

Figure 16.2: Two body $\left(\pi^{+} \pi^{-}\right)$mass plot without particle identification. Different decay channels are normalized by their production cross sections. The arrows indicate the range of the signal mass window. (Note the log scale.)

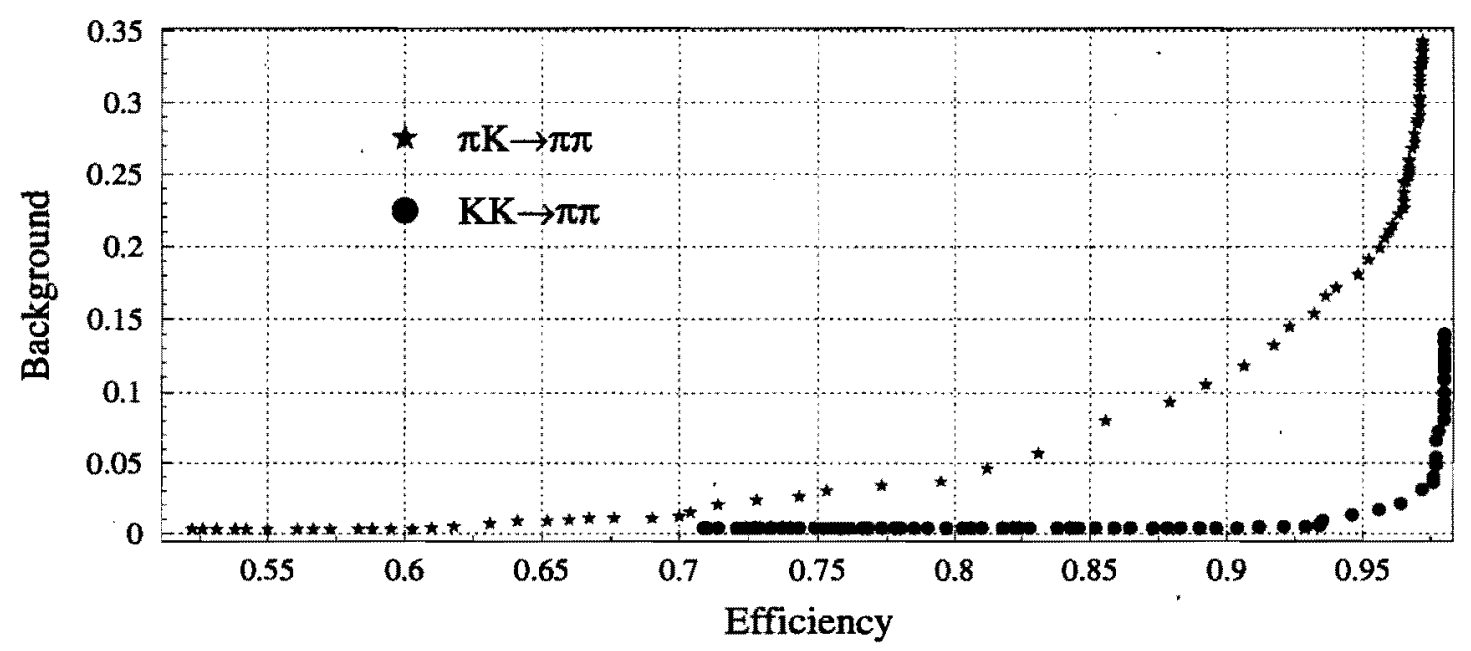

Figure 16.3: RICH event selection: $\pi^{+} \pi^{-}$signal efficiency vs contamination from other two-body decay modes 


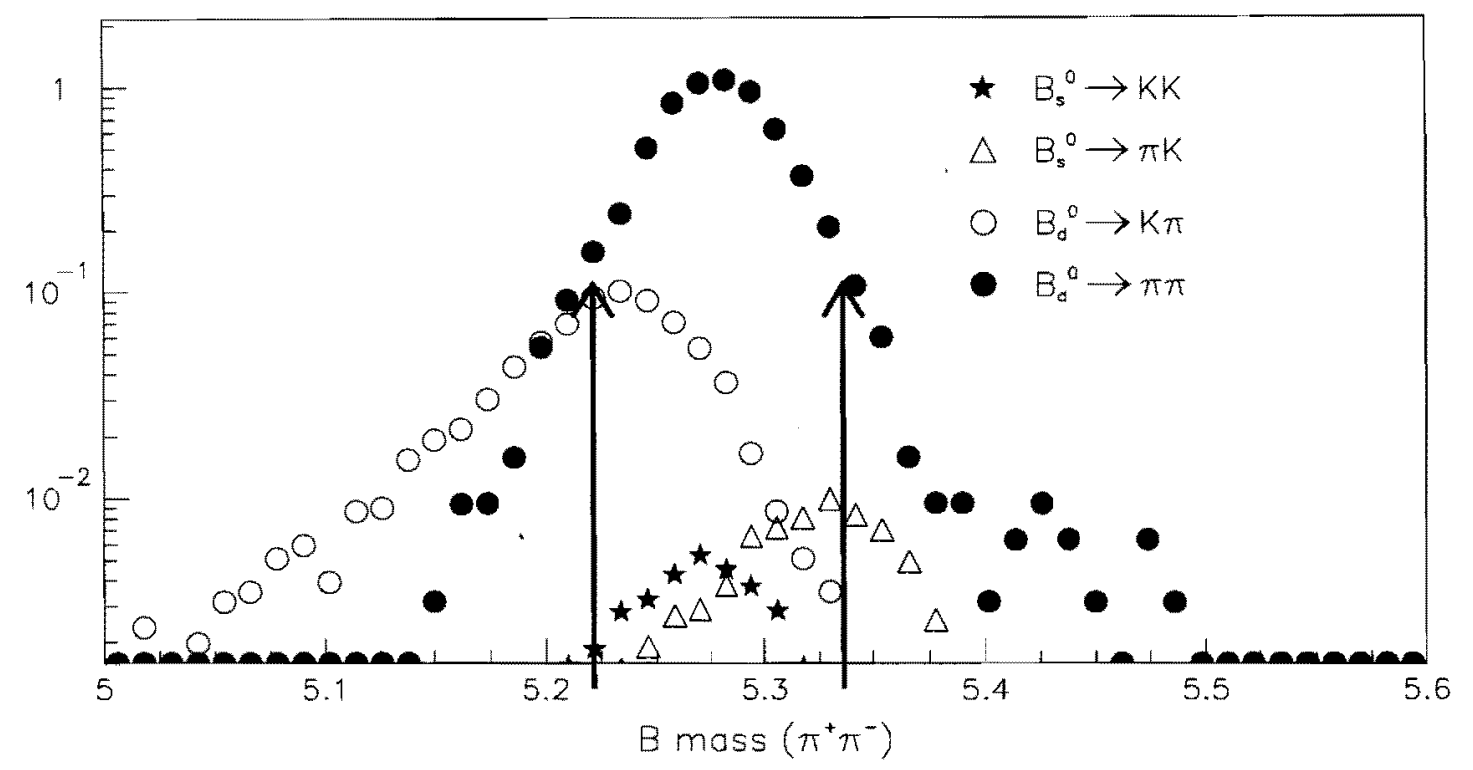

Figure 16.4: Two body $\left(\pi^{+} \pi^{-}\right)$mass plot with particle identification, normalized by their production cross sections. The arrows indicate the range of the signal mass window. (Note the $\log$ scale.)

Besides the two-body $B$ decay background samples, we did a full BTeVGeant simulation of $b \bar{b}$ backgrounds. In order to reduce the CPU time required to simulate a sufficiently large data sample of $b \bar{b}$ decays, we investigated a method to preselect events at the generator level which are likely to cause difficulties. We found that the difference between the reconstructed and generated $P_{T}$ of the tracks is fairly small and Gaussian (in the low $P_{T}$ region, where we have our selection criteria). This is shown in Fig. 16.5. On the basis of the small observed differences, we preselected the generator events before the BTeVGeant simulation. The preselection criteria are based on the $P_{T}(>0.4 \mathrm{GeV} / \mathrm{c})$ of each track, the sum of the $P_{T}(>1.8 \mathrm{GeV} / \mathrm{c})$ of two tracks, the opening angle of the tracks, the extrapolation of tracks to the RICH chamber, etc. In order to reject background at the generator level, we had to sacrifice a small fraction of event selection efficiency.

These preselection requirements reduce the generic $b \vec{b}$ event sample by a factor of 100 . From this sample, only 4 events (two $\pi^{+} \pi^{-}$, one $K^{+} \pi^{-}$and one $\pi^{+} K^{-}$) have a $\pi^{+} \pi^{-}$mass that lies within $200 \mathrm{MeV} / \mathrm{c}^{2}$ of $M_{B^{0}}$. Applying the RICH identification leads to an $80 \%$ efficiency for the two $\pi^{+} \pi^{-}$events and a $4 \%$ efficiency for each of the $K^{+} \pi^{-}$and $\pi^{+} K^{-}$ events. Thus, we have 1.68 background events. If we scale to the $B$ signal region which is $115 \mathrm{MeV} / \mathrm{c}^{2}$ and multiply by the combined Level 1 and Level 2 trigger efficiency (64\% $\times$ $90 \%$ ), we expect $\approx 4,600 b \bar{b}$ background events from one year of running BTeV at the design luminosity of $2 \times 10^{32} \mathrm{~cm}^{-2} \mathrm{~s}^{-1}$.

The remaining contributions (from the two-body decay channels) are listed in Table 16.1 


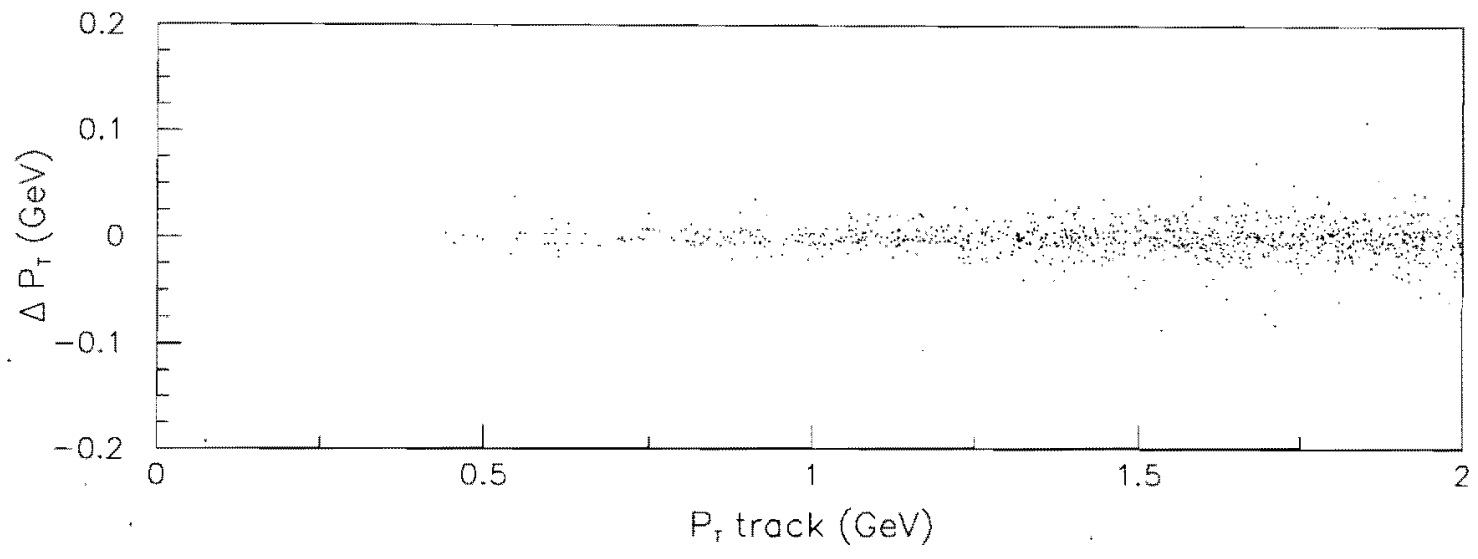

Figure 16.5: $\Delta P_{T}$ vs $P_{T}$ for pion tracks where $\Delta P_{T}$ is the difference between the generated and reconstructed $P_{T}$.

and add up to 3,200 events per year. Therefore, the total background is 7,600 events per year leading to a signal-to-background ratio of $3: 1$ with a $25 \%$ error.

The effective tagging efficiency $\left(\epsilon D^{2}\right)$, discussed in Chapter 15 , is estimated to be $10 \%$. Using the tagging efficiency and the $B^{\circ} \rightarrow \pi^{+} \pi^{-}$yield, we can obtain an uncertainty on the CP asymmetry. Based on one year of running at the design luminosity, we expect an uncertainty on $a_{C P}$ of 0.024 , as summarized in Table 16.1 .

We have repeated this analysis using the MCFast simulation. The reconstruction efficiencies obtained from each simulation agree (8.0\% in BTeVGeant and $8.8 \%$ in MCFast). This result shows that the simulations of the tracking system in BTeVGeant and MCFast are quite similar.

As meritioned in Part $\mathrm{I}$, measuring both $B^{o} \rightarrow \pi^{+} \pi^{-}$and $B_{s} \rightarrow K^{+} K^{-}$may allow an extraction of $\gamma$. To this end, we have also looked for $B_{s} \rightarrow K^{+} K^{-}$signal events. This analysis is nearly identical to the $B^{o} \rightarrow \pi^{+} \pi^{-}$analysis after interchanging $B^{o} \rightarrow \pi^{+} \pi^{-}$and $B_{s} \rightarrow K^{+} K^{-}$samples from signal to background (and vice versa). As in the $B^{o} \rightarrow \pi^{+} \pi^{-}$ analysis, other two-body decay modes can mimic our signal as shown in Fig. 16.6.

From the RICH simulation, we find that at an $80 \%$ signal efficiency for $B_{s} \rightarrow K^{+} K^{-}$, we accept $5 \%(1.5) \% \pi^{+} \pi^{-}\left(K^{+} \pi^{-}, \pi^{+} K^{-}\right)$background events as $K^{+} K^{-}$. It is clear from Fig. 16.7 that by using the $\mathrm{RICH}$ information we can reject most of the backgrounds which are coming from other two-body decay modes.

The expected $B_{s} \rightarrow K^{+} K^{-}$yield, including the acceptance, reconstruction efficiency, trigger efficiency, and particle ID efficiency is 33,000 events per year at the design luminosity. This is summarized in Table 16.1. 


\begin{tabular}{|l|c|}
\hline Luminosity & $2 \times 10^{32} \mathrm{~cm}^{-2} \mathrm{sS}^{-1}$ \\
Running time & $10^{7} \mathrm{sec}$ \\
Integrated Luminosity & $2000 \mathrm{pb}^{-1}$ \\
$\sigma_{b \bar{b}}$ & $100 \mu \mathrm{b}$ \\
Number of $B \bar{B}$ events & $2 \times 10^{11}$ \\
Number of $B^{0}$ events & $1.5 \times 10^{11}$ \\
$\mathcal{B}\left(B^{0} \rightarrow \pi^{+} \pi^{-}\right)$ & $0.43 \times 10^{-5}$ \\
\hline Reconstruction efficiency & $8.0 \%$ \\
Trigger efficiency (Level 1$)$ & $64 \%$ \\
Trigger efficiency (Level 2$)$ & $90 \%$ \\
RICH I. D. efficiency & $80 \%$ \\
Number of reconstructed $(\pi \pi)$ & \\
$B^{o} \rightarrow \pi^{+} \pi^{-}$ & $2.37 \times 10^{4}$ \\
\hline Background after RICH rejection & \\
$B^{o} \rightarrow K^{+} \pi^{-}$ & $0.27 \times 10^{4}$ \\
$B_{s} \rightarrow \pi^{+} K^{-}$ & $0.03 \times 10^{4}$ \\
$B_{s} \rightarrow K^{+} K^{-}$ & $0.02 \times 10^{4}$ \\
$B$-generic & $0.46 \times 10^{4}$ \\
\hline $\mathrm{S} / \mathrm{B}$ & 3 \\
Tagging efficiency $\epsilon \mathrm{D}^{2}$ & $10.0 \%$ \\
$\delta a_{C P}$ & $2.36 \times 10^{-2}$ \\
\hline
\end{tabular}

Table 16.1: Projected yield of $B^{\circ} \rightarrow \pi^{+} \pi^{-}$and the uncertainty on $a_{C P}$ from the BTeVGeant simulation. 


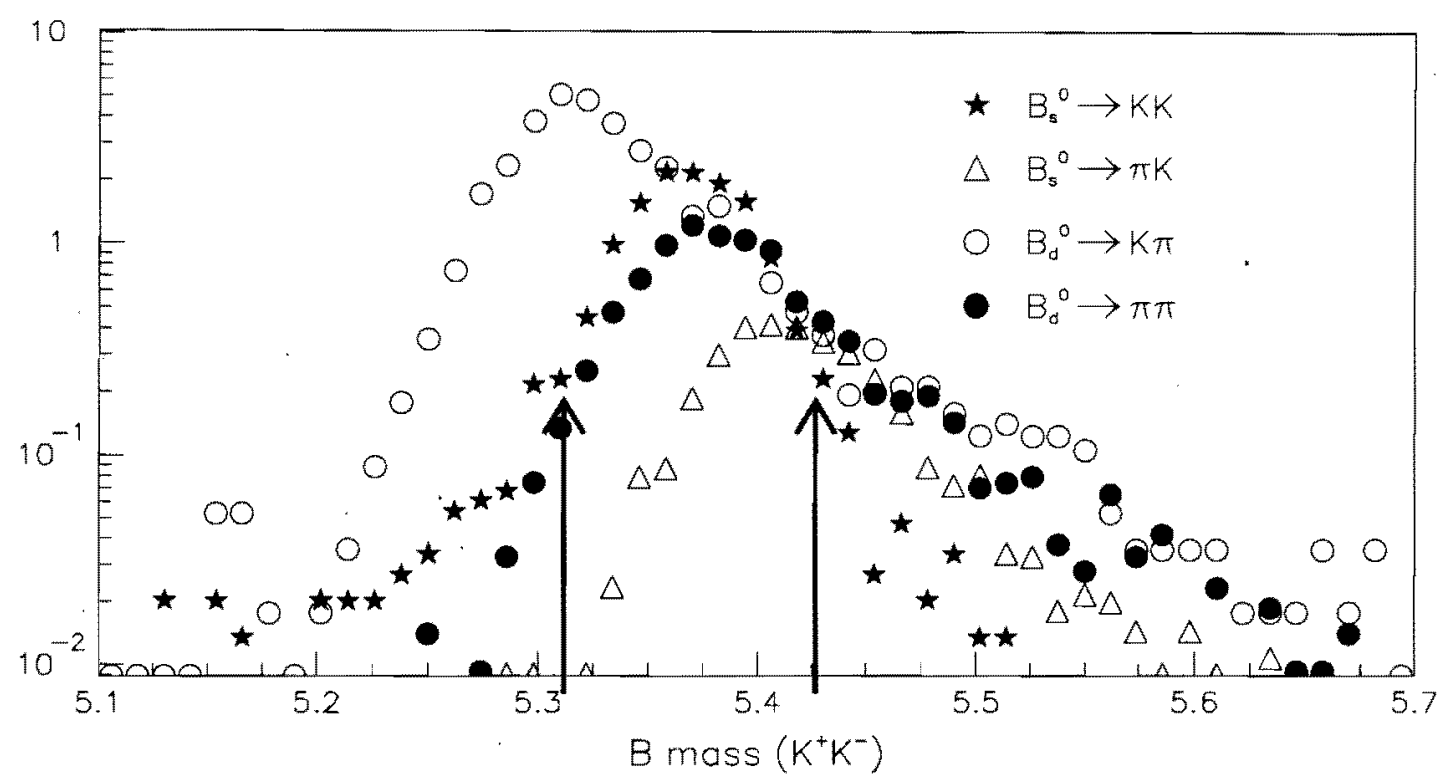

Figure 16.6: Two body $\left(K^{+} K^{-}\right)$mass plot without particle identification. Different decay channels are normalized by their production cross sections. The arrows indicate the range of the signal mass window. (Note the log scale.)

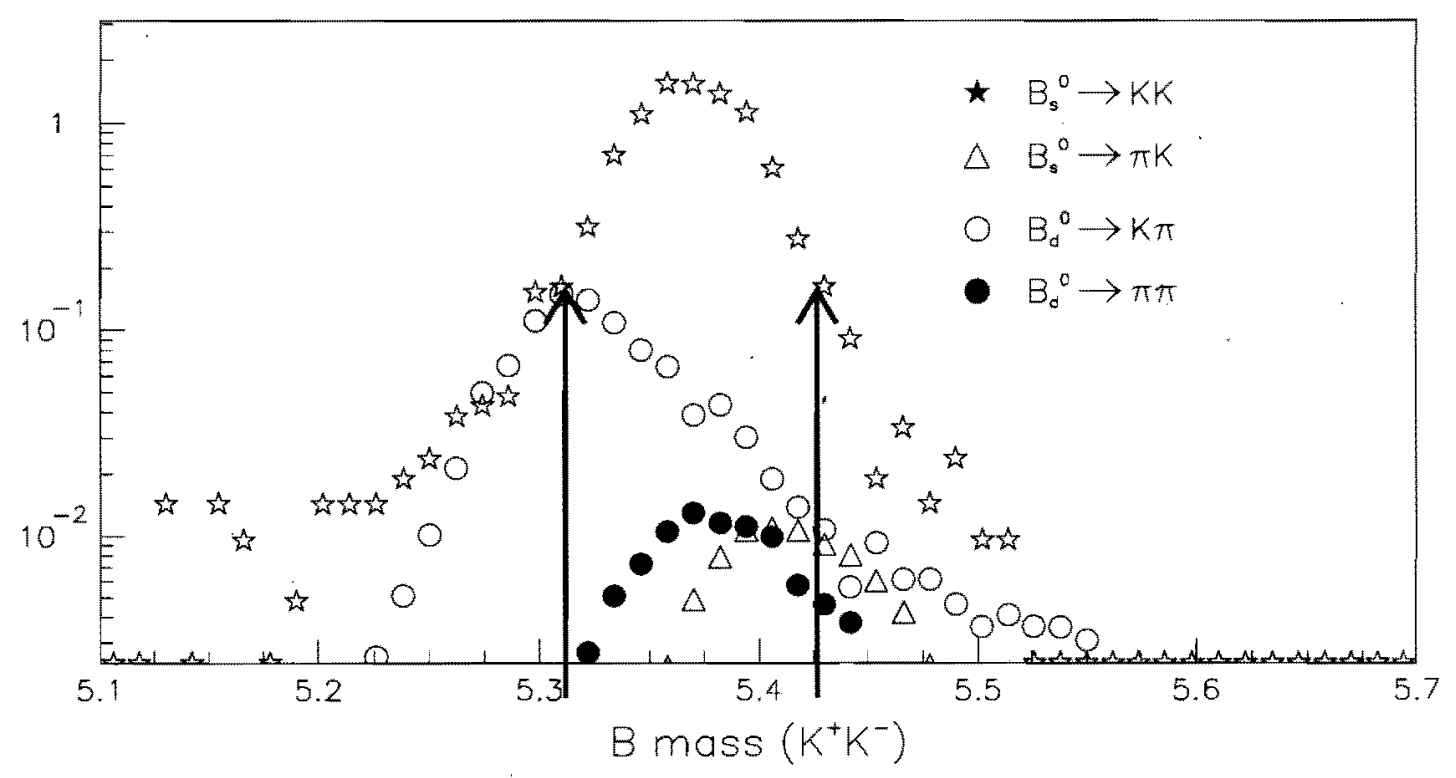

Figure 16.7: Two body $\left(K^{+} K^{-}\right)$mass plot with particle identification and normalized by their production cross sections. The arrows indicate the range of the signal mass window. 


\begin{tabular}{|l|c|}
\hline Luminosity & $2 \times 10^{32} \mathrm{~cm}^{-2} \mathrm{~s}^{-1}$ \\
$10^{7} \mathrm{sec}$ \\
Running time & $2000 \mathrm{pb}^{-1}$ \\
$\sigma_{b \bar{b}}$ & $100 \mu \mathrm{b}$ \\
Number of $B \bar{B}$ events & $2 \times 10^{11}$ \\
Number of $B_{s}$ events & $0.52 \times 10^{11}$ \\
$\mathcal{B}\left(B_{s} \rightarrow K^{+} K^{-}\right)$ & $1.7 \times 10^{-5}$ \\
\hline Reconstruction efficiency & $8.1 \%$ \\
Trigger efficiency (Level 1) & $64 \%$ \\
Trigger efficiency (Level 2) & $90 \%$ \\
RICH I. D. efficiency & $80.0 \%$ \\
Number of reconstructed $(\mathrm{K} \mathrm{K})$ & \\
$B_{s} \rightarrow K^{+} K^{-}$ & $3.29 \times 10^{4}$ \\
\hline Background after RICH rejection & \\
$B^{0} \rightarrow K^{+} \pi^{-}$ & $0.39 \times 10^{4}$ \\
$B_{s} \rightarrow \pi^{+} K^{-}$ & $0.04 \times 10^{4}$ \\
$B^{0} \rightarrow \pi^{+} \pi^{-}$ & $0.04 \times 10^{4}$ \\
$B$-generic & $0.04 \times 10^{4}$ \\
\hline $\mathrm{S} / \mathrm{B}$ & 6.6 \\
\hline
\end{tabular}

Table 16.2: Projected yield of $B_{s} \rightarrow K^{+} K^{-}$and fake rates. 


\subsection{Measuring $\sin 2 \beta$ using $B \rightarrow J / \psi K_{s}^{0}$}

As discussed in Section 1.5, the decay $B_{d}^{0} \rightarrow J / \psi K_{s}^{0}$ is the golden mode for measuring the angle $\beta$ in the unitarity triangle. While we expect that $\sin 2 \beta$ will have been measured before the $\mathrm{BTeV}$ experiment begins, the collaboration aims to significantly improve that measurement. This section will present the reconstruction efficiency, trigger efficiency and signal to background ratio for the decay chain $B_{d}^{0} \rightarrow J / \psi K_{s}^{0}, J / \psi \rightarrow \mu^{+} \mu^{-}, K_{s}^{0} \rightarrow \pi^{+} \pi^{-}$.

For this study, Monte Carlo events were generated using Pythia and QQ as discussed in Section 12.3 and the detector response was simulated using BTeVGeant. The output of BTeVGeant was analyzed as would be real data.

When designing analysis cuts, it is important to understand both the efficiency of the cuts on signal events and the power of the cuts to reject background. Because of the narrow widths of the $J / \psi$ and the $K_{s}^{0}$, the dominant source of background entries is combinations of real $J / \psi \rightarrow \mu^{+} \mu^{-}$decays with real $K_{s}^{0} \rightarrow \pi^{+} \pi^{-}$decays. CDF found that prompt $J / \psi$ 's are a large fraction of total $J / \psi$ production [4] and, extrapolating from their results, one expects that $J / \psi$ 's from $\mathrm{B}$ decays comprise only about $5 \%$ of the total $J / \psi$ production. However, the background from prompt $J / \psi$ production is strongly suppressed by the topological cuts, leaving decays of the type $b \rightarrow J / \psi X$ as the dominant source of background.

The analysis was performed as follows. Each event was required to have an identified primary vertex that was successfully fitted with an acceptable $\chi^{2}$. A track'was identified as a muon candidate provided the Monte Carlo truth table indicated that it was a muon, it had a momentum of more than $5.0 \mathrm{GeV} / c$ and it had a hit in the most downstream muon detector. $J / \psi$ candidates were formed by combining pairs of oppositely charged muon candidates and requiring that the invariant mass of the $\mu^{+} \mu^{-}$pair be within $3 \sigma$ of the known mass of the $J / \psi$. It was also required that the $\mu^{+} \mu^{-}$pair pass a fit to a common vertex, that the vertex fit return an acceptable $\chi^{2}$ and that the vertex be detached from the primary vertex by at least $L / \sigma_{L}>4$, where $L$ is the distance between the two vertices and $\sigma_{L}$ is the error on $L$. As illustrated in Figure 16.8, this cut rejects $99.95 \%$ of the background from prompt $J / \psi$ 's while keeping $80 \%$ of the signal. A fit was performed to constrain the mass of the candidate to that of the $J / \psi$.

All other tracks with a momentum of at least $0.5 \mathrm{GeV} / c$ were accepted as pion candidates, provided they missed the primary vertex by $b>3 \sigma$, where $b$ is the impact parameter between the track and the primary vertex and where $\sigma$ is the error on $b . K_{s}^{0}$ candidates were selected by combining oppositely charged pairs of pion candidates and requiring that the invariant mass of the $\pi^{+} \pi^{-}$. pair be within $3 \sigma$ of the known mass of the $K_{s}^{0}$. It was also required that $K_{s}^{0}$ candidates pass a fit to a common vertex and that the vertex fit return an acceptable

$\chi^{2}$. A fit was performed to constrain the mass of the candidate to that of the $K_{s}^{0}$.

A $B$ candidate was defined as the combination of a $J / \psi$ candidate and a $K_{s}^{0}$ candidate which pointed back to the primary vertex. To reduce combinatorial background it was required that the $K_{s}^{0}$ candidate point back to the $J / \psi$ vertex within $3 \sigma$ and that the $K_{s}^{0}$ impact parameter with respect to the $J / \psi$ vertex divided by its impact parameter with respect to the primary vertex be less than 2.0 . 

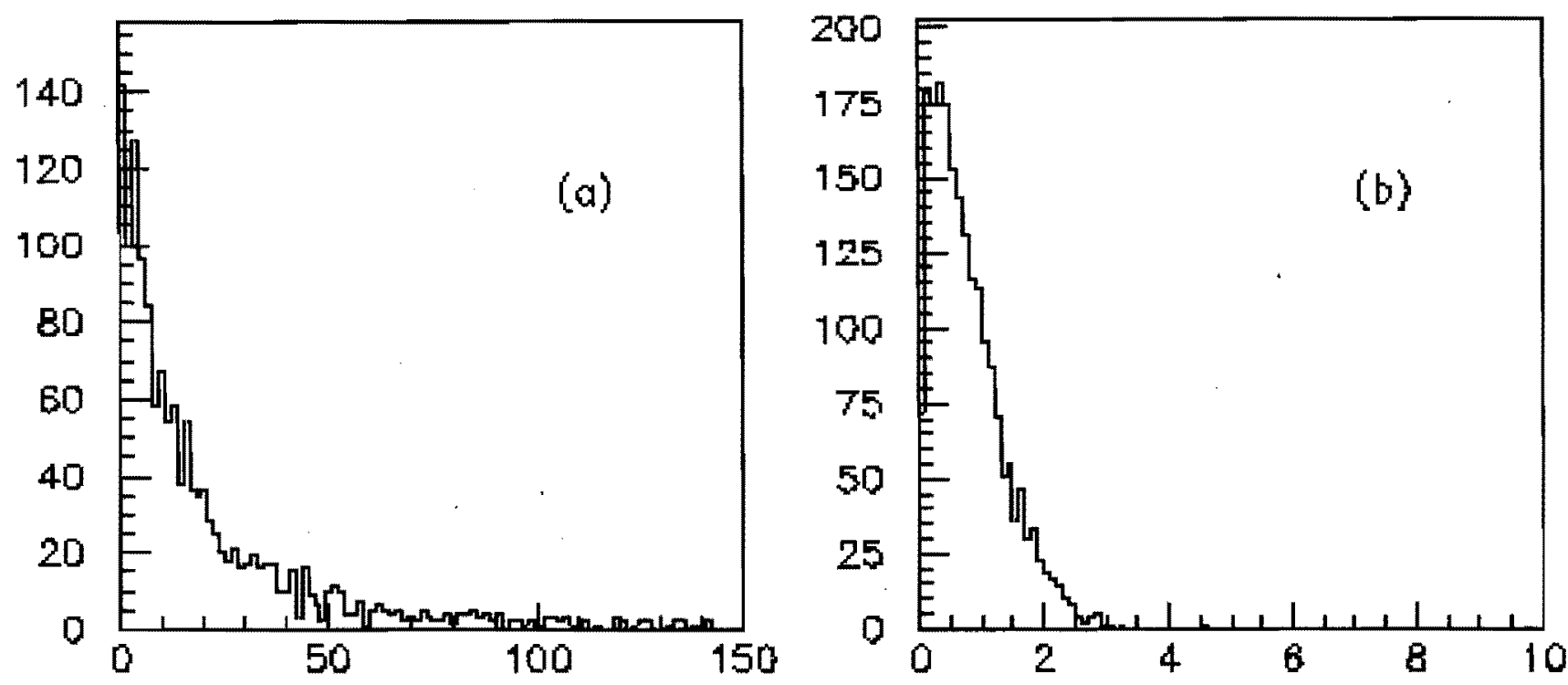

Figure 16.8: Distributions of $L / \sigma_{L}$ for (a) $J / \psi$ candidates from the decays of b-hadrons and (b) prompt $J / \psi$ candidates. The prompt candidates are suppressed by requiring $L / \sigma_{L}>4$.

The invariant mass spectrum of $B$ candidates which pass the above criteria is shown in Figure 16.9. A clear signal with a width of $\sigma=9.3 \mathrm{MeV} / c^{2}$ is seen at the mass of the $B_{d}$. The efficiency for a $B_{d} \rightarrow J / \psi K_{S}^{0}$ decay to fall into the mass peak is $0.040 \pm 0.002$ and the mean resolution on the proper decay time is 50 fs.

As mentioned above, the dominant source of background is decays of the type $b \rightarrow J / \psi X$. This background was studied by generating large samples of such decays, using Pythia and QQ; these samples were passed through the MCFast based detector simulation and analyzed as real data. This study predicted that the signal to background ratio in this channel is approximately $S / B=10$.

The BTeV Level 1 trigger, described in chapters 9 and 14 , was run on events which passed the analysis cuts, and it was found to have an efficiency of $52 \pm 3 \%$. This decay mode can also be triggered by muon and dimuon triggers. Section 8.3 , which describes the algorithms and performance of the muon trigger, estimates a trigger efficiency of $50 \%$ for this decay mode. There is, as yet, no calculation of the total Level 1 trigger efficiency which takes into account the correlations between the two triggers. For this proposal it will be estimated that the combined Level 1 trigger efficiency is $75 \%$.

Section 14.3.3 discusses the performance of the Level 2 tracking trigger. There is, as yet, no calculation of the total Level 2 trigger efficiency which takes into account the correlations between the muon and tracking triggers. For this proposal it will be estimated that the combined Level 2 trigger efficiency is $90 \%$.

In Section 15, it is estimated that the tagging efficiency for $B_{d}$ decays is $\epsilon D^{2}=0.10$.

There are two methods which can be used to extract $\sin (2 \beta)$ from the reconstructed, 


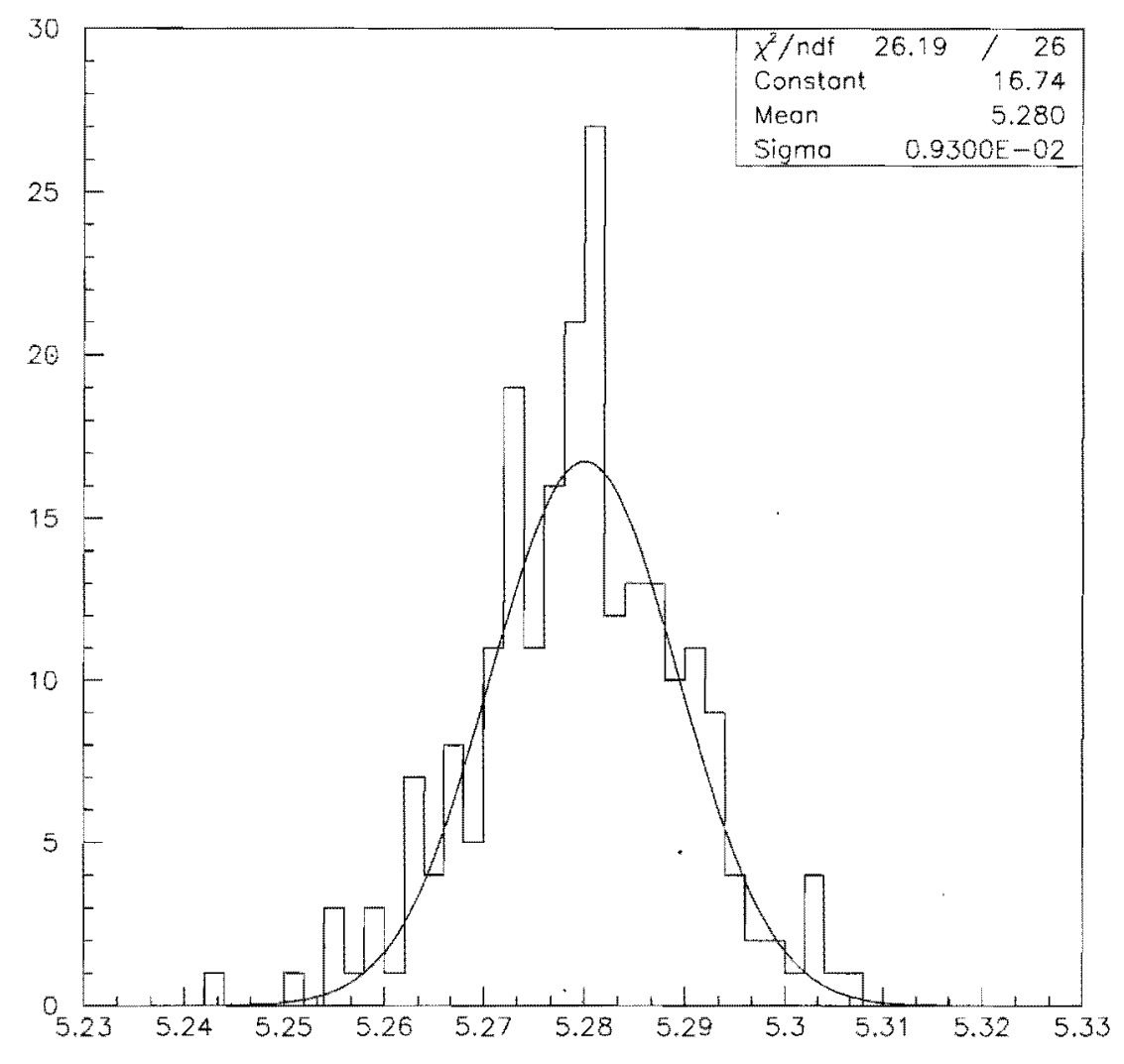

Figure 16.9: The $J / \psi K_{s}^{0}$ invariant mass distribution for candidates which survive the selection criteria described in the text.

tagged $J / \psi K_{s}^{0}$ candidates, a time integrated method and a time dependent method. The sensitivity of the time integrated method is given by,

$$
\delta(\sin (2 \beta))=\frac{1+x_{d}^{2}}{x_{d}} \sqrt{\frac{1}{\epsilon D^{2} N}} \sqrt{\frac{S+B}{S}},
$$

while the sensitivity of the time dependent method is given by,

$$
\delta(\sin (2 \beta))=e^{\frac{x_{d}^{2} \Gamma_{d}^{2} \sigma_{t}^{2}}{2}} \sqrt{\frac{1+4 x_{d}^{2}}{2 x_{d}^{2}}} \sqrt{\frac{1}{\epsilon D^{2} N}} \sqrt{\frac{S+B}{S}},
$$

where $N$ is the number of tagged decays, $x_{d}=0.723 \pm 0.032$ [5] is the $B_{d}$ mixing parameter, $\sigma_{t}$ is the resolution on the proper decay time and where $\Gamma_{d}=0.641 \pm 0.016 \times 10^{12} \mathrm{~s}^{-1}[5]$ is the natural width of the $B_{d}$. For the $B_{d}$, the time dependent method yields a sensitivity which 
is about $20 \%$ better than that given by the time integrated method. In previous documents the $\mathrm{BTeV}$ collaboration has reported the sensitivity on $\sin (2 \beta)$ using the time integrated method but in this document the superior, time dependent, method will be used.

The above discussion is summarized in Table 16.3 which reports a sensitivity of $\delta \sin (2 \beta)=0.025$.

\begin{tabular}{c|c}
\hline Luminosity & $2 \times 10^{32} \mathrm{~cm}^{-2} \mathrm{~s}^{-1}$ \\
Running time & $10^{7} \mathrm{sec}$ \\
$\sigma_{b \bar{b}}$ & $100 \mu \mathrm{b}$ \\
Number of $B \bar{B}$ events & $2 \times 10^{11}$ \\
$\mathcal{B}\left(\bar{b} \rightarrow B_{d}\right)$ & 0.4 \\
Numberr of $B_{d}^{0}$ or $B_{d}^{0}$ & $1.6 \times 10^{11}$ \\
$\mathcal{B}\left(B_{d}^{0} \rightarrow J / \psi K_{s}^{0}\right)$ & $4.45 \times 10^{-4}$ \\
$\mathcal{B}\left(J / \psi \rightarrow \mu^{+} \mu^{-}\right)$ & 0.061 \\
$\mathcal{B}\left(K_{s}^{0} \rightarrow \pi^{+} \pi^{-}\right)$ & 0.6861 \\
$\epsilon($ Geometric + cuts $)$ & 0.04 \\
Level 1 Trigger efficiency & 0.75 \\
Level 2 Trigger efficiency & 0.90 \\
Number of reconstructed $B_{d}^{0} \rightarrow J / \psi K_{s}^{0}$ & $80,500$. \\
Tagging efficiency $\epsilon D^{2}$ & $10 \%$ \\
$S / B$ & 10 \\
Resolution on proper decay time & $0.043 \mathrm{ps}$ \\
$B_{d}$ Lifetime & $1.56 \mathrm{ps}$ \\
$x_{d}$ & 0.723 \\
\hline$\delta \sin (2 \beta)$, time integrated & 0.030 \\
$\delta \sin (2 \beta)$, time dependent & 0.025 \\
\hline
\end{tabular}

Table 16.3: Summary of the sensitivity to $\sin (2 \beta)$ using $B \rightarrow J / \psi K_{s}^{0}$. The time dependent method provides the better sensitivity. 


\section{3 $\quad B^{o} \rightarrow D^{*-} \rho^{+}$}

\subsubsection{Introduction}

We have studied the performance of the BTeV detector for the decay $B^{\circ} \rightarrow D^{*-} \rho^{+}$, which has been adopted as a benchmark decay containing a $\pi^{\circ}$. This decay has been observed and studied in $e^{+} e^{-}$collisions on the $\Upsilon(4 \mathrm{~S})$ resonance by CLEO. It is therefore possible to compare the predicted performance of $\mathrm{BTeV}$ with the CLEO result, which currently defines the state of the art in $\gamma$ and $\pi^{\circ}$ detection in heavy quark decays. The branching ratio $B^{\circ} \rightarrow D^{*-} \rho^{+}$is measured to be $0.0068 \pm 0.0034[5]$.

This decay, and the closely related decay $B^{\circ} \rightarrow D^{*-} \pi^{+}$, also provide a method, albeit a difficult one, of measuring the CKM angle $\alpha[6]$. This is done by studying the differences in the four related rates

$$
\Gamma\left(B_{d}(t) \rightarrow f\right), \Gamma\left(B_{d}(t) \rightarrow \bar{f}\right), \Gamma\left(\bar{B}_{d}(t) \rightarrow \bar{f}\right), \Gamma\left(\bar{B}_{d}(t) \rightarrow f\right)
$$

where $f$ represents the various final states $D^{* \mp} \pi^{ \pm}, \rho^{ \pm}, a_{1}^{ \pm}$, etc. These processes have an interference term which is governed by

$$
\lambda=\frac{q}{p} \frac{<f \mid \bar{B}_{d}>}{<f \mid B_{d}>}=\rho \exp ^{i(\phi+\Delta)}
$$

where $\rho$ is the amplitude ratio and $\Delta$ is a strong phase. The weak phase can be shown to be

$$
\phi=-2 \beta-\gamma=-\pi+\alpha-\beta \text {. }
$$

Since $\beta$ will be known at the time this measurement will be made, this can be viewed as a method of measuring $\alpha$ which is quite different from other methods. The main difficulty in this method lies in the small size of the amplitude ratio, $\rho$ :

$$
\rho \approx\left|\frac{V_{u b}}{V_{c b}}\right| \times\left|\frac{V_{c d}}{V_{u d}}\right| \approx 0.02 .
$$

The small asymmetry means that a large number of events and an excellent understanding of systematics is required for this measurement. If the measurement can be made, its great virtue is that it is not contaminated by Penguin diagrams.

\subsubsection{Analysis}

For this study, we have generated the signal events with Pythia and QQ using the correct helicity structure of the decays determined by CLEO. For our study of the background, we make the assumption that the main source of background events is assumed to be from true $B$ decays of all kinds (i.e. all types of $B$ hadrons) which produce a $D^{*-}$ in the decay chain. For both the signal and background simulation, the $B$ event is accompanied by minimum bias interactions, with the average per generated $B$ being 2 . The detector simulation is performed using BTeVGeant with the thresholds and conditions described in chapter 12 . The analysis relies especially on the Kalman filter, the vertex package and the electromagnetic cluster finder. 
The signal we are studying follows the decay chain

$$
\begin{array}{rll}
B^{o} \quad \rightarrow \quad & D^{*-} \rho^{+} \\
\text {where } & D^{*-} \rightarrow \overline{D^{o}} \pi^{-} \\
\text {and } & \overline{D^{o}} \rightarrow K^{+} \pi^{-} \\
\text {and } & \rho^{+} \rightarrow \pi^{+} \pi^{o} .
\end{array}
$$

At present, we have only looked at $\overline{D^{\circ}}$ decaying into $K^{+} \pi^{-}$, although $K^{+} \pi^{-} \pi^{+} \pi^{-}$should also be copious. The 'signal' sample we are using consists of 40,000 events in which there is a $B^{\circ}$ which is forced to decay according to the above chain, while the away side $\bar{B}$-hadron is decayed generically by QQ. The 'background' sample consists of 200,000 events in which there is a $D^{*-}$ in the final state. The $D^{*-}$ may come from either the $B$ or $\bar{B}$. The $\bar{D}^{\circ}$ which is the daughter of the $D^{*-}$ always decays to $K^{+} \pi^{-}$.

The analysis goes as follows: For the $\overline{D^{0}}$, we require a $K^{+} \pi^{-}$combination with invariant mass between 1.840 and $1.890 \mathrm{GeV} / \mathrm{c}^{2}$. It must decay downstream of the primary vertex and must have a pion (bachelor pion) with the correct sign with which it makes a mass difference of $145.5 \pm 2.0 \mathrm{MeV} / \mathrm{c}^{2}$.

With these requirements, we obtain the $D^{*-}-D$ Q-value shown in Fig. 16.10. The fitted width of this peak is $583 \mathrm{keV}$.

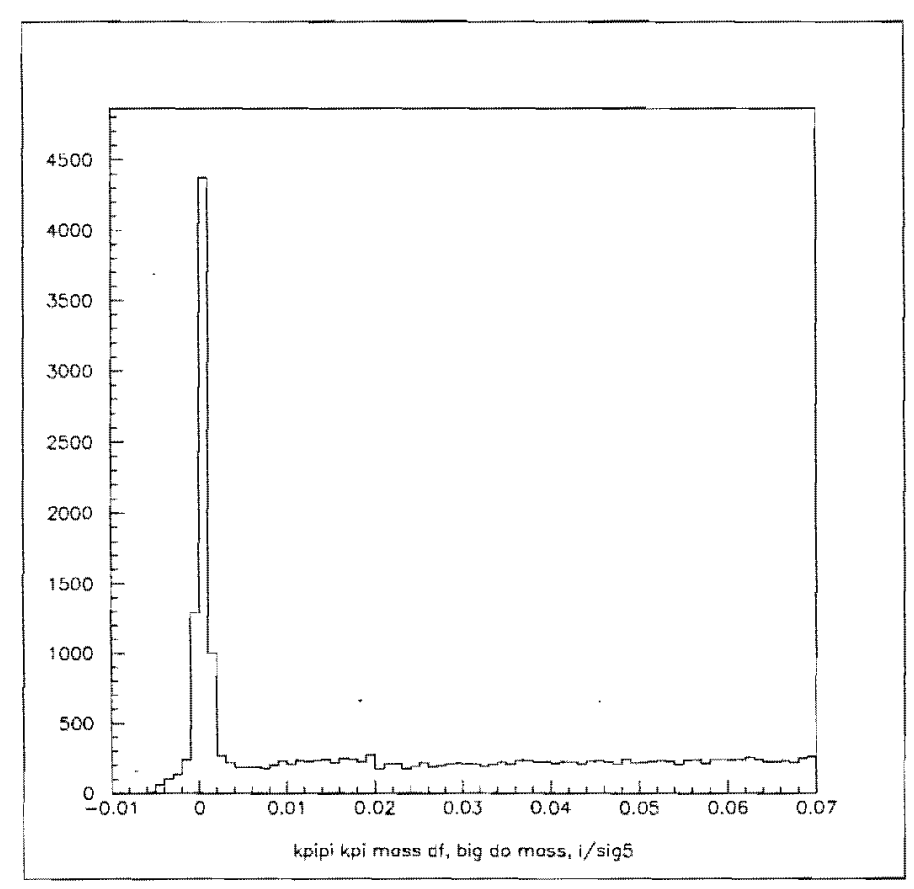

Figure 16.10: The $D^{*-}-D$ Q-value, $\mathrm{M}\left(K^{+} \pi^{-} \pi^{-}\right)-\mathrm{M}\left(K^{+} \pi^{-}\right)-0.1455$, in $\mathrm{GeV} / \mathrm{c}^{2}$.

The $\overline{D^{\circ}}$ must form a vertex with the bachelor pion and an oppositely charged pion with 
total mass less than $5.15 \mathrm{GeV} / \mathrm{c}^{2}$. The vertex formed by these tracks must have at least an 8 standard deviation separation from the primary vertex.

The $\pi^{o}$ is formed by taking pairs of reconstructed showers, "bumps" in the language of our cluster finder, found in the same arm, with mass between 126 and $140 \mathrm{MeV} / \mathrm{c}^{2}$ and which make a good transverse momentum balance about the $B$ direction with the charged tracks in the $B$ candidate (This last requirement is discussed below). To find the $\pi^{\circ}$, we run the calorimeter cluster finder described above. We exclude all shower candidates whose energies are less then $0.750 \mathrm{GeV}$ or which occur within $20 \mathrm{~cm}$ of the beam axis (to stay away from the region of very high occupancy near the beam). We eliminate showers from hadrons or electrons by excluding showers if any charged track points within $10 \mathrm{~cm}$ of the shower's centroid - an 'isolation' cut. We eliminate showers for which the ratio of energy in the 9 blocks around the shower center to the energy in the 25 blocks around the shower is less than 0.85 - a 'shower shape' cut. Showers failing this cut are more spread out than normal electromagnetic showers and may be due to neutral hadrons, hadrons overlapping genuine electromagnetic showers, or partially merging electromagnetic showers. In any case, their energies and angles are likely to be incorrectly measured. In Fig. 16.11, we show the invariant mass distributions of all pairs of neutral showers for various values of the isolation cut. A clear $\pi^{\circ}$ peak is evident. The width of the $\pi^{\circ}$ mass peak is $3.5 \mathrm{MeV} / \mathrm{c}^{2}$. The background in these plots is combinatoric background due to the presence of many additonal photons in the event underlying the $B$ and in the accompanying minimum bias events.

At this point, we form the invariant mass of the oppositely charged pion and all $\pi^{o}$ candidates and require the $\pi^{+} \pi^{o}$ mass to be compatible with the $\rho^{+}$mass, that is, between $0.540 \mathrm{GeV} / \mathrm{c}^{2}$ and $1.0 \mathrm{GeV} / \mathrm{c}^{2}$. This is not a very powerful cut because of the large natural width of the $\rho^{+}$.

If we plot the invariant mass of the $D^{*-}$ and the $\rho^{+}$using only the requirements described so far, the background levels are unacceptably high. The reason for this is that we have to consider all $\pi^{o}$ candidates which, in combination with the bachelor pion, satisfy the very loose " $\rho$ " requirement. This leads to many false $\rho$ combinations which eventually produce a large combinatoric background in the final $D^{*-} \rho^{+}$invariant mass plot.

To defeat this background, we exploit the fact that the pixel detector provides information about the parent $B^{\circ}$ which can be used to eliminate $\pi^{\circ}$ s which are not associated with the $B^{o}$ decay. There are two equivalent ways to understand this. One way is to consider that the $B$ direction is determined by the line connecting the primary vertex with the intersection of the $D^{*}$ and the charged pion from the $\rho$. The direction of this line is known with an accuracy which varies but is often better than a few milliradians. We accept only $\rho \rightarrow \pi^{+} \pi^{\circ}$ candidates which balance the transverse momentum around the direction of the $B$ as determined from the pixel detector. One can implement this by taking the cross product of the $\rho$ momentum vector against the $B$ direction and then requiring it to "balance", i.e. be equal in magnitude and opposite in direction, within resolution, the cross product of the $D^{*}$ momentum vector against the $B$ direction. We have used this method. Alternatively, the 'pointback' method is to form a composite object out of the $D^{*} \rho$ combination and to require the resulting $B$ candidate to project through the $B$ vertex as determined by the 


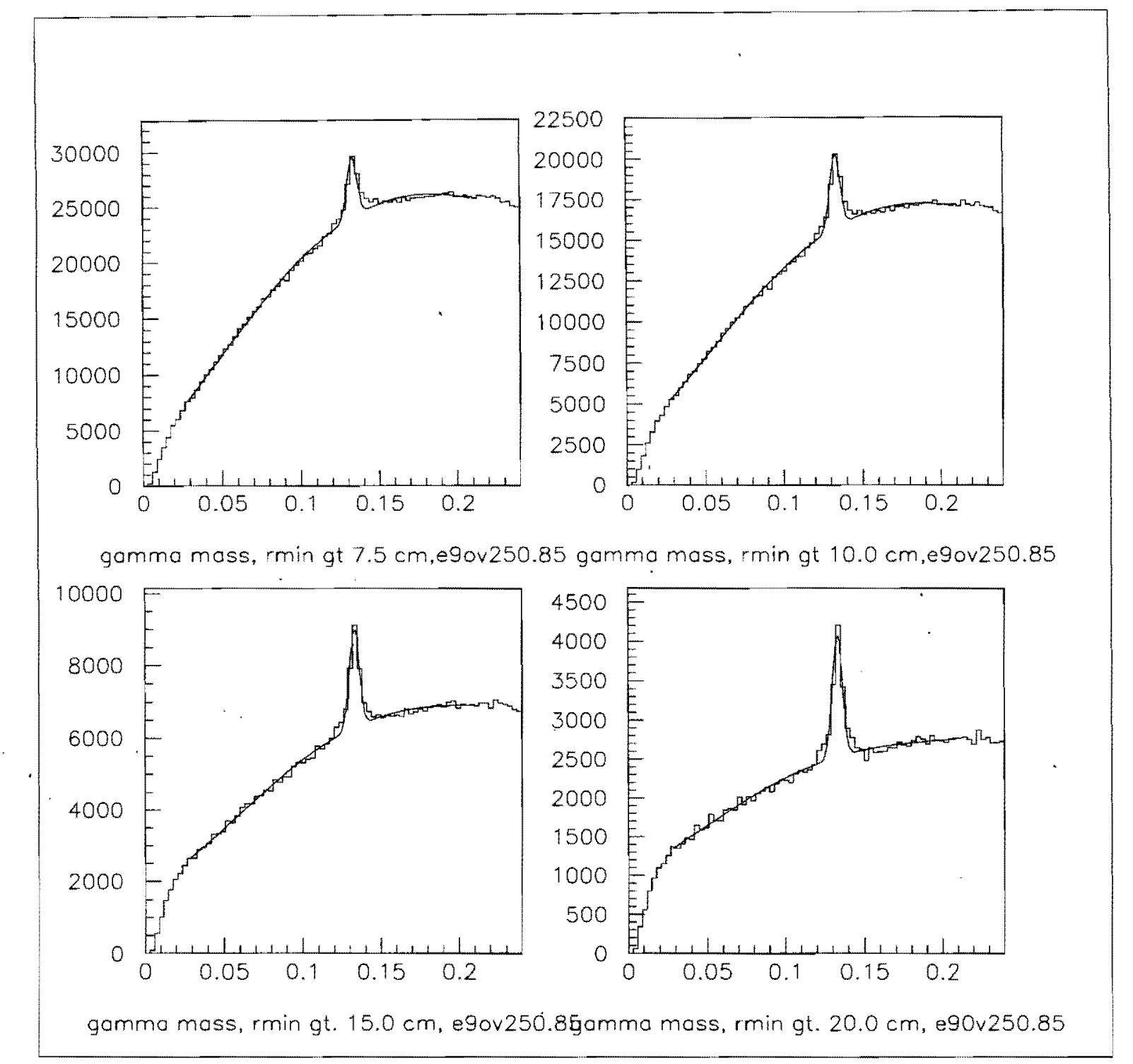

Figure 16.11: Four different sets of cuts on the $\gamma-\gamma$ mass spectrum: (upper left) $R_{\min }<$ $7.5 \mathrm{~cm}$ and $E 9 / E 25<0.85$; (upper right) $R_{\min }<10.0 \mathrm{~cm}$ and $E 9 / E 25<0.85$; (lower left) $R_{\min }<15.0 \mathrm{~cm}$ and $E 9 / E 25<0.85$; and (lower right) $R_{\min }<20.0 \mathrm{~cm}$ and $E 9 / E 25<$ 0.85 . The fit is to a Gaussian and a fourth order polynomial. Most analysis work used the cuts of the plot in the upper right corner. 
pixel detector to the primary vertex position (also determined by the pixel detector). The $P_{t}$ balance method and the pointback requirement are equivalent and serve to reduce the combinatoric background because random $\pi^{\circ} \mathrm{S}$ will rarely combine with the charged pion to satisfy the balance requirement. Figure 16.12 shows schematically how these requirements work. Figure 16.13 shows the polar angle resolution on the $B$ direction for the final signal events in this analysis. The resolution is about 1 milliradian. Figure 16.14 shows the $P_{t}$ balance variable we cut on:

$$
\frac{\left|P_{t}\left(D^{*}\right)\right|-\left|P_{t}(\rho)\right|}{\left|P_{t}\left(D^{*}\right)\right|+\left|P_{t}(\rho)\right|}
$$

The $D^{*}$ and $\rho$ are also required to be produced back-to-back in the plane perpendicular to the $B$ direction.

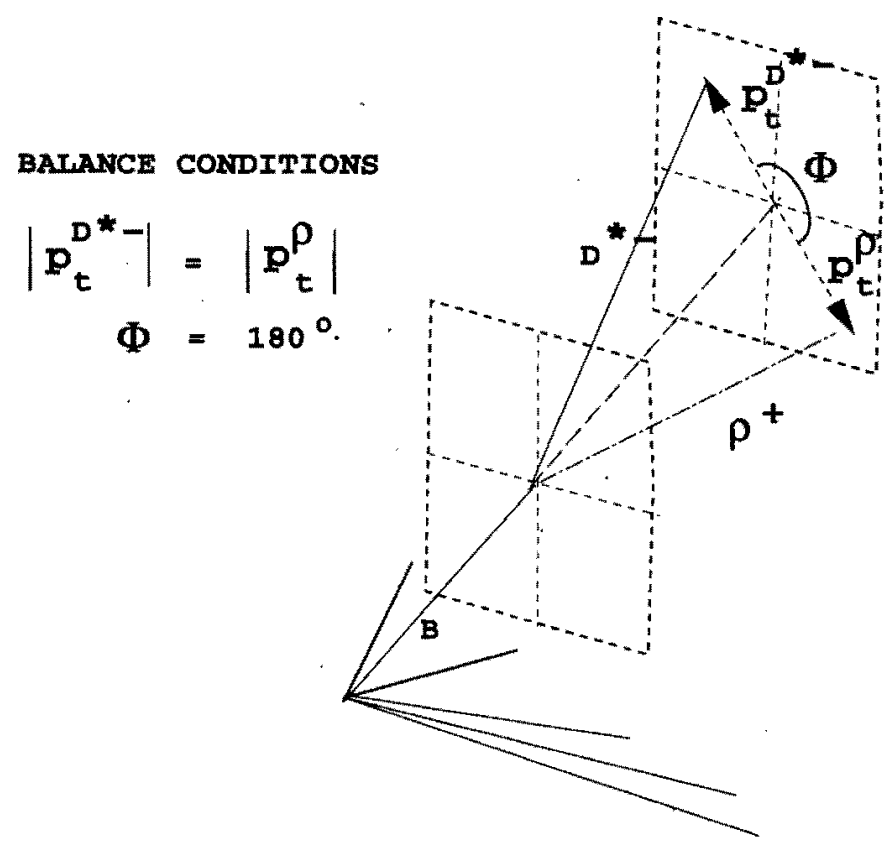

Figure 16.12: $P_{t}$ balance about the $B$ direction.

Figures 16.15 and 16.16 show the effect of the $P_{t}$ balance cut on the signal and background, respectively. The cuts used are 0.15 on the $P_{t}$ balance variable and a requirement that the cosine of the azimuthal angle between the $P_{t}$ vector of the $D^{*}$ relative to the $B$ and the $P_{t}$ vector of the $\rho$ relative to the $B$ be less than -0.98 . The invariant mass peak of the final state signal, (Fig. 16.15), which contains 4 charged tracks and two photons has a width of about $35 \mathrm{MeV} / \mathrm{c}^{2}$. The number of background combinations in the vicinity of the signal, (Fig. 16.16), is reduced by a factor of more than 15 by this technique. The yield of signal events is hardly affected by these cuts. 


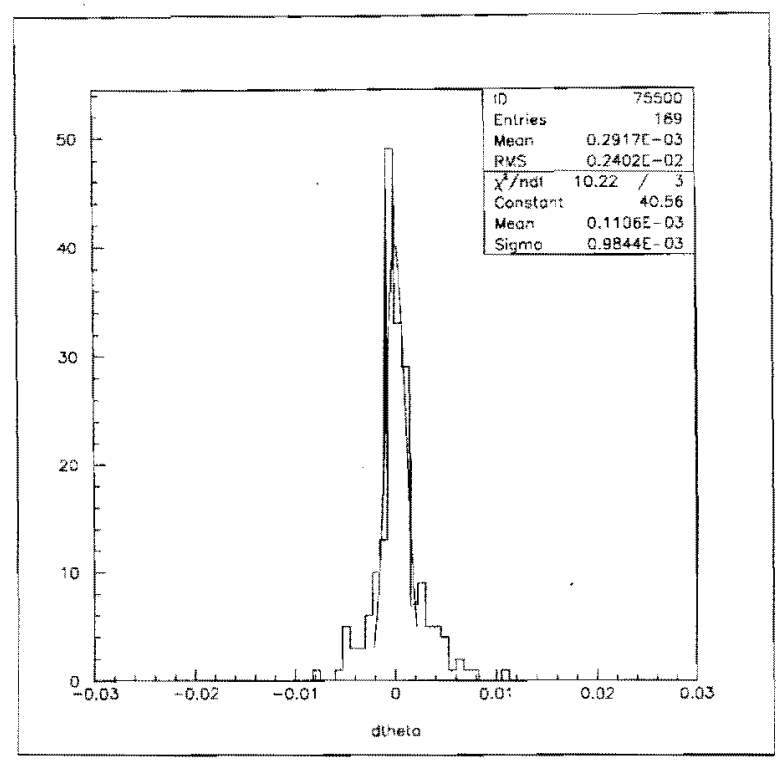

Figure 16.13: Resolution on $B$ polar angle, $\theta$, measured from the fitted primary and $B$ vertices. The quantity plotted is $\theta_{\text {measured }}-\theta_{\text {generated }}$. The width of the "core" of the distribution is about 1 milliradian.

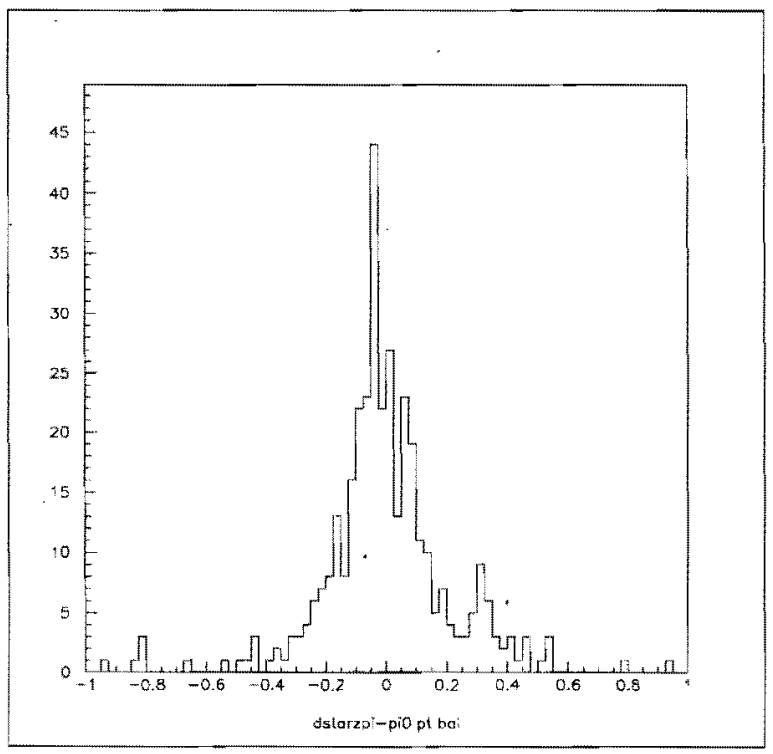

Figure 16.14: Distribution of the $P_{t}$ balance variable for signal events. 


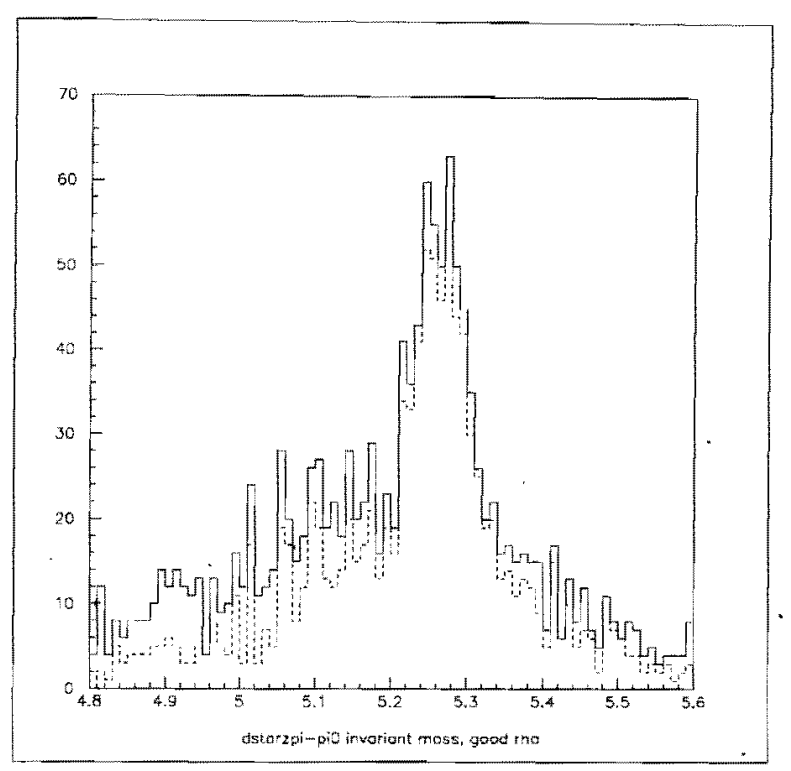

Figure 16.15: Effect of $P_{t}$ balance cuts on signal events. Both histograms show the invariant mass of the $D^{*} \rho$ system. The solid histogram is before the $P_{t}$ balance cuts and the dashed histogram is after the cuts. The number of events is only slightly affected.

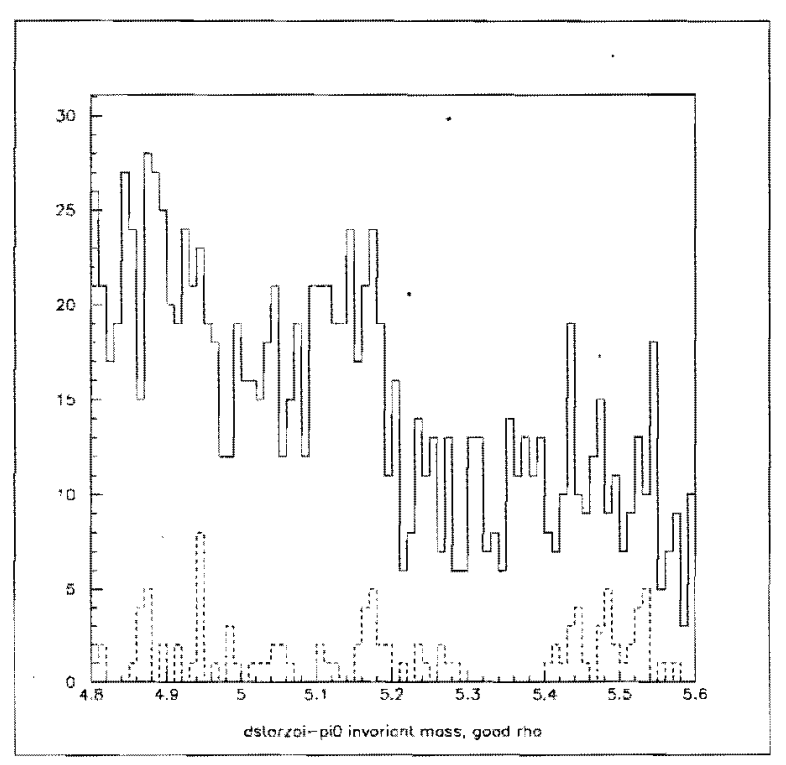

Figure 16.16: Effect of $P_{t}$ balance cuts on background events. Both histograms show the invariant mass of the $D^{*} \rho$ system. The solid histogram is before the $P_{t}$ balance cut and the dashed histogram is after the cuts. The number of events is reduced by a factor of more than 15 . 
The invariant mass distribution shown in Fig. 16.15 for signal events has a peak sitting on top of a broad "background". Comparison of the events in this distribution with generator information reveals that almost all the events in the broad side structures are real signal events with the correct charged track assignments. The background is due to either correct photon assignments where one of the photon's energy has been mismeasured due to the presence of other showers nearby, or to pairings of one correct photon with an incorrect, very low energy, photon which replaces the correct one.

The $\pi^{\circ}$ s expected for this state have an average energy of only about $6 \mathrm{GeV}$. They are rather fragile in the sense that any energy from other sources that overlaps the signal photons will either cause signal to be lost or its resolution to be degraded. Fig. 16.17 shows the effect on the signal shape when different cuts are placed on the energy of the $\pi^{\circ}$. Both size and quality of the signal are affected by these cuts. Sensitivities are quoted below for each of these four signals.

The background is obtained by subjecting the $D^{*}$ inclusive $B$ decay events, generated as described above, to the same analysis as the signal events. We use the calculation below to establish the correct absolute normalization of the signal and the background distributions and to derive the total yield and the signal-to-background ratio.

The calculation of the number of events per year at the BTeV design luminosity of $2 \times 10^{32} \mathrm{~cm}^{-2} \mathrm{~s}^{-1}$ and the signal-to-background ratio is shown in Table 16.4. The number of signal events per year is expected to be about $70,000-100,000$. The signal-to-background ratio depends on the cuts but is about $2: 1$. If we simply restrict the mass interval over which we define the signal, this improves to over $3: 1$ for the tightest cut, with a loss of about $7 \%$ of the signal. We have now run enough background events so that we have a reasonable sample for studying the cuts to try to reduce the background further.

\subsubsection{Background from Charm}

At the Tevatron, charm production is expected to be an order of magnitude larger than $B$ production. The copious production of $D^{*} \mathrm{~s}$ from charm is a potential background to the $B^{o} \rightarrow D^{*-} \rho^{+}, \pi^{+}$signals. We have generated 100,000 events with a $D^{*}$ which we then force to decay to $D^{o} \pi$ and the $D^{o}$ to $K \pi$. We pass these through our analysis programs and no events survive. We place a $90 \%$ confidence level upper limit on the yield per year of 25,000 . The $90 \%$ confidence level upper limit on the signal-to-background from charm is $4 / 1$ and is probably much better. The conclusion is that the background will be mainly from $B$ decays, not charm.

\subsubsection{Effect of Multiple Interactions}

We have described how $\mathrm{BTeV}$ defeats the combinatoric photon background even in the presence of an average of two minimum bias events per crossing. We studied the signalto-background ratio as a function of the number of interactions per crossing. The result is shown in Fig. 16.18, where we plot the $D^{*-} \pi^{+}$invariant mass for various values of the number 


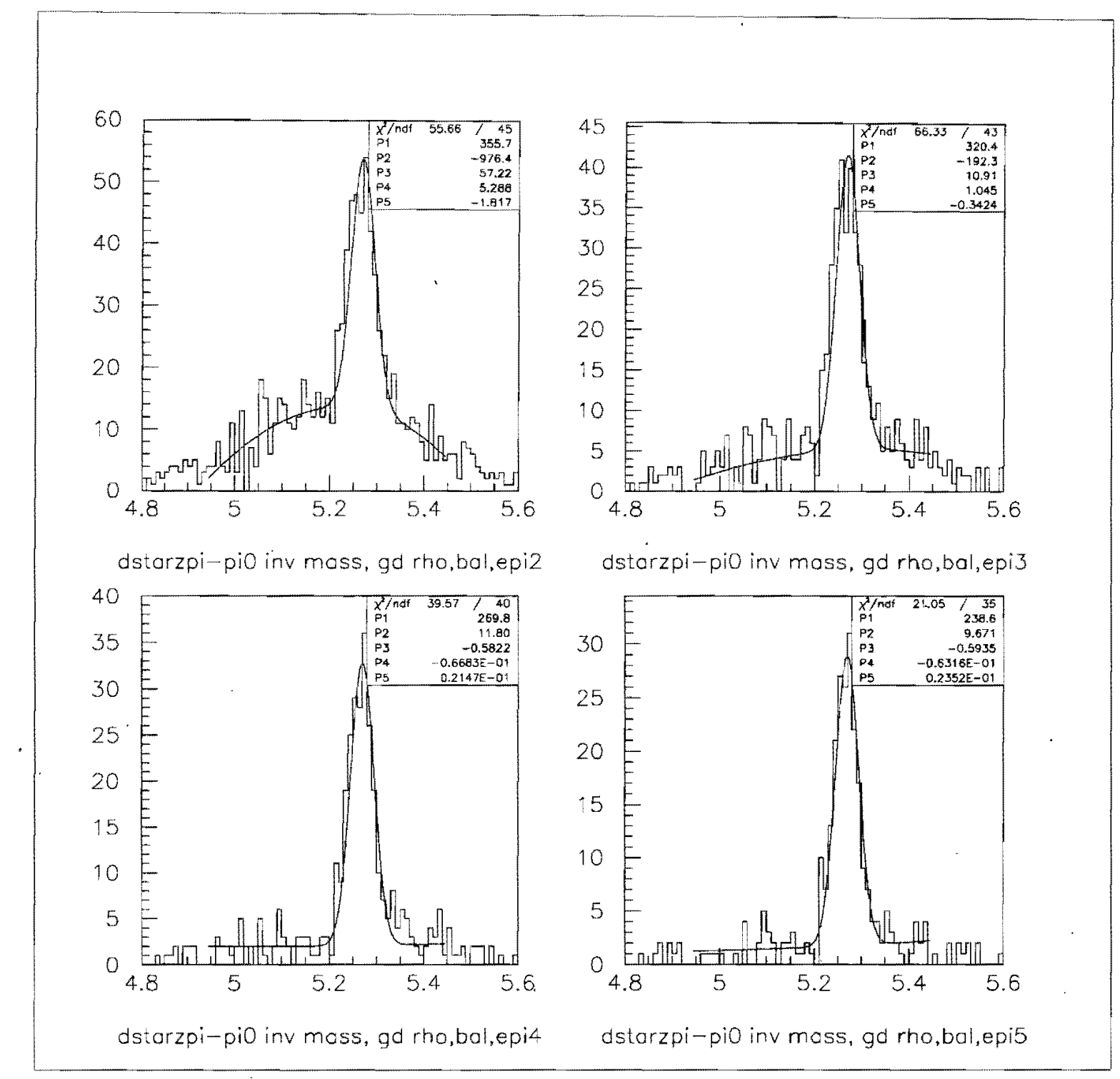

Figure 16.17: $D^{*} \rho^{+}$invariant mass for four values of the minimum allowed $\pi^{o}$ energy: (upper right) $2 \mathrm{GeV}$; (upper left) $3 \mathrm{GeV}$; (lower right) $4 \mathrm{GeV}$; and (lower left) $5 \mathrm{GeV}$. As the $\pi^{o}$ energy requirement is raised, the tails of the mass peak disappear. 


\begin{tabular}{|c|c|}
\hline Quantity & Value \\
\hline$\overline{b \bar{b} \text { events/year }}$ & $2 \times 10^{11}$ \\
\hline \multicolumn{2}{|l|}{ Signal: } \\
\hline$\overline{B^{\circ} / \overline{B^{\circ}} / \text { year }}$ & $1.5 \times 10^{11}$ \\
\hline $\mathcal{B}\left(B^{o} \rightarrow D^{*-} \rho^{+}\right)$ & 0.007 \\
\hline $\mathcal{B}\left(D^{*-} \rightarrow \overline{D^{o}} \pi^{-}\right)$ & 0.68 \\
\hline $\mathcal{B}\left(\overline{D^{o}} \rightarrow K^{+} \pi^{-}\right)$ & 0.04 \\
\hline \multicolumn{2}{|l|}{ No. events produced per year } \\
\hline$B^{o} \rightarrow D^{*-} \rho^{+}, D^{*-} \rightarrow \overline{D^{o}} \pi^{-}, \overline{D^{o}} \rightarrow K^{+} \pi^{-}+\mathrm{CC}$ & $2.86 \times 10^{7}$ \\
\hline Width of signal & $32 \mathrm{MeV} / \mathrm{c}^{2}$ \\
\hline Mass window & $5.20-5.34 \mathrm{GeV} / \mathrm{c}^{2}$ \\
\hline \multicolumn{2}{|l|}{ Number of MC signal events recon. in mass window: } \\
\hline$\pi^{\circ}$ Energy $>2.0 \mathrm{GeV}($ efficiency $=0.79 \%)$ & 314 \\
\hline$\pi^{o}$ Energy $>3.0 \mathrm{GeV}($ efficiency $=0.69 \%)$ & 277 \\
\hline$\pi^{o}$ Energy $>4.0 \mathrm{GeV}($ efficiency $=0.58 \%)$ & 232 \\
\hline$\pi^{o}$ Energy $>5.0 \mathrm{GeV}($ efficiency $=0.53 \%)$ & 212 \\
\hline Trigger efficiency. - Level 1 & 0.63 \\
\hline Trigger efficiency - Level 2 (estimate) & 0.90 \\
\hline Overall Trigger Efficiency & 0.57 \\
\hline \multicolumn{2}{|c|}{ Expected no. events reconstructed and triggered per year: } \\
\hline$\overline{\pi^{\circ} \text { Energy }>2.0 \mathrm{GeV}(\mathrm{S} / \mathrm{N}=1.07)}$ & $12.8 \times 10^{4}$ \\
\hline$\pi^{o}$ Energy $>3.0 \mathrm{GeV}(\mathrm{S} / \mathrm{N}=1.56)$ & $11.2 \times 10^{4}$ \\
\hline$\pi^{o}$ Energy $>4.0 \mathrm{GeV}(\mathrm{S} / \mathrm{N}=1.95)$ & $9.4 \times 10^{4}$ \\
\hline$\pi^{o}$ Energy $>5.0 \mathrm{GeV}(\mathrm{S} / \mathrm{N}=2.39)$ & $8.6 \times 10^{4}$ \\
\hline \multicolumn{2}{|l|}{ Background (in $140 \mathrm{MeV} / \mathrm{c}^{2}$ ): } \\
\hline$D^{*}$ production fraction & 0.22 \\
\hline $\mathcal{B}\left(D^{*-} \rightarrow \overline{D^{o}} \pi^{-}\right)$ & 0.68 \\
\hline $\mathcal{B}\left(\overline{D^{o}} \rightarrow K^{+} \pi^{-}\right)$ & 0.04 \\
\hline$D^{*-} \rightarrow \overline{D^{\circ}} \pi^{-}, \overline{D^{o}} \rightarrow K^{+} \pi^{-}+\mathrm{CC}$ & $2.4 \times 10^{9}$ \\
\hline \multirow{2}{*}{\multicolumn{2}{|c|}{$\begin{array}{l}\text { Number of MC BG events in mass window } 4.8-5.6 \mathrm{GeV} \text { : } \\
\pi^{o} \text { Eneroy }>2.0 \mathrm{GeV} \text { (efficiency }=0.05 \% \text { ) }\end{array}$}} \\
\hline & 102 \\
\hline$\pi^{o}$ Energy $>3.0 \mathrm{GeV}($ efficiency $=0.03 \%)$ & 58 \\
\hline$\pi^{\circ}$ Energy $>4.0 \mathrm{GeV}$ (efficiency $=0.02 \%$ ) & 40 \\
\hline$\pi^{o}$ Energy $>5.0 \mathrm{GeV}($ efficiency $=0.015 \%)$ & 30 \\
\hline Trigger effic. assumed to be same as signal & 0.57 \\
\hline$\pi^{\circ}$ Energy $>2.0 \mathrm{GeV}$ & $12.0 \times 10^{4}$ \\
\hline$\pi^{\circ}$ Energy $>3.0 \mathrm{GeV}$ & $7.2 \times 10^{4}$ \\
\hline$\pi^{\circ}$ Energy $>4.0 \mathrm{GeV}$ & $4.8 \times 10^{4}$ \\
\hline$\pi^{o}$ Energy $>5.0 \mathrm{GeV}$ & $3.6 \times 10^{4}$ \\
\hline
\end{tabular}

Table 16.4: Details of the calculation of the signal and background events obtained in one year of running for the decay $B^{o} \rightarrow D^{*-} \rho^{+}$ 
of generated primary vertices in the crossing. The signal-to-background ratio obviously degrades after about 4 interactions/crossing.

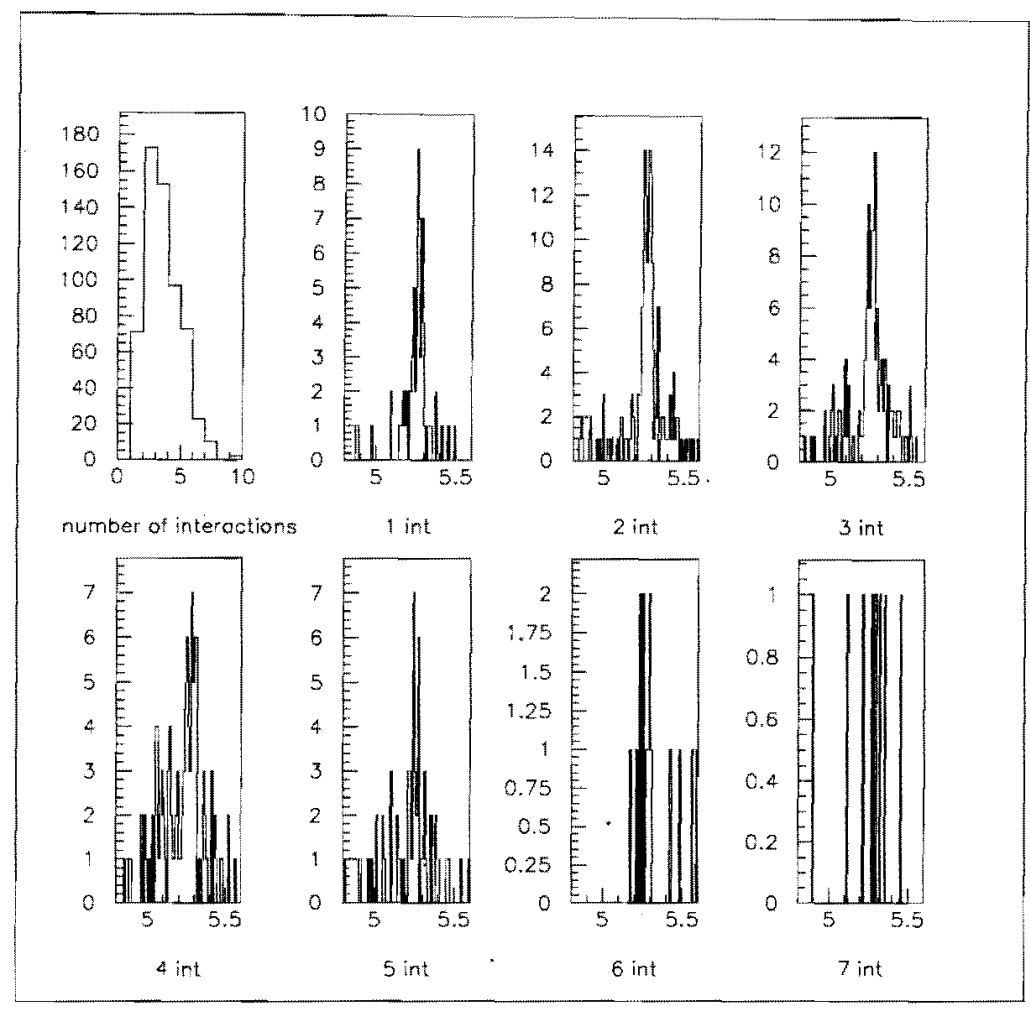

Figure 16.18: The top left plot shows the number of interactions per crossing for this analysis. The next seven plots show the $D^{*}-\rho^{+}$invariant mass plot when there are 1 to 7 interactions in the crossing.

\subsubsection{The CLEO result}

We can compare BTeV's signal-to-background and yield to CLEO's. The most up-to-date CLEO data are presented in B. Barish et al, CLEO CONF 97-01, EPS 97-339 [7].

The CLEO data sample consists of $3.1 \mathrm{fb}^{-1}$ taken on the $\Upsilon(4 S)$ and $1.4 \mathrm{fb}^{-1}$ taken on the continuum at a center-of-mass energy $60 \mathrm{MeV}$ lower. CLEO has always taken on-resonance and off-resonance data with approximately 2:1 ratio of luminosities. In the next generation of $e^{+} e^{-} b$-factories, the design luminosities are approximately $3 \times 10^{33} \mathrm{~cm}^{-2} \mathrm{~s}^{-1}$, which could yield $30 \mathrm{fb}^{-1}$ in a "Snowmass" year of $10^{7} \mathrm{~s}$. Thus the reported CLEO yields, given below, should be multiplied by a factor of 6.7 to compare with the expected yields at the time that BTeV could run.

For the mode $\bar{B} \rightarrow D^{*+} \rho^{-}$, CLEO uses three $D^{o}$ decay modes: $K^{-} \pi^{+}, K^{-} \pi^{+} \pi^{o}$ and $K^{-} \pi^{+} \pi^{+} \pi^{-}$. The reconstructed $B$ mass plots are shown in Fig. 16.19. 

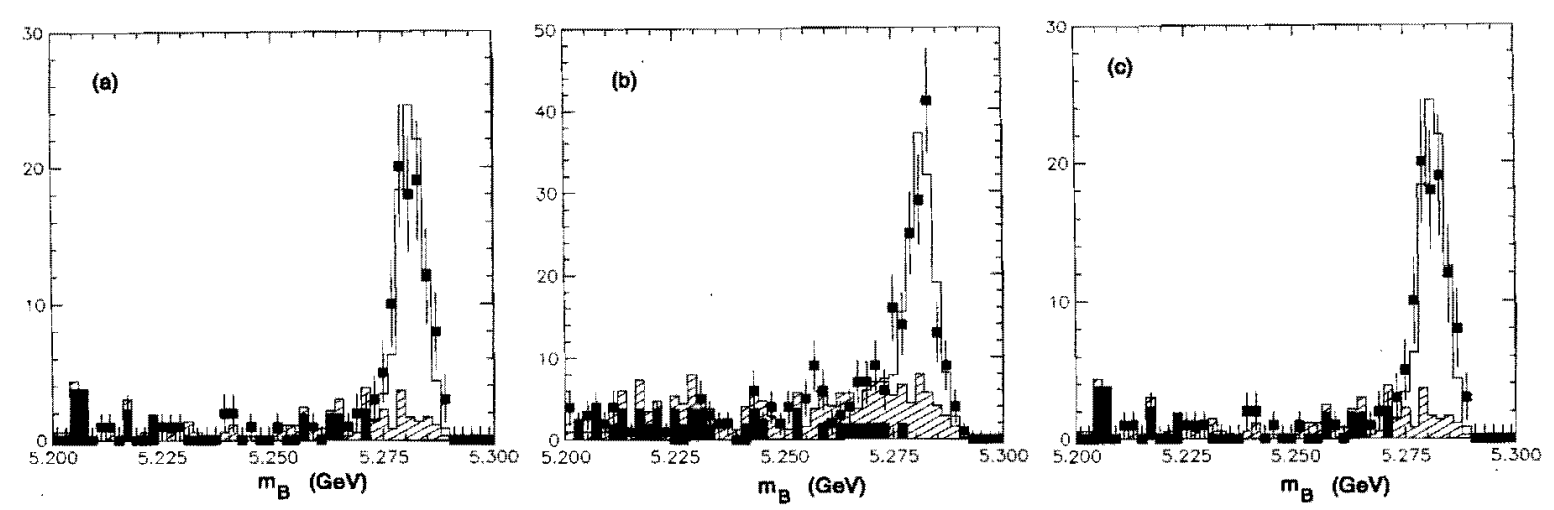

Figure 16.19: Reconstructed mass plots for $\bar{B} \rightarrow D^{*+} \rho^{-}$from CLEO. The data measured on the $\Upsilon(4 S)$ are shown as points with error bars, the scaled continuum data are shown as filled rectangles and the estimated $B \bar{B}$ background in cross-hatch. The solid histogram is the result of the fit to a signal Gaussian and the two background components. The three plots are for different $D^{a}$ decay modes: (a) $K^{-} \pi^{+}$, (b) $K^{-} \pi^{+} \pi^{o}$ and (c) $K^{-} \pi^{+} \pi^{+} \pi^{-}$.

In Table 16.5 we give the measured CLEO yields. The signal to background (S:B) ratio for the $D^{o} \rightarrow K^{-} \pi^{+}$mode is approximately $4.5: 1$ and is worse for the other decay modes.

\begin{tabular}{lrrr}
\multicolumn{4}{c}{ Table 16.5: CLEO yields for $\bar{B} \rightarrow D^{*+} \rho^{-}}$. \\
\hline \hline$D^{o}$ mode & Yield & efficiency & $\mathcal{B}(\%)$ \\
\hline$K^{-} \pi^{+}$ & 77 & $10.8 \%$ & $0.805 \pm 0.107$ \\
$K^{-} \pi^{+} \pi^{o}$ & 110 & $4.9 \%$ & $0.666 \pm 0.086$ \\
$K^{-} \pi^{+} \pi^{+} \pi^{-}$ & 63 & $4.6 \%$ & $0.749 \pm 0.136$ \\
\hline \hline
\end{tabular}

\subsubsection{Comparison and Summary}

The signal to background expected in $\mathrm{BTeV}$ compares very favorably with that obtained by CLEO, and is expected to improve simply by optimizing the cuts after the backgrounds are studied in detail. The $\mathrm{BTeV}$ event yield per year is more than 230 times higher than is anticipated from CESR/CLEO III, BaBar, and BELLE.

This analysis, obtained with a full and realistic GEANT simulation, demonstrates BTeV's ability to study states with $\gamma_{\mathrm{S}}$ and $\pi^{\circ} \mathrm{s}$. We have shown that even with a complicated underlying event and accompanying minimum bias events, we can successfully reconstruct the very low energy photons from these decays and can suppress the combinatoric background. 


\subsubsection{Implications for Measurement of $\alpha$}

Since the CP asymmetry in this class of decays is expected to be only of order $2 \%$, a measurement requires many tens of thousands of tagged events. The final state $D^{*-} \pi^{+}$does not have an additional $\pi^{\circ}$ and so will have a higher reconstruction efficiency than $D^{*-} \rho^{+}$. Because it is a vector-pseudoscalar decay rather than a vector-vector decay, there are fewer decay helicity amplitudes to deal with. The $D^{*-} \pi^{+}$branching fraction is $0.00276 \pm 0.00021$ [5], which is only $40 \%$ of the $D^{*-} \rho^{+}$'s, but the reconstruction efficiency is $4-6$ times more (depending on the cuts) so there will be more events. We expect $7-10,000$ tagged $D^{*-} \rho^{+}$and $15,000-25,000$ $D^{*-} \pi^{+}$per year, using just the $K \pi$ decay mode of the $D^{\circ}$. We expect that the use of the $K 3 \pi$ decay mode of the $D^{\circ}$ will contribute an equal number for both decays. Finally, it should be possible to use the $K^{+} \pi^{-} \pi^{o}$ decay mode of the $D^{\circ}$ for the $D^{*-} \pi^{+}$decay. It should be possible to reconstruct 50,000 tagged decays per year. A measurement of $\alpha$ by this technique may be feasible over a period of a few years. 


\subsection{Measurement of $\gamma$}

Several suggestions on how to measure the CKM angle $\gamma$ have been summarized in Chapter 1. While discrete ambiguities are inherent in each of these methods, using several methods will help remove some of these ambiguities as well help control systematic errors. We report here our simulations of three methods.

\subsubsection{CP Violation in $B_{s} \rightarrow D_{s} K^{-}$}

\subsubsection{Introduction}

When a final state $f$ can come from both a pure $B^{0}$ and a pure $\bar{B}^{0}$, the amplitudes for the direct decay $B^{0} \rightarrow f$ and the mixing induced sequence $B \rightarrow \bar{B}^{0} \rightarrow f$ can interfere. A time dependent CP-violating effect can thus appear. Consider the following decay widths [8]:

$$
\begin{aligned}
\Gamma(B \rightarrow f)= & \frac{|M|^{2}}{2} e^{-t}\left\{\left(1+\rho^{2}\right) \cosh (y t)+\left(1-\rho^{2}\right) \cos (x t)\right. \\
& +2 \rho \cos (\delta+\phi) \sinh (y t)-2 \rho \sin (\delta+\phi) \sin (x t)\} \\
\Gamma[\bar{B} \rightarrow \bar{f}]= & \frac{|M|^{2}}{2} e^{-t}\left\{\left(1+\rho^{2}\right) \cosh (y t)+\left(1-\rho^{2}\right) \cos (x t)\right. \\
& +2 \rho \cos (\delta-\phi) \sinh (y t)-2 \rho \sin (\delta-\phi) \sin (x t)\} \\
\Gamma[B \rightarrow \bar{f}]= & \frac{|M|^{2}}{2} e^{-t}\left\{\left(1+\rho^{2}\right) \cosh (y t)-\left(1-\rho^{2}\right) \cos (x t)\right. \\
& +2 \rho \cos (\delta-\phi) \sinh (y t)+2 \rho \sin (\delta-\phi) \sin (x t)\} \\
\Gamma[\bar{B} \rightarrow f]= & \frac{|M|^{2}}{2} e^{-t}\left\{\left(1+\rho^{2}\right) \cosh (y t)-\left(1-\rho^{2}\right) \cos (x t)\right. \\
+ & +2 \rho \cos (\delta+\phi) \sinh (y t)+2 \rho \sin (\delta+\phi) \sin (x t)\}
\end{aligned}
$$

where:

$$
\begin{gathered}
M=\langle f \mid B\rangle \\
M^{\prime}=\langle f \mid \bar{B}\rangle \\
\rho=\left|M^{\prime} / M\right| \\
x=\Delta m / \Gamma \\
y=\Delta \Gamma / 2 \Gamma
\end{gathered}
$$




$$
\begin{aligned}
& \delta=\text { strong phase } \\
& \phi=\text { CKM phase }
\end{aligned}
$$

An example of this type of decay is $B_{s}$ which can decay into both $D_{s}^{+} K^{-}$and $D_{s}^{-} K^{+}$. The branching fractions are expected to both be $\sim 10^{-4}$ and in this case the weak angle $\phi=\gamma$. If $\Delta \Gamma=0$ then the quantities $\rho, \sin (\gamma+\delta)$ and $\sin (\gamma-\delta)$ can be extracted from a time dependent study and $\sin \gamma$ can be extracted up to a 2 -fold ambiguity. If $\Delta \Gamma$ is significantly different from zero then both $\sin (\gamma+\delta)$ and $\cos (\gamma+\delta)$ are determined from the fit and $\gamma$ can be determined without ambiguities. It is assumed that the value of $x_{s}$ will have been determined in a separate measurement, and $\Delta \Gamma$ can probably be determined more accurately from other measurements.

\subsubsection{Reconstruction Efficiency}

A study of the reconstruction efficiency has been done for the decay modes

$$
\begin{gathered}
B_{s} \rightarrow D_{s} K, D_{s} \rightarrow \phi \pi, \phi \rightarrow K^{+} K^{-} \text {and } \\
B_{s} \rightarrow D_{s} K, D_{s} \rightarrow K^{*} K, K^{*} \rightarrow K^{+} \pi^{-}
\end{gathered}
$$

The events were generated by Pythia and the detector modeled using BTeVGeant. Each event consists of a $b \bar{b}$ interaction and a mean of 2 minimum bias interactions, to simulate a luminosity of $2 \times 10^{32} \mathrm{~cm}^{-2} \mathrm{~s}^{-1}$. Loose cuts were applied initially and the tighter cuts were chosen after the background was studied.

For the $D_{s} \rightarrow \phi \pi$ decay mode the following cuts were used:

- At least one of the kaons from the $\phi$ decay and also the $K$ from the $B_{s}$ decay were required to be identified in the $\mathrm{RICH}$.

- All tracks have at least 3 hits in the silicon pixel detector.

- The impact parameter with respect to the primary vertex was $>3 \sigma$ for all 4 charged tracks.

- To reduce the background due to "detached" tracks that really come from other interactions we require that the impact parameter with respect to the primary vertex be less than $0.2 \mathrm{~cm}$ for all tracks.

- The $\phi$ and $D_{s}$ were required to be within $\pm 2.5 \sigma$ of the nominal mass.

- The distance between the primary vertex and $D_{s}$ decay vertex $L<8.0 \mathrm{~cm}$ and $L / \sigma_{L}\left(D_{s}\right)>10.0$.

- $L / \sigma_{L}\left(B_{s}\right)>4.0$.

- The transverse momentum of the $B_{s}$ with respect to its line of flight from the primary vertex was required to be less than $1.0 \mathrm{GeV} / \mathrm{c}$. 
Table 16.6: Projected Number of Reconstructed $B_{s}^{0} \rightarrow D_{s} K$ per year

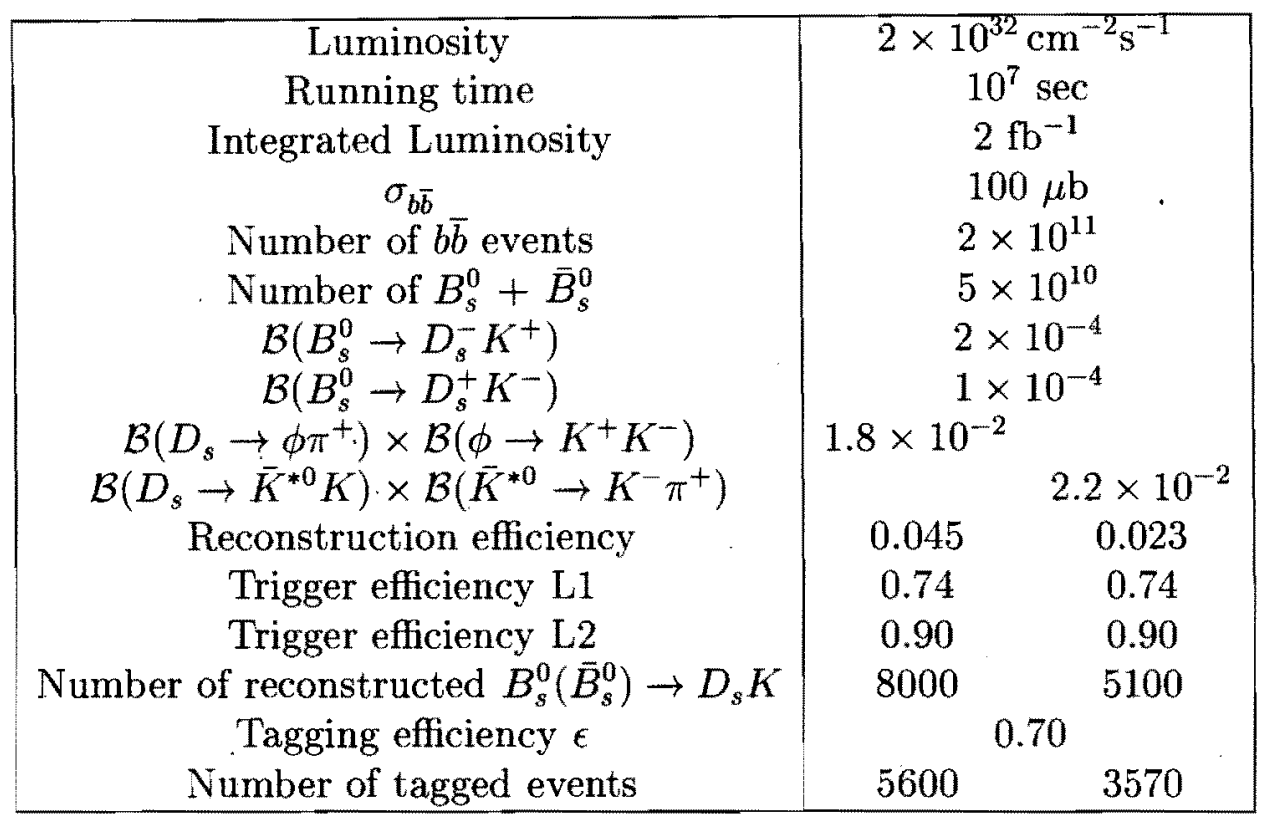

- The impact parameter with respect to the primary vertex was required to be less than $3 \sigma$ for the reconstructed $B$.

The distributions of $L / \sigma_{L}$ and the mass peaks for the $D_{s}$ and $B_{s}$ are shown in Fig 16.20.

The combined geometric acceptance and reconstruction efficiency was found to be $4.5 \%$. If we require both kaons from the $\phi$ decay to be identified in the RICH, the efficiency drops to $2.5 \%$. Of the events that passed these analysis cuts, $74 \%$ passed the secondary vertex trigger. For the $D_{s} \rightarrow K^{*} K$ mode we used the same cuts except that both kaons from the $D_{s}$ decay were required to be identified in the RICH. The combined reconstruction efficiency and geometric acceptance for the $D_{s} \rightarrow K^{*} K$ mode was found to be $2.3 \%$, and the trigger efficiency for the events passing the analysis cuts was $74 \%$.

The results of the tagging study described in Chapter 15 indicate that we can expect a tagging efficiency $\epsilon=0.70$ and a dilution $D=0.37$ giving an effective tagging efficiency $\epsilon D^{2}=0.01$.

The expected number of events in $10^{7}$ seconds is shown in Table 16.6.

As the CP asymmetry is diluted by a factor of $e^{-\sigma_{t}^{2} x_{s}^{2} / 2}$ it is important to have good time resolution. Fig 16.21 is a plot of the generated proper time minus the reconstructed proper time for events passing the cuts described above. A Gaussian fit to the $t_{g e n}-t_{r e c}$ distribution gives $\sigma_{t}=0.043 \mathrm{psec}$. Given that $\tau_{B_{s}}=1.54 \mathrm{psec}$, then $\sigma_{t} / t=0.03$. 

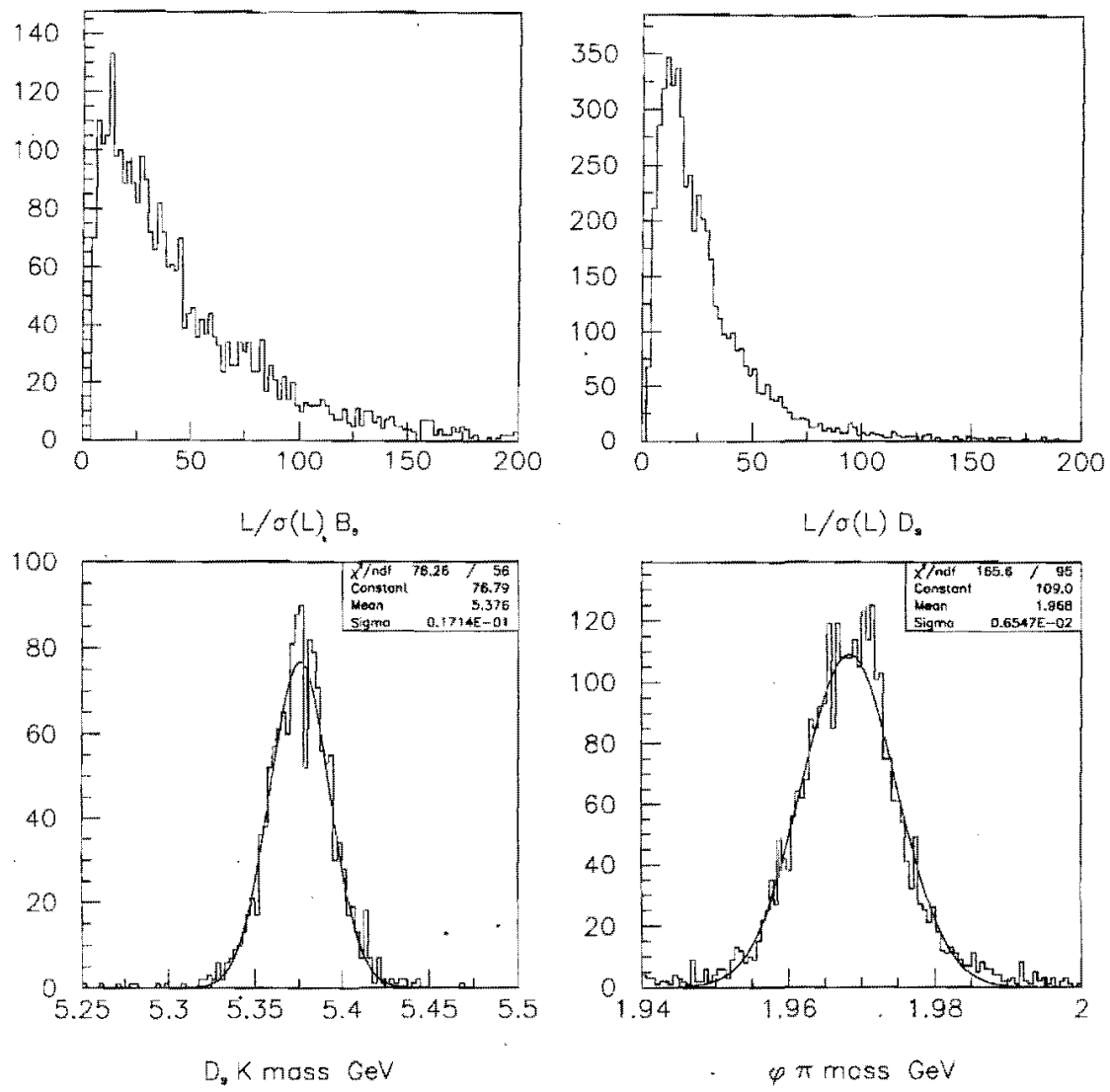

Figure 16.20: $L / \sigma_{L}$ and mass peaks for $B_{s}$ and $D_{s}$.

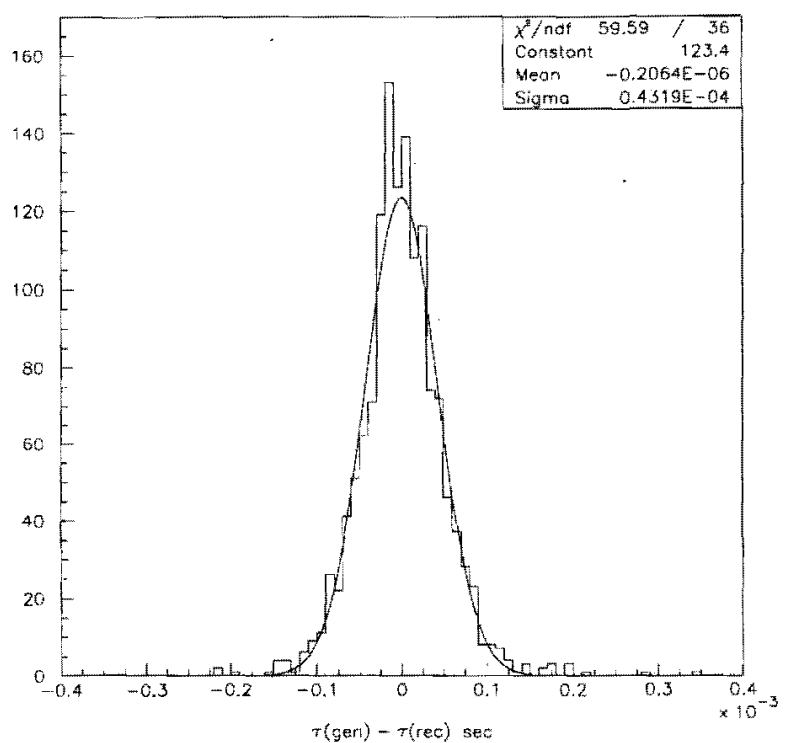

Figure 16.21: Proper time resolution for $B_{s}: t_{g e n}-t_{r e c}$ (nsec). 


\subsubsection{Background Studies}

Background can arise from real physics channels such as $B_{s} \rightarrow D_{s} \pi$ and $B_{s} \rightarrow D_{s}^{*} \pi$ where the $\pi$ is misidentified as a kaon or from random combinations of a real $D_{s}$ with a $K$ from the other $B$ or a primary interaction.

Combinatoric Background The combinatoric background was studied in two steps. First, generic $b \bar{b}$ events were generated in order to study the signal to background of $D_{s} \rightarrow \phi \pi$. Preliminary results indicate we can achieve $S / B \sim 1$ and we estimate that most of the combinatoric background will come from real $D_{s}$.

Second, " $B$ " $\rightarrow D_{s} X, D_{s} \rightarrow \phi \pi$ events were generated to determine the background from real $D_{s}$ 's combining with other tracks in the event. The $D_{s}$ can be from directly produced charm or from $B$ decays. Although the charm production cross-section is expected to be about a factor of 10 higher that the $b \bar{b}$ production cross-section, the trigger efficiency for charm events is much lower.

The background events were reconstructed as described above for the signal except that all pion tracks were used as kaon candidates to simulate misidentification in the RICH, then a pion misidentification rate was imposed later.

For $900 \mathrm{~K} " \bar{B}$ " $\rightarrow D_{s} X, D_{s} \rightarrow \phi \pi$ events, 10 events remained in the mass window $5.0-$ $6.0 \mathrm{GeV} / \mathrm{c}^{2}$ after all the cuts above were applied. In all these events the kaon candidate was really a pion. We then use a pion misidentification rate of $2 \%$ and estimate that the combinatoric background is about $1 \%$ of the signal.

Background from $B_{s} \rightarrow D_{s} \pi \quad$ Background can also come from decays such as $B_{s} \rightarrow D_{s} \pi$, $B_{s} \rightarrow D_{s}^{*} \pi$ where the pion is misidentified as a kaon. Most of the background comes from $B_{s} \rightarrow D_{s} \pi$. For decays where there is a missing particle there is very little overlap of the reconstructed mass with the signal region. The signal and scaled background are shown in Fig. 16.22. We expect that this will be the largest source of background and estimate $\mathrm{S} / \mathrm{B} \sim 7$. These results assume that pions are misidentified as kaons at a rate of $2 \%$. We have used the stand-alone simulation of the RICH detector described in Chapter 6 to study the efficiency of the signal vs. efficiency of the background from misidentified pions. The results are shown in Table 16.7 .

\subsubsection{Extracting $\rho$ and $\sin \phi$ using Maximum Likelihood fits}

A mini Monte Carlo study was performed to determine the expected error on $\gamma$.

For the first study, the input values of the parameters were chosen to be $x_{s}=30.0$, $\rho=0.7, \sin (\gamma)=0.75, \delta=10 \operatorname{deg}$ and $\Delta \Gamma=0.16$.

A set of "events" (i.e. proper times) was generated, split into the 4 decay modes with correct time distributions. The proper times were then smeared with a Gaussian of width $\sigma_{t}=0.03 \tau$, and a cutoff at low $t$ which simulated an $L / \sigma_{L}$ cut: $t_{\min }=0.25 \tau$. A fraction of the events were assigned to come from the "wrong flavor" parent. A mistag fraction of 


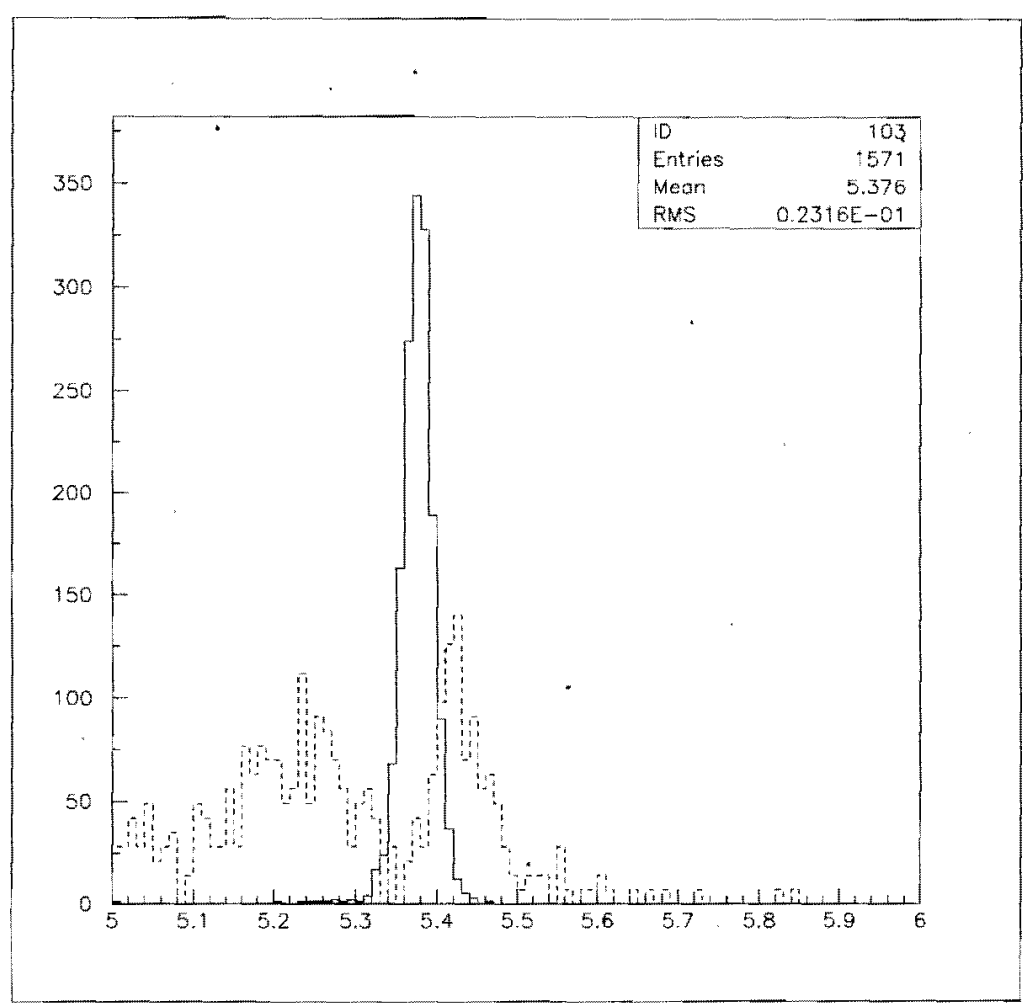

Figure 16.22: Comparison of $B_{s} \rightarrow D_{s} K$ signal and background from $B_{s} \rightarrow D_{s} X$, where X contains at least one pion misidentified as a $K$. 
Table 16.7: RICH efficiency.

\begin{tabular}{|cc|}
\hline$B_{s} \rightarrow D_{s} K$ & $B_{s} \rightarrow D_{s} \pi$ \\
\hline 0.62 & 0.00000 \\
0.66 & 0.00184 \\
0.73 & 0.00551 \\
0.75 & 0.00735 \\
0.76 & 0.00919 \\
0.78 & 0.01287 \\
0.79 & 0.01471 \\
0.80 & 0.01654 \\
0.81 & 0.01838 \\
0.82 & 0.04596 \\
0.84 & 0.07700 \\
0.85 & 0.12132 \\
0.86 & 0.17647 \\
\hline
\end{tabular}

$32 \%$ is used. Background events with a pure exponential time distribution are added to the "signal" events. The background is assumed to have the same lifetime as the signal.

A maximum likelihood fit was used to find the values of $\rho, \gamma, \delta$ and $\Delta \Gamma$. One thousand trials were done, each of 6,800 events. The fitted values of the parameters are shown in Fig 16.23.

The values of the input parameters were varied to study the impact on the error. The results of the fits are shown in Table 16.8 .

\subsubsection{Conclusions}

The ability of $\mathrm{BTeV}$ to measure the angle $\gamma$ of the unitarity triangle depends on several factors which are not well known at the moment, in particular the branching fractions for $B_{s} \rightarrow D_{s} K$ and the $B_{s}$ mixing parameter $x_{s}$.

Using the estimates of branching fractions given by Aleksan et al. [9], we expect to have about 9200 reconstructed, tagged events per year at a luminosity of $2 \times 10^{32} \mathrm{~cm}^{-2} \mathrm{~s}^{-1}$. The study of the sensitivity to $\gamma$ presented above was done assuming 6800 tagged events and gave error on $\gamma$ of about $7^{\circ}$. We expect that this will improve with the increased number of events. 

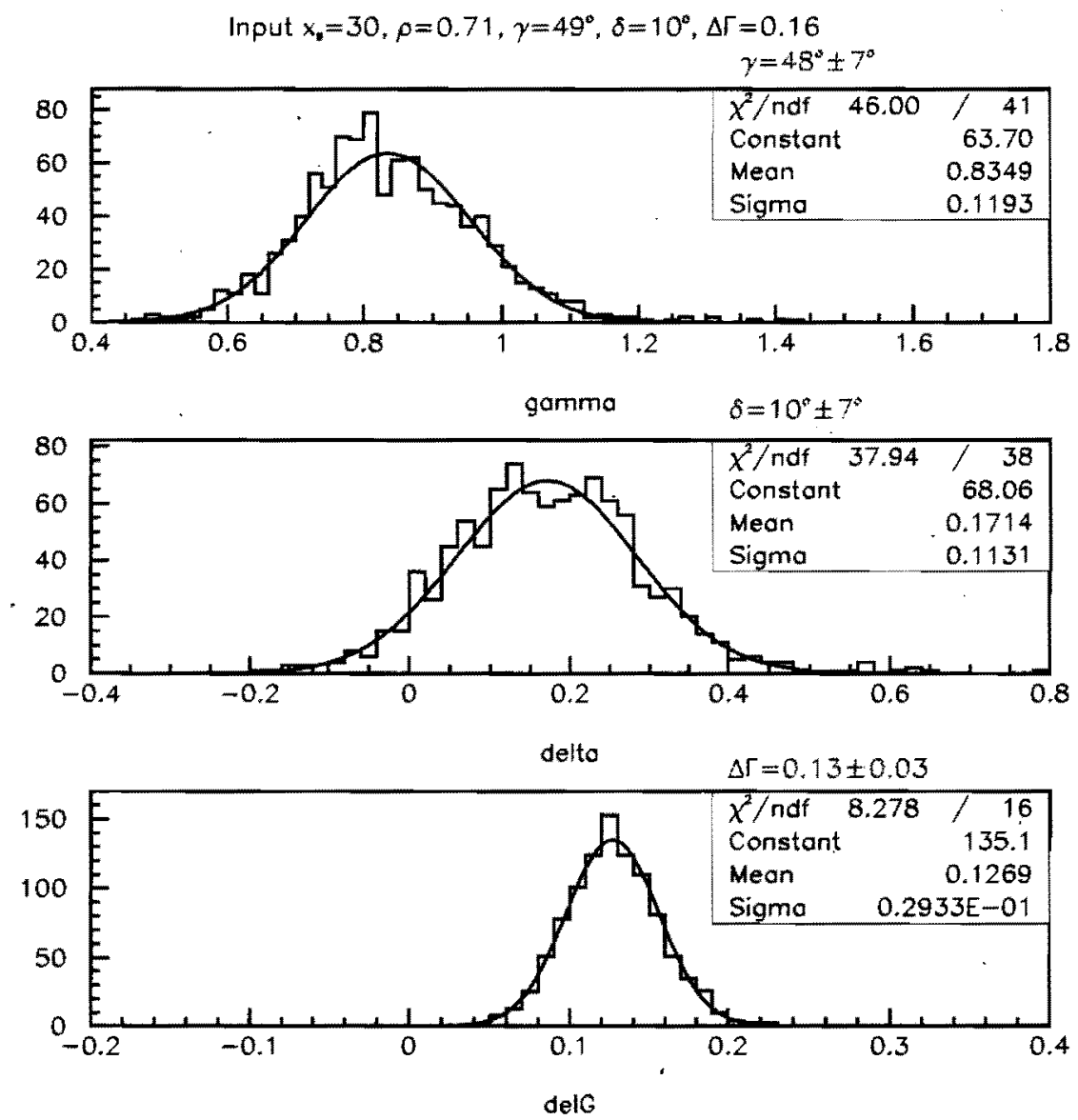

Figure 16.23: Fitted values of $\gamma, \delta$, and $\Delta \Gamma$ 
Table 16.8: Results of fits with variation of input parameters.

\begin{tabular}{|ccccc|cc|}
\hline$x_{s}$ & $\rho$ & $\delta$ & $\gamma$ & $\Delta \Gamma$ & $\sigma(\gamma)$ & $\sigma(\Delta \Gamma)$ \\
\hline 20 & 0.71 & $10^{\circ}$ & $49^{\circ}$ & 0.16 & $6^{\circ}$ & 0.03 \\
30 & 0.71 & $10^{\circ}$ & $49^{\circ}$ & 0.16 & $7^{\circ}$ & 0.03 \\
40 & 0.71 & $10^{\circ}$ & $49^{\circ}$ & 0.16 & $8^{\circ}$ & 0.03 \\
30 & 0.50 & $10^{\circ}$ & $49^{\circ}$ & 0.16 & $8^{\circ}$ & 0.03 \\
30 & 0.71 & $10^{\circ}$ & $30^{\circ}$ & 0.16 & $6^{\circ}$ & 0.03 \\
30 & 0.71 & $10^{\circ}$ & $90^{\circ}$ & 0.16 & $15^{\circ}$ & 0.04 \\
30 & 0.71 & $0^{\circ}$ & $49^{\circ}$ & 0.16 & $6^{\circ}$ & 0.03 \\
30 & 0.71 & $20^{\circ}$ & $49^{\circ}$ & 0.16 & $6^{\circ}$ & 0.03 \\
30 & 0.71 & $10^{\circ}$ & $49^{\circ}$ & 0.06 & $8^{\circ}$ & 0.04 \\
30 & 0.71 & $10^{\circ}$ & $49^{\circ}$ & 0.26 & $6^{\circ}$ & 0.03 \\
\hline
\end{tabular}




\subsubsection{CP Violation in $B^{ \pm} \rightarrow D^{o} K^{ \pm}$}

\subsubsection{Introduction}

In the Standard Model, $b \rightarrow c \bar{u} s$ and $b \rightarrow \bar{c} u s$ transitions have a relative CKM phase $\gamma$. In order to measure CP violation we must find a way for seemingly distinct final states to interfere. The Gronau-London-Wyler (GLW) method [10] extracts $\gamma$ by measuring the $B^{ \pm}$ decay rates to $D^{0} / \bar{D}^{0}$ mesons. If the $D^{0}$ and $\bar{D}^{0}$ decay to a CP eigenstate then the two decays $B^{-} \rightarrow K^{-} D^{0}$ and $B^{-} \rightarrow K^{-} \bar{D}^{0}$ lead to a common final state and can give rise to CP violating effects. However, the two interfering amplitudes are very different in magnitude and thus the interference effects are limited to $O(10 \%)$. Another problem is that it is necessary to measure separately the branching ratios $\mathcal{B}\left(B^{-} \rightarrow K^{-} D^{0}\right)$ and $\mathcal{B}\left(B^{-} \rightarrow K^{-} \bar{D}^{0}\right)$. While the former can be measured in a straightforward way, the latter is very difficult to measure.

Recently Atwood, Dunietz and Soni [11] have pointed out that $C P$ violation can be greatly enhanced for decays to final states that are common to both $D^{0}$ and $\bar{D}^{0}$ that are not CP eigenstates. In particular, large asymmetries are possible for final states $f$ such that $D^{0} \rightarrow f$ is doubly Cabibbo suppressed and $\bar{D}^{0} \rightarrow f$ is Cabibbo allowed.

\subsubsection{Description of method}

The Atwood, Dunietz and Soni method requires the determination of branching ratios for at least two distinct final states $f 1$ and $f 2$.

Define the following quantities:

$$
\begin{gathered}
a=\mathcal{B}\left(B^{-} \rightarrow K^{-} D^{0}\right) \\
b=\mathcal{B}\left(B^{-} \rightarrow K^{-} \bar{D}^{0}\right) \\
c(f 1)=\mathcal{B}\left(D^{0} \rightarrow f 1\right), \quad c(f 2)=\mathcal{B}\left(D^{0} \rightarrow f 2\right) \\
c(\overline{f 1})=\mathcal{B}\left(D^{0} \rightarrow \overline{f 1}\right), \quad c(\overline{f 2})=\mathcal{B}\left(D^{0} \rightarrow \overline{f 2}\right) \\
d(f 1)=\mathcal{B}\left(B^{-} \rightarrow K^{-} f 1\right), \quad d(f 2)=\mathcal{B}\left(B^{-} \rightarrow K^{-} f 2\right) \\
\bar{d}(f 1)=\mathcal{B}\left(B^{+} \rightarrow K^{+} f 1\right), \quad \bar{d}(f 2)=\mathcal{B}\left(B^{+} \rightarrow K^{+} f 2\right)
\end{gathered}
$$

Assume that we can measure the quantities $a, c(f 1), c(f 2), c(\overline{f 1}), c(\overline{f 2}) d(f 1), d(f 2)$, $\bar{d}(f 1)$ and $\bar{d}(f 2)$ but not $b$.

We can express $d(f 1)$ in terms of $a, b, c(f 1), c(\overline{f 1})$, the strong phase $\xi_{1}$ and the weak phase $\gamma$. 


$$
\begin{aligned}
& d(f 1)=a * c(f 1)+b * c(\overline{f 1})+2 \sqrt{a * b * c(f 1) * c(\overline{f 1})} \cos \left(\xi_{1}+\gamma\right) \\
& \bar{d}(f 1)=a * c(f 1)+b * c(\overline{f 1})+2 \sqrt{a * b * c(f 1) * c(\overline{f 1})} \cos \left(\xi_{1}-\gamma\right) \\
& d(f 2)=a * c(f 2)+b * c(\overline{f 2})+2 \sqrt{a * b * c(f 2) * c(\overline{f 2})} \cos \left(\xi_{2}+\gamma\right) \\
& \bar{d}(f 2)=a * c(f 2)+b * c(\overline{f 2})+2 \sqrt{a * b * c(f 2) * c(\overline{f 2})} \cos \left(\xi_{2}-\gamma\right)
\end{aligned}
$$

These four equations contain the 4 unknowns $\xi_{1}, \xi_{2}, b, \gamma$ which can be determined up to discrete ambiguities. Adding additional decay modes will reduce the ambiguities. The strong phases $\xi_{i}$ are related to the $D$ decay phase shifts $\delta_{i}$ by the relation:

$$
\xi_{1}-\xi_{2}=\delta_{1}-\delta_{2}
$$

If the $D$ decay phase shifts can be determined elsewhere then we have an extra constraint on equations. This method measures direct $\mathrm{CP}$ violation and does not require tagging or time-dependent measurements.

If we add a third decay mode we have 6 equations in 5 unknowns which will help to resolve ambiguities.

\subsubsection{Acceptance and Reconstruction Efficiency}

The reconstruction efficiency of the proposed $\mathrm{BTeV}$ detector for $B^{-} \rightarrow K^{-} D^{0}$ has been studied for two decay modes of the $D^{0}: D^{0} \rightarrow K^{+} \pi^{-}$and $D^{0} \rightarrow K^{-} K^{+}$. Note that the second decay mode is a CP eigenstate. In this case even though the branching fraction for $B^{-} \rightarrow K^{-} \overline{D^{0}}, \overline{D^{0}} \rightarrow K^{+} K^{-}$is expected to be only $1 \%$ of $B^{-} \rightarrow K^{-} D^{0}, D^{0} \rightarrow K^{+} K^{-}$we could still get up to $20 \% \mathrm{CP}$ asymmetry. The events are generated with Pythia and the detector is modeled with MCFast.

The final analysis cuts are selected to give a clean $D^{0}$ signal and reduce background from random combinations with kaons. The cuts are shown in Table 16.9. All tracks are required to be within the geometric acceptance of the RICH and have a momentum between 3 and $70 \mathrm{GeV} / \mathrm{c}$. The reconstructed signal is shown in Fig 16.24. The fitted Gaussian has a width of $17 \mathrm{MeV}$.

The combined geometric acceptance and reconstruction efficiency of the $B$ is $2.6 \%$ for the $D^{0} \rightarrow K \pi$ mode and $2.4 \%$ for the $D^{0} \rightarrow K K$ mode.

The requirement that all tracks be identified in the RICH was studied separately and found to be $70 \%$ efficient. The trigger efficiency for events that pass the final analysis cuts is $70 \%$ for both modes.

The expected number of events is shown in Table 16.10 . 


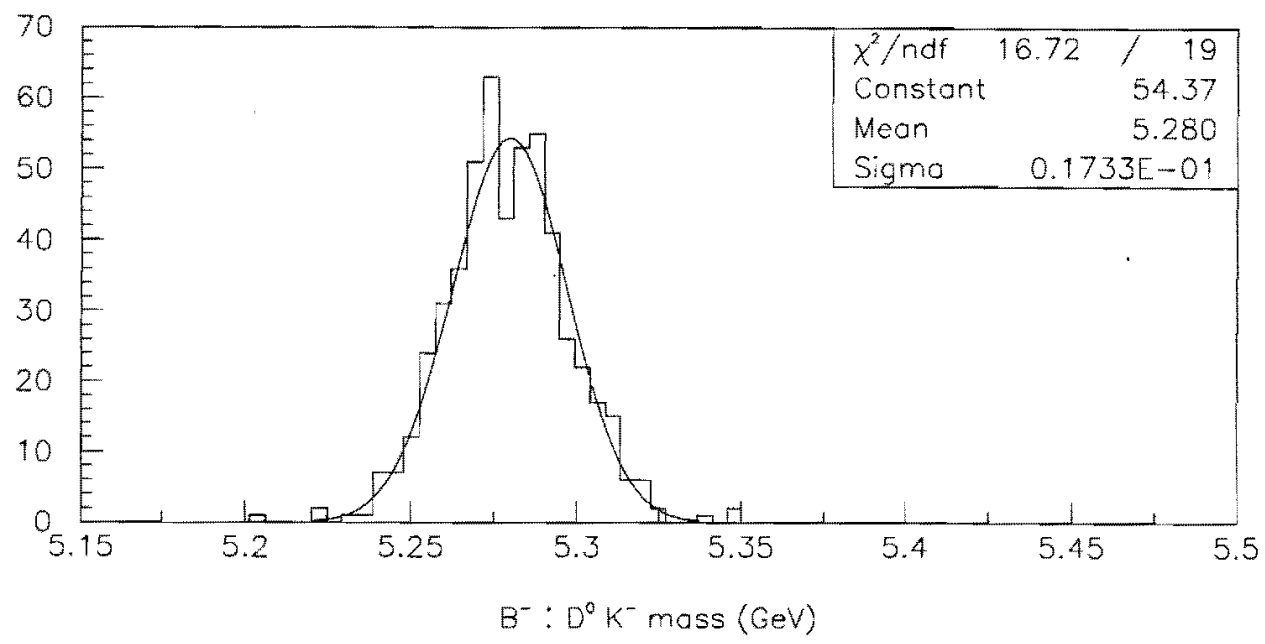

Figure 16.24: Invariant mass of $B^{-} \rightarrow \overline{D^{0}} K^{-}$.

\subsubsection{Background Studies}

Generic $b \bar{b}$ and $c \bar{c}$ events were studied and it was found that for both types of events the $D^{0} \rightarrow K^{-} \pi^{+}$and $D^{0} \rightarrow K^{-} K^{+}$signals had $S / B>5$ using the same cuts as for the $D^{0}$ in the $B^{-} \rightarrow K^{-} D^{0}$ decays. Therefore only background arising from real $D^{0} \mathrm{~s}$ need be considered.

Charm events with a $D^{0} \rightarrow K^{-} \pi^{+}$have a probability of $3.3 \%$ of passing the $D^{0}$ analysis cuts. The events which pass the cuts have a trigger efficiency of $10 \%$ and $0.6 \%$ of these events have another detached $K$. Generic $b \bar{b}$ events with a $D^{0}$ have a $7.0 \%$ probability of passing the $D^{0}$ analysis cuts. These events have a trigger efficiency of $35 \%$ and $4.0 \%$ of these have another detached $K$. Therefore we estimate that a generic $b \bar{b}$ event is 50 times more likely to contribute to background that a $c \bar{c}$ event. Thus even though the charm production cross-section is much larger than the $b \bar{b}$ cross-section, most of the background will come from $b \bar{b}$ events.

Table 16.9: Cuts for $D^{0} \rightarrow K \pi$ and $D^{0} \rightarrow K^{+} K^{-}$

\begin{tabular}{|c|c|}
\hline$L / \sigma_{L}\left(B^{-}\right)$ & $>10$ \\
$L / \sigma_{L}\left(D^{0}\right)$ & $>4$ \\
DCA to primary vertex (all tracks) & $>3 \sigma$ \\
$\chi^{2}(B$ vertex $)$ & $<5$ \\
$\chi^{2}(D$ vertex $)$ & $<10$ \\
$B$ point back to primary vertex & $<2 \mathrm{GeV} / \mathrm{c}$ \\
$p_{t}$ balance & $<2 \sigma$ \\
DCA & $1.85-1.88 \mathrm{GeV} / \mathrm{c}^{2}$ \\
$D^{0}$ mass window
\end{tabular}


Table 16.10: Projected number of reconstructed $B^{-} \rightarrow K^{-} D$ per year.

\begin{tabular}{|c|cc|}
\hline Decay Mode & $K^{-}\left(K^{+} \pi^{-}\right)$ & $K^{-}\left(K^{+} K^{-}\right)$ \\
Luminosity & $2 \times 10^{32} \mathrm{~cm}^{-2} \mathrm{~s}^{-1}$ \\
Running time & \multicolumn{2}{|c}{$10^{7} \mathrm{sec}$} \\
Integrated Luminosity & \multicolumn{2}{|c}{$2 \mathrm{fb}^{-1}$} \\
$\sigma_{b \bar{b}}$ & \multicolumn{2}{|c}{$100 \mu \mathrm{b}$} \\
Number of $B^{ \pm}$ & \multicolumn{2}{|c}{$1.5 \times 10^{11}$} \\
Branching ratio & $1.7 \times 10^{-7}$ & $1.1 \times 10^{-6}$ \\
Reconstruction efficiency & 0.026 & 0.024 \\
RICH efficiency & 0.70 & 0.70 \\
Trigger efficiency L1 & 0.70 & 0.70 \\
Trigger efficiency L1 & 0.90 & 0.90 \\
Number of reconstructed $B^{ \pm}$ & 300 & 1800 \\
\hline
\end{tabular}

Background in both modes, $B^{-} \rightarrow K^{-}\left[K^{+} \pi^{-}\right]$and $B^{-} \rightarrow K^{-}\left[K^{+} K^{-}\right]$, could arise from:

- $B^{-} \rightarrow \pi^{-} D^{0}$ where the $\pi^{-}$is misidentified as a $K$, and similar decays such as $B^{-} \rightarrow$ $\pi^{-} D^{* 0}$ and $B^{-} \rightarrow \rho^{-} D^{0}$ where there is a missing $\pi^{0}$ and the $\pi^{-}$is misidentified. These decays all have significantly higher branching fractions than the signal. If we assume that the probability of misidentifying a $\pi^{-}$as a $K^{-}$is $2 \%$, the relative signal

- and background from these modes is shown in Fig 16.25. This is the most significant source of background for the $D^{0} \rightarrow K^{+} K^{-}$mode.

- " $B$ " $\rightarrow \overline{D^{0}} X$ events where the $\overline{D^{0}}$ makes a good vertex with a $K^{-}$from the other $B$ or from the underlying event. This was studied by generating " $B$ " $\rightarrow \overline{D^{0}} X, \bar{D}^{0} \rightarrow$ $K^{+} \pi-$ events and using the same reconstruction as for the signal. 1.6 million " $B$ " $\rightarrow$ $\overline{D^{0}} X, \bar{D}^{0} \rightarrow K^{+} \pi$ - events were generated and after cuts as described for the signal, no events remained in the mass window $5.0-5.5 \mathrm{GeV} / \mathrm{c}^{2}$, and 1 event in the $5.5-6.0$ $\mathrm{GeV} / \mathrm{c}^{2}$ window.

We assume this type of background has the same trigger efficiency as the signal. We estimate we can achieve $\mathrm{S} / \mathrm{B} \sim 1$ in the $D^{0} \rightarrow K^{+} \pi^{-}$mode, and we expect this to be the dominant source of background for this mode. This type of background will be much less significant in the $D^{0} \rightarrow K^{+} K^{-}$mode because both the signal and background come from singly Cabibbo suppressed decays.

\subsubsection{Extracting $\gamma$ from decay rates}

In order to estimate our ability to measure $\gamma$, several sets of input parameters $\left(b, \gamma, \xi_{1}, \xi_{2}\right)$ were chosen and for each set the expected number of events in each channel was calculated. Then, 1000 trials were done for each set, smearing the number of events by $\sqrt{N+B}$. For 


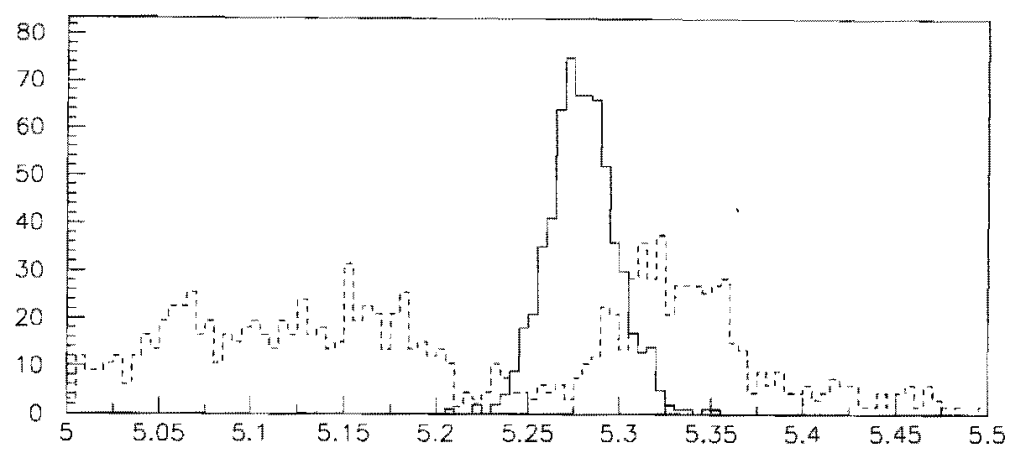

Figure 16.25: Signal (solid line) and background (dashed line) from $B^{-} \rightarrow \pi^{-} D^{0}$ and $B^{-} \rightarrow$ $\pi^{-} D^{0} X$ where the $\pi^{-}$is misidentified as a $K^{-}$.

each trial, values for $b$ and $\gamma$ are calculated. The fitted values of $b$ and $\gamma$ are shown in Table 16.11 and Fig. 16.26.

\begin{tabular}{|c|cccc|}
\hline & Test 1 & Test 2 & Test 3 & Test 4 \\
\hline $\mathcal{B}\left(\times 10^{-6}\right)$ & 2.2 & 2.2 & 2.2 & \\
$\xi_{1}$ & $45^{\circ}$ & $0^{\circ}$ & $90^{\circ}$ & $70^{\circ}$ \\
$\xi_{2}$ & $30^{\circ}$ & $45^{\circ}$ & $10^{\circ}$ & $30^{\circ}$ \\
$\gamma$ & $65^{\circ}$ & $75^{\circ}$ & $85^{\circ}$ & $50^{\circ}$ \\
$\gamma$ fit & $(67 \pm 10)^{\circ}$ & $(75 \pm 7)^{\circ}$ & $(85.0 \pm 2.4)^{\circ}$ & $(50.0 \pm 3.2)^{\circ}$ \\
\hline
\end{tabular}

Table 16.11: Input values of parameters and results of fit for $\gamma$.

\subsubsection{Conclusions}

We expect to reconstruct about $300 B^{ \pm} \rightarrow(K \pi) K^{ \pm}$and 2,000 $B^{ \pm} \rightarrow(K K) K^{ \pm}$per year at the design luminosity of $2 \times 10^{32} \mathrm{~cm}^{-2} \mathrm{~s}^{-1}$. With this number of events, $\gamma$ can be measured to $\pm 10^{\circ}$ for most values of $\gamma, \xi_{1}$ and $\xi_{2}$. The error on $\gamma$ depends on the value of $\gamma$ and the strong phases, in particular the error decreases with increasing difference in the strong phases. If we assume that the ratio of Cabibbo favored to doubly Cabibbo suppressed branching fractions is the same for the two decay modes then the equations have no solution when $\left|\xi_{1}\right|=\left|\xi_{2}\right|$. 


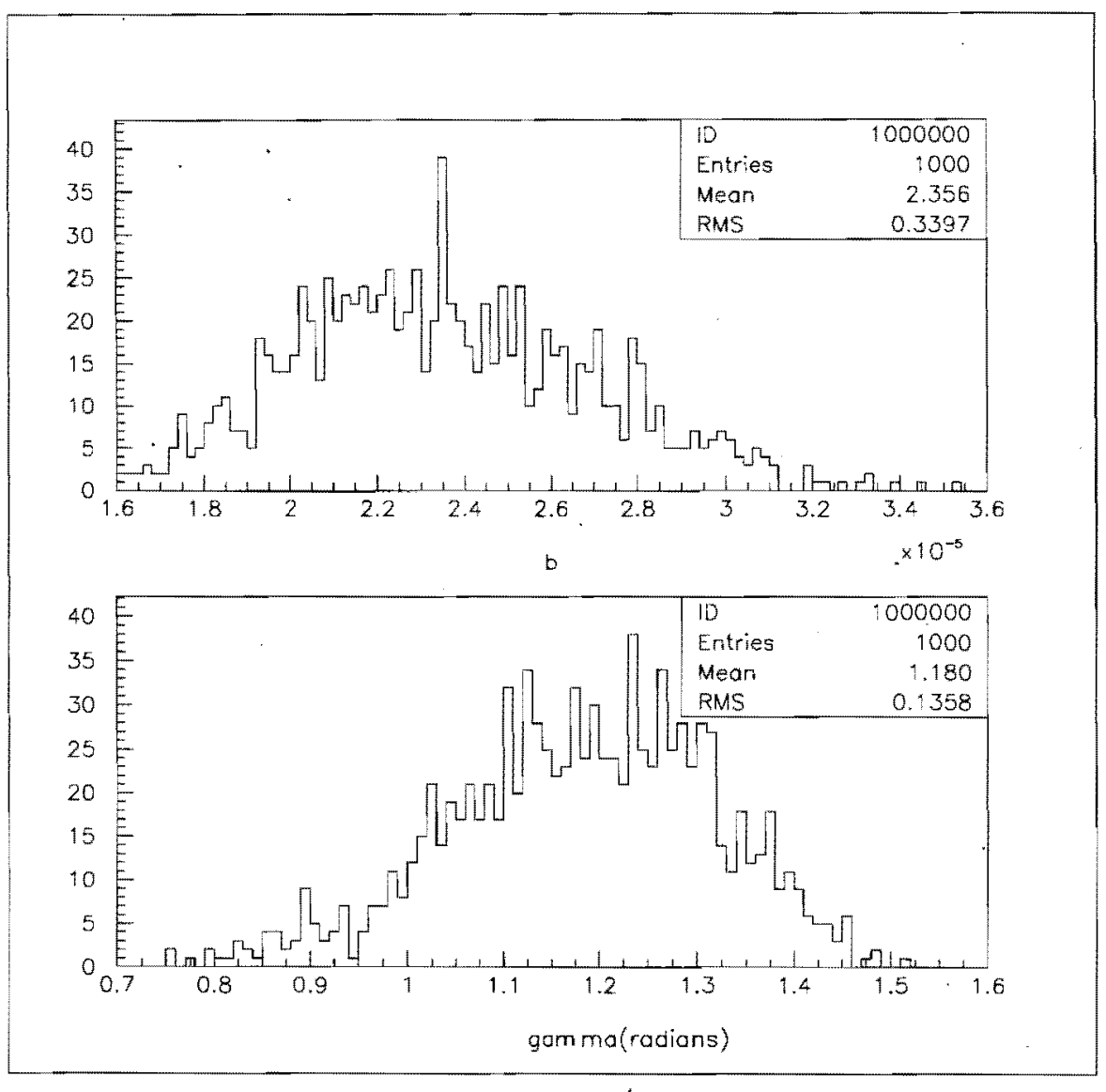

Figure 16.26: Calculated values of $\gamma$ and $b$ for input values $\gamma=65^{\circ}(1.13 \mathrm{rad}), b=2.2 \times 10^{-6}$. 


\subsubsection{CP Violation in $B \rightarrow K \pi$}

This method uses the branching ratios of the decays $B^{+} \rightarrow \pi^{+} K^{0}$ and $B^{0} \rightarrow \pi^{-} K^{+}$and their complex conjugates as explained in a paper by Gronau and Rosner [12]. The decay $B^{+} \rightarrow$ $\pi^{+} K^{0}$ proceeds only through a penguin amplitude and the decay $B^{0} \rightarrow \pi^{-} K^{+}$proceeds through both penguin and tree amplitudes. Ignoring electroweak penguin contributions, the penguin amplitudes in the charged and neutral $B$ decays to $K \pi$ are equal.

It is useful to define

$$
\begin{gathered}
R \equiv \frac{\Gamma\left(B^{0} \rightarrow K^{+} \pi^{-}\right)+\Gamma\left(\bar{B}^{0} \rightarrow K^{-} \pi^{+}\right)}{\Gamma\left(B^{+} \rightarrow K^{0} \pi^{+}\right)+\Gamma\left(B^{-} \rightarrow \bar{K}^{0} \pi^{-}\right)} \\
A^{\prime} \equiv \frac{\Gamma\left(B^{0} \rightarrow K^{+} \pi^{-}\right)-\Gamma\left(\bar{B}^{0} \rightarrow K^{-} \pi^{+}\right)}{\Gamma\left(B^{+} \rightarrow K^{0} \pi^{+}\right)+\Gamma\left(B^{-} \rightarrow \bar{K}^{0} \pi^{-}\right)} .
\end{gathered}
$$

An expression for $\gamma$ can be given in terms of $R, A^{\prime}$ and the ratio of tree to penguin amplitudes $r \equiv|T| /|P|$.

If we define $\delta$ to be the phase difference between the penguin and tree amplitudes, the ratios $R$ and $A^{\prime}$ can be rewritten :

$$
\begin{gathered}
R=1-2 r \cos \gamma \cos \delta+R^{2} \\
A^{\prime}=2 r \sin \delta \sin \gamma
\end{gathered}
$$

Combining these equations to eliminate $\delta$ we get

$$
R=1+r^{2} \pm \sqrt{4 r^{2} \cos ^{2} \gamma-A^{\prime 2} \cot ^{2} \gamma}
$$

Fleischer and Mannel[13] have claimed that if $R<1$ a useful bound can be obtained regardless of the value of $r$ or $\delta$ :

$$
\sin ^{2} \gamma \leq R
$$

If we have information on $r$ we can get a more precise estimate of $\gamma$.

CLEO has determined $R=0.65 \pm 0.40$, which suggests that the ratio could be less than 1.0 [14]. If this result holds with improved statistics then this method compliments other methods in that it will begin to exclude some of the region around $\gamma=\pi / 2$. A recent updated result from CLEO gives $R=0.95 \pm 0.31$ [15].

There has been much discussion about the effect of final state interactions on the measured branching ratios. Falk et al. [16] have shown that contributions to $B^{+} \rightarrow \pi^{+} K^{0}$ from rescattering of channels such as $B^{+} \rightarrow \pi^{0} K^{+}$lead to a modification of the Fleischer-Mannel bound: 


$$
\sin ^{2} \gamma \leq R(1+2 \epsilon \sqrt{1-R})
$$

where $\epsilon$ is the ratio of the rescattered amplitude to the penguin amplitude. They estimate $\epsilon=O(0.1)$. This rescattering contribution can be determined by measuring the $K^{+} K^{-}$final state branching ratio, which $\mathrm{BTeV}$ is well equipped to do.

To study the reconstruction efficiency of $B^{+} \rightarrow \pi^{+} K_{s}$ Monte Carlo events were generated using Pythia and the detector modeled with MCFast. First, the primary vertex was found, then $K_{s}$ candidates were selected by combining all pairs of oppositely charged tracks not identified as kaons. These tracks were required to have an impact parameter greater than $5 \sigma$ with respect to the primary vertex, to form a vertex with $\chi^{2}<5$ and to be within 6 $\mathrm{MeV} / \mathrm{c}^{2}$ of the nominal $K_{s}$ mass. The $K_{s}$ vertex was required to have $\ell / \sigma_{\ell}>6$ with respect to the primary vertex. $B$ candidates were selected by combining the $K_{s}$ candidates with all other charged tracks not identified as kaons and having an impact parameter greater than $3.5 \sigma$ with respect to the primary vertex. The reconstructed $\mathrm{B}$ candidate was required to have $\ell / \sigma_{\ell}>4$, and point back to the primary with an impact parameter less than $2.5 \sigma$. The combined geometric acceptance and reconstruction efficiency of the $B^{+} \rightarrow \pi^{+} K_{s}$ mode is $3.3 \%$ and the trigger efficiency (Level 1 plus Level 2) is $24 \%$. The reconstructed signal is shown in Fig. 16.27.

The background has been studied using $500 \mathrm{~K}$ generic $b \bar{b}$ events with minimum bias events added to give an average of two interactions per beam crossing. Only one event with a mass above $5 \mathrm{GeV} / \mathrm{c}^{2}$ survived the cuts as described above. From these limited statistics we estimate we can achieve a signal-to-background ratio of about 1:1.

The decay mode $B^{0} \rightarrow \pi^{-} K^{+}$has also been simulated. The two prong vertex can be distinguished from background with an $\ell / \sigma_{\ell}$ cut and the requirement that the reconstructed $B$ point back to the primary vertex. The reconstruction efficiency is found to be $8.4 \%$ and the trigger efficiency (Level 1 plus Level 2) for events which pass the analysis cuts is $57 \%$. The background has been studied using $12 \times 10^{6} b \vec{b}$ events and the signal-to-background ratio was found to be $20: 1$.

We expect to reconstruct $8,000 B^{ \pm} \rightarrow \pi^{ \pm} K_{s}$ and $108,000 B^{0} / \bar{B}^{0} \rightarrow \pi^{-1+} K^{+1-}$ decays in $10^{7} \mathrm{~s}$ of running at $\mathcal{L}=2 \times 10^{32} \mathrm{~cm}^{-2} \mathrm{~s}^{-1}$. Gronau and Rosner [12] have estimated that a $5^{\circ}$ precision in $\gamma$ can be achieved with 2,400 events in each channel. This of course, doesn't take into account the aforementioned theoretical criticisms of the technique. 
$2000 / 05 / 05 \quad 13.45$

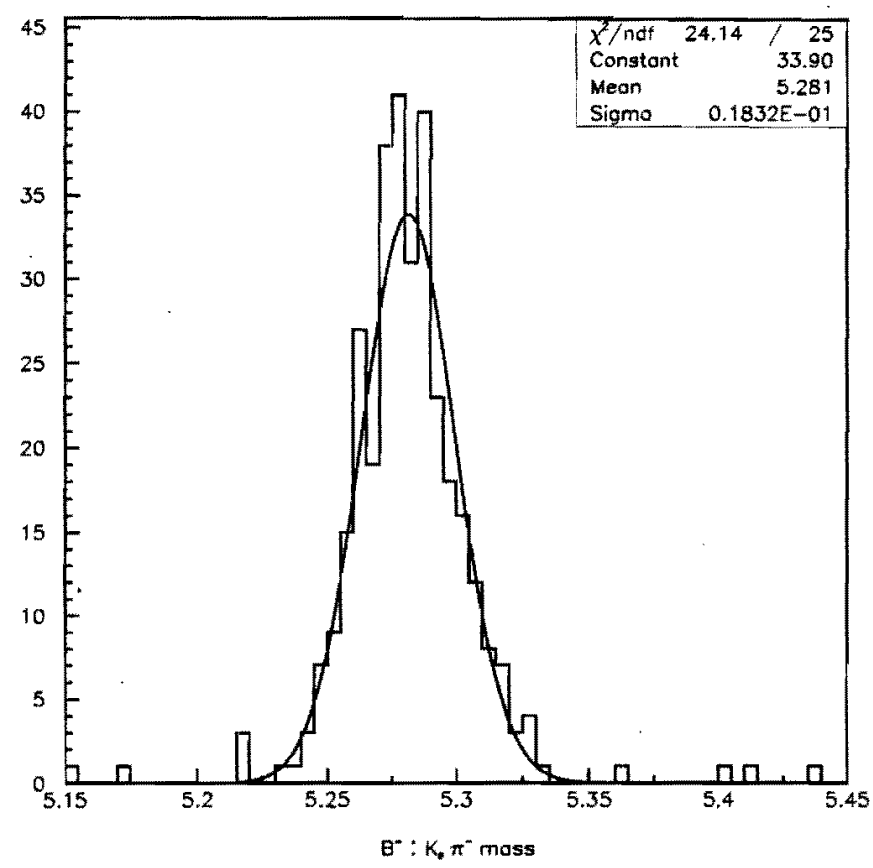

Figure 16.27: $K_{s} \pi$ mass 


\subsection{Analysis of $B \rightarrow \rho \pi$}

The CKM angle $\alpha$ cannot be extracted from the $B^{o} \rightarrow \pi^{+} \pi^{-}$asymmetry in a model independent way because of the large "penguin pollution" problem. However, asymmetries in the decays $B^{o} \rightarrow \rho \pi$ can be used to measure this angle (see Chapter 1) [17]. There are three final states in this decay: $B^{o} \rightarrow \rho^{o} \pi^{o}, B^{o} \rightarrow \rho^{+} \pi^{-}$and $B^{o} \rightarrow \rho^{-} \pi^{+}$. CLEO has measured the average branching ratio of the latter two modes to be $2.8_{-0.7}^{+0.8} \pm 0.4 \times 10^{-5}$ and limits the $\rho^{o} \pi^{o}$. to $<5.1 \times 10^{-6}$ at $90 \%$ confidence level [18]. BTeV, with its crystal calorimeter, should be able to collect and reconstruct a substantial sample of $B \rightarrow \rho \pi$ events.

The reconstruction efficiencies for $B \rightarrow \rho \pi$ and backgrounds were studied using a full GEANT simulation, for $\rho^{ \pm} \pi^{\mp}$ and $\rho^{o} \pi^{o}$, separately. All signal and background samples were generated with a mean of two interactions per crossing. While signal events are relatively easy to generate, backgrounds are more difficult. For channels with branching ratios on the order of $10^{-5}$ and efficiencies on the order of $1 \%$, it is necessary to generate at least $10^{7} b \bar{b}$ background events. This is a difficult task that requires large amounts of CPU time and data storage. Since almost $90 \%$ of the time spent in generating the events is in the electromagnetic calorimeter, we pass all the generated events through the tracking system and perform a preliminary analysis on the charged tracks before generating the calorimeter information. The output of this proceedure is as realistic as running all the events through the entire GEANT process but saves a factor of three in computing time.

We look for events containing a secondary vertex formed by two oppositely charged tracks. One of the most important selection requirements for discriminating the signal from the background is that the events have well measured primary and secondary vertices. We demand that both the primary and the secondary have vertex fits with $\chi^{2} /$ dof $<2$. We also make a cut on the the distance between the primary and the secondary vertices, divided by the error, $L / \sigma_{L}>4$. The two vertices must also be separated from each other in the plane transverse to the beam. We define $r_{\text {transverse }}$ in terms of the primary interaction vertex position $\left(x_{P}, y_{P}, z_{P}\right)$ and the secondary decay vertex position $\left(x_{S}, y_{S}, z_{S}\right)$ as $r_{\text {transverse }}=$ $\sqrt{\left(x_{P}-x_{S}\right)^{2}+\left(y_{P}-y_{S}\right)^{2}}$ and cut out events where the secondary vertex is close to the reconstructed primary. Furthermore, to insure that the charged tracks do not originate from the primary, we require that both the $\pi^{+}$and the $\pi^{-}$candidate have an impact parameter with respect to the primary vertex (DCA) $>100 \mu \mathrm{m}$.

Events passing these selection criteria are passed through the electromagnetic calorimeter simulation which uses GEANT. Our aim is to find good $\pi^{\circ}$ candidates. We select candidate "bumps" in the calorimeter using the cluster finder code described in Chapter 12.4. Photon candidates are required to have a minimum bump energy of $1 \mathrm{GeV}$ and pass the shower shape cut which requires $E 9 / E 25>0.85$. The shower shape cut is used to select electromagnetic showers. We reduce the background rate by insuring that the photon candidates are not too close to the projection of any charged tracks on the calorimeter. For $\rho^{ \pm} \pi^{\mp}$, the minimum distance requirement is $>2 \mathrm{~cm}$, while for $\rho^{\circ} \pi^{\circ}$, we require the minimum distance $>5.4$ $\mathrm{cm}$. Candidate $\pi^{\circ}$ 's are two-photon combinations with invariant mass between 125 and $145 \mathrm{MeV} / \mathrm{c}^{2}$. More details of $\pi^{o}$ selection are given in the description of the analysis of the 
Table 16.12: Selection Criteria

\begin{tabular}{|l|c|c|}
\hline Criteria & $\rho^{ \pm} \pi^{\mp}$ & $\rho^{0} \pi^{0}$ \\
\hline Primary vertex criteria & $\chi^{2}<2$ & $\chi^{2}<2$ \\
Secondary vertex criteria & $\chi^{2}<2$ & $\chi^{2}<2$ \\
$r_{\text {transverse }}(\mathrm{cm})$ & 0.0146 & 0.0132 \\
Normalized distance $L / \sigma$ & $>4$ & $>4$ \\
Distance $L, \mathrm{~cm}$ & $<5$ & $<5$ \\
DCA of track, $\mu \mathrm{m}$ & $>100$ & $>100$ \\
$t_{\text {proper }} / t_{0}$ & $<5.5$ & $<5.5$ \\
$E_{\pi^{+}}, \mathrm{GeV}$ & $>4$ & $>4$ \\
$E_{\pi^{-}}, \mathrm{GeV}$ & $>4$ & $>4$ \\
$p_{t}\left(\pi^{+}\right), \mathrm{GeV} / c$ & $>0.4$ & $>0.4$ \\
$p_{t}\left(\pi^{-}\right), \mathrm{GeV} / c$ & $>0.4$ & $>0.4$ \\
Isolation for $\gamma, \mathrm{cm}$ & $>2.0$ & $>5.4$ \\
$E_{\pi^{0}}, \mathrm{GeV}$ & $>5$ & $>9$ \\
$p_{t}\left(\pi^{0}\right), \mathrm{GeV} / c$ & $>0.75$ & $>0.9$ \\
$\Delta p_{t} / \Sigma p_{t}$ & $<0.06$ & $<0.066$ \\
$m_{\pi^{0}}, \mathrm{MeV} / c^{2}$ & $125-145$ & $125-145$ \\
$m_{\rho}, \mathrm{GeV} / c^{2}$ & $0.55-1.1$ & $0.55-1.1$ \\
\hline
\end{tabular}

channel $B^{o} \rightarrow D^{*-} \rho^{+}$in Section 16.3.

Kinematic cuts can greatly reduce the background to $B \rightarrow \rho \pi$ while maintaining the signal efficiency. Minimum energy and transverse momentum $\left(p_{t}\right)$ requirements are placed on each of the three pions. Here $p_{t}$ is defined with respect to the $B$ direction which is defined by the position of the primary and secondary vertices. We demand that the momentum vector of the reconstructed $B$ candidate point back to the primary vertex. The cut is implemented by requiring $p_{t}$ balance among the $\pi^{+}, \pi^{-}$, and $\pi^{\circ}$ candidates relative to the $B$-direction and then divided by the sum of the $p_{t}$ values for all three particles $\left(\Delta p_{t} / \Sigma p_{t}\right)$. We also make a cut on the $B$ decay time requiring that the $B$ candidate live no more than 5.5 proper lifetimes $\left(t_{\text {proper }} / t_{0}<5.5\right)$. The selction criteria for the two modes are summaried in Table 16.12 .

For this study, we generated and analyzed three large samples of events using BTeVGeant: $125,000 B \rightarrow \rho^{0} \pi^{0}$ events, $125,000 B \rightarrow \rho^{+} \pi^{-}$events, and 4,450,000 generic $b \bar{b}$ background events. The results of the analysis after applying the cuts in Table 16.12 are presented in Fig. 16.28 (for $\rho^{o} \pi^{o}$ ) and Fig 16.29 (for $\rho^{+} \pi^{-}$). The background mass spectra are on the left side of the figures, and the signal events are on the right side.

The mass resolution for the $B$ is $\approx 28 \mathrm{MeV} / c^{2}$. The mean $\pi^{\circ}$ mass value in the $B \rightarrow \rho \pi$ events is $135 \mathrm{MeV} / c^{2}$ with a resolution of about $3 \mathrm{MeV} / c^{2}$. The relevant yields for $\rho \pi$ are shown in Table 16.13. The reconstruction efficiency is $(0.36 \pm 0.02) \%$ for $\rho^{0} \pi^{0}$ and $(0.44 \pm 0.02) \%$ for $\rho^{+} \pi^{-}$. The background was obtained by considering the mass interval 

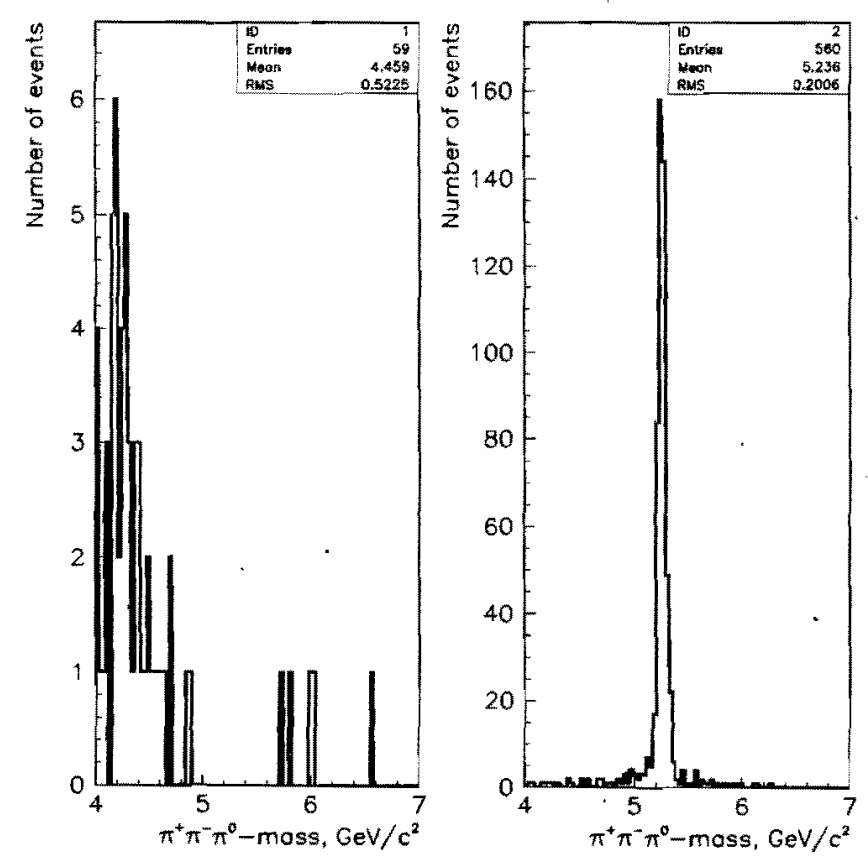

Figure 16.28: Invariant $\pi^{+} \pi^{-} \pi^{\circ}$ mass distributions for background (left) and signal (right) events for $B \rightarrow \rho^{o} \pi^{o}$.
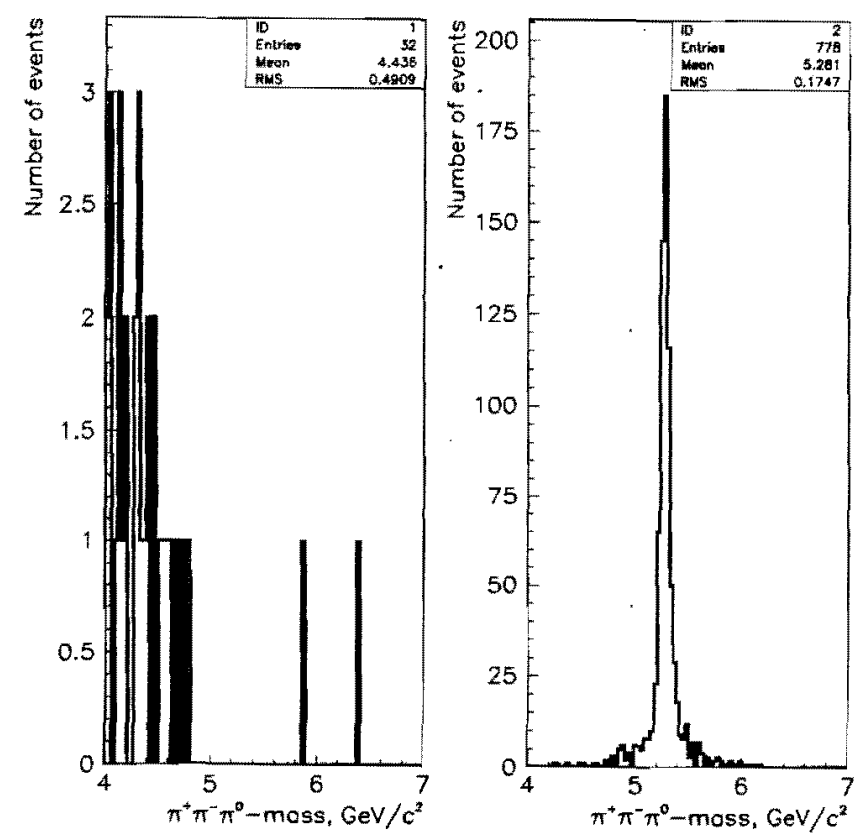

Figure 16.29: Invariant $\pi^{+} \pi^{-} \pi^{\circ}$ mass distributions for background (left) and signal (right) events for $B \rightarrow \rho^{+} \pi^{-}$. 
Table 16.13: $B \rightarrow \rho \pi$ Yields

\begin{tabular}{|l|c|c|}
\hline Quantity & $\rho^{ \pm} \pi^{\bar{F}}$ & $\rho^{o} \pi^{o}$ \\
\hline Branching ratio & $2.8 \times 10^{-5}$ & $0.5 \times 10^{-5}$ \\
Efficiency & 0.0044 & 0.0036 \\
Trigger efficiency (Level 1) & 0.6 & 0.6 \\
Trigger efficiency (Level 2) & 0.9 & 0.9 \\
S/B & 4.1 & 0.3 \\
Signal $/ 10^{7} \mathrm{~s}$ & 9,400 & 1,350 \\
$\epsilon D^{2}$ & 0.10 & 0.10 \\
Flavor tagged yield & 940 & 135 \\
\hline
\end{tabular}

between 5 and $7 \mathrm{GeV} / c^{2}$. The signal interval is taken as $\pm 2 \sigma$ around the $B$ mass or 112 $\mathrm{MeV} / \mathrm{c}^{2}$.

The final numbers of both signal and background events are reduced by including the Level 1 and Level 2 trigger efficiency, but the S/B ratio is not signifcantly changed. From this study we find that we can expect to reconstruct about $9,400 \rho^{ \pm} \pi^{\mp}$ events and $1,350 \rho^{\circ} \pi^{o}$ events per year.

We can, therefore, expect to collect a sample of $\sim 1000$ flavor tagged $\rho^{ \pm} \pi^{\mp}$ events and $\sim 150 \rho^{\circ} \pi^{o}$ per year with signal-to-background levels of approximately $4: 1$ and 1:3, respectively. A comparison with other experiments is given in Part IV. We have not yet done a full simulation to the sensitivity to $\alpha$. Final results will depend on several unknown quantities including the branching ratio for $\rho^{o} \pi^{o}$ and the ratio of tree to penguin amplitudes. Analysis by Snyder and Quinn [17] showed that with 2,000 background free events they could always find a solution for $\alpha$ and the accuracy was in the range of $5-6^{\circ}$. We can collect these 2,000 events in $2 \times 10^{7}$ seconds, but we will have some background. Quinn and Silva have also proposed using non-flavor tagged rates as input which should improve the accuracy of the $\alpha$ determination [19]. 


\subsection{Measuring $\chi$ with $B_{s} \rightarrow J / \psi \eta\left(\eta^{\prime}\right)$}

\subsubsection{Introduction}

The CP violating angle, $\chi$, defined in Chapter 1 , can be measured by using $B_{s}$ decay modes. The all-charged mode $B_{s} \rightarrow J / \psi \phi$ is one way to measure this, but due to the fact that this is a vector-vector final state of mixed-CP, a complicated angular analysis is required and therefore a very large data sample must be obtained. The channels $B_{s} \rightarrow J / \psi \eta^{\prime}$ and $B_{s} \rightarrow J / \psi \eta$, can be used to determine the angle $\chi$ from a simple asymmetry measurement.

We estimate the branching ratios using the quark model [20]. The $\eta$ and $\eta^{\prime}$ wave functions are given in terms of the quark wave functions as:

$$
\begin{aligned}
\psi(\eta) & =(u \bar{u}+d \bar{d}-s \bar{s}) / \sqrt{3} \\
\psi\left(\eta^{\prime}\right) & =(u \bar{u}+d \bar{d}+2 s \bar{s}) / \sqrt{6} .
\end{aligned}
$$

Thus the branching ratios are related to the measured decay $B^{o} \rightarrow J / \psi K^{o}$, taking equal lifetimes as

$$
\begin{aligned}
\mathcal{B}\left(B_{s} \rightarrow J / \psi \eta\right) & =\frac{1}{3} \mathcal{B}\left(B_{s} \rightarrow J / \psi K^{o}\right) \\
\mathcal{B}\left(B_{s} \rightarrow J / \psi \eta^{\prime}\right) & =\frac{2}{3} \mathcal{B}\left(B_{s} \rightarrow J / \psi K^{o}\right)
\end{aligned}
$$

It should be noted that a large enhancement in one of these rates is possible, as implied by the large branching fraction for $B \rightarrow \eta^{\prime} K$.

We consider only the decays $\eta \rightarrow \gamma \gamma, \eta^{\prime} \rightarrow \rho^{o} \gamma$ and $\eta^{\prime} \rightarrow \pi^{+} \pi^{-} \eta$. The $J / \psi$ was found in the $\mu^{+} \mu^{-}$decay mode. All relevant branching ratios are listed in Table 16.14

\begin{tabular}{|l|c|}
\hline Decay & Branching Fraction \\
\hline $\mathcal{B}\left(B_{s} \rightarrow J / \psi \eta\right)$ & $3.3 \times 10^{-4}$ \\
$\mathcal{B}\left(B_{s} \rightarrow J / \psi \eta^{\prime}\right)$ & $6.7 \times 10^{-4}$ \\
$J / \psi \rightarrow \mu^{+} \mu^{-}$ & 0.059 \\
$\eta \rightarrow \gamma \gamma$ & 0.392 \\
$\eta^{\prime} \rightarrow \rho \gamma$ & 0.308 \\
$\eta^{\prime} \rightarrow \pi^{+} \pi^{-} \eta$ & 0.438 \\
\hline
\end{tabular}

Table 16.14: Relevant branching fractions.

\subsubsection{Signal Selection}

We now discuss selection requirements for signal events. First of all, the signal channels contain photons. They are selected as isolated neutral bumps in the $\mathrm{PbWO}_{4}$ calorimeter that are at least $7 \mathrm{~cm}$ away from any track intersection and satisfy the following criteria: 


$\begin{array}{ll}E_{\gamma} & >0.5 \mathrm{GeV} \\ \mathrm{E} 9 / \mathrm{E} 25 & >0.95 \\ \text { \# of cell hit } & >4 \\ \text { Second moment mass } & <100 \mathrm{MeV} / \mathrm{c}^{2}\end{array}$

We now list the criteria for the individual particles.

$J / \psi \rightarrow \mu^{+} \mu^{-}$

- Both muons should have hits in the rear end of the RICH and at least one must be identified in the muon system.

- $P_{T}$ of each muon $>0.2 \mathrm{GeV} / \mathrm{c}$ and at least one with $P_{T}>1.0 \mathrm{GeV} / \mathrm{c}$.

- $\chi^{2}$ of common vertex of both muons $<4$.

- Invariant mass within $100 \mathrm{MeV}$ of the $J / \psi$ mass.

$\eta \rightarrow \gamma \gamma$

- Each photon has $E_{\gamma}>4 \mathrm{GeV}$ and $P_{T}>0.4 \mathrm{GeV} / \mathrm{c}$.

- Invariant mass of two-photon combinations must be within $15 \mathrm{MeV} / \mathrm{c}^{2}$ of the $\eta$ mass.

$\eta^{\prime} \rightarrow \rho^{o} \gamma$

- Two oppositely charged tracks, each with momenta greater than $1 \mathrm{GeV} / \mathrm{c}$ are taken as $\pi^{+} \pi^{-}$candidates.

- The $\pi^{+} \pi^{-}$invariant mass must be within $0.55 \mathrm{GeV} / \mathrm{c}^{2}$ of the $\rho$ mass.

- The $\pi^{+} \pi^{-}$must form a common secondary vertex with the $\mu^{+} \mu^{-}$from the $J / \psi$ with a fit $\chi^{2}<10$.

- Addition of a single photon $\left(P_{T}>0.3 \mathrm{GeV} / \mathrm{c}\right)$ to these tracks produces an invariant mass within $15 \mathrm{MeV} / \mathrm{c}^{2}$ of the $\eta^{\prime}$ mass.

$\eta^{\prime} \rightarrow \pi^{+} \pi^{-} \eta$

- The same selection criteria for the $\eta$ defined above, except that $P_{T}>0.2 \mathrm{GeV} / \mathrm{c}$ for each photon is required.

- Two oppositely charged tracks, each with momenta greater than $1 \mathrm{GeV} / \mathrm{c}$ are taken as $\pi^{+} \pi^{-}$candidates.

- The $\pi^{+} \pi^{-}$must form a common secondary vertex with the $\mu^{+} \mu^{-}$from the $J / \psi$ with a fit $\chi^{2}<10$.

- The $\eta$ and the $\pi^{+} \pi^{-}$have an invariant mass within $15 \mathrm{MeV}$ of the $\eta^{\prime}$ mass. 
Signal events are also required to satisfy the following general criteria:

- A good primary vertex $\chi^{2} / N_{p}<4$, where $N_{p}$ is the number of charged tracks in the primary vertex.

- Distance between the primary and secondary vertices $(L)>50 \mu \mathrm{m}$ for $\eta^{\prime}$ and $>100$ $\mu \mathrm{m}$ for $\eta$.

- $L / \sigma_{L}>3$

- Normalized distance of closest approach with respect to the primary vertex $\left(\mathrm{DCA} / \sigma_{D C A}\right)$ of each charged track $>3$.

- No additional track is consistent the $B_{s}$ vertex with $\chi^{2}<10$ for the the $\eta$ final state, or $\chi^{2}<20$ for the $\eta^{\prime}$ final states.

- Opening angle between ' $B$ '-direction and the particle direction $<10 \mathrm{mrad}$ and $15 \mathrm{mrad}$ for $J / \psi \eta^{\prime}$ and $J / \psi \eta$ respectively. Here the ' $B$ '-direction is defined by the vector joining the primary and secondary vertices and the particle direction is defined as the vector sum of the 3-momenta of all measured particles.

- Invariant mass of $J / \psi \eta$ or $J / \psi \eta^{\prime}$ within $40 \mathrm{MeV} / \mathrm{c}^{2}$ of the $B_{s}$ mass $\left(\sigma_{M_{B}}=19 \mathrm{MeV} / \mathrm{c}^{2}\right)$.

We show in Fig. 16.30 the invariant mass distributions of signal candidates for $\gamma \gamma, \rho^{o} \gamma$ and $\pi^{+} \pi^{-} \eta$.
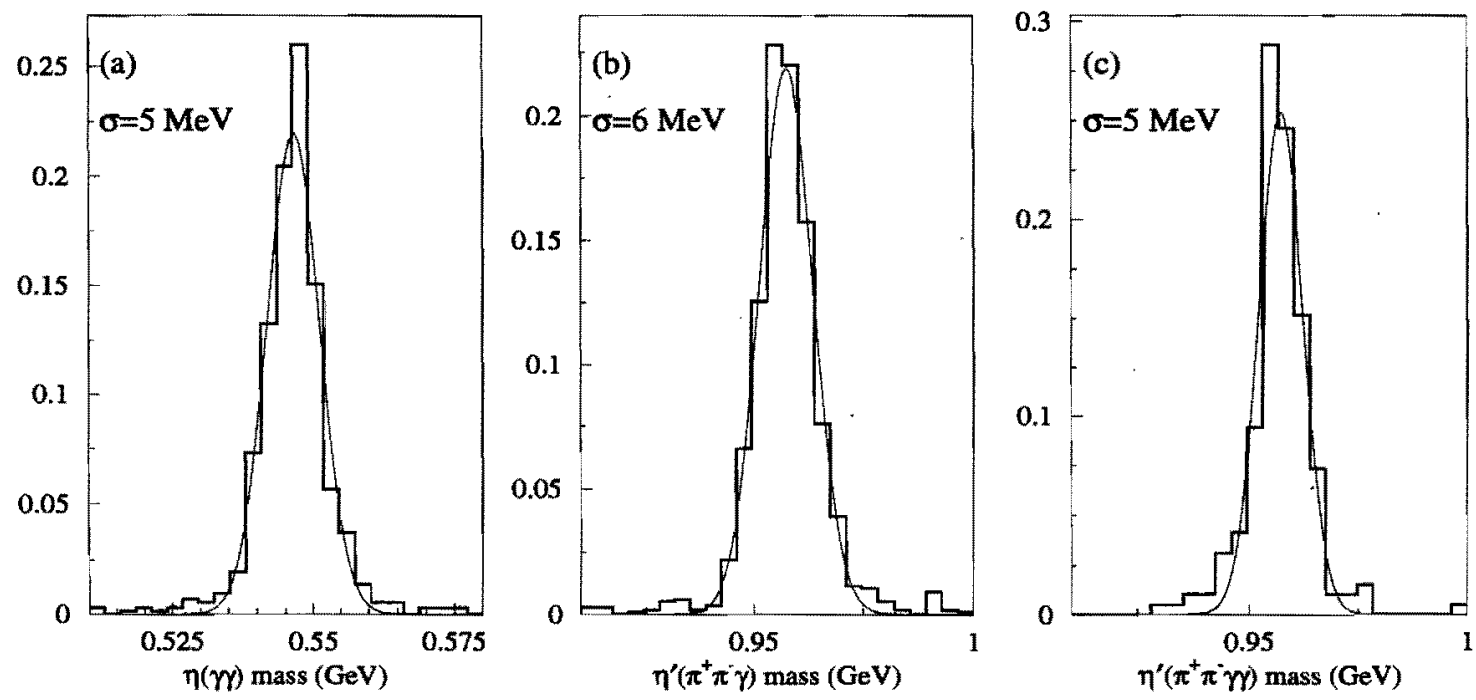

Figure 16.30: The invariant mass distributions for (a) $\eta \rightarrow \gamma \gamma$, (b) $\eta^{\prime} \rightarrow \pi^{+} \pi^{-} \gamma$, and $\eta^{\prime} \rightarrow \pi^{+} \pi^{-} \eta, \eta \rightarrow \gamma \gamma$. The Gaussian mass resolutions are indicated. 
The $\mu^{+} \mu^{-}$mass distribution from $J / \psi$ decays is shown in Fig. 16.31(a). We can improve the $B$ mass distributions by constraining the dimuons to be at the nominal $J / \psi$ mass. This greatly improves the four-vector of the reconstructed $J / \psi$. After applying this constrained fit we find the $B_{s}$ mass distributions shown in Fig. 16.31(b). Note, that we could also constrain the $\eta$ and $\eta^{\prime}$ masses to their nominal values using the same fitting technique. This will be done for future analyses.
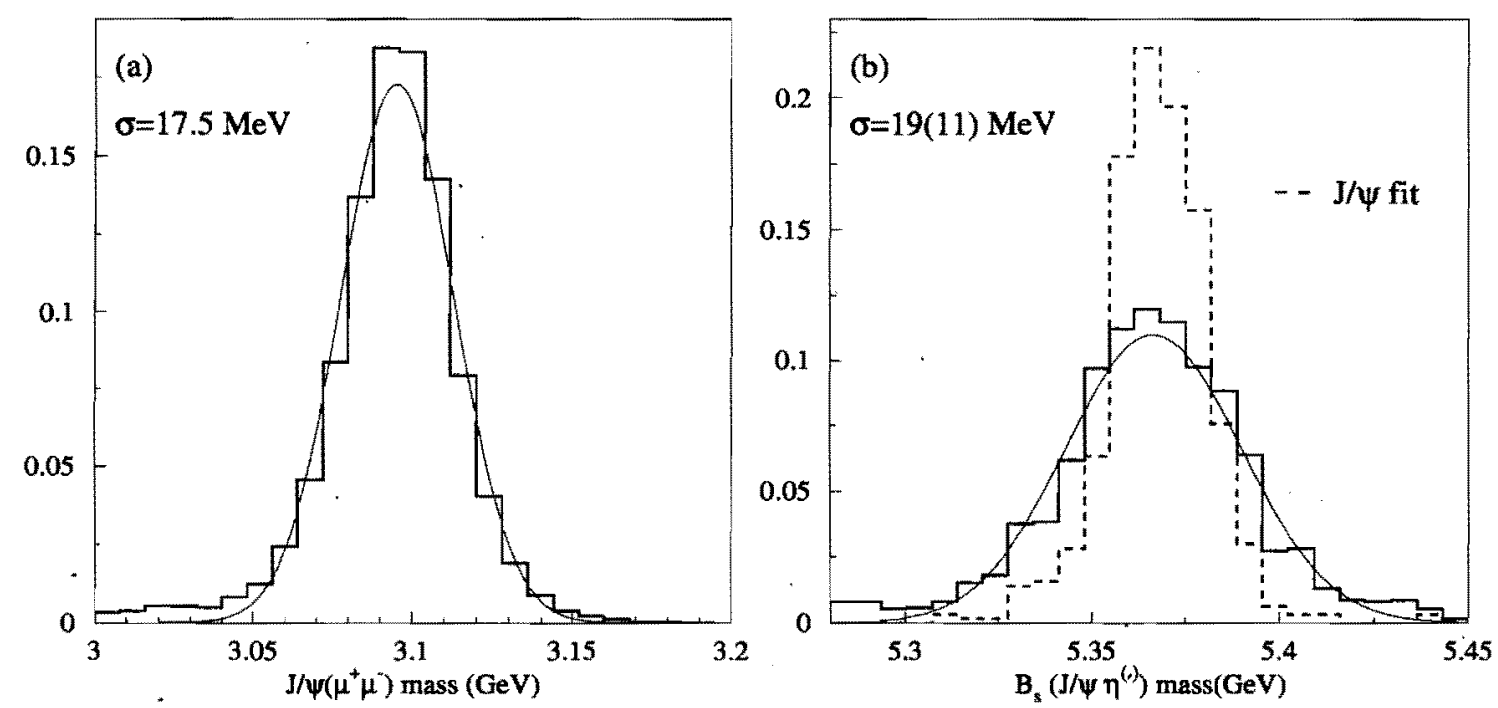

Figure 16.31: (a) The dimuon invariant mass. (b) The reconstructed $B_{s}$ mass for all three final states of $\eta$ and $\eta^{\prime}$ summed together. The solid curve is the done with without constraining the the $\mu^{+} \mu^{-}$to the $J / \psi$ mass, while the dashed curve is done using the constraint. The $B_{s}$ mass resolution improves from 19 to $11 \mathrm{MeV} / \mathrm{c}^{2}$.

\subsubsection{Background Estimation}

The dominant background to these decay modes is from $b(\bar{b}) \rightarrow J / \psi X$. (This is discussed in section 2.2 of this chapter.) To calculate reconstruction efficiencies of signals and estimation of background, Monte Carlo events were generated using Pythia and QQ to decay the heavy particles. Only events with real $J / \psi \rightarrow \mu^{+} \mu^{-}$decays were kept for further analysis. The $\mathrm{BTeV}$ detector simulation was done using the GEANT simulation package. We add to the $b \bar{b}$ background events another set of light quark background distributed with a mean Poisson multiplicity of two.

Distributions of several variables for both signal and background are compared in Fig. 16.32.

These results are based on $\sim 4,500$ detector simulated signal events (each channel), which were preselected in generator level using the criteria that all particles of these signals are within geometrical acceptance region of detector. Similarly, 40,000 background events are 

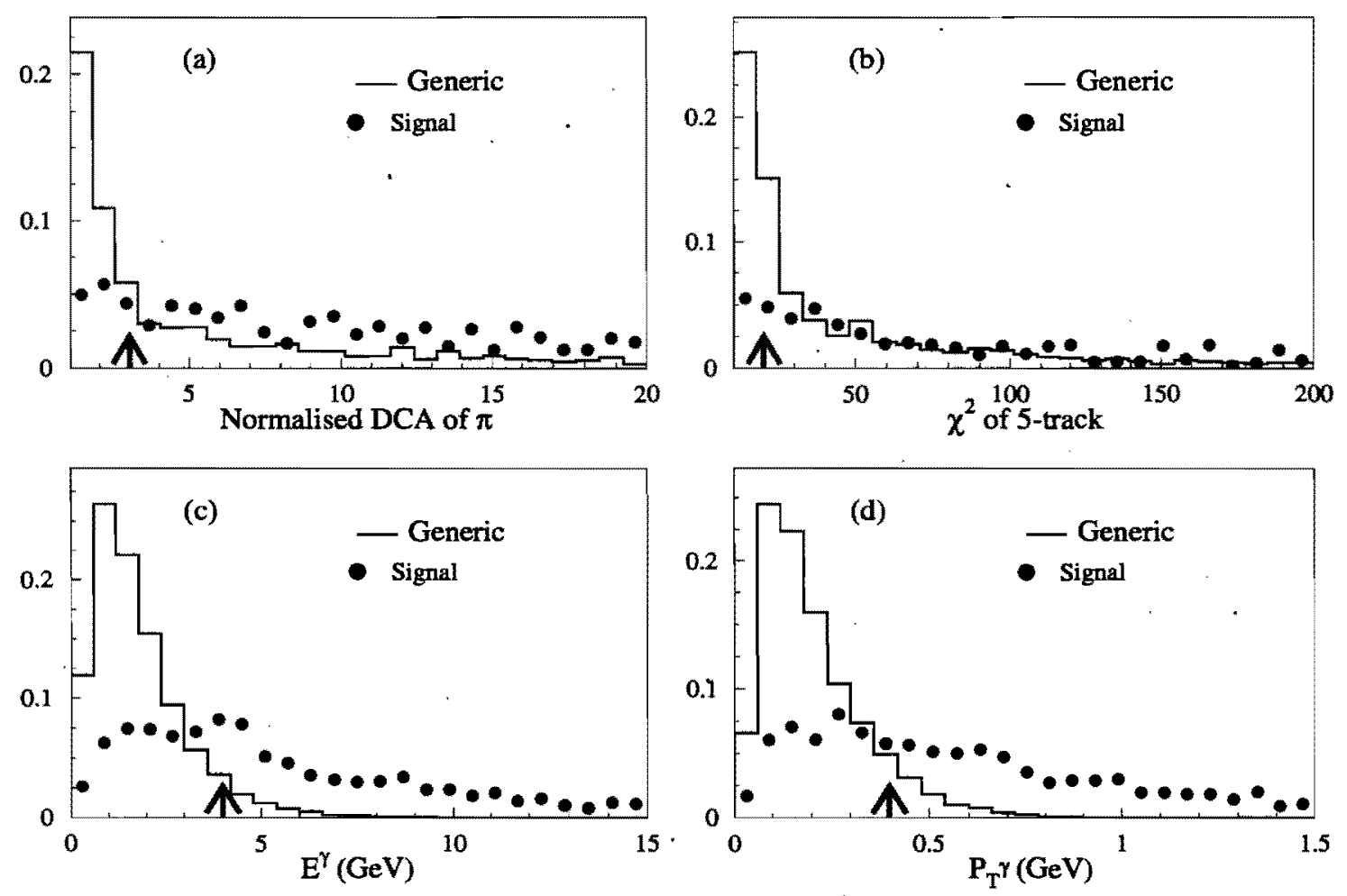

Figure 16.32: Distributions of backgrounds in several variables compared with signal. For $\eta^{\prime} \rightarrow \rho \gamma$ (a) DCA $/ \sigma_{D C A}$, (b) $\chi^{2}$ of adding an additional track to the $J / \psi \pi^{+} \pi^{-}$vertex; for $\eta \rightarrow \gamma \gamma(\mathrm{c})$ energy of the photons and (d) the transverse momentum of the photons with respect to the beam direction. The arrows show the position of the cuts. 
also preselected from 5.8 million generic $b \bar{b}$ events. To determine backgrounds we only looked at the dimuon channels, and the $\gamma \gamma$ decay of the $\eta$ and the $\rho^{\circ} \gamma$ decay of the $\eta^{\prime}$.

After all selection criteria, 1 event survived in each of the $J / \psi \eta$ and $J / \psi \eta^{\prime}$ channels within a wide $B_{s}$ mass window of $400 \mathrm{MeV} / \mathrm{c}^{2}$ (signal mass window is $44 \mathrm{MeV} / \mathrm{c}^{2}$ ). This leads to a signal-to-background expectation for $J / \psi \eta$ and $J / \psi \eta^{\prime}$, of 15:1 and 30:1. That the backgrounds are so low is not surprising. We therefore feel confident that we can add the $\eta^{\prime} \rightarrow \pi^{+} \pi^{-} \eta$ modes in without significant background.

\subsubsection{Sensitivity to $\sin (2 \chi)$}

The expected yield of signal events and the resulting asymmetry measurement are given in Table 16.15. The trigger efficiency consists of Level 1 efficiencies from the detached vertex trigger and the dimuon trigger and the Level 2 trigger.

\begin{tabular}{|c|c|c|c|}
\hline $\begin{array}{l}\text { Luminosity } \\
\text { Running time } \\
\text { Integrated Luminosity } \\
\sigma_{b \bar{b}} \\
\text { Number of } b \bar{b} \text { events } \\
\text { Number of } B_{s} \text { events }\end{array}$ & & $\begin{array}{c}2 \times 10^{32} \mathrm{~cm}^{-2} \\
10^{7} \mathrm{sec}^{-1} \\
2000 \mathrm{pb}^{-1} \\
100 \mu \mathrm{b} \\
2 \times 10^{11} \\
0.5 \times 10^{11}\end{array}$ & \\
\hline & \multicolumn{2}{|c|}{$B_{s} \rightarrow J / \psi \eta^{\prime}$} & $B_{s} \rightarrow J / \psi \eta$ \\
\hline & $\eta^{\prime} \rightarrow \rho^{0} \gamma$ & $\eta^{\prime} \rightarrow \pi^{+} \pi^{-} \eta$ & $\eta \rightarrow \gamma \gamma$ \\
\hline Reconstruction efficiency (\%) & 1.2 & 0.60 & 0.71 \\
\hline$S / B$ & $30: 1$ & - & $15: 1$ \\
\hline Level 1 Trigger efficiency (\%) & 85 & 85 & 75 \\
\hline Level 2 Trigger efficiency (\%) & 90 & 90 & 90 \\
\hline Number of reconstructed signal events & 5670 & 1610 & 1920 \\
\hline Tagging efficiency, $\epsilon \mathrm{D}^{2}$ & \multicolumn{3}{|c|}{0.1} \\
\hline Total Number tagged & \multirow{2}{*}{\multicolumn{3}{|c|}{$\begin{array}{c}994 \\
0.033\end{array}$}} \\
\hline$\delta(\sin 2 \chi)$ & & & \\
\hline
\end{tabular}

Table 16.15: Projected yield of $B_{s} \rightarrow J / \psi \eta^{\prime}\left(B_{s} \rightarrow J / \psi \eta\right)$ and the uncertainty on $\chi$.

The accuracy on $\sin (2 \chi)$ is not precise enough to measure the standard model predicted value, which is comparable to the error, in $10^{7}$ seconds of running. Nevertheless, we are optimistic. The low background level makes it possible to loosen the cuts and gain acceptance. We could also add in the $J / \psi \rightarrow e^{+} e^{-}$decay mode. This will not be as efficient as $\mu^{+} \mu^{-}$due to radiation of the electrons, but will be useful. We also believe that ways can be found to improve flavor tagging efficiency, especially for $B_{s}$. Furthermore, we will have many years of running, and we can expect some improvement from the use of $B_{s} \rightarrow J / \psi \phi$. 


\subsection{Measurement of $B_{s}$ Mixing Using $B_{s} \rightarrow D_{s}^{-} \pi^{+}$and $B_{s} \rightarrow J / \psi \bar{K}^{* 0}$}

As discussed in Section 1.2.2, the measurement of $B_{s}$ mixing allows a determination of $\left|V_{t s} / V_{t d}\right|$, which corresponds to one side of the $B$ unitarity triangle. While the theoretical discussion was given in terms of the mass splitting between the light and heavy $B_{s}$ mass eigenstates, $\Delta m_{s}$, the discussion here will be in terms of $x_{s}$, where $x_{s}=\Delta m_{s} / \Gamma$.

In this section, the $x_{s}$ reach of $\mathrm{BTeV}$ will be demonstrated. This study was carried out in several steps, the first step being a simulation of the $\mathrm{BTeV}$ detector response to signal events. The output of this step was treated as real data and passed through a physics analysis program to determine the yield, the time resolution and the signal-to-background ratio in each mode. This information was then passed to a separate program which computed the $x_{s}$ reach; this program is discussed in Section 16.7.2. A separate background study was performed.

\subsubsection{Yields, Resolutions and Signal-to-Background Ratios}

The mode for which $\mathrm{BTeV}$ has the most sensitivity to $x_{s}$ is $B_{s} \rightarrow D_{s}^{-} \pi^{+}$, where the $D_{s}^{-}$ decays either by $D_{s}^{-} \rightarrow \phi \pi^{-}, \phi \rightarrow K^{+} K^{-}$, or by $D_{s}^{-} \rightarrow K^{* 0} K^{-}, K^{* 0} \rightarrow K^{+} \pi^{-}$. Both of these $D_{s}^{-}$modes have narrow intermediate states and characteristic angular distributions, both of which can be used to improve the signal-to-background ratio.

For this study, Monte Carlo events were generated using Pythia and QQ, as described in Section 12.3, and the detector response was simulated using BTeVGeant. The output of BTeVGeant was analyzed in a fashion similar to that described for $B_{s} \rightarrow D_{s}^{ \pm} K^{\mp}$ in Section 16.4.1. The main difference is that the particle ID cuts were loosened significantly; the bachelor pion was not required to be identified by the RICH and it was required that only one of the two kaons be strongly identified. These changes were made because a state with a larger branching ratio requires less stringent background rejection in order to obtain a reasonable signal-to-background ratio. From this study the efficiency for a $B_{s}$ candidate to survive all of the analysis cuts was found to be $2.7 \%$ for the $\phi \pi$ mode and $2.3 \%$ for the $K^{* 0} K$ mode. For both modes the resolution on the mass of the $B$ was found to be $18 \mathrm{MeV} / c^{2}$ and the mean resolution on the proper decay time was found to be $43 \mathrm{fs}$.

The $\mathrm{BTeV}$ Level 1 trigger simulation, described in chapters 9 and 14, was run on the $B_{s} \rightarrow D_{s}^{-} \pi^{+}$events which passed the analysis cuts; the trigger was found to have an efficiency of $74 \%$ for both the $\phi \pi^{-}$and $K^{* 0} K^{-}$final states. Section 14.3 .3 discusses the performance of the Level 2 trigger and reports an estimated efficiency of $90 \%$.

The flavor tagging power of $\mathrm{BTeV}$ was discussed in Chapter 15 , in which it was argued that the nominal tagging power is $\epsilon D^{2}=0.1$ which arises from $\epsilon=0.70$ and $D=0.37$.

It is believed that the dominant source of backgrounds will be events of the form $X_{b} \rightarrow D_{s}^{-} X$, where $X_{b}$ may be any $b$ flavored hadron. The background combinations arise when a true $D_{s}^{-}$combination is combined with some other track in the event. An MCFast based study of 1 million $B \rightarrow D_{s}^{-} X$ events was performed using an older version of the de- 
tector geometry, the one used for the BTeV Preliminary Technical Design Report (PTDR). Comparisons between BTeVGeant and MCFast, and comparisons between the old and new detector geometries, show that these background studies remain valid. When the 1 million $B \rightarrow D_{s}^{-} X$ events were passed through MCFast and analyzed as real data, 8 entries remained in a mass window 6 times larger than the mass window used to select signal $B_{s}$ candidates. From this it is estimated that the signal-to-background ratio in this channel is 8.4:1. This study was performed without the proper treatment of multiple interactions in one beam crossing. To account for this, the signal-to-background ratio used in the estimate of the $x_{s}$ reach is $3: 1$.

The background from direct charm production has not yet been investigated. While direct charm production has a cross-section about 10 times higher than that for production of charm via $B$ decay, it is triggered much less efficiently. Moreover the the requirement of two, distinct detached vertices greatly reduces the background from direct charm. In the end it is expected that the background from $B \rightarrow D_{s}^{-} X$ will dominate.

Table 16.16 gives a summary of the preceeding results and discusses a list of all assumptions which went into the computation of the yield. In one year it is expected that 72,000 events will trigger, survive all analysis cuts and have their birth flavor tagged. This number is substantially higher than that reported in the BTeV Preliminary Technical Design Report (PTDR). The main reason is that the earlier result used only lepton tagging and kaon tagging, the tagging methods with the best dilutions but the smallest efficiencies.

Another mode with good $x_{s}$ sensitivity is $B_{s} \rightarrow J / \psi \bar{K}^{* 0}, J / \psi \rightarrow \mu^{+} \mu^{-}, \bar{K}^{* 0} \rightarrow K^{-} \pi^{+}$. Although this mode is Cabibbo suppressed, other factors are in its favor: the final state consists of a single detached vertex and the state is triggerable with several independent strategies, including impact parameter triggers, secondary vertex triggers and dimuon triggers [21]. While this mode does not have the $x_{s}$ reach of $D_{s}^{-} \pi^{+}$it does cover much of the expected range and it provides a powerful check with partly independent systematics.

For reasons of time limitations, the simulation of the $J / \psi \widetilde{K}^{* 0}$ mode used MCFast, not BTeVGeant. The analysis of this mode proceeded as follows. To be considered as part of a signal candidate, a track was required to have at least 20 total hits and at least 4 pixel hits. The only further requirement placed on $\pi^{ \pm}$candidates was that they have a momentum greater than $0.5 \mathrm{GeV} / c$. In order to be considered a muon candidate, a track was required to have a momentum $p>5 \mathrm{GeV} / c$, to penetrate the hadron filter and to leave hits in the most downstream muon chambers. Kaon candidates were required to satisfy a simplified model of the RICH system: the track was required to have a momentum in the range $3<p<70 \mathrm{GeV} / c$ and was required to have hits in the tracking station downstream of the RICH mirror. True kaons which satisfied this criteria were identified as kaons with an efficiency of $90 \%$; other hadrons which satisfied this criteria were identified as kaons $3 \%$ of the time.

A $\mu^{+} \mu^{-} K^{-} \pi^{+}$combination was accepted as a $B_{s}$ candidate if the confidence level of fitting all four tracks to a single vertex was greater than 0.005 . It was also required that the resonant substructure requirements be satisfied. Combinations were considered for further analysis provided the decay length of the $B_{s}$ candidate, $L$, satisfied $L / \sigma_{L}>10$ and the impact 


\begin{tabular}{lll}
\hline Quantity & Value & $\begin{array}{c}\text { Yield } \\
\text { (Events/year) }\end{array}$ \\
\hline \hline Luminosity: & $2 \times 10^{32} \mathrm{~cm}^{-2} \mathrm{~s}^{-1}$ & \\
One Year: & $10^{7} \mathrm{~s}$ & \\
$\sigma_{b \dot{b}}:$ & $100 \mu \mathrm{b}$ & \\
$\mathcal{B}\left(B_{s} \rightarrow D_{s}^{-} \pi^{+}\right):$ & $3.0 \times 10^{-3}$ & \\
$\mathcal{B}\left(D_{s}^{-} \rightarrow \phi \pi^{-}\right):$ & 0.030 & \\
$\mathcal{B}\left(D_{s}^{-} \rightarrow K^{* 0} K^{-}\right):$ & 0.036 & \\
$\mathcal{B}\left(\phi \rightarrow K^{+} K^{-}\right):$ & 0.49 & \\
$\mathcal{B}\left(K^{* 0} \rightarrow K^{+} \pi^{-}\right):$ & 0.67 & \\
$\mathcal{B}\left(\bar{b} \rightarrow B_{s}\right)$ & 0.13 & \\
$\epsilon\left(\right.$ Geometry + cuts : $\left.\phi \pi^{-}\right)$ & 0.027 & \\
$\epsilon\left(\right.$ Geometry + cuts : $\left.K^{* 0} K^{-}\right)$ & 0.023 & \\
$\epsilon$ (Trigger) Level 1 & 0.74 & 72,000 \\
$\epsilon($ Trigger) Level 2 & 0.90 & \\
$\epsilon$ (Tag) & 0.70 & \\
Tagging Dilution & 0.37 & \\
$S / B$ & $3: 1$ & \\
$\sigma($ Proper Decay time $)$ & $43 \mathrm{fs}$ & \\
\hline
\end{tabular}

Table 16.16: Projected yield for $B_{s} \rightarrow D_{s}^{-} \pi^{+}$in one year of $\mathrm{BTeV}$ running. The numbers in the third column give the expected yield when all of the factors down to and including that line have been considered. The branching fraction $\mathcal{B}\left(B_{s} \rightarrow D_{s}^{-} \pi^{+}\right)$was estimated to be the same as $\mathcal{B}\left(B_{d} \rightarrow D^{-} \pi^{+}\right)$. The value for $\mathcal{B}\left(\bar{b} \rightarrow B_{s}\right)$ was obtained from [3].

parameter of the $B_{s}$ candidate with the primary vertex, $d$, satisfied $d<3 \sigma_{d}$. Each of the four $B_{s}$ granddaughters were required to have an impact parameter with the primary vertex, $d$, of $d>2 \sigma_{d}$. Candidates with poor time resolution were rejected by demanding $\sigma_{t} \leq 0.09 \mathrm{ps}$. Also the mass of the $J / \psi$ was constrained to its PDG value. The above procedure found that the efficiency for the 4 tracks to be within the fiducial volume of the tracking system was $14.2 \pm 0.3 \%$ and the efficiency for the remaining candidates to pass the analysis cuts was $0.29 \pm 0.01$. The resolution on the mass of the $B_{s}$ was found to be $8.6 \pm 0.3 \mathrm{MeV} / c^{2}$ and the mean resolution on the proper decay time was found to to be $36 \mathrm{fs}$.

The $\mathrm{BTeV}$ Level 1 trigger simulation was run on the $J / \psi \bar{K}^{* 0}$ sample and, of the candidates which passed all analysis cuts, $68 \pm 2 \%$ also passed the trigger; the error is statistical only. However, this mode can also be triggered by the dimuon trigger. Section 8.3 , which describes the algorithms and performance of the muon trigger, estimates a trigger efficiency of $50 \%$ for this decay mode. There is, as yet, no calculation of the total Level 1 trigger efficiency which takes into account the correlations between the two triggers. For this proposal it will be estimated that the combined Level 1 trigger efficiency is $85 \%$. Section 14.3 .3 discusses the performance of the Level 2 tracking trigger and reports an estimated efficiency 


\begin{tabular}{llr}
\hline Quantity & Value & $\begin{array}{c}\text { Yield } \\
\text { (Events/year) }\end{array}$ \\
\hline \hline Luminosity: & $2 \times 10^{32} \mathrm{~cm}^{-2} \mathrm{~s}^{-1}$ & \\
One Year: & $10^{7} \mathrm{~s}$ & \\
$\sigma_{b \bar{b}}:$ & $100 \mu \mathrm{b}$ & \\
$\mathcal{B}\left(B_{s} \rightarrow J / \psi \bar{K}^{* 0}\right):$ & $8.5 \times 10^{-5}$ & \\
$\mathcal{B}\left(J / \psi \rightarrow \mu^{+} \mu^{-}\right):$ & 0.061 & \\
$\mathcal{B}\left(\bar{K}^{* 0} \rightarrow K^{-} \pi^{+}\right):$ & 0.667 & \\
$\mathcal{B}\left(\bar{b} \rightarrow B_{s}\right)$ & 0.13 & \\
$\epsilon($ Geometric $)$ & 0.142 & \\
$\epsilon$ (Analysis cuts) & 0.26 & \\
$\epsilon($ Trigger) Level 1 Tracking only & 0.60 & 5100 \\
$\epsilon($ Trigger) Level 1 Total & 0.85 & 3600 \\
$\epsilon($ Trigger) Level 2 & 0.90 & 5300 \\
$\epsilon$ (Tag) & 0.70 & \\
Include $J / \psi \rightarrow e^{+} e^{-}$ & 1.5 & \\
Tagging Dilution & 0.37 & \\
$S / B$ & $2: 1$ & \\
$\sigma$ (Proper Decay time) & $36 \mathrm{fs}$ & \\
\hline
\end{tabular}

Table 16.17: Projected yield for $B_{s} \rightarrow J / \psi \bar{K}^{* 0}$ in one year of BTeV running. The numbers in the third column give the expected yield when all of the factors down to and including that line have been considered. The estimate for $\mathcal{B}\left(B_{s} \rightarrow J / \psi \bar{K}^{* 0}\right)$ was obtained from [21] and that for $\mathcal{B}\left(\bar{b} \rightarrow B_{s}\right)$ was obtained from [3]. The trigger efficiency is quoted as a fraction of those events which pass the analysis cuts.

of $90 \%$.

By far the dominant background is expected to come from decays of the form $X_{b} \rightarrow J / \psi X, J / \psi \rightarrow \mu^{+} \mu^{-}$, where $X_{b}$ is any $b$ flavored hadron. An MCFast based simulation of 500,000 such decays was performed and the signal-to-background level was estimated to be about $2: 1$. Some sources of background that one might, at first, think to be important turn out not to be a problem. First, the more copious $B_{s} \rightarrow J / \psi \phi$ final state is not a significant source of background because of the excellent particle ID provided by the RICH system. Second, the mass resolution is sufficient to separate the decay $B_{d} \rightarrow J / \psi \bar{K}^{* 0}$.

Finally, the expected yield can be increased by at least $50 \%$ by using the decay mode $J / \psi \rightarrow e^{+} e^{-}$. This mode will have an efficiency for secondary vertex triggers which is comparable to that for $J / \psi \rightarrow \mu^{+} \mu^{-}$; an electron trigger, which is not yet specified, is part of the BTeV baseline design.

The information reported here is summarized in Table 16.17 and is used in the mini Monte Carlo described in the next section. 


\subsubsection{Computation of the $x_{s}$ Reach}

The final step in the study was to use a mini-Monte Carlo to study the $x_{s}$ reach of BTeV. This mini-Monte Carlo generates two lifetime distributions, one for mixed events and one for unmixed events, smears the distributions and then extracts a measured value of $x_{s}$ from a simultaneous fit of the two distributions. The time smearing is a Gaussian of fixed width, using the mean time resolutions determined above. The model includes the effects of mistagging, background under the signal, and the minimum time cut which is implied by the $L / \sigma_{L}$ cut. It is assumed that the lifetime distribution of the background is an exponential with the same mean lifetime as that of the $B_{s}$.

Figures $16.33 \mathrm{a}$ ) and b) show the proper time distributions which result from one run of the mini-Monte Carlo for a generated value of $x_{s}=40$. The simulation is for the decay mode $B_{s} \rightarrow D_{s}^{-} \pi^{+}$for one month of $\mathrm{BTeV}$ running. Part a) shows the proper time distribution for unmixed decays while part b) shows the distribution for mixed decays. Part c) of the figure shows, as a function of $x_{s}$, the value of the unbinned negative log likelihood function computed from the simulated events. A clear minimum near the generated value of $x_{s}$ is observed and the likelihood function determines the fitted value to be $x_{s}=39.96 \pm 0.08$. A step of 0.5 in the negative log likelihood function determines the $1 \sigma$ error bounds and a line is drawn across the figure at the level of the $5 \sigma$ error bound.

This figure nicely illustrates the distinction between two quantities which are often confused, the significance of the result and the error on $x_{s}$. The significance of the signal is determined by how far the depth of the global minimum falls below that of the next most significant minimum. The error on $x_{s}$ is determined by the curvature of the likelihood function at the global minimum. While these quantities are clearly related, they are distinct; in particular, the significance of the signal is not the relative error on $x_{s}$.

The error returned by the fit was checked in two ways. First, an ensemble of miniMonte Carlo experiments was performed and the errors were found to correctly describe the dispersion of the measured values about the generated ones. Second, the errors returned by the fit were found to be approximately equal to the Cramer-Rao minimum variance bound.

The mini-Monte Carlo was also used to study the level of statistics below which the experiment is unable to measure $x_{s}$. As the number of events in a trial is reduced, the negative log likelihood function becomes more and more ragged and the secondary minima become more pronounced. Eventually there are secondary minima which reach depths within 12.5 units of negative $\log$ likelihood $(5 \sigma)$ of the global minimum. When this happens in a sufficiently large fraction of the trials, one must conclude that only a lower limit on $x_{s}$ can be established. In the region of the parameter space which was explored, the absolute error on $x_{s}$ was approximately 0.1 when this limit was reached. This was independent of the generated value of $x_{s}$; that is, the discovery measurement of $x_{s}$ will have errors of something like \pm 0.1 , even if $x_{s}$ is large, say 40 .

It is awkward to map out the $x_{s}$ reach of the apparatus by running a large ensemble of mini-Monte Carlo jobs; instead the following automated procedure was used. Following ideas from McDonald [22], the sum over events in the likelihood function was replaced with an integral over the parent distribution. Because the parent distribution does not have any 


\section{Simulation of $X_{s}=40$ with $43 \mathrm{fs}$ Smearing}
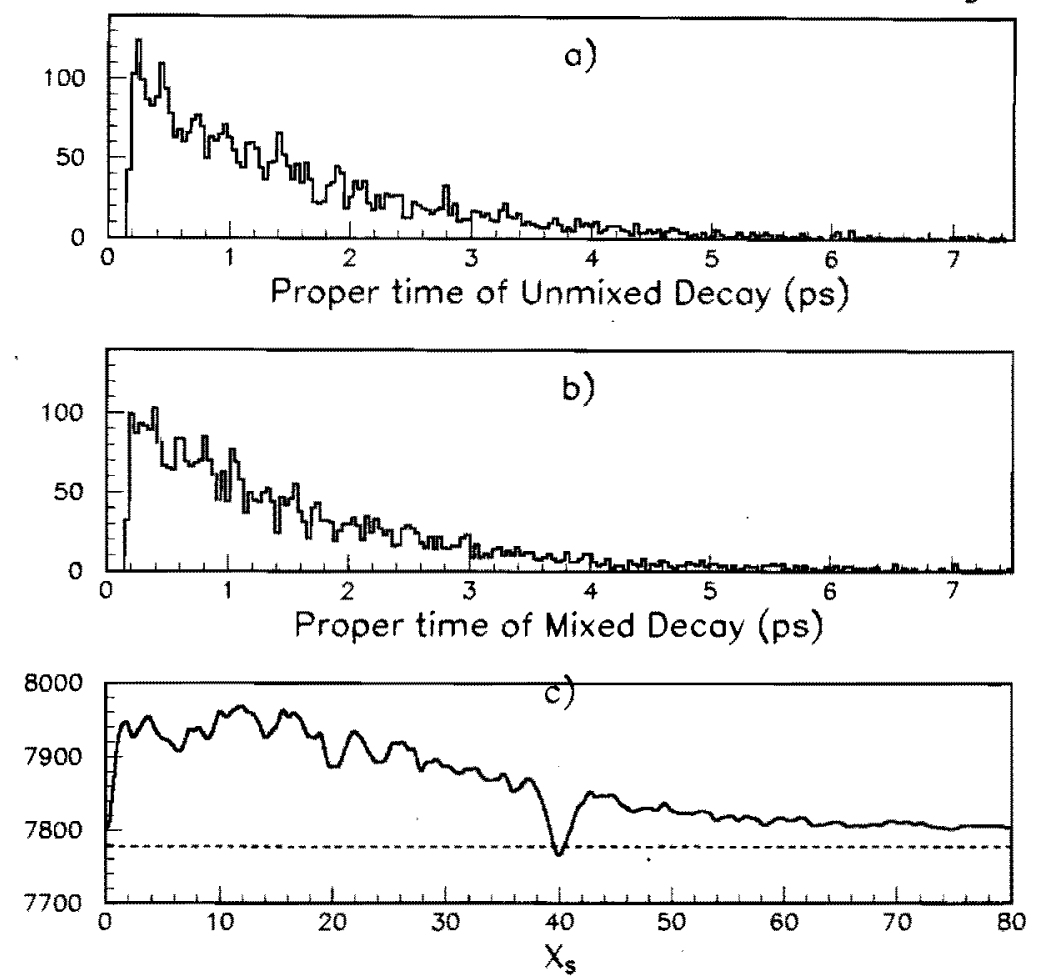

Figure 16.33: Mini-Monte Carlo proper lifetime plots of a) unmixed and b) mixed decays for a generated value of $x_{s}=40$. The plots simulate the results of the $B_{s} \rightarrow D_{s}^{-} \pi^{+}$channel after one month of running. The oscillations are prominent. Part c) shows the negative log likelihood function which was obtained from the entries in parts a) and b). A prominent minimum is seen at the generated value of $x_{s}$. The dashed line marks the level above the minimum which corresponds to $5 \sigma$ significance.

statistical fluctuations, the fluctuations in the likelihood function are removed, leaving only the core information. An example of such a likelihood function is shown in Fig. 16.34.

A likelihood function computed in this way has the property that it scales linearly with the number of events being simulated. This can be stated formally as follows. Let $x_{0}$ denote the generated value of $x_{s}$ and let $\mathcal{L}\left(x ; x_{0}, N\right)$ denote the value of the likelihood function, evaluated at $x$, for a sample which has a true value of $x_{0}$ and which contains $\mathrm{N}$ events. Then,

$$
\mathcal{L}\left(x ; x_{0}, N\right)=N \mathcal{L}\left(x ; x_{0}, 1\right)
$$

Now, one can define the significance of the minimum, $n$, as,

$$
n^{2}=2.0 N\left[\mathcal{L}\left(\infty ; x_{0}, 1\right)-\mathcal{L}\left(x_{0} ; x_{0}, 1\right)\right]
$$




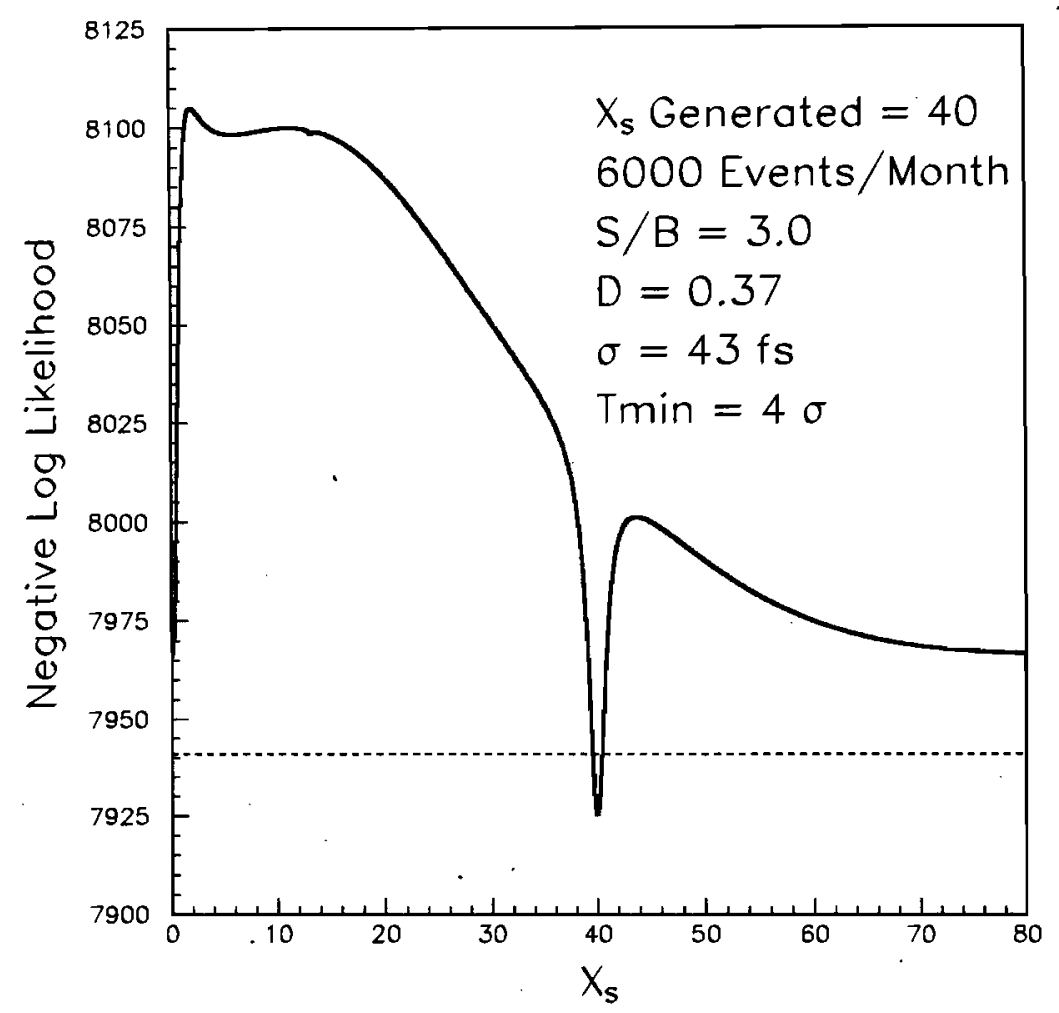

Figure 16.34: The same likelihood function as in part c) of the previous figure but obtained using the integral method described in the text. The overall shape is the same but the statistical fluctuations have been removed. There is also an overall level shift which is related to the goodness of fit in the previous figure.

For practical purposes $\infty$ was chosen to be 160 . If one did not have to worry about the missing statistical fluctuations it would be normal to define a significant signal as $5 \sigma$, or $n^{2}=25$. Instead, sufficient significance was defined as $n^{2}=31.25$, by adding a somewhat arbitrary safety margin; this allows for the usual $5 \sigma$ plus a downwards fluctuation of up to $2.5 \sigma$ anywhere else in the plot. Equation 16.22 was solved for $N$, which was then converted into the running time required to collect $N$ events. This procedure was repeated for many different values of $x_{0}$ to obtain Fig. 16.35. The solid line shows, for the $D_{s}^{-} \pi^{+}$mode, the number of years needed to obtain a measurement with a significance of $5 \sigma$ plus the safety margin. The safety margin reduces the $x_{s}$ reach at 3 years by only 3 or 4 units of $x_{s}$. For small values of $x_{s}$, the effect of the safety margin is not visible. The dashed line shows the same information but for the $J / \psi \bar{K}^{* 0}$ mode; for this mode the effect of the safety margin is similarly small.

Inspection of Fig. 16.35 shows that, using the $D_{s}^{-} \pi^{+}$mode, $\mathrm{BTeV}$ is capable of observing 


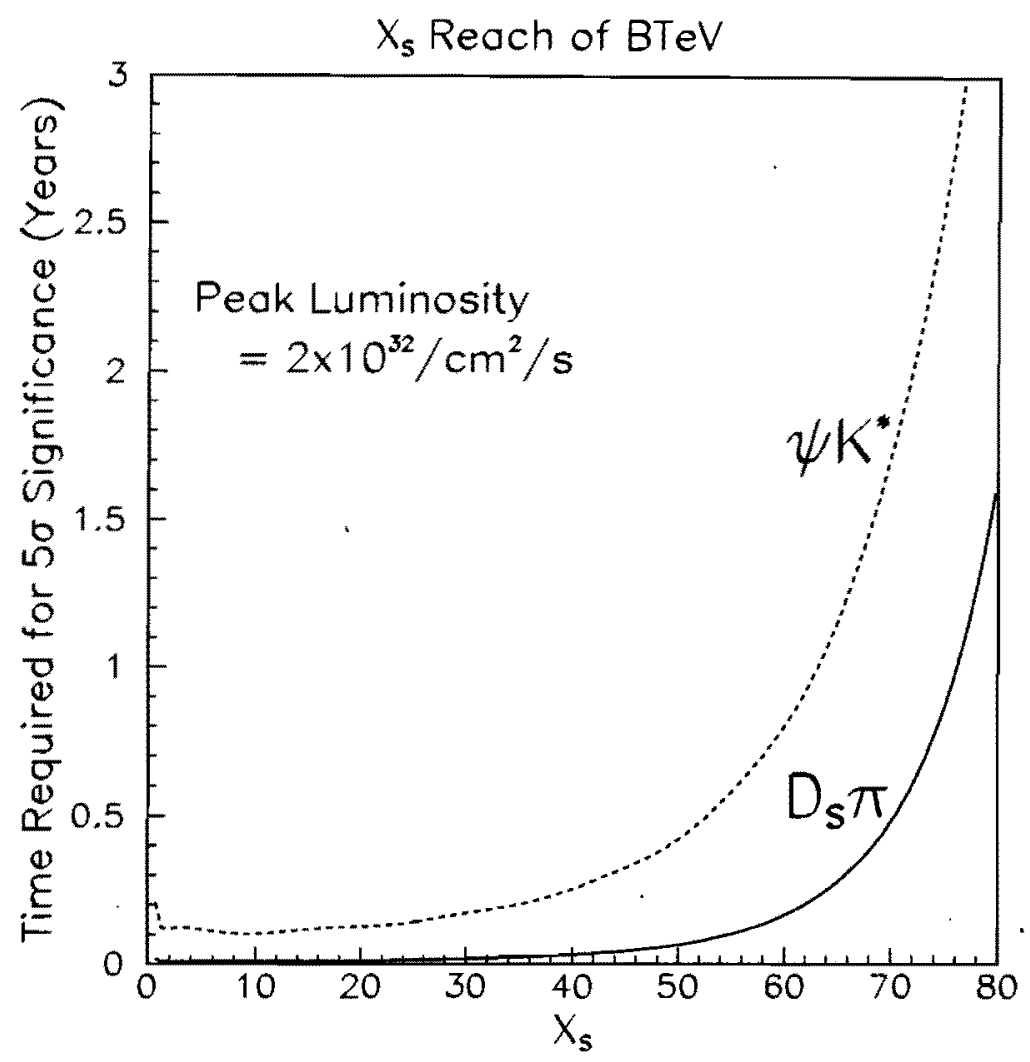

Figure 16.35: The $x_{s}$ reach of the $\mathrm{BTeV}$ detector. The curves indicate the number of years of running which are required to make a measurement of $x_{s}$ with a statistical significance of $5 \sigma$; a safety margin, discussed in the text, has been included in the definition of $5 \sigma$. The curves are for the two different decay modes indicated on the figure.

all $x_{s}$ less than 75 in one year of running. The improvement since the PTDR comes from two sources: there is an overall improvement which comes from using the full tagging power of $\mathrm{BTeV}$; there is an additional improvement at large values of $x_{s}$ which comes from the improved resolution on the proper decay time, which, in turn, was achieved by reducing the amount of material in the pixel detectors. 


\subsection{Flavor-changing Rare $B$ Meson Decays}

\subsubsection{The Physics Significance of Rare $B$ Meson Decays}

Within the Standard Model, flavor-changing neutral current decays of $b$ quarks may occur through loop diagrams, such as penguin diagrams or box diagrams. Such decays are suppressed in the Standard Model. If some of these decays occur at a much higher level than predicted by the Standard Model, this would be evidence for new particles which contribute to the loop or box diagrams. This would provide a path to observe new physics at a higher mass scale than can be probed directly at existing or planned accelerators. Table 16.18 gives a list of some interesting rare decays, their estimated Standard Model branching fractions, and current upper limits [23]:

\begin{tabular}{|l|c|c|}
\hline \hline Decay Mode & Est. BR(SM) & $\begin{array}{c}\text { Measurements and } \\
90 \% \text { CL upper limits }\end{array}$ \\
\hline$\left(B_{d}, B_{u}\right) \rightarrow X_{s} \mu^{+} \mu^{-}$ & $(5.7 \pm 1.2) \times 10^{-6}$ & $<3.6 \times 10^{-5}$ \\
$\left(B_{d}, B_{u}\right) \rightarrow X_{d} \mu^{+} \mu^{-}$ & $(3.3 \pm 1.9) \times 10^{-7}$ & - \\
$\left(B_{d}, B_{u}\right) \rightarrow K \mu^{+} \mu^{-}$ & $(4.0 \pm 1.5) \times 10^{-7}$ & $<0.9 \times 10^{-5}$ \\
$\left(B_{d}, B_{u}\right) \rightarrow K^{*} \mu^{+} \mu^{-}$ & $(1.5 \pm 0.6) \times 10^{-6}$ & $<2.5 \times 10^{-5}$ \\
$B_{s} \rightarrow \mu^{+} \mu^{-}$ & $(3.5 \pm 1.0) \times 10^{-9}$ & $<8.4 \times 10^{-6}$ \\
$B_{d} \rightarrow \mu^{+} \mu^{-}$ & $(1.5 \pm 0.9) \times 10^{-10}$ & $<1.6 \times 10^{-6}$ \\
\hline$\left(B_{d}, B_{u}\right) \rightarrow X_{s} e^{+} e^{-}$ & $(8.4 \pm 2.2) \times 10^{-6}$ & - \\
$\left(B_{d}, B_{u}\right) \rightarrow X_{d} e^{+} e^{-}$ & $(4.9 \pm 2.9) \times 10^{-7}$ & - \\
$\left(B_{d}, B_{u}\right) \rightarrow K e^{+} e^{-}$ & $(5.9 \pm 2.3) \times 10^{-7}$ & $<1.2 \times 10^{-5}$ \\
$\left(B_{d}, B_{u}\right) \rightarrow K^{*} e^{+} e^{-}$ & $(2.3 \pm 0.9) \times 10^{-6}$ & $<1.6 \times 10^{-5}$ \\
$B_{s} \rightarrow e^{+} e^{-}$ & $(8.0 \pm 3.5) \times 10^{-14}$ & - \\
$B_{d} \rightarrow e^{+} e^{-}$ & $(3.4 \pm 2.3) \times 10^{-15}$ & - \\
\hline \hline
\end{tabular}

Table 16.18: Estimated branching fractions and current measurements of flavor-changing neutral current decays with muons and electrons.

Given the enormous production rate of $B$-mesons at the Tevatron, we should be able to observe some of these decays and set stringent limits on others. The precise vertexing of the silicon pixel detector will easily allow us to differentiate $b$ decays from non- $b$ backgrounds. We present expected sensitivities from studies of some of these decay channels.

\subsubsection{Exclusive Channel $B^{0} \rightarrow K^{* 0} \mu^{+} \mu^{-}$}

There is great interest in studying $B^{0} \rightarrow K^{* 0} \ell^{+} \ell^{-}$as a window to new physics [24]. Observation of a total rate for $B^{0} \rightarrow K^{* 0} \mu^{+} \mu^{-}$different than the Standard Model prediction would be evidence for non-Standard Model particles. In fact, new physics may lead to qualitative differences in the distributions of the differential decay rate and the forward-backward asymmetry, $A_{f b}$, of the $\ell^{+} \ell^{-}$system as a function of $Q^{2}$. It has been pointed out by several 
theorists [25] that the Standard Model predicts $A_{f b}=0$ for a certain value of $Q^{2}$, as shown in Fig. 16.36. Furthermore, the position of this zero appears to be model-independent within the Standard Model. Many extensions to the Standard Model predict this asymmetry to be non-zero for all values of $Q^{2}$. Figure 16.36 shows the predictions of one of these models for different values of the Wilson coefficients [26].
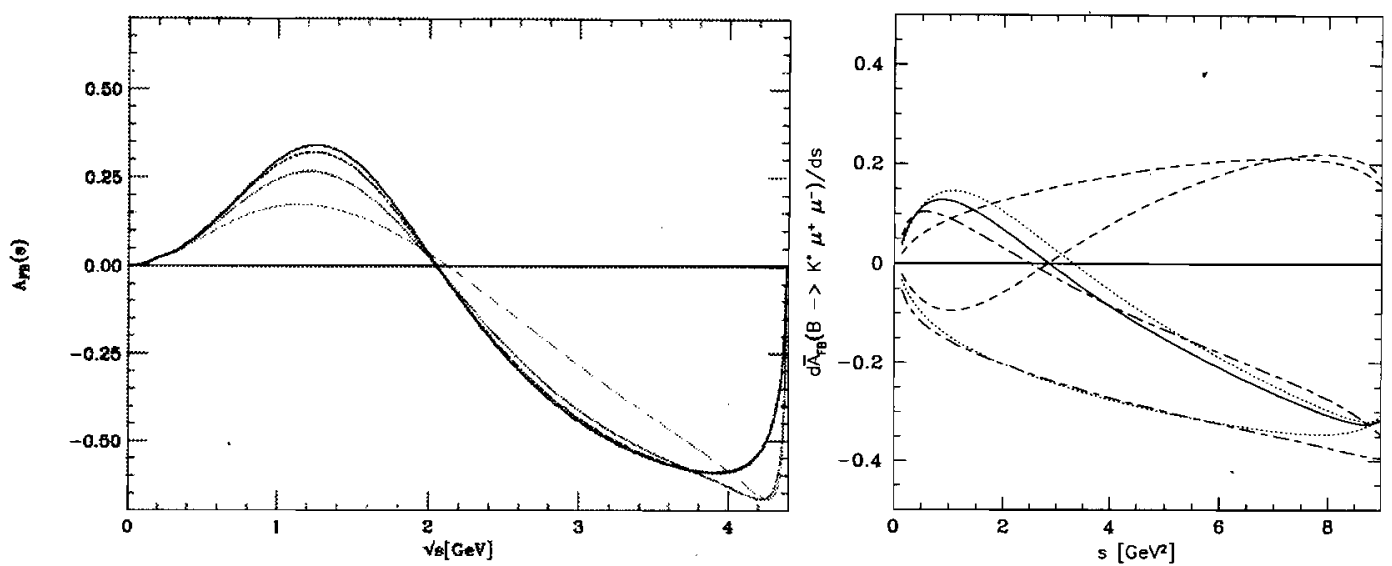

Figure 16.36: Forward-backward asymmetry in the Standard Model (left) [25]. Predictions of the SUGRA (MIA-SUSY) model [26], with various values of the Wilson coefficients (right).

It is unlikely that D0 or CDF will have sufficient sensitivity in Run II to confirm or deny the existence of a zero crossing in the $A_{f b}$ versus $Q^{2}$ distribution. In addition, the rates at $e^{+} e^{-} B$ factories are of order 10 events/year at design luminosity. Therefore, the main competition to $\mathrm{BTeV}$ on these modes will come from LHC-b.

Since we expect large backgrounds under the signal, an understanding of these backgrounds is critical to understanding our sensitivity. The various sources of background are:

- $b$-events where portions of the two $b$ hadrons in the event appear to form a vertex downstream of the production point. In approximately $1 \%$ of all $b \bar{b}$ events both $B$ hadrons decay semileptonically producing two real muons. In addition, there is a charged kaon from at least one of the $b$ 's over $90 \%$ of the time.

- Minimum bias events where three particles conspire to fake a secondary vertex and two of the particles either decay downstream of the magnet or make hadronic showers which leave a signal in the muon detector (hadron punch-through).

- Charm events with one or more real muons and kaons.

- More generally, any combination of the above.

- Decays from single $B$ mesons where two charged pions fake muons.

The basic weapons to combat these backgrounds are:

- Excellent discrimination between the primary and secondary vertices, which eliminates backgrounds from minimum bias events and from the underlying event within a true $b$ 
event. Tracks which are not part of the $b$ vertex are easily rejected by requiring a nonzero vertex fit probability, as shown in Fig. 16.37(a). Also, the normalized decay length $\left(L / \sigma_{L}\right)$, shown in Fig. 16.37(c), provides additional discrimination against background.

- Excellent mass resolution $\left(\sigma \approx 17 \mathrm{MeV} / c^{2}\right)$ on the final state, shown in Fig. 16.38.

- Excellent "point-back" resolution of the reconstructed $b$ candidate with respect to the primary vertex. This will help to reject vertices that have been artificially pieced together from particles from the two separate $b$ 's in the event. The normalized $B$ impact parameter $\left(b_{B} / \sigma_{b_{B}}\right)$ with respect to the primary vertex is quite different for signal and background events, as shown in Fig. 16.37(b).

- The ability to reject combinations which include tracks that are from the primary vertex or other vertices in the event, by cutting on the impact parameter of the track with respect to that vertex. Figures $16.37(\mathrm{e})$ and (f) show the normalized impact parameter of the kaon and pion with respect to the primary vertex $\left(b_{K} / \sigma_{b_{K}}\right.$ and $\left.b_{\pi} / \sigma_{b_{\pi}}\right)$.

- Excellent particle ID for the hadron (RICH) and the leptons (electromagnetic calorimeter and muon detector).

The $B^{0} \rightarrow K^{* 0} \mu^{+} \mu^{-}$events were selected using the following criteria:

- Two muons of opposite charge, each with momentum greater than $5 \mathrm{GeV} / c$. Both muon tracks were required to have at least one hit in the muon chambers.

- Kaon track momentum greater than $3 \mathrm{GeV} / c$. The kaon track was also required to have at least one hit in the forward drift chamber between the RICH and calorimeter. Perfect $\pi / K$ separation and $100 \%$ efficiency for reconstructing the Cherenkov photons of tracks which traverse the RICH is assumed.

- Good primary vertex with confidence level greater than $1 \%$.

- Good $b$ vertex with confidence level greater than $1 \%$.

- Decay length greater than $7 \sigma$.

- $B$ impact parameter with respect to the primary vertex less than $2.5 \sigma$.

- $K$ impact parameter with respect to the primary vertex greater than $2.5 \sigma$.

- $\pi$ impact parameter with respect to the primary vertex greater than $2.5 \sigma$.

- $B$ momentum greater than $20 \mathrm{GeV} / c$.

- $\left|m(K \pi)-m_{K^{* 0}}\right|<50 \mathrm{MeV} / c^{2}$.

- Cut $100 \mathrm{MeV} / c^{2}$ about the $J / \psi$ and $\psi^{\prime}$ nominal masses to remove regions dominated by $B \rightarrow \psi K^{*}$ and $B \rightarrow \psi^{\prime} K^{*}$, which interfere with the signal.

Of $4.4 \mathrm{pb}^{-1}$ of MCFast $b \bar{b}$ background events generated (about one million events), nine pass the selection criteria. These events were not required to pass the trigger. For $2 \mathrm{fb}^{-1}$ of data (one year of running at a luminosity of $2 \times 10^{32} \mathrm{~cm}^{-2} \mathrm{~s}^{-1}$ ), this would correspond 


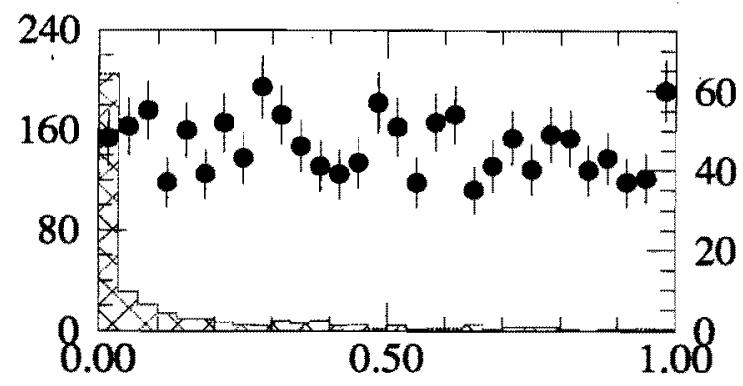

a) B Vertex Probability

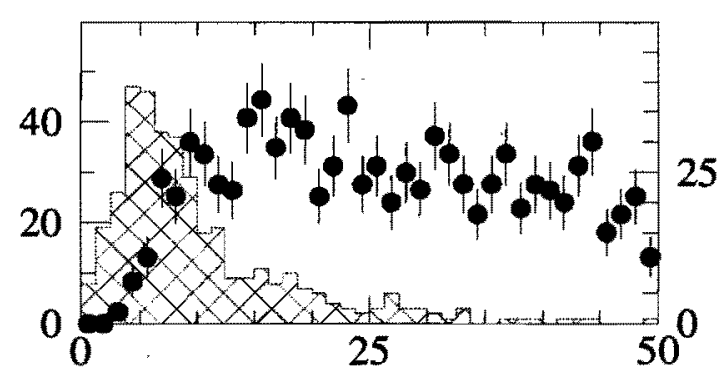

c) Norm. Decay Length

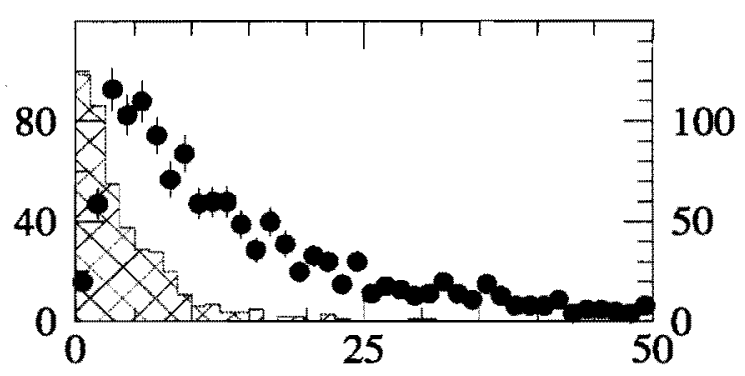

e) Norm. K Impact Param.

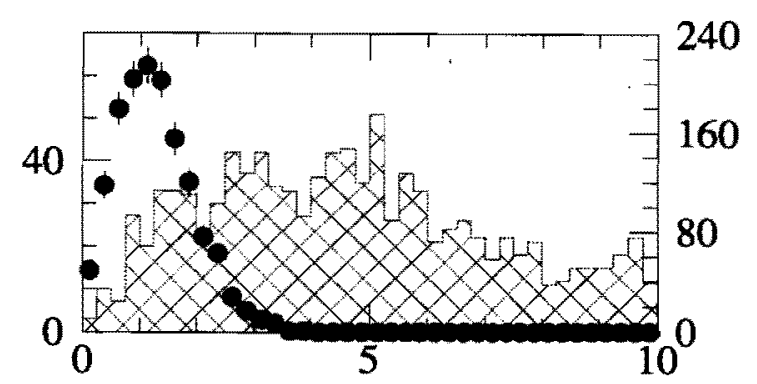

b) Norm. B Impact Param.

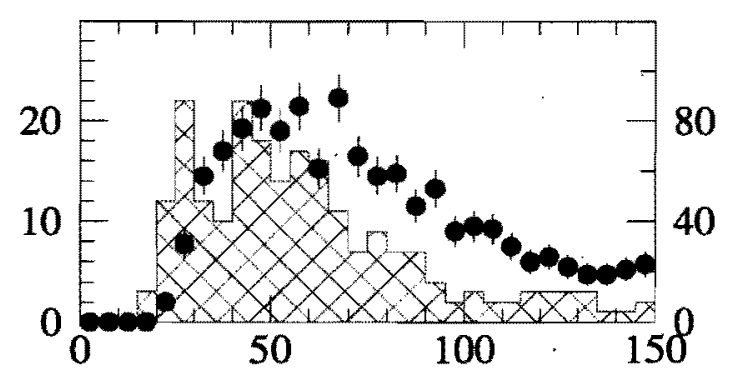

d) $\mathrm{p}(\mathrm{K} \pi \mu \mu)(\mathrm{GeV} / \mathrm{c})$

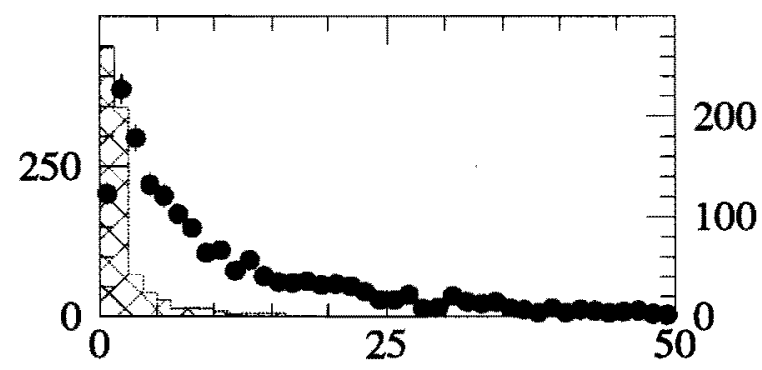

f) Norm. $\pi$ Impact Param.

Figure 16.37: Distributions of cut variables for signal (points) and $b \bar{b}$ background (hatched) MCFast events. 
to 4090 events in the range $4.7 \mathrm{GeV} / c^{2}<m(K \pi \mu \mu)<5.7 \mathrm{GeV} / c^{2}$, shown in Fig. 16.38. The width of the $B^{0}$ mass peak obtained from the MCFast signal Monte Carlo sample is $17 \mathrm{MeV} / c^{2}$. Thus, after the trigger efficiency is applied, we can expect about 200 background events from semileptonic $b \bar{b}$ decays under the $B^{0}$ mass peak, as shown in Table 16.19. With an expected yield of $\sim 2200$ signal events, this corresponds to a signal-to-background ratio of about 11 .
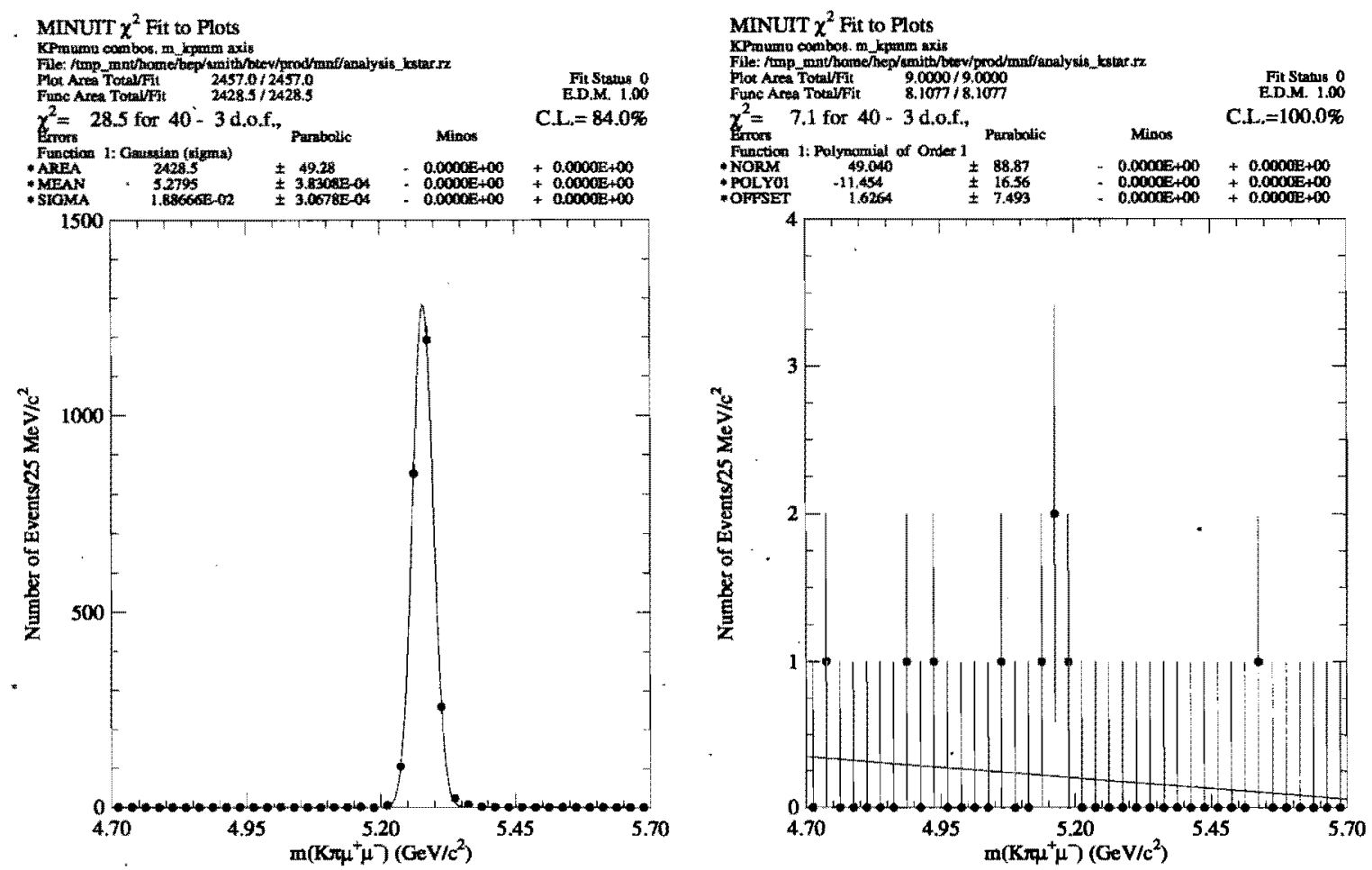

Figure 16.38: Distributions of tagged $B^{0} \rightarrow K^{* 0} \mu^{+} \mu^{-}$signal (left) and $4.4 \mathrm{fb}^{-1}$ of $b \bar{b}$ background (right) MCFAST events.

We did not include the $B^{0} \rightarrow \psi K^{* 0}$ as a background. That decay is large compared to the rare decay considered here and will interfere with the rare decay and distort the dimuon mass distribution in the vicinity of $3 \mathrm{GeV} / \mathrm{c}^{2}$. In this study, a cut was applied to remove the regions around the $J / \psi$ and ' $\psi^{\prime}$. In fact, this state can be used to calibrate the efficiency of the analysis and as a normalization for a measurement of the relative branching fraction.

A sample of $2 \mathrm{fb}^{-1}$ of signal MCFast Monte Carlo events were generated according to the Standard Model prediction for $A_{f b}$ and $Q^{2}$ [24]. Figure 16.39 shows the distributions of reconstructed $A_{f b}$ and number of events as a function of $m\left(\mu^{+} \mu^{-}\right)$for this sample, after all cuts and the trigger efficiency have been applied. We have included our anticipated $b \bar{b}$ semileptonic background, generated with $A_{f b}=0$, in the asymmetry plot. We should be able to easily observe and measure the position of a zero in the asymmetry if it exists, or make a strong case for non-Standard Model physics, if it does not. 


\begin{tabular}{|c|c|}
\hline Luminosity & $2 \times 10^{32} \mathrm{~cm}^{-2} \mathrm{~s}^{-1}$ \\
\hline Running Time/Year & $10^{7} \mathrm{~s}$ \\
\hline$b \bar{b}$ Cross Section & $100 \mu \mathrm{b}$ \\
\hline Number of $b \bar{b}$ Pairs Produced & $2 \times 10^{11}$ \\
\hline$N_{B^{0}}$ Produced & $1.5 \times 10^{11}$ \\
\hline Est. $\mathcal{B}\left(B^{0} \rightarrow K^{* 0} \mu^{+} \mu^{-}\right)$ & $(1.5 \pm 0.6) \times 10^{-6}$ \\
\hline $\mathcal{B}\left(K^{0 *} \rightarrow K^{+} \pi^{-}\right)$ & 0.67 \\
\hline Number of Signal Events Produced & $1.5 \times 10^{5}$ \\
\hline Level 1 Trigger Efficiency (vertex + dimuon) & $80 \%$ \\
\hline Level 2 Trigger Efficiency & $90 \%$ \\
\hline Reconstruction Efficiency & $2.0 \%$ \\
\hline Number of Signal Events & 2200 \\
\hline Number of Background in Signal Box & 200 \\
\hline Signal/Background & 11 \\
\hline \hline
\end{tabular}

Table 16.19: Estimate of sensitivity to $B^{0} \rightarrow K^{* 0} \mu^{+} \mu^{-}$based on one year of running. Only backgrounds from $b \bar{b}$ semileptonic decays were included in this study.
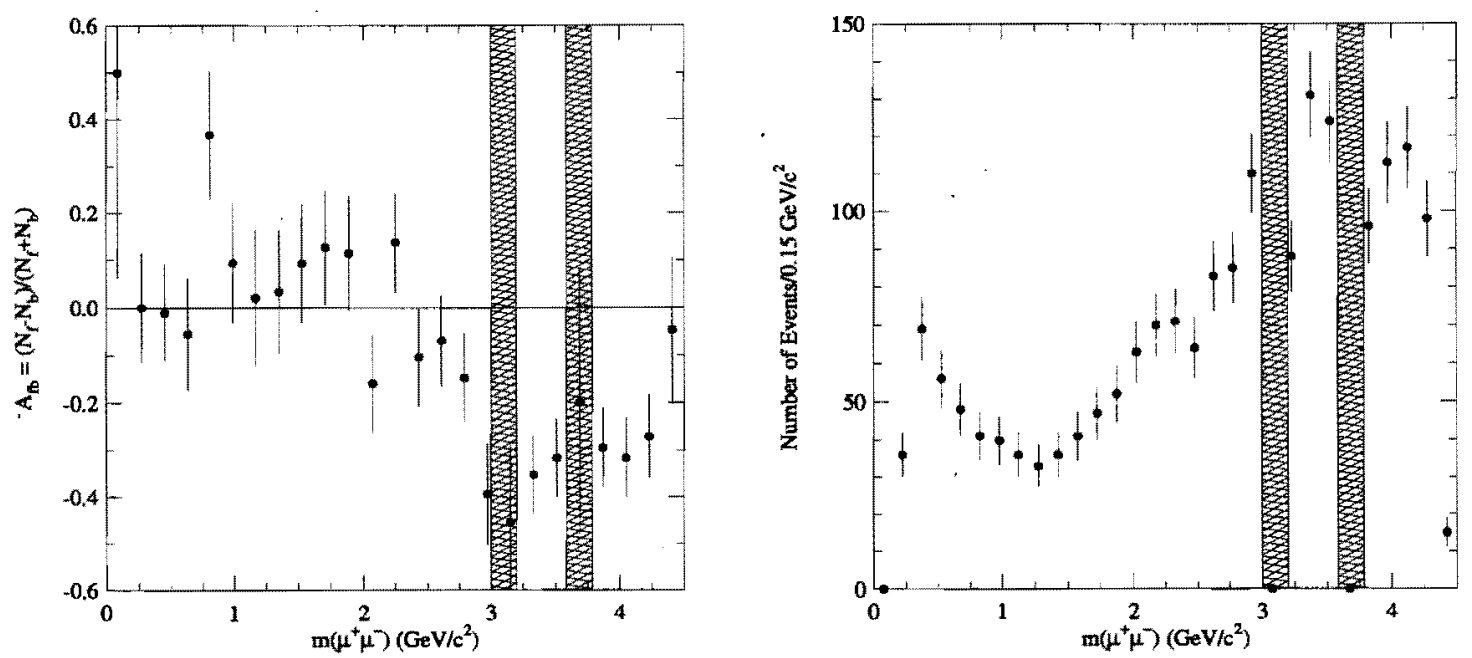

Figure 16.39: Expected forward-backward asymmetry distribution, including background, (left) and number of events (not including background) (right) as a function of $m\left(\mu^{+} \mu^{-}\right)$ after one year of running. 


\subsubsection{Exclusive Channel $B^{+} \rightarrow K^{+} \mu^{+} \mu^{-}$}

While the channel $B^{+} \rightarrow K^{+} \mu^{+} \mu^{-}$is not as rich as $B^{0} \rightarrow K^{* 0} \mu^{+} \mu^{-}$, in that the asymmetry $A_{f b}$ is expected to be small within the Standard Model and beyond the Standard Model, a measurement of the decay rate is still a sensitive probe of new physics. In particular, measurement of the differential decay rate will provide input to determine the magnitude and sign of the Wilson coefficients $C_{7}, C_{9}$, and $C_{10}$.

Most of the backgrounds to this channel are the same as those listed for the $B^{0} \rightarrow K^{* 0} \mu^{+} \mu^{-}$analysis in Section 16.8.2. Events for this study were selected using nearly the same criteria as the $B^{0} \rightarrow K^{* 0} \mu^{+} \mu^{-}$analysis:

- Kaon track momentum greater than $4 \mathrm{GeV} / c$. The kaon track was also required to have at least one hit in the forward drift chamber between the RICH and calorimeter. Perfect $\pi / K$ separation and $100 \%$ efficiency for reconstructing the Cherenkov photons is assumed.

- Two muons with momentum greater than $5 \mathrm{GeV} / c$. Both muon tracks were required to have at least one hit in the muon chambers.

- Good primary vertex with confidence level greater than $1 \%$.

- Good $b$ vertex with confidence level greater than $1 \%$.

- Decay length greater than $7 \sigma$.

- $B$ impact parameter with respect to the primary vertex less than $2.5 \sigma$.

- $K$ impact parameter with respect to the primary vertex greater than $2.5 \sigma$.

- $B$ momentum greater than $20 \mathrm{GeV} / c$.

- Cut $100 \mathrm{MeV} / c^{2}$ about the $J / \psi$ and $\psi^{\prime}$ nominal masses to remove regions dominated by $B \rightarrow \psi K$ and $B \rightarrow \psi^{\prime} K$, which interfere with the signal.

We have not simulated all sources of background. Our estimates indicate that the most serious background is from events with pairs of $b$ 's, each of which undergoes semileptonic decay. The background contribution was estimated by applying the selection criteria to a sample of 2.5 million MCFast semileptonic $b \bar{b}$ events, corresponding to a luminosity of $10 \mathrm{pb}^{-1}$. Of these events, 41 passed the selection cuts and fell within a $1 \mathrm{GeV} / c^{2}$ window centered on the $B^{+}$mass. Extrapolating to a year of running at a luminosity of $2 \times 10^{32} \mathrm{~cm}^{-2} \mathrm{~s}^{-1}\left(2 \mathrm{fb}^{-1}\right)$, we expect about 8200 events in this window. Assuming a uniform distribution across the $B$ mass window (this is conservative, since it is falling, as shown in Fig. 16.40), we can expect about 400 events within $2 \sigma$ of the $B^{+}$mass after applying the trigger efficiency.

The overall efficiency for this state, with cuts designed to achieve good background rejection, is about $3.0 \%$. Table 16.20 gives a calculation of the yield obtained in a one year run at a luminosity of $2.0 \times 10^{32} \mathrm{~cm}^{-2} \mathrm{~s}^{-1}$. The number of signal events passing the trigger and all selection criteria is approximately 1280 . Given the impressive signal-to-background ratio of 3.2 , we will certainly observe this decay, even if the branching fraction is lower than theory predicts. 

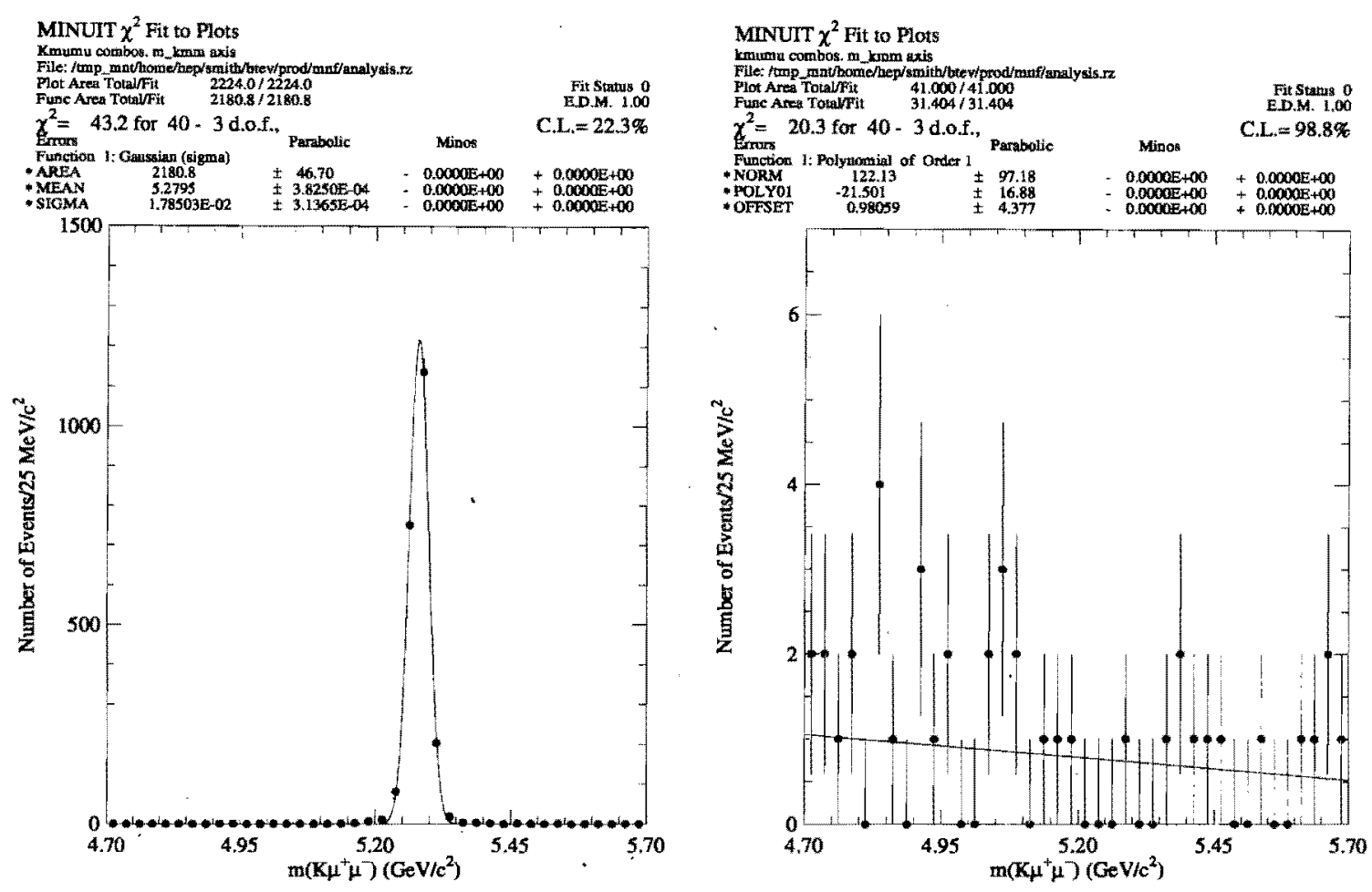

Figure 16.40: Distributions of $B^{+} \rightarrow K^{+} \mu^{+} \mu^{-}$signal (left) and $10 \mathrm{fb}^{-1}$ of $b \bar{b}$ background (right) events.

\begin{tabular}{|c|c|}
\hline \hline Luminosity & $2 \times 10^{32} \mathrm{~cm}^{-2} \mathrm{~s}^{-1}$ \\
\hline Running Time/Year & $10^{7} \mathrm{~s}$ \\
\hline$b \bar{b}$ Cross Section & $100 \mu \mathrm{b}$ \\
\hline Number of $b \bar{b}$ Pairs Produced & $2 \times 10^{11}$ \\
\hline Number of $B^{+}$Produced & $1.5 \times 10^{11}$ \\
\hline Est. $\mathcal{B}\left(B^{+} \rightarrow K^{+} \mu^{+} \mu^{-}\right)$ & $(4.0 \pm 1.5) \times 10^{-7}$ \\
\hline Number of Signal Events Produced & $6.0 \times 10^{4}$ \\
\hline Level 1 Trigger Efficiency (vertex + dimuon) & $80 \%$ \\
\hline Level 2 Trigger Efficiency & $90 \%$ \\
\hline Reconstruction Efficiency & $3.0 \%$ \\
\hline Number of Background Events in Signal Box & 400 \\
\hline Number of Signal Events & 1280 \\
\hline Signal/Background & 3.2 \\
\hline \hline
\end{tabular}

Table 16.20: Estimate of sensitivity to $B^{+} \rightarrow K^{+} \mu^{+} \mu^{-}$based on one year of running. 
The CDF II TDR [27] predicts a signal of $100-300 B^{+} \rightarrow K^{+} \mu^{+} \mu^{-}$events in Run II. CDF's lack of particle ID for tracks above $1 \mathrm{GeV} / c$ leaves it exposed to background from all pions in an event conspiring with muons to create background. It is unlikely that CDF's signal-to-background in Run II (0.1 in Run I) will approach that expected at BTeV. In BTeV, only kaons contribute to the background, due to the $\mathrm{RICH}$, and there are fewer of them. $\mathrm{BTeV}$ will have intrinsically less background than detectors without powerful particle ID.

\subsubsection{Inclusive $\left(B_{d}^{\dot{*}}, B_{u}\right) \rightarrow X_{s} \mu^{+} \mu^{-}$}

We have studied the ability of the $\mathrm{BTeV}$ detector to measure inclusive $\left(B_{d}, B_{u}\right) \rightarrow X_{s} \mu^{+} \mu^{-}$. The method used is similar to that used by CLEO [28][29], in which a kaon and 0-4 pions are combined with the dilepton pair. For this study, no neutral pion or kaon candidates were included. Thus, the efficiency for modes containing a $\pi^{0}$ or $K^{0}$ was assumed to be zero.

Monte Carlo samples of signal and $0.24 \mathrm{pb}^{-1}$ of $b \bar{b}$ semileptonic background events were generated with MCFast. The signal sample only includes decays to charged kaons and pions. In calculating the efficiency, we naively assume all final states contribute equally. This results in an efficiency of approximately $0.3 \%$ for a truly inclusive sample. We expect approximately 4000 reconstructed events in all-charged modes after one year of running.

The selection criteria are nearly identical to those used in the exclusive modes, generalized to $0-4$ pions. We find that 68 of the $b \bar{b}$ semileptonic background events pass all selection criteria and fall within a $1 \mathrm{GeV} / \mathrm{c}^{2}$ window about the $B$ mass, as shown in Fig. 16.41. This extrapolates to 20,500 events in a $2 \sigma$ signal region under the $B$ mass after applying the trigger efficiency. The combinatoric background was assessed by analyzing anti-tagged combinations in an MCFast signal Monte Carlo sample corresponding to about $100 \mathrm{pb}^{-1}$, shown in Fig. 16.41. We find 399 events in a $1 \mathrm{GeV} / c^{2}$ window about the $B$ mass, for a sample of 205 signal events. From this, we estimate a combinatoric contribution in the signal region of 400 events in one year of running. Furthermore, this contribution is insensitive to the number of multiple interactions. We find signal/background $\sim 0.13$, as summarized in Table 16.21.

In this analysis, we have demonstrated that the precise vertex reconstruction in $\mathrm{BTeV}$ is very effective at removing combinations involving non- $b$ daughters. As a result, we will have adequate sensitivity to measure the inclusive $\left(B_{d}, B_{u}\right) \rightarrow X_{s} \mu^{+} \mu^{-}$branching fraction. Of course, significant improvements can be made to this simple analysis, which has not been optimized. More inclusive measurements which include combinations with a $K_{s}^{0}$ or $\pi^{0}$ may also be feasible, although the backgrounds associated with the $\pi^{0}$ are expected to be large.

Other planned searches for inclusive $\left(B_{d}, B_{u}\right) \rightarrow X_{s} \mu^{+} \mu^{-}$at D0 and CDF only use the dilepton mass and are likely limited to the endpoint phase space region above the $\psi^{\prime}$. Below this, backgrounds from semileptonic $b$ decays overwhelm the signal. Since we include the entire phase space except where the $J / \psi$ and $\psi^{\prime}$ dominate the cross section, we avoid the model dependence introduced by measuring only the endpoint region [30]. Therefore, this measurement will provide a long-awaited model-independent determination of the Wilson coefficients $C_{7}^{\text {eff }}$ and $C_{10}$. 

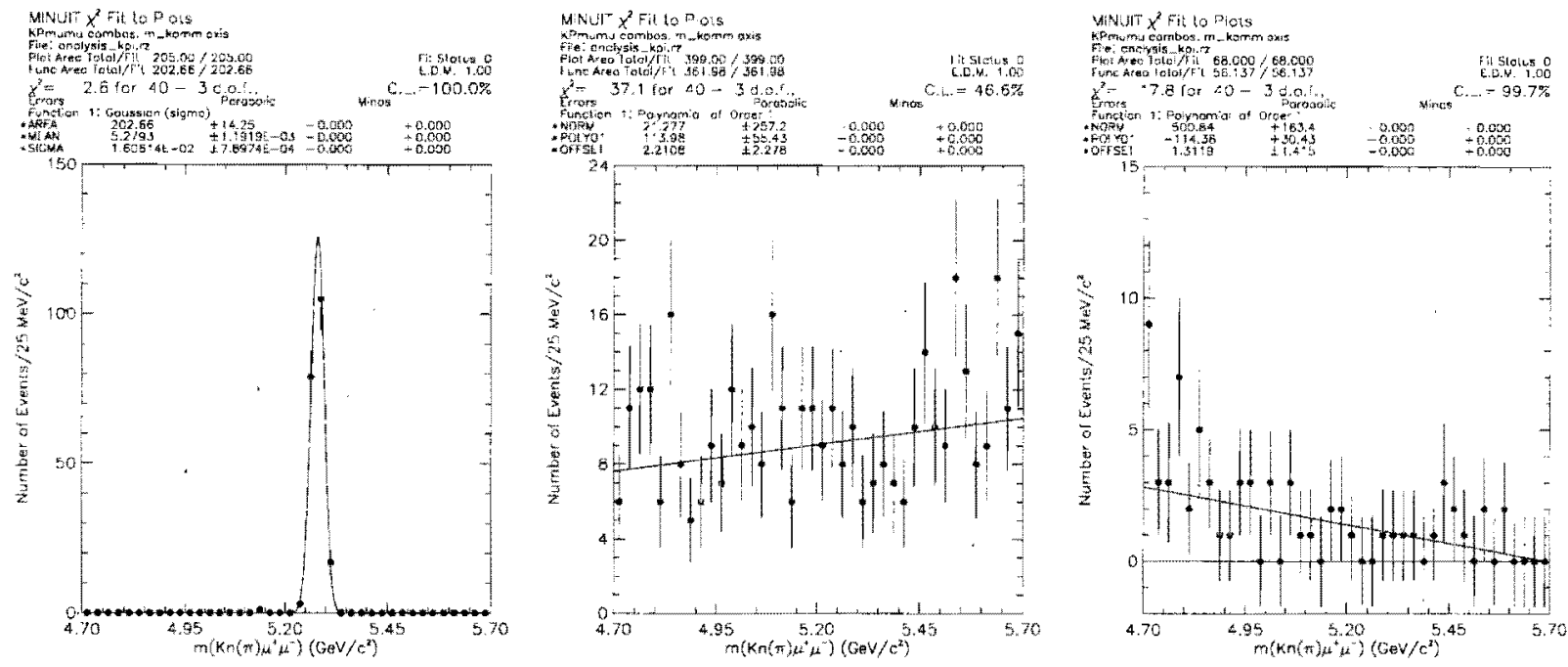

Figure 16.41: $B$ mass distributions of $B^{+/ 0} \rightarrow K^{+} n\left(\pi^{+/-}\right) \mu^{+} \mu^{-}(n=0-4)$ from $100 \mathrm{pb}^{-1}$ of tagged signal (left) and anti-tagged combinatoric background (center) events. $B$ mass distribution of $0.24 \mathrm{pb}^{-1}$ of $b \bar{b}$ background Monte Carlo events (right), where both $b$ 's decay semileptonically.

\begin{tabular}{|c|c|}
\hline \hline Luminosity & $2 \times 10^{32} \mathrm{~cm}^{-2} \mathrm{~s}^{-1}$ \\
\hline Running Time/Year & $10^{7} \mathrm{~s}$ \\
\hline$b \bar{b}$ Cross Section & $100 \mu \mathrm{b}$ \\
\hline Number of $b \bar{b}$ Pairs Produced & $2 \times 10^{11}$ \\
\hline Number of $B_{d}, B_{u}$ Produced & $3.0 \times 10^{11}$ \\
\hline Est. $\left(B_{d}, B_{u}\right) \rightarrow X_{s} \mu^{+} \mu^{-}$ & $(5.7 \pm 1.2) \times 10^{-6}$ \\
\hline Number of Signal Events Produced & $1.7 \times 10^{6}$ \\
\hline Level 1 Trigger Efficiency (vertex + dimuon) & $80 \%$ \\
\hline Level 2 Trigger Efficiency & $90 \%$ \\
\hline Selection Cut Efficiency & $0.3 \%$ \\
\hline Number of Background Events in Signal Box & 27,800 \\
\hline Number of Signal Events & 3600 \\
\hline Signal/Background & 0.13 \\
\hline Significance of Signal & $20 \sigma$ \\
\hline
\end{tabular}

Table 16.21: Estimate of sensitivity to $\left(B_{d}, B_{u}\right) \rightarrow X_{s} \mu^{+} \mu^{-}$based on one year of running. 


\subsection{Consequences of Degraded Detector Performance}

\subsubsection{Introduction}

A specific PAC request from the Aspen meeting of June 1999 is:

Simulations should also be used to analyze how much the physics reach of the experiment is degraded if various components of the detector do not meet design specifications.

Every effort will be made by $\mathrm{BTeV}$ collaborators to meet or exceed design specifications. Indeed, we believe the design of $\mathrm{BTeV}$ to be conservative with room for improvement in many areas within the budget envelope. Here, we summarize the studies that specifically address the PAC question.

\subsubsection{Trigger Performance}

Degradation in the trigger could, in principle, arise from either a loss in efficiency or a worsening of pixel resolution. In fact, the full pixel resolution is not used at the trigger stage since the algorithm does not take the time to do all the corrections, so modest changes in the resolution should not be important. We worsened the pixel resolution by assuming that we had no help from the analog information, and thus used pure digital resolution. The rejection for minimum bias events, worsened by less than $10 \%$, while efficiencies on $b$ decays were unaffected.

On the other hand, significant losses in efficiency would worsen the trigger efficiency and could only be fixed by adding more layers in the algorithm. That would increase the time required by the trigger and would be costly in terms of requiring more processors:

We have evaluated the trigger efficiency as a function of the pixel efficiency for the final state $B_{s} \rightarrow D_{s}^{+} K^{-}$and minimum bias events. To make things really interesting, we added a substantial (and unreasonable) number of noise hits randomly to each pixel plane. While we believe the final pixel efficiency will be well in excess of $99 \%$, we allowed the efficiency to be as low as $98 \%$. Fig. 16.42 shows the effect of these changes on signal efficiency and background rejection. While some loss is observable on signal events, it does not seriously degrade the physics performance.

\subsubsection{Decay Time Resolution}

The decay time resolution, $\sigma_{t}$, is a critical element of BTeV and will be negatively affected by having a degraded position resolution in the pixel detector. $\sigma_{t}$ is improved both by having less material and by having better position resolution. Let us consider, for example, the time resolution in $B_{s} \rightarrow J / \psi \bar{K}^{*}, \bar{K}^{*} \rightarrow K^{-} \pi^{+}$. The resolution we obtain for the current baseline detector is $\sigma_{t}=36 \mathrm{fs}$.

If the position resolution were degraded by a factor of two, $\sigma_{t}$ would increase by about $25 \%$. We do not expect any degradation to approach this level. If additional material were added to the pixel detector, there would be deterioration due to multiple scattering. If the spatial resolution of the detector were zero, this deterioration would increase as $\sqrt{\text { thickness. }}$ 


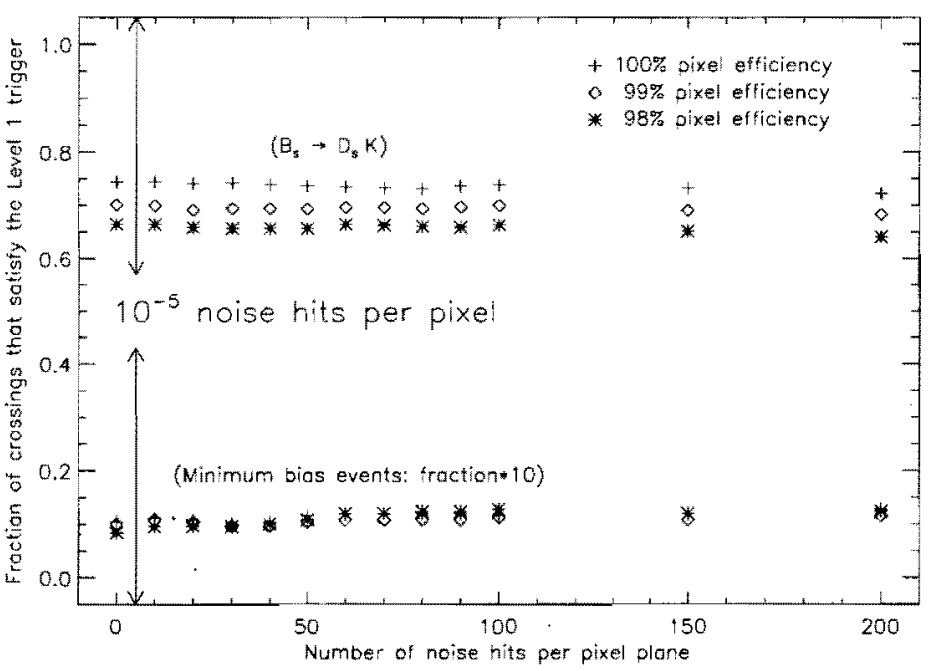

Figure 16.42: The Level 1 trigger efficiency for $B_{s} \rightarrow D_{s}^{+} K^{-}$and minimum bias events (multiplied by 10 for better viewing) for three values of assumed pixel efficiencies as a function of the number of added noise hits per plane. We expect, nominally, 5 noise hits per plane, corresponding to a density of $10^{-5}$ noise hits.

Since we have a finite spatial resolution, the dependence is somewhat weaker; in any case, we do not expect to have to increase the detector thickness. These results were abstracted from a detailed evaluation of the change in $\sigma_{t}$ as a function of spatial resolution and material thickness that was done for the original baseline pixel detector, with 3 planes per station. We expect the results to be similar for the 2-plane per station design.

We mention in passing that it is possible to substantially improve the decay time resolution by moving the pixel detector closer to the beam line. Our design calls for the pixel detector to be $6 \mathrm{~mm}$ away. This is limited by radiation damage. New advances in pixel technology suggest that we can move the pixel detector closer. The practical limit determined by the machine is $3 \mathrm{~mm}$. This would improve the decay time resolution by about $30 \%$.

\subsubsection{Ring Imaging Particle Identification}

The primary way of degrading performance here is to not get as many photons as we expect. This could be caused by bad mirrors, poorer than specified quantum efficiency or contamination of the gas. The latter is hard to imagine because the detector operates in the visible light regime. In any case, we have evaluated the $\mathrm{RICH}$ performance on the decay mode $B^{o} \rightarrow \pi^{+} \pi^{-}$using a detector with $50 \%$ fewer photons than design specification. (We do not view this as a realistic loss.)

Fig. 16.43 shows the efficiency for detecting $B^{\circ} \rightarrow \pi^{+} \pi^{-}$versus the efficiency for misidentifying the competing decay $B^{\circ} \rightarrow K^{+} \pi^{-}$, for our design and a detector degraded by a $50 \%$ loss of photons. 


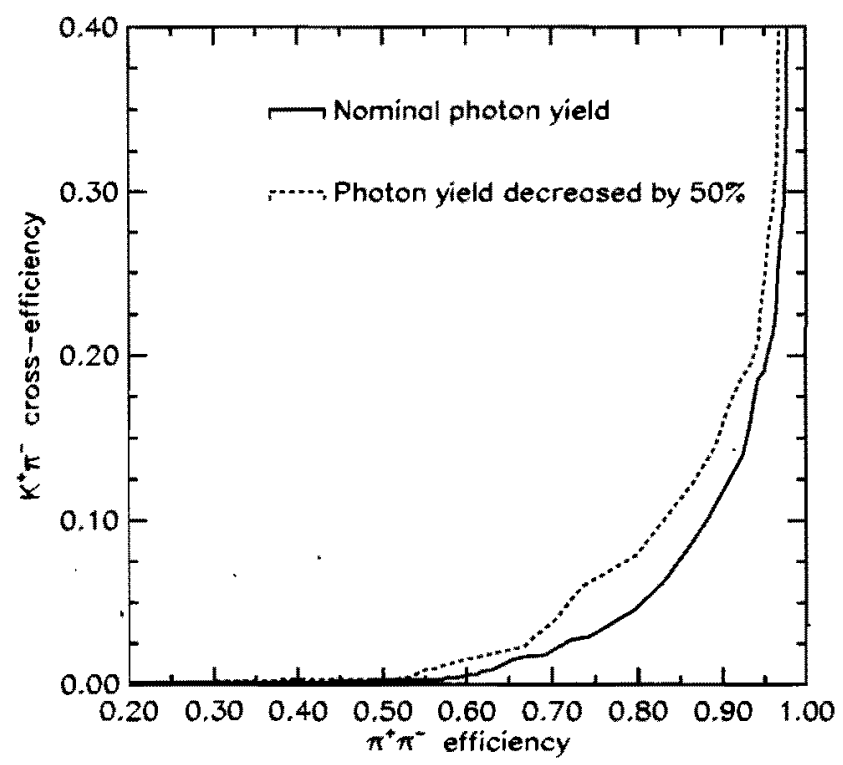

Figure 16.43: The efficiency for identifying both pions in the reaction $B^{o} \rightarrow \pi^{+} \pi^{-}$versus the efficiency for misidentifying $B^{\circ} \rightarrow K^{+} \pi^{-}$as $\pi^{+} \pi^{-}$. The solid-line is for the baseline design, while the dashed line is for a $50 \%$ loss of photons.

While there is a significant loss of efficiency for small fake rates, the interpolation to a more reasonable loss of $\sim 10 \%$ would not be a disaster.

\subsubsection{Electromagnetic Calorimeter}

The performance of the EM calorimeter would be degraded if we failed to obtain the expected energy resolution

$$
\frac{\sigma_{E}}{E}=\sqrt{\frac{a^{2}}{E}+b^{2}}=\frac{a}{\sqrt{E}} \oplus b,=\frac{1.6 \%}{\sqrt{E}} \oplus 0.55 \% \text {. }
$$

Our physics reach in final states with single photons will have increased backgrounds since the signal-to-background ratio depends directly on the energy resolution. Doubling the energy width would cut the single photon signal-to-background ratio in half.

The effects on $\pi^{\circ}$ mass resolution are more subtle because the position resolution is important here and that is also degraded by a worsening of the energy resolution. We evaluated the changes that would occur if the constant term, $b$, in the energy resolution changed from the nominal value of $0.55 \%$ for $10 \mathrm{GeV} \pi^{o}$ 's, which is a typical value in many of our important processes. The mass resolution is $2.9 \mathrm{MeV}, 3.0 \mathrm{MeV}, 4.6 \mathrm{MeV}$ and 7.5 $\mathrm{MeV}$, for $b$ values of $0.55 \%, 1.0 \%, 5.0 \%$ and $10.0 \%$, respectively. For a reasonable range of constant terms, the mass resolution is unaffected.

We also evaluated the effect of changing the photon yield for $10 \mathrm{GeV} \pi^{o}$ 's. Changing the photon yield from a nominal 7 photoelectrons/MeV to 4 photoelectrons/MeV, changes the $\pi^{\circ}$ mass resolution from $2.9 \mathrm{MeV}$ to $3.2 \mathrm{MeV}$. 


\section{Bibliography}

[1] A. Gritsan "Charmless Hadronic B Meson Decays with CLEO," presented at Lake Louise Winter Institute 2000, to appear in proceedings.

[2] O.R. Long et. al., "Monte Carlo Simulation of $B^{o} \rightarrow \pi^{+} \pi^{-}$from $p p$ Interactions at $\sqrt{s}=40 \mathrm{TeV} ", \mathrm{UPR} / 216 \mathrm{E}, \mathrm{PRINCETONE} / \mathrm{HEP} / 92-07, \mathrm{SSCL} / \mathrm{PP} / 139(1992)$.

[3] F. Abe et. al., The CDF Collaboation, Phy. Rev. D54, 6596 (1996). FERMILAB-PUB-96/199-E.

[4] " $J / \psi$ and $\psi(2 S)$ Production in $p \bar{p}$ Collisions at $\sqrt{s}=1.8 \mathrm{TeV}$ ", F. Abe et al., The CDF Collaboration, Phys. Rev. Lett. 79, 572 (1997):

[5] Particle Data Group, C. Caso et al., The European Physical Journal C3, 1 (1998).

[6] I. Dunietz, Phys.Lett. B427, 179 (1998).

[7] B. Barish et al., CLEO CONF 97-01, EPS 97-339. This is available on the web at, http://www.lns.cornell.edu/public/CONF/1997/Bhad-conf.ps

[8] Proceedings of the "Workshop on B Physics at the Tevatron, Run II and Beyond", in preparation. See also, http://www-theory.fnal.gov/people/ligeti/Brun2/ .

[9] "Determining the CP-violating phase $\gamma$ ",

R. Aleksan, I. Dunietz, B. Kayser, Z. Phys. C54, 653 (1992).

[10] M. Gronau and D. London, Phys. Lett. B253, 483 (1991) and M. Gronau and D. Wyler, Phys. Lett. B265, 172 (1991).

[11] D. Atwood, I. Dunietz and A. Soni; Phys. Rev. Lett. 78, 3257 (1997).

[12] M. Gronau and J. Rosner, CALT-68-2142, hep-ph/9711246 (November 1997).

[13] R. Fleischer and T. Mannel, hep-ph/9704423.

[14] R. Godang et al., Phys. Rev. Lett. 803456 (1998).

[15] D. Cronin-Hennessy et al., hep-ex/0001010. 
[16] A. Falk, A. Kagan, Y. Nir and A. Petrov, JHU-TIPAC-97018 (December 1997).

[17] A. E. Snyder and H. R. Quinn, Phys. Rev. D48, 2139 (1993).

[18] M. Bishai et al., (CLEO) "Charmless Hadronic B Decays to Exclusive Final States with a $K^{*}, \rho, \omega$ or $\phi$ Meson," CONF 99-13 (1999).

[19] H. R. Quinn and J. P. Silva, "The Use of Early Data on $B \rightarrow \rho \pi$ Decays," hep$\mathrm{ph} / 0001290(2000)$.

[20] Private communication from Jon Rosner.

[21] P. McBride and S. Stone; Nucl. Instrum. Meth. A368, 38 (1995).

[22] K.T. McDonald, "Maximum Likelihood Analysis of CP Violating Asymmetries", PRINCETON-HEP-92-04, Sep 1992. 12pp, unpublished.

[23] A. Ali, "Rare B Decays in the Standard Model", Beauty '96, North Holland Press, 1996, the Netherlands, Table 1, page 13 and references therein.

[24] C. Greub, A. Ioannissian, and D. Wyler, Phys. Lett. B346, 149 (1995).

[25] G. Burdman, Phys. Rev. D57, 4254 (1998).

[26] A. Ali, P. Ball, L. Handoko, and G. Hiller, Phys. Rev. D61 (2000).

[27] "The CDF II Detector Technical Design Report", CDF collaboration, FERMILAB-Pub-96/390-E (1996).

[28] CLEO Collaboration, M.S. Alam et al, Phys. Rev. Lett. 74, 2885 (1995).

[29] CLEO Collaboration, S. Glenn et al, Phys. Rev. Lett. 80, 2289 (1998).

[30] C. Bauer, C. Burrell, Phys. Lett. B469, 248 (1999). 


\section{Part IV}

\section{Summary of Physics reach}




\section{Chapter 17}

\section{Summary of Physics Reach and Comparisons With Other Experiments}

\subsection{Sensitivities to CP Violating Angles}

$\mathrm{BTeV}$ will have outstanding performance in determining $\mathrm{CP}$ violating asymmetries. The results of our simulations are summarized in Table 17.1 for a luminosity of $2 \times 10^{32} \mathrm{~cm}^{-2} \mathrm{~s}^{-1}$ and $10^{7}$ seconds.

Table 17.1: Yearly sensitivities for CP violating quantities.

\begin{tabular}{lll}
\hline \hline Quantity & Decay Mode(s) & Sensitivity \\
\hline $\sin (2 \beta)$ & $B^{o} \rightarrow J / \psi K_{S}$ & \pm 0.025 \\
$\alpha$ & $B^{o} \rightarrow \rho \pi$ & $\sim \pm 10^{\circ}$ \\
$\gamma$ & $B_{s} \rightarrow D_{s}^{ \pm} K^{\mp}$ & $\sim \pm 7^{\circ}$ \\
$\gamma$ & $B^{-} \rightarrow \bar{D}^{\circ} K^{-}$ & $< \pm 10^{\circ}$ \\
$\gamma$ & $B \rightarrow K \pi$ & $\pm<5^{\circ}$ (plus theoretical errors) \\
$\sin (2 \chi)$ & $\left.B_{s} \rightarrow J / \psi \eta^{(}\right)$ & \pm 0.033 \\
Asymmetry & $B^{o} \rightarrow \pi^{+} \pi^{-}$ & \pm 0.024 \\
\hline \hline
\end{tabular}

We briefly discuss each of these measurements:

- The error in $\sin (2 \beta)$ includes the $20 \%$ improvement (over a time-integrated measurement) we obtain by fitting the time distribution.

- We expect to have $\sim 1000$ effective flavor tagged $\rho^{ \pm} \pi^{\mp}$ events and $\sim 150 \rho^{\circ} \pi^{o}$ per year. The signal/background levels are 4.1 and 0.3 , respectively. We have not done a full simulation of our sensitivity to $\alpha$. Final results will depend on several unknown quantities including the branching ratio for $\rho^{\circ} \pi^{\circ}$, and the ratio of tree to penguin amplitudes. 
Analysis by Snyder and Quinn [1] showed that with 2000 background free events they always found a solution for $\alpha$ and the accuracy was in the range of $5-6^{\circ}$. We can collect these events in $2 \times 10^{7}$ seconds, but we will have some background. Furthermore Quinn and Silva have proposed using non-flavor-tagged rates as input that should improve the accuracy of the $\alpha$ determination [2].

- Although the $B \rightarrow K \pi$ modes provide the smallest experimental error in determining $\gamma$, there is model dependent error associated with this method. On the other hand, the other two methods, which use $B_{s} \rightarrow D_{s}^{ \pm} K^{\mp}$ and $B^{-} \rightarrow \bar{D}^{\circ} K^{-}$, provide model independent results and can be averaged. The interplay of the three methods can be used to resolve ambiguities.

- The error in $\sin (2 \chi)$ averaged over both $J / \psi \eta$ and $J / \psi \eta^{\prime}$ decay modes of the $B_{s}$ is $\pm 3.3 \%$, using only $J / \psi \rightarrow \mu^{+} \mu^{-}$decays. Since we expect an asymmetry of $\sim 3 \%$, it will take us a few years to make this important measurement. Including $J / \psi \rightarrow e^{+} e^{-}$ and $B_{s} \rightarrow J / \psi \phi$ would reduce the time.

- The asymmetry in $B^{o} \rightarrow \pi^{+} \pi^{-}$may be useful to gain insight into the value of $\alpha$ with theoretical input or combined with $B_{s} \rightarrow K^{+} K^{-}$and theory to obtain $\gamma$. This study was done both with MCFast and GEANT. The signal efficiency is $10 \%$ higher in MCFast and the background levels the same in both, within statistics.

\section{7:2 Sensitivity to $B_{s}$ Mixing}

BTeV can definitively reach $x_{s}$ values of 75 in $10^{7}$ seconds of running. Put another way, it will take us only 5 days of steady running to reach $x_{s}$ of 20 . These estimates are based on the decay mode $B_{s} \rightarrow D_{s}^{+} \pi^{-}$, with $D_{s}^{+} \rightarrow \phi \pi^{+}$and $K^{* o} K^{+}$. Definitive is defined here as the ability to make a measurement where the best solution for a fit to the oscillation frequency is better by " 5 standard deviations" than the next best fit. Thus BTeV can cover the entire range of $x_{s}$ values allowed in the Standard Model.

\subsection{Reach in Rare Decays}

$\mathrm{BTeV}$ has excellent reach in rare decays. We have investigated the exclusive decays $B^{o} \rightarrow K^{* o} \mu^{+} \mu^{-}, B^{+} \rightarrow K^{+} \mu^{+} \mu^{-}$and the inclusive decay $B \rightarrow X_{s} \mu^{+} \mu^{-}$.

We acquire $\sim 2200 K^{* o} \mu^{+} \mu^{-}$decays in $10^{7}$ seconds, enough to measure the lepton-forwardbackward asymmetry and test the Standard Model. Although the asymmetry is expected to be small in $K^{+} \mu^{+} \mu^{-}$, we test the Standard Model expectation, due to our large sample of $\sim 1300$ events per year.

We also expect to be able to measure the inclusive rate $b \rightarrow s \mu^{+} \mu^{+}$with $20 \sigma$ significance. This inclusive rate is very important. It could either show non-Standard Model physics or greatly constrain alternative models. 


\subsection{Comparison with CDF, D0, CMS, and ATLAS}

Both CDF and D0 have measured the $b$ production cross section [3]. CDF has contributed to our knowledge of $b$ decay mostly by its measurements of the lifetime of $b$-flavored hadrons [4], which are competitive with those of LEP [5] and recently through its discovery of the $B_{c}$ meson [6]. CDF has also seen the first hint for CP violation in the $b$ system [7]. These detectors were designed for physics discoveries at large transverse momentum. It is remarkable that they have been able to accomplish so much in $b$ physics. They have shown that it is possible to do $b$ physics in the environment of a hadron collider.

However, these detectors, and the new central detectors ATLAS and CMS are very far from optimal for $b$ physics. BTeV has been designed with $b$ physics as its primary goal. To have an efficient trigger based on separation of $b$ decays from the primary, BTeV uses the large $|\eta|$ region where the $b$ 's are boosted. The detached vertex trigger allows collection of interesting purely hadronic final states such as $\pi^{+} \pi^{-}, \rho \pi, D_{s}^{+} \pi^{-}$and $D_{s}^{+} K^{-}$. It also allows us to collect enough charm to investigate charm mixing and $\mathrm{CP}$ violation.

The use of the forward geometry also allows for excellent charged hadron identification over a wide momentum range, with a gaseous RICH detector. This is crucial for many physics issues such as separating $K \pi$ from $\pi \pi, D_{s} \pi$ from $D_{s} K$, kaon flavor tagging, etc.

Furthermore an experiment that plans on answering all the open questions in $b$ physics, requires a high quality electromagnetic calorimeter. Installation of such a calorimeter in the CLEO detector made new physics vistas possible and such a device in BTeV allows for the measurement of several crucial final states such as $B^{\circ} \rightarrow \rho \pi$, and $B_{s} \rightarrow J / \psi \eta^{\prime}$. The only central detector that is planning to have a high quality electromagnetic calorimeter is CMS.

Finally, BTeV has all the crucial elements required to study any newly suggested $b$ or charm process or uncover new physics. The crucial elements are:

- a detached vertex trigger in the first trigger level,

- highly efficient particle identification across the entire momentum range with good $(\approx 100: 1)$ background rejection,

- an electromagnetic calorimeter with sufficiently good energy resolution and efficiency to fully reconstruct rare $B$ decay final states with single photons or neutral pions.

BTeV will have a physics reach substantially beyond that of CDF, D0, CMS, and ATLAS.

\subsection{Comparison with $e^{+} e^{-} B$ Factories}

Most of what is known about $b$ decays has been learned at $e^{+} e^{-}$machines [8]. Machines operating at the $\Upsilon(4 S)$ found the first fully reconstructed $B$ mesons (CLEO), $B^{o}-\bar{B}^{o}$ mixing (ARGUS), the first signal for the $b \rightarrow u$ transition (CLEO), and Penguin decays (CLEO). Lifetimes of $b$ hadrons were first measured by experiments at PEP, slightly later at PETRA, and extended and improved by LEP [8]. 
The success of the $\Upsilon(4 S)$ machines has led to the construction at KEK and SLAC of two new $\Upsilon(4 S)$ machines with luminosity goals in excess of $3 \times 10^{33} \mathrm{~cm}^{-2} \mathrm{~s}^{-1}$. These machines will have asymmetric beam energies so they can measure time dependent $\mathrm{CP}$ violation. They will join an upgraded CESR machine at Cornell that has symmetric beam energies. These machines will investigate only $B^{o}$ and $B^{ \pm}$decays. They will not investigate $B_{s}, B_{c}$ or $\Lambda_{b}$ decays [9].

Table 17.2 shows a comparison between $\mathrm{BTeV}$ and an asymmetric $e^{+} e^{-}$machine for measuring the $\mathrm{CP}$ violating asymmetry in the decay mode $B^{o} \rightarrow \pi^{+} \pi^{-}$. Here we use the recently reported measurement of the branching ratio by CLEO [10]. In Table 17.3 we show a similar comparison for the final state $B^{-} \rightarrow \bar{D}^{o} K^{-}$, a mode that could be used to determine the CKM angle $\gamma$. It is clear that the large hadronic $b$ production cross section can overwhelm the much smaller $e^{+} e^{-}$rate. Furthermore, the $e^{+} e^{-} B$ factories do not have access to the important $\mathrm{CP}$ violation measurements that need to be made in $B_{s}$ decays.

Table 17.2: Number of tagged $B^{o} \rightarrow \pi^{+} \pi^{-}\left(\mathcal{B}=0.43 \times 10^{-5}\right)$.

\begin{tabular}{rrcrrrr}
\hline \hline & & & & Signal & Tagging & \\
& $\mathcal{L}\left(\mathrm{cm}^{-2} \mathrm{~s}^{-1}\right)$ & $\sigma^{\prime}$ & $\# B^{o} / 10^{7} \mathrm{~s}$ & Efficiency & $\epsilon D^{2}$ & $\#$ tagged $/ 10^{7} \mathrm{~s}$ \\
\hline$e^{+} e^{-}$ & $3 \times 10^{33}$ & $1.2 \mathrm{nb}$ & $3.6 \times 10^{7}$ & 0.3 & 0.3 & 13 \\
$\mathrm{BTeV}$ & $2 \times 10^{32}$ & $100 \mu \mathrm{b}$ & $1.5 \times 10^{11}$ & 0.037 & 0.1 & 2370 \\
\hline
\end{tabular}

Table 17.3: Number of $B^{-} \rightarrow \bar{D}^{\circ} K^{-}\left(\mathcal{B}=1.7 \times 10^{-7}\right)$.

\begin{tabular}{lrcrrr}
\hline & & & & Signal & \\
& $\mathcal{L}\left(\mathrm{cm}^{-2} \mathrm{~s}^{-1}\right)$ & $\sigma$ & $\# B^{-} / 10^{7} \mathrm{~s}$ & Efficiency & Events $/ 10^{7} \mathrm{~s}$ \\
\hline$e^{+} e^{-}$ & $3 \times 10^{33}$ & $1.2 \mathrm{nb}$ & $3.6 \times 10^{7}$ & 0.5 & 2 \\
$\mathrm{BTeV}$ & $2 \times 10^{32}$ & $100 \mu \mathrm{b}$ & $1.5 \times 10^{11}$ & 0.012 & 300 \\
\hline \hline
\end{tabular}

\subsection{Comparison with LHC-b}

\subsubsection{General Comparisons}

LHC-b [11] is an experiment planned for the LHC with almost the same physics goals as $\mathrm{BTeV}$. Here we show how BTeV can compete with $\mathrm{LHC}-\mathrm{b}$ in many areas and why it is a superior experiment in some very important areas. Both experiments intend to run at a luminosity of $2 \times 10^{32} \mathrm{~cm}^{-2} \mathrm{~s}^{-1}$. There are several inherent advantages and disadvantages that LHC-b has compared with BTeV. The issues that favor LHC-b are: 
- The $b$ production cross-section is expected to be about five times larger at the LHC than at the Tevatron, while the total cross-section is only 1.6 times as large.

- The mean number of interactions per bunch crossing is expected to be about 3 times lower at the LHC than at the Tevatron (at 132 ns bunch spacing).

The issues that favor $\mathrm{BTeV}$ are:

- $\mathrm{BTeV}$ is a two-arm spectrometer, which increases the signal by a factor of two compared with LHC-b.

- The short bunch spacing at the LHC, $25 \mathrm{~ns}$, has serious negative effects on all their detector subsystems. There are occupancy problems if the sub-detector integration times are long. This can be avoided by having short integration times, but that markedly increases the electronics noise. For example, in a silicon detector these considerations make first level detached vertex triggering more difficult than at the Tevatron; $\mathrm{BTeV}$ has a more relaxed $132 \mathrm{~ns}$ bunch spacing, 5.3 times longer. In fact, the current plan of LHC-b is to trigger in their first trigger level on muons, electrons or hadrons of moderate $p_{t}$, and detect detached vertices in the next trigger level.

- The seven times larger beam energy at the LHC makes the range of track momenta that need to be momentum analyzed and identified much larger and therefore more difficult. The larger energy also causes a large increase in track multiplicity per event, which makes pattern recognition and triggering more difficult.

- The interaction region at the Tevatron is three to six times longer along the beam direction than at LHC $\left(\sigma_{z}=5 \mathrm{~cm}\right)$, which allows BTeV to be able to accept collisions with a mean of two interactions per crossing, since the interactions are well separated in $z$. LHC-b plans to veto crossings with more than one interaction.

- $\mathrm{BTeV}$ is designed to have the vertex detector in the magnetic field, thus allowing the rejection of low momentum tracks at the trigger level. Low momentum tracks are more susceptible to multiple scattering which can cause false detached vertices leading to poor background rejection in the trigger.

- $\mathrm{BTeV}$ is designed with a high quality $\mathrm{PbWO}_{4}$ electromagnetic calorimeter, that provides high resolution and acceptance for interesting final states with $\gamma^{\prime} \mathrm{s}, \pi^{o}$ 's, and $\eta^{\left({ }^{\prime}\right)}$ 's.

- Use of a detached vertex trigger at Level 1 allows for an extensive charm physics program absent in LHC-b. It also allows for a more uniform collection of $b$ triggers.

- The LHC-b data acquisition system is designed to output $200 \mathrm{~Hz}$ of $b$ decays, while $\mathrm{BTeV}$ is designed for a $4,000 \mathrm{~Hz}$ output bandwidth where we estimate $1,000 \mathrm{~Hz}$ of $b$ 's and $1,000 \mathrm{~Hz}$ of charm; $\mathrm{BTeV}$ potentially has access to a much wider range of heavy quark decays. 
- The BTeV electromagnetic calorimeter is superior in energy resolution and segmentation to LHC-b's. BTeV uses $\mathrm{PbWO}_{4}$ crystals while LHC-b has a Shaslik-style Pbscintillating fiber device, following a preshower detector. The LHC-b energy resolution is $10 \% / \sqrt{E} \oplus 1.5 \%$, which compares poorly with BTeV's $1.6 \% / \sqrt{E} \oplus 0.55 \%$. The LHCb detector segmentation is $4 \mathrm{~cm} \times 4 \mathrm{~cm}$ up to $\sim 90 \mathrm{mr}, 8 \mathrm{~cm} \times 8 \mathrm{~cm}$ to $\sim 160 \mathrm{mr}$ and $16 \mathrm{~cm} \times 16 \mathrm{~cm}$ at larger angles. (The distance to the interaction point is $12.4 \mathrm{~m}$.) Thus the segmentation is comparable to $\mathrm{BTeV}$ only in the inner region. (BTeV has $2.6 \mathrm{~cm} \times 2.6 \mathrm{~cm}$ crystals $7.4 \mathrm{~m}$ from the center of the interaction region.)

We have more than compensated for LHC-b's initial advantages in $b$ cross-section due their higher center-of-mass energy. In fact, the high energy actually works in many ways as a disadvantage. For example, LHC-b needs two RICH counters to cover the momentum range in their one arm. Particle identification and other considerations force LHC-b to be longer than BTeV. Its single arm is as long as both BTeV arms put together. As a result, LHC-b's transverse size is four times that of $\mathrm{BTeV}$, but it covers the same solid angle as only one of BTeV's two arms. It is expensive to instrument all of this real estate with high quality particle detectors. Thus, the total cost for LHC-b based only on instrumented area, (a naive assumption) would be twice the total cost for $\mathrm{BTeV}$, even though LHC-b would cover only half the solid angle.

We have done a detailed comparison between BTeV and LHC-b using two modes of great importance because they give direct determinations of the CP violating angles $\alpha$ and $\gamma$, and report our results here.

\subsection{2 $\quad B^{o} \rightarrow \rho \pi$}

We base our comparison on the total number of untagged events quoted by both experiments. The BTeV numbers come from Part III of this document. The LHC-b numbers are found in their Technical Design Report [11]. Both sets of numbers are calculated for $10^{7}$ seconds at a luminosity of $2 \times 10^{32} \mathrm{~cm}^{-2} \mathrm{~s}^{-1}$. We have corrected the LHC-b numbers by normalizing them to the branching ratios used by BTeV. In Table 17.4 we compare the relevant quantities [12].

Table 17.4: Event yields and signal/background for $B^{\circ} \rightarrow \rho \pi$.

\begin{tabular}{|c|c|c|c|c|c|}
\hline \multirow[t]{2}{*}{ Mode } & \multirow{2}{*}{ Branching Ratio } & \multicolumn{2}{|c|}{$\mathrm{BTeV}$} & \multicolumn{2}{|c|}{$\overline{\mathrm{LHC}-\mathrm{b}}$} \\
\hline & & Yield & $\mathrm{S} / \mathrm{B}$ & Yield & $S / B$ \\
\hline $\bar{B}^{o} \rightarrow \rho^{ \pm} \pi^{\overline{7}}$ & $2.8 \times 10^{-5}$ & 9400 & 4.1 & 2140 & 0.8 \\
\hline$B^{o} \rightarrow \rho^{o} \pi^{0}$ & $0.5 \times 10^{-5}$ & 1350 & 0.3 & 880 & - \\
\hline
\end{tabular}

LHC-b has done a background estimate based on a heavily preselected sample of events [13]. These include: 
- a preselection for charged pions and photons which required the momentum or energy to exceed a value depending on the polar angle of the candidate. For charged pions, the momentum cut varied between 1 and $2 \mathrm{GeV} / \mathrm{c}$ and for photons the energy cut varied between 2 and $6 \mathrm{GeV}$;

- selection of signal-like events based on a discriminant variable built from kinematic variables of the $\pi, \rho$ and $B^{o}$;

- selection based on the reconstructed secondary vertex for a $\pi^{+} \pi^{-}$combination;

- Dalitz plot cuts to eliminate low energy $\pi^{o}$ combinatorial background due to particles from the primary vertex.

These cuts are applied to the generator event sample before the events are processed through GEANT [14]. The BTeV simulation was carried out without any preselection cuts. We were worried that the preselection would bias us to lower background rates. For example, if two photons overlapped or interactions of charged tracks put energy into photon clusters these can well become part of our background sample. Thus the LHC-b background estimate may well be only a lower limit.

We note that their $\pi^{\circ}$ mass resolution varies between 5 and $10 \mathrm{MeV} / \mathrm{c}^{2}$ (r.m.s.) and their $B^{o}$ mass resolution is $50 \mathrm{MeV} / \mathrm{c}^{2}$ (r.m.s.). The corresponding numbers for $\mathrm{BTeV}$ are 3.1 $\mathrm{MeV} / \mathrm{c}^{2}$ and $28 \mathrm{MeV} / \mathrm{c}^{2}$.

With this analysis, LHC-b claims signal/background (S/B) of 1.3 for $\rho^{ \pm} \pi^{\mp}$, where they have assumed a branching ratio of $4.4 \times 10^{-5}$. For our assumed branching ratio, $\mathrm{S} / \mathrm{B}$ is 0.8 ; The $\mathrm{S} / \mathrm{B}$ for $\mathrm{BTeV}$ is 4.1 . Furthermore, the $\mathrm{BTeV}$ background analysis was done without preselection and therefore is likely to be more realistic. For the final state $\rho^{\circ} \pi^{\circ} \mathrm{LHC}-\mathrm{b}$ has not produced a background estimate; in our experience it is difficult to estimate signal efficiencies without evaluating how restrictive the selection criteria need to be to reduce backgrounds.

It is not surprising that BTeV's superior crystal calorimeter and detached vertex trigger produce a large advantage in this final state over LHC-b. BTeV has a factor of 7 advantage in signal yield in $\rho^{ \pm} \pi^{\mp}$ and a better $\mathrm{S} / \mathrm{B}$ by a factor of 5 .

\subsection{3 $B_{s} \rightarrow D_{s}^{ \pm} K^{\mp}$}

A comparison of the estimated total efficiencies (excluding $D_{s}$ decay branching ratios), $B_{s}$ mass resolutions, and S:B ratios are given in Table 17.5. Here $D_{s}^{+} \rightarrow K^{+} K^{-} \pi^{+}$can be reconstructed via either $\phi \pi^{+}$or $K^{* o} K^{-}$. Here BTeV and LHC-b differ somewhat. LHC-b has the same efficiency in both modes, whereas $\mathrm{BTeV}$ analyzes them somewhat differently. For $K^{* o} K^{-} \mathrm{BTeV}$ requires both charged kaons to hit the RICH detector, while for $\phi \pi^{+}$only one charged kaon is required to be identified in the RICH. (The reconstruction efficiency for $\phi \pi^{+}$is $4.5 \%$, while for $K^{* o} K^{-}$it is $2.5 \%$ ).

We are a factor of 1.7 better in this mode. This is not unexpected. The LHC-b trigger efficiency is 4.1 times lower than $\mathrm{BTeV}$ and their acceptance a factor of two lower, since $\mathrm{BTeV}$ has two arms and the apertures of the two experiments are nearly equal. This factor 
Table 17.5: Comparison of BTeV and LHC-b sensitivities for $B_{s} \rightarrow D_{s}^{ \pm} K^{\mp}$.

\begin{tabular}{lcccc}
\hline \hline Branching Ratio & \multicolumn{2}{c}{$\overline{\mathrm{BTeV}}$} & \multicolumn{2}{c}{ L $\overline{\mathrm{LH}}-\mathrm{b}$} \\
& Yield & S/B & Yield & S/B \\
\hline $3 \times 10^{-4}$ & 13,100 & 7 & 7,660 & 7 \\
\hline
\end{tabular}

of 8 should more-than neutralize the LHC-b cross-section advantage, of a factor of 5 , and in this study it has. We note however, that details of the analysis come into play. If we require both kaons from the $\phi$ to hit the RICH, we reduce our number of events to 9550 , still 1.25 times larger than LHC-b.

\subsection{Summary}

$\mathrm{BTeV}$ is far superior to $e^{+} e^{-}$colliders operating on the $\Upsilon(4 S)$ because of the enormous difference in the $b$ rate. For reconstructed $B^{+}$and $B^{o}$ decays, BTeV has a factor of $\sim 200$ more rate. Furthermore, the important $B_{s}$ physics cannot be done at the $e^{+} e^{-}$machines [9].

CDF, D0, CMS, and ATLAS cannot compete in areas where particle identification or photon detection are important; as a result, the $b$-physics reach of $\mathrm{BTeV}$ is substantially greater.

$\mathrm{BTeV}$ is competitive with LHC-b in 'high-priority' final states with all charged particles. For final states with $\gamma$ 's, $\pi^{o}$ 's, $\eta$ 's or $\eta^{\prime}$ 's, BTeV has a factor of $\approx 7$ advantage. Furthermore, $\mathrm{BTeV}$ will write to tape a factor of 5 more $b$ events than LHC-b, allowing for more physics studies.

$\mathrm{BTeV}$ has all the components necessary to measure the most important quantities in heavy quark decays. These include spectacular vertex detection, triggering, particle identification, photon detection, and electron and muon identification. The studies presented here were done on what is currently believed to be the most important modes. What's in fashion, however, changes. $\mathrm{BTeV}$ is a powerful enough detector to be able to test new and interesting ideas. 


\section{Bibliography}

[1] A. E. Snyder and H. R. Quinn, Phys. Rev. D 48 (1993) 2139.

[2] H. R. Quinn and J. P. Silva, "The Use of Early Data on $B \rightarrow \rho \pi$ Decays," hep$\mathrm{ph} / 0001290(2000)$.

[3] K. Abe et al., (CDF), Phys. Rev. Lett. 75, 1451 (1995); S. Abachi et al., (D0), Phys. Rev. Lett. 74, 3548 (1995). See also the UA1 measurement C. Albajar et al., Phys. Lett. B186, 237 (1987); B213, 405 (1988); B256, 121 (1991).

[4] K. Abe et al., (CDF), Phys. Rev. Lett. 76, 4462 (1996); ibid 77, 1945 (1996); K. Abe et al., (CDF), Phys. Rev. D 57, 5382 (1998).

[5] T. Junk, "A Review of $B$ Hadron Lifetime Measurements from LEP, the Tevatron and SLC," in Proceedings of the 2nd Int. Conf. on B Physics and CP Violation, Univ. of Hawaii, (1997), ed. T. E. Browder et al., World Scientific, Singapore (1998).

[6] K. Abe et al., (CDF), "Observation of $B_{c}$ Mesons in $p-\bar{p}$ Collisions at $\sqrt{s}=1.8 \mathrm{TeV}$," hep-ex/9804014 (1998).

[7] M. Paulini, "B Lifetimes, Mixing and CP Violation at CDF," Review article to appear in the Int. Journal of Modern Physics A, hep-ex/9903002 (1999).

[8] See B Decays, revised 2nd Edition ed. S. Stone, World Scientific, Singapore, (1994).

[9] In principle the $e^{+} e^{-}$machines could run on the $\Upsilon(5 S)$, which is likely to be a source of $B_{s}$ mesons. However, the predicted cross-section for $B_{s}$ production is only $\sim 0.1$ of that of $B$ production on the $\Upsilon(4 S)$. Furthermore the decay time resolution necessary to resolve $B_{s}$ oscillations cannot be obtained using the relatively slow $B_{s}$ mesons produced at the $\Upsilon(5 S)$.

[10] Y. Kwon et al., (CLEO), "Study of Charmless Hadronic B Decays into the Final States $K \pi, \pi \pi$ and $K K$, with the First Observation of $B \rightarrow \pi^{+} \pi^{-}$ and $B \rightarrow K^{\circ} \pi^{\circ}$." CLEO CONF 99-14 (1999). Paper can be found at http://www.Ins.cornell.edu/public/CONF/1999/cleo_conf99-14.ps .

[11] "LHCb Technical Proposal," CERN/LHCC 98-4, LHCC/P4 (1998), available at http://lhcb.cern.ch . 
[12] We have confirmed with T. Nakada, the LHC-b spokesperson, that the yields for this mode as quoted in their Technical Proposal are their current values that we should use in our comparisons. The branching ratio numbers used by LHC-b were taken from Table 15.11 on page 157. The number of events were taken from Table 15.12. Since these numbers are quoted as being "tagged," we divided by the 0.40 tagging efficiency ' given on page 145 . The two final states $\rho^{+} \pi^{-}$and $\rho^{-} \pi^{+}$are given separately by LHC-b; we added them together. The same proceedure was followed for $B_{s} \rightarrow D_{s} K$.

[13] P. Ball et al., "B Decays at the LHC," CERN-TH/2000-101, hep-ph/0003238.

[14] Although they state a $1 \%$ efficiency here, this is only a partial efficiency according to T. Nakada. 


\section{Part V}

\section{Cost Summary}




\section{Chapter 18}

\section{Cost Summary}

This proposal is accompanied by a detailed Work Breakdown Structure (WBS) and cost estimate, which includes several hundred pages of material. In this section of the proposal, we explain briefly the methods used and present the high level summary (rollup) of the cost estimate.

\subsection{Methodology}

The BTeV collaboration has prepared a detailed costing of the proposal following procedures prepared by E. Barsotti (entitled 'Cost \& Schedule Procedures for the BTeV Proposal'). The procedures document is based on the procedures developed for the SDC collaboration (see SDC document SDT-000009). These procedures are currently being used for US CMS projects.

\subsubsection{Documentation}

The proposed BTeV costs are documented in a Work Breakdown Structure (WBS) Excel spreadsheet. The WBS spreadsheet is accompanied by two Microsoft Word documents, the WBS Level 2 System Summaries, and the WBS dictionary. All three documents are available on a $\mathrm{BTeV}$ website.

\subsubsection{Inclusivity}

We tried to include all the essential elements of the detector, the electronics, the trigger and the data acquisition system and all infrastructure required to carry out a project of this size and complexity. We used recent experience to try to avoid omissions which have led to problems for other projects in the past. In particular, based on discussion with electronics engineers, we have included an appropriate number of prototype development runs on the electronics development projects. We have also included large contingencies on these runs since they sometimes go awry. We have costs for a reasonble number of test stands to support 
R\&D activities and testing at multiple sites. There are significant resources assigned to project management and ES\&H both for the whole project and for each subproject. We have included all calibration and monitoring systems and many infrastructure items which are commonly overlooked such as control room electronics. The data acquisition and trigger software are also included in the construction project since the detector can not be shown to be working without them.

While it has been traditional (BaBar being a recent exception) to treat the offline computing as a separate entity, we at least wanted to describe the resources needed for it. We have included it in our WBS but have split it out into a separate cost table. The estimates for hardware at this point should be viewed as approximately correct. The estimates for resources to develop software are difficult to make and should be viewed as informed guesses based on recent experience.

\subsubsection{Labor Costs}

We have followed the rule of accounting for the effort of collaboration physicists but not costing it. It is considered part of the 'base program' cost.

We have costed all engineers and lead technicians at Fermilab rates. Where we plan to use less skilled technicians, student, or term labor, we have used a lower rate. These are

called out clearly in the spreadsheets. It should be noted that software professionals and computer scientists are treated under the engineer category.

All labor is split into development and manufacturing. For salaries, we include fringe benefits for all labor except term labor. We do not include overhead.

\subsubsection{Handling of Inflation and Deflation}

To handle the effects of inflation on personnel costs, we assume that the average year for development is 2002 and inflate all personnel costs to that year. We assume that the average year for production manufacturing is 2004 and inflate all personnel costs to that year.

Most major cost drivers involve discussions with vendors. We have tried to make clear to them when the main acquisitions would be made and have asked for prices applicable during that period. Since their responses are only quotes, not committments, it is very hard for us to do more than this.

Many high technology components undergo deflation at least on a price/performance basis. We have used standard trending practices in each industry to estimate the costs of CPUs, disks, computer memory, FPGAs, optical links, network switches, etc. Many of these items are purchased late in the $\mathrm{BTeV}$ construction period. We have been fairly conservative in the trending lines we have used. 


\subsubsection{Contingency}

For most WBS elements we have used the formula provided in the handbook. This has led to a consistent picture of contingency but may appear to be low. We believe that this is because the plan emphasizes so many costed $R \& D$ steps whose main goal is to reduce risk. We have also been fairly conservative in trending the cost of electronics and computer costs into future years. Thus, there exists the equivalent of additional hidden contingency in the number of design cycles provided, and in the trending curves used.

\subsection{The Detector Cost Estimate}

Since BTeV is only at the proposal submission stage, we do not have complete engineering designs for our systems. However, we have a stable and complete baseline design and an active program of R\&D on all parts of the detector. Many of our systems are similar to ones that have been built recently or are being built for future experiments. We have drawn on our own experience with these types of systems, and that of others, to develop the cost estimate for $\mathrm{BTeV}$. In many cases, the large cost drivers are procurements and, for these, we have had direct discussions with vendors and have obtained price quotes.

Below, we describe briefly the basis of the cost estimate for each major WBS Level 2 project. We break it into two parts: the first describes the tasks associated with the construction of the detector, including the hardware and software for the trigger and data acquisition; the second describes costs associated with providing facilities to carry out the offline analysis and to develop the offline framework and analysis programs. More details can be found in the accompanying WBS Spreadsheet and Dictionary entries (note, the subsection numbers here correspond to the Level 2 WBS cost estimate sections).

\subsubsection{Vertex and Toroidal Magnets and Beam Pipe}

This section covers the three major mechanical subsystems of the proposed BTeV spectrometer. They are the vertex analysis magnet, the four toroidally magnetized iron absorber walls for the muon detectors, and the beam pipes necessary to connect the silicon pixel detector vacuum box to the rest of the Tevatron accelerator.

The vertex magnet will be obtained by reusing the existing SM3 analysis magnet from experiment E866 in the MEast beam line. The muon toroids (and their shielding insert) will be assembled from large soft-iron slabs machined on two sides only. The silicon vacuum box to beampipe transition window will be a 0.030 " thick, hemispherical, spun aluminum transition piece. The beampipes will be thin-walled, 3.8 or $6.4 \mathrm{~cm}$ diameter aluminum beam pipes.

The rigging costs and the assembly and manufacturing techniques involved here are standard and well understood at Fermilab. The mechanical costs include $\$ 470 \mathrm{~K}$ for the vertex magnet, $\$ 1.14 \mathrm{M}$ for the 4 magnetized muon toroids, $\$ 32 \mathrm{~K}$ for the beam pipes, and $\$ 160 \mathrm{~K}$ for $\mathrm{ES} \& \mathrm{H}$ and management. 


\subsubsection{Pixel Detector}

The pixel vertex detector provides high resolution space points near the interaction that are used both online and offline to reconstruct tracks and associate them with their parent vertices. The pixel sensors will be very similar to those developed for use in the ATLAS pixel detector. They will be fabricated with $\mathrm{n}+$ pixels $(50 \times 400$ micron cell size) on low resistivity n-bulk silicon. An Application Specific Integrated Circuit (ASIC) being designed at Fermilab (FPIX) will read out the pixel sensors. A non-radiation hard prototype of this chip has performed very well in recent beam tests. We plan to implement the final version in a commercial process, $0.25 \mu \mathrm{m}$ CMOS, which appears to be capable of withstanding the radiation dose. The pixel sensors will be connected to the readout chips using bumpbonding technology. The mechanical supports will be all-carbon composite structures that will include integrated cooling tubes. These structures will be developed and manufactured by Energy Science Laboratories, Inc. A relatively high contingency is assigned to this item. This contingency is intended to cover the possibility that all-carbon structures cannot be successfully developed, and beryllium support and cooling frames must be used instead.

The costs of the pixel detector are based on: our own experience developed during the $R \& D$ phase, which includes extensive interaction with vendors, experience in building silicon strips for Run II, knowledge of the cost estimates of the LHC experiments CMS and ATLAS, and engineering input on production issues (SCIdet) and electronics design (front end and data acquisition). These costs include $\$ 2.9 \mathrm{M}$ for the sensors, $\$ 4.0 \mathrm{M}$ for the front-end electronics, $\$ 2.0 \mathrm{M}$ for mechanical systems, $\$ 570 \mathrm{~K}$ for test beam studies, and $\$ 520 \mathrm{~K}$ for ES\&H and management.

\subsubsection{RICH Detector}

The purpose of the BTeV Ring Imaging Cerenkov Detectors (RICH) is to provide good and efficient identification of hadrons with momentum from a few $\mathrm{GeV} / \mathrm{c}$ to over $70 \mathrm{GeV} / \mathrm{c}$. A main cost driver has been the sensors, which are Hybrid Photo Diodes (HPD).

The price of the HPDs is based on discussions with the vendor, DEP. The cost of the readout is based on our collaborator's recent experience in developing a similar readout for the CLEO RICH in collaboration with a vendor. Cost of the high voltage units has been obtained by discussions with CAEN. Costs for mirrors, mounts, gas vessel etc were derived with the help of the experience of HERA-B. The RICH costs include $\$ 11.2 \mathrm{M}$ for the photon detectors, $\$ 2.6 \mathrm{M}$ for electronics, $\$ 2.2 \mathrm{M}$ for mechanical systems, and $\$ 620 \mathrm{~K}$ for ES\&H and management.

\subsubsection{Electromagnetic Calorimeter Detector}

The $\mathrm{BTeV}$ electromagnetic calorimeters (ECAL) are designed to detect and measure the energy of electrons and photons by total absorption of their resulting electromagnetic showers in a highly segmented array of $\mathrm{PbWO}_{4}$ crystals. Each crystal is read out with a photomultiplier tube (PMT). 
The costs of crystal production were obtained by direct discussion with vendors in Russia and China who are making the nearly identical CMS crystals and with other potential suppliers. Costs for other items were derived from: discussions with a PMT vendor (Hamamatsu), quotes on high voltage systems from CAEN, discussions about the mechanical support with BaBar and CMS, and discussions about the calibration system with $\mathrm{KTeV} / \mathrm{KAMI}$ and CMS. The ECAL costs include $\$ 15.0 \mathrm{M}$ for the crystals, $\$ 3.4 \mathrm{M}$ for electronics, $\$ 1.6 \mathrm{M}$ for mechanical systems, $\$ 490 \mathrm{~K}$ for testing, and $\$ 500 \mathrm{~K}$ for ES\&H and management.

\subsubsection{Muon Detector}

The BTeV muon detectors are an array of $3 / 8$ " proportional tubes positioned between and behind the muon toroids. The basic building blocks of the detector are "planks" of thirtytwo proportional tubes arranged in a double layer with an offset of half a tube. Each set of tubes is soldered at each end to a brass gas manifold, and supported in the middle by soldering to a brass rib piece. The electronics is based on a chip used in the Run-II CDF central outer tracker. Prototype chambers have been operated in a test beam at Fermilab and have resulted in design modifications to the electronics. The design of this system is viewed as nearly complete.

Costs of the muon system, based on the current design, are derived from detailed discussions with vendors and pricing of all components. The costs include $\$ 2.7 \mathrm{M}$ for the proportional tubes, $\$ 3.0 \mathrm{M}$ for electronics, $\$ 80 \mathrm{~K}$ for mechanical systems, $\$ 320 \mathrm{~K}$ for test beam studies, and $\$ 420 \mathrm{~K}$ for ES\&H and management.

\subsubsection{Forward Tracker Straw Detector}

The straw tube tracker consists of 7 stations of straw tube proportional wire detectors in each $\mathrm{BTeV}$ spectrometer arm with a total of some 86,000 individual straw tube detectors. The design and construction of the straw tubes closely follows the design of the ATLAS TRT straw tubes currently under construction at a $\mathrm{BTeV}$ collaborator's university.

The costs of the straw tube system are derived from the, very detailed cost baseline of the ATLAS detector. The costs include $\$ 6.5 \mathrm{M}$ for the straw detectors, $\$ 4.2 \mathrm{M}$ for electronics, $\$ 320 \mathrm{~K}$ for mechanical systems, $\$ 300 \mathrm{~K}$ for test beam studies, and $\$ 600 \mathrm{~K}$ for ES\&H and management.

\subsubsection{Forward Tracker Silicon Microstrip Detector}

The Forward Tracker Silicon Microstrip Detector consists of a set of small angle silicon microstrip detectors. The silicon planes will be constructed from single sided silicon wafers, of dimension $12 \mathrm{~cm} \times 4.8 \mathrm{~cm}$ and $4.8 \mathrm{~cm} \times 9 \mathrm{~cm}$, and thickness 200 microns. The silicon front end electronics will require several custom designed integrated circuits.

The costs of the silicon system are derived from the costs of building the Run II CDF silicon detectors at SCIDET. Members of the BTeV group have also built similar systems. 
The costs include $\$ 1.1 \mathrm{M}$ for the silicon detectors, $\$ 2.2 \mathrm{M}$ for electronics, $\$ 430 \mathrm{~K}$ for mechanical systems, $\$ 400 \mathrm{~K}$ for test beam studies, and $\$ 660 \mathrm{~K}$ for $\mathrm{ES} \& \mathrm{H}$ and management.

\subsubsection{Unassigned}

WBS level 1.8 is unassigned

\subsubsection{Level 1 and Global Triggers}

The Level 1 vertex trigger uses the pixel detecetor to search for decay vertices in every beam crossing $(7.6 \mathrm{MHz})$ and provides the basic trigger for the experiment. A major R\&D effort has developed the algorithms and carried out the timing studies required to establish the initial design and size of this system. The Level 1 trigger also includes a dimuon trigger from the muon system, and other prescale and beam crossing triggers. The triggers process data stored in hit lists in fast memory. The algorithms and trigger decisions are implemented in field-programmable gate arrays (FPGA) and digital signal processors (DSP). The Global Level 1 Trigger (GLV1) controls the flow of data from the front-end detector electronic subsystems into the data acquisition system. Monitoring of the rates, luminosity, etc. are also done in this unit.

We recognize that rapid progress in electronics implies that the present design DSPS, FPGAs, fast memory, etc., will be superseded by devices with greater performance per dollar. We have attempted to estimate these trends in costing the trigger system. The costs of the Level 1 and Global Trigger include $\$ 4.8 \mathrm{M}$ for the vertex trigger system, $\$ 1.1 \mathrm{M}$ for the muon trigger system, $\$ 270 \mathrm{~K}$ for the GLV1 and other trigger processors, and $\$ 200 \mathrm{~K}$ for ES\&H and management.

\subsubsection{Data Acquisition Electronics and Links}

The BTeV data acquisition system (DAQ) must digitize and buffer all detector data at the beam crossing rate. The data is transferred from the detector subsystems to the Level 1 Buffers in the counting room (a distance of 30 to 70 meters) by optical links. Level 1 Buffers store data while Level 1 trigger decisions are being made and Level $2 / 3$ buffers hold data while processor farms process further trigger logic. A set of large switches under control of the processors direct the data flows.

The costs of the DAQ system are based on current experience in purchasing similar systems for Run II. These costs include, $\$ 3.2 \mathrm{M}$ for electronics, $\$ 1.0 \mathrm{M}$ for the optical links, $\$ 400 \mathrm{~K}$ for installation and hardware, and $\$ 150 \mathrm{~K}$ for ES\&H and management.

\subsubsection{Level 2/3 Processor Array}

The Level 2/3 Processor array consists of a large number of independent general-purpose processors $(2,500$ in the baseline system). They interface to the DAQ network through 
standard fast ethernet links and will be standard low-cost consumer PCs with minimal addon features. They will probably run a Linux variant to reduce software costs. The links to the data acquisition system are included in the Data Acquisition subproject. This project includes mainly the processors.

The costs of this system are based on using the cost of Run II purchases as a fixed starting point and are extrapolated using a conservative model of industry trends (that is, we use the classic performance/price slope, rather than the much steeper one that has been applicable in recent years). The costs include $\$ 2.3 \mathrm{M}$ for processors, $\$ 300 \mathrm{~K}$ for disks and network connections, $\$ 370 \mathrm{~K}$ for tape drives and external data connections, and $\$ 20 \mathrm{~K}$ for ES\&H and management.

\subsubsection{Analysis and Simulation Facilities}

These costs occur within the Computing Division and are discussed in the section below on Offline Computing Cost Estimates.

\subsubsection{Controls/Monitoring and Timing}

The goal of the monitor and control system is to provide access to all electronic systems in the $\mathrm{BTeV}$ experiment via a single host computer. It integrates the configuration, firmware management, monitoring, timing, and diagnostics functions into a single system. It is implemented as a tree network of links and PC processing nodes.

The costs of this monitor/control system are based on similar systems recently designed and built at Fermilab. These costs include $\$ 1.3 \mathrm{M}$ for electronics, $\$ 410 \mathrm{~K}$ for rad hard ICs, and $\$ 140 \mathrm{~K}$ for ES\&H and management.

\subsubsection{Control Room Electronics and Equipment Infrastructure}

The control room information infrastructure necessary for personnel to run the experiment and the electronics tying all the disparate pieces together into a coherent system are included in this section. The usual consoles and monitor screens are included, along with some common electronics items. Two accelerator interfaces are provided, one in the control room and a duplicate in the pit.

The costs of this system are based on similar systems recently designed and built at Fermilab. These costs include $\$ 470 \mathrm{~K}$ for electronics, $\$ 570 \mathrm{~K}$ for infrastructure, and $\$ 120 \mathrm{~K}$ for ES\&H and management.

\subsubsection{Control, Monitoring and Event Readout Software}

This section includes the software and hardware framework to control and monitor the data flow in the data acquisition system. It also includes Test Stand software and Control/Monitoring and Timing System software. 
Cost estimates for these systems are based on experience with similar software projects at Fermilab. Contingencies are small because nothing in this system is considered risky technically. The costs include, $\$ 1.5 \mathrm{M}$ for DAQ software, $\$ 320 \mathrm{~K}$ for test stand software, $\$ 400 \mathrm{~K}$ for slow controls, and $\$ 410 \mathrm{~K}$ for ES\&H and management.

\subsubsection{Algorithm and Other Software and Integration Facilities}

These costs occur within the Computing Division and are discussed in the section below on Offline Computing Cost Estimates.

\subsubsection{Test Stands and Test Equipment}

The BTeV Test Stands and Test Equipment are used to facilitate development and testing of the individual components of the entire system. The BTeV Test Stand will be a convenient and efficient way to perform component level debugging throughout the entire design and development stages and will be built to identical specifications, yet contain the flexibility the user needs to customize their test stand. The test stand will be based on a PC or workstation that can accept standard PCI-based plugin cards.

The major components of the BTeV Test Stands and Test Equipment are mostly vendor supplied stock items. The costs for these items are based on either current catalog prices or on purchase orders from recently purchased equipment. The costs include $\$ 950 \mathrm{~K}$ for test stand hardware, $\$ 450 \mathrm{~K}$ for test equipment, $\$ 105 \mathrm{~K}$ for test stand software, and $\$ 120 \mathrm{~K}$ for ES\&H and management.

\subsubsection{Unassigned}

WBS level 1.18 is unassigned.

\subsubsection{System Installation, Integration Testing and Commission- ing}

This section includes those items that have to do with bringing together the various elements of the BTeV Detector and making them operate in concert with each other. Some of the tasks included here are: environmental safety and health $(\mathrm{ES} \& \mathrm{H})$, and the management of the system installation and commissioning.

The cost estimates for this item are almost entirely labor. The labor estimates are based on discussions with personnel from CDF and D0. A uniform contingency of $25 \%$ was added to the time estimates. The costs include $\$ 850 \mathrm{~K}$ for system installation, and $\$ 300 \mathrm{~K}$ for ES\&H and management. 


\subsubsection{BTeV Project Management}

The project management section includes the project office, its staff, and its expenses. It is assumed that a Project Manager, Deputy Project Manager, Project Mechanical Engineer, Project Electronics Engineer, Project Safety Officer, and an Administrative Assistant/Project Budget Officer are included as well as a travel budget and consultant expenses.

The costs for $\mathrm{BTeV}$ Project Management include $\$ 2.9 \mathrm{M}$ for the $\mathrm{BTeV}$ project office, $\$ 300 \mathrm{~K}$ for project management by physicists, $\$ 500 \mathrm{~K}$ for external reviews and consultants, and $\$ 200 \mathrm{~K}$ for documentation and software.

\subsection{The Offline Computing Cost Estimate}

The cost of development for the data acquisition software is included in the detector portion of the project. Here we present cost estimates for the acquisition and commissioning of the data analysis hardware and the development of the offline software. The software includes both the infrastructure and framework for the reconstruction, data access, and physics analysis as well as the development of the reconstruction and physics analysis algorithms. While we expect that physicists and software professionals will collaborate closely in both parts of the effort, we expect that the infrastructure and framework will have a large component of computing scientists and software professionals while the algorithm and analysis development will have a large component of physicists.

The costs are driven by the amount of data recorded by the experiment, which is discussed in detail in this document, the number of processing steps, the processing time per step, the number of parallel analyses and the number of data analysts. The efforts on the trigger and the intense simulation efforts, along with the group experience with analysis of some of the largest datasets recorded in HEP so far form part of the basis for these estimates. Experience with Run 2 and Computing Division experience and tracking of trends form the other major input.

\subsubsection{Analysis and Simulation Facilities}

The simulations, reconstructions, and analyses needed to produce physics, results from the $\mathrm{BTeV}$ data will be carried out on farms of PCs. The costs in this section are based on the assembly, coding, and operation of the PC farms recently built for CDF and D0. The costs include $\$ 1.3 \mathrm{M}$ for analysis computing, $\$ 2.9 \mathrm{M}$ for data archiving, and $\$ 600 \mathrm{~K}$ for desktop systems.

\subsubsection{Algorithm and Other Software and Integration}

This section addresses the costs associated with developing the software needed to translate the raw data stream into reconstructed HEP event samples. The software costs associated 
with the efficient storage and retrieval of data, pattern recognition algorithms, code management, and event visualization tools are included. The costs include $\$ 2.9 \mathrm{M}$ for algorithm and code development, and $\$ 3.5 \mathrm{M}$ for software tools and code management.

\subsection{Cost Summaries}

\subsubsection{Detector Cost Summary}

The total of the baseline cost estimate is $105 \mathrm{M} \$$, as detailed in Table 18.1.

Table 18.1: Baseline BTeV Detector Cost Estimate (\$)

\begin{tabular}{lrl}
\hline & Cost & \\
Item & BTeV Baseline & Comment \\
\hline Magnets, beampipes & $1.80 \mathrm{M}$ & \\
Pixels & $14.25 \mathrm{M}$ & based on CMS and ATLAS projections \\
RICH & $17.14 \mathrm{M}$ & based on HERA-B RICH \\
EM Calorimeter & $21.02 \mathrm{M}$ & based on PbWO 4 from CMS \\
Muon. & $6.72 \mathrm{M}$ & \\
Straw Tubes & $12.14 \mathrm{M}$ & based on ATLAS staw tubes \\
Silicon Strips & $5.10 \mathrm{M}$ & based on Run II silicon \\
Trigger Level 1 & $6.20 \mathrm{M}$ & based on 3200 DSP processors \\
DAQ+links & $4.82 \mathrm{M}$ & based on 2500 processors, 20 tape drives \\
Level 2/3 & $2.95 \mathrm{M}$ & \\
Controls/timing & $1.97 \mathrm{M}$ & \\
Control room & $1.16 \mathrm{M}$ & \\
DAQ software & $2.87 \mathrm{M}$ & \\
Test stands & $1.62 \mathrm{M}$ & \\
Installation, commissioning & $1.45 \mathrm{M}$ & \\
Project management & $3.86 \mathrm{M}$ & \\
\hline Total & $105.07 \mathrm{M}$ & \\
\hline \hline
\end{tabular}

\subsubsection{Computing Cost Summary}

The total of the baseline cost estimate of the computing hardware and software, $12.4 \mathrm{M} \$$, is detailed in Table 18.2 . 


\begin{tabular}{|c|c|c|}
\hline Item & $\begin{array}{c}\text { Cost } \\
(2 \mathrm{arms})\end{array}$ & comment \\
\hline Hardware & $5.3 \mathrm{M}$ & based on industry trends \\
\hline Software Dev. & $7.1 \mathrm{M}$ & includes $50 \%$ contingency \\
\hline Total & $12.4 \mathrm{M}$ & \\
\hline
\end{tabular}




\section{Appendix A Detector Design Details and R\&D Plans}




\section{A.1 Pixel Detector}

In this section, we first present a review of our R\&D on pixel sensors (A.1.1). This is followed by a review of our testbeam results, with emphasis on charge collection, charge sharing, and position resolution (A.1.2). Finally, we present short sections on the bench top apparatus which we have built to explore ways to provide electromagnetic shielding for the $\mathrm{BTeV}$ pixel detector and its readout against pickup from the circulating beams (A.1.3), and on our recently completed radiation exposure of prototype $0.25 \mu \mathrm{m}$ CMOS pixel readout circuits (A.1.4).

In the past two years, we have made great progress in the development of the individual components and enabling technologies required to build the $\mathrm{BTeV}$ pixel detector. Our R\&D focus is now shifting to system engineering problems. We believe that it is very important for us to test our design ideas in real systems, and to gain experience with large scale pixel detectors. In the immediate future, our focus will be on subsystems, specifically pixel modules of a sensor bonded to 5 readout chips and to a readout "HDI." Soon after that, we will start tests of modules "shingled" onto a carbon-carbon "half-plane." Longer term, we expect to validate our BTeV design in " $3 \%$ " and " $10 \%$ " system tests in $\mathrm{C} 0$ or a test beam. 


\section{A.1.1 Sensor Design and Simulation}

\section{A.1.1.1 Introduction}

A key element driving the pixel sensor design is the necessity to put these detectors as close as possible to the beam axis in order to optimize the vertex resolution. In our baseline configuration, the innermost edge is located $6 \mathrm{~mm}$ away from the beam line. This requirement focuses our choices on technologies optimized for an extended lifetime of the detector in a harsh radiation environment. This challenge is not unique to $\mathrm{BTeV}$; it is also quite important for the LHC experiments. This allows us to progress in a cost effective way utilizing joint developments with other research groups.

The geometrical parameters of the sensor are the result of a careful optimization. The dimensions of the pixel unit cell determine the hit resolution and occupancy. In turn, they affect the complexity of the system, the space available for the pixel electronics, and the demands posed on the cooling system. The sensor thickness affects the signal to noise achievable in the course of the detector lifetime, and the resolution achievable for large angle tracks that share the charge signal among several pixel cells. The material budget is affected not only by the thickness of the active elements in this system (sensor and readout electronics), but also by the mechanical support and cooling system.

Finally, a key element to ensure that the sensor can provide useful information after high radiation dose is the ability to withstand a high reverse bias voltage. For example, the ROSE collaboration finds that the ATLAS "first layer," located at a radius of $10.1 \mathrm{~cm}$ from the beam axis, will have a depletion voltage of about $800 \mathrm{~V}$ after 10 years of LHC operation, if fabricated with conventional silicon, but this can be reduced to $400 \mathrm{~V}$ using oxygenated wafers [1]. Note that ATLAS is planning to use a layer even closer to the beam axis, the so-called "b-layer" located at $4.3 \mathrm{~cm}$. Our situation is closer to the "first layer." One of the key features required for such high voltage operation is a careful guard ring design. Several multi-ring designs that fulfil this requirement have been developed [2], [3].

\section{A.1.1.2 Radiation Hardness}

Radiation induced changes in silicon sensors have been the subject of detailed studies[4]. These changes are generally divided into two classes; changes in surface properties and in bulk properties. The latter changes are generally expressed in terms of an equivalent fluence of $1 \mathrm{MeV}$ neutrons. Conversion factors as a function of the particle types and their energies, known as NIEL (non ionizing energy loss) scaling coefficients, have been compiled by the ROSE collaboration [5]. Above $1 \mathrm{MeV}$, protons, neutrons and pions have NIEL factors less than 1. Bulk damage effects include increased leakage current, reduced charge collection efficiency and change in effective doping concentration. This eventually leads to the so called "type inversion" in $n$ type substrates, when their effective doping changes sign, leading to effective p-type substrates. ATLAS and CMS both have chosen a sensor technology for their pixel detectors commonly identified as $n^{+} n p^{+}$, in which the pixels are $n^{+}$cells on an n-type substrate and the backplane is a $p^{+}$junction. These sensors can be operated after type 
inversion at bias voltages less than the full depletion voltage. In the simpler $p^{+} n n^{+}$sensors, after type inversion, if the sensor is operated at less than the full depletion voltage, then the charge spreads across many pixels, which quickly leads to a loss of position resolution and efficiency. However, a more complex sensor fabrication procedure is needed in order to provide electrical isolation between the $n^{+}$pixels, which otherwise would be shorted together by the n-channel induced in the surface by positive charges trapped in the oxide layer.

A dedicated R\&D effort towards the development of substrates capable of withstanding large radiation doses, the ROSE collaboration, made systematic studies of "defect engineering," with the goal of better performance through extended periods of heavy radiation exposure [6]. Their main results are:

1. The introduction of controlled amounts of oxygen has several advantages. In particular, it slows the change in free carrier concentration due to radiation damage.

2. Reverse annealing saturates at high proton fluence $\left(\geq 2 \times 10^{14} \mathrm{p} / \mathrm{cm}^{2}\right)$ and its time constant is found to be a factor of 4 larger for oxygenated wafers. This allows the detector to remain at room temperature for a longer period of time without adverse effects.

The promising results from this effort have lead the LHC experiments to consider the adoption of oxygenated silicon for the sensors used in their inner tracking systems. BTeV is already working with companies capable of producing pixel sensors from oxygenated wafers.

\section{A.1.1.3 Radiation Levels}

The simulation of the expected radiation level for the $\mathrm{BTeV}$ pixel sensor has been performed using the BTeV-GEANT code, including a detailed geometry of our baseline detector, including support structures and services. Figure A.1 shows the spatial distribution of the expected particle fluence. Note that the fluence falls very rapidly with radial distance from the beam axis, making the radiation damage a crucial issue only for the innermost portion of our sensors. The maximum particle fluence is $\sim 10^{14} / \mathrm{cm}^{2} / \mathrm{yr}$, assuming a luminosity of $2 \times 10^{32} \mathrm{~cm}^{-2} \mathrm{~s}^{-1}$. This corresponds to an integrated dose similar to the one quoted for the ATLAS "first layer" pixel system, located at $10.1 \mathrm{~cm}$ from the beam. The dominant component of radiation dose are relatively high energy pions, with a NIEL factor less than 1.

\section{A.1.1.4 Sensor Design}

The $\mathrm{BTeV}$ pixel cell size is $50 \mu \mathrm{m}$ by $400 \mu \mathrm{m}$, where the small dimension is dictated by the needed spatial resolution. The technology chosen is $n^{+} n p^{+}$.

Different $\mathrm{n}$-side isolation techniques have been developed for double-sided silicon microstrip detectors. In particular $p^{+}$implants, generally called "p-stops", have been implemented with various geometries. Recent developments [7] have shown that the implant 


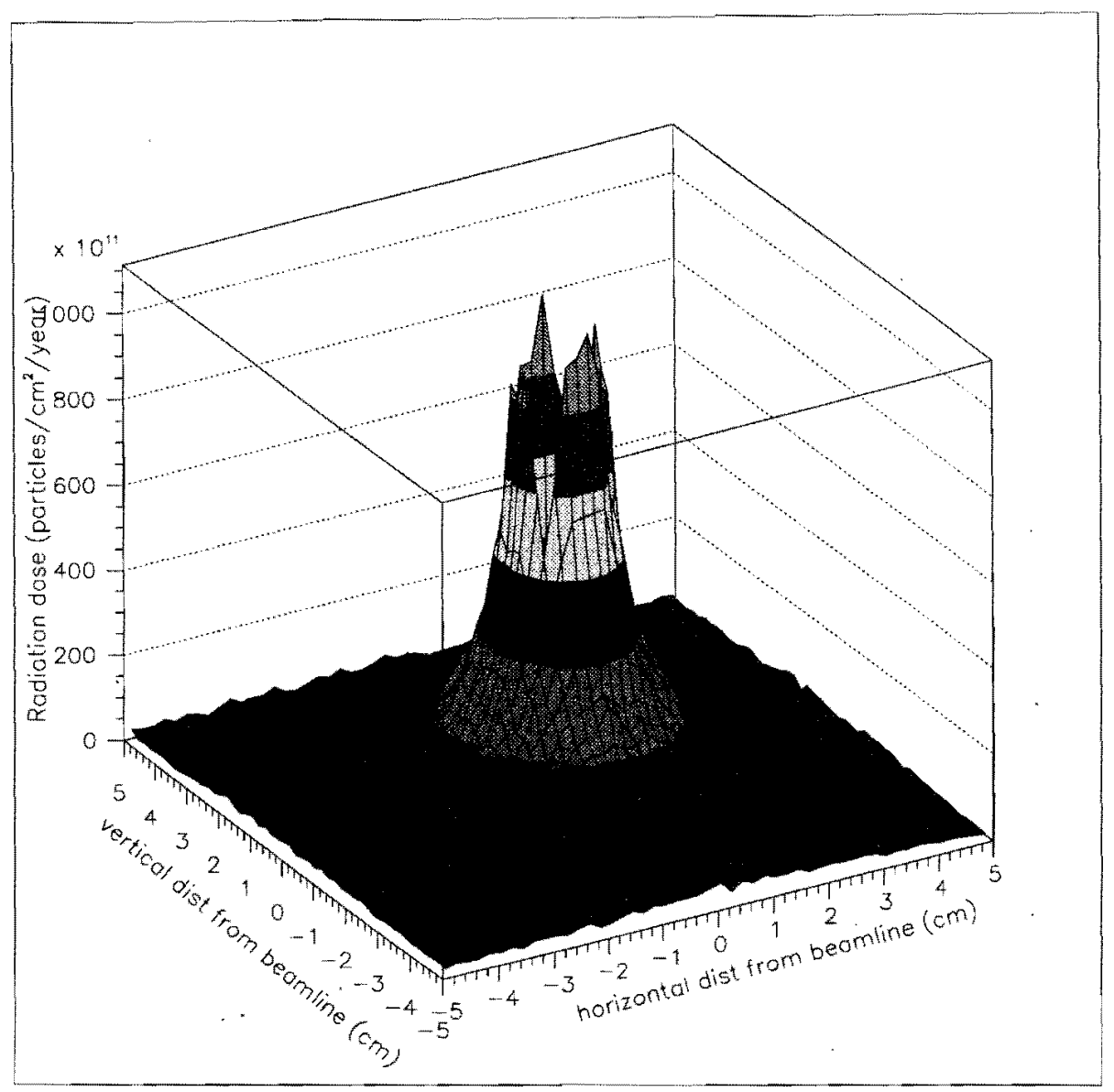

Figure A.1: Spatial distribution of the particle fluence in a pixel plane.

geometry can influence the interstrip capacitance and the noise performance. Moreover they can modulate the charge sharing between strips beyond the the spread due to diffusion.

An alternative approach, known as "p-spray", has been developed by the ATLAS collaboration. This consists of a medium dose boron implantation covering the entire $n$-side of the sensor. In the first implementation of this isolation technique, the ATLAS collaboration added a bias structure used for testing the sensor before bump bonding. Test beam results from ATLAS and BTeV have shown charge losses [9] in region of this bias network. A more refined solution is already implemented in new p-spray devices. We have acquired some of these new ATLAS devices, and thus will be able to verify whether they meet our specifications, both in terms of electrical performance and radiation tolerance.

We are also developing sensors of our own design. Our first effort was a joint development with the US CMS group led by B. Gobbi. We made a joint submission in Spring 1999 to two vendors, SINTEF and CSEM. These wafers contain $n^{+} n p^{+}$sensors with different $p$-stop isolation geometries. Both submissions include wafers from oxygen doped silicon. 


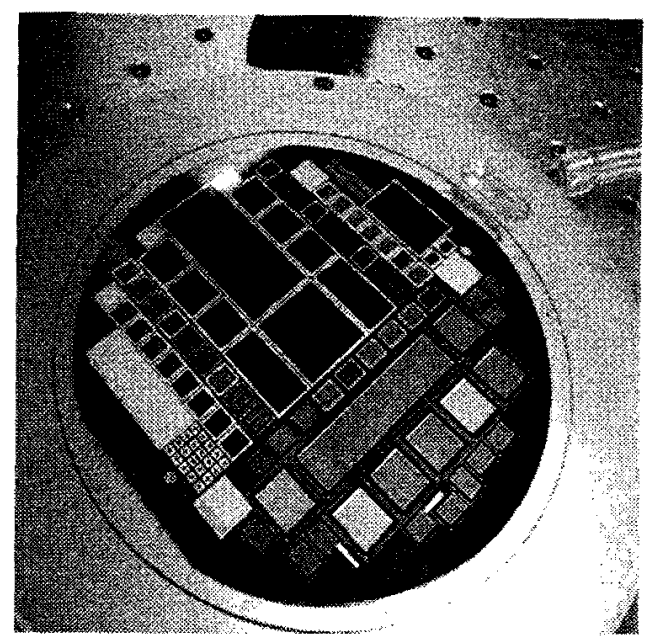

Figure A.2: SINTEF wafer from joint BTeV/CMS submission.

The specifications for the sensor prototypes in the joint BTeV-CMS submission are summarized below.

The following geometrical tolerances need to be met:

- Misalignment of $p^{+}$implant, $n^{+}$implant and metal layers $\leq 1.5 \mu \mathrm{m}$,

- uniformity of wafer thickness (wafer to wafer) $\pm 10 \mu \mathrm{m}$,

- thickness $300 \mu \mathrm{m}$,

The following electrical specifications need to be met:

- Leakage current $25 \operatorname{deg} \mathrm{C} \leq 50 \mathrm{nA} / \mathrm{cm}^{2}$ at $1.5 \times$ Depletion Voltage,

- resistivity $1.5-2.5 \mathrm{~K} \Omega$,

- breakdown voltage $\geq 300 \mathrm{~V}$.

We have recently received the first wafers from this submission from SINTEF (see Figure A.2). Figure A.3 shows I-V curves measured for two of the smaller BTeV sensors on one of these wafers. These curves are as expected for wafer-probed pixel detectors, and show very small leakage current and high reverse breakdown voltage (500 $\mathrm{V}$ or higher).

We are planning to subject these devices to different types and doses of radiation. We will measure their static electrical characteristics and their signal collection properties before and after irradiation. In addition, we will investigate radiation effect on sensors bump bonded to front end electronics and study their properties in the laboratory and in beam tests.

\section{A.1.1.5 Sensor simulation}

A detailed understanding of the factors affecting the sensor performance is crucial to its design. We have studied a number of issues through simulation. 


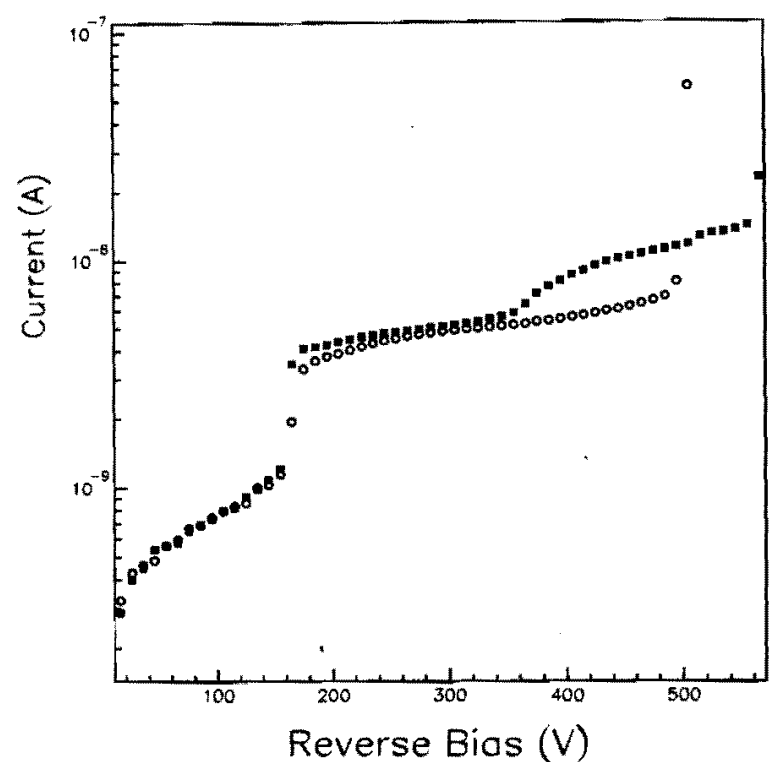

Figure A.3: $I-V$ characterization of two different sensor cells on a SINTEF wafer recently received at Fermilab.

In an $n^{+} n p^{+}$detector, the charge carriers collected at the pixel electrodes are electrons. We have simulated the properties of these $n^{+} n p^{+}$sensors, as well as more conventional $p^{+} n n^{+}$ pixel devices, which have holes as the collected charge. In addition, we have considered radiation damage effects. These include increased leakage current and the change in charge collection efficiency induced by the change in the effective donor concentration.

Other factors that affect the ultimate resolution achievable in this system are related more closely to the design approach and the performance of the readout electronics. In particular, the electronic noise, and the threshold that determines the minimal charge deposition that will be recorded as a signal hit, are important. The sensitivity to these parameters has been studied, as well as the tradeoff between analog and digital readout.

In order to understand these effects, we have developed a stand-alone simulation, based on a two-dimensional model of the signal formation in silicon. This program has been interfaced with the Monte Carlo software used to study our physics reach. This integration has been used to predict detector occupancy, crucial in trigger simulation, and to determine the required readout bandwidth. We have validated some of our results by comparing them with our recently acquired test beam data (see next section).

\section{A.1.1.6 Spatial resolution studies}

Our approach to silicon sensor modeling [10] is similar to previous studies performed for silicon microstrip detectors [11], [12]. We have investigated the interdependence of charge diffusion, magnetic field effects, electronic noise, discriminator threshold and number of bits in the back-end electronics.

The ability to achieve a spatial resolution better than the nominal "digital" resolution $(\sigma=$ width $/ \sqrt{12}$ ) is closely related to how accurately we can interpolate between the location of the centers of individual pixels using information on measured charge on each pixel in a 
cluster.

We have modeled the signal induced by minimum ionizing tracks traversing silicon using a charge straggling distribution function supported by experimental data and a detailed theoretical model of the interactions responsible for the energy loss in silicon [13]. The detector has been divided into $30 \mu \mathrm{m}$ thick slices to model fluctuations in the energy deposition along the path of the charged particle.

Electrons and holes produced by the energy deposited by a traversing charged particle drift along the electric field lines $(\vec{E})$ in the detector. The equations describing this drift motion are:

$$
\begin{aligned}
& \vec{J}_{n}=q \rho_{n} \mu_{n} \vec{E} \\
& \vec{J}_{h}=q \rho_{h} \mu_{h} \vec{E}
\end{aligned}
$$

where $q$ is the electron charge, $\mu_{n}$ and $\mu_{h}$ are the mobility of electrons and holes respectively, $\rho_{n}$ is the number of free electrons per unit volume, and $\rho_{h}$ is number of free holes per unit volume.

In parallel, the charge cloud spreads laterally due to diffusion. The parameters characterizing the drift in the electric field $\left(\mu_{h}, \mu_{e}\right)$ are related to the parameter describing the diffusion of the charge cloud $\left(D_{h}, D_{e}\right)$ by the Einstein equation:

$$
D_{h(e)}=\frac{k T}{q} \mu_{h(e)}
$$

where $D_{h}$ and $D_{e}$ are the diffusion coefficients, $q$ is the electron charge and $k T$ is the product of the Boltzmann constant and the absolute temperature of the silicon. The average square deviation with respect to the trajectory of the collected charge without diffusion is $\left\langle\Delta r^{2}\right\rangle=$ $2 D \Delta t$. In our study we have used $\mu_{h}=400 \mathrm{~cm}^{2} / V s, \mu_{e}=1450 \mathrm{~cm}^{2} / V s$.

When the silicon detector is located inside a magnetic field, there is an additional source of charge spreading; the lateral motion induced on electrons and holes by the magnetic field. This effect shifts the centroid of the detection point of the charged cloud and also widens it, because the amount of drift is proportional to the path length of the detected electrons (holes). This so called " $E \times B$ effect" depends on a parameter called the Hall mobility, $\mu_{H}$, which is proportional to the charge carrier mobility discussed above. In order to take into account the effect of the magnetic field in the bend plane, the following values of the Hall mobility have been taken from experimental data quoted in [14]:

$$
\begin{array}{r}
\mu_{H, h}=0.72 \mu_{h}, \\
\mu_{H, e}=1.15 \mu_{e} .
\end{array}
$$

The charge-cloud image appears on more pixels when the incident track crosses the detector at an angle, as the generation points of the electron-hole pairs spread out along the track pathlength. These various effects are illustrated in Figure A.4, which shows the charge broadening due to diffusion, the magnetic field and the combination of the these two effects together with track inclination with respect to the normal to the detector. 
Charge Distribution
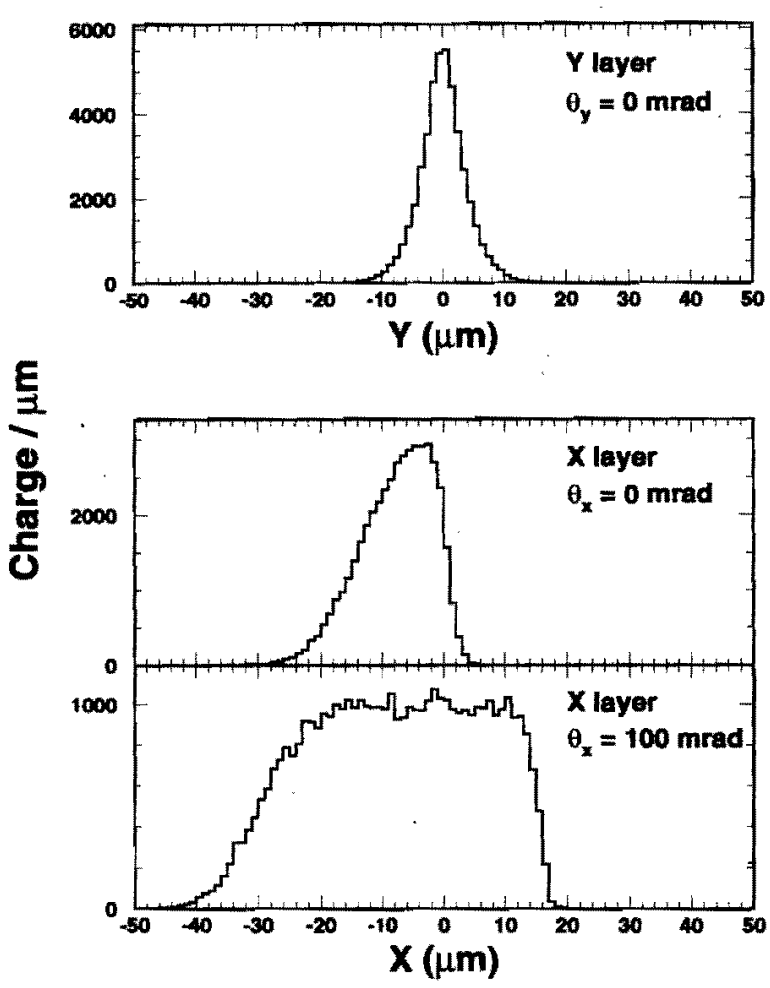

Figure A.4: Collected charge spreading in a $300 \mu \mathrm{m}$ silicon detector a) produced by diffusion, b) produced by the interplay of diffusion and the magnetic field for $\mathrm{B}=1.6 \mathrm{~T}$, and $\mathrm{c}$ ) produced by diffusion and magnetic field effects when the charged track is incident at an angle of 100 $\mathrm{mr}$ in the bend plane.

An analog readout improves the spatial accuracy through the use of charge weighting. low intrinsic noise is obviously a key element in this approach, together with a low and uniform threshold.

Two assumptions are common to all the configurations used in our study of the interdependence between threshold, noise and digitization accuracy . We have considered a $300 \mu \mathrm{m}$ thick Si detector biased in over-depleted mode $(200 \mathrm{~V}$ for a detector that is fully depleted at $120 \mathrm{~V}$ ). We have assumed full charge collection and negligible capacitive cross talk between adjacent pixels. These assumptions will be relaxed in future studies.

\section{A.1.1.7 Factors affecting the spatial resolution}

We have carefully examined the various factors affecting the hit resolution in the small pixel direction, both in the bend plane and in the direction orthogonal to it, with reference to a pixel geometry of $50 \mu \mathrm{m} \times 400 \mu \mathrm{m}$, using several track orientations. 
Figure A.5 shows the relationship between the position of incidence at the pixel plane and the reconstructed coordinate for tracks at normal incidence on the pixel detector, whereas Figure A.6 assumes a track inclined at an angle $\theta_{x}=300 \mathrm{mr}$. We have started our study assuming a readout scheme with the intrinsic electronic noise switched off, and we consider both a binary and an analog readout (with 6-bit ADC resolution). We can see that if the electronics noise is negligible, a digital readout actually can achieve a better resolution than the naive expectation of the width of the cell divided by $\sqrt{12}$. This is due to the fact that when the charge is shared between two or more pixels it is possible to do a digital charge weighting algorithm that provides some interpolation between the position of individual pixels.

Let us start our discussion of analog readout with tracks at normal incidence. Figure A.5 shows that, in the presence of a magnetic field, the charge can actually be "focused" inside the pixel instead of spread out by virtue of the magnetic field. This makes the analog and digital readout equivalent, because there is not a second pixel helping to interpolate the track position using charge sharing. On the other hand, tracks incident on the pixel region where the magnetic field spreads the charge further with respect to diffusion, are helped significantly by analog readout. In order to take full advantage of the analog information, it is necessary to adopt an optimal choice of the charge weighting algorithm that reflects the expected distribution of the charge profile. In particular, for tracks incident at very small angles, it is necessary to perform a non-linear weighting [12].

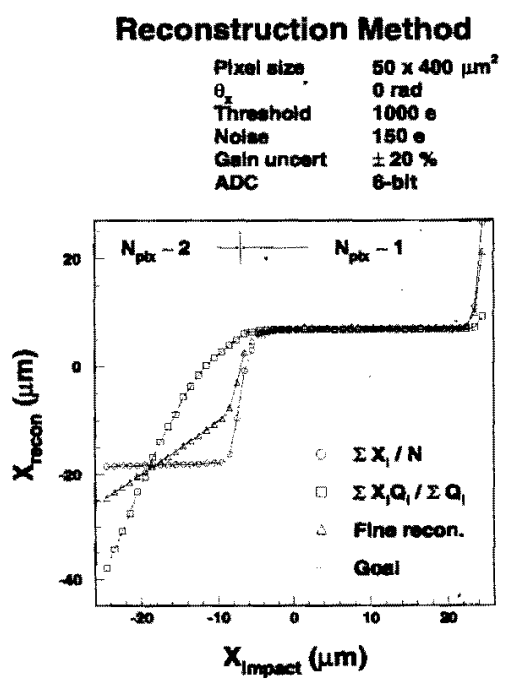

Figure A.5: The reconstructed $x$ coordinate as a function of the charged track impact point on the pixel cell in a $1.6 \mathrm{~T}$ magnetic field, for a pixel size of $50 \times 400 \mu \mathrm{m}^{2}$, and normal incidence. Both these coordinates are measured with respect to the pixel center.

An additional important constraint in the achievable resolution is the discriminator threshold. In this study we have assumed that only the pixels having a signal above threshold 


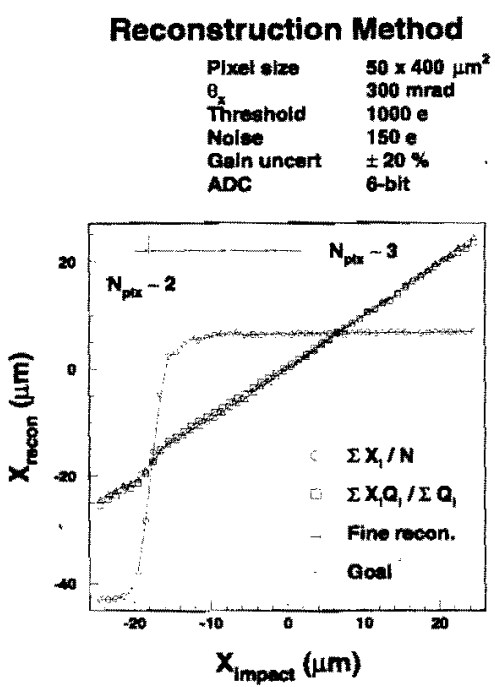

Figure A.6: The reconstructed $\mathrm{x}$ coordinate as a function of the charged track impact point on the pixel cell in a $1.6 \mathrm{~T}$ magnetic field, for a pixel size of $50 \mathrm{x} 400 \mu \mathrm{m}^{2}$, and incidence angle in the bend plane $\theta_{X}=300 \mathrm{mr}$. Both these coordinates are measured with respect to the pixel center.

are read out, and we have varied the threshold for analog and binary readout. Figure A.7.a shows the effect of increasing the threshold for an incident angle $\Theta_{x}=300 \mathrm{mr}$, for analog and digital readout respectively. Figure A.7.b shows the corresponding pixel occupancy. Each curve shows the fraction of events having $N$ pixels hit for a given threshold. For instance, for a threshold of 1000 electrons, essentially all events have either two or three pixels above threshold, with $\sim 60 \% 3$-pixel clusters, and $40 \% 2$-pixel clusters. The resolution achievable for binary readout shows a characteristic oscillatory behavior as we change the threshold. The worst resolution is always at the thresholds for which most of the events have clusters with the same size, corresponding to less interpolation power. In the analog readout case, the higher the threshold, the coarser is the charge sharing process.

The slope of the threshold dependence of the resolution is not very high below 3000 electrons, as illustrated by Figure A.7.

Figure A.8 shows the number of pixels hit as a function of the track angle for electron and hole collection. Figure A.9 shows the resolution as a function of the angle in the bending plane $\left(\theta_{x}\right)$, and in the non-bend plane $\left(\theta_{y}\right)$ expected with digital readout and with a 4 bit ADC respectively. At angles above $\pm 50 \mathrm{mr}$ the resolution achieved with analog readout is significantly better.

An understanding of the resolution and occupancy expected for a given sensor and readout electronics choice is very important to optimize the performance of the final system. In 

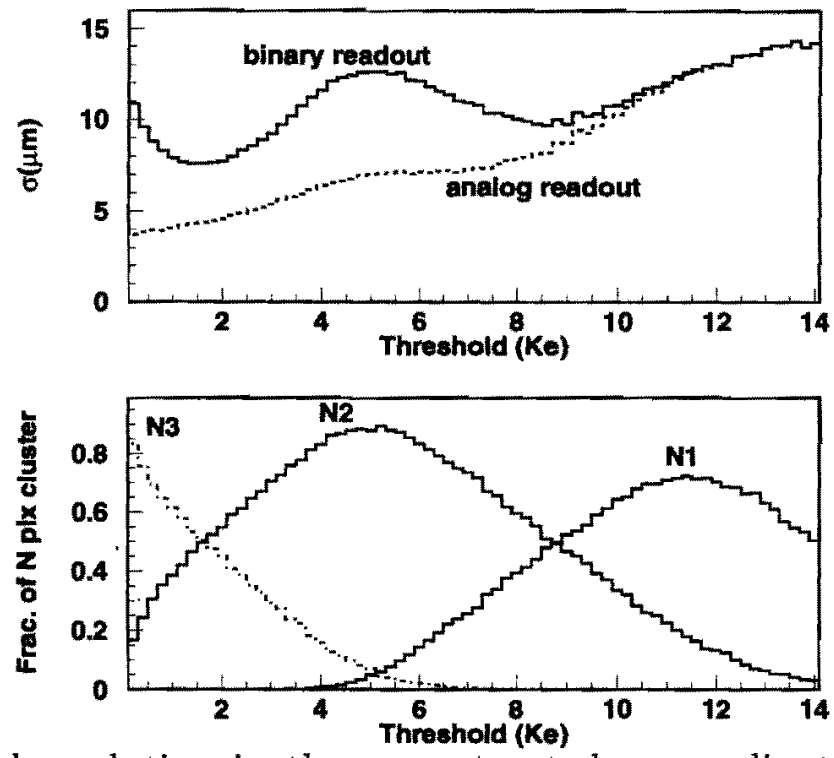

Figure A.7: a) Spatial resolution in the reconstructed $\mathrm{x}$ coordinate as a function of the threshold for a pixel size of $50 \times 400 \mu \mathrm{m}^{2}$ and incidence angle in the bend plane $\theta_{X}=300$ $\mathrm{mr}$. b) percentage of events having $\mathrm{N}$ pixels hit as a function of the threshold for the same configuration.

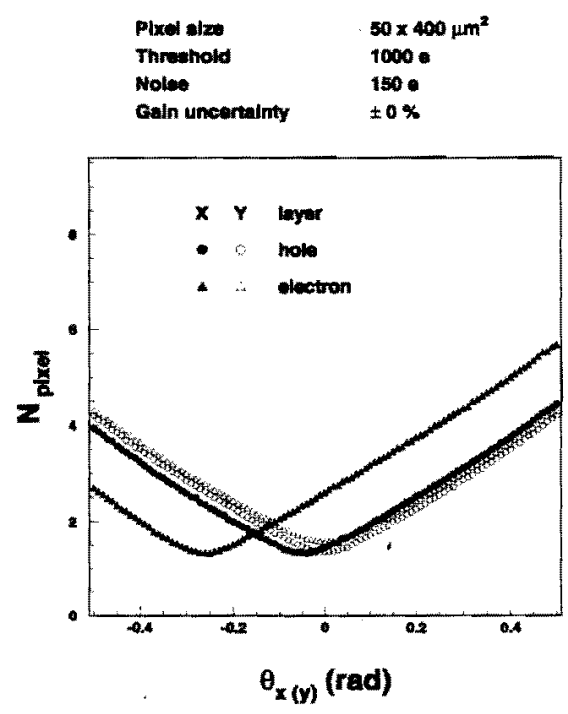

Figure A.8: Number of pixels hit for a $50 \mu \mathrm{m}$ pixel small dimension as a function of the track orientation. The magnetic field is parallel to the $\hat{x}$ direction.

order for this tool to be effective, its accuracy must be checked with experimental data. We have done a systematic study of the performance expected from various sensor and readout 


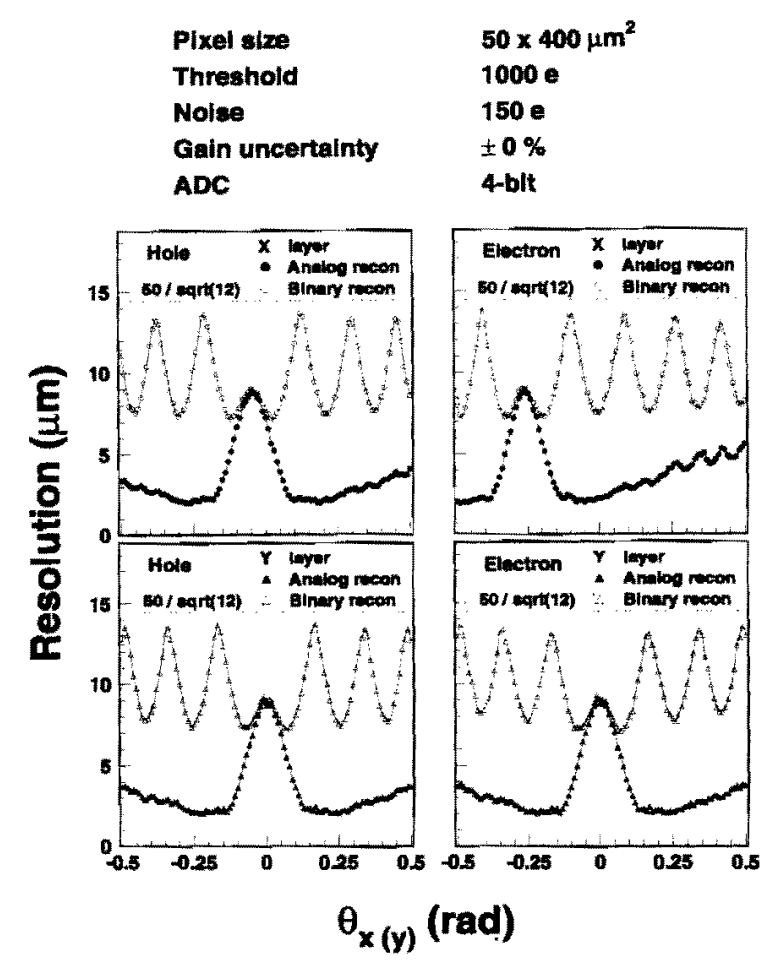

Figure A.9: Resolution for binary readout and 4-bit ADC readout for $50 \mu \mathrm{m}$ pixel small dimension. The magnetic field is parallel to the $\hat{x}$ direction.

electronics combinations used in the test beam run recently completed. Details on the data taken and the analysis procedure will be given in the next section. The comparison between predicted and measured resolution is shown in Figures A.18 and A.19 for two different digitization accuracies ( 8 bits and 2 bits) and different thresholds. The agreement is very good, if we take into account that effects like imperfect alignment, track projection errors and angular resolution are so far neglected. We will introduce them in a Monte Carlo simulation of our beam set-up presently under development.

\section{A.1.1.8 Occupancy studies}

An additional application of our sensor model is a more accurate information on the hit multiplicity associated with a given track angle. We have used this information to achieve a better understanding of several key features of our detector performance.

We have investigated[15] the occupancy in the inner portion of our detector for different event classes and different assumptions on the interaction rate using GEANT. This study has been a crucial input for the engineering group responsible for the design of the pixel readout chip and to test the readout architecture and identify possible bottlenecks in the 

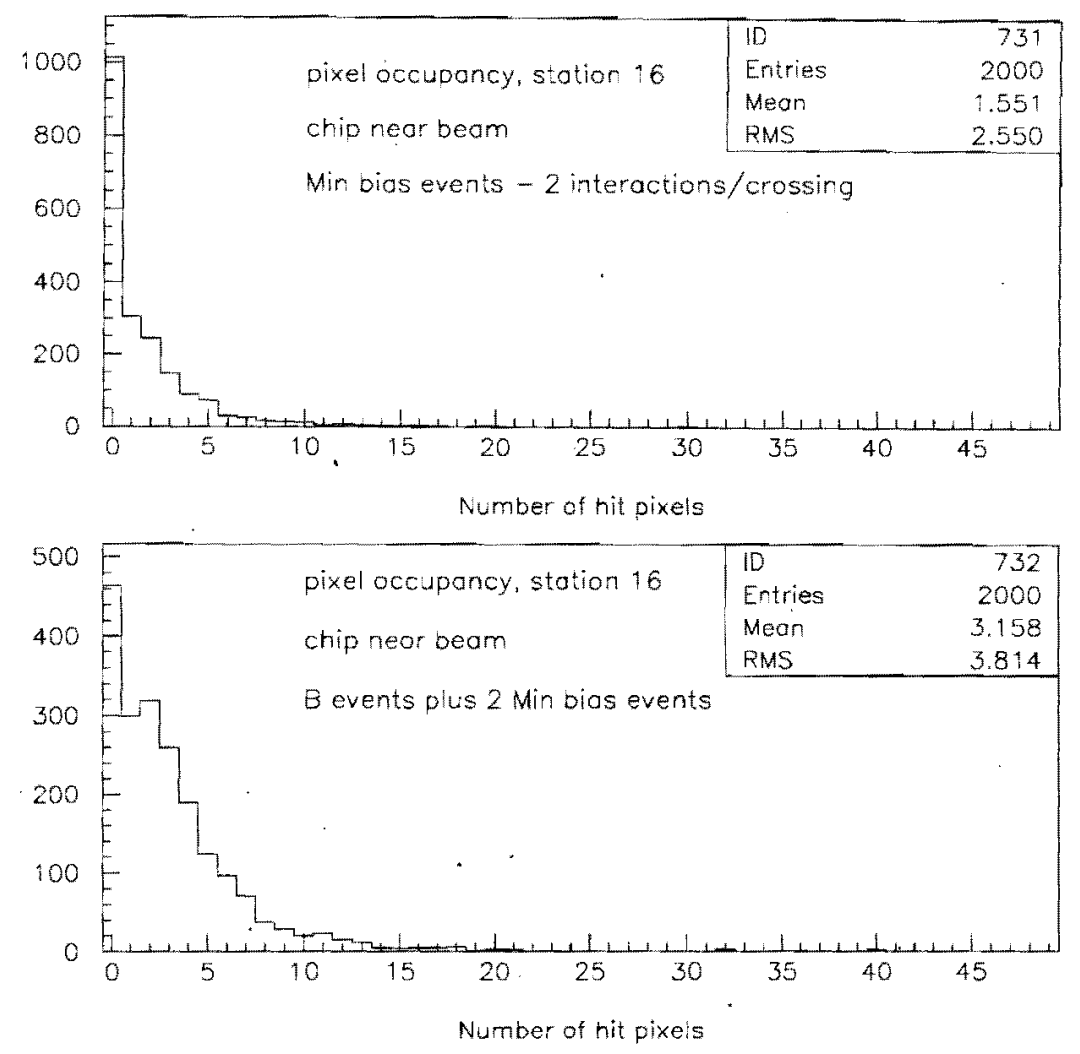

Figure A.10: Number of pixels hit per crossing in the hottest pixel readout chip (nearest to the beam). The upper histogram shows the occupancy expected at a luminosity of $2 \times 10^{32} \mathrm{~cm}^{-2} \mathrm{sec}^{-1}$. The lower histogram shows the occupancy expected in $b$ events at this luminosity.

data throughput. The pixel occupancy has been studied for a chip located with its inner edge $6 \mathrm{~mm}$ from the beam pipe. The chip has been assumed to be $12 \mathrm{~mm} \times 12 \mathrm{~mm}$, with a $50 \mu \mathrm{m} \times 400 \mu \mathrm{m}$ pixel cell. The results are shown in Figure A.10, based on an expected luminosity of $2 \times 10^{32} \mathrm{~cm}^{-2} \mathrm{~s}^{-1}$.

This study also provides data samples for the readout architecture simulation and the trigger processor generator. Initial studies have shown that, assuming a pixel size of 50 $\mu \mathrm{m} \times 400 \mu \mathrm{m}$ and 3 bit flash ADC's digitizing the pixel cell signal, the proposed columnbased data-driven architecture should be capable of achieving the hit readout rate required by the $\mathrm{BTeV}$ experiment [16]. 


\section{A.1.1.9 Assessment of the Sensor Performance}

The characterization program that will enable us to finalize the design of our sensor includes laboratory measurements and beam tests. We successfully started this research program with $p$-stop and $p$-spray sensors of ATLAS design. We have studied the I-V performance of the detectors bump-bonded to FPIX0 and FPIX1 readout chips. Our measurements show that the static properties of these sensors are quite good prior to irradiation.

The results obtained through the large sample of data acquired in an extensive test beam run at Fermilab is discussed in the following section.

This summer we will start a program of irradiation of detector samples. We will start by exposing selected samples to a Co source and measuring the I-V curve following irradiation. Samples will also be exposed to a neutron beam. This will allow us to measure the change in leakage current and breakdown voltage. It is also very important to determine the change in collection properties upon irradiation. To this purpose we are planning to use the transient current and charge technique developed at BNL by V. Eremin, N. Strokan, E. Verbitskaya and $\mathrm{Z} . \mathrm{Li}[17]$. These techniques are based on the analysis of the current and charge pulse shapes which arise when carriers are produced inside the electric field region inside the material under study. These techniques have been shown to provide the most accurate measurement of the effective carrier concentration at high radiation dose and to measure carrier drift mobility.

When radiation hard electronics will be available we will complete our study by irradiating samples of detectors bump bonded to readout electronics. It is important to proceed in a systematic fashion to identify potential problems.

\section{A.1.1.10 Interface with other R\& $D$ efforts}

We have engaged in several collaborative efforts with other groups pursuing the development of sensors that meet our requirements. In particular, we are engaged in joint submissions with the US-CMS and ATLAS pixel groups and we have acquired samples of the ATLAS p-stop and p-spray devices.

We are particularly interested in the ATLAS development effort as the unit cell geometry of their choice matches our needs quite well. We will investigate how close these sensors come to satisfying our needs and we will develop our strategy for future submissions accordingly.

Some of us have also joined the ROSE collaboration and we will adopt any proven technological solution that will emerge from their work. Lastly, we are in close contact with the solid state sensor development group at BNL and we are planning collaborative efforts to develop a deeper understanding on some of the key issues affecting sensor optimization. 


\section{A.1.2 Test Beam Results}

\section{A.1.2.1 Introduction}

A test of several pixel detectors was performed in the Fermilab MTest beam line during the fixed target run of 1999-2000. The purposes of the beam test were to:

- Gain operational experience with pixel detectors, utilizing both p-stop and p-spray varieties of ATLAS sensors, and look for potential problems and sensitivities in the use of pixel detectors for $\mathrm{BTeV}$.

- Determine the spatial measurement resolution of $50 \mu \mathrm{m} \times 400 \mu \mathrm{m}$ pixel elements as a function of particle angle of incidence, number of readout bits describing the charge deposited in each pixel element, sensor bias, readout threshold, and combinations of these parameters.

- Determine the validity of our sensor simulations as predictors of pixel detector performance for future hardware choices.

The beam test was successful in all these aims. The main results are summarized in the body of this proposal. In the following pages, we describe the experimental setup, and present some of the results in greater detail.

\section{A.1.2.2 Pixel Devices Tested}

The pixel detectors tested are all from the "first ATLAS prototype submission [18]," and all have $50 \mu \mathrm{m} \times 400 \mu \mathrm{m}$ pixels. Two of the sensors come from a single wafer manufactured by $\mathrm{CiS}$, and the other three are from a single Seiko wafer. The CiS sensors (one p-stop "ST1" and one p-spray "ST2") were indium bump bonded to FPIX0 readout chips by Boeing North America. FPIX0 is the first generation pixel readout chip developed at Fermilab. Its primary purpose was to establish a front end design appropriate for use at the Tevatron collider with 132 ns between crossings. The FPIX0 readout pixel was designed to match the sensor pixel size of the ATLAS prototypes, but FPIX0 is much smaller than a prototype "single chip" sensor. The instrumented portion of the sensor is 11 columns $\times 64$ rows. Each FPIX0 readout pixel contains an amplifier, a comparator, and a peak sensing circuit. When any comparator fires, a CHIP_OR signal is asserted. FPIX0 provides a zero-suppressed readout of hit pixels. The information read out consists of hit row and column numbers, together with a voltage level which is proportional to the peak pulse height recorded by the peak sensing circuit in the hit cell. For this beam test, the analog output was connected to a buffer amplifer mounted next to the pixel detector. The output of the buffer amplifier was digitized by an 8-bit flash ADC.

The Seiko sensors (two p-stop ST1's and one p-spray ST2) were indium bump bonded to FPIX1 red lout chips by Advanced Interconnect Technology Ltd. (AIT). FPIX1 is the second generation of pixel readout chip developed at Fermilab, and is the first implementation of a high speed readout architecture designed for $\mathrm{BTeV}$. Each cell contains an amplifier very 


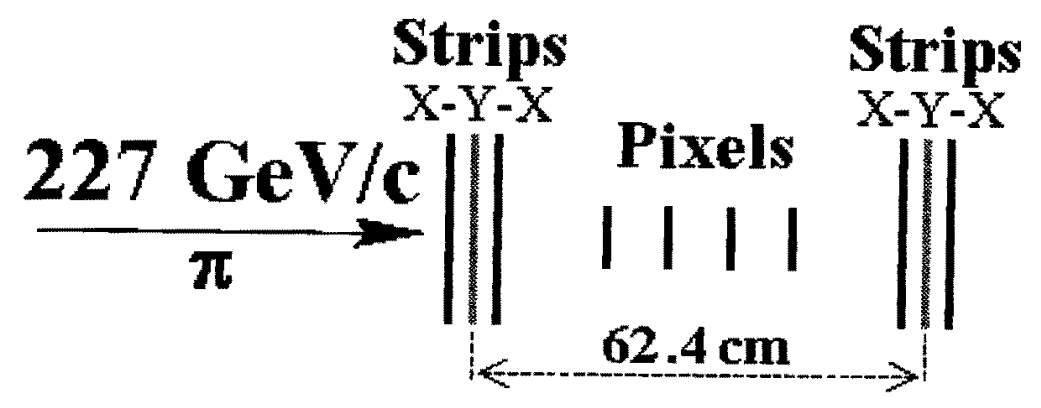

Figure A.11: Schematic drawing of the silicon telescope.

similar to the FPIX0 amplifier. Instead of a single comparator and a peak sensing circuit, each FPIX1 cell contains four comparators, which form a 2-bit flash ADC. FPIX1 is much larger than FPIX0. It contains 18 columns of 160 rows, and is the same size as the ATLAS single chip sensors. However, a minor design error limits the number of rows which may be read out to $\sim 90$ per column.

\section{A.1.2.3 Experimental Setup}

All data described here were collected using a $227 \mathrm{GeV} / \mathrm{c}$ pion beam. This was the highest momentum available in the MTest beamline, and was chosen to minimize multiple scattering. The pixel devices under test were located between two stations of single sided silicon strip detectors (SSD's) as shown in Figure A.11. The pixel detectors were mounted on printed circuit boards which fit tightly into slots machined in an aluminum box. The box was connected rigidly to the mechanical support structure for both SSD stations. A number of slots were provided in the pixel box which allowed detectors to be positioned normal to the incident beam direction. One set of slots allowed a single detector to be positioned at angles of $5,10,15,20$, and 30 degrees with respect to the beam direction. Up to four pixel detectors were tested in the beam at one time. As is discussed below, at this point in our analysis, there is a small uncertainty of 1-2 degrees in the overall rotation of the SSD telescope and pixel test box with respect to the incident beam direction. The pixel detectors were oriented so that the $50 \mu \mathrm{m}$ pitch measured the horizontal (X) coordinate. Each silicon microstrip station contained two planes which measured the $\mathrm{X}$ coordinate, and one plane which measured the vertical $(\mathrm{Y})$ coordinate. Three of the four $\mathrm{X}$-measuring microstrip planes were $20 \mu \mathrm{m}$ strip pitch. The remaining X-plane, and the two Y-planes were $25 \mu \mathrm{m}$ strip pitch. The silicon microstrip detectors were read out using SVX-IIb ASIC's [19]. Because of common mode noise problems, the sparse-scan feature of the SVX-II was not used (every strip was read out). The extrapolation accuracy of this silicon microstrip telescope at the pixel detectors under test was $\sim 2.1 \mu \mathrm{m}$ for tracks with shared charge in adjacent SSD channels.

The readout was triggered by the coincidence of signals from two $15 \mathrm{~cm} \times 15 \mathrm{~cm}$ scintillation counters, positioned upstream and downstream of the silicon telescope and separated from each other by about $10 \mathrm{~m}$. In order to select tracks incident on the active area of the 

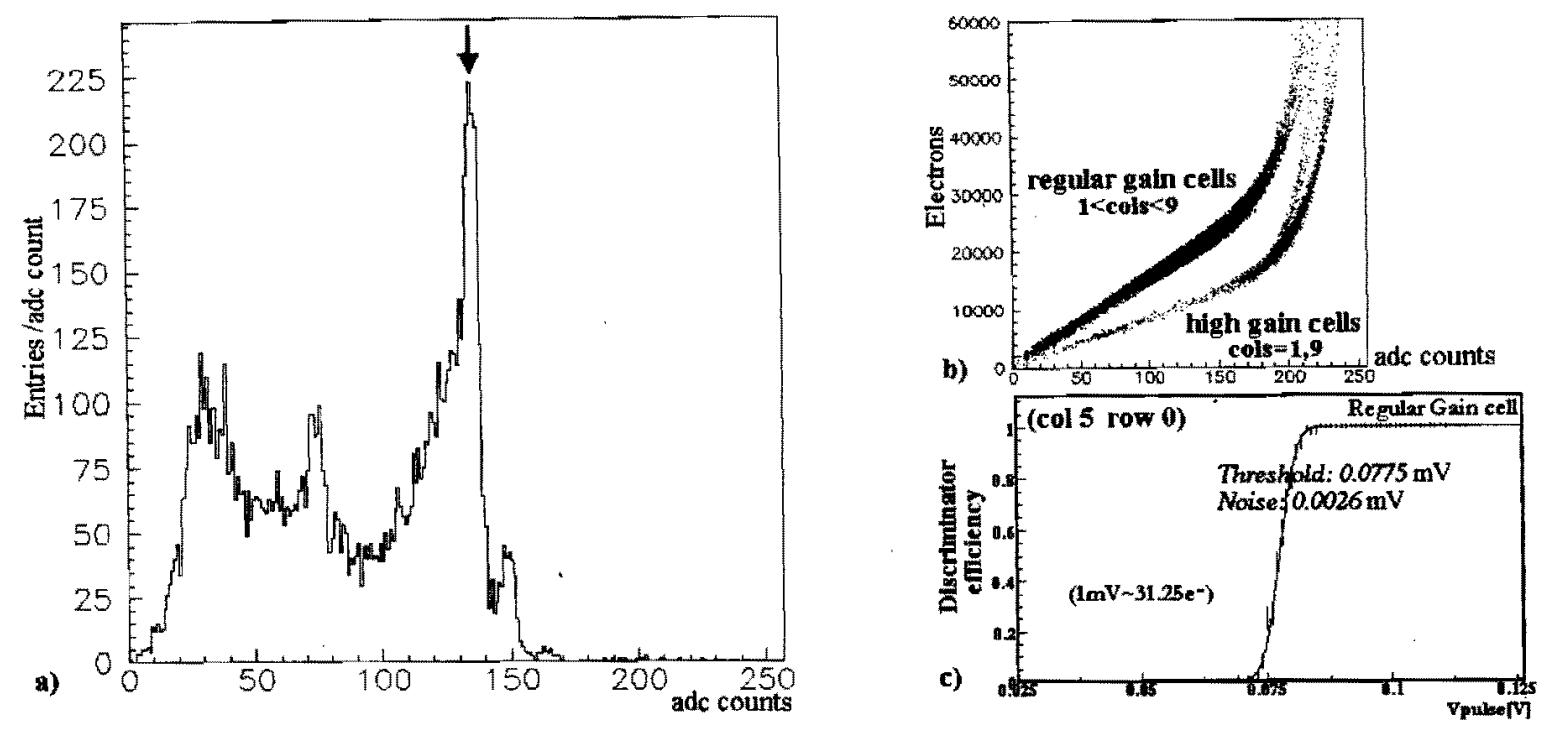

Figure A.12: Calibration for the FPIX0 p-spray (CiS) sensor. Plot a) shows a Tb x-ray spectrum. The arrow indicates the $K_{\alpha}$ peak. Plot $b$ ) shows the pulser calibration data for all channels, and plot c) shows a discriminator threshold curve for a single channel. Curves like the one shown in c) are used to determine the amplifier noise and discriminator threshold dispersion.

pixel detectors, the FPIX0 p-stop CHIP_OR output signal was also required in coincidence with the scintillator signals. The data acquisition system was based on VME, adapted from the CDF SVX test stand. The typical event size was $\sim 6.3$ Kbyte, more than 6.1 Kbyte of which was the unsparsified SSD data. The DAQ system was able to record about 800 events per spill. A total of about 3,000,000 useful events were collected.

\section{A.1.2.4 Calibration}

The pixel detectors were calibrated using a pulser and two x-ray sources ( $\mathrm{Tb}$ and $\mathrm{Ag}$ foils excited by an Am $\alpha$ emitter). The threshold efficiency curve and the analog pulse response for each cell were determined by injecting charge through the integrated calibration capacitance in each FPIX cell, and sweeping the injected pulse amplitude in small steps. For FPIX0instrumented detectors, the absolute calibration of the discriminator thresholds, amplifier noise, and ADC scale, were established by measuring the ADC peak of the known $\mathrm{K}_{\alpha}$ line of the sources. Figure A.12 illustrates the calibration of the FPIX0 p-spray sensor.

FPIX0 contains two types of amplifiers, "high gain" and "standard gain." For most of the data taking, the discriminator threshold for the FPIX0 p-stop was set to a voltage equivalent to $2500 \pm 400 \mathrm{e}^{-}$for the standard gain cells, and $1500 \pm 230 \mathrm{e}^{-}$for the high gain cells. For the FPIX0 p-spray device the corresponding thresholds were typically $2200 \pm 350$ $\mathrm{e}^{-}$and $1250 \pm 160 \mathrm{e}^{-}$. The amplifier noise was measured to be $105 \pm 15 \mathrm{e}^{-}$for standard gain 
cells, and $83 \pm 15 \mathrm{e}^{-}$for for high gain cells of the FPIX0 p-stop sensor. The corresponding noise values for the FPIX 0 p-spray sensor were $80 \pm 10 \mathrm{e}^{-}$for standard gain cells, and $67 \pm 8$ $\mathrm{e}^{-}$for high gain cells. In addition, we found an equivalent charge noise due to the external buffer amplifier and ADC of $400 \pm 150 \mathrm{e}^{-}$for FPIX0 p-stop standard gain cells, and $205 \pm 95$ $\mathrm{e}^{-}$for FPIX0 p-stop high gain cells. The corresponding external noise values for the FPIX0 p-spray sensor were $185 \pm 20 \mathrm{e}^{-}$and $100 \pm 20 \mathrm{e}^{-}$.

The calibration of the four thresholds input to the FPIX1 chips (one for each comparator in the 2-bit FADC implemented in every cell) and the amplifier noise measurement for FPIX1instrumented detectors, was done by sweeping the thresholds and measuring the differential counting rate due to the $\mathrm{x}$-ray source. We found a set of four average threshold values in nominal running conditions for the FPIX1 p-stop of about $3780 e^{-}, 4490 e^{-}, 10290 e^{-}$, and $14680 e^{-}$, with a spread of about $380 e^{-}$. The amplifier noise was measured to be $110 \pm 30 e^{-}$. The quoted spread refers to channel to channel variation on a single chip and is an rms value.

It should be noted that FPIX1 can be operated successfully at very low threshold. We are currently doing bench tests with an ATLAS tile-1 sensor, bump bonded to five FPIX1 chips, and read out through a prototype "High Density Interconnect" (HDI) flexible circuit. This test module can be operated without oscillation with discriminator threshold set below $1500 \mathrm{e}^{-}$. The FPIX1 sensor hybrids that we tested in the beam were mounted on a printed circuit board which was designed for bench tests. In order to interface this board to the readout system which we designed for FPIX0, we fabricated a daughter board which was mounted on the FPIX1 printed circuit board. This arrangement suffered from noise and pickup problems that we never fully overcame. Consequently, we were not able to test the FPIX1-instrumented sensors in the beam with very low discriminator thresholds.

\section{A.1.2.5 Charge collection}

Charge collection can be studied in detail for the FPIX0 instrumented sensors, thanks to the 8-bit analog information, and the absolute calibration provided by the use of x-ray sources. The measured pulse height distributions were fit using a Landau function convoluted with a Gaussian [20]:

$$
f(E)=N \int_{-\infty}^{+\infty} d E^{\prime} \frac{e^{-\frac{\left(E-E^{\prime}\right)^{2}}{2 \sigma_{g}^{2}}}}{\sqrt{2 \pi \sigma_{g}^{2}}} \frac{\phi\left(\frac{E^{\prime}-E_{m p}}{\xi}+\lambda_{0}\right)}{\xi}
$$

In this expression, $\mathrm{N}$ is a normalization constant, $\frac{\phi\left(E^{\prime}\right)}{\xi}$ is an ordinary Landau function, with $\lambda_{0}=-0.223$, so that $E_{m p}$ is the most probable energy loss ${ }^{1}$, and $\sigma_{g}$ is the standard deviation of the Gaussian function. The Landau curve is derived in the literature assuming that the energy loss is due to Rutherford scattering between the charged particle and free electrons. The Gaussian takes into account the smearing due to the bonding of electrons in the silicon crystal, and to the finite resolution of the detector and readout electronics. The fit is done with $\mathrm{N} / \xi, E_{m p}, \xi$, and $\sigma_{g}$ as free parameters (P1, P2, P3, and P4 in the fit), using the energy

\footnotetext{
${ }^{1}$ When $E^{\prime}=E_{m p}$ the function $\phi(\lambda)$ reaches its maximum; the Gaussian shifts the maximum only by a small amount.
} 
required to create an electron-hole pair in silicon $(3.6 \mathrm{eV})$ to convert energy in $\mathrm{eV}$ to charge in $\mathrm{e}^{-}$.

Figures A.13a and A.14a show the pulse height distributions for the FPIX0 p-spray and p-stop detectors respectively. The "improved Landau" function fits the experimental data quite well, except that there is an obvious peak at low pulse height in Figure A.13a, indicating charge collection inefficiency in the p-spray sensor, and a bump at $\sim 50000 \mathrm{e}^{-}$in Figure A.14a, which is due to saturation of the off-chip buffer amplifier/ADC combination. Figure A.13b shows that the p-spray sensor suffers sizeable charge collection inefficiency between columns, especially on the column boundaries which include the "punch-through biasing" network. Our measurement of this charge loss ${ }^{2}$ is consistent with previous measurements made by the ATLAS pixel collaboration[21]. Figure A.14b shows the spatial distribution of the tracks for which the charge collected in the FPIX0 p-stop sensor is less than 15000 $\mathrm{e}^{-}$(under this value the Landau distribution predicts a very small fraction of events). This plot shows that the p-stop sensor also suffers from a very small amount of charge collection inefficiency, and that this inefficiency is concentrated at the four corners of the sensor pixels.

We find that the charge collected by the CiS p-spray sensor is about $24 \%$ less than the charge collected by the p-stop sensor. This measurement is also in agreement with the ATLAS test beam results [21]. The most probable and average charge collected are respectively $20000 \pm 70 \mathrm{e}^{-}$and $23100 \pm 70 \mathrm{e}^{-}$for the CiS p-spray sensor ${ }^{3}$. For the CiS p-stop sensor, the most probable charge collected is $24730 \pm 30 \mathrm{e}^{-}$, and the average charge collected is $30100 \pm 30 \mathrm{e}^{-}$.

\section{A.1.2.6 Charge Sharing}

As is discussed above in the pixel sensor simulation section, the charge deposited by a single track is often shared by more than one pixel. The amount of charge sharing is determined by geometry (the number of cells crossed by the track), and by diffusion of the electrons drifting in the silicon. The fraction of the time that each cluster size is observed (for the regular gain cells of the FPIX0 p-stop sensor) are listed in table A.1. The data show clearly the dominance of two cluster sizes for each incident beam angle, as expected from geometry.

For each angle, we studied the influence on charge sharing of different detector bias voltages and readout discriminator threshold settings (see Figure A.15). Since charge-sharing is determined primarily by geometry at large track angles, the effect of changing the bias voltage is negligible for track angles of 10 .degrees and above. However, at small angles, for which charge sharing is primarily a function of electron diffusion, lower detector bias increases charge-sharing. As expected, the discriminator threshold is important at all track angles; lower threshold always translates directly into more charge sharing.

\footnotetext{
${ }^{2}$ These charge losses are not intrinsic to the p-spray technology, but are a feature of this particular sensor design. The ATLAS pixel group has reported that their new p-spray sensor has charge collection properties very similar to what we observe for the CiS p-stop sensor.

${ }^{3}$ This measurement is done using only tracks far away from the inter-pixel boundary.
} 

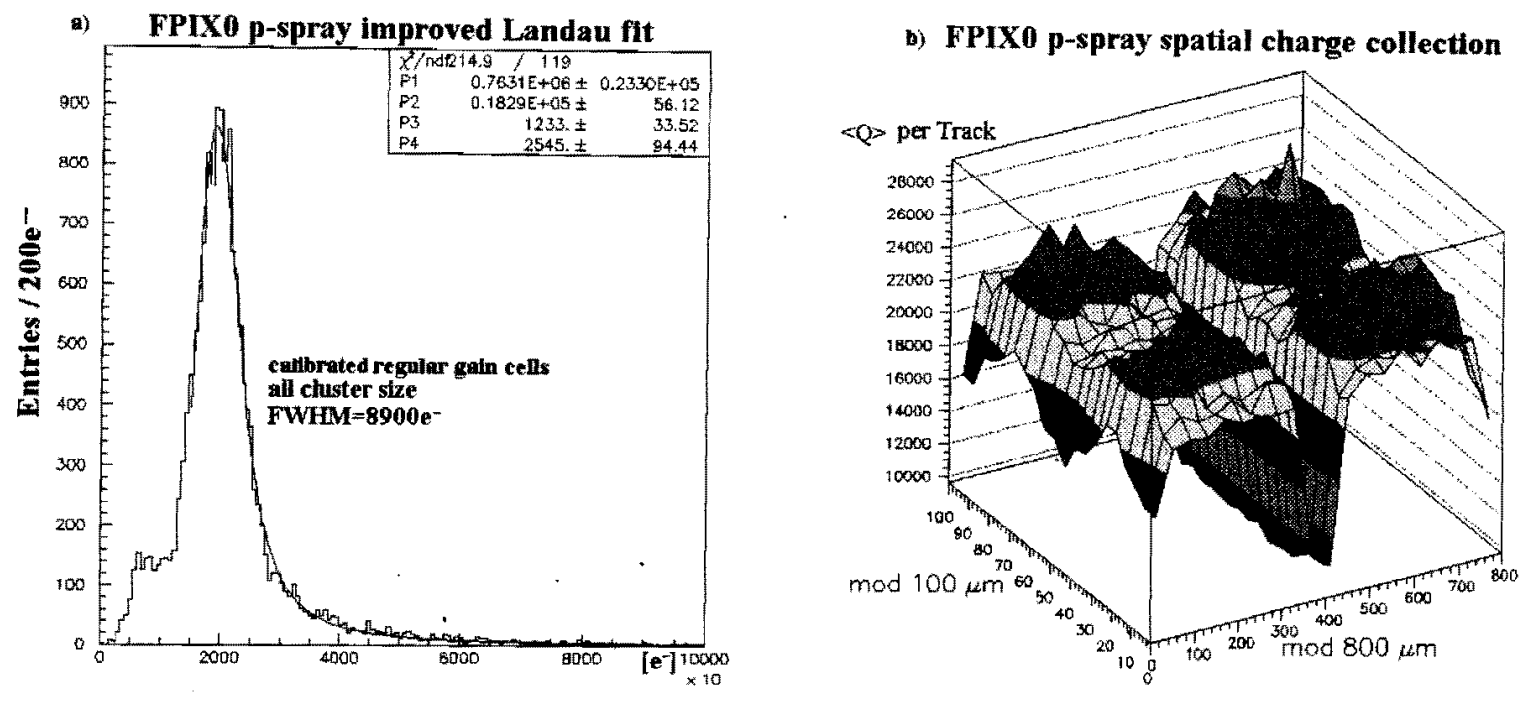

Figure A.13: The plot on the left shows the "improved Landau" fit of the pulse height distribution for the CiS p-spray sensor bump-bonded to an FPIX0 chip, when the track is at normal incidence. The plot on the right shows the average charge collected as a function of track position.
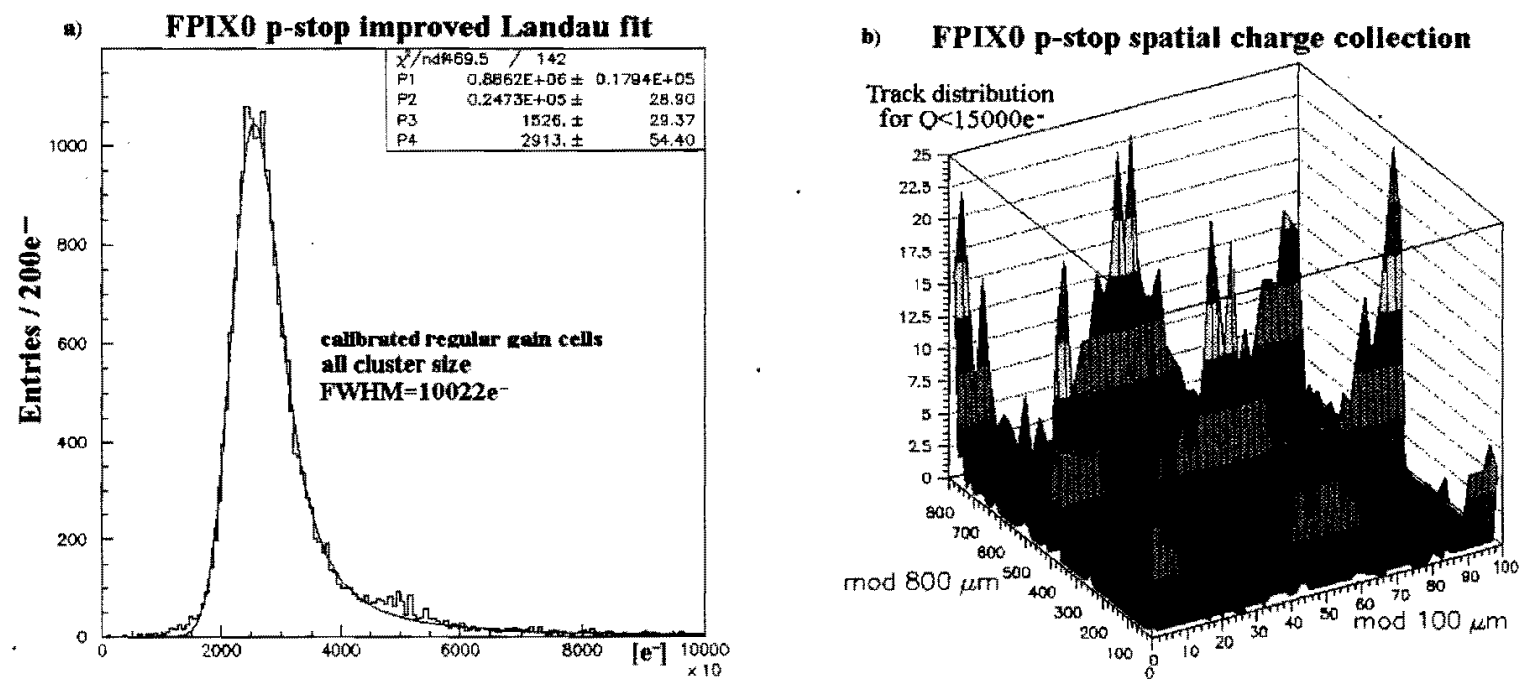

Figure A.14: The plot on the left shows the "improved Landau" fit of the pulse height distribution for the CiS p-stop sensor bump-bonded to an FPIX0 chip, when the track is at normal incidence. The plot on the right shows the distribution of tracks that leave a signal less than $15000 \mathrm{e}^{-}$in the detector.

\section{A.1.2.7 Spatial Resolution}

The tracks used to study pixel resolution were fit using data from the SSD telescope and from pixel detectors other than the device under test, using a Kalman-filter [22]. The prediction 


\begin{tabular}{|l|l|l|l|l|l|l|}
\hline$[$ degs $]$ & $\mathrm{CS}=1$ & $\mathrm{CS}=2$ & $\mathrm{CS}=3$ & $\mathrm{CS}=4$ & $\mathrm{CS}=5$ & $C S \geq 6$ \\
\hline 0 & $.639 \pm .0034$ & $.328 \pm .0033$ & $.017 \pm .0010$ & $.009 \pm .0019$ & $.0034 \pm .0004$ & $.0025 \pm .0004$ \\
5 & $.433 \pm .0035$ & $.527 \pm .0035$ & $.022 \pm .0011$ & $.010 \pm .0011$ & $.0041 \pm .0004$ & $.0028 \pm .0004$ \\
10 & $.090 \pm .0018$ & $.846 \pm .0025$ & $.040 \pm .0016$ & $.015 \pm .0009$ & $.0055 \pm .0005$ & $.0029 \pm .0005$ \\
15 & - & $.635 \pm .0034$ & $.332 \pm .0034$ & $.022 \pm .0011$ & $.0080 \pm .0007$ & $.0034 \pm .0004$ \\
20 & - & $.209 \pm .0027$ & $.741 \pm .0030$ & $.031 \pm .0014$ & $.0124 \pm .0009$ & $.0060 \pm .0006$ \\
30 & - & - & $.178 \pm .0024$ & $.769 \pm .0029$ & $.041 \pm .0018$ & $.0115 \pm .0009$ \\
\hline
\end{tabular}

Table A.1: Cluster size fraction for various angles of incidence for FPIX0 p-stop regular gain cells. For each angle, the sample size is about 20000 tracks. These data were collected with a sensor bias voltage of $-140 \mathrm{~V}$, and a discriminator threshold of $2500 \mathrm{e}^{-}$.
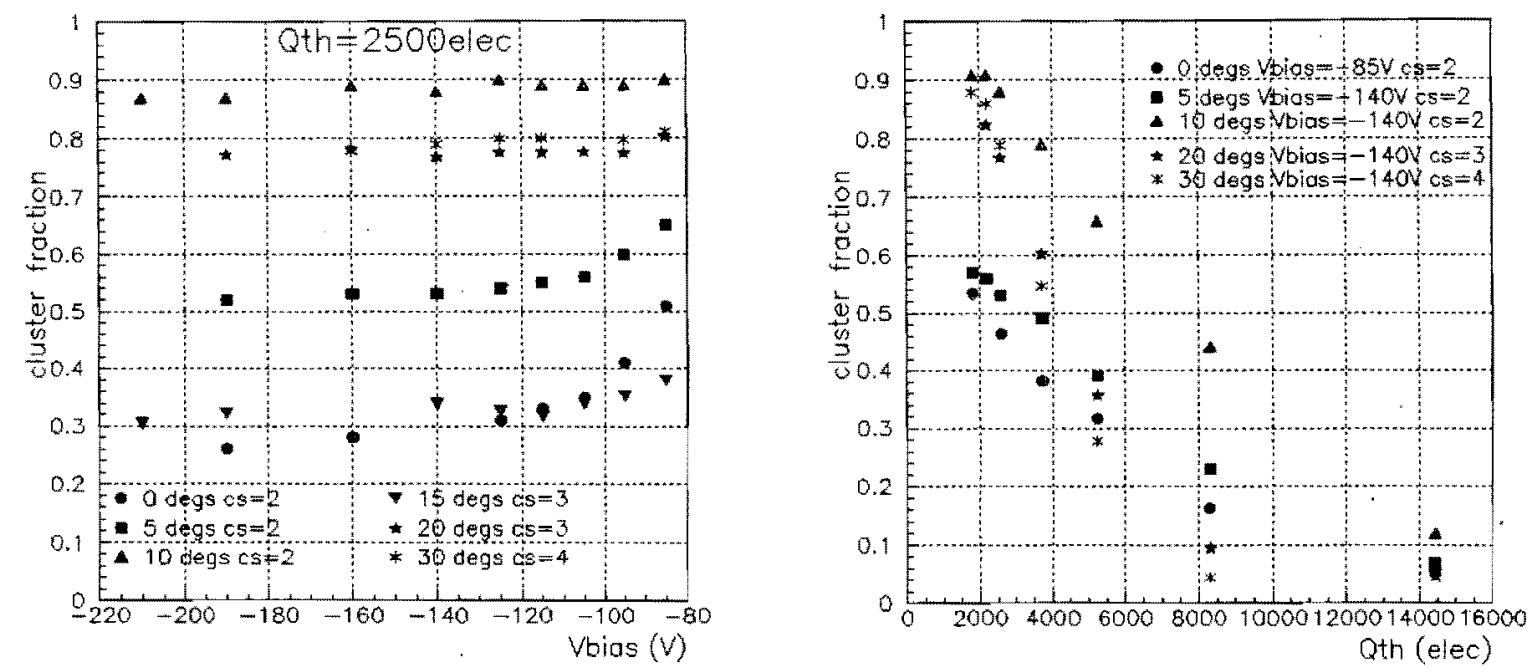

Figure A.15: The plot on the left shows the fraction of the time that the indicated cluster size was recorded as a function of detector bias for a fixed readout threshold and a variety of angles. The plot on the right shows the same fraction as a function of the detector readout threshold for a fixed bias voltage and a variety of angles. These data were recorded by the p-stop FPIX0 detector, which has a depletion voltage of $\sim-85 \mathrm{~V}$.

error at the pixel device under test varied, depending on the cluster width (and therefore the measurement precision) of each of the points included in the fit, and on the number of pixel planes in the test setup for a given data set (and therefore the amount of multiple scattering). For the bulk of the data taking, two pixel planes were tested at a time. In this configuration, the average extrapolation error along the short pixel dimension (X) was about $2.5 \mu \mathrm{m}$. The prediction error was reduced to $2.1 \mu \mathrm{m}$ if tracks were required to have cluster size two in both the most upstream and the most downstream X-measuring SSD planes.

No projection error has been subtracted from the measurement resolutions reported here. 
For all angles except 30 degrees, the results presented were obtained by demanding cluster size two in the most upstream and downstream X-measuring SSD planes. Less data were collected at 30 degrees than at the other angles. Consequently, no cluster size cut was made in the analysis of the 30 degree data.

The coordinate measured by a pixel detector is given by the position of the center of the cluster of hit pixels associated with a track, plus a correction (conventionally called the $\eta$ function) which is a function of the charge sharing, the cluster width, and the track angle. For this analysis, we have used a "head-tail" algorithm for computing the $\eta$ function, which ignores the charge deposited in pixels on the interior of a cluster, and uses only the charge deposited on the edges of the cluster. The histogram on the left side of Figure A.16 shows the distribution of $\eta=\frac{\left(q_{R}-q_{L}\right)}{\left(q_{R}+q_{L}\right)}$, where $q_{R}$ is the charge measured in the "right" hand pixel in a cluster of two, and $q_{L}$ is the charge measured in the "left" hand pixel in the cluster, for data taken with beam normally incident on the FPIX0 p-stop sensor. The asymmetry of this distribution indicates that, in fact, there was a small angle between the beam and the normal to the pixel detector. The plot on the right shows the difference between the predicted X position (in the plane of the pixel detector) and the edge between the two hit pixels (the "digital position"), as a function of $\eta$. The $\eta$ function for cluster size two and normal incidence is the function required to make this distribution a constant, with an average value of zero. For each detector, we have determined a set of $\eta$ functions using plots like the one shown on the right hand side of Figure A.16.
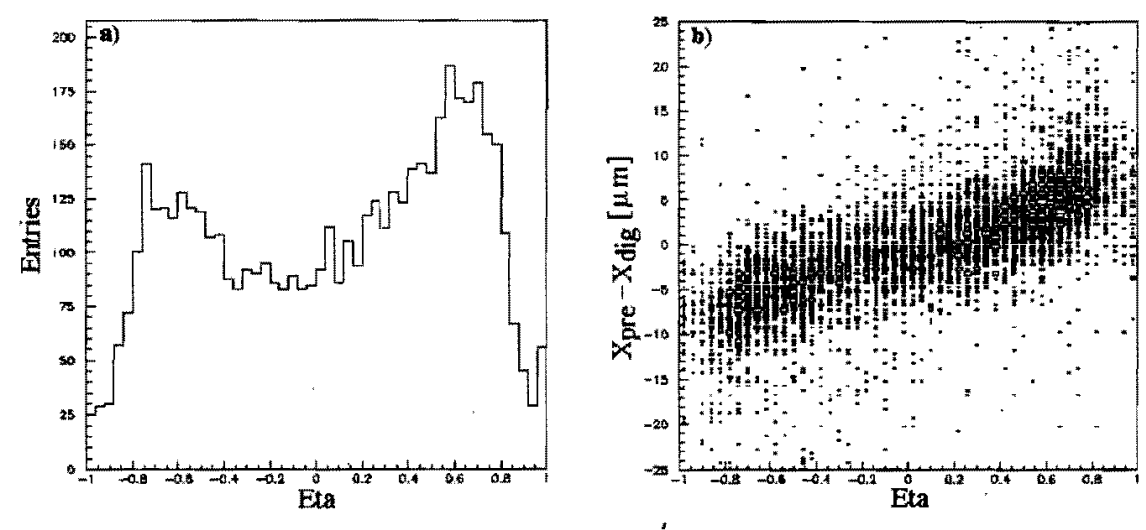

Figure A.16: The histogram on the left is the $\eta$ distribution for data collected with beam normally incident on the FPIX0 p-stop sensor. An entry is made in this histogram only if exactly two adjacent pixels in a single column are hit. The plot on the right shows the difference between the predicted track X-position and the position of the edge between the two hit pixels, as a function of $\eta$ for the same data.

The residual distributions for the FPIX0 p-stop detector, for data taken with $\mathrm{V}_{\text {bias }}=$ $-140 \mathrm{~V}$, and $\mathrm{Q}_{t h}=2500 \mathrm{e}^{-}$, are shown in Figure A.17. Each residual distribution is fit to a Gaussian (shown superimposed in Figure A.17). Clearly, the residual distributions are not Gaussian. This is true especially at zero and five degrees, where no charge sharing 


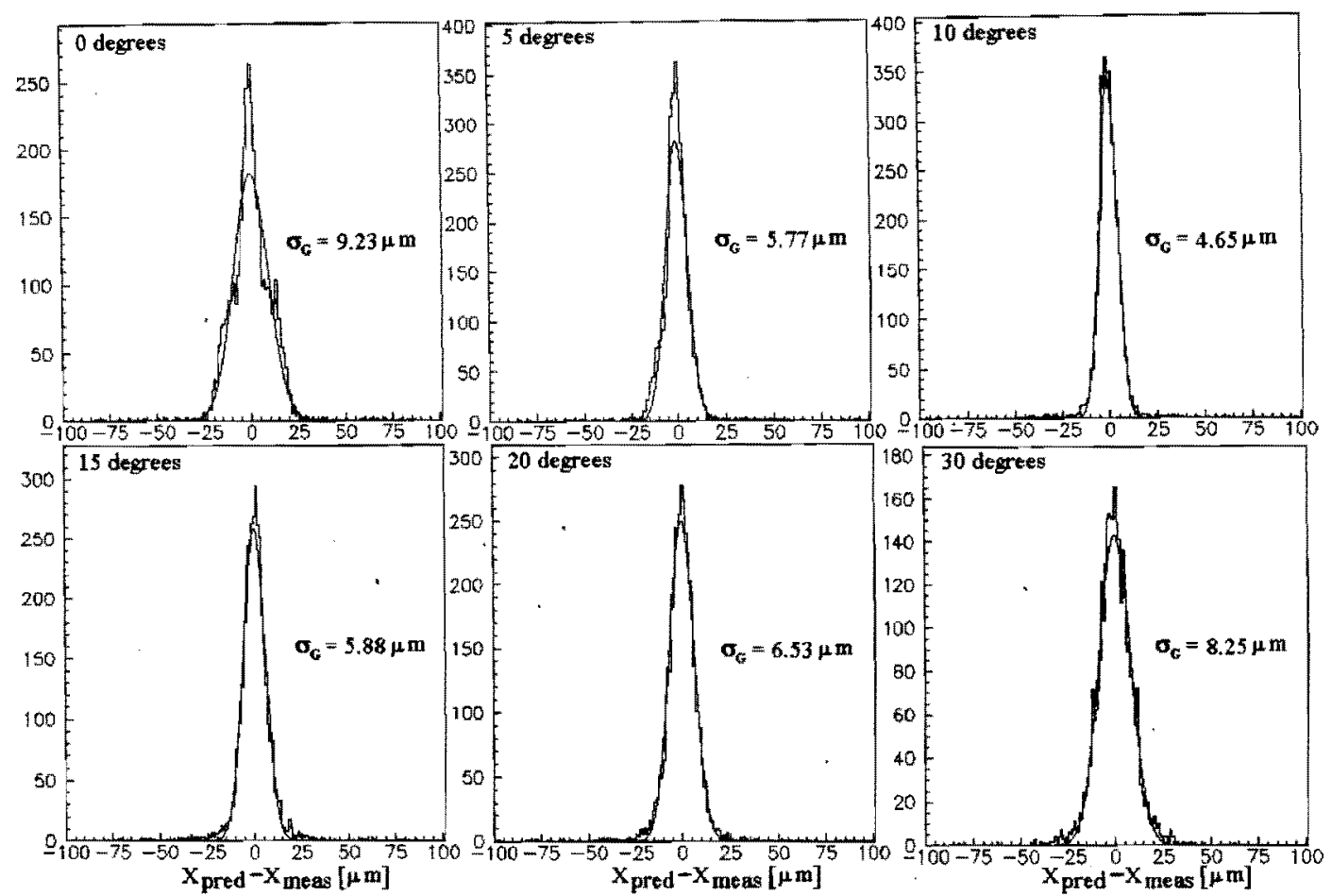

Figure A.17: Residual distributions for the FPIX0 p-stop detector. $\sigma_{G}$ is the standard deviation of the Gaussian fit to each residual plot.

information is available a significant fraction of the time, since only one pixel is hit. The residual distributions also have more entries far from zero than the Gaussian fits. These "tails" are due to the emission of $\delta$-rays, and are discussed below. Nonetheless, the Gaussian standard deviations provide a reasonably good characterization of the width of the central peak for all of the plots.

Figure A.18 shows our simulation result for the resolution as a function of angle, given a discriminator threshold of $2500 \mathrm{e}^{-}$. The points shown superimposed on the simulation result are the Gaussian standard deviations from the fits of Figure A.17. We have also computed residual distributions for this data set without using any charge sharing information. These "digital" resolution results are included in Figure A.18, superimposed on our simulated digital resolution.

Using this method, we have accumulated residual distributions as a function of incident beam angle for all five pixel sensors. All of the results are in reasonable agreement with our simulations. Figure A.19 shows the simulation and experimental results for the FPIX1 p-stop detector. These results are slightly worse than the results that we obtained by degrading the FPIX0 p-stop pulse height information to 2-bits equivalent (see Figure 4.6 in the body of this proposal). This is because the FPIX1-instrumented detector was operated with a discriminator threshold of $\sim 3780 \mathrm{e}^{-}$, while the FPIX0-instrumented detector was operated 


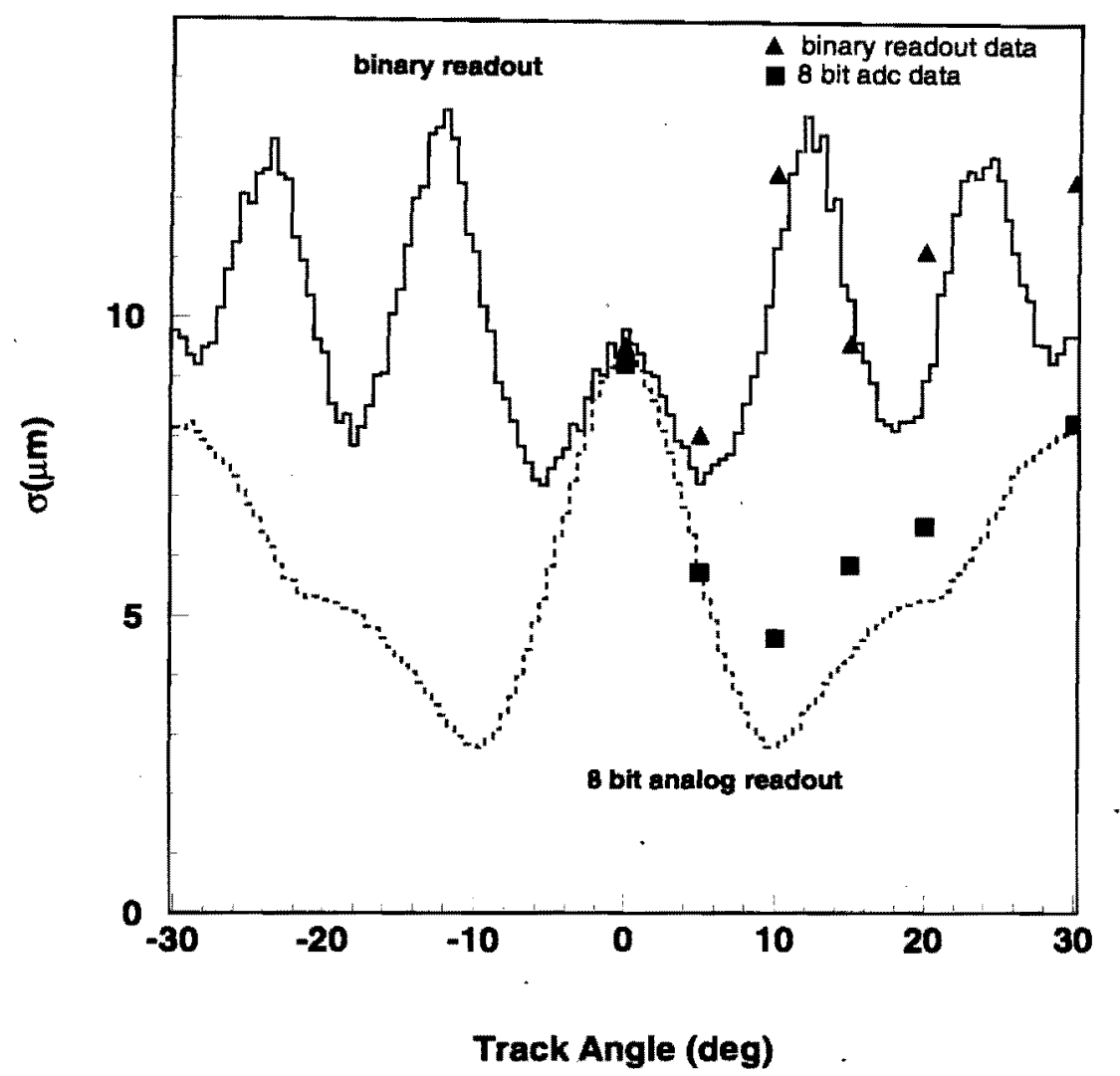

Figure A.18: Position resolution as a function of beam incidence angle for the FPIX0instrumented CiS p-stop sensor. The curves are the simulated RMS for $\mathrm{Q}_{\text {thres }}=2500$ $\mathrm{e}^{-}$. The oscillating curve is the simulated digital resolution; the lower curve assumes 8-bit charge digitization. The squares are the Gaussian $\sigma$ 's shown in Figure A.17, and the triangles are the $\sigma$ 's extracted from fits to residual distributions made without using charge sharing information.

with a discriminator threshold of $\sim 2500 \mathrm{e}^{-}$. Figure A.19 shows that our simulation correctly describes the degradation of position resolution with increased discriminator threshold.

Figure A.20 shows how the position resolution is affected by changes in the sensor bias voltage and the discriminator threshold. As expected, these curves are highly correlated to the data presented in Figure A.15. At a given angle, more charge sharing translates directly into better position resolution.

The $\mathrm{BTeV}$ pixel detector will be exposed to a very non-uniform radiation dose. In our 


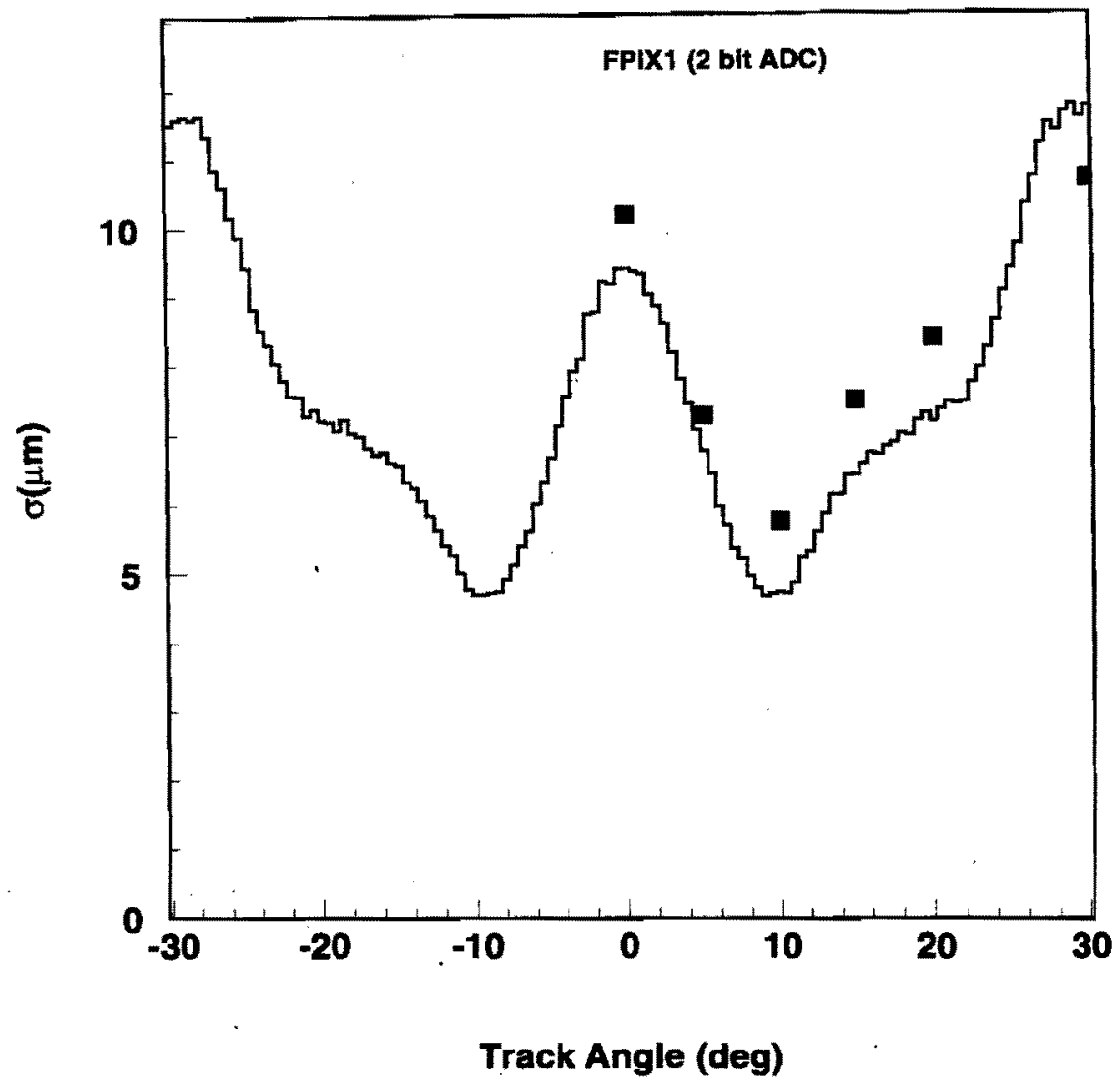

Figure A.19: Position resolution as a function of beam incidence angle for an FPIX1instrumented Seiko p-stop sensor. The curve is the simulated RMS for $\mathrm{Q}_{\text {thres }}=3780 \mathrm{e}^{-}$, assuming 2-bit charge digitization. The squares are $\sigma$ 's from Gaussian fits to the residual distributions.

baseline design, a single sensor will extend from the outer edge of the detector, $5 \mathrm{~cm}$ from the beamline, to the inner edge of the detector, $6 \mathrm{~mm}$ from the beamline. As shown in Figure A.1, this will mean that the inner section of the sensor will radiation damage much faster than the outer section. Consequently, the depletion voltage of the inner section will change (first decreasing, and then increasing) much faster than the outer section. One bias voltage will be applied to the sensor, but the outer section will generally operate very overdepleted, while the inner section will likely be only slightly over-depleted. The insensitivity of the pixel position resolution to the degree of over-depletion shown in Figure A.20 is thus very encouraging. These data suggest that there will be little, if any, degradation in the position resolution as a function of radiation exposure until it is no longer possible to fully deplete the inner section of the sensor. At this point, the degradation in resolution due to loss of signal can be estimated using the results presented in Figure A.20. 

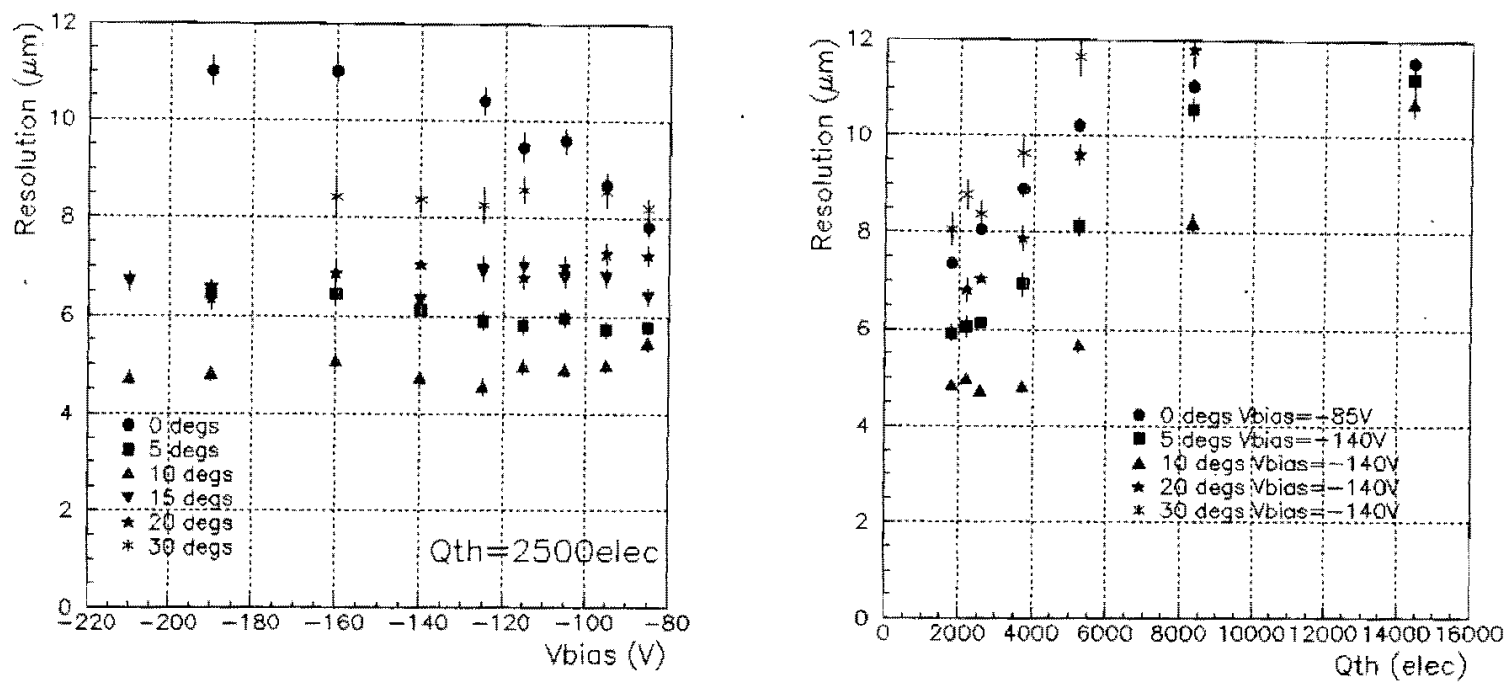

Figure A.20: Spatial resolution versus bias voltage for readout threshold and track angle fixed (left plot). Spatial resolution versus readout threshold for bias voltage and track angle fixed (right plot). The data are from FPIX0 p-stop.

\section{A.1.2.8 More Accurate Fits to the Residual Distributions}

The pixel residual distributions deviate from Gaussian in two important ways. First, for angles less than $\operatorname{Arctan}(50 / 300)=9.5$ degrees, some tracks pass through one pixel only. For these single pixel clusters, the residual distributions are almost square. In this case, the residual can be fit by the function:

$$
F_{1}(x)=\int_{-\frac{p}{2}+W_{D}}^{\frac{p}{2}-W_{D}} d x \frac{F_{1}^{0}}{\sqrt{2 \pi \sigma_{W_{D}}^{2}}} e^{-\frac{x^{2}}{2 \sigma_{W_{D}}^{2}}}
$$

This is a square convoluted with a Gaussian representing the fact that for tracks near an edge, diffusion spreads a fraction of the charge into the neighboring pixel. The total charge lost, which fluctuates from track to track, determines whether or not the neighbor receives enough charge to fire its discriminator.

The second factor that makes the pixel residual distributions non-Gaussian is the emmision of $\delta$-rays. For relativistic particles, $\delta$-rays are emitted at a large angle with respect to the particle direction. Very low energy $\delta$-rays are emitted perpendicular to the track direction. Even $200 \mathrm{keV} \delta$-rays are emitted at an angle of $\sim 66$ degrees[23]. These $\delta$-rays travel a long distance in the pixel detector, and deposit energy as they do so. Low energy $\delta$-rays which stop in one of the pixels crossed by the particle skew the charge sharing and degrade the resolution. Higher energy $\delta$-rays cross one or more pixel boundaries and skew the position measurement even more. We have found that our experimental residual distributions for cluster sizes greater than one can be fit using a function which is the sum of a Gaussian term 
and a term which is a square with edges that decrease like a power of $1 / \mathrm{x}$ :

$$
F(x)=F_{\text {Gauss }}(x)+F_{\text {power-law }}(x)
$$

where $F_{\text {Gauss }}$ is a Gaussian, and $F_{\text {power-law }}$ is defined in the following way:

$$
F_{\text {power-law }}(x)=\left\{\begin{array}{c}
\frac{A_{p l}}{\left|r_{c u t-o f f}\right| \gamma} \text { for }|x|<r_{c u t-o f f} \\
\frac{A_{p l} f}{|x| \gamma} \text { for }|x|>r_{\text {cut-off }}
\end{array}\right.
$$

$A_{p l}$ is a normalization constant, $r_{c u t-o f f}$ is the half width of the constant term, and $\gamma$ is the exponent of the power law.

Figure A.21 shows the residual distributions for the FPIX0 p-spray detector taken with the beam (nominally) at normal incidence. The plot on the left shows the residual for onepixel clusters, with a fit to equation A.7 superimposed. The plot on the right shows the residual for clusters of two or more pixels, with a fit to equation A.8 superimposed. The distributions are shown using a log scale to emphasize the fact that these functions provide good fits to the tails of the distributions.
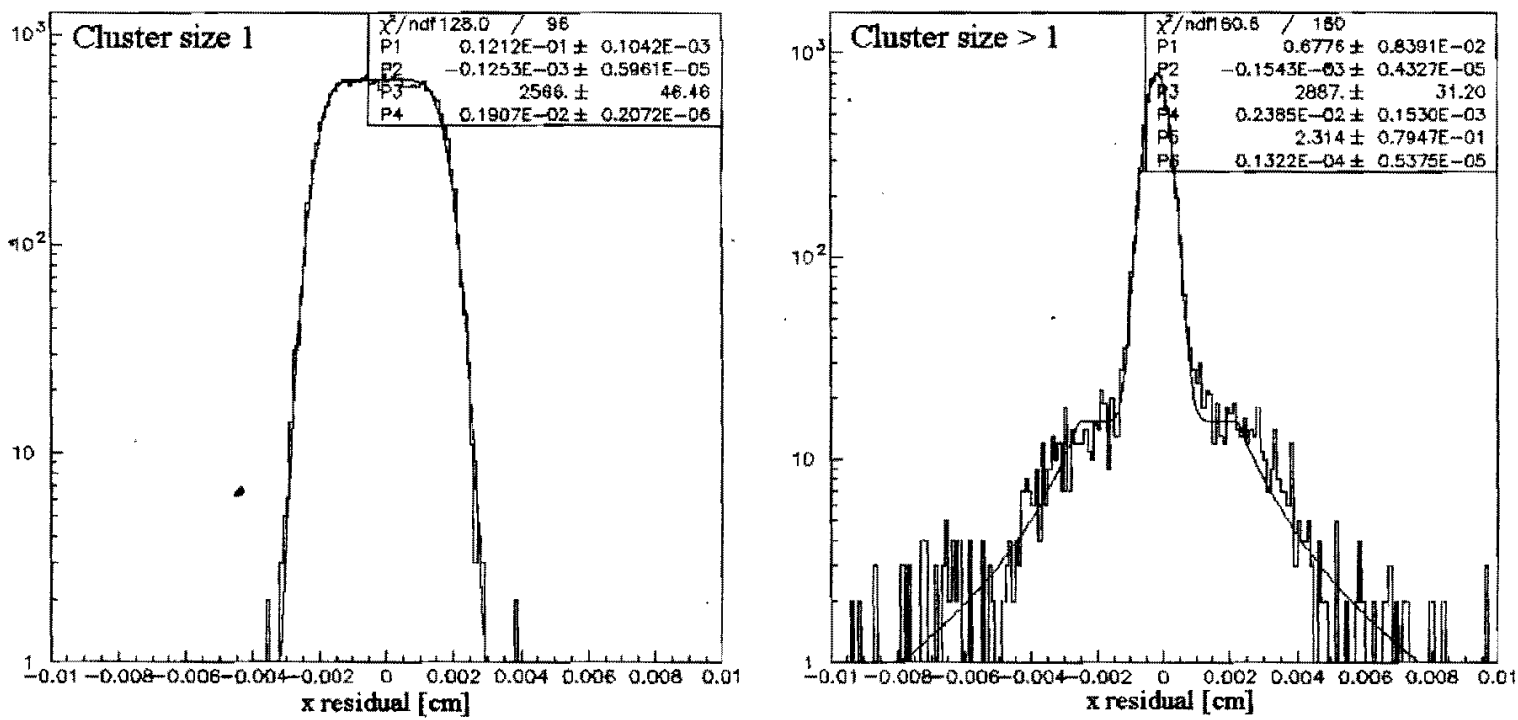

Figure A.21: Residual distributions of FPIX0 p-spray detector at zero degrees fitted with the functions described in this chapter. The plot on the left shows the distribution for cluster size 1 and the plot on the right for clusters having more than one hit.

All of our residual distributions are fit well using these functional forms. Moreover, we find that all of the residual distributions can be reasonably well described by equation A.8, using one set of parameters for $F_{\text {power-law }}(x)$. For all of these distributions, we find a satisfactory representation of the data with $\gamma=2, r_{\text {cut-off }}=15 \mu \mathrm{m}$, and $A_{p l}$ set so that $F_{\text {power-law }}$ accounts for $20 \%$ of the total number of entries in the distribution. 
For the simulations of physics processes discussed in the body of this proposal, the pixel resolution has been approximated by a Gaussian whose width is a piecewise linear function of the track angle. This function is shown in Figure 4.6 in the body of this proposal. For all Level 1 trigger simulations, we have added tails to this representation of the pixel resolution function using equation A.8, with the power-law parameters listed above. This slightly overestimates the tails for most angles of incidence, as can be seen in Figure A.22, and we are encouraged by the fact that the inclusion of these tails has only a very small affect on the Level 1 trigger.
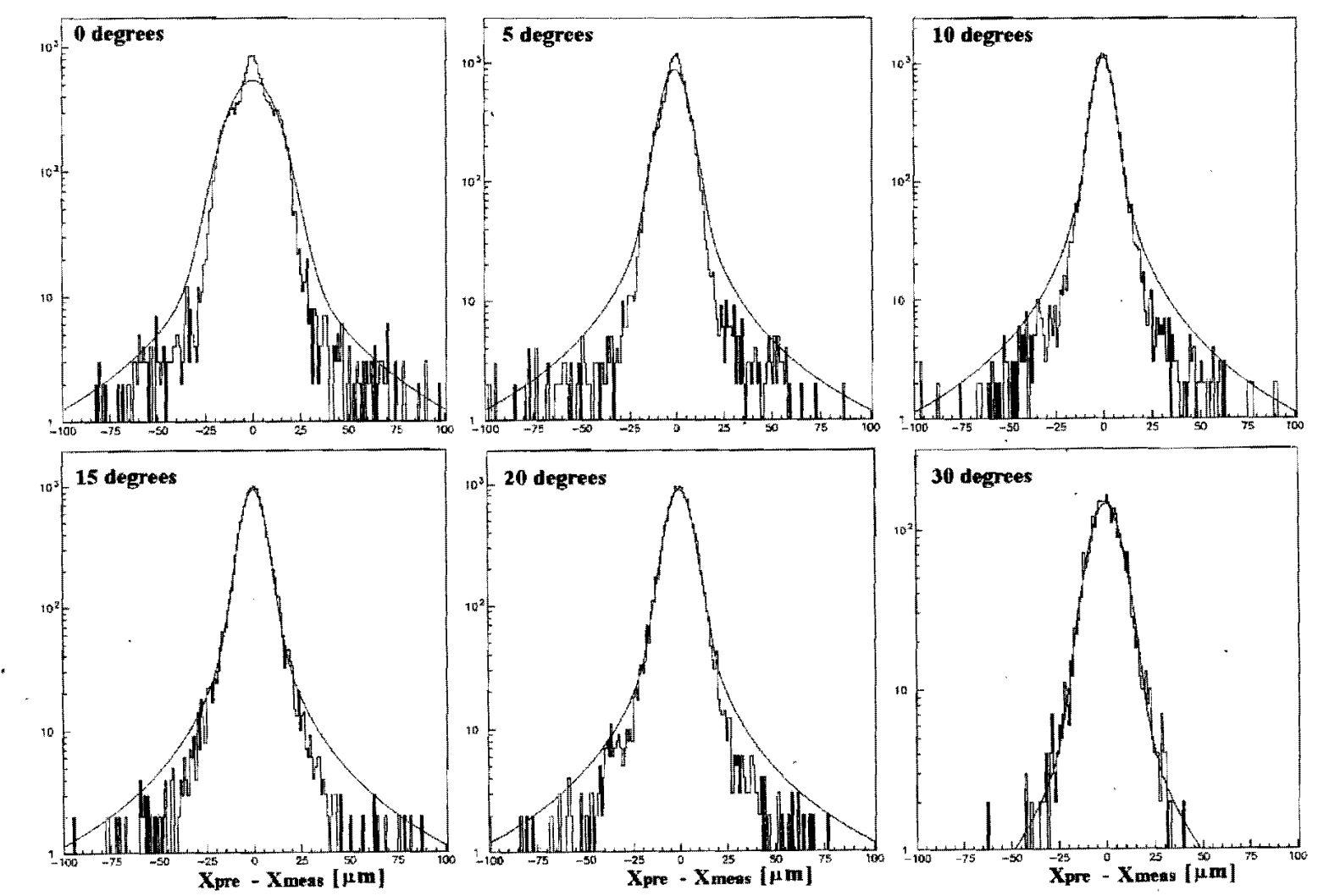

Figure A.22: Residual distributions for the FPIX0 p-stop detector. The data are the same as shown in Figure A.17, plotted here using a log scale to make the tails of the distributions easier to see. The curves superimposed on the plots are the resolution functions which were used in Monte Carlo studies of the BTeV trigger. 


\section{A.1.3 Electromagnetic Pickup Study}

In order to study the effects of electromagnetic pickup on electronics located within the beam pipe, a bench top apparatus has been assembled which simulates the fields due to a circulating beam. This was accomplished using concentric cylinders. The center cylinder is pulsed using an Amplifier Research Model 3500A100 RF amplifier, and the outer cylinder is used as a return path for the current. The current along the center conductor approximates the circulating beam, while the outer conductor represents the beam pipe. The fields between these cylinders should approximate the expected fields in and around the BTeV pixel detector. The characteristic impedance for the apparatus is $50 \mathrm{ohms}$ [24]. A 5 inch wide by half inch thick slot was cut into the outer pipe orthogonal to the apparatus axis to allow access to the field region between the pipes.

The input to the amplifier is driven by a pulse generator. The input pulse is roughly Gaussian with a width of $7 \mathrm{~ns}$. The pulse is repeated every $132 \mathrm{~ns}$. The output pulse has a width of $8 \mathrm{~ns}$ and a peak amplitude of 320 volts, corresponding to a peak current of 6.4 amps or $3 \times 10^{11}$ electrons per pulse (which closely approximates the proton bunch density for the Tevatron). This pulse corresponds to an amplification factor of $20 \%$ of the maximum allowed by the RF amplifier.

A loop of wire was placed in the field region to measure the induced voltage with respect to apparatus ground as a function of the peak output power. This loop was terminated either with a $50 \mathrm{ohm}$ resistor on each end or with a $10 \mathrm{kilo-ohm} \mathrm{termination} \mathrm{on} \mathrm{both} \mathrm{ends.}$ The data is shown below.

\begin{tabular}{|c|c|c|c|}
\hline \multicolumn{2}{|c|}{$50 \bar{\Omega}$ Termination } & \multicolumn{2}{c|}{$10 \mathrm{k} \Omega$ Termination } \\
\hline RF Power (Watts) & Peak Voltage & RF Power (Watts) & Peak Voltage \\
\hline 80 & 2.7 & 60 & 7.1 \\
\hline 180 & 3.3 & 160 & 9.0 \\
\hline 340 & 4.1 & 320 & 11.5 \\
\hline 580 & 5.0 & 560 & 14.7 \\
\hline 1180 & 7.1 & 840 & 17.5 \\
\hline
\end{tabular}

The first test utilized an existing data source (the Gazelle Developmental System or GDS). GDS sends and receives an ECL 40 bit random data pattern over a serial link and checks for errors. It can operate at $250 \mathrm{Mbps}, 500 \mathrm{Mbps}$, and $1 \mathrm{Gbps}$. For our tests, the speed was $250 \mathrm{Mbps}$. The signal goes from the Gazelle System to the Finisar Card where the ECL signal is converted to an $850 \mathrm{~nm}$ fiber optic signal. The signal goes over 50/125 micron multimode fiber to the test card. On the test card, the signal is converted to differential ECL and goes over twisted pair wire through the field region. The signal is then sent back to the Gazelle System via optical fiber where it is checked for errors. The system is controlled by a Macintosh host computer which also displays the error rate.

With $20 \%$ RF amplification, no data errors were observed provided that the test card ground plane was tied to the outer shell of the apparatus. When the test card was isolated 
from the outer conductor, data errors began to appear when the peak output power exceeded 1000 watts. At higher amplification, the error rate was essentially $100 \%$. A metal plate was then added as a shield on the "upstream" side of the test card which reduced the error rate to zero.

A test card with traces of various length and width was manufactured to systematically study induced voltages and shielding techniques. In general, it was found that the induced voltage is weakly correlated with the trace width and length. Furthermore, grounded guard rings were not found to provide effective shielding from electromagnetic pickup. A solid metal foil connected to the apparatus ground (the outer cylinder) did dramatically reduce the pickup. Furthermore, simple low impedance resistive termination reduced the observed induced voltage by a factor of 10 .

A simple test board without any shielding was built to study the operation of standard TTL chips in the field region. The test board consisted of a 555 timer and 7404 TTL inverter. The oscillation frequency was $30 \mathrm{kHz}$. Power and ground were brought into the field region using PCB traces, with the power and ground lines coupled using two $0.1 \mu \mathrm{F}$ capacitors. Signals were brought out of the field region using micro coaxial cables.

During the first test, a ground strap was bolted between the board ground and apparatus ground. The circuit was found to perform flawlessly. Pickup was evident; however, the induced RF noise was measured to be only $400 \mathrm{mV}$ (peak to peak). The grounding strap was then removed and precautions were taken to insure that the test board was electrically isolated from the apparatus ground. The 555 timer and 7404 inverter outputs were then measured with respect to the negative terminal of the DC power supply supplying power to the test circuit. As before, the circuit performed flawlessly with an RF noise of $400 \mathrm{mV}$ evident. By measuring the board ground voltage with respect to the apparatus ground, it was found that the test circuit power and ground lines were floating with respect to the apparatus by more than 10 volts.

The TTL logic study will be extended to surface mount CMOS technology. A custom printed circuit board has been constructed using a $60 \mathrm{MHz}$ CMOS oscillator connected to a CMOS inverter, connected to a PECL interface and Finisar optical transmitter. All components will be positioned within the field region. Future studies will also include prototype $\mathrm{BTeV}$ pixel boards along with an array of boards mounted within the apparatus to study the effects due to the unique $\mathrm{BTeV}$ pixel geometry. The initial studies are encouraging and indicate that RF pickup due to the circulating beams will not preclude the safe and reliable operation of pixel electronics inside the beam pipe. 


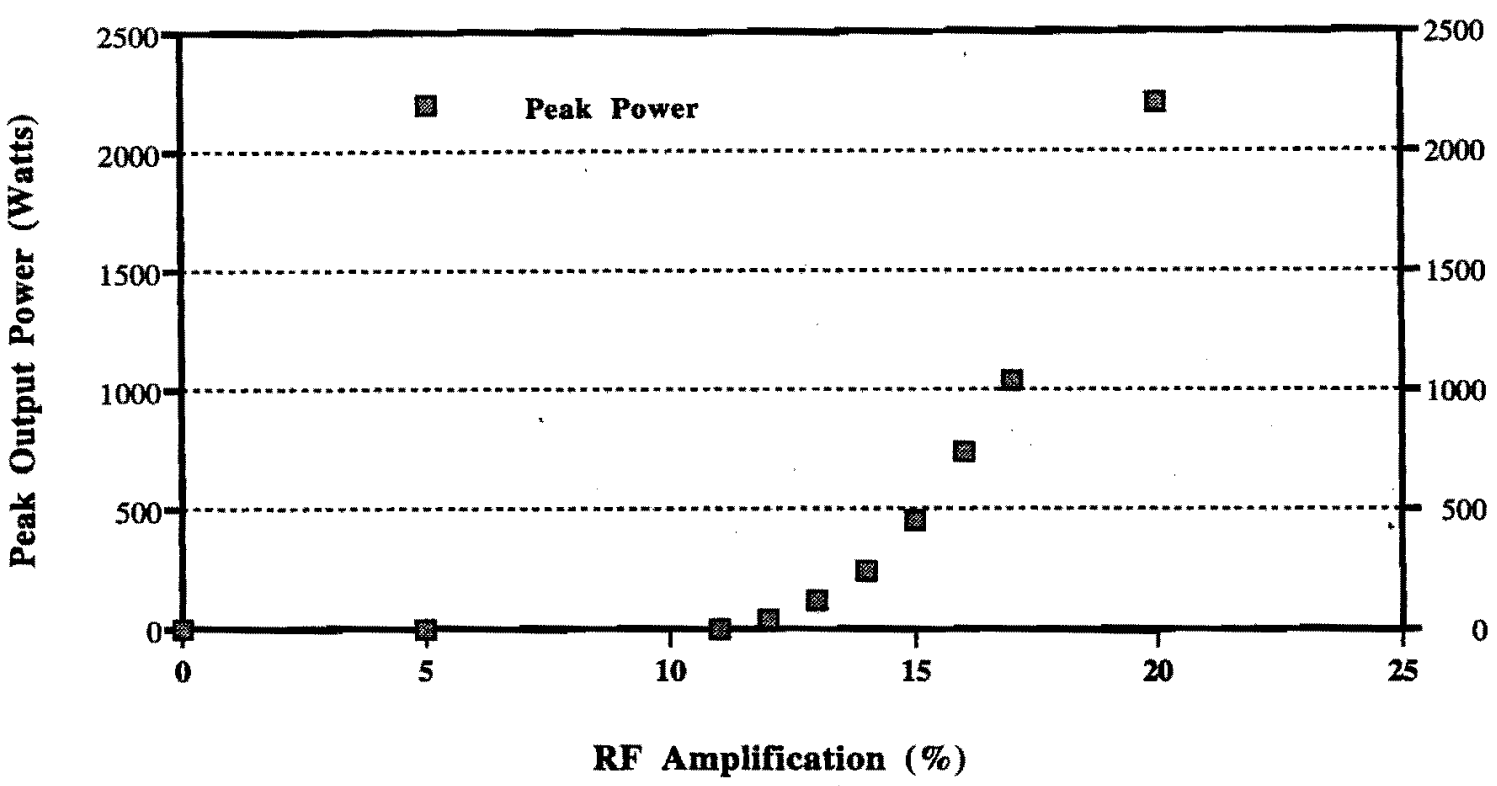

Figure A.23: Peak output power for the RF amplifier as a function of the RF amplification.

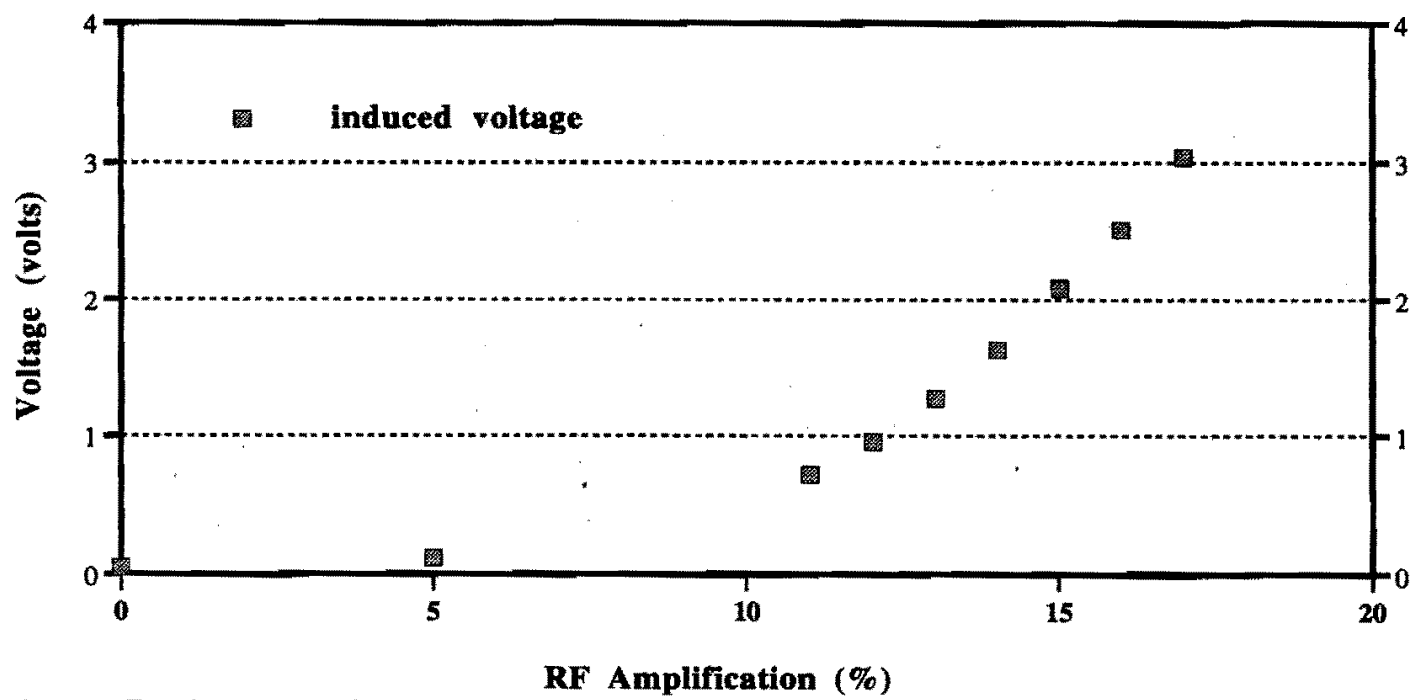

Figure A.24: Peak induced voltage on an unterminated trace as a function of the RF amplification. 


\section{A.1.4 FPIX2 Radiation Damage Tests}

The first two generations of pixel readout chips designed at Fermilab (FPIX0 and FPIX1) were implemented in standard CMOS technologies (HP $0.8 \mu \mathrm{m}$ and HP $0.5 \mu \mathrm{m}$, respectively). The R\&D plan established in 1997 called for an eventual migration of the final FPIXn design to a military radiation hard technology, probably the Honeywell $0.5 \mu \mathrm{m}$ CMOS SOI process.

In December, 1998, motivated by the results of RD49, which showed that commercial deep submicron CMOS devices could be made radiation hard, we modified our pixel chip R\&D plan to focus on implementation in a commercial $0.25 \mu \mathrm{m}$ CMOS process. Fermilab engineers joined RD49, and Fermilab initiated negotiations that have resulted in our gaining access, through CERN, to IBM's $0.25 \mu \mathrm{m}$ CMOS technology. Since the Taiwan Semiconductor Manufacturing Corporation (TSMC) $0.25 \mu \mathrm{m}$ CMOS, which is available through MOSIS, is similar to the IBM process, we decided to try to develop it as a second source. Starting with the radiation tolerant design rules developed at CERN for use with the IBM process, Fermilab engineers developed a set of design-rule files for their CADANCE IC design program which satisfy both IBM and TSMC design constraints. FPIX2 is being designed for implementation in $0.25 \mu \mathrm{m}$ CMOS, either using the IBM process, or the TSMC process, or both.

Two prototype circuits have already been designed and fabricated using TSMC $0.25 \mu \mathrm{m}$ CMOS. The first (preFPIX2) included sample transistors, and a small number of redesigned FPIX amplifiers and comparators. The second (preFPIX2T) includes two columns of 160 rows of complete FPIX2 pixel readout cells. Bench tests of these circuits verify that the analog sections of the new FPIX2 perform at least as well as previous FPIX circuits.

Very recently (April 18 - April 24, 2000), preFPIX2 and preFPIX2T chips were irradiated using a $\mathrm{Co}^{60}$ source at Argonne. The preFPIX2T chips received a dose of approximately 33 MRad. This is roughly equivalent to the $10^{15}$ particles $/ \mathrm{cm}^{2}$ exposure anticipated at the inner edge of the BTeV pixel detector after 10 years of running at a luminosity of $2 \times 10^{32} \mathrm{~cm}^{-2} \mathrm{sec}^{-1}$. A preliminary analysis of data collected before, during, and after this irradiation is very encouraging. Figure A.25 shows the measured amplifier noise and discriminator threshold for the 320 cells in one preFPIX2T, both before and after irradiation. The circuit was biased using the same voltage and current levels after irradiation as before. After irradiation, the amplifier noise increased by less than $5 \%$. The average discriminator threshold, expressed in equivalent $\mathrm{e}^{-}$, for the applied threshold voltage, went down from $\sim 1100 \mathrm{e}^{-}$to $\sim 1000$ $\mathrm{e}^{-}$, and the discriminator threshold RMS did not increase at all. More analysis is required to determine whether or not the apparent decrease in threshold dispersion is statistically significant.

Previous RD49 radiation damage tests have all been performed on parts made using IBM $0.25 \mu \mathrm{m}$ CMOS. Our results verify that circuits fabricated using TSMC $0.25 \mu \mathrm{m}$ CMOS are similarly radiation tolerant. 

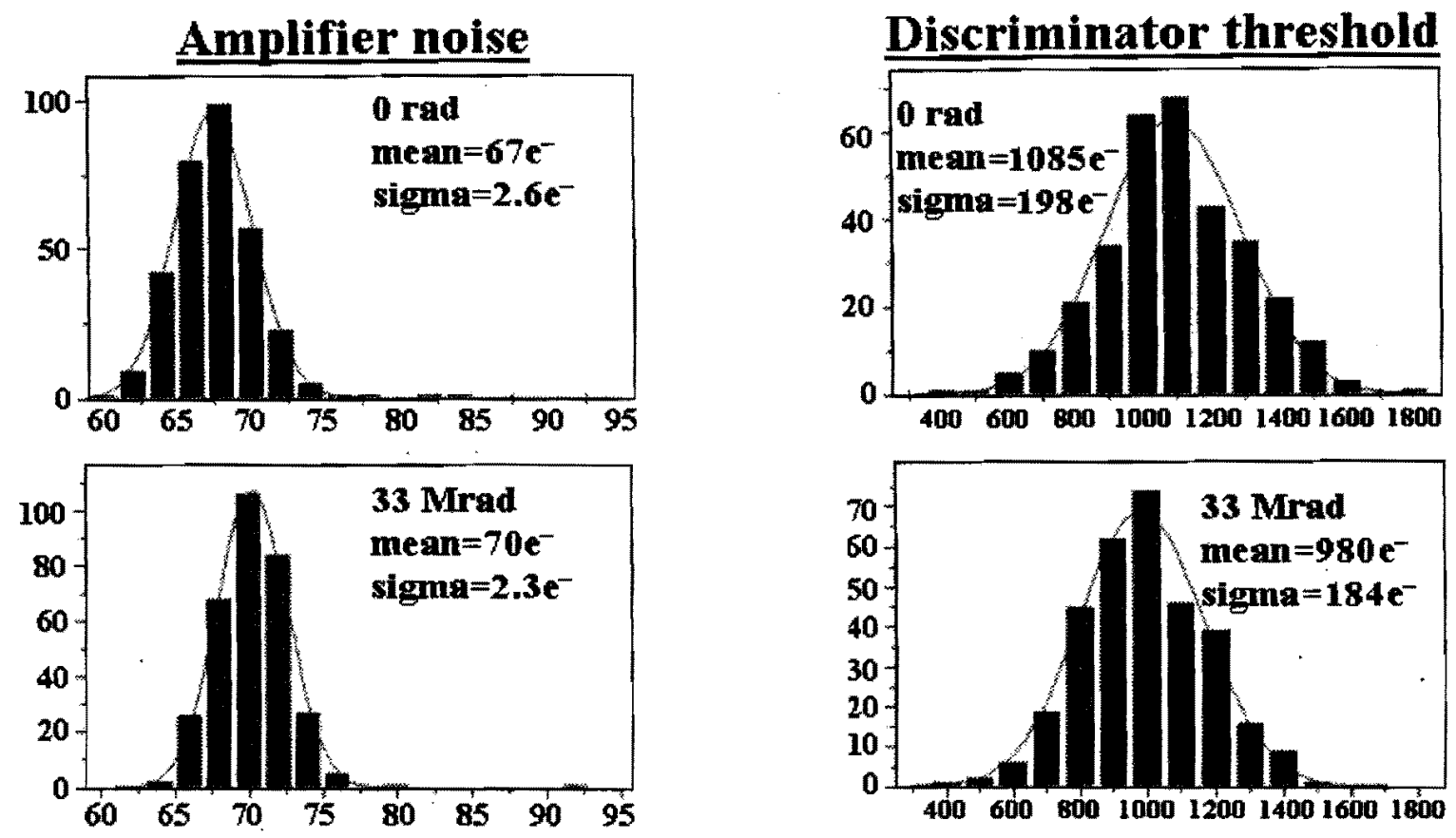

Figure A.25: PreFPIX2T radiation tolerance. The plots on the left show the measured amplifier noise for the 320 cells of a preFPIX2T chip. The average noise increased from 67 $\mathrm{e}^{-}$to $70 \mathrm{e}^{-}$after $33 \mathrm{MRad}$. The plots on the right show the distribution of discriminator thresholds for the same 320 cells, before and after irradiation. The plots show that with the same external threshold voltage applied, the effective threshold decreased from $\sim 1100 \mathrm{e}^{-}$to $\sim 1000 \mathrm{e}^{-}$after $33 \mathrm{MRad}$. The threshold dispersion did not increase after irradiation. 


\section{Bibliography}

[1] M. Moll, Summary of the 3rd Rose Status Report, 5th Rose Workshop, March 2000.

[2] The ATLAS Pixel Detector Technical Design Report, CERN/LHCC-98-13.

[3] The CMS Tracker Project Technical Design Report, CERN/LHCC-98-6.

[4] E. Fretwurst et al., Nucl. Instr. Meth. A326 (1993) 357; V. Eremin, Z. Li Nucl. Instr. Meth. A362, (1995) 338; Z. Li Nucl. Instr. Meth. A360 (1995) 445, Z. Li et al. , Nucl. Instr. Meth. 277 (1996) 265; Z. Li Nucl. Instr. Meth. A368 (1996) 353.

[5] The ROSE Collaboration RD48 Status Report, CERN/LHCC 97-39 (1997).

[6] The Rose Collaboration CERN Report LHCC-2000-09, Dec. 1999.

[7] M. Artuso, Presented at 29th International Conference on High-Energy Physics (ICHEP 98), Vancouver, Canada, 23-29 Jul 1998, Syracuse Un. Preprint HEPSY-98-2, (1998).

[8] F. Hügging et al., Nucl. Instr. Meth. A439 (2000) 529.

[9] F. Ragusa contributed talk to Vertex '99, Preprint submitted to Nucl. Instr. Meth. (1999).

[10] M. Artuso and J. Wang, BTeV Internal Report BTeV-Int-97-18, February 1998

[11] E. Belau et al, Nucl. Instr. and Meth. 124, (1983) 253.

[12] R. Turchetta, Nucl. Instr. and Meth. A 335, (1993) 44.

[13] H. Bichsel Rev. of Mod. Phys., (1988) 663.

[14] L.H.H. Scharfetter (RD19), Active Pixel Detectors for Large Hadron Colliders, CERN Thesis, 1997, and CERN 99-09 (1999)

[15] P. Kasper, BTeV Internal Report BTeV-int-98/3, March 1998.

[16] G. Cancelo et al., BTeV Internal Report BTeV-int-98/16, September 1998.

[17] C.J.Li and Z. Li, Nucl. Instr. Meth. A364 (1995) 108. 
[18] T. Rohe, et al., "Sensor Design for the ATLAS Pixel Detector", Nucl. Instr. and Meth. A409 (1998) 224.

[19] T. Zimmerman, et al., "Design of an Advanced Readout Chip for Silicon Strip Detectors", IEEE Trans. Nucl. Sci. Vol.40, No.4 (1993) 736.

[20] S. Hancock, et al., "Energy Loss Distributions for Single Particles and Several Particles in a Thin Silicon Absorber", Nucl. Instr. and Meth. B1:16 (1984)

[21] The Atlas silicon pixel sensors, ATLAS-INDENT-NO-XXX September 10, 1999

[22] R. Kutschke, "Coordinate Systems in the 1999 Test Beam Run", BTeV Internal Report, BTeV-int-1999/10+ (1999).

[23] M. Pindo, "Simulating Charge Collection in Silicon Pixel", Nucl. Instr. and Meth. A 395 (1997) 360 .

[24] Specifications and a schematic of the beam simulator apparatus can be found at http://www-ap.fnal.gov/ gpj/BTeV/pixelshield.html. 


\section{A.2 RICH Design and R\&D}

\section{A.2.1 Hybrid Photodiode R\&D}

The PP0380V HPD tube from DEP [1] (see Fig 5.5 in Part II) which we plan to use to detect Cherenkov photons has been described in Part II of this proposal.

DEP, the manufacturer of the HPD, is an electro-optics company with over 20 years of experience in the field of image intensifiers. Its manufacturing capacity is about 6000 such devices per year; no image intensifier tube has ever been rejected after delivery, according to DEP. They have been producing hybrid tubes for several years. Last year, they produced 30 HPDs of the 61-channel variety.

HPDs are also the photon detector of choice for the CMS HCAL (about 500 HPDs of 19 and 73 channels), and the LHC-b RICH (about 500 HPDs of 2048 channels).

There are two issues in the tube construction which require development work: material to block short wavelengths and segmentation of the silicon diode. They are actually coupled to each other. We would like to operate with wavelengths above $280 \mathrm{~nm}$, which makes the chromatic error somewhat smaller than the emission point error. This scheme also requires that the silicon diode segmentation is increased from the 61 pixels presently used by DEP to 163 pixels in order to bring the segmentation error down to a value comparable with the emission point and chromatic errors. Such an increase of diode segmentation can be achieved with the present manufacturing process. The development would be done by DEP and their subcontractors. The cost of this development is included in our budget.

The presently manufactured tube has a quartz window which cuts off at $160 \mathrm{~nm}$. To increase the cut-off value to $280 \mathrm{~nm}$, we plan to investigate alternative window materials, various coatings of the quartz window or a separate window between the gas volume and the HPDs. The latter solution is attractive from the point of view of sealing the RICH vessel, but would induce $\sim 8 \%$ loss in photon yield due to the reflective losses.

The alternative approach would be to make use of the large wavelength sensitivity of the HPDs with quartz windows. This makes the chromatic error much larger but leaves per track Cherenkov angle resolution almost unchanged (about $\sim 10 \%$ deterioration) because of the larger number of detected Cherenkov photons. In this approach, there is no need to develop a new silicon diode, since the 61 pixel version already produces a satisfactory segmentation error. Making use of short-wavelength photons imposes much stricter requirements on gas purity, mirror quality, etc., which is why we do not favor this solution. Such a possibility illustrates, however, that the performance of the RICH detector does not crucially depend on the success of the developments mentioned above.

There are, of course, a large number of tests which need to be performed on the HPDs. These are tests of linearity, gain, and uniformity of response. In addition, it is critical to study issues of tube aging, which are known to be caused by either Si diode instability, or photocathode degradation with time. These are not expected to be a problem in our design, however, due to the low radiation dose at the photon detector plane and the low light levels. 


\section{A.2.2 HPD High Voltage System}

The Hybrid Photon Detector requires a very high applied voltage in order to provide the ejected photoelectron with the kinetic energy necessary to produce a sufficiently large signal, as there is no intrinsic charge amplification. The HPD design [1] requires three very high voltages: $20 \mathrm{kV}$ for the photocathode, $19.9 \mathrm{kV}$ for electrostatic focus, and $4.2 \mathrm{kV}$ for final demagnification. There is essentially no current draw $(\sim 10 \mathrm{nA})$ for these electrodes. In addition, the HPD requires a $60 \mathrm{~V}$ low voltage for reverse-biasing the Si diode.

Our choice for a high voltage system is the commercially available CAEN SY 1527 system. CAEN either has developed or is currently developing modules for this system which suit our needs, with the exception of the $20 \mathrm{kV}$ requirement. However, they are very interested in pursuing development of such a module, which would also be useful to LHC-b for their HPDs. For a previous system (SY 127) they already produce a $20 \mathrm{kV}$ module, so the technical issues of very high voltage are well-understood by them.

Since there is effectively no current draw by the HPD, one can simply fan out each HV channel to a number of different tubes. Therefore our design for the distribution of the high voltage uses cable assemblies to bus the voltage from the SY 1527 or SY 127 modules to each HPD of a gang of HPDs. Currently we envision ganging 15 HPDs per single HV channel separately for each of the four voltages required.

The high voltage issues to be addressed by future $R \& D$ are:

1. Development of the $20 \mathrm{kV}$ CAEN module. This development will be done by CAEN. With some inconvenience we could also use the existing SY 127 module.

2. Development of robust HV cable assemblies. The high voltage assemblies must fanout a given voltage to a number of HPDs. They consist of cables, connector, and custom-made fan-outs and feed-throughs. Some of these components are commercially available, such as the cable and connectors. Other components such as the fan-outs, feed-throughs, and the assembly design itself must be developed and tested, and must be mechanically and electrically robust. We have to determine if the HV cable must be shielded, what material to use for the fan-outs, and how to design the feed-throughs.

3. Testing and Integration of the HV cable assemblies. In conjunction with the mechanical design, described elsewhere, the cable assemblies must be properly integrated to avoid arcing and corona discharges. Each assembly also needs to be tested beforehand at voltage in a test station.

\section{A.2.3 Photon Detector Mechanical Design}

The mechanical support structure for the photon detectors must support the tubes themselves, plus the analog electronics, digital electronics, high voltage cable assemblies, and cooling pipes. Additionally, the front face of the HPDs will make a gas seal to the gas radiator volume. 
This structure must be designed in an integrated manner, taking into consideration all of the requirements of each of the parts it supports, i.e., mechanical, electronic, and thermal elements.

The current design calls for triads of HPDs to be bundled together as a unit, with readout electronics. This triad will be tested together and mounted as a single module.

There are several issues to be resolved in future $R \& D$ of the mechanical design.

1. The faces of the HPDs are hexagonally packed together on the detector plane, and should be maintained at the closest possible packing fraction to avoid loss of Cherenkov photons. The optimal way of accomplishing this needs to be determined.

2. The exterior of the HPD is at voltage, so it needs to be coated with Kapton or an equivalent material. The HPD triad bundling and support should not be metallic. There can be no possible corona points in the design.

3. The front of the HPD must make a gas seal with the gas radiator volume. The amount of pressure required and the mechanical support to deliver the compression needs to be studied. However, to ease the necessity of a high-quality seal, the photon detectors will be enclosed in a separate gas volume containing clean gas.

4. There must be sufficient support for the electronics and cooling at the rear of the HPDs. Transverse space for this and the high voltage cabling is at a premium, and a detailed design with realistic materials and standoff is needed.

Again, these issues are intimately connected to the overall mechanical structure for the photon detectors, which is non-trivial and needs to be engineered as an integrated whole.

\section{A.2.4 HPD Readout Electronics}

The signal produced by the hybrid photodiode (HPD) is a narrow charge pulse of $\sim 5000$ electrons. Our goal is to achieve a signal to noise ratio of about 7 , corresponding to an equivalent noise charge (ENC) of about $700 e^{-}$. The charge pulse duration depends upon the bias voltage of the silicon pad detector and its input capacitance and is typically around $10 \mathrm{~ns}$. Thus, the readout electronics determines the time development of the analog signal. Our goal is to process the information coming from this detector element within the bunch crossing it originates from. This means that the information must be transferred to the data combiner chip discussed elsewhere [2] within $132 \mathrm{~ns}$. The maximum number of readout channels to be instrumented is 339,040 corresponding to 2,080 readout chips.

Fortunately, there are some front end devices already developed and produced with their characteristics tuned to a whole variety of different applications that we can adapt to our system. These ASICs belong to the so called VA family [3], developed and produced by IDE AS, Norway [4]. They have been used in a variety of systems including particle physics detectors, both accelerator based [5] and in space [6], as well as for medical applications [7]. We have worked with this company on a previous project, the CLEO III RICH, to develop a 


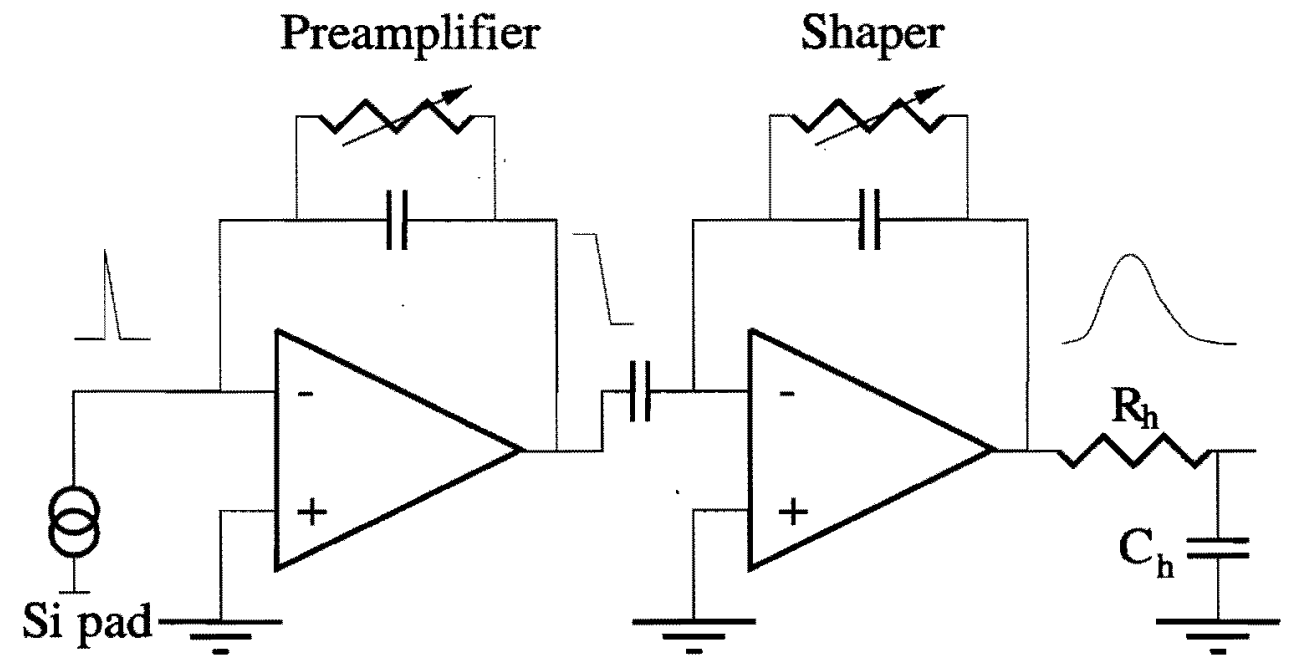

Figure A.26: Block diagram of the analog cell in VA circuits.

custom made ASIC, the VA_RICH and associated hybrids, that will be described below [8]. While this particular chip is not appropriate for $\mathrm{BTeV}$, our experience has been extremely positive, both in terms of reliability of the products and in terms of meeting the production schedule and this gives us confidence that this effort will proceed equally smoothly. Thus we are planning to employ a custom made adaptation of an ASIC of the VA family, that we will refer to as VA_BTeV, as the front end device for HPD readout.

This conservative approach of decoupling the HPD and the readout electronics production is possible because the segmentation optimal for our needs allows us to bring the pad signals out of the HPD tube as pins that can be mated with a multi-chip module containing the readout electronics. On the other hand, LHC-b is pursuing an integrated sensor-chip system inside the tube as their preferred solution because they require a much finer segmentation than $\mathrm{BTeV}$. This couples the tube manufacturing with the silicon pad detector and the readout electronics production which makes all stages of the project more difficult.

The most common solution for low noise analog front end chips for solid state and gas pad detectors is based on a RC-CR preamplifier and shaper circuit, which is at the core of the analog cell in the VA circuits. Fig. A.26 shows a block diagram of this circuit and the profile of the voltage signal at the output of the individual processing elements. This analog cell consists of a charge sensitive preamplifier with a large voltage-controlled resistor in the feedback loop to insure DC stability, followed by an AC coupled shaper that limits the bandwidth of the system. The bandwidth is related to the peaking time of the system: a short peaking time corresponds to larger bandwidth, making it more challenging to keep the ENC low as the dominant source of intrinsic electronic noise has a uniform power density spectrum. We are seeking peaking times below $100 \mathrm{~ns}$.

Our goal is an equivalent noise charge (ENC) of 700 electrons or better. This would be a challenging task for new, undeveloped readout electronics, especially at the short peaking times that we envisage. The ASIC called VA_32/75 [4], already fully engineered and available from IDE AS as a 32 channel chip, has a nominal peaking time of 75 ns and a noise 
performance given by:

$$
\mathrm{ENC}=400 e^{-}+25 e^{-} / \mathrm{pf} \times C_{i n}
$$

This would correspond to an ENC of $500 e^{-}$at our expected input capacitance of $4 \mathrm{pF}$. It is very likely that a tuning of the chip parameters for our specific application will achieve better noise performance for VABTeV, even though an ENC of $500 e^{-}$is already suitable for our needs.

We have opted for a binary readout as we can achieve adequate spatial resolution with binary charge weighting and the readout system is much simpler this way. Thus, the analog section is followed by a discriminator to perform zero suppression. This component of the VA_BTeV chip is based on the TA32C fast triggering ASIC [4], developed by IDE AS for medical imaging applications. Each channel will feature a discriminator with its threshold individually adjusted with an internal DAC. The zero suppressed output will be sent to the $\mathrm{BTeV}$ data combiner multichip module. The low occupancy of the system allows us to fan in 3 VA BTeV circuits to a single data combiner chip.

We are planning to purchase the VA_BTeV ASICs mounted in a multichip module. IDE AS has experience on a variety of packaging solutions. In particular, some packaging previously implemented by IDE AS match the HPD pinout structure quite closely. Possibly, 3 such modules could be integrated in a single printed circuit board to reduce the number of interconnections between individual ASICs and the data combiner chip hybrid.

\section{A.2.4.1 The CLEO III RICH front end electronics}

A brief summary of the process that we followed in acquiring the CLEO III RICH electronics and the performance that we achieved may be interesting to put this new effort in perspective. The CLEO III RICH includes 230,400 channels that need to be processed with low noise electronics to achieve good efficiency also for the low level photon signals that are quite copious in this device as the probability distribution of the charge produced in the avalanche initiated for a single photon is exponential.

The chip that was developed by IDE AS, VA_RICH, is an adaptation of the VA design concept to optimize simultaneously the noise performance and the dynamic range. This chip features 64 readout channels. IDE AS assembled them in G10 multi-layer hybrid circuits hosting two ASICs.

The VA_RICH chip is described by a block diagram similar to the one shown in Fig. A.26. The resistor $R_{h}$ in this case is a switch and the capacitor $C_{h}$ is the analog memory in this sample and hold circuit. Upon receiving a logical signal that signals that the charge is ready to be read out, the switch opens and the charge signal is stored in $C_{h}$. In our case we could afford to use a serial readout for 128 channels. Thus, two chips are daisy-chained and the signals stored in the capacitors $C_{h}$ which are sent to the remote processing electronics as differential current signals. The clock driving the shift register that connects individual channels to the output has a frequency of $7 \mathrm{MHz}$.

The noise of this chip, measured in a laboratory test setup is [8]:

$$
E N C=117 e^{-}+8.1 e^{-} / \mathrm{pf} \times C_{i n}
$$


This performance was achieved with a peaking time of $2 \mu \mathrm{s}$. The input capacitance is relatively large because the signal from the cathode pad needed to be fanned in to a high density connector and then routed from the connector to the input pad on the chip. An average input capacitance of the order of about $15 \mathrm{pF}$ is our estimate, with some spread due to different trace lengths. Thus we would predict an average noise figure of about $240 e^{-}$. Note that the task of maintaining this low noise performance is very challenging because the power supply and all the control bias voltages and currents are provided by boards residing O. a VME crate which feature mixed analog and digital circuitry and are transmitted by cables about $20 \mathrm{ft}$ long [9]. This system has an additional component of coherent noise, due to ground fluctuations, that is subtracted on line, and some incoherent noise due to some small coupling between analog and digital ground in the data boards. However, the mean noise measured in the whole detector is about 400 electrons, which is good for such a large system with the constraints discussed above. The long cable and the vicinity of complex VME digital activity in the vicinity of the analog section will be missing in the BTeV design.

The VA_RICH production followed the initially projected schedule quite well. We produced a total of 2,200 hybrids, 1,800 of which are installed in the RICH detector. A smaller number (16) are employed to read out the main CLEO drift chamber cathodes. The hybrids were produced and tested at IDE AS, Oslo. They were subsequently tested at Syracuse University upon arrival and underwent a 1 week burn-in running at elevated temperature to eliminate the hybrids susceptible to early failures during detector operation. Almost no hybrids were discarded after this test. In summary, the production went quite smoothly and the performance achieved in the complete system is quite consistent with the expectations from our initial prototype characterization.

\section{A.2.5 Silica Aerogel Radiator}

The physics rationale for the use of a silica aerogel radiator in addition to a $\mathrm{C}_{4} \mathrm{~F}_{10}$ gas radiator has been presented in detail in Part II. Kaon/proton identification is extended below $9 \mathrm{GeV} / c$ to $\sim 3 \mathrm{GeV} / c$, and also the fake-rate for a given efficiency is reduced compared to a gas radiator alone. The dual radiator approach has been proposed for one of the LHC-b RICH detectors [10] and has been adopted by HERMES for its RICH [11]. We now discuss some of the technical issues associated with the use of silica aerogel.

The most advantageous feature of silica aerogel is that its refractive index is in the range 1.01 to 1.10 , placing it between that of the heaviest gas $\left(\mathrm{C}_{4} \mathrm{~F}_{10}, n=1.00138\right)$ and the lightest liquid $\left(\mathrm{C}_{5} \mathrm{~F}_{12}, n=1.26\right)$. The implication for a $\mathrm{RICH}$ detector is that this material allows hadron identification in the difficult momentum region of a few $\mathrm{GeV} / c$. Aerogel is very light and does not add substantially to the material budget in front of the EM calorimeter. It is difficult to handle since it is extreme fragile, and can cleave with very little applied stress.

\section{A.2.5.1 Fabrication Issues}

Traditionally, the utility of silica aerogel has been limited due to the pore size acting as a strong Rayleigh scattering center. Recently a new fabrication technique has been developed 
by KEK for BELLE which results in aerogel of better transparency [12, 13]. The strength of the Rayleigh scattering component was reduced by approximately a factor of two over previous values. This technique has been transferred to manufacturer Matsushita Electric Works, Ltd (Japan), who subsequently manufactured the aerogel for HERMES [11].

The Syracuse University group has had extensive discussions with Matsushita about their aerogel production and capabilities, which were very favorable. HERMES required $\sim 100$ liters of aerogel, which Matsushita successfully provided. In fact, their production output totaled 1500 liters of aerogel last year. From them we have a price quotation for their standard material, and furthermore we have had discussions on the possibility of modifying their technique to obtain even clearer aerogel. They are currently evaluating this possibility.

In addition, there is another source of very good quality aerogel from the Boreskov Institute of Catalysis (Novosibirsk, Russia) [14, 15]. The capacity of this group is currently quite small, and thus far they have only produced aerogel with a different refractive index than we require, so we choose to stay with Matsushita but retain them as a backup source.

\section{A.2.5.2 Transmittance Measurements}

We have obtained six samples of standard SP-30 aerogel manufactured by Matsushita, and have performed a number of measurements on them.

\section{Transmittance Measurement Technique}

Transmittance measurements were made at Syracuse University with a Visible Light Spectrophotometer, a system consisting of an intense light source, chopper, monochromator, $\mathrm{XY}$ position control stage, PMT, ADC, and LabVIEW readout. This system, originally designed for measurements in the VUV (Vacuum Ultra Violet) of crystals for the CLEO-III RICH, has been modified to work in the visible spectrum.

The external transmittance is measured by taking the ratio of measured PMT photovoltages for the "sample-in" over "sample-out" conditions, i.e.,

$$
T_{\mathrm{ext}}=\frac{\Delta V_{\mathrm{in}}}{\Delta V_{\mathrm{out}}}
$$

where $\Delta V$ indicates photo-voltage amplitude, with automatic baseline subtraction provided by the chopper, and the denominator is sampled periodically during the measurement procedure.

Currently, we are able to measure transmittance as a function of wavelength in the range 280-530 $\mathrm{nm}$, with an accuracy of about 0.01 . (We expect the HPD quantum efficiency to have an effective bandwidth 280-650 nm:) Systematic studies and wavelength calibration have been performed. 


\section{Transmittance Spectrum}

The external transmittance was measured as a function of wavelength for all six Matsushita samples. At each wavelength, nine points over the face of the sample were measured and averaged. The result for all samples is shown in Figure A.27. The rms spread among the samples was under a few percent.

Also shown is a fit of the data as a function of wavelength, given by the standard parameterization of the external transmittance,

$$
T_{\text {ext }}=A_{H} e^{-C_{H} d / \lambda^{4}}
$$

where the "Hunt parameters" are $A_{H}$, the asymptotic value of the transmittance, typically 0.95 , that of a good glass, and $C_{H}$, the "clarity coefficient", the strength of the Rayleigh scattering component, typically 100 in units of $10^{-4} \mu \mathrm{m}^{4} \mathrm{~cm}^{-1}$ for good aerogel. The wavelength dependence in this formula indicates the dominance in the attenuation process of the Rayleigh scattering component. Our fit yields the values

$$
A_{H}=0.977, \quad C_{H}=67.9 \times 10^{-4} \mu \mathrm{m}^{4} \mathrm{~cm}^{-1},
$$

which corresponds to a typical Rayleigh scattering length at $400 \mathrm{~nm}$ of $L_{\text {scatt }}^{(400)}=3.77 \mathrm{~cm}$. This nearly matches our design thickness of $4 \mathrm{~cm}$.

\section{XY Uniformity Scan}

A two-dimensional scan of some of the tiles was made, in which the external transmittance was measured at an array of positions over the surface of the piece. The result of the scan indicates that the external transmittance varies over the surface by about $5 \%$. This variation is dominated by an area which has a filmy residue on the surface, clearly apparent on each of the samples, and probably the consequence of handling during the manufacturing process. There is no indication of any systematic drop in transparency near the edges.

\section{Comparison of Different Aerogels}

The external transmittance was also measured for a number of other samples, obtained courtesy of KEK. These were samples from the BELLE Endcap Aerogel Cherenkov Counter, labeled EACC herein, and a sample from the Novosibirsk group, which has $n=1.05$.

From measurements of these samples, the Hunt parameters were extracted, and compared to other results available in the literature (either the published parameters or parameters extracted from published plots). Figure A.28 and Table A.2.5.2 summarize the results of our measurements and comparison.

The Matsushita samples we have obtained are in fact quite good, compared to the average Hermes and KEK EACC aerogels. They are not as good as the Novosibirsk aerogel, or the best samples that KEK has produced. We believe that further improvement can be obtained by the modification to the manufacturing technique mentioned above. 


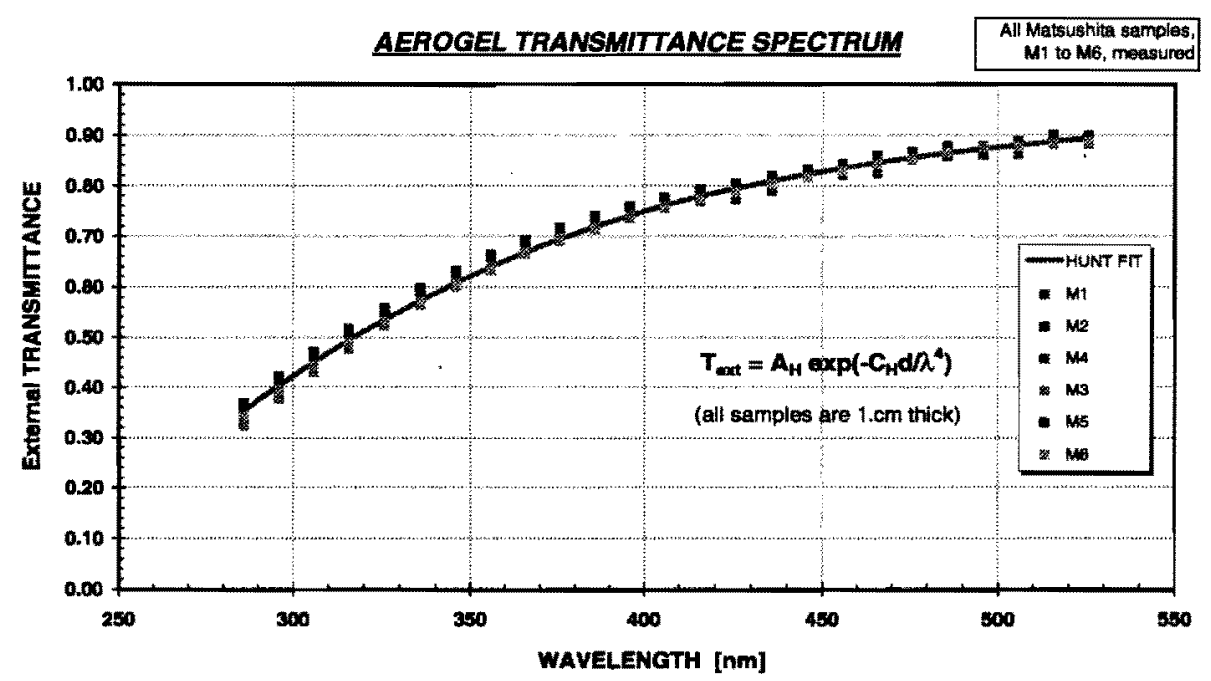

Figure A.27: Aerogel Transmittance as a function of wavelength, for six Matsushita samples. Points represent the data, the line represents a fit made to the standard Hunt formula.

\section{Transmittance Variation with Thickness}

As a test of the variation of the transmittance with thickness, we have measured the transmittance of a stack of Matsushita aerogel tiles, from 1 to 5 tiles (approximately 1 to $5 \mathrm{~cm}$ ). This is not an accurate measurement of the bulk absorption of aerogel, rather it provides a comparison of a stack of tiles, with gaps, to a single thick tile, with the bulk absorption calculated from the Hunt formula extrapolated to the given thickness.

Figure A.29 shows the result of this comparison for our nominal design thickness of 4

Table A.2.5.2. Comparison of Different Aerogels.

\begin{tabular}{l||l|c|c|c|l}
\hline \hline $\begin{array}{l}\text { Aerogel } \\
\text { Source }\end{array}$ & $n$ & $A_{H}$ & $\begin{array}{c}C_{H} \\
{\left[10^{-4} \mu \mathrm{m}^{4} \mathrm{~cm}^{-1}\right]}\end{array}$ & $\begin{array}{c}L_{\text {scatt }}^{(400)} \\
{[\mathrm{cm}]}\end{array}$ & Comments \\
\hline \hline Matsushita & 1.03 & $0.977 \pm 0.002$ & $67.9 \pm 0.4$ & 3.77 & SU measurements \\
Novosibirsk & 1.049 & $0.983 \pm 0.011$ & $54.0 \pm 1.2$ & 4.74 & SU measurements \\
Novosibirsk & 1.050 & $0.926 \pm 0.012$ & $47.8 \pm 2.4$ & 5.36 & fit from data, [14] \\
HERMES & 1.03 & 0.964 & 94 & 2.72 & published fit, [11] \\
KEK EACC & 1.030 & $0.980 \pm 0.004$ & $78.6 \pm 0.7$ & 3.26 & SU measurements \\
KEK best & 1.028 & $0.949 \pm 0.006$ & $49.9 \pm 1.1$ & 5.13 & fit from data, [12] \\
Airglas Ltd & 1.03 & 0.96 & 180 & 1.42 & old one-step method [16] \\
\hline \hline
\end{tabular}




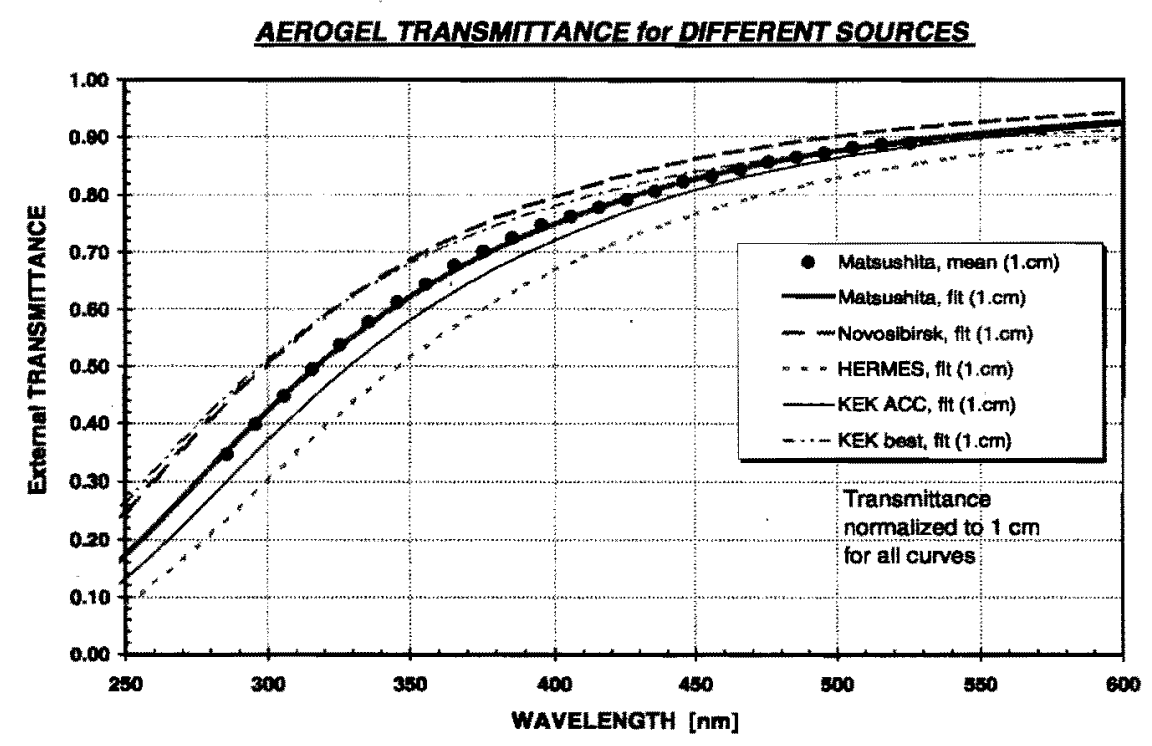

Figure A.28: Aerogel Transmittance as a function of wavelength, for samples from different sources. The data points indicate the mean transmittance of the Matsushita samples, whereas the lines are Hunt parameter fits as described in the text, all normalized to $1 \mathrm{~cm}$ thickness.

$\mathrm{cm}$. As expected, the result is that the stack has a transmittance lower than the bulk by about $5 \%$ at $400 \mathrm{~nm}$ and about $10 \%$ at $500 \mathrm{~nm}$ (where the HPD quantum efficiency starts to drop). This scaling approximation may overestimate the transmittance due to multiple scattering in the thicker bulk. In any case, we conclude that we can reasonably stack the tiles mechanically in the detector and still obtain good transparency.

\section{A.2.5.3 Future Aerogel Radiator R\&D}

The major issues for the aerogel radiator are:

1. Is the aerogel sufficiently transparent? Is it uniform over the face of the tile?

Our measurements so far indicate that the Matsushita aerogel has good transparency, which should provide slightly larger photon yield than the 8 photons per track detected in the HERMES experiment [11]. Further improvements in transparency would increase the photon yield per track. As noted above, we must continue to pursue this issue with the manufacturer.

We have provisional evidence that transmittance is uniform to about $5 \%$ over the face of the tile, however there are mechanical variations at the edges of the tile which provide non-uniform boundaries between tiles. This too needs to be improved by the manufacturer. 


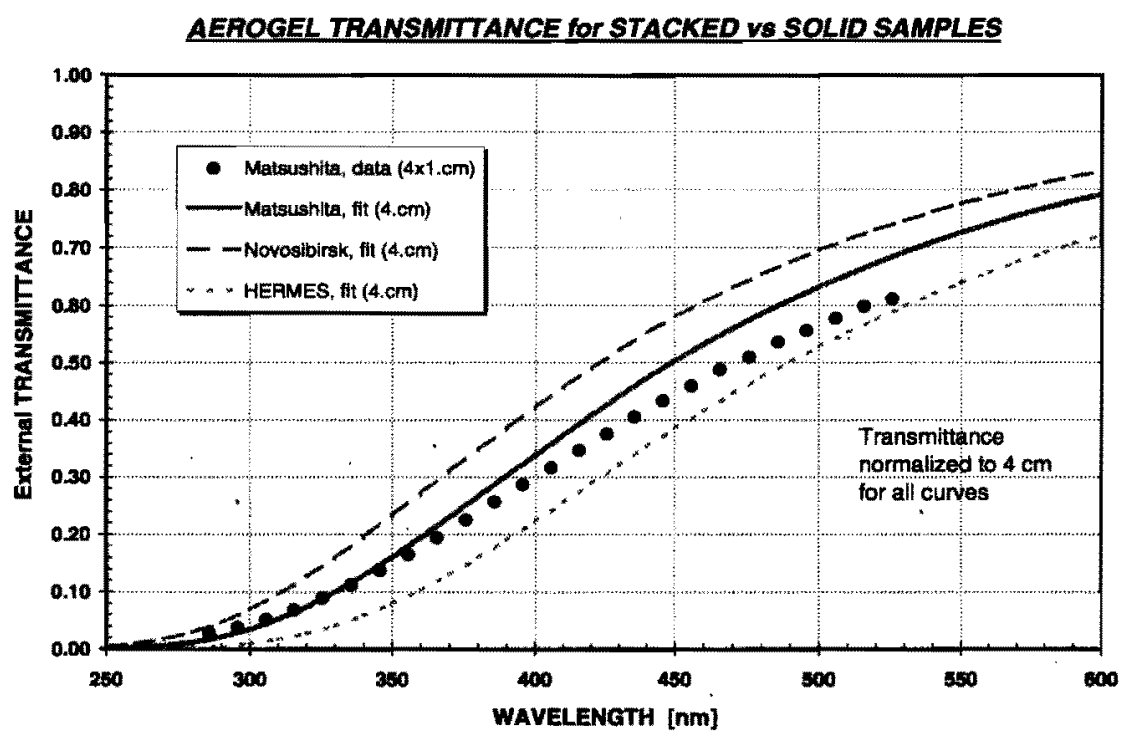

Figure A.29: Aerogel Transmittance as a function of wavelength, for a stack of 4 Matsushita tiles (data points), and for the Matsushita samples extrapolated to $4 \mathrm{~cm}$ bulk thickness (thick line). Other samples are shown for comparison.

2. Choice of a suitable window for aerogel- $C_{4} F_{10}$ interface.

The Rayleigh scattering cross-section increases for shorter wavelengths. Many scattered photons are eventually absorbed. Those which exit to the gas volume become background hits for Cherenkov ring reconstruction. The majority of the scattered photons can be eliminated by a suitable choice of window material to be inserted between aerogel and the gas radiator which would block short wavelengths. The type of material must be radiation hard. Material type and thickness need to be studied. This can be done with the existing transmission measurement setup.

3. Is the aerogel refractive index sufficiently uniform over a tile?

We have not yet made this measurement. There are reported in the literature refractiveindex variations of 0.003 over the face of a tile, peaking at the edges [14]. We need to construct a test station for this measurement and investigate the variations for the Matsushita samples.

4. Does aging affect either the transmittance or the refractive index? We will need to know if the aerogel properties are stable with time. In the past, aerogel transmittance has degraded after a few years in use which has been attributed to moisture absorption. However, the KEK method produces hydrophobic aerogel, which is expected to prevent this effect [12]. This is a situation which needs to be tested and monitored during 
production.

In addition to these issues, we will need to construct a test station to measure each aerogel tile manufactured in the production runs, in order to be sure it meets our specifications and in order to be able to monitor the production process for possible difficulties. We have done this in the past for the crystals for the CLEO-III RICH, so we are well aware of the issues involved and the possibilities of production processes drifting out of tolerance.

\section{A.2.6 Alternative Photon Detector System: MAPMTs}

In addition to the hybrid photodiodes, we have considered multianode photomultipliers as an alternative choice for photon detectors in the RICH detectors. As illustrated in Fig 5.6, the HPDs offer a cheaper solution. However, acceptable performance can be achieved with the PMT system as well. Thus, it is useful to describe PMT based detector which we consider a back-up option in case the price structure changes or the HPD solution runs into some unexpected obstacles.

In the PMT approach, multianode tubes must be used in order to reach the required accuracy for the detected photon position. Hamamatsu has recently developed the R5900 multianode phototubes which are about 1 inch $\times 1$ inch in cross-section and are segmented into four (R5900-M4), sixteen (R5900-M16) or sixty-four (R5900-M64) separate anodes. The active area of these tubes is only about $40 \%$. Some kind of light focusing system in front of the PMTs is needed to recover the dead area. The R5900-M16 tubes were adopted for the HERA-B RICH detector [17]. HERA-B used a two-lens system providing demagnification by a factor of two. In the HERA-B solution, the tubes are not closely packed, reducing the cost of the detector but allowing the segmentation error to dominate the achievable resolution. In addition, the photon yield suffers from the reflective losses at each lens surface, further deteriorating particle identification. Light loss for aerogel photons would be even larger than for gaseous photons because of the small angular acceptance of the two lens system. Our simulations show that the two lens system would fall far short of our goals without any cost saving compared to the scheme described below.

A different demagnification system was proposed by R. Forty [18] (see Fig. A.30). A single refractive boundary in front of each Hamamatsu tube provides enough demagnification to recover the dead area of the tube. The convex-plano lens is in direct optical contact with the PMT window preserving good photon yield. High quality acrylic lenses can be formed by injection molding. Many lenses could be molded together as the tubes are closely packed in this approach. We have simulated such a system in our ray tracing Monte Carlo. Variation of the lens' refractive index with photon wavelength was taken into account. When using the M64 tubes, the segmentation error was found to be $0.35 \mathrm{mrad}$ per photon, somewhat smaller than the emission point error, but larger than naïvely expected from the anode size and the demagnification factor. Geometrical and chromatic aberrations in the lens contribute to this error. The M64 tubes are too expensive to make them practical in our experiment. The M16 tubes are a factor of two cheaper than the M64 tubes. They increase the segmentation error

by less than a factor of two as the aberration effects become unimportant compared to the 
larger anode size. The photon position error for the M16 system is $0.55 \mathrm{mrad}$ per photon (about the same as the emission point error). Overall resolution is only $18 \%$ worse for the M16 system than for the M64 tubes. Therefore, R5900-M16 tubes are a possible choice for the $\mathrm{BTeV}$ RICH detector.
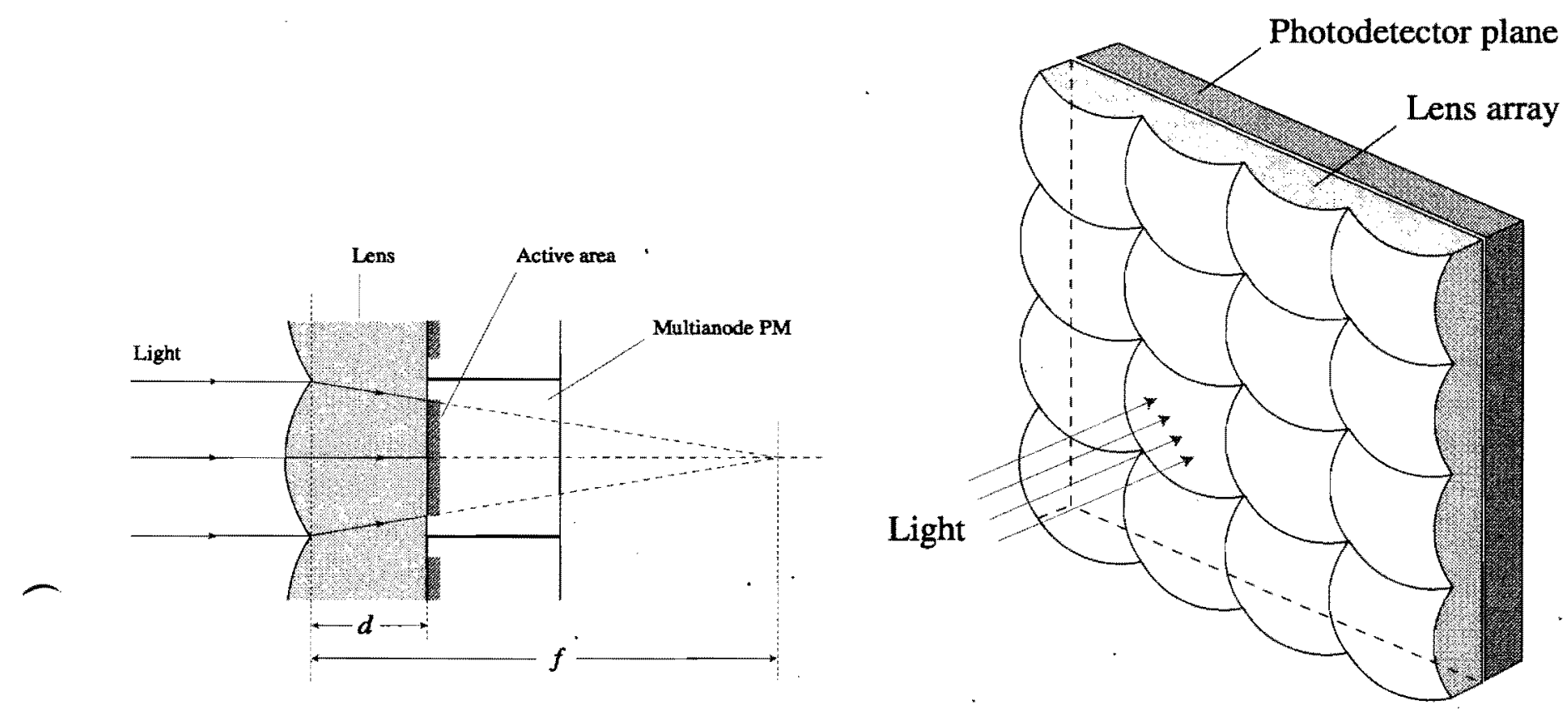

Figure A.30: Two views of the lens system proposed by R. Forty for use with closely packed arrays of Hamamatsu R5900 MAPMTs. The pictures are taken from Ref.[18].

In the PMT approach the wavelengths are limited to visible range by the affordable lens material. PMTs with sensitivity extended to the UV are more expensive since the sealing of quartz windows with a PMT glass envelope is complicated. Thus, standard bialkali photocathode on a borosilicate glass window (with the wavelength cutoff at $300 \mathrm{~nm}$ ) is adopted, resulting in a chromatic error of $0.33 \mathrm{mrad}$ per photon. On average, 58 photons are expected in the PMT system for a fully contained gaseous Cherenkov ring emitted by a fast particle. The total Cherenkov angle resolution is $0.83 \mathrm{mrad}$ per photon and $0.11 \mathrm{mrad}$ per track.

The particle ID resolution in the PMT option is only slightly worse than projected for the HPD system. Therefore, our choice is dictated by the cost of the photo-detectors. The price of PP0380V HPD tubes is 30\% less than for the R5900-M16 multi-anode PMTs for the same area covered. Counting the cost of PMT bases and the lens system would only enlarge the difference. Even though HPDs require much higher voltages than PMTs, they don't draw any current making the HV system more affordable. The number of electronic channels is comparable between the two systems. The lifetime of photo-detectors, radiation hardness and resistance to remnant magnetic fields is sufficient for both types of photo-detectors. 


\section{A.2.7 RICH Simulation and Reconstruction Software}

Charged tracks that exit the central dipole magnet are input to our RICH simulation package. The physics simulations included an average of two minimum bias events in addition to the $b \bar{b}$ event. Photon conversions in front of the RICH detector were also included. The same RICH simulation package is used in the BTeVGeant and MCFast environments. Cherenkov photons are radiated along charged track intersections with aerogel and gas radiators limited by their geometrical extent, which includes a simulation of the beampipe hole. The dependence of the refractive indices of the $C_{4} F_{10}$ and aerogel radiators on the photon wavelength is taken from previous measurements. Cherenkov threshold effects are simulated exactly by explicit integration of the Cherenkov formula over wavelengths for each value of the particle velocity. Each Cherenkov photon is traced through the RICH geometry. The light reflection and refraction depends on the photon polarization induced by the Cherenkov radiation process. Absorption at the beampipe, vessel walls and in the gas radiator itself are taken into account. Photons reaching one of the spherical mirrors are reflected towards the photo-detectors. The wavelength dependence of the reflection probability is simulated. The average reflectivity was set to agree with the results achieved in the HERA-B experiment. Each photo-detector tube is represented in the ray tracing, including all geometrical details. The HPD tube has a spherical quartz window which focuses photons onto the photocathode. (In the PMT system, optical focusing by acrylic lenses plays an even bigger role.) We have included the wavelength dependence of the refractive indices and absorption coefficients for quartz and acrylic. The wavelength dependence of the quantum efficiency of the photocathodes is simulated according to the benchmark curves supplied by DEP and Hamamatsu. For HPDs, we introduced a $280 \mathrm{~nm}$ cutoff, which can be achieved with an appropriate window coating. Electrostatic focusing of photoelectrons onto the silicon diode is assumed to be linear. The hexagonal pixel geometry is assumed, with 163 pixels per HPD tube. For the multianode tubes we simulate the dependence of the photoelectron collection efficiency on the first dynode, which depends on the point of photon incidence at the photocathode (using data supplied by Hamamatsu).

In the analysis of the Monte Carlo data, we start from charged tracks reconstructed in the tracking system. For each hit recorded in the photo-detectors we calculate a Cherenkov angle for a given track and each mass hypothesis $(e, \mu, \pi, K, p)$, assuming that the photon was radiated at the middle point of the track intersection with the radiator. We store a list of such calculated Cherenkov angles if the reconstructed angle is within $\pm 3 \sigma$ of any of these mass hypotheses. Since Cherenkov rings from different tracks intersect each other (see Fig. 5.7), we remove hits from the intersection regions before calculating the per track average Cherenkov angle. This is done in two steps. In the first pass, all hits within $\pm 3 \sigma$ to a mass hypothesis are included in the per track average, excluding those hits which are within $\pm 3 \sigma$ of the pion hypothesis for any other track. The second pass is essentially the same except that instead of assuming that all tracks are pions in the hit exclusion, the most likely mass hypothesis based on the first pass result is used.

Variables used to discriminate between two mass hypotheses for the same track are described in Section 5.6 of Part II of this proposal. We do not yet use all of the available 
information in the most efficient way. Particle identification algorithms need to be refined, especially at lower momenta, where the threshold effects become important. Therefore, we believe that the simulation results presented in this proposal are conservative. Clearly more work on the reconstruction algorithms is needed. 


\section{Bibliography}

[1] Delft Electronic products B.V., Specification of $72 \mathrm{~mm}$ Electrostatically Focused Hybrid Photodiode, PP0380, 184-2114A0 (Sep. 1998).

[2] BTeV, BTeV proposal - Appendix A - Data Acquisition System.

[3] E. Nygard et al., Nucl. Instr. and Meth. A301 (1991) 506.

[4] IDE AS, IDE Front End ASICS, (1997).

[5] M. Tanaka et al., Nucl. Instr. and Meth. A432 (1999) 422.

[6] B. Alpat et al., Nucl. Instr. and Meth. A439 (2000) 53.

[7] M. Overdick et al., Nucl. Instr. and Meth. A392 (1997) 173.

[8] M. Artuso Nucl. Instr. and Meth.A409 (1998) 375.

[9] M. Artuso et al., Nucl. Instr. and Meth. A419 (1998) 577.

[10] A. Braem et al., Nucl. Instr. and Meth. A433 (1999) 153.

[11] D. Ryckbosch et al., Nucl. Instr. and Meth. A433 (1999) 98.

[12] T. Sumiyoshi et al., Nucl. Instr. and Meth. A433 (1999) 385.

[13] I. Adachi et al., Nucl. Instr. and Meth. A355 (1995) 390.

[14] A.R. Buzykaev et al., Nucl. Instr. and Meth. A433 (1999) 396.

[15] A.F. Danilyuk et al., Nucl. Instr. and Meth. A433 (1999) 406.

[16] E. Nappi, ICFA Instrumentation Bulletin 17 (Fall 1998).

[17] J.L. Rosen, Nucl. Inst. and Meth. A408 (1998) 191; S. Kopar, Proceedings of the The 3rd Int. Workshop on Ring Imaging Cherenkov Detectors Weizmann Institute of Science Ein-Gedi, Dead-Sea, Israel, Nov., 1998.

[18] R. Forty, "Use of lenses to increase the RICH photo-detector coverage", LHCb Internal Note LHCB/98-038. 


\section{A.3 Electromagnetic Calorimetry}

\section{A.3.1 Introduction}

A thorough investigation of $B$ decays requires the ability to study final states including single photons, $\pi^{0}$ 's, and $\eta$ 's. Among these are some of the important decay modes mentioned in Part 1:

- $B^{0} \rightarrow(\rho \pi)^{0} \rightarrow \pi^{+} \pi^{-} \pi^{0}$

- $B^{0} \rightarrow K^{* 0} \gamma$ and $\rho^{0} \gamma$

- $B_{s}^{0} \rightarrow \psi \pi^{0}, \psi \eta$, and $\psi \dot{\eta}^{\prime}$

- $B^{0} \rightarrow K^{* 0} \pi^{0} ;$ and

- $B^{ \pm} \rightarrow K^{ \pm} \pi^{0}$ and $\pi^{ \pm} \pi^{0}$.

In addition to reconstructing photons, the electromagnetic calorimeter will be used to identify electrons. This has critical application in CP studies: $B$-tagging with the semileptonic decays $B \rightarrow D^{(*)} e \nu$, and the reconstruction of exclusive $B$ decays into $J / \psi$ or $\psi^{\prime}$.

To study these decays, one needs to separate small signals from background, a major challenge in the hadron-collider environment. The requirements for the BTeV calorimeter are similar to those for the CMS experiment at the LHC: excellent energy and position resolution (little inactive materials), compact shower size to minimize overlapping showers (small Moliere-radius materials), fast signal (to minimize shower overlaps in time), and radiation hardness. Among the materials that were known to have potential to satisfy these requirements were inorganic scintillation crystals such as $\mathrm{PbWO}_{4}(\mathrm{PWO})$ and $\mathrm{CeF}_{3}$. CMS chose PWO scintillation crystals. Taking advantage of the enormous R\&D effort already carried out for CMS, we have decided to develop a design for an electromagnetic calorimeter based on PWO crystals. Since we can use PMT's (photomultiplier tubes) instead of CMS's APD (avalanche photodiodes) or VPT (vacuum phototriodes), our resolution will be better than that of CMS, particularly at energies below $10 \mathrm{GeV}$. This energy range is particularly important for $\mathrm{BTeV}$.

\section{A.3.2 Radiation Tolerance and Radiation Levels}

Radiation damage of PWO crystals is a serious issue. Detailed studies [2] reveal that the light transmission of crystals deteriorates due to formation of color centers by radiation, while the scintillation mechanism itself seems unaffected. When a PWO crystal no longer receives radiation, its color centers (semi-stable excited states) disappear, and it recovers from transmission degradation by natural room-temperature annealing. In fact, this annealing goes on even during radiation exposure. Therefore, when crystals are exposed to a constant radiation level, they lose light only up to the point where the rates of radiation damage and natural recovery balance. 
For the majority of crystals at CMS (all barrel crystals and $25 \%$ of endcap crystals), the expected radiation dose rate is less than $0.15 \mathrm{~Gy} /$ hour [3]. At this rate the CMS production crystals lose only a few per cent of their light output. For about $10 \%$ of the endcap crystals, the radiation dose rate will be as high as $18 \mathrm{~Gy} /$ hour. Even at this rate, the light loss saturates at less than $20 \%$ [4]. This reflects the fact that the density of crystal defects, which can turn into color centers, is limited in these high-quality crystals.

This level of radiation sensitivity is perfectly acceptable for BTeV, where most crystals will incur less than $0.1 \mathrm{~Gy} /$ hour, and only a few crystals receive more than $1 \mathrm{~Gy} /$ hour. To make these estimates, we have carried out a full calculation of the radiation environment, accounting for both the direct products of collisions and the secondary particles produced in interactions with the material of the $\mathrm{BTeV}$ detector [5]. To calculate the radiation levels in the crystals and the phototubes, a full Monte Carlo simulation using the MARS (IHEP) code was performed [6]. MARS is the standard IHEP package used for radiation environment calculations for both the CMS and LHC-b projects at CERN [7]. We used Pythia to generate minimum bias events for input to MARS. At a luminosity of $2 \times 10^{32} \mathrm{~cm}^{-2} \mathrm{~s}^{-1}$, assuming an inelastic cross-section of $60 \mathrm{mb}$, the Tevatron produces $1.2 \times 10^{7}$ inelastic $\bar{p} p$ events per second. Together with particles produced in interactions with detector material, this produces a decidedly hostile radiation environment.

Each year of BTeV operation was assumed to be $10^{7}$ seconds, the same unit as was used in the physics sensitivity estimates. In Fig. A.31 we show the annual per-crystal dose (at shower maximum, 4-7 cm behind the front face of the EMcal) as a function of position transverse to the Tevatron beamline.

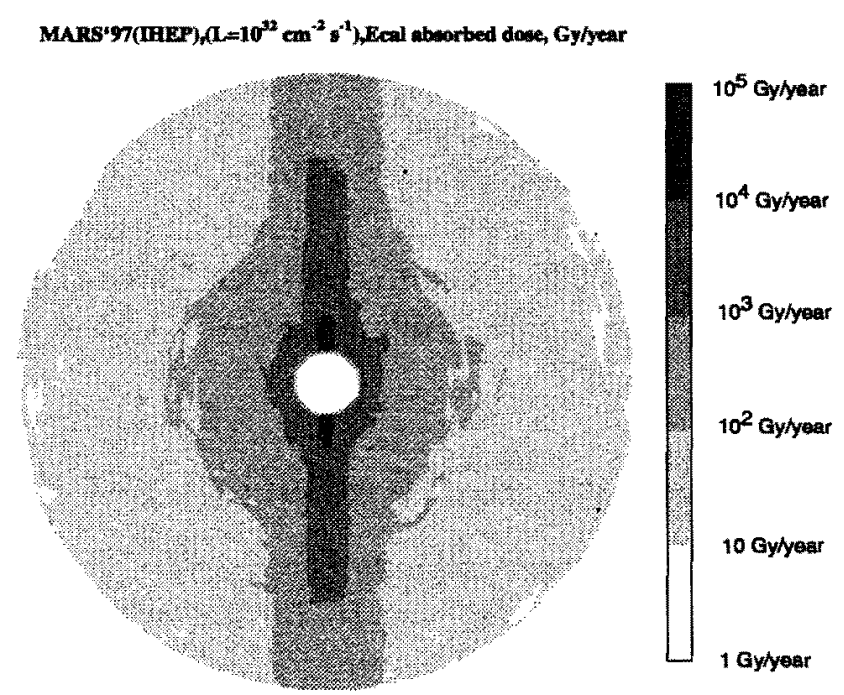

Figure A.31: Annual radiation dose in crystals at shower maximum. Note that a yearly dose of $1 \mathrm{kGy}$ corresponds to a dose rate of $0.3 \mathrm{~Gy} /$ hour. 


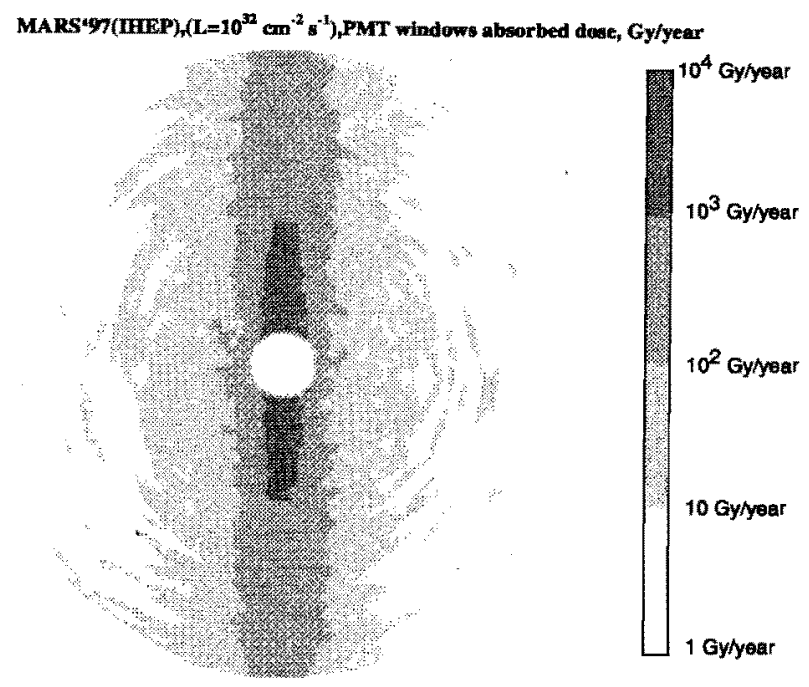

Figure A.32: Annual radiation dose at PMT windows.

The annual radiation dose exceeds $10 \mathrm{kGy}$ ( $3 \mathrm{~Gy} /$ hour) for a small number of crystals at the smallest radii just above and below the beamline. This is because a portion of the intense flow of charged particles near the beamline, which mostly pass through the hole of $15 \mathrm{~cm}$ radius in the middle of the calorimeter, are deflected vertically by the dipole magnet. The highest dose is $30 \mathrm{kGy} /$ year or $10 \mathrm{~Gy} /$ hour. This is still only $1 / 2$ of the maximum dose rate CMS expects. Fig. 6.3 (in Part 2) shows the fraction of the $\mathrm{BTeV}$ crystals below a given dose rate, which is shown on the horizontal axis. One can see that $90 \%$ of the crystals receive dose rates less than those for the CMS barrel crystals.

The PMT windows can potentially also suffer radiation damage. Here our concern is the total dose, since there is no recovery mechanism. The same simulation as described above indicates that the maximum dose near the Tevatron beamline is $7 \mathrm{kGy} /$ year. Fig. A.32 shows the radiation level at the PMT's as a function of their location. From this study, we conclude that the majority of the PMT's must have either quartz or radiation-tolerant glass windows [9].

\section{A.3.2.1 Expected Energy Resolution and Efficiencies}

The energy resolution that we can expect from a PWO system will depend on several factors, but it can be parameterized as a function of the incident energy $E$ as

$$
\frac{\sigma_{E}}{E}=\sqrt{\frac{S^{2}}{E}+C^{2}} \equiv \frac{S}{\sqrt{E}} \oplus C .
$$


The first term, the "stochastic term," is due to fluctuations in the leakage of shower energy to crystals surrounding the main cluster of crystals (transverse shower leakage). Since the number of photoelectrons detected by the PMT's fluctuates appreciably, it also contributes to this stochastic term. We will evaluate this term below. The second term, the "constant term," arises from variations in light output along the crystal length and errors in the relative calibration between crystals. Fluctuations in the energy escaping from the rear of the crystals also contribute to this term.

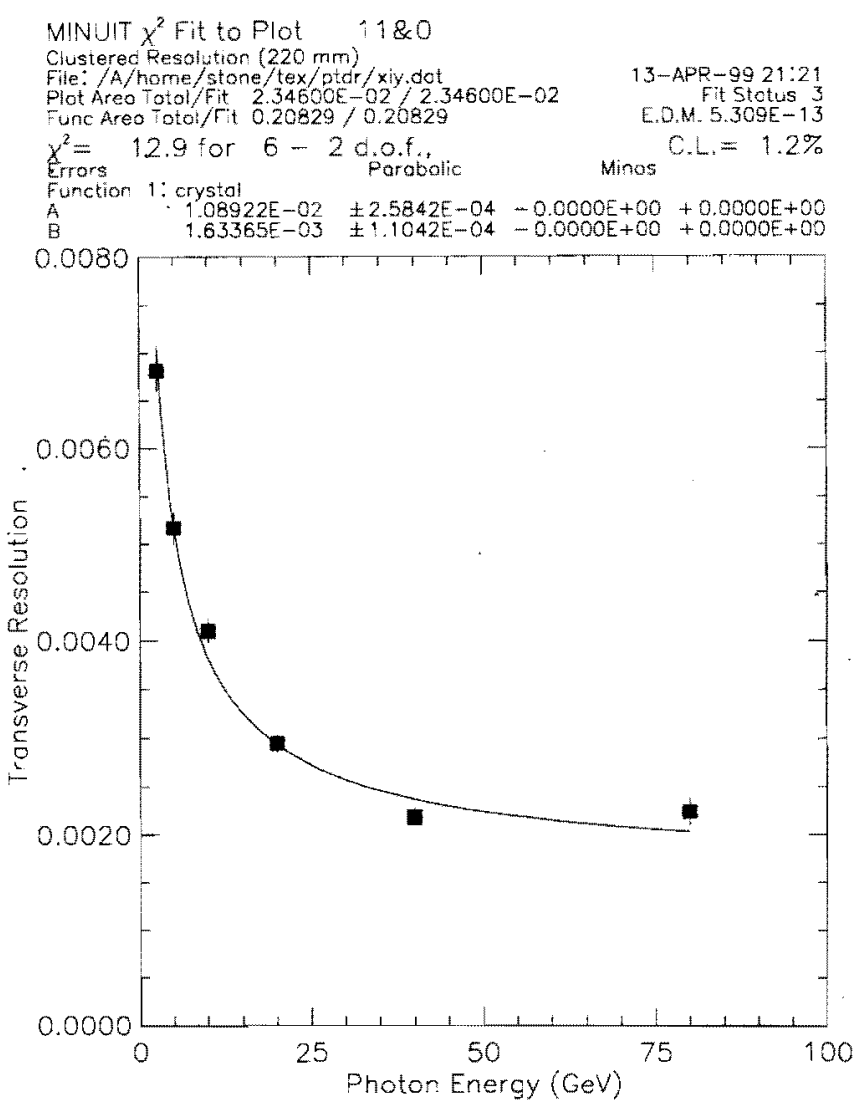

Figure A.33: Energy resolution as a function of incident photon energy for $220 \mathrm{~mm}$ long crystals and $5 \times 5$ clustering.

We can use the energy resolution expected by CMS to guide our expectations. CMS based their study on an assumption of 4 photoelectrons/MeV read out by two APD's. There is an excess noise factor in the APD's due to correlated gain, effectively giving a factor of two reduction in the number of photons. Thus photostatistics gives a contribution of $2.3 \% / \sqrt{E}$ to the expected CMS resolution. The term for lateral containment was studied for a $5 \times 5$ array of $22 \times 23 \mathrm{~mm}^{2}$ crystals, for which CMS found a contribution of $1.5 \% / \sqrt{E}$. Adding these together in quadrature gives $2.7 \% / \sqrt{E}$. They estimated the constant term to be $0.55 \%$. CMS also included extra noise, which is constant in $\sigma_{E}$, due to leakage currents in the photodiodes, and the effect of summing over $\sim 25$ interactions per crossing, each with 


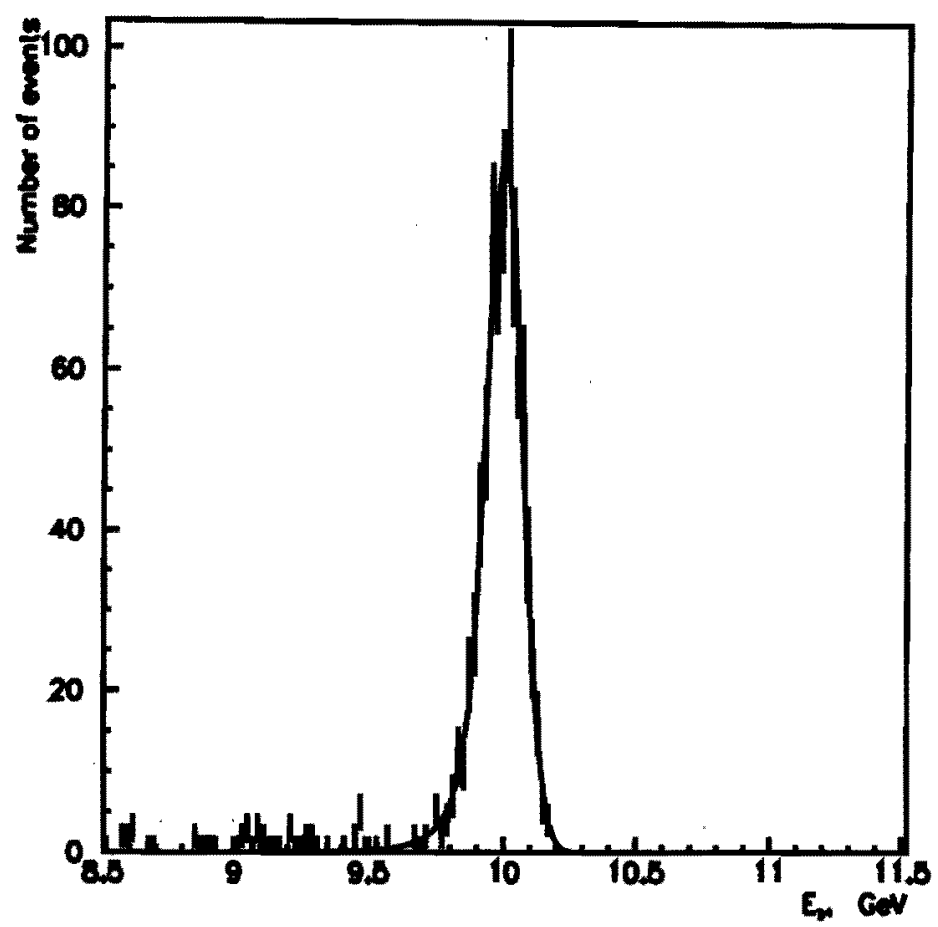

Figure A.34: Distribution of measured photon energies for $10-\mathrm{GeV}$ photons using a realistic clustering algorithm. The curve shows the result of a fit to a resolution function.

7 times more energy than the Tevatron. These effects are not present for BTeV.

Additional guidance can be found by reviewing the performance of the KTeV CsI calorimeter. They have achieved $[10]$

$$
\frac{\sigma_{E}}{E}=\frac{2 \%}{\sqrt{E}} \oplus 0.45 \%
$$

The dominant contribution to the constant term seems to be dependence of the response on the position on the face of the crystal where the photon is incident. This effect was not observed in the CLEO CsI calorimeter [11].

The photon statistics term for $\mathrm{BTeV}$, taking 7 photoelectrons/MeV, is expected to be $1.3 \% / \sqrt{E}$, including the correlated gain factor for the PMT of 1.2 .

The energy resolution determined with a GEANT-based simulation of $220 \mathrm{~mm}$ long crystals, without photon statistics or electronic noise, is shown in Fig. A.33. It can be parameterized as

$$
\frac{\sigma_{E}}{E}=\frac{a}{\sqrt{E}} \oplus b
$$

where $a=1.1 \%$ and $b=0.2 \%$, and $E$ is in GeV. The stochastic term $a$ comes mainly from transverse leakage, while the constant term arises mainly from longitudinal leakage. 


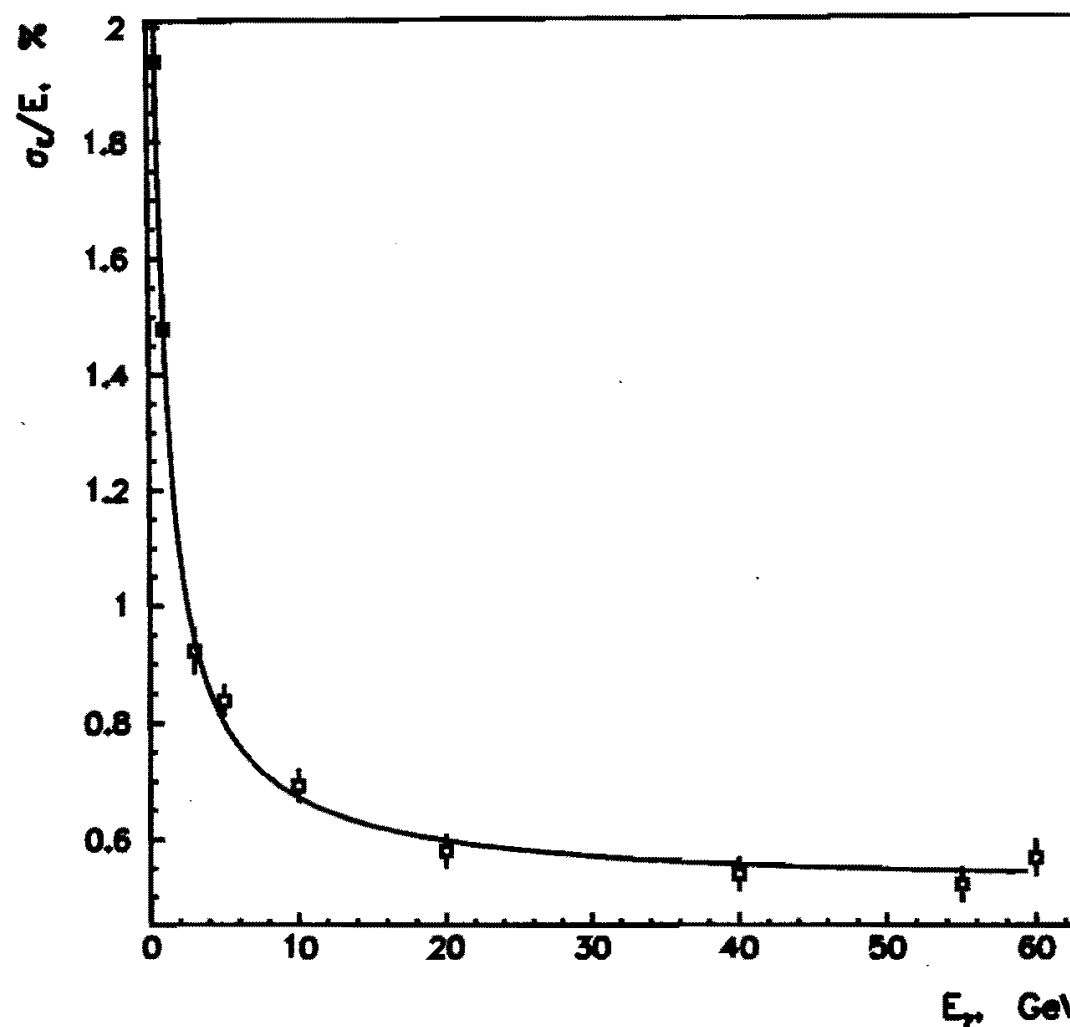

Figure A.35: Energy-dependent energy resolution. The curve shows the result of a fit to a resolution function.

Adding the two stochastic components in quadrature gives $1.6 \% / \sqrt{E}$. We take the CMS estimated value of $0.55 \%$ for the constant term and find an expected energy resolution for $\mathrm{BTeV}$ of

$$
\frac{\sigma_{E}}{E}=\frac{1.6 \%}{\sqrt{E}} \oplus 0.55 \% .
$$

Fig. A.34 shows the distribution of measured energies for a Monte Carlo simulation of $10 \mathrm{GeV}$ photons. The effect of photon statistics was included in the simulation. The curve shows the result of a fit to a photon resolution function [13]. The resolution is about $0.7 \%$ at $10 \mathrm{GeV}$. Fig. A.35 shows energy dependence of the energy resolution, and the result of the fit to Equation (1). The fit results indicate that the constant term of the resolution is $0.5 \%$ and stochastic term is $1.4 \%$ at $1 \mathrm{GeV}$, which is consistent with Equation A.18 above.

The efficiency for detecting photons in a clean event environment is about $80 \%$, independent of energy. Most of the loss comes from conversions in material in front of the calorimeter. This efficiency clearly depends additionally on the event environment and the number of interactions per crossing. 


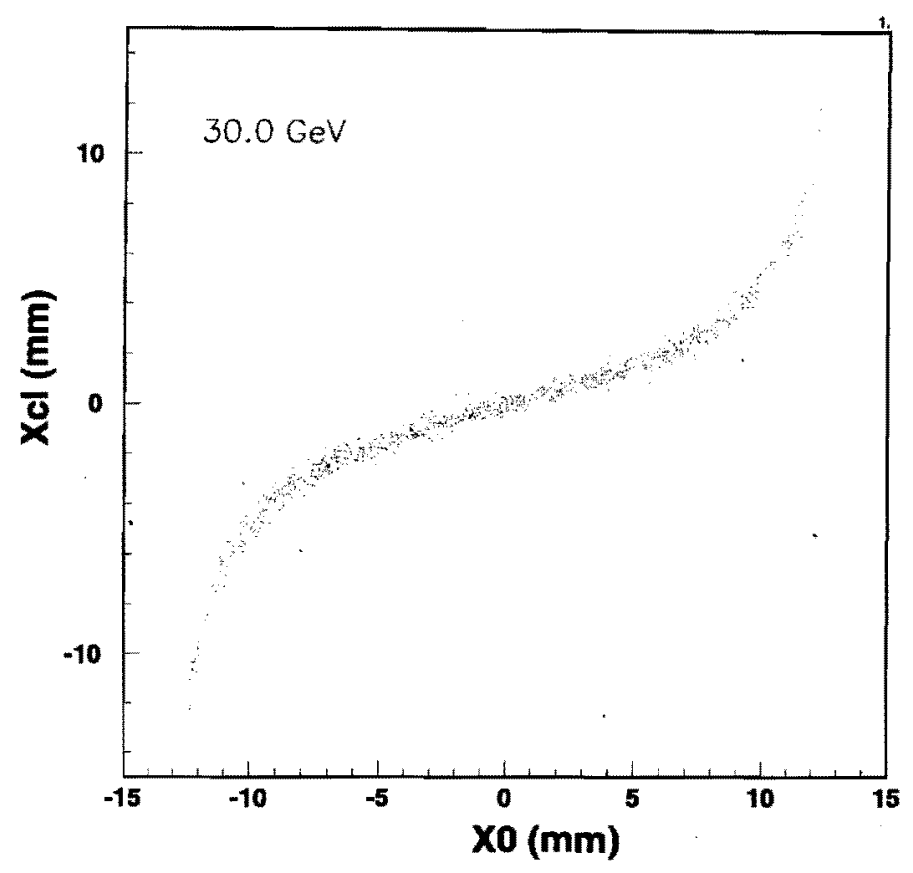

Figure A.36: Measured photon position $x_{\mathrm{Cl}}$ versus known photon position $x_{0}$ for $30 \mathrm{GeV}$ photons.

\section{A.3.2.2 Position Resolution}

The $\mathrm{BTeV}$ electromagnetic calorimeter is 7 meters from the center of the dipole magnet, so the resolution of the projected angle is given to first order by the error in the transverse position divided by 7 meters. This is particularly important for high-energy $\pi^{0}$ 's, since the opening angle of the photon pair is small. We used GEANT to simulate the energy deposit in $220 \mathrm{~mm}$ long crystals. Positions were determined in both directions transverse to the crystal axes. The position of the reconstructed photon is determined from the energy deposited by using the formula:

$$
x_{\mathrm{cl}}=\frac{\sum_{i=1}^{25} x_{i} \cdot E_{i}}{\sum_{i=1}^{25} E_{i}},
$$

where $E_{i}$ is the energy reconstructed in each crystal, $x_{i}$ is the position of the center of each crystal, and the sum extends two crystals in each direction from the highest energy crystal.

Fig. A.36 shows $x_{\mathrm{cl}}$ versus the true position of the photon, $x_{0}$, for a sample of simulated $30 \mathrm{GeV}$ incident photons. The characteristic "S-curve" shape is apparent. To evaluate the position resolution, the overall shape of the S-curve is removed by a fit to give a corrected cluster position $x_{\text {corr }}$. In Fig. A.37, the distribution in $\Delta x=x_{\text {corr }}-x_{0}$ is plotted. The width of this distribution is the r.m.s. position resolution. 


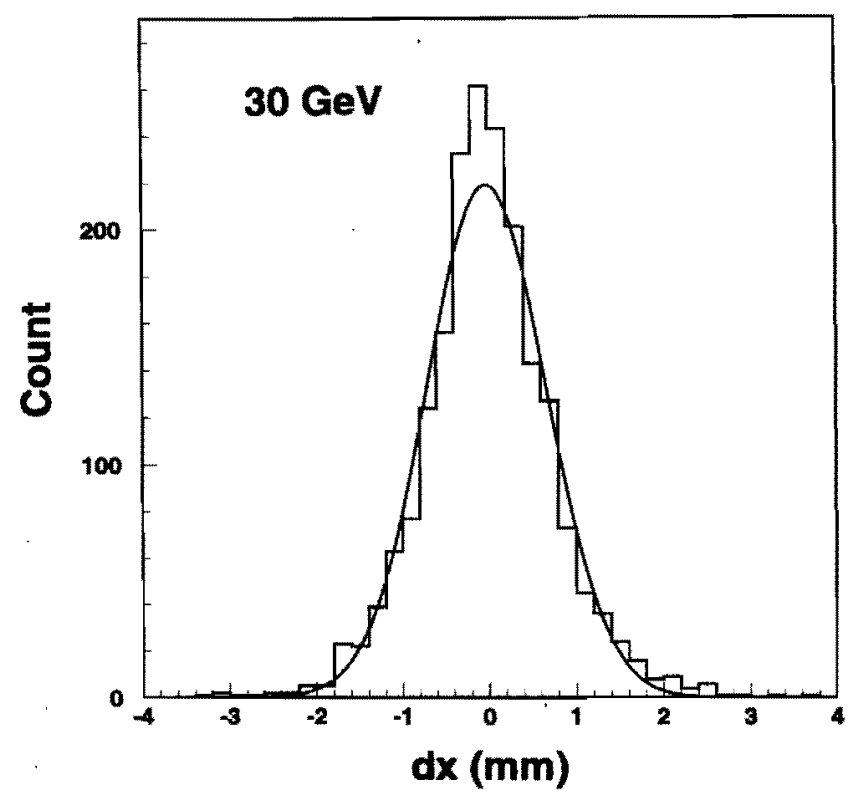

Figure A.37: Distribution of $\Delta x=x_{\text {corr }}-x_{0}$ for $30 \mathrm{GeV}$ photons. The fit is a Gaussian with mean consistent with zero and $\sigma=0.7 \mathrm{~mm}$.

The results of performing this study over a range of energies are shown in Fig. A.38, which shows the r.m.s. position resolution as a function of energy using full. BTeVGeant simulations and a realistic clustering algorithm. The fit gives

$$
\sigma_{x}=\frac{3.1 \mathrm{~mm}}{\sqrt{E}} \oplus 1.7 \mathrm{~mm} .
$$

This position resolution implies excellent angular resolution. At $6 \mathrm{GeV}$, the r.m.s. angular resolution is $0.3 \mathrm{mrad}$.

\section{A.3.3 Design Parameters}

\section{A.3.3.1 Projective vs. Non-projective Geometry}

It is well known that calorimeters with projective geometry perform better than nonprojective ones. The projective structure minimizes shower overlaps and provides better position resolution. Fabrication of the calorimeter, on the other hand, may be more complex with a projective geometry and may result in higher cost.

CLEO's endcap calorimeter has a non-projective geometry. This decision was made to simplify its construction, since it was possible simply to stack crystals one on top of another. Detailed Monte Carlo studies showed the performance of this calorimeter to be comparable to that of a projective-geometry alternative. This is because the angular resolution plays a negligibly small role compared to the energy resolution for all measurements relevant to the 


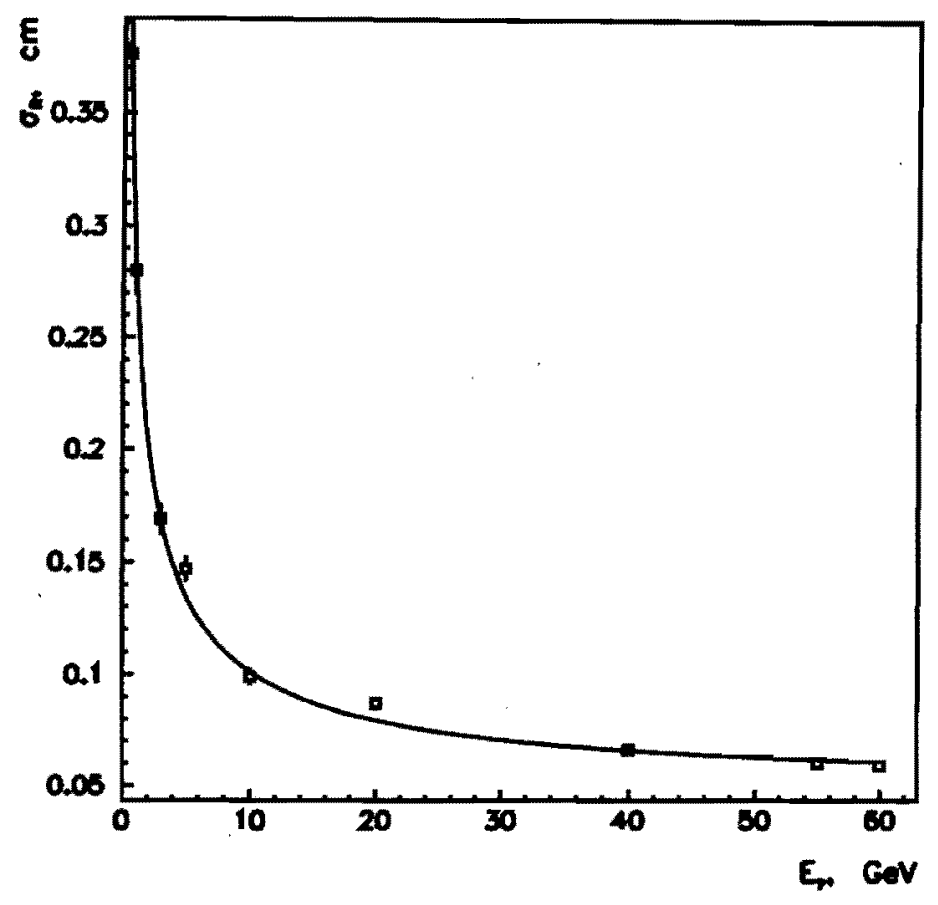

Figure A.38: Energy-dependent position resolution. The curve shows the result of a fit to resolution function.

CLEO physics program, including the $\pi^{0}$ mass resolution. This conclusion is specific to the physics of an $e^{+} e^{-}$storage ring at a center-of-mass energy of $\sim 10 \mathrm{GeV}$, where all particles decaying into photons are of quite low energy.

For $\mathrm{BTeV}$, the deterioration of position resolution for rectangular crystals is as much as $2 \mathrm{~mm}$. This will degrade the $\pi^{0}$ mass resolution above $10 \mathrm{GeV}$. No other negative effects of a non-projective calorimeter design have been identified at the time of this writing.

PWO crystals are too fragile to stack up one on top of another - with sufficient weight over them the crystals would fracture. In the absence of clear cost advantages, we have therefore decided to adopt a projective geometry as our baseline design for the $\mathrm{BTeV}$ calorimeter. If we find a way to make a non-projective (rectangular crystal) calorimeter more cheaply, and if there is no additional physics case for the projective geometry, we will likely change our baseline design. 


\section{A.3.3.2 Lateral Size}

The lateral size of the crystals should be comparable to the Moliere radius of PWO to provide the best performance. We use $25.4 \times 25.4 \mathrm{~mm}^{2}$ in the front and $26 \times 26 \mathrm{~mm}^{2}$ in the back, somewhat smaller than the design for the CMS endcap crystals [12]. Anything larger would compromise the position resolution and increase overlapping showers, but would reduce the cost of PMT's and electronics. A smaller lateral size would increase the cost substantially, particularly because we would need to use custom-size PMT's.

\section{A.3.3.3 Crystal Length}

The crystal length for the BTeV calorimeter has been determined with a study of GEANTsimulated showers, the results of which are summarized in Fig. A.39. The distributions of the detected energies for samples of simulated $80-\mathrm{GeV}$ photons (normalized to the true energy) are shown for crystal lengths ranging from $170 \mathrm{~mm}$ to $230 \mathrm{~mm}$. It is clear that the loss in energy resolution in going from $230 \mathrm{~mm}$ to $220 \mathrm{~mm}$ is minimal, but as one goes to shorter lengths, the energy resolution suffers.
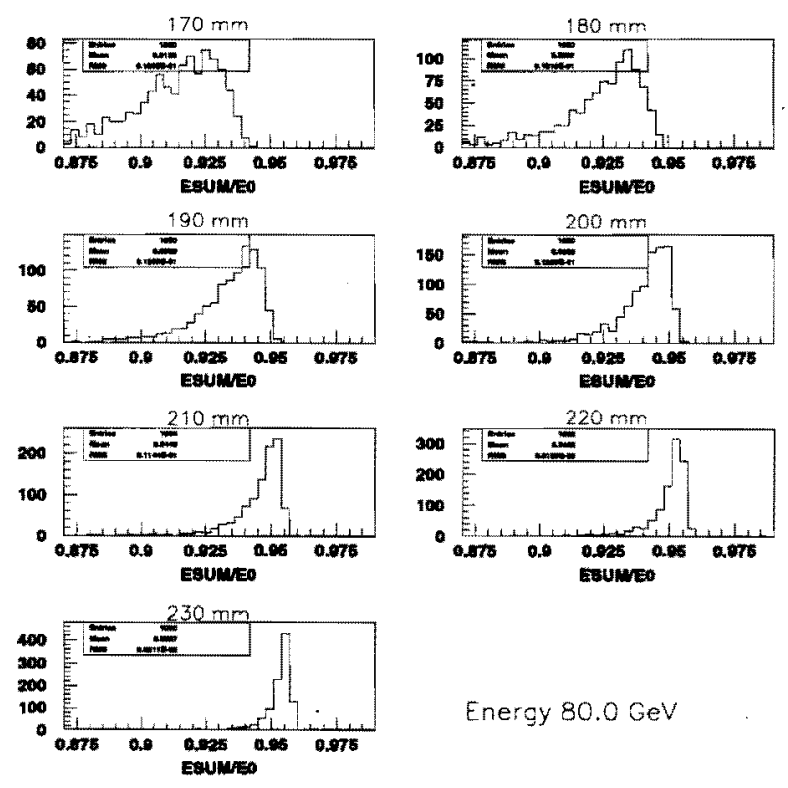

Energy $80.0 \mathrm{GeV}$

Figure A.39: Energy deposited for $80 \mathrm{GeV} / \mathrm{c}$ photons normally incident on $\mathrm{PbWO}_{4}$ crystals of the labeled lengths.

The normalized energy distributions for GEANT events with different energy photons and crystal lengths between 210 and $230 \mathrm{~mm}$ were fitted with resolution functions developed for the Crystal Ball experiment [13]. An example of such a fit is shown in Fig. A.40. The values of the energy resolution for the cases considered in this study are shown in Fig. A.41: The energy resolution is minimally affected by shortening the crystals from $230 \mathrm{~mm}$ to 220 


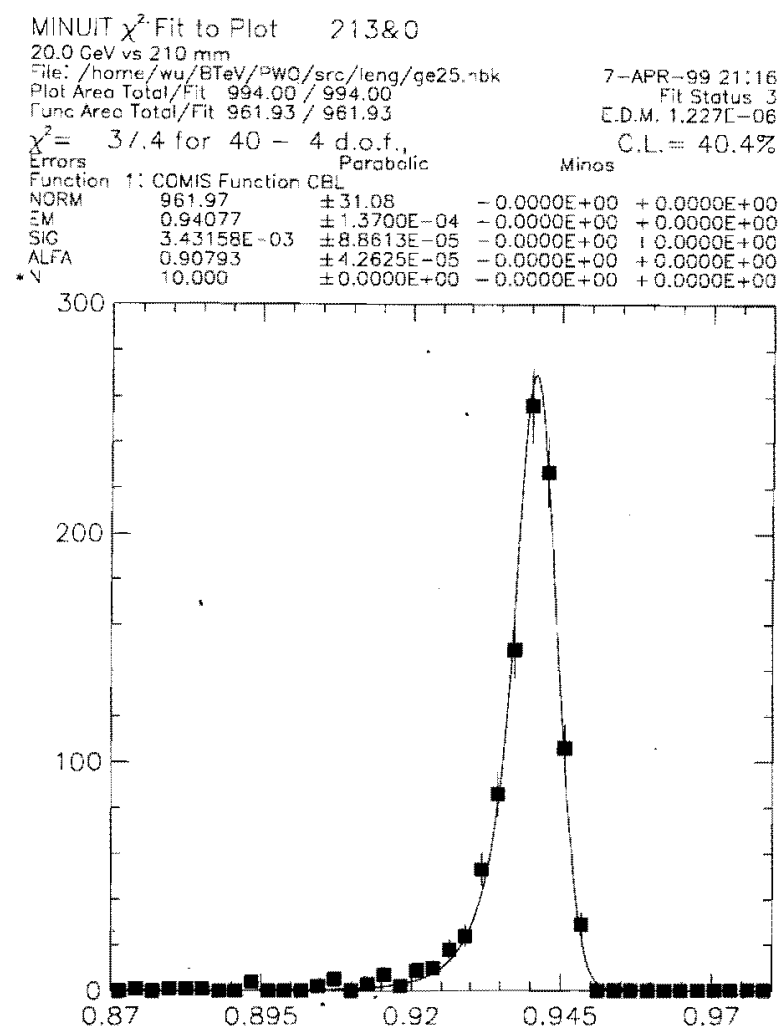

Figure A.40: Fit of the normalized clustered energy deposit for normally incident $20 \mathrm{GeV}$ photons in crystals of length $210 \mathrm{~mm}$. The fitted line shape is from the Crystal Ball experiment.

$\mathrm{mm}$, but there is a significant degradation in going to $210 \mathrm{~mm}$. This study uses the energy deposited in $5 \times 5$ arrays of crystals. Smearing due to photon statistics has not been included, so the resolutions are unreasonably good. Based on these studies, we decided to use $220 \mathrm{~mm}$ long crystals.

\section{A.3.3.4 $\pi^{0}$ Mass Resolution}

We have also studied the mass resolution for $\pi^{0}$ decays to photon pairs. Fig. A.42 shows the distributions of two-photon invariant masses when the initial $\pi^{0}$ 's have energies of 5,10 and $30 \mathrm{GeV}$. We observe an excellent resolution of less than $3 \mathrm{MeV}$ when the $\pi^{0}$ energy is between 2 and $10 \mathrm{GeV}$ [15]. It degrades slightly at higher energies where the angular resolution starts to contribute. At energies lower than $2 \mathrm{GeV}$, the mass resolution also degrades, in this case because the stochastic term in the photon-energy resolution becomes significant. 


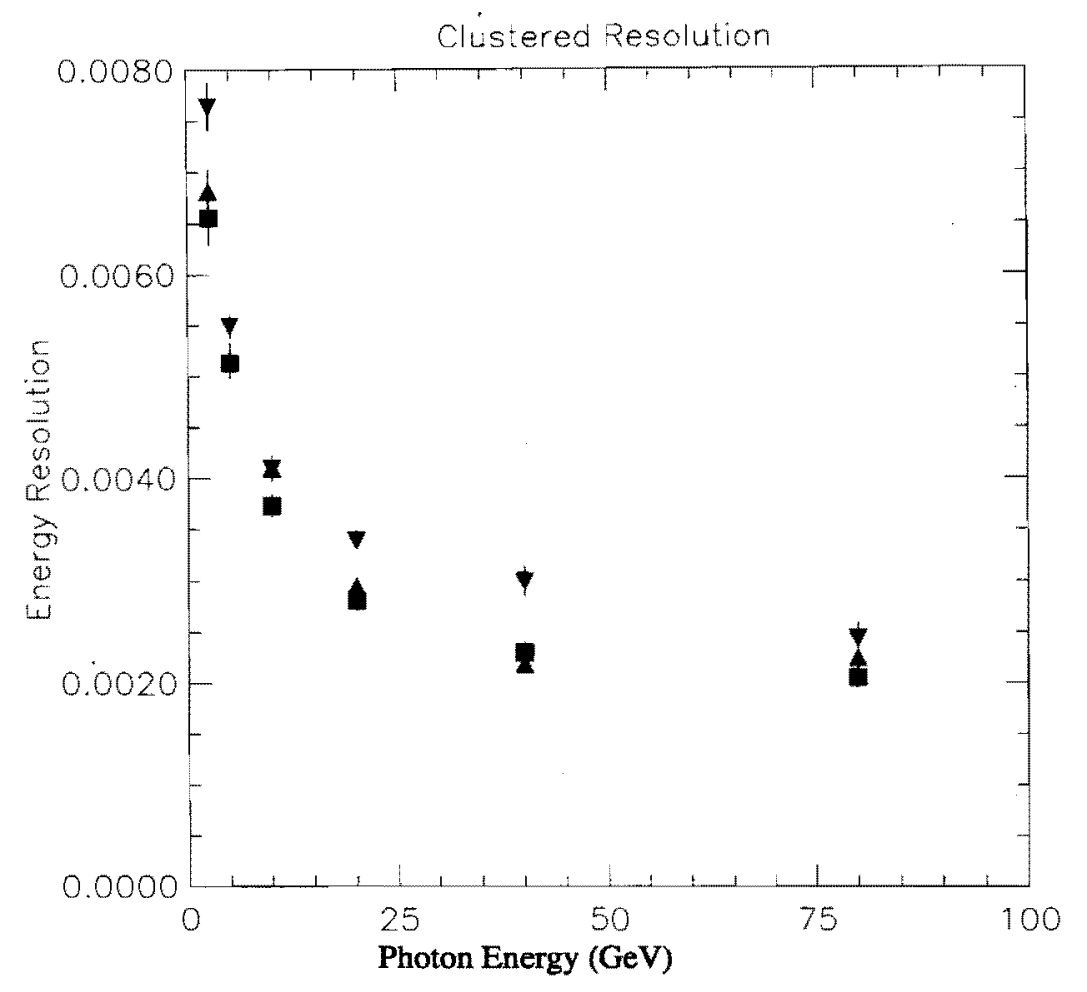

Figure A.41: Energy resolution as a function of incident photon energy for different crystal lengths using $5 \times 5$ clustering. The squares are for a crystal length of $230 \mathrm{~mm}$, the triangles for $220 \mathrm{~mm}$ and the inverted triangles for $210 \mathrm{~mm}$.

\section{A.3.3.5 Merged $\pi^{0}$}

It is clearly quite important that $\mathrm{BTeV}$ be able to distinguish photons from $\pi^{0}$ 's. Many of the $\pi^{0}$ 's will be fast and could in principle appear as single clusters. To study how well we could separate these two components with the proposed transverse segmentation, we generated samples of both $\gamma^{\prime}$ s and $\pi^{0}$ 's at a variety of energies.

To study the probability of overlaps quantitatively, we calculated the second-moment mass or cluster mass, which is described in Section 14.3 (ECAL Cluster Finder). The cluster mass distributions for both photons and $\pi^{0}$ 's of fixed energies are compared in Fig. A.43. They are well separated up to $60 \mathrm{GeV}$, but they start overlapping at $70 \mathrm{GeV}$. In Table A.3 we show the photon detection efficiency for selection criteria with $\pi^{0} \rightarrow \gamma \gamma$ rejection probabilities of $90 \%$ and $99 \%$. Since most of the photons and neutral pions of interest in $\mathrm{BTeV}$ are below $60 \mathrm{GeV}$, the photon- $\pi^{0}$ discrimination achieved with this design is excellent. 

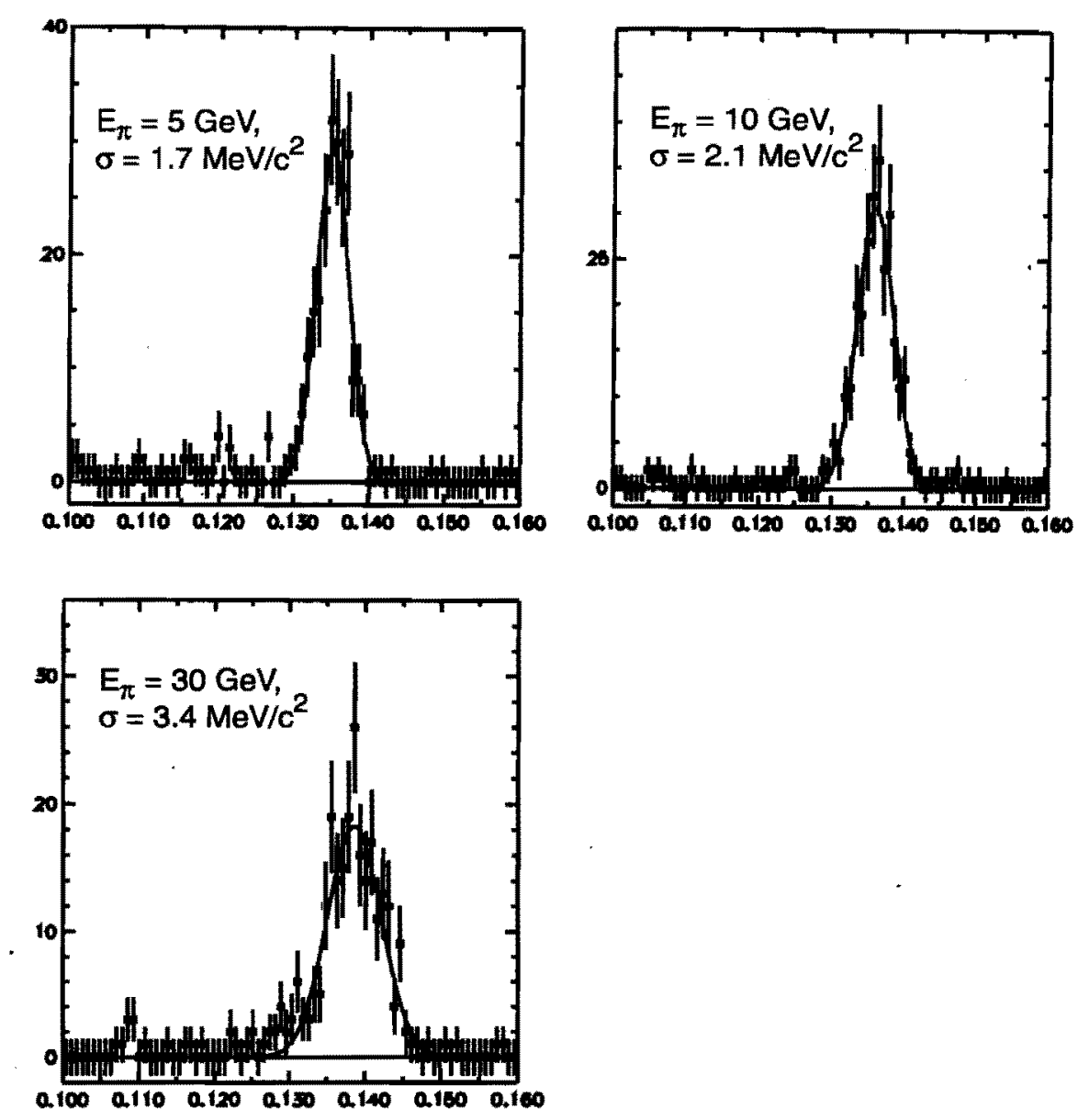

Figure A.42: Two-photon invariant mass distributions for simulated $\pi^{0}$ 's of energy 5,10 and $30 \mathrm{GeV}$.

\section{A.3.4 Mechanical Systems}

Since no design work on the BTeV calorimeter mechanical support system has yet been done, the baseline assumes that we will adapt the CMS endcap design. It has many nice features, but is expensive. If we can use a CLEO-like approach, the cost could go down substantially. One of the major obstacles to this is the fragility of PWO crystals, which are very sensitive to mechanical stress. As has been mentioned, they cannot simply be stacked, because the crystals near the bottom are likely to crack from the weight above.

CMS chose to use a structure made of "alveolar cells." These are molded from glass fiber and resin for the barrel and carbon fiber for the endcaps. Crystals are held individually, so that the alveolar cells bear the weight. In the CMS endcap design, the alveolar cells are cantilevered from the strong back, which is the major structure member of the endcaps. 
Table A.2: Reconstructed $\pi^{0}$ mass resolution.

\begin{tabular}{cc}
\hline \hline & \\
$\pi^{0}$ Energy (GeV) & Resolution $(\mathrm{MeV})$ \\
\hline 1 & 4.6 \\
2 & 2.6 \\
5 & 1.7 \\
10 & 2.1 \\
30 & 3.4 \\
40 & 4.9 \\
\hline
\end{tabular}

Table A.3: Photon detection efficiencies for $99 \% \pi^{0}$ rejection

\begin{tabular}{ccc}
\hline \hline & & \\
Energy $(\mathrm{GeV})$ & $\gamma$ efficiency for $90 \% \pi^{0}$ rejection & $\gamma$ efficiency for $99 \% \pi^{0}$ rejection \\
\hline 40 & 100 & 99.8 \\
50 & 99.7 & 99.7 \\
60 & 99.5 & 93 \\
70 & 93 & 64 \\
\hline \hline
\end{tabular}

There is $0.4 \mathrm{~mm}$ of alveolar materials between crystals, but this design avoids any materials in front of the endcaps.

The major cost for this design is in the production of alveolar cells, over $\$ 1 \mathrm{M}$ for the 80,000 crystals in CMS. The Babar experiment used a similar design for their CsI calorimeter, and their cost was comparable to CMS.

\section{A.3.5 Crystal Acquisition}

For PWO crystals to be fast and radiation hard, they need to be produced very carefully. Any impurities and crystal defects, like $\mathrm{Pb}$ and $\mathrm{O}$ vacancies, increase both the slower components of the light output, and the radiation susceptibility. Therefore, establishing mass production procedures to produce good crystals has been one of the major goals of the R\&D program of the CMS calorimeter group and.its vendors at the Bogoroditsk Plant in Russia and the Shanghai Ceramic Institute.

They have realized this goal by concentrating on the following five factors:

- economical raw material purification methods

- adjustment of the stoichiometric ratio between $\mathrm{PbO}$ and $\mathrm{WO}_{3}$ in the raw material to compensate for the evaporation of $\mathrm{PbO}$ during crystal growth

- environmental gas during the crystal growth 
- annealing methods

- doping of $\mathrm{Y}, \mathrm{La}$ and/or $\mathrm{Nb}$ to compensate residual crystal defects.

The Russian manufacturer uses the Czochralski method to grow crystals. Before embarking on their PWO R\&D project with CMS, they already owned more than 100 ovens, which had previously been used to grow other kinds of crystals. They have already succeeded in producing good PWO crystals for the CMS barrel calorimeter, and have in fact produced more than 6000 production crystals in the past year. The Russian manufacturer still needs to complete additional $R \& D$ before it can produce larger and radiation-hard crystals with high production yield for higher-radiation endcap calorimeters (up to $18 \mathrm{~Gy} /$ hour or 25 $\mathrm{kGy} /$ year).

The Chinese producer uses the Bridgeman method to grow crystals. Due to its prior commitment to other HEP experiments, it did not start production R\&D until after the Russians. Nevertheless, the Chinese can already produce good barrel crystals, although it needs to improve its production yield more to be able to reduce the production cost below the quoted price.

We have visited both the Bogoroditsk and Beijing/Shanghai production facilties, hosted by our Russian IHEP and Chinese colleagues. Both companies are interested in growing crystals for BTeV at the same price they have promised to CMS, $\$ 1.60 / \mathrm{cc}$. They plan to finish production for CMS by the middle of 2005 by producing 7000 crystals per year at Bogoroditsk and 10000 crystals per year in China. Some capital investment on our part will be necessary to start crystal production prior to the CMS completion. We believe an upfront investment of $\$ 0.8-1.6 \mathrm{M}$ is sufficient to start $\mathrm{BTeV}$ production either at Russian and Chinese plant, and will provide enough crystals for one calorimeter by 2005.

There are two other potentially interested sources for PWO crystals. One new source is at Shandong University. One of the physicists there has joined BTeV, and more are interested in joining BTeV. The Shandong University HEP group forged a collaboration with their university's Institute of Crystal Materials when it became clear that PWO crystals may be powerful tools to study $b$ hadrons. The institute has grown a multitude of crystals for different purposes, many of which have been for laser applications. They have mastered various crystal growing technologies, including the Czochralski method. They have grown a handful of PWO crystals which are visually clearer than the first crystals grown in Russia several years ago, indicating that they already have good control of impurities and crystal defects. Although those crystals were smaller in size than we need, the Institute is eager to carry out an R\&D program this year to produce full-size sample crystals by the end of 2000 .

Many of the results of the R\&D work done by the collaboration of CMS and the Bogoroditsk plant have been published, but some of the important details are proprietary. It therefore may take some time for Shandong University to be able to produce truly radiation hard full-size crystals at an acceptable production cost.

Meanwhile, it is crucial that $\mathrm{BTeV}$ develop capabilities to measure important crystal characteristics such as radiation tolerance, so that we can give quick feedback on the qualities of its crystals to the Crystal Institute at Shandong. 
The Japanese firm, Furukawa, has worked with a KEK physicist to produce radiation hard PWO crystals for almost as long as Russians and CMS. They are the largest producer of Cadmium Tungstate crystals in the world, but they have not done much work to minimize production cost of PWO crystals. Consequently, their current production cost of PWO is about 3 times higher than the Russian and the Chinese. They are interested in producing PWO crystals for BTeV and are working on lowering their cost. One attractive option for them as well as for us is a possible collaboration between Furukawa and Shandong University, combining Furukawa's existing know-how about PWO production with lower Chinese labor costs. This collaboration has potential for producing good and affordable crystals in a relatively short time scale.

\section{A.3.5.1 Calibration Systems}

Good calibration systems and procedures are essential to realize the BTeV calorimeter's potential for excellent resolution. It is a challenging task to maintain the calibration to better than $0.5 \%$, particularly when changes in radiation levels can affect the calibration. Since the radiation levels are different for different parts of the detector, the resulting changes in calibration also vary over the calorimeter. Fortunately, the maximum variation is expected to be less than $2 \%(0.5 \%)$ for $90 \%(50 \%)$ of our crystals.

Although the light output of PWO crystals depends strongly on the temperature $(-2 \% / \mathrm{C})$, we will manage this problem by maintaining a very stable temperature for all of the crystals. This requires careful monitoring, but poses no serious obstacles.

Ultimately, we need to use physics events to calibrate every crystal so that we know how to convert the signals from the crystals to photon energies. We plan to use the electron sample from $B$ semileptonic decays to calibrate every crystal.

The calibration can be checked using $\pi^{0}, \eta$ and $J / \psi$ particles, since their masses are well measured. Fig.A.44 shows a clear $\pi^{0}$ peak in $B$ events at outer radii.

Unfortunately, we will not have enough of these physics events to calibrate every crystal every hour. So we will need to have a calibration system that will track the changes in the calibration over time, and relative to neighboring crystals.

Since we have not done any $R \& D$ on such a calibration system, we again draw on CMS R\&D results. CMS plans to use a laser light source and optical fiber light distribution system to monitor the time variation of calibration. The light source needs to simulate both the color and the time structure of PWO scintillation light $(\sim 500 \mathrm{~nm}$ in wavelength, pulse width of $100 \mathrm{~ns}$ ) and powerful enough to light many crystals at the same time. If the power of a light pulse simulates $100-\mathrm{GeV}$ photons (i.e. $10^{7}$ scintillation photons), the light source needs to produce $0.2 \mathrm{~mJ}$ of energy in each pulse. The output is sent to one of the 80 sections of about 1000 crystals through a "switch." After the switch, the light travels through two levels of splitters and quartz fibers to reach individual crystals. The light intensity is monitored at the source as well as after the two levels of splitters using PIN photodiodes. The overall objective is to maintain calibration of individual crystals to within $0.2 \%$ accuracy over time. $\mathrm{KTeV}$ as well as other FNAL experiments employ similar systems. 


\section{A.3.6 BTeV Electromagnetic Calorimeter R\&D Program}

We plan to acquire a small number of crystals from Russia and Shanghai, and some PMT's from Hamamatsu to get experience by looking at signals from radioactive sources. Performing our own studies on the effect of intense radiation on the light output and light transmission of these crystals will be valuable.

Since the stability of the PMT's is important for the performance of our detector, we also plan to study this.

When a test beam becomes available, we will want to expose a $5 \times 5$ (or better $7 \times 7$ ) stack of crystals to an electron beam to study the resolution, leakage, temperature sensitivity, and other parameters. We will also conduct further studies of the radiation effects. This study may be possible with a high-energy radioactive source, which produce photons or electrons of energies larger than $10 \mathrm{MeV}$.

We need to build equipment to characterize crystals (measuring transmittance and emission spectrum analyser). A radiation facility to test the radiation hardness is also important. It is urgent to help Shandong University Crystal Institute to make fast progress in their PWO crystal production R\&D. One way to accomplish this would be to provide them with equipment for crystal characterization, particularly to test radiation hardness.

Although PWO crystals are "radiation hard", their light output varies as the radiation level varies. We need to study whether the light-injection calibration method can track this variation with sufficient precision. The planned calibration system will not simulate the spectrum of the light perfectly, nor are the mean path lengths that light travels in the crystals the same. By choosing the wavelength properly, the CMS people find that the system is likely to work well [14]. It will be necessary for us to test this ourselves.

We need to start studying the accuracy of the calibration of the energy scale using the physics processes mentioned above.

We also need to start designing a realistic mechanical structure, and the temperature control system. We will have to explore cost issues with phototube manufacturers. 

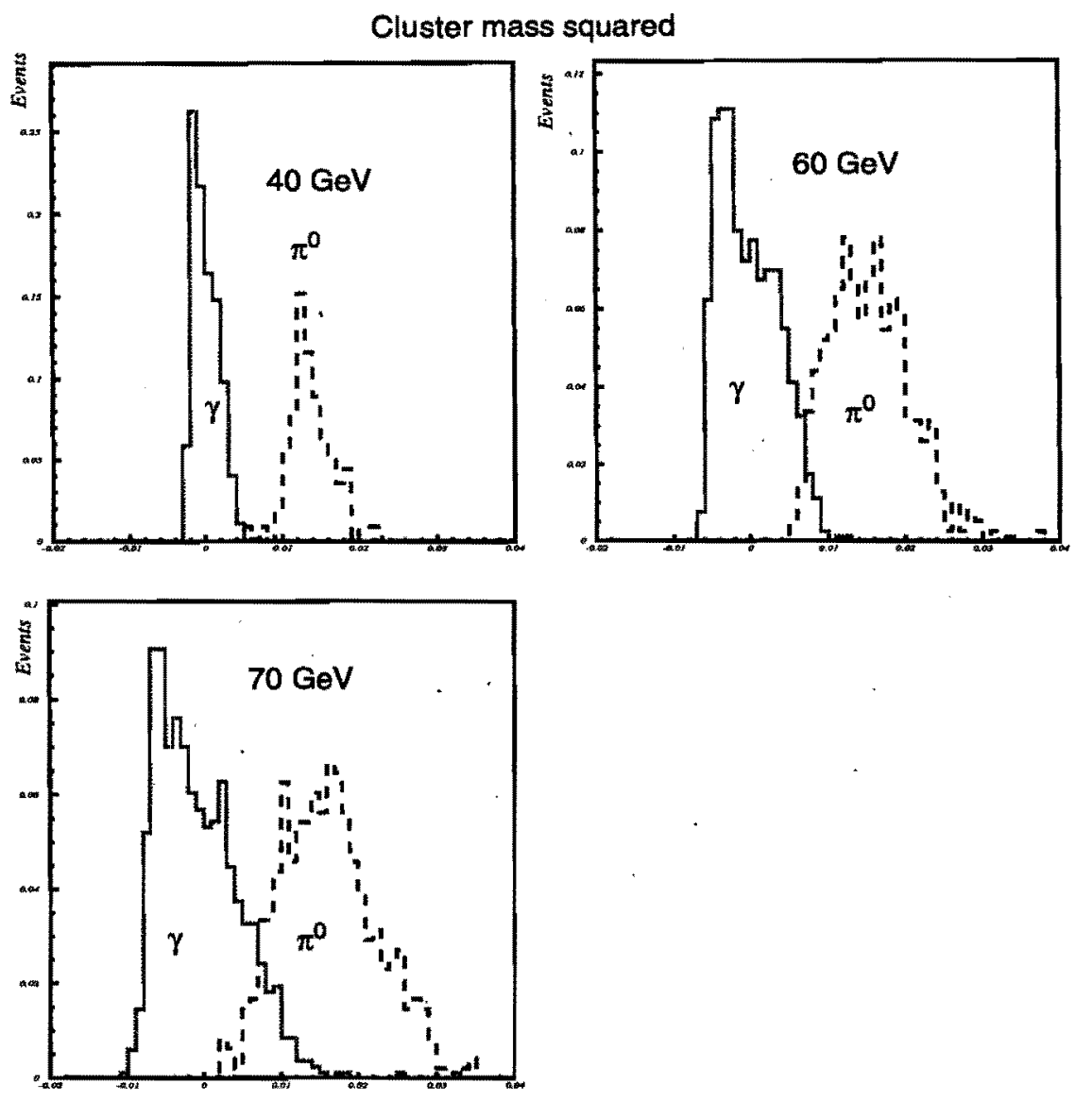

Figure A.43: Cluster mass distribution for $\gamma$ 's and $\pi^{0}$ s obtained with a full BTeVGeant simulation. Energy of the photons and $\pi^{0}$ 's is $40 \mathrm{GeV}, 60 \mathrm{GeV}$ and $70 \mathrm{GeV}$, as indicated. 


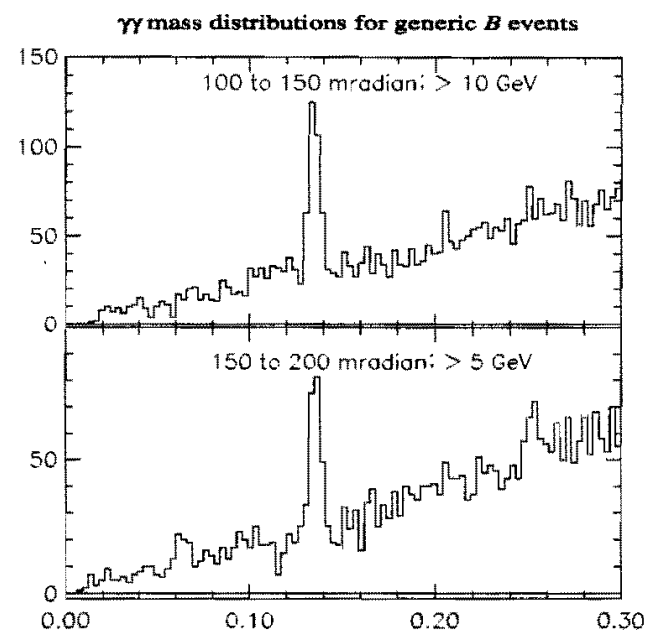

Figure A.44: $\gamma \gamma$ mass distribution for events containing a $B$ event as well as a few minimumbias events. The energy of the $\pi^{0}$ candidates are required to be greater than $10 \mathrm{GeV}$ (top) and $5 \mathrm{GeV}$ (bottom), respectively. 


\section{Bibliography}

[1] Although the quantum efficiencies of photocathodes of PMT's are lower than APD's that CMS uses, they cover much larger areas of the end of PWO crystals.

[2] A.N. Annenkov et al., "Systematic Study of the Short-Term Instability of $\mathrm{PbWO}_{4}$ Scintillator Parameters under Irradiation," CMS Note 1997-055.

[3] The output results are given in units of absorbed dose (Gy/year). Note that 1 Gray $[\mathrm{J} / \mathrm{kg}]$ is equivalent to 100 rads.

[4] "Radiation damage Kinetics in PWO crystals," CMS Note 1997-008.

[5] To a much lesser degree, some radiation results from beam halo and when beam particles which are deflected at small angles get backscattered by accelerator elements outside the $\mathrm{BTeV}$ detector. Their contributions are small enough to be ignored.

[6] I.Azhgirey,I.Kurochkin and V.Talanov, "Development of MARS Code Package for Radiation Problems: Solution of Electro-Nuclear Installations Design, Proc. XV Conf. on Charged Particles Accelerators, Protvino, October 1996.

[7] CMS Collaboration, "The Muon Project Technical Design Report", CERN/LHCC 9732, CMS TDR 3 (1997); A.Uzunian," CMS Radiation Studies Results'99", Fourth Annual RDMS CMS Collaboration Meeting, CMS Document 1999-113, CERN (1999).

[8] CMS, "The Electromagnetic Calorimeter Project Technical Design Report," CERN/LHCC 97-33, CMS TDR 4 (1997).

[9] Hamamatsu Photonics K. K., "Photomultiplier Tube," p. 176 (1994). Beware the units are Roentgens not rads. Here 1 Roentgen is approximately 2 rads. U. V. glass windows lose about $10 \%$ of their transparency with a $3 \mathrm{kGy}$ dose, and therefore are not an acceptable solution except on the outside in the horizontal plane, where there are alot of crystals.

[10] Private communication from Ed Blucher.

[11] E. Blucher, B. Gittelman, B. K. Heltsley, J. Kandaswamy, R. Kowalewski, Y. Kubota, N. Mistry, A. Bean, and S. Stone, "Tests of Cesium Iodide Crystals for an Electromagnetic Calorimeter," Nuclear Instruments \& Methods A249, 201 (1986). 
[12] CMS Endcap crystal size has changed to $30 \times 30 \mathrm{~mm}^{2}$ to reduce electronics cost after they confirmed that Bogoroditsk Plant can grow larger crystals.

[13] T. Skwarnicki, "A Study of the Radiative Cascade Transitions Between the UpsilonPrime and Upsilon Resonances," DESY F31-86-02 (thesis, unpublished) (1986).

[14] "A Study of the Monitoring of Radiation Damage to CMS ECAL Crystals, Performed at X5-GIF," CMS NOTE 2000-020

[15] In part 2, chapter 6 , we quote $2.6 \mathrm{MeV}$ mass resolution for $10 \mathrm{GeV} \pi^{0}$ 's, which was found using a non-optimized photon position calculation method. 


\section{A.4 Muon Detector Design, R\&D and Test Beam Re- sults}

In Chapter 8, we gave an outline of our proposed muon detector design and evidence for a workable standalone dimuon trigger. In this section we provide details of the detector design, the assembly, the front-end electronics, the method of readout as well as a summary of the results of the beam test conducted during the summer of 1999 .

\section{A.4.1 Detector Design and Fabrication}

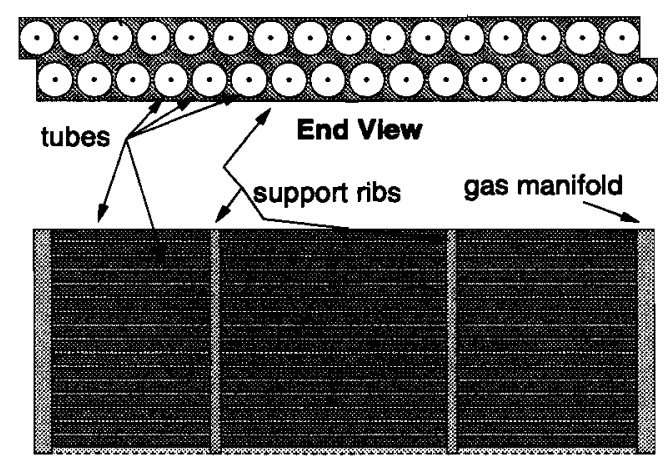

Top Vlew

Figure A.45: End and top views of one "plank" of proportional tubes.

\section{A.4.1.1 Basic Building Block: Proportional Tube Plank}

The basic building block in the construction of a detector station is a "plank" of $3 / 8$ " stainless steel proportional tubes as shown in Fig. A.45. Thirty-two tubes are arranged in a double layer with an offset of half a tube ("picket fence" geometry). The tubes will be soldered to brass gas manifolds at each end. For the longer tubes, additional brass support braces will also be soldered to the tubes. This soldering technique has been tested by us and is simple and efficient. This system provides a sturdy, self-supporting building block. Soldering the tubes to brass also provides an excellent Faraday cage for the tubes. Proportional tubes have been selected as the detector technology because they are robust and have the necessary rate capability. We intend to use a fast gas (such as $88 \% \mathrm{Ar}, 10 \% \mathrm{CF}_{4}, 2 \% \mathrm{CO}_{2}$, which has a drift velocity of roughly $9 \mathrm{~cm} / \mu \mathrm{s}$ [1]) so the maximum collection time (drift plus charge integration) for a signal should be less than $60 \mathrm{~ns}$. Use of thin walled ( 0.01 ") stainless steel tubes has been proven to work by the CDF collaboration where similar tubes [2] had a long lifetime with low failure rate.

The $0.5 \mathrm{~cm}$ effective wire spacing of this design has a spatial resolution of $1.5 \mathrm{~mm}(5 \mathrm{~mm}$ $/ \sqrt{12}$ ), with no dead regions between tubes. 


\section{A.4.1.2 Design of a Detector Station}
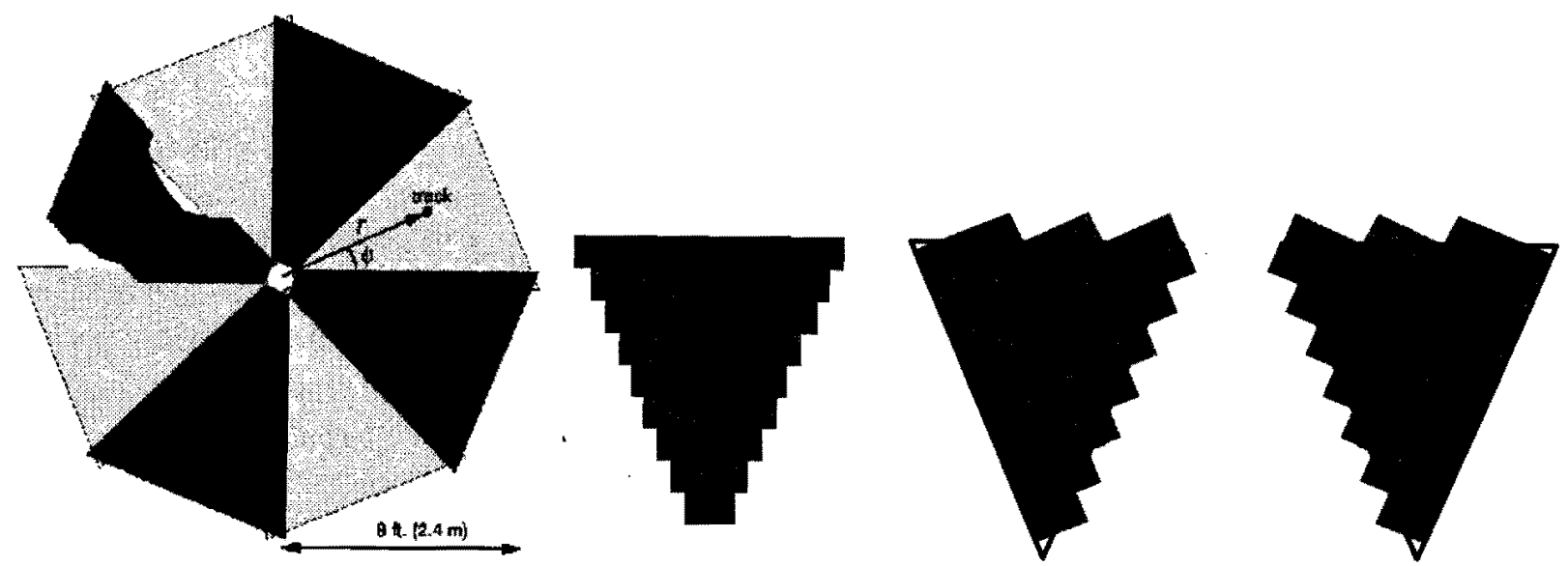

Figure A.46: (left) Beams-eye view of one muon detector station, which consists of eight overlapping octants arranged in two layers. One octant is cut away in places to show the overlap between adjacent octants. (right) Arrangement of planks to form the four views in an octant ( $r$ view is repeated). There will be 13 planks per view (more than shown).

To minimize occupancy at small radii and to minimize pattern recognition confusion, each detector station will consist of eight overlapping pie shaped "octants," as shown in Fig. A.46a. The four views $(r, u, v$, and $r)$ in each octant are shown in Fig. A.46b. The $r$ (radial) view is repeated to provide redundancy for the most important (bend) view and help reject fake tracks. The $u$ and $v$ stereo views are rotated $\pm 22.5^{\circ}$ from the $r$ view and are used to measure $\phi$ to resolve hit ambiguities. This geometry gives excellent resolution and redundancy in the bend direction $(\hat{\theta})$ of the toroids, and the resolution in the $\hat{\phi}$ direction is reasonable $\left(\sigma_{\phi}=2.8 \mathrm{~mm}\right.$, which is approximately twice the single plank resolution).

To construct an octant, the views are stacked on top of each other and are built from the planks described above. There will be 13 planks in each view of an octant ranging in length from 1 foot to 6 feet. This octant geometry has several advantages. In all views, the planks are short near the beam pipe and get longer at larger radii. This gives us shorter tubes where the occupancy is high and longer tubes away from the beam pipe which keeps the cost down. In addition to spreading the occupancy in a sensible way, splitting the system into octants should also help minimize pattern recognition confusion.

Pairs of octants will be combined into quads which will be the structure moved in and out of the spectrometer. These quads will be composed of $1 / 8$ " sheets of aluminum on which all of the planks are attached. Since the two octants that make up a quad are staggered in $z$, there are actually 8 (4 views for 2 octants) layers of planks in $z$ (although the actual overlap is small). Each of the 8 layers of planks will be attached (front and back) to a 1/8" thick aluminum support sheet. This sheet will cover the entire quad area and will also act as a $\delta$-ray shield.

With 32 channels per plank, 13 planks per view, 4 views per octant, 8 octants per station, 
3 stations per arm, and 2 arms in the spectrometer, we arrive at 79,872 total channels in the system.

\section{A.4.1.3 Proportional Tube Details}

Each of the 32 tubes in a plank share a common gas manifold at each end which is made of brass and soldered to the tubes. The wires will be held at positive high voltage and read out through a blocking capacitor by front-end electronics which will attach at one end. High voltage will be fed to the plank at the same end as the electronics. We intend to use a "fast" gas mixture of $88 \% \mathrm{Ar}, 10 \% \mathrm{CF}_{4}, 2 \% \mathrm{CO}_{2}$ or something similar. For more details on the front-end electronics, expected signals, etc., see the section on front-end electronics below.

The tubes in the system will vary from 1-6 feet in length. During the beam test in the summer of 1999 we tested 10 planks with tubes ranging in length from 1-6 feet strung with 30 and 50 micron wire. There was no sign of wire slippage or breakage in the 320 channels. We plan on using 30 micron wire because it allows one to use lower high-voltage to achieve the same gain (see Section A.4.2.3). Our calculations and tests indicate no wire supports are necessary, even for 6 -foot tubes.

After soldering the tubes to the gas manifold, the tubes will be strung and the wires held in place via a crimp pin. The arrangement is very similar to that used in the CDF muon system [2]. The crimp pins will extend out of the gas manifold and will attach to a circuit board which contains the blocking capacitors and an edge connector for the front-end cards.

\section{A.4.1.4 Miscellaneous Mechanical Details}

Because of the overlapping octant geometry of each detector station, there is plenty of room for cabling and front-end electronics everywhere except near the beam pipe. Space limittions at small radii limits the inner radius of the system to approximately $16 \mathrm{~cm}$. GEANT studies of our detector at design luminosity (described in 8.3.1) indicate the innermost $r$-view tube (at $r=16 \mathrm{~cm}$ ) of the first station will have an occupancy of $35 \%$ (the $u$ and $v$ views and downstream stations have significantly less occupancy). Therefore an inner radius of $16 \mathrm{~cm}$ is reasonable from a physics perspective as well as from a space constraint perspective. The hits from the minimum bias background have a $\sim 1 / r^{2}$ dependence; as a result, at a radius of $r=32 \mathrm{~cm}$ the $r$-view occupancy of the first station drops to $7 \%$. For stations $2 \& 3$, the $r$-view occupancy is $10 \%(2 \%)$ for $r=16 \mathrm{~cm}(r=32 \mathrm{~cm})$.

Weight is a potential concern because we are using stainless tubes. Using thin walled tubes reduces this concern significantly. We estimate the weight of the stainless tubes in one octant (all four views) to be $250 \mathrm{lbs}$, the same as if we were to use 1/32" thick aluminum tubes. However, the hanging frame used to support the octants and the supporting Aluminum sheets will probably double the weight of each octant, so that each detector station will weigh $1.5-2$ tons.

We plan on building one extra station of spares $(1 / 6=16 \%$ of those in the total system). The spares will be in the form of loose planks. If a problem develops (more than a handful of broken wires, etc.), we will take out a quad, replace the plank, and put the quad back. 
Fabrication will take place at the three collaborating muon institutions, Illinois, Puerto Rico, and Vanderbilt.

\section{A.4.2 Front End Electronics}

In order to capture all the charge coming from a typical proportional tube for a single incident ionizing particle, one must integrate the charge due to the positive ion drift over a long period of time relative to the expected beam crossing for the $\mathrm{BTeV}$ experiment. The time needed to collect a portion of the total charge is a function of the gas used in the proportional tube, the geometry of the tube, and the high voltage bias of the center wire, relative to the outer conductor $[3],[4]$ :

$$
\frac{Q}{Q_{o}}=\frac{\ln \left(1+t / t_{o}\right)}{2 \ln (b / a)}
$$

where $Q$ is the charge collected in an amount of time, $t, Q_{o}$ is the total charge from the ionization (depends on the gain of the tube), $b$ and $a$ are the outer and inner radius, respectively, of the conductors in the proportional tube, and [4]:

$$
t_{o} \sim \frac{a}{2 v_{d}}
$$

where $v_{d}$ is the drift velocity of the gas for the chosen value of the gain in the tube [1]. Alternatively, for a very fast signal, one could try to just capture the fast signal coming from the electron amplification, but this is only $1-2 \%$ of $Q_{o}$.

In $\mathrm{BTeV}$ we intend to use the muon proportional tubes in the Level 1 Trigger. The natural cutoff for integrating a signal then is on the order of a beam crossing, or 100 ns. However, we would like to reduce backgrounds associated with the collider beams, which pass through 60-70 ns before the interaction products arrive. For a fast gas like $\mathrm{ArCO}_{2} \mathrm{CH}_{4}$ [1], and an effective wire-to-wire spacing of $0.5 \mathrm{~cm}$, the maximum drift time before the first signals from a track arrive is about $30 \mathrm{~ns}$. This means that if we would like to be able to separate the various beam crossings, our electronics should be able to resolve 2 hits occurring 30 ns apart.

\section{A.4.2.1 Amplifier choice}

Fortunately, electronics to amplify and digitize the analog signal from a chamber hit in a high rate environment were developed for the SSC [3], and continue to be refined for use in detectors for the LHC [5] and at Fermilab [6]. We will take full advantage of these developments.

We intend to mimic the setup used for the CDF COT [6]. There will be a PC board soldered to the end of the gas manifold to deliver high voltage to each proportional tube. Connected to this is another PC board with electronics to amplify and digitize the tube signal. The high voltage will be delivered to each plank by one SHV cable. Therefore, high voltage will be adjustable on a plank-by-plank basis but not on a tube-by-tube basis. 
We plan to utilize the ASDQ chip developed at the University of Pennsylvania to amplify and digitize the signals coming from the proportional tubes. The family of ASD chips (and applications) is described in detail elsewhere [3],[6], but we briefly mention some of the more important features here.

The ASD8B, which is being used for evaluation and beam tests is a predecessor of the ASDQ chip. The ASD8B (ASDQ) chip amplifies the first $6 \mathrm{~ns}(10 \mathrm{~ns})$ of the signal and outputs an LVDS (equivalent) differential logic signal. The ASD8B (ASDQ) chip, when mounted on a PC board, has a low effective threshold of about $1 \mathrm{fC}(2 \mathrm{fc})$. The ASD8B noise level was verified in tests we performed on an evaluation board supplied by the University of Pennsylvania in December of 1998. Both chips features a double pulse resolution of $\sim 20 \mathrm{~ns}$. This family of chips seem to be an ideal choice for the BTeV muon system, and it is important to determine if we have sufficient signal to utilize them.

\section{A.4.2.2 Practical Considerations}

To calculate the signal expected in the proportional tube, we use the data presented in reference [1]. For the same gain as quoted in the paper $\left(\sim 1.8 \times 10^{5}\right)$, we can use the quoted results to determine our operating parameters for different tube geometries and gas mixtures. In Table A.4.2.2, we summarize the results for different gases and inner conductor radii.

Table A.4: Operating parameters for different gases and inner wire radii (a). The values of $t_{d r i f t}^{M}$ assume a wire space of $0.5 \mathrm{~cm}$.

\begin{tabular}{|l|llllll|}
\hline \hline Gas & $\left.E_{\text {field }}\right|_{a}$ & $v_{d}$ & $V_{\text {bias }}$ & $Q_{6 \mathrm{~ns}}$ & $t_{\text {drift }}^{M}$ & $\mathrm{a}$ \\
\hline$\frac{\mathrm{ArC} \mathrm{H}_{6}}{50 / 50}$ & $150\left(\frac{\mathrm{kV}}{\mathrm{cm}}\right)$ & $4.8\left(\frac{\mathrm{cm}}{\mu \mathrm{s}}\right)$ & $3545(\mathrm{~V})$ & $7.8(\mathrm{fC})$ & $50(\mathrm{~ns})$ & $50(\mu \mathrm{m})$ \\
$\frac{\mathrm{ArCO}}{80 / 20}$ & 164 & 2.4 & 3768 & 5.9 & 100 & 50 \\
$\frac{\mathrm{ArCO} \mathrm{CF}_{4}}{88 / 2 / 10}$ & 150 & 8.7 & 3454 & 9.5 & 30 & 50 \\
$\frac{\mathrm{ArC}_{2} \mathrm{H}_{6}}{50 / 50}$ & 150 & 4.8 & 1656 & 8.8 & 50 & 20 \\
$\frac{\mathrm{ArCO}_{2}}{80 / 20}$ & 164 & 2.4 & 1806 & 7.1 & 100 & 20 \\
$\frac{\mathrm{ArCO} \mathrm{CF}_{4}}{88 / 2 / 10}$ & 150 & 8.7 & 1656 & 10.4 & 30 & 20 \\
\hline \hline
\end{tabular}

In the table, we have made some assumptions. We've assumed that the most important factor in calculating the gain is sensitivity to a single ionization cluster of a single electronion pair; we haven't taken into account the effect of adding a termination resistor (see below); we haven't taken into account the effect of a magnetic field; and we've neglected aging effects in the tube. We've also taken for granted that the electronics will be located at the end of the proportional tubes: lowering the electronic noise and increasing the amount of signal we get in the small integration time available. Even so, we can learn a great deal from looking at the table. 


\section{A.4.2.3 Amplifier Observations}

A smaller wire size is desirable from an electronics standpoint: the operating voltage $\left(V_{\text {bias }}\right)$ is lower and the integrated charge collected $\left(Q_{6 n s}\right)$ is a little higher. The lower operating voltage gives us more flexibility in the choice of a decoupling capacitor, allowing the option of surface mount components in the high voltage system. The higher integrated charge means we can safely raise the threshold of the electronics without losing signal, allowing greater flexibility in our noise budget. Additionally, it is expected that the gain of a tube has less sensitivity to the operating voltage with a smaller wire [1].

Also evident is the advantage of having a fast gas. Not only can one integrate more charge in a smaller period of time, one has less sensitivity to beam crossings. We can achieve our goal, stated previously; of the first signal to arrive after a maximum time of about $t_{\mathrm{drift}}^{M}=30 \mathrm{~ns}$.

Another way to look at the table is to reason that all the combinations calculated will produce a working detector, and that using the ASD chip allows us flexibility in our choice of wire diameter, gas, and operating voltage.

At most, a termination resistor will raise our noise by $1 \mathrm{fC}$ and lower our gain by a factor of 2. This is to be offset by the expectation that about 10 primary ion-pair clusters will be produced, on average, for a MIP passing through $1 / 3 \mathrm{~cm}$ of gas volume.

One final consideration in evaluating the ASD8B for $\mathrm{BTeV}$ is that the process used to make the chip is obsolete and the chip will not be available in production quantities [7]. We plan to use the improved version (ASDQ). It integrates for a slightly longer period, has the same double pulse resolution, and has a higher intrinsic noise level of $\sim 2 \mathrm{fC}$.

\section{A.4.2.4 Results of the Beam Test}

In the summer of 1999 we contructed 320 channels in 10 planks, reading out 128 channels in 8 planks (due to limited electronics). The front part of the setup showing 3 one-foot planks followed by 2 six-foot planks with high-voltage and readout cables is shown in Fig. A.47. This construction did not have the Faraday cage properties of our current design. The gas manifolds were made of an insulator and glued to the tubes. The other supports, although made of aluminum, were glued, increasing the resistance between tubes up to $50 \Omega$. We used the ASD8B chip to amplify and digitize the signal. In our initial tests, we found that the ASD8B was very sensitive to RF noise and, in our configuration, required a nearly complete Faraday cage around the amplifier card. This was accomplished by wrapping the tubes with aluminum foil and constructing boxes made of copper plated G10 in which the amplifier card resided. These boxes were connected to the aluminum foil with copper tape. With the Faraday cage in place it was possible to reduce the random noise to sufficiently low levels. This is the motivation for our current design which naturally creates a Faraday cage by soldering the tubes to a brass gas manifold and connecting these to aluminum sheets which will extend out to the end of the electronics allowing a Faraday cage around the electronics.

With the makeshift Faraday cage in place we determined that the ASD8B chip gave enough gain as demonstrated by the plateau curves in Fig. A.48. Unfortunately, further 


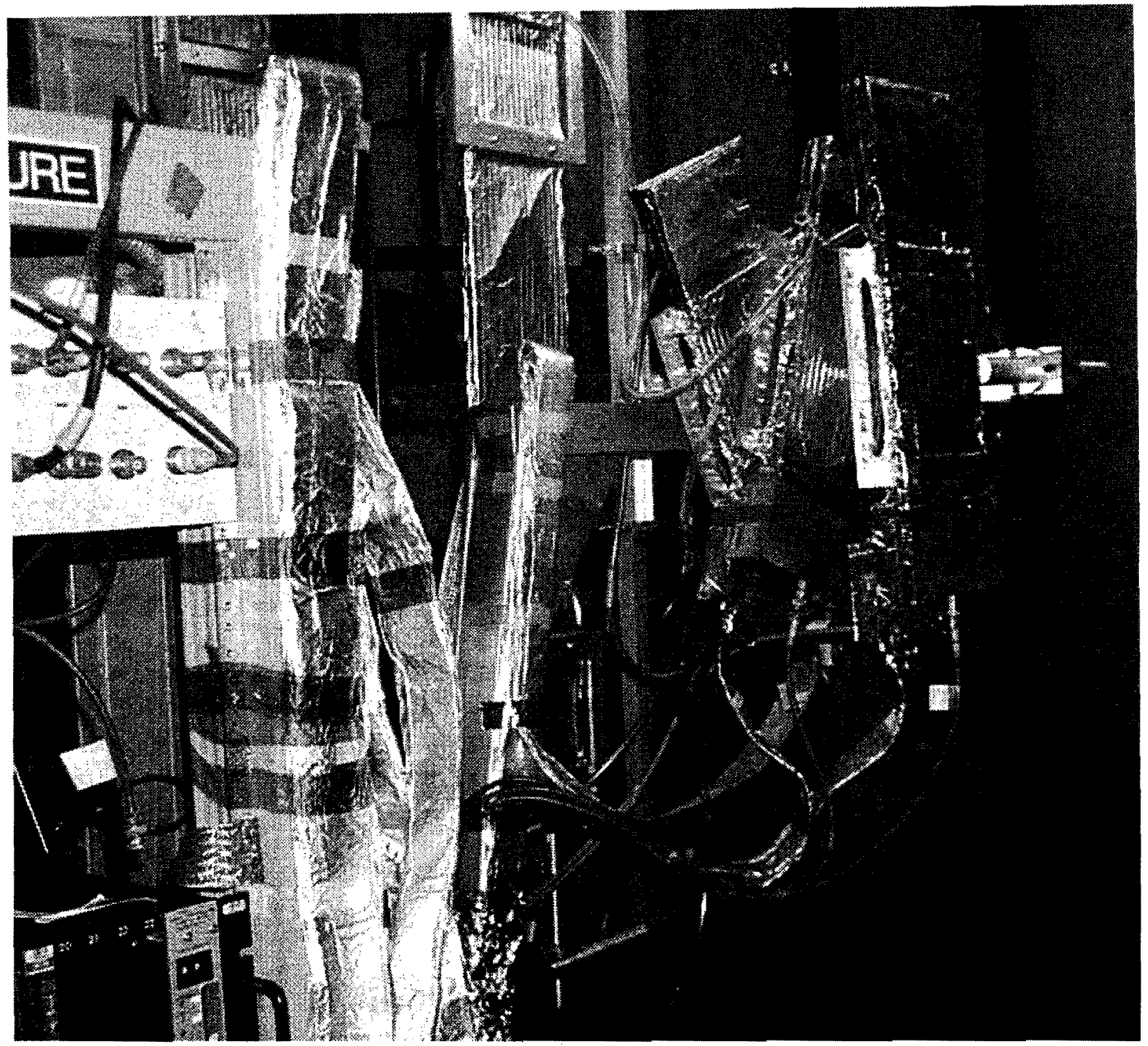

Figure A.47: Photo of the muon testbeam setup. The beam direction was from right to left. Three of the one-foot planks and the two six-foot planks can be seen. The remaining planks (two three-foot planks and three more one-foot planks) are further back. The planks and cables are wrapped in aluminum foil to provide sheilding. The amplifiers are inside boxes made of copper-plated G10.

tests revealed that with the front-end design we implemented, the level of cross-talk was quite high. That is, when only one or two tubes should be firing when a single particle passed through, oftentimes, five or six would fire. At the end of the beam test, the cross-talk was reduced by adding termination resistors to the open end of the tube. We believe we can reduce the noise even further with a more intelligent signal routing scheme and/or additional small components. 


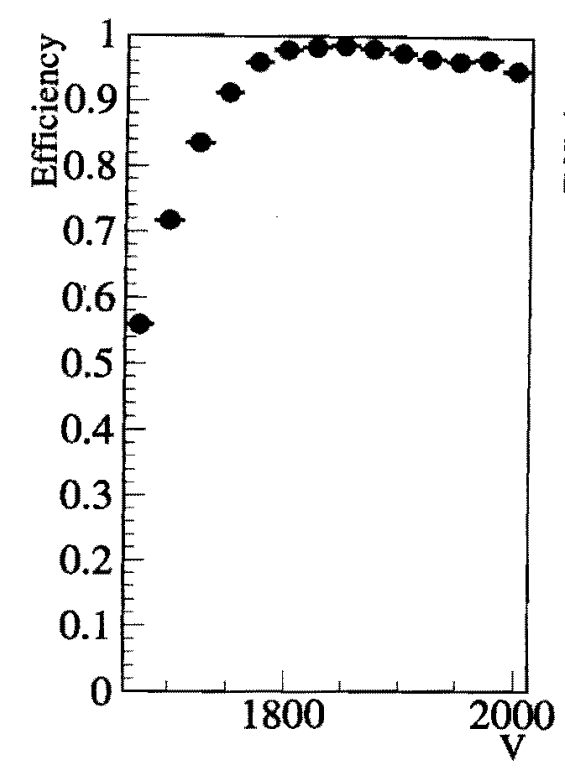

(a) one-foot plank

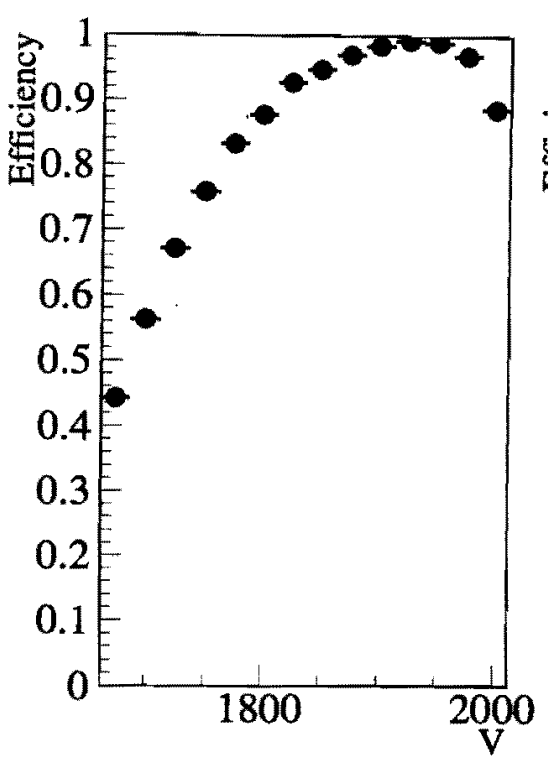

(b) three-foot plank

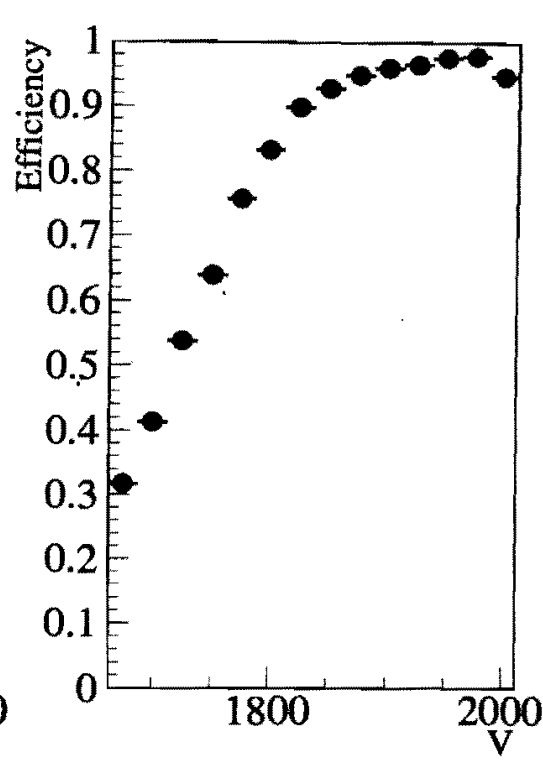

(c) six-foot plank

Figure A.48: Plateau curves (efficiency versus voltage) for a one-foot, three-foot, and six-foot plank of tubes. We require hits in scintillators upstream and downstream of the muon planks and for these events we read out single-hit LeCroy TDC's to determine if a hit occurred at the proper time. The drop in efficiency above the plateau is due to the particular readout electronics (single-hit TDC's with a long gate) when high hit rates are encountered. These data were taken using the tubes strung with 30 micron wire with $80 \% / 20 \% \mathrm{Ar} / \mathrm{CO}_{2}$ gas.

\section{A.4.2.5 Front-end Readout}

As mentioned previously, the ASD chips produce differential logic signals (LVDS) suitable for driving up to $10 \mathrm{~m}$ of cable. The LVDS signal consists of $1 \mathrm{bit} / \mathrm{channel}$ signifying whether or not a channel is on or off. The $\mathrm{BTeV}$ experiment plans to use optical fiber to deliver data to the counting room upstairs. We plan to convert the signals to optical at the octant level. Converting each plank of 32 channels to optical would require 2496 optical connections which is quite expensive and inefficient. Similarly, bringing all 79,872 channels out of the muon system to the north wall would require a large amount of cables and many racks of serializing boards. We propose to use copper-based twisted-flat cables to connect the 13 planks that make up one view of one octant to a serializer board(s). These 13 planks (416 channels) would be sparsified, serialized, and delivered to the counting room on an opticalfiber link. Other links will also be used to run the slow control (mainly threshold setting) and monitoring. 


\section{A.4.3 Future prototyping}

We plan to continue developing prototypes over the next year. We are planning on building on the successful construction of the first prototypes for the beam test in the summer of 1999 . The basic construction change that we will be making is to solder brass gas manifolds to the tubes instead of gluing plastic gas manifolds. This was motivated by the requirement of a good Faraday cage for the tubes and the amplifier. The basic soldering technique has been verified on a small test sample. Since this soldering technique requires heating the tubes, it was felt that rather than stringing the tubes first and then attaching the manifold (as was done in the first prototypes) it would be safer to solder the manifold (and any support pieces) and then string the tubes. This requires a slight adjustment of the tools and methods used to string the tubes, especially in crimping the wire to the crimp pin which holds the wire at tension.

In our next round of prototypes we will test the basic changes to the construction (soldering instead of gluing and crimping after instead of before). We would like to verify that the new construction method is as efficient and reliable as the old method. In addition, we would like to determine how much this helps reduce the noise problems we saw with unshielded tubes in the previous prototype. Finally, we would like this to proceed rapidly so that we can conduct long-term tests of the system including such potential problems as wire "creep," gas "poisoning," etc.

We will also require new prototypes for the front-end electronics. The new prototype will be using the ASDQ chip instead of the ASD8B chip, used previously. We are also planning a new prototype for the card which uses the ASDQ chip. We have many ideas for improving the layout of this card compared to its predecessor which was used for the 1999 test beam run. These improvements include better signal routing and possibly an analog buffer. 


\section{Bibliography}

[1] J.M. Butler et al., "Study Of Fast Gases, Resolutions And Contaminants In The D0 Muon System," Nucl. Instrum. Meth. A290, 122 (1990).

[2] S. Bhadra, S. Errede, L. Fishback, H. Keutelian and P. Schlabach, "The Design And Construction Of The CDF Central Drift Tube Array," Nucl. Instr. Meth. A268, 92 (1988).

[3] "A Fast, Low Power, Amplifier-Shaper-Discriminator for High Rate Straw Tracking Systems," F.M. Newcomer et al., Trans. Nuclear Science, Vol 40, N4, P630-636, August, 1993.

[4] F. Sauli in EXPERIMENTAL TECHNIQUES IN HIGH-ENERGY NUCLEAR AND PARTICLE PHYSICS (2nd ed.), edited by T. Ferbel, World Scientific, Singapore 1991, p. 79 .

[5] ATLAS Collaboration, "ATLAS inner detector: Technical design report. Vol. 2," CERNLHCC-97-17.

[6] R. Blair et al. [CDF-II Collaboration], "The CDF-II detector: Technical design report," FERMILAB-PUB-96-390-E.

[7] M. Newcomer, Private Communication. 


\section{A.5 Level 1 Trigger Timing Studies}

\section{A.5.1 Introduction}

During the past year, considerable effort has been devoted to timing studies of the Level 1 vertex trigger. Since the vertex trigger must perform a large number of calculations in a very short time, the number of processors required to do the job is large. It is important to have an accurate determination of the number of processors, because factors of two or more can have a significant impact on whether or not a particular design is feasible. For example, a farm of 50,000 digital signal processors (DSPs) might be difficult to maintain, whereas farms of $\approx 10,000$ DSPs are being operated successfully [1].

Fortunately, the timing studies for the calculations that we plan to perform using DSPS indicate that Level 1 requires fewer than 2300 DSPs. This number will shrink as processor speeds increase.

To obtain an accurate estimate of the processing needs for Level 1, we adopted an aggressive approach aimed at achieving maximum performance for the type of DSP selected for our design study. This approach was called for, since early estimates based on Level 1 trigger code suggested that very large numbers of processors might be needed. For our studies we selected the Texas Instruments $200 \mathrm{MHz}$ TMS320C67X floating-point DSP, rated at 1200 MFLOPS. We are also considering the ADSP-2106x family of DSPs made by Analog Devices for use in the trigger, but all of our detailed timing studies were done assuming the TI DSP.

The software for our timing studies is the Level 1 trigger code. This is the same track and vertex reconstruction software that is used to determine trigger efficiencies for physics simulations, and to study the Level 1 trigger performance (see Chapter 14). The trigger code that finds track segments is not included in the timing studies, since the segment finding is performed by the FPGA tracker, not by DSPs.

Three key decisions made it possible for us to achieve our final timing estimate for Level 1. First, we decided to develop the trigger code using a Windows PC development and debugging environment. This made it possible to develop the trigger code for three different purposes: (i) physics simulations running under UNIX, (ii) detailed trigger studies using the Windows development environment, and (iii) vendor-supplied DSP simulators, which are often able to run the code developed for the Windows environment (without too many platform-specific modifications). Second, we decided to write the trigger code using the lowest-level assembly language for the TMS320C67X DSP. This leads to a signficant increase in the effort required to implement the trigger code in the DSP, but it provides the maximum performance gains. The resulting code is called expert-optimized assembly-language code, in contrast to the code that is generated by an optimizing compiler. The expert-optimized code development involves interleaving calculations, rearranging the order of calculations compared to the original $\mathrm{C}$ code, and tailoring the code to the specific architecture of the TMS320C67X. Third, we included timing estimates for "operating-system software," so that our time estimates are representative of all software required for a complete implementation of the Level 1 trigger code. This includes code for memory management, I/O and DMA. 
support, as well as interrupt and error handling. Time estimates for the operating-system software are based on code developed for the BTeV hardware trigger prototype.

Our final result for the number of processors required to execute the Level 1 trigger code using the TMS320C67X is 2300 DSPs. This represents a factor of 20-50 improvement (depending on the level of optimization achieved for different subroutines) compared to a high-level C-code implementation of the trigger algorithm. This result is significant, since it means that a much smaller number of processors (a factor of 20-50 fewer processors) is required for Level 1, compared to an estimate that is based on a high-level implementation of our trigger algorithms.

\section{A.5.2 Timing Studies}

As shown in Fig. 9.3 and described in Chapter 9, there are two distinct processor farms in the design of the Level 1 trigger. One farm is used to process tracks, the second is used for vertexing. The results from our timing studies are presented for the track and vertex farms in Tables A.5 and A.6, respectively.

Table A.5: DSP cycle counts for each subroutine in the track processing code. The cycle counts are calculated per pixel detector quadrant per beam crossing.

\begin{tabular}{lr}
\hline \hline Subroutine & cycles/quadrant/crossing \\
\hline bb33-triplet-sorting & 650 \\
bb33-forward-matching & 980 \\
bb33-backward-matching & 980 \\
bb33-faketrack-removal & 1940 \\
bb33-radius-of-curvature & 1500 \\
calculate-angles & 660 \\
extrapolate-yz-to-beamline & 1440 \\
extrapolate-xz-to-beamline & 300 \\
estimate-vertexing-errors & 300 \\
estimate-vertex-z-coord & 240 \\
calculate-momentum & 660 \\
\hline Total DSP cycle count & 9650 \\
\hline \hline
\end{tabular}

Table A.5 lists the subroutines that are executed by DSPs in the track farm. The table shows the estimated number of DSP cycles required by each subroutine. The estimates are based on an accurate determination of the number of machine-code operations, and the average number of trigger primitives handled by each subroutine (assuming an average of two interactions per beam crossing). We tend to overestimate the numbers of trigger primitives, and to assume maximum loop counts. For example, if a particular subroutine operates on an average of 5.2 tracks, we use 6 tracks for our time estimates. With regard to loop counts, the code often involves loops with conditional statements that permit loops to terminate before 
completing all iterations. An example of this is a double loop over all interior and exterior track segments (ordered by pixel-station number) that finds matching track segments. As soon as a match is found by the Level 1 trigger code, the loop terminates. We simplify our estimate of the time required to execute this double loop by assuming that the loop does not terminate until all combinations of interior and exterior track segments have been considered. This overestimates the required time, but it is a conservative result that we are comfortable with.

Our estimate for the number of TMS320C67X DSPs required for the track farm is calculated as follows. We multiply the total number of DSP cycles required by the track subroutines by the cycle time ( $5 \mathrm{~ns}$ ) for the TMS320C67X DSP. This gives us an average time of $48.25 \mu$ s per quadrant per beam crossing. We add $6.75 \mu$ s for the "operating system" overhead, and obtain $55 \mu \mathrm{s}$. Finally, we require a large enough number of DSPs working in parallel so that a processor is available every $132 \mathrm{~ns}$, on average. Therefore, we need 417 DSPs per quadrant, or a total of 1668. For faster DSPs that Texas Instruments will unveil in the coming years, the cycle counts shown in Table A.5 should remain the same (TI promises code compatibility), and the time per cycle will be reduced.

Table A.6: DSP cycle counts for each subroutine in the vertex processing code. The cycle counts are calculated per beam crossing.

\begin{tabular}{lr}
\hline \hline Subroutine & cycles/crossing \\
\hline setup-tracks & 1925 \\
get-ip-miss-at-primary & 3060 \\
compare-tr-to-clst & 4788 \\
calculate-cluster-vertex & 360 \\
add-to-cluster & 1080 \\
vtxp1-init & 50 \\
vtxp2-clusters & 120 \\
vtxp3-primary & 90 \\
vtxp4-add-tracks & 1200 \\
vtxp5-detach-tracks & 600 \\
vtxp6-detach-tracks & 600 \\
vtxp7-count-dtracks & 300 \\
vtxp8-count-dtracks & 300 \\
vtxp9-trigger & 200 \\
\hline Total DSP cycle count & 14673 \\
\hline \hline
\end{tabular}

For the vertex farm we use the cycle counts listed in Table A.6. This gives us an average time of $73.4 \mu \mathrm{s}$ per beam crossing. The processors in the vertex farm are not subdivided by quadrants, so we do not multiply this result by 4 . We add $3.8 \mu$ s for the "operating system" overhead, and obtain $77.2 \mu \mathrm{s}$. The total DSP count is 585 .

Thus, the total DSP count for Level 1 is 2253 . 


\section{Bibliography}

[1] D. Chen et al., Nucl. Phys. Proc. Suppl. 73 (1999) 898. 


\section{Appendix B}

\section{Roadmap: Answers to PAC Questions}

After reviewing the BTeV PTDR submitted in May of 1999, the PAC made several useful observations and noted several issues that warranted special priority for further study. In this appendix we summarize the results of the studies recommended by the PAC, and point to the portions of the proposal where each issue is addressed in detail.

Before addressing each of the issues raised, we would like to emphasize a few of the more interesting developments that have occurred since the PTDR:

- The number of planes in each station of the baseline pixel detector is now two instead of three. This significantly reduces the amount of material and complexity of the system without diminishing the tracking and triggering performance.

- The performance of the BTeV Level 1 pixel trigger is similar to that described in the PTDR even with $2 / 3$ as many pixel planes. Unlike in the PTDR, we have used a full GEANT event simulation and introduced non-Gaussian tails to the hit distributions based on data from our 1999 beam tests. The simulation included underlying events, multiple interactions, and full pattern recognition. Selecting Level 1 trigger "cuts" to give us a rejection for minimum bias events of 100 , we find that the trigger efficiency for several modes of interest are generally much better than $50 \%$. We have found that this performance is robust with respect to pixel inefficiencies and noise, multiple interactions, and beam misalignments.

- BTeV's ability to study states with $\gamma$ 's and $\pi^{0}$ 's using its $\mathrm{PbWO}_{4}$ electromagnetic calorimeter has been validated. Using full GEANT simulations with a complicated underlying event and accompanying minimum bias events, we can successfully reconstruct even the very low energy photons from these decays and can suppress the combinatoric background. We expect to reconstruct a factor of 7 more $B \rightarrow \rho \pi$ decays than LHC-b with a factor of 5 better signal-to-background.

- We were asked to include beam related backgrounds in our studies. At present, the $\mathrm{C} 0$ interaction region is still being designed. However, the Beams Division assured us that they now know how to reduce these backgrounds to levels much smaller than the 
physics related backgrounds. We do show that our Level 1 pixel trigger is extremely robust, even at noise levels much larger than expected. Another detector system that could conceivably suffer is the muon system, and there the problems would be close to the beam. Our muon trigger does not rely on the tubes near the beam pipe.

The items requested by the PAC can be broken down into four categories: (1) physics studies, (2) trigger/detector studies, (3) comparisons to other experiments, and (4) staging and commissioning scenarios.

\section{B.1 Physics Studies}

The PAC requested a thorough study of six processes that "have a particularly important role to play both in the extraction of fundamental physics and in the decision of whether the proposal will be approved." For three processes a full GEANT simulation was recommended $\left(B \rightarrow \rho \pi, B_{s} \rightarrow D_{s} K\right.$, and $\left.B \rightarrow D^{*} \rho\right)$ and for the other three $\left(B \rightarrow J / \psi K_{s}, B^{-} \rightarrow D^{0} K^{-}\right.$, and $\left.B_{s} \rightarrow D_{s} \pi\right)$ a thorough study using MCFast was thought to suffice.

The following elements were to be included in each study:

- Assumptions of a luminosity of $2 \times 10^{32} \mathrm{~cm}^{-2} \mathrm{~s}^{-1}$ and a running time of $10^{7} \mathrm{sec} /$ year.

- Detailed and realistic simulations, including both physics and beam related backgrounds, secondary interactions and decays, all significant environmental effects such as multiple interactions, and detector/electronics noise.

Part III of this proposal discusses the studies we have made of the six requested modes. Each includes the above elements. In particular, we have used BTeVGeant (described in Section 12.1) for modes where a full GEANT simulation was requested. A summary of each is given below, along with pointers to where in the proposal one can find detailed descriptions.

\section{B.1.1 $B \rightarrow \rho \pi$ (BTeVGeant study)}

The PAC noted that this was a mode for which we would be expected to do substantially better than LHC-b. They pointed out that in our studies for the PTDR we did not include backgrounds, and requested "a convincing statement of the uncertainty in $\sin (2 \alpha)$ which could be expected from BTeV."

This study is described in detail in Section 16.5, and is summarized again in Part IV. We expect to reconstruct a factor of 7 more $B \rightarrow \rho \pi$ decays than LHC-b with 5 times better signal-to-background (see Part IV).

The reconstruction efficiencies for $B \rightarrow \rho \pi$ and backgrounds were studied using a full GEANT simulation (BTeVGeant). All signal and background samples were generated with a mean of two interactions per crossing. For the backgrounds, this required generating $4.5 \times 10^{6} b \bar{b}$ events. Almost $90 \%$ of the time spent in generating these events is in the electromagnetic calorimeter simulation. 
We expect to have $\sim 1000$ effective flavor tagged $\rho^{ \pm} \pi^{\mp}$ events and $\sim 150 \rho^{0} \pi^{0}$ per year with signal-to-background ratios of approximately $4: 1$ and 1:3, respectively. We expect to continue our Monte Carlo production in order to build up a large enough sample of background events to study how to improve this. We have not done a full simulation of our sensitivity to $\alpha$. Final results will depend on several unknown quantities including the branching ratio for $\rho^{0} \pi^{0}$ and the ratio of tree to penguin amplitudes. Analysis by Snyder and Quinn [1] showed that with 2,000 background free events they always found a solution for $\alpha$ and the accuracy was in the range of $5-6^{\circ}$. We can collect these events in $2 \times 10^{7}$ seconds, but we will have some background. Furthermore, Quinn and Silva have proposed using non-flavor tagged rates as input that should improve the accuracy of the $\alpha$ determination [2].

\section{B.1.2 $B_{s} \rightarrow D_{s} K$ (BTeVGeant study)}

The PAC pointed out that since this final state must be distinguished from $D_{s} \pi$, excellent particle identification is crucial. They requested that we study this mode using a more detailed simulation of our $K / \pi$ separation so that a more realistic assessment can be made.

We have presented this study in Section 16.4.1. We used a detailed simulation of the $\mathrm{RICH}$ detector described in Chapter 6 to study the efficiency of the signal vs. efficiency of the background from misidentified pions. We expect that $B_{s} \rightarrow D_{s} \pi$ will be the largest source of background (although we investigated others) and estimate a signal-to-background level of $\sim 7$. Using the estimates of branching fractions given by Aleksan et al. [3], we expect to have about 13,100 reconstructed events per year. This will result in a measurement error on $\gamma$ of about $7^{\circ}$.

We note that we expect to reconstruct $70 \%$ more of these decays than LHC-b at the same signal-to-background (see Part IV).

\section{B.1.3 $B \rightarrow D^{*} \rho$ (BTeVGeant study)}

We were asked to fully investigate the decay $B \rightarrow D^{*} \rho$ as a benchmark of our ability to reconstruct modes with $\pi^{0}$ 's. The results of this study, which used BTeVGeant, are presented in Section 16.3 and compared to the CLEO result for this decay.

We find that the signal to background expected in BTeV compares very favorably with that obtained by CLEO, and is expected to improve simply by optimizing the cuts after the backgrounds are studied in detail. The event yield per year is more than 230 times higher than that expected from each of the $e^{+} e^{-} B$-factories.

This analysis demonstrates BTeV's ability to study states with $\gamma^{\prime}$ s and $\pi^{0}$ s. We have shown that even with a complicated underlying event and accompanying minimum bias events, we can successfully reconstruct the very low energy photons from these decays and can suppress the combinatoric background. 


\section{B.1.4 $B \rightarrow J / \psi K_{s}$ (MCFast study)}

The PTDR quoted a sensitivity to $\sin (2 \beta)$ based on a time integrated measurement and stated that a time dependent measurement would be more sensitive. The PAC asked that the time dependent sensitivity be determined, and asked that we make a more detailed study of tagging efficiencies.

The tagging study is presented in Chapter 15. The sum of all our tagging efficiencies is $15 \%$ but there are correlations. Our initial attempt to account for these correlations gives us a tagging efficiency of $9 \%$ but there are known deficiencies in the way we have handled events with more than one tag. As a result, we have continued to use the $10 \%$ we used in the PTDR but expect to do better when we complete our study.

The time dependent determination of $\sin (2 \beta)$ from $B \rightarrow J / \psi K_{s}$ is presented in Section 16.2. We find that in one year of running our uncertainty on $\sin (2 \beta)$ is 0.025 . As expected from analytic calculations, this is $20 \%$ better than the time integrated method.

\section{B.1.5 $\quad B^{-} \rightarrow \overline{D^{0}} K^{-}$(MCFast study)}

In the PTDR a study of this mode was presented without a discussion of backgrounds. The committee requested a complete analysis in order to judge BTeV's sensitivity in this mode.

- It was found (see Section 16.4.2) that only background arising from real $D^{0}$ 's need be considered, and that $b \bar{b}$ events were the major contributor (as opposed to $c \bar{c}$ ). The expected signal-to-background ratio for the $(K \pi) K^{-}$mode is 1:1. The signal-to-background for the $(K K) K^{-}$mode is better.

We expect to reconstruct about $300 B^{ \pm} \rightarrow(K \pi) K^{ \pm}$and $2,000 B^{ \pm} \rightarrow(K K) K^{ \pm}$per year at the design luminosity of $2 \times 10^{32} \mathrm{~cm}^{-2} \mathrm{~s}^{-1}$. With this number of events, $\gamma$ can be measured to $\pm 10^{\circ}$ for most values of $\gamma$ and strong phase shifts.

\section{B.1.6 $x_{s}$ from $B_{s} \rightarrow D_{s} \pi$ (BTeVGeant study)}

The PAC pointed out that the measurement of $x_{s}$ was useful as a benchmark for assessing the experiment's ability to integrate its capabilities in proper-time resolution, tagging, and particle identification, as well as its overall sensitivity to $B_{s}$ physics.

As described in Section $16.7, \mathrm{BTeV}$ is capable of observing all $x_{s}$ values less than 75 in one year of running. The improvement since the PTDR comes from two sources: there is an overall improvement which comes from using the full tagging power of BTeV; there is an additional improvement at large values of $x_{s}$ which comes from the improved resolution on the proper decay time, which was achieved by reducing the amount of material in the pixel detectors. 


\section{B.2 Trigger/Detector Studies}

We were asked to present a "clear and convincing case that all major detector systems are realizable and that the associated budgets are justified." The budget for the BTeV detector is justified in Part V of this proposal and supporting documents (the BTeV Work Breakdown Structure document). The first half of this request (all systems are realizable) is the subject of Part II and Appendix A of this proposal. We believe we have provided a convincing case.

The committee also raised specific questions about (1) the Vertex Trigger, (2) Pixel R\&D, (3) EMCal, (4) Particle ID, (5) Muon Trigger, and (6) R\&D Issues. These are addressed below. The committee requested that the following be included:

- Detailed and realistic simulations, including both physics and beam related backgrounds, secondary interactions and decays, all significant environmental effects such as multiple interactions, and detector/electronics noise.

- Clear and quantitative discussions of the trade-off between cost and performance for each detector subsystem, whenever substantial cost differences are involved.

- Studies should show how much the physics reach of the experiment is degraded if various components of the detector do not meet their design specification.

These have been included. The last two items are addressed in separate subsections below.

\section{B.2.1 Vertex Trigger}

The PAC commented that the vertex trigger is very ambitious, and that a convincing case based on careful studies was needed. They requested that we study the effects of non-perfect pattern recognition, beam misalignments and noise.

A more detailed discussion of this subject is given in Chapter 14. The results are summarized here.

The Level 1 vertex trigger has been extensively modified since the PTDR. This trigger is designed to work with the redesigned pixel system, which has less material than the PTDR system due to a reduction in the number of pixel planes per tracking station (two instead of three, see Chapter 4).

Our simulations are now significantly more realistic. They use GEANT (BTeVGeant) which includes effects from hadronic interactions, photon conversions, decays in flight, and delta rays. We introduce non-Gaussian tails to the hit distributions which are motivated by data from our 1999 beam tests. Our trigger simulation works at the "hit level" and therefore performs full pattern recognition. All studies are performed with an average of two interactions per beam crossing (the expected amount). One concern expressed by the PAC was that the doublet configuration would require too much time and swamp the trigger. The solution to this problem is to restrict the pattern recognition to a subset of pixel clusters to reduce the combinatorics. 
We find that the trigger performance is at least as good as that described in the PTDR. We desire a rejection for minimum bias events of a factor of 100. Selecting Level 1 trigger "cuts" to give this level of rejection, we have looked at the trigger efficiency for several modes (see Table 14.1, Chapter 14) and find that they are generally much better than $50 \%$. One mode $\left(B_{s} \rightarrow D_{s}^{+} K^{-}\right)$has an expected efficiency of $74 \%$.

We have found that this performance is exceptionally robust with respect to pixel inefficiencies and noise. Even adding 40 times the expected noise, we find only a slight decrease in trigger efficiency and a slight decrease in minimum bias rejection (see Figure 14.5 of Chapter 14). Pixel inefficiency does reduce trigger efficiency (Figure 14.5 again) but not dramatically.

We find minimal sensitivity to multiple interactions per event. If the two interactions are very close to each other, the trigger does get confused a small fraction of the time, but the effect on the trigger rate is insignificant (see Figure 14.6 of Chapter 14).

Finally, since we reconstruct full vertices and never rely on a beam constraint, beam misalignment is not a problem.

\section{B.2.2 Pixel R\&D}

The PAC commended BTeV on "impressive progress" made on the pixel system in 1998-99. This progress has continued in 1999-2000. In a beam test, we have demonstrated that $50 \mu \mathrm{m}$ $\times 400 \mu \mathrm{m}$ pixels can provide the precise position measurements required by BTeV (details are provided in Appendix A). Moreover, we have verified that our pixel simulation program, also described in Appendix $\mathrm{A}$, provides an accurate representation of the performance of real devices. Appendix A also describes our ongoing R\&D on RF shielding and very recent results on the radiation hardness of FPIX readout circuits implemented in $0.25 \mu \mathrm{m}$ CMOS.

The PAC encouraged $\mathrm{BTeV}$ to evaluate pixel detector designs using a smaller number of sensors to reduce the cost and complexity of the system. As stated above, our design now has two pixel planes per station, instead of three. Moreover, the design concept of a "shingled" support and cooling structure allows two planes of pixel detectors to be held on

a single support. Together, these changes represent a significant reduction in the amount of material in the pixel detector, and a reduction in both the channel count and the system complexity.

The PAC noted that we were behind the timeline we presented in 1998, which called for us 1) to choose the sensor material and implant type by June, 1999, and 2) to have final sensor specifications by May, 2000. We have now made the decision that the pixel detector will use sensors fabricated from low-resistivity oxygenated n-type silicon, with n-implant pixels. We have not yet finalized our sensor specifications, but we expect to do so in the next year.

The PAC noted the progress that we made in 1998-99 towards establishing a reliable bump bonding capability, and we are continuing to make progress in this regard. The most notable recent development is the promising results we have obtained in tests of circuits bonded by NCMC using fluxless solder bumps. 
The PAC stated that "a major decision remains as to which rad-hard" technology will be used for the pixel readout chip. In December, 1998, we made the decision to implement the third generation FPIX readout chip (FPIX2) in a commercial $0.25 \mu \mathrm{m}$ CMOS process, using guard rings and enclosed-geometry transistors for radiation hardness. Our recent radiation damage test verifies that this was a good decision. We expect to complete the FPIX2 design in Fall, 2000, and have devices in hand by early 2001. We expect this device will be radiation hard and will meet all of our pixel readout requirements.

The PAC stated that it would like to understand better the extent to which the FPIX1 readout chip met BTeV's requirements, and what types of improvements were planned. A fully functional, radiation hard, FPIX1 would have met all of BTeV's requirements. However, in addition to being radiation hard, FPIX2 will be superior to FPIX1 in the following ways:

- A 3-bit FADC, instead of 2-bit, will provide better sensor performance monitoring ability, as well as excellent charge-sharing information.

- Smaller discriminator threshold dispersion will allow a lower threshold to be used, probably under $2000 \mathrm{e}^{-}$. This will be especially important for the readout of radiation damaged sensors, which may yield a smaller signal than undamaged sensors.

- Better leakage current compensation will allow FPIX2 to be used to read out heavily damaged sensors without having to.adjust the amplifier feedback current.

- Simplified end-of-column logic will increase the readout bandwidth of FPIX2 by approximately a factor of two over FPIX1.

- An increase in token passing speed made possible by the use of smaller feature size transistors will allow even higher readout bandwidth, or columns containing more than 160 rows of pixels, or both.

- A significantly reduced I/O pin count will allow a less complex high density interconnect to be used.

As is described briefly in Chapter 4, progress is also being made on the development of a low-mass integrated cooling and mechanical support structure. We have received, and are thermally testing, a first "fuzzy carbon" prototype. By the end of this summer, we expect to receive two new prototypes that can be used in tests of sensor modules.

In general, our R\&D focus is shifting from the level of individual components and technologies to the system level. The PAC encouraged us to "push towards a system prototype that would test not only the electrical performance, but also the mechanical and thermal properties." We agree that this is a high priority and we intend to mount such a test as soon as possible. In addition, our R\&D plan, and the cost estimate for this proposal, both anticipate a " $3 \%$ " system test, followed by a " $10 \%$ " system test. Assuming that these tests can be done in $\mathrm{C} 0$, we expect to include as part of the tests an RF shielding solution that we will have verified using the beam simulator described in Appendix A. We believe that these tests will allow us to set achievable goals for the pixel detector, and to formulate a detailed and reliable construction plan. 


\section{B.2.3 EMCal}

The PAC requested comprehensive studies of $\gamma$ and $\pi^{0}$ reconstruction using a full (GEANT) simulation of the electromagnetic calorimeter. They also noted that the difficulty in obtaining the required quantities of $\mathrm{PbWO}_{4}$ is a serious concern.

We have implemented a full GEANT simulation of the electromagnetic calorimeter, and used it to study the efficiency and backgrounds for final states with $\pi^{0} \mathrm{~s}$. The results are presented in Chapter 16 and were summarized above (Sections B.1.1 and B.1.3). BTeV's ability to study states with $\gamma$ 's and $\pi^{0}$ 's using its $\mathrm{PbWO}_{4}$ electromagnetic calorimeter has been vindicated. Using full GEANT simulations with a complicated underlying event and accompanying minimum bias events, we can successfully reconstruct even the very low energy photons from these decays and can suppress the combinatoric background. We expect to reconstruct a factor of 7 more $B \rightarrow \rho \pi$ decays than LHC-b with 5 times better signal-tobackground (see Part IV).

As for the difficulty in obtaining the required quantities of $\mathrm{PbWO}_{4}$, we have begun disci. is ions with both Russian (the Bogoroditsk Techno Chemical Plant) and Chinese suppliers in Beijing and Shanghai. CMS production is scheduled to finish in 2005. We have visited the crystal production facilities in Russia and in China. Our Russian and Chinese collaborators have been most helpful in setting up these visits. The Russians have already been producing production crystals $(>6000)$ and are eager to have our business. They have supplied prices and possible schedules. The Chinese have not started production as of this writing, but they are very close to doing so. They are also very interested in BTeV production. Our collaborators at Shandong University are also interested and capable of producing $\mathrm{PbWO}_{4}$ crystals. We would like to initiate a startup program with them as soon as possible. Generally we think it important to have more than one supplier of crystals. Because of the open nature of the $\mathrm{BTeV}$ detector, crystal installation can proceed in place over a long period of time.

\section{B.2.4 RICH}

The PAC commented that high quality particle identification, primarily $K / \pi$ separation, is essential for BTeV's physics objectives, both for signals and tagging. They felt that the effort on this detector should be substantially strengthened over the next year.

The effort on the RICH has been strengthened over the past year. For more information see Chapter 6 and Appendix A. A more detailed simulation of this detector has also been implemented and used in the physics simulations performed for this proposal (see Chapter 16 and Section B.1.2 above).

\section{B.2.5 Muon Trigger}

The PAC expressed concern that only three (high segmentation) detector stations were planned for the muon system in each arm. Their primary concern was the resistance of the muon trigger to spurious tracks and backgrounds, effects which were not included in the studies performed for the PTDR. 
We have added a view to each detector station, so that there are now 4 views instead of 3. This adds redundancy and should help with the rejection of spurious hits and tracks. We have also performed a full BTeVGeant simulation for minimum bias events and $B \rightarrow J / \psi K_{s}$. Hadronic interactions, conversions, etc., are all included in the simulation. These simulations predict that the innermost proportional tubes in our system will have occupancies of $\sim 20 \%$, although the occupancy falls off rapidly $\left(\sim 1 / r^{2}\right)$ with distance from the beam.

Our simulations show that a realizable Level 1 trigger with a rejection factor for minimum bias events of $500-600$ is possible with high efficiency for modes of interest $(50 \%$ for $B \rightarrow$ $\left.J / \psi K_{s}\right)$. These simulations were generated with only 3 views per stations instead of 4 . The fourth view can only improve the results. More detailed information on this trigger simulation is given in Section 8.3.

\section{B.2.6 R\&D Issues}

After the April 2000 PAC meeting, the committee requested that we discuss major remaining $\mathrm{R} \& \mathrm{D}$ issues. All of the detector subsystems have detailed plans for future $\mathrm{R} \& \mathrm{D}$; these are discussed in Appendix A.

\section{B.2.7 Cost/Performance Trade-offs .}

The PAC requested that we discuss the trade-off between cost and performance "whenever substantial cost differences are involved."

In the RICH detector, the cost of photo-detectors is a significant fraction of the total cost. We have performed a study of efficiency for a specific state $(B \rightarrow \pi \pi)$ versus photo-detector coverage (i.e. the number of photo-detectors we have to buy). The results are presented in Figure 6.6 of Chapter 6.

In the EMCal, we have also made a compromise between coverage and cost. As discussed in Chapter 7 , the calorimeter cost can be parameterized roughly as

$$
T(\$)=700(\$) \times N_{c}+3,400,000(\$),
$$

where $N_{c}$ is the number of crystals and $T$ the total cost. The fixed costs represent mainly startup of crystal production, the crystal container, the light calibration pulsar and electronics development. Figure 7.1 of that chapter shows the efficiency as a function of calorimeter radius for the reaction $\bar{B}^{o} \rightarrow D^{*+} \rho^{-}$. Weighing this efficiency versus total cost, we adopted an outer radius of $160 \mathrm{~cm}$, corresponding to 23,700 crystals for both arms.

The pixel detector is the heart of the experiment. The number of pixel planes is defined by beam size. Preliminary studies indicate that there is minimal gain in performance from increasing the size of the pixel planes. These studies indicate that decreasing the size of the planes may be possible, but this is a complicated analysis. Because of the importance of this detector, we are taking a conservative approach and we have not decreased plane size. We intend to perform a detailed analysis, taking into account the important issues. If at that time there is strong evidence indicating minimal impact, we will reduce the plane size. 


\section{B.2.8 Degradation of Performance and Physics Reach}

The PAC commented: "Simulations should ... be used to analyze how much the physics reach of the experiment is degraded if various components of the detector do not meet design specification."

Section 16.9 of this proposal is devoted to a discussion of how degraded performance of detector subsystems would affect our physics reach. The section is reasonably short and we will not attempt to summarize it here.

\section{B.3 Comparisons to Other Experiments}

The PAC concluded:

- $\mathrm{BTeV}$ has a substantial physics reach beyond that of $e^{+} e^{-}$experiments at the $\Upsilon(4 \mathrm{~S})$.

- $\mathrm{BTeV}$ will likely have a physics reach substantially beyond that of CDF and D0, including their beyond-the-baseline upgrades.

- $\mathrm{BTeV}$ may be superior to LHC-b in three main areas: vertexing, triggering, and photon energy resolution.

Their main request was: "The Committee would like to see a clear and convincing demonstration that BTeV has a physics reach superior to LHC-b."

This issue is addressed in Part IV of this proposal. Comparing to our detailed simulations, we show that $\mathrm{BTeV}$ is better than LHC-b in 'high-priority' final states with all charged particles. For final states with $\gamma^{\prime}$ s, $\pi^{0}$ 's, $\eta^{\prime}$ 's or $\eta^{\prime \prime}$ s, BTeV is far superior. Furthermore, $\mathrm{BTeV}$ will write to tape 5 times more $b$ events than LHC-b, allowing for a wider range of physics studies. This is important because we cannot anticipate all the physics that will be interesting at the time $\mathrm{BTeV}$ actually runs, and new ideas and phenomena may appear or become relevant.

\section{B.4 Staging and Commissioning Scenarios}

The PAC has requested a staging and commissioning scenario for $\mathrm{BTeV}$ compatible with minimal luminosity reduction to CDF and Do.

A more detailed discussion of our plans for deployment is given in Chapter 11 of the proposal. We request the following:

- We would like to be installed with at least one arm operational and receive enough luminosity to make at least one measurement (such as $\sin (2 \beta), B_{s}$ mixing, and/or studies of $K^{*} \gamma$ ) before LHC turns on. 
- We would prefer to finish our installation and checkout of the first arm prior to installation of the second. Our physics program can begin as soon as the first arm is completed.

We assume that the various detector subsystems in the first arm can be installed individually as they become ready. As discussed in Chapter 11, this is possible because the detector is not monolithic but is made up of individually mounted and independent subsystems. Short term accelerator shutdowns could be used for this purpose.

As subsystems are installed, we will initially want short runs (at the end of stores, etc.) for checkout and commissioning studies. It would be acceptable to us if these runs were kept brief so that the net impact on delivered luminosity to CDF and D0 was minimal (a 1\% diminishment or so). A principle goal during this period will be to debug and commission the level 1 and 2 triggers and data acquisition system, which can't be tested in external beam tests. Some of this can be done with a wire that can be moved into the beam halo without disturbing the luminosity in the other two collision regions.

Once the full arm is installed we request a period during which we receive longer, high luminosity runs. This period will allow us to accomplish a limited physics program prior to LHC turn-on (as discussed above), and we anticipate this period will take roughly four months. 'BTeV believes that this running will have a huge impact on our ability to commission the detector and believes that the impact on CDF and D0 can be kept low so that their physics reach is not noticeably affected. A reasonable goal might be to make sure that the net effect on CDF and D0 during this period was no more than $10 \%$. 


\section{Bibliography}

[1] A. E. Snyder and H. R. Quinn, Phys. Rev. D 48 (1993) 2139.

[2] H. R. Quinn and J. P. Silva, "The Use of Early Data on $B \rightarrow \rho \pi$ Decays," hep$\mathrm{ph} / 0001290(2000)$.

[3] "Determining the CP-violating phase $\gamma$ ",

R. Aleksan, I. Dunietz, B. Kayser, Z. Phys C 54, 653-659 (1992) 